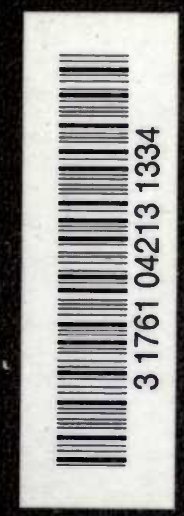



AN INTRODUCTION TO THE STRUCTURE AND REPRODUCTION OF PLANTS 


\section{AN INTRODUCTION TO THE STUDY OF PLANTS}

By F. E. Fritchi, D.Sc., Ph.D., F.L.S., and E. J. SalisbuRy, D.Sc., F.L.S. With over 250 Illustrations. Fourth Edition. Demy 8vo. $7 s$. $6 d$. net. This volume completely covers the London University Matriculation Syllabus.

"This book is one of a very small class, the kind that makes teachers wonder whatever their pupils did in the past without them."-Educational Times.

"Certainly one of the very best of the elementary textbooks of botany we have seen."-Journal of Education.

\section{ELEMENTARY STUDIES IN PLANT LIFE}

By F. E. FriTCH, D.Sc., Ph.D., F.L.S., and E. J. Salisbury, D.Sc., F.L.S. With over 150 Illustrations. Crown 8vo. Third Edition, 3s. $6 d$. net.

"It would not be easy to find a book better suited to the needs of junior students." - Nature.

"There is plenty of practical work, the style is easy and natural, the illustrations are good and numerous. Altogether this is one of the soundest and most attractive books for beginners we have seen."-School World.

LONDON : G. BELL AND SONS LTD.

York House, Portugal St., W.C. 2 
PLATE I

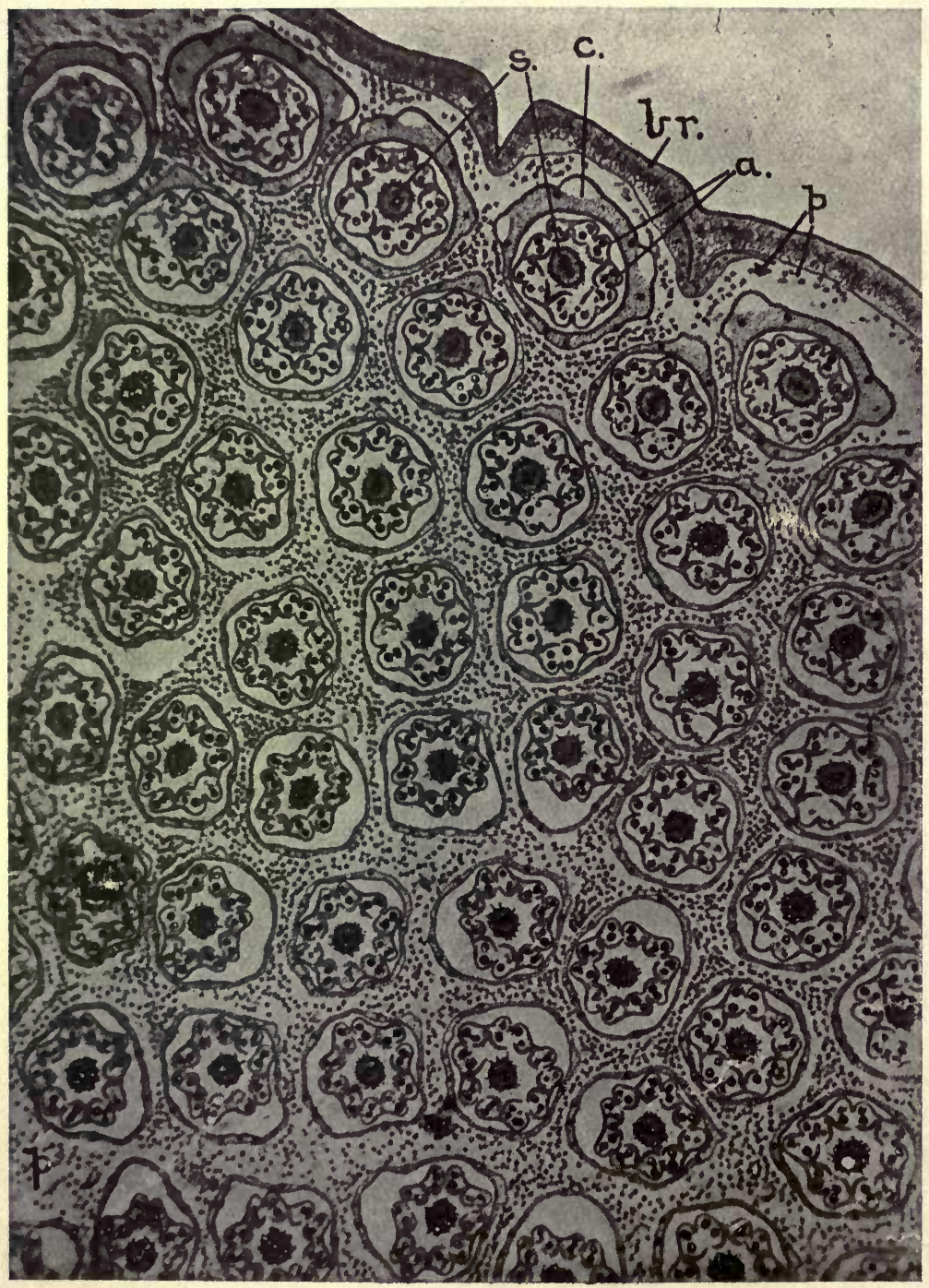

Fig. 210.-Photomicrograph of transverse section through the young capitulum of the Dandelion (Taraxacum). [Photo: E. J.S.] 


\section{AN INTRODUCTION TO THE STRUCTURE AND REPRODUCTION OF PLANTS}

BY

F. E. FRITCH, D.Sc., Рн.D., F.L.S. PROFESSOR OF BOTANY, EAST LONDON COLLEGE, UNIVERSITY OF LONDON

AND

E. J. SALISBURY, D.Sc., F.L.S. LECTURER IN BOTANY AND FELLOW OF UNIVERSITY COLLEGE, UNIVERSITY OF LONDON
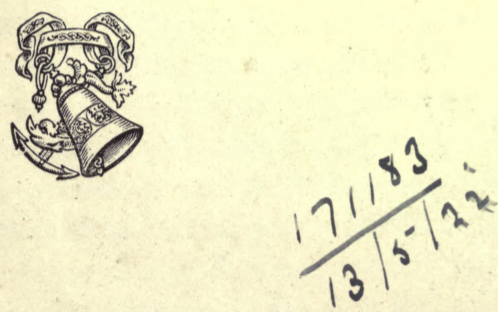

LONDON

G. BELL AND SONS LTD. 
First Published, 1920

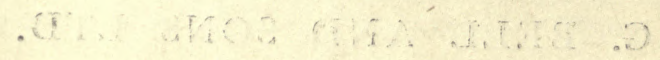

$$
\begin{aligned}
& \text { C.: I }
\end{aligned}
$$




\section{P REFACE}

THis volume has been prepared in response to the demand for a sequel to our Introduction to the Study of Plants, from which the minute structure and details of life-history, that require the use of a microscope for their proper comprehension, were purposely omitted.

Whilst the present volume is complete in itself, frequent references to the earlier book are included, and the two together form a comprehensive introduction to the science of Botany. Not only is the syllabus for all Higher School Examinations amply covered, but the two volumes will also be found to meet the needs of the first-year student at the Universities. Like its predecessor, however, the present work was compiled for those who really desire to learn something of the rôle of plants in nature, of their structure and of their mode of life, rather than merely to furnish another examination textbook.

With the aim of giving a better survey of the Vegetable Kingdom and presenting a more balanced concept, we have abandoned the study of isolated types in favour of a more general account, indicating the range of form and reproductive methods within each group. This has not resulted in an undue increase in the subject-matter, since we have omitted much developmental detail which has little educational value or significance, except for the specialist. With the object of widening the outlook of the student on the two aspects of botanical science treated in this volume, special chapters have been included dealing with ecological anatomy, and variation and heredity. Moreover, we have introduced such physiology as is more appropriately considered in relation to microscopic structure.

Features, whether of plant-anatomy or plant-chemistry, which are of commercial importance are emphasised throughout, and it is hoped that this may serve to combat the frequent ignorance 
of botanical students with respect to the economic aspects of their subject. We have again been at pains to illustrate the subjectmatter, as far as possible, by reference to British or commonly cultivated plants, especially in that part of the book dealing with the different groups.

The index has been compiled with the greatest care, and includes numerous subject-headings with a view to rendering the large number of facts dealt with of the widest possible use. Some twothirds of the illustrations are original, and have been especially prepared for this book. For a number of these we are indebted to Mrs. F. E. Fritch, who is responsible for those initialled H. F. ; the remainder are the work of one or other of the authors. The source of all illustrations that have been copied is acknowledged in the figure descriptions. For the use of the photograph illustrating Fig. 222 we are greatly indebted to Prof. Bateson, F.R.S., and Miss Pellehew. We also have to thank Mr. W. B. Johnson for the photograph illustrating Fig. 200, Prof. F. W. Oliver, F.R.S., for Figs I88-rgr, Mr. E. M. Cutting, M.A., for Figs. I36-I39, the proprietors of the Indiarubber Journal (through the instrumentality of Mr. H. Wright, A.R.C.S.) for Fig. 79, and Dr. H. C. I. Gwynne-Vaughan for permission to reproduce Figs. IO-I2.

We are glad to have this opportunity of expressing our gratitude to the following specialists, who have kindly read through various sections of the manuscript and offered criticisms and suggestions : Prof. A. E. Boycott, F.R.S. (the section on Bacteria), Mr. E. M. Cutting, M.A. (the chapter on Fungi), Prof. F. W. Oliver, F.R.S. (the Cycads and Pteridosperms), and Dr. F. G. Pope (chemical sections). We are also indebted to the works of numerous authorities on special branches of the subject, and, among these, would especially mention that of Haas and Hill on The.Chemistry of Plant Products, which we have repeatedly consulted in connection with the chemical matter.

LoNDON,

$$
\begin{aligned}
& \text { F. E. F. } \\
& \text { E. J. S. }
\end{aligned}
$$

May 1920. 


\section{O N T E N T S}

\section{PART I.-THE ANATOMY OF PLANTS}

CHAPTER ? PAGE

I. The Structure and Physiology of the PlantCELL • • . . . . . . . .

II. Growing Points and Cell-division . . . i i6

III. The Differentiation of Plant-cells · : 27

iV. The Non-living Contents of Cells. . . 40

V. Cell-contents (By-Products, etc.) . . . 58

VI. The Structure of Roots • . . . 65

VII. The Structure of the Young Stem • . 76

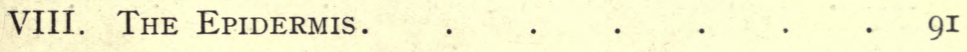

IX. The Structure of the Leaf • . . . . iO7

X. Secondary Thickening • • . . . . Iif

Xi. Cork-formation, Etc. $\quad \cdot \quad \cdot \quad \cdot \quad \cdot \quad \cdot \quad$ I35

XiI. Secretory Organs • .

XiII. Anatomy in Relation to the Habitat • ${ }^{5} 8$ 
PART II.-THE LIFE-HISTORIES AND REPRODUCTION OF PLANTS

CHAPTER

XIV. Simple Vegetable Organisms

PAGE

XV. Pond-scums, Seaweeds, etc. (Algete) $/ \cdot$ I95

XVI. Reproduction among the Alge . V. 2 I2

XVII. The Structure and Reproduction of the FUNGI . . . . . . . 230

XVIII. Physiology of Fungi, Lichens, Bacteria . 252 XIX. Liverworts AND Mosses f. : • • 269 XX. FERNS $\cdot \cdot \cdot \cdot \cdot \cdot 289$

XXI. Horsetalls and Clubmosses . . . . 3Io

XXII. The Cycads and Certain Extinct Seed-bearing

Plants • . . . . . . . 322

XXIII. The Conifers _. $\quad . \quad . \quad . \quad . \quad 334$

XXIV. The Angiosperms . . • . 354

XXV. Heredity and Evolution $V$. • • • 374

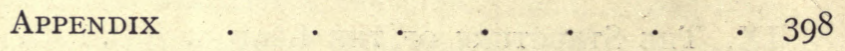

INDEX $\quad . \quad . \quad . \quad . \quad \ldots \quad . \quad .4 \mathrm{II}$ 


\title{
THE
}

\section{STRUCTURE AND RE- PRODUCTION OF PLANTS}

\author{
PART I \\ THE ANATOMY OF PLANTS
}

\section{CHAPTER I}

\section{The Structure and Physiology of the Plant-cell}

EVERY living organism, whether animal or plant, is composed of one or more minute units called cells, and this applies even to those forms of life which are so small that they can only be seen with the help of a microscope. The green powdery covering so often present on tree-trunks and palings consists of multitudes of single-celled plants (Pleurococcus, Fig. I02, p. I92), whilst millions of cells together form the body of a garden weed or tree. Organisms of the latter type are described as multicellular, whilst those consisting of a single cell are termed unicellulur.

The structure of a cell will best be realised if a typical example is studied, such as that obtained by stripping off the skin (or epidermis) from the inner surface of an Onion-scale. If a small portion of this be mounted in water, and examined under the low power of a microscope (see Appendix I), it will be seen to consist of a large number of oblong cells connected together without any intervening spaces to form a tissue (Fig. I, A). The network of delicate lines separating the individual units is constituted by the cell-walls, which are all joined to one another. In each cell a colourless, somewhat dense granular substance (the cytoplasm, Fig. I, B, Cy.) is visible, particularly around the 
edge, whilst near the centre or against one side is situated a rounded shining body, the nucleus (Fig. I, B, N).

These cells consist, then, of three parts, the cell-wall (Fig. I, $\mathrm{B}, C . W$.$) , which is not living and is merely a protective case,$ the cytoplasm, and the nucleus; the last two constitute the

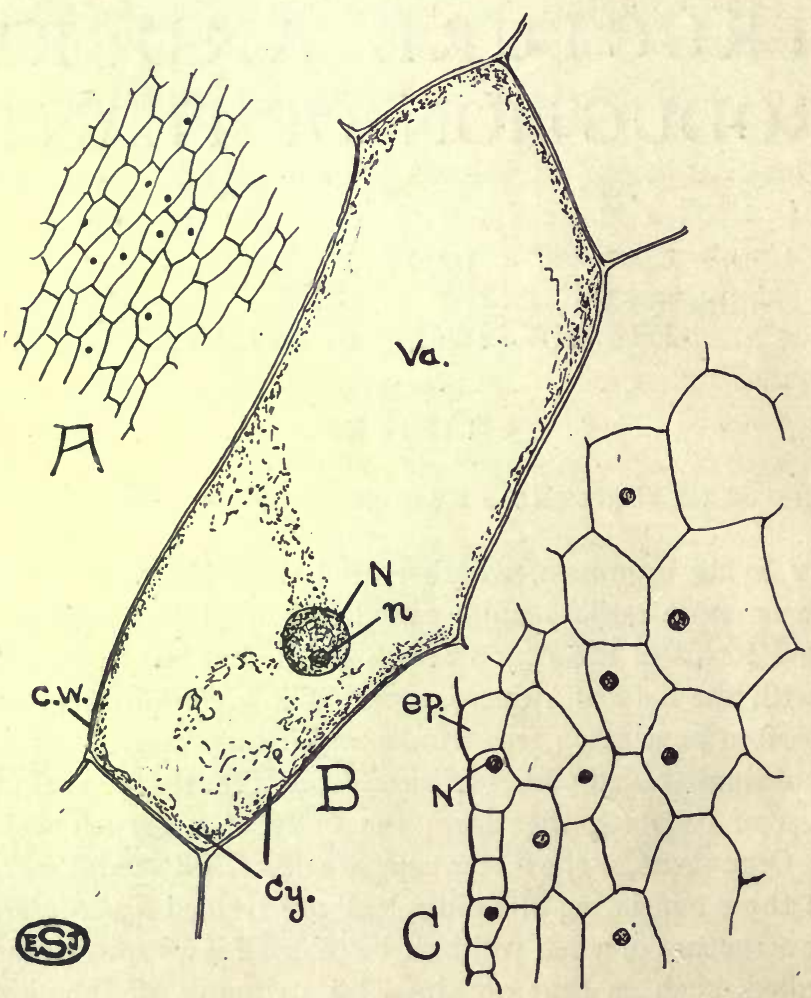

FIG. 1.-Epiderm is of Onion bulb-scale. A, small portion in surface view. $\mathrm{B}$, a single cell much enlarged. $\mathrm{C}$, a scale in vertical section. In $\mathrm{A}$ and $\mathrm{C}$ the nuclei are shown black. $C y$., cytoplasm; $C . W$., cellwall ; $e p$. , epidermis ; $N$, nucleus ; $n$, nuc'eolus ; $V a$., vacuole.

living part or protoplast, composed of protoplasm. The protoplasm is probably a very complex mixture.of proteins, fatty bodies, etc., composed mainly of the elements carbon, hydrogen, oxygen, nitrogen, and sulphur (often together with phosphorus, especially in the nucleus). It has somewhat the consistency 
of the white of an unboiled egg, and usually contains large numbers of small granules which are partly of the nature of food-bodies and partly waste-products. The fact that not all, though some, of the properties of living protoplasm are exhibited by cells which are ground to pulp suggests that the particular characteristics of the cytoplasm are not entirely an outcome of its chemical constitution, but are to some extent a consequence of its ultimate structure. The nucleus is the most important part of the protoplast, a fact which will become more apparent in later chapters (Part II), when the reproductive processes of plants are studied. It appears to be essential for many of the vital activities of the cell. A demonstration of this is afforded by the fact that, if the unicellular animal Stentor is broken into pieces, the fragments which contain portions of the nucleus develop into new individuals, whilst the others, after a short time, perish. Moreover, the nucleus is generally found in that part of the cell which is in process of active growth, e.g. at the tip of a growing root-hair.

The structure of the Onion-cell can be more clearly distinguished if the strip of epidermis be stained with a drop of iodine (see Appendix II), and a single cell examined under the highpower objective. The protoplasm will have assumed a yellowish tint, whilst the nucleus appears pale brown. This colour reaction of the protoplast is one characteristic of proteins generally. 'The nucleus (Fig. I, B, N) is bounded by a thin nuclear membrane, whilst within it one or two small round bodies, the nucleoli $(n)$, are now plainly visible, since they are stained more deeply than the rest. The cytoplasm does not completely fill the cell, but there is a large space or vacuole ( $V a$.) occupying the greater part of the central region; this vacuole, apparently empty, is in reality filled with a watery fluid, the cell-sap. Close observation shows that the cytoplasm is'not evenly granular throughout, but that there is a very thin clear layer immediately within the cell-wall. This layer is a result of physical forces operating at the surface of the cytoplasm, and a similar clear layer can be detected at the surface abutting on the vacuole. These layers are spoken of as the plasmatic membranes.

If another strip of Onion epidermis be mounted in concentrated sulphuric acid, the cell-walls will swell and disappear. 
Subsequent addition of iodine gives a blue colouration to the dissolving walls, a reaction characteristic of cellulose, of which most thin cell-membranes largely consist.

It must be realised that the cells just examined, like all plant-cells, are developed in three dimensions, a fact which can be verified by cutting a section transversely across the Onionscale, when the epidermal cells will appear as flat tabular structures (Fig. I, C).

As a second example, one of the cells forming the purple hairs (Fig. 2, A) on the stamens of the Common Spiderwort (Tradescantia) may be examined. ${ }^{1}$ These show the same structure as those of the Onion, but the cell-sap is here of a purple colour. The nucleus, surrounded by a small mass of cytoplasm, is not uncommonly suspended in the middle of the vacuole, the enveloping cytoplasm being connected with that lining the cell-wall by a number of protoplasmic strands (Fig. 2, B). If the granules in these strands are closely observed, they will be seen to exhibit a continuous streaming movement which can be accelerated by slight warming, but ceases on the addition of a poison (e.g. alcohol), whereby the cell is killed. A temporary cessation of the movement can be brought about by mounting the cells in water to which a trace of some anæsthetic (e.g. chloroform) has been added. On returning the hairs to ordinary water, however, streaming of the granules is soon resumed. A similar effect is obtained if the cells are mounted in water which has been boiled and subsequently cooled, the result showing the necessity of oxygen for the performance of such movements. The movement is really due to a flowing of the cytoplasm, and this phenomenon betrays the fluid consistency of the latter. No movement can be recognised, however, in the plasmatic membranes, which are therefore probably of a denser character.

All living plant-cells display the features above described, but in many cases other structures are present, and of these the commonest are bodies known as plastids. In those cells of the plant which are exposed to the light the plastids become the depositories of the green pigment, or chlorophyll, and are then

1 If material of the Spiderwort is not available, a good substitute is furnished by the unicellular hairs lining the inner surface of the corollatube of the White Dead-nettle, in which,however, the cell-sap is uncoloured. 
known as chloroplasts. On examining a leaf of the Canadian Pondweed (Elodea canadensis) under the high power, each of the more or less rectangular cells will be found to contain a number of chloroplasts, which will be seen from one of two aspects (Fig. 3). Some, situated along the sides of the cell, are viewed in profile and appear as flattened ellipses (O.S.),

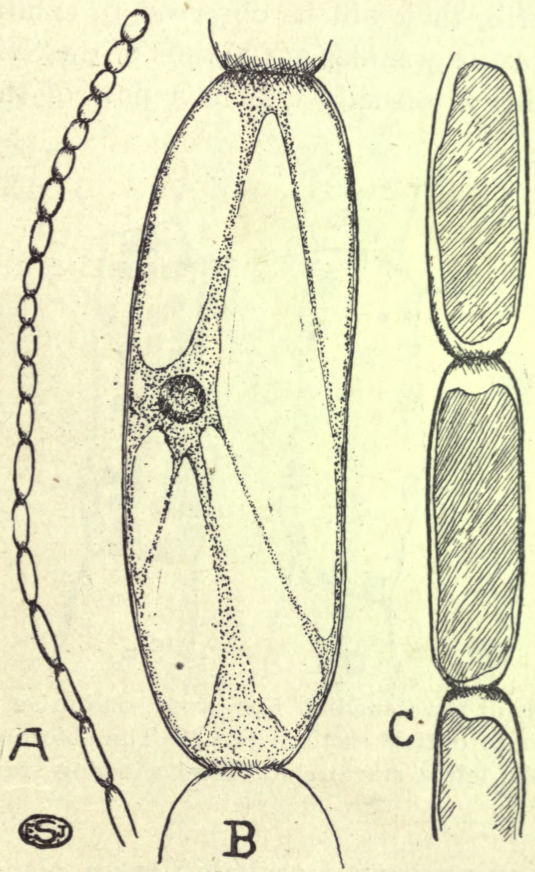

FIG. 2.- Staminal hairs of the Spiderwort (Tradescantia). A, a single hair ; B, a single cell showing the nucleus and strands of cytoplasm passing across the vacuole; C, a hair plasmolysed with sea-water.

whilst others lying against the upper or lower walls, and consequently seen from the surface, present a round or oval form $(S u$.). Each chloroplast is thus a solid body which has more or less the shape of a biconvex lens.

If attention be centred on a single cell, the chloroplasts lying against the upper face can be brought clearly into view by turning, the fine adjustment; on focussing to a lower level, 
however, another layer of chloroplasts appears belenging to the same cell. We can thus liken each cell to an oblong box lined along the whole of the inner surface with a thick viscid fluid (the cytoplasm) in which are embedded the chloroplasts, the cavity of the box representing the vacuole (Fig. 3, Va.) with its cell-sap.

By watching the chloroplasts, particularly in the elongated cells of the midrib, they will be observed to exhibit movement similar to that of the granules in the cells of the Spiderwort, and here as there this is actually due to a flow of the cytoplasm.

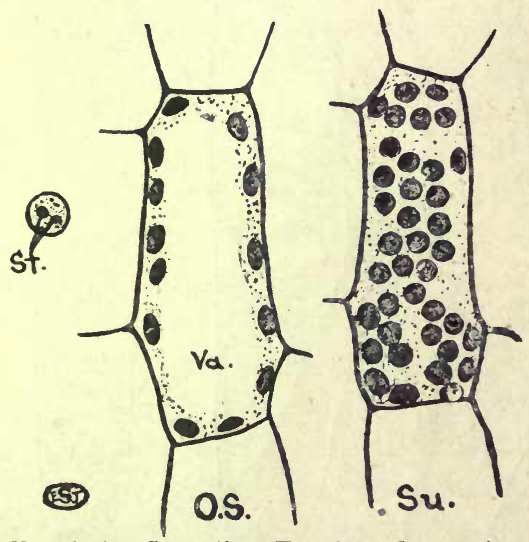

Fig. 3.-Two cells of the Canadian Pondweed, one in surface view (Su.), and the other in optical section (O.S.). The chloroplasts are shown black. At the left a single chloroplast showing starch grains (St.). $V a$., vacuole.

Such a streaming movement probably takes place in the cytoplasm of all living cells, but in many cases it is so slow that it cannot be demonstrated. By its means food-substances, etc., are more quickly distributed from one part of the cell to the other than by mere diffusion.

When leaves of the Canadian Pondweed, or those of other plants, are kept in spirit for some little time, all the chlorophyll is removed in solution. The chloroplasts, though now colourless, have undergone no change of shape, and are therefore merely specialised parts of the cytoplasm which held the chlorophyll.

The green cells of all the higher plants contain numerous 
chloroplasts, essentially similar in form to those just studied. In the simple types of plant-life (e.g. among the Algæ), however, the cells are often provided with but a single chloroplast or a limited number of them, and these are frequently of a much more elaborate pattern. Spirogyra, which is exceedingly common in stagnant water, furnishes an extreme instance. The plant

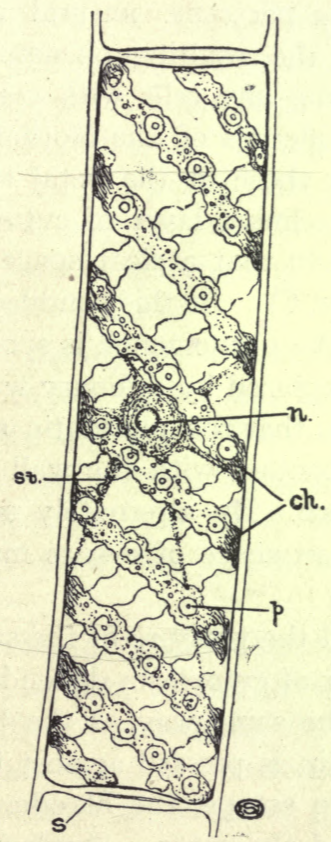

Fig. 4.- Single cell of a Spirogyra filament showing the spiral chloroplast $(c h$.$) , the pyrenoids (p.) surrounded by starch, and the nucleus (n$. supported by cytoplasmic strands (st.). $S$, separating wall between adjacent cells.

here consists of a single thread or filament composed of cylindrical cells joined end to end. According to the species, each cell contains, apart from the cytoplasm and nucleus, one or more chloroplasts in the shape of green spiral bands, usually with a jagged edge (Fig. 4, ch.). These spiral chloroplasts are here, again, situated in the lining layer of cytoplasm. At intervals bright bodies, consisting of protein and known as pyrenoids, are 
embedded within their substance (Fig. 4, p.); but these are more evident after treatment with iodine, whereupon they become blue, owing to the fact that each is surrounded by a layer of starch. Pyrenoids are not found in the chloroplasts of the higher plants, but are quite frequent amongst the Algæ (see p. 207).

Returning to the cells in the hairs of the Spiderwort, we will mount them in 2 per cent. natural or artificial sea-water (see Appendix III) ; the resulting phenomena could be equally well observed by using any cells with coloured sap, e.g. those forming the lower epidermis of the Mother-of-Thousands (Saxifraga sarmentosa), or those in the petal of a Pæony. In the cells thus treated the lining layer of cytoplasm' has contracted away from the wall, so that a clear space is visible between it and the latter (Fig. 2, C). In this condition the cell is said to be plasmolysed, and the phenomenon is spoken of as plasmolysis. If the sea-water be replaced by ordinary water, the cell-contents increase in volume, so that the plasmatic membrane regains its original position in contact with the wall and the cell resumes its normal appearance. By alternately substituting sea-water and tap-water, this sequence of events may be repeatedly observed.

In the normal cell the pressure of the sap within the vacuole keeps the lining layer of cytoplasm distended and forced against the wall, in much the same way as the bladder of a football, when inflated with air, is pressed against the leather cover. If the air be allowed to escape, the bladder shrinks and a space is left between it and the cover. Similarly, the contraction of the protoplast of the cell, when surrounded by sea-water, must . be attributed to a decrease in volume of the cell-sap owing to leakage into the outside liquid. The recovery, when placed in water, obviously implies an increase in volume of the sap, and this can only be due to some of the water around having passed into it.

It is a well-known physical phenomenon (osmosis) that, when two solutions of different concentrations are separated by certain kinds of membranes termed "semi-permeable membranes" (which may be of a fluid consistency), there is a passage of water from the weaker solution to the stronger until both attain the same 
concentration. This is due to the fact that such membranes, whilst readily permeable to water, are for all practical purposes impermeable to the dissolved substances. The lining layer of protoplasm, or probably more correctly the plasmatic membranes, exhibit these features. Consequently, when cells are surrounded by solutions which are more concentrated than the cell-sap, water passes out and the protoplast contracts. On the other hand, when replaced in water, the cell-sap is the more concentrated solution, and the protoplast becomes distended until its further expansion, and further increase in the volume of the sap, is prevented by the cell-wall. In a healthy plant, supplied with sufficient water, all the living cells are thus distended to their utmost, that is to say they are turgid, a condition which plays an important part in maintaining the stability of herbaceous organs (cf. F. \& $\mathrm{S}^{1}$., p. I02).

These phenomena plainly show that the cytoplasmic lining is readily permeable to water, but not appreciably to the substances dissolved in the sap, nor to those in the solution around. If it were permeable, the concentration inside and outside the cell would rapidly become equal as a result of diffusion and plasmolysis could not occur, nor would turgescence of the cells be possible.

The plasmatic membranes exhibit their semi-permeable character, however, only so long as the protoplast remains alive ; hence after death both the substances dissolved in the cell-sap can pass outwards, and external solutions can diffuse inwards. If cells of the Spiderwort, etc., mounted in water, are killed by gently heating the slide over a spirit-lamp, the coloured sap will be found to escape into the surrounding liquid; moreover, it will be found impossible to bring about plasmolysis or to render such killed cells turgid. These results, apart from showing that the semi-permeable character of the cytoplasm is altered by death, demonstrate that the cell-wall is permeable to substances in solution. The permeability of the cytoplasm, when dead, to the cell-sap may be exhibited on a large scale by placing slices of Beetroot in hot water.

A moment's thought, however, will show that the plasmatic

1 I.e. Fritch and Salisbury, An Introduction to the Study of Plants, which in the subsequent pages will always be briefly referred to in this way. 
membranes cannot be completely impermeable, since most of the substances found dissolved in the soil-water can be detected within the plant. Moreover, plants will thrive for months or years in a water-culture solution, ${ }^{1}$ from which analysis shows that mineral salts have been absorbed (cf. also below, p. I5).

Were it not for the restraining influence of the wall, which is only slightly elastic, but possesses considerable strength, a plant-cell placed in pure water would increase in size until it became ruptured. This actually occurs when the root-hairs of salt-marsh plants are suddenly transferred to water, for these, like other marine plants, have a highly concentrated sap. Owing to the restriction on the dimensions of the cell, under normal circumstances, the intake of water is arrested when the wall has reached the limit of its stretching capacity. The more concentrated the sap the greater its attraction for water, and hence, when a cell is surrounded by water, ${ }^{2}$ the cytoplasm will be forced against the cell-wall with a pressure proportional to the strength of the sap. This pressure is spoken of as osmotic pressure, and in many plant-cells is very considerable, as is well illustrated by the following figures, which give the osmotic pressures in atmospheres in a few selected cases:

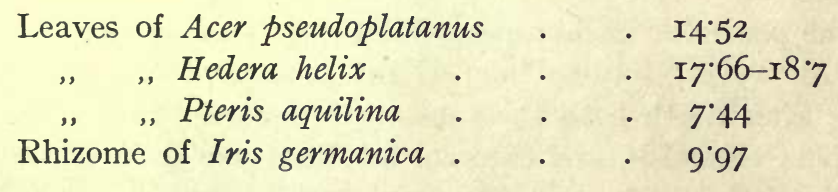

Owing to the small size of the cell, it is not possible to estimate the osmotic pressure of the sap directly, but an indirect measure is afforded by the strength of the solution necessary to bring about plasmolysis. By using artificially prepared semi-permeable membranes (see Appendix IV), the osmotic pressure of any given solution can be calculated in terms of atmospheric pressure. In

1 Cf., F. \& S., Fp. 125, 126, see footnote on p. 9.

2 Even when a cell forms one of a tissue, it will receive water from all sides as long as the water-supply is normal, since all parts of a healthy plant imbibe water (cf. p. 26). Until a cell has reached the limit of its stretching capacity, water will be withdrawn into its vacuole from any of the surrounding cells which are more turgid. 
general the osmotic strength of a solution depends upon the number of molecules it contains (though this does not apply to dilute solutions of many salts), so that when these are present in equal proportions, two solutions (e.g. of cane-sugar and grape-sugar) have the same osmotic pressure. Such equimolecular solutions are obtained by dissolving substances, in a litre of distilled water, in proportions equivalent to their molecular weights. If the number of grammes of the compound dissolved in a litre of water is equivalent to the molecular weight, we have a so-called molecular solution, briefly indicated by $M$. Solutions of other strengths are indicated as $0.5 M, 0.2 M$, etc. Since the weight of a substance depends on the number and mass of the atoms composing its molecule, a ro per cent. solution of a complex compound, such as inulin or dextrin, will contain fewer molecules than a ro per cent. solution of a simpler compound, such as grape-sugar or cane-sugar; moreover, cane-sugar, which has a higher molecular weight than grape-sugar, will exhibit a lower osmotic pressure than the latter when in solutions of the same percentage strength. These important facts are illustrated in the following table:

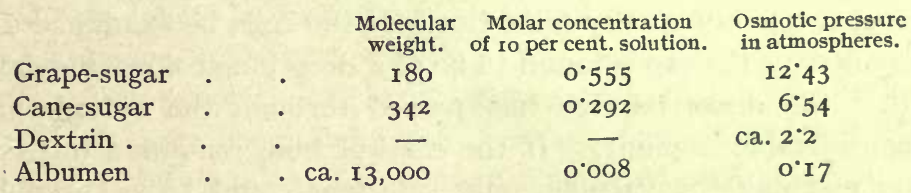

In order to determine the osmotic pressure of any cell, a solution of sea-water (see Appendix III), strong enough to bring about plasmolysis, is first obtained. We next proceed to find a slightly weaker solution such as will fail to plasmolyse the cell. By experimenting with a series of solutions of intermediate strengths, one can eventually be found which causes very little contraction of the protoplast, the plasmatic membrane only receding very slightly from the angles of the cell. The osmotic strength of the solution which produces this effect is somewhat stronger than that of the cell-sap, since, at first, the contraction of the protoplast is accompanied by shrinkage of the stretched cell-wall. The osmotic pressure of the sap may, however, be taken as approximately that of the solution which just fails to 
produce any visible plasmolysis (for equivalent pressures, see Appendix V). ${ }^{1}$

Another method, in which the average osmotic pressure of a whole tissue is determined, depends upon the curvatures consequent upon the different stretching capacities of the walls of the component cells. ${ }^{2}$ For this purpose one can make use of short lengths of the stalk of a Dandelion-inflorescence which are split lengthwise into four portions. When placed in water liquid is absorbed by the cells, but, since those towards the outside of the stem have thicker walls than the inner ones, the latter have a greater stretching capacity; as a consequence the strips curl up and form rings. A strength of sea-water that causes neither increase nor decrease in the curvature will have approximately the same osmotic pressure as the cell-sap. Stems of many herbaceous plants can be utilised in this way.

Attention has already been drawn to the fact that the protoplasm must be permeable to the compounds dissolved in the soil-water or in a water-culture solution. The permeability of the protoplasm can be demonstrated by placing young shoots of the Canadian Pondweed in a solution of methyl blue, so weak that it has but a very faint tint. If the cells be examined after some days the sap is found to be of a deep blue colour, indicating that the dissolved dye has passed through the cytoplasm in considerable amount. If the methyl blue remained unaltered on reaching the vacuole, only sufficient could have entered to bring about a concentration equivalent to that of the solution outside the cells. But the deep blue colour shows that the dye has accumulated within the sap, and this is due to the combination of the methyl blue with the tannin in the latter to form a substance to which the plasmatic membrane is impermeable. In this way the concentration of the dye which enters the cellsap is continually being reduced to a strength below that outside. As a consequence more and more methyl blue diffuses in, and thus the deep blue colour is gradually produced.

A. further demonstration of permeability of the cytoplasm is

1 Very accurate determinations of osmotic pressure are made by indirect means depending on the relation between osmotic pressure and the temperature at which a liquid (e.g. the expressed sap of a plant-organ) freezes.

2 Cf. F. \& S., pp. 103, 104, see footnote on p. 9. 
obtained when filaments of Spirogyra (and many other Algæ) are placed in a dilute ( $\mathrm{I}$ per cent.) solution of caffein. If the effect be observed under the microscope, a very fine greyish precipitate is seen to appear in the vacuole, which, as it accumulates, renders the cell more and more opaque. This precipitate is again a consequence of combination between the tannin in the cell-sap and the caffein.

Both examples illustrate a very important phenomenon, viz. that plant-cells can absorb and accumulate considerable quantities of various substances from very dilute solutions, provided that within the cell they are changed into some other form which does not readily pass through the plasmatic membrane. This fact is significant when it is remembered that many compounds are only present in the soil-water in very small amounts. The phenomena just discussed also explain the accumulation of food-reserves (e.g. insoluble starch) in large quantities in the cells of storage-organs. When these reserves are utilised, it is clear that they must be changed into a form which can diffuse to the growing organs, starch, for example, being transformed into sugar. This process can be simulated by placing leaves of the Canadian Pondweed, whose cell-sap has acquired a deep blue colour in the way above described, in a very dilute solution of citric acid ( $I$ per cent.). The latter passes into the cells and changes the blue pigment into a form capable of diffusing through the plasmatic membrane. Since its concentration inside the cell is greatly in excess of that outside, diffusion takes place and the sap gradually loses its deep blue colouration.

Although, in the numerous processes of diffusion that occur between the living cells of the plant, complex organic substances, analogous to methyl blue and caffein, are often involved, the mode of absorption of the simple inorganic compounds in the soil-water is of primary importance. It is a well-known fact that mineral salts bring about contraction of the protoplast of plant-cells (including root-hairs), when used singly and in a sufficiently concentrated solution. In recent years, however, the American botanist Osterhout has shown that many, perhaps all, of these cases of so-called plasmolysis are due, not to impermeability of the plasmatic membrane to the compound in question, 
- but to a poisonous or toxic effect of the latter upon the protoplasm. Two compounds, however, which separately exert such a harmful effect can often, if mingled in certain proportions, provide a solution which produces no plasmolysis and is not poisonous.

For example, if germinating zoospores of Vaucheria (cf. p. 2I7) are placed in weak solutions of sodium chloride (ranging from 0.0937 to $0^{\circ}$ oor $M$ ), plasmolysis ensues more or less rapidly (in the case of the weaker solutions only after an interval of a day or more). If, however, calcium chloride is added to the salt solution in the proportion of one molecule of the former to one hundred of the latter, the young plants of Vaucheria can be subjected to strengths up to as much as O.I $M$ without any contraction of the cell-contents. Indirectly the same effects can be observed by studying the growth and duration of life of young plants of the same Alga in the different solutions: in sodium chloride alone or calcium chloride alone there is practically no growth and death soon takes place; whilst in a mixture of the two in the proportions mentioned above, growth is as vigorous, and the plants remain alive as long, as in glass-distilled ${ }^{1}$ water or in much diluted sea-water.

Similar observations can be made on the development of the root-system of Wheat-seedlings, although these are not so sensitive to the poisonous solutions. In the same way potassium chloride and magnesium chloride are toxic when used separately, though when mixed in suitable proportions the poisonous effect largely disappears. Analogous results have been obtained with animals and, animal-cells.

It is not only the chlorides that give such results, for similar effects have been obtained with nitrates, sulphates, etc. All these compounds are employed in such dilute solutions that dissociation of the molecules into ions will have taken place. Since in the different experiments much the same amount of chloride is present, the striking results obtained must be due to some effect of the metallic ions, probably upon the proteins of the plasmatic membrane. The prevention of the poisonous action of one ion by one or more other ions is spoken of as

1 Ordinary distilled water often contains traces of copper, etc., which are exceedingly harmful to most vegetable organisms. 
antagonism, and is probably greatest between ions of different valencies. A mixture of ions in solution in such proportions that they exert no toxic effect is called a balanced solution, and when a solution of this nature produces contraction of the protoplast, the effect is a true plasmolysis. In this category may be placed sea-water, which is a mixture consisting mainly of sodium chloride, magnesium chloride, magnesium sulphate, potassium chloride, and calcium chloride, and for this reason sea-water suitably diluted forms the best medium for determining osmotic pressures in the plant-cell.

The fact that many mineral salts produce false plasmolysis proves that they are capable of penetrating the protoplast; and even when in so dilute a solution as to have no deleterious effects, there is no reason to suppose that their power of penetration is diminished. The compounds in the soil-water are similarly capable of passing into the plant, although so many are present that the influence of the one upon the other cannot be overlooked. As yet, however, our knowledge on this point is quite inadequate. The complexity of the phenomena is illustrated by the fact that plants do not absorb compounds in the same proportions as they occur naturally in the soil-solution. One of the most striking instances of such selective absorption is furnished by Seaweeds, which contain as much as 0.5 per cent. of iodine in their ash, whilst iodides are present in sea-water in almost imperceptible amount. 


\section{CHAPTER II}

\section{Growing Points and Cell-division}

IN some of the simpler forms of plants (e.g. Spirogyra) almost every cell may exhibit growth and division, but, in the vast majority, the cells originate in the first instance by division from

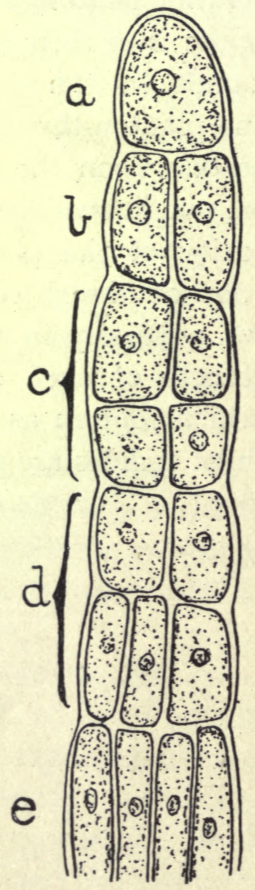

FIG. 5.-End of a branch of Sphacelaria. The successive segments cut off from the apical cell $(a)$ are lettered in order from the youngest to the oldest $(b-e)$, and show increasing subdivision.

so-called growing points. These, in the case of a Flowering Plant, are situated at the tips of the stem and root and of their 
respective branches. A similar apical position of the growing point usually obtains amongst the lower plants.

A good example is afforded by the little Seaweed Sphacelaria, which is not uncommon in rock-pools along the sea-shore. Here each branch ends in a single large cell (Fig. 5, a), which constitutes the growing point. During the active season of the year this cell is constantly increasing in size, and, when it has attained a certain length, it becomes halved by the formation of a cross-

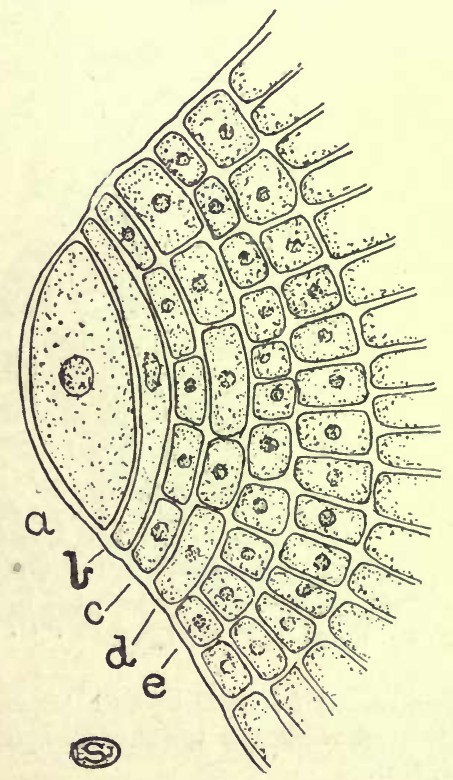

FIG. 6.-Growing point $(a)$ and adjacent part of the thallus of Dictyota. Lettering as in Fig. 5 .

wall (Fig. 5, b). The upper portion again enlarges until a new septum arises, whilst the lower half (termed a segment) undergoes further division (Fig. 5,c-e) to form the cells of the mature plant. The Seaweed Dictyota furnishes another good instance of a unicellular growing point, whose mode of division will be understood by reference to Fig. 6 . The daughter-cells or segments in these two cases are cut off in a single series parallel to one face of the cell.

In most plants having a unicellular growing point the apical 
cell has the form of a three-sided pyramid with a rounded base directed outwards (Fig. 7, a.c.); such a cell is described as tetrahedral. Segments are here cut off in succession from each of the three flat inner faces, these segments undergoing further subdivision to form the mature tissues. This type is found in Mosses and Ferns, and can be readily examined in a longitudinal section through the tip of the stem of a Horsetail Fern (Equisetum) (Fig. 7). In such a section the apical cell (a.c.) of course appears

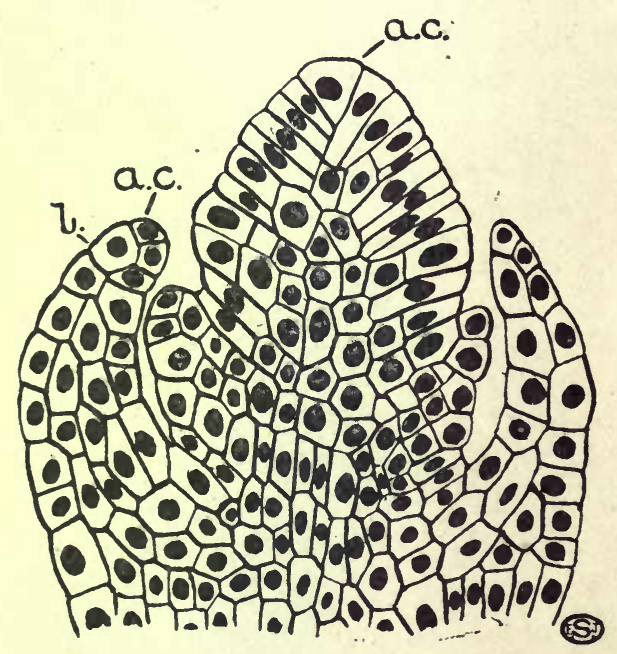

FIG. 7.-Growing apex of the Giant Horsetail (Equisetum maximum) in longitudinal section, showing the tetrahedral apical cells of the stem and leaf (a.c.) ; $l$, leaf.

triangular, and two only of the three series of segments are visible. In the roots of Ferns and other Cryptogams which possess such a unicellular growing point its mode of division is similar, but segments are also cut off parallel to the rounded base, the cells produced by their subdivision forming the root-cap.

The growing point of the higher plants consists of a tissue of small actively growing and dividing elements, which takes the place of the single cell of the lower forms. Such a tissue is called a meristem, and is best examined by cutting longitudinal sections through the apex of stem or root. In both organs the 
cells are arranged in several layers (Fig. 8) which can be traced back into the different regions of the mature plant. As the cells of the growing point multiply, those that remain near the apex retain their power of division, whilst those that come to lie further back gradually pass over into permanent tissue.

In the stem the outermost layer of the meristem usually divides only by walls at right angles to the surface to produce a single layer of cells which in the more mature region can be recognised as the epidermis (Fig. 8 , ep.). The innermost cells

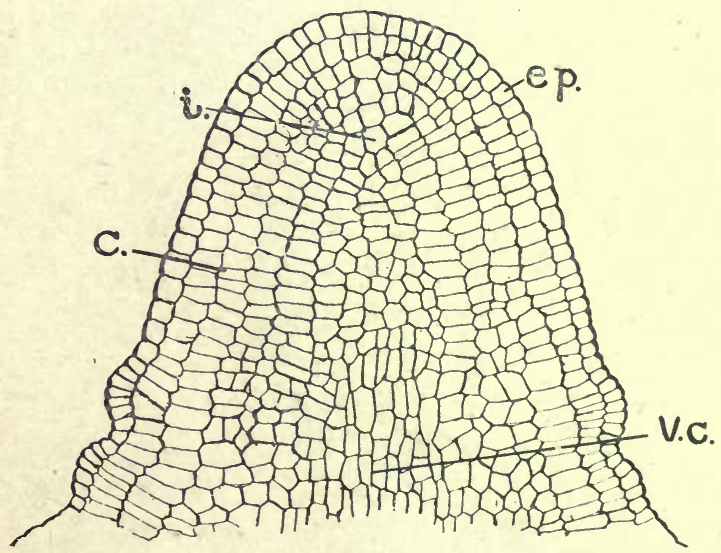

E.J.S.

FIG. 8.-Growing point of the stem of the Mare's Tail (Hippuris) in longitudinal section, showing the regions of vascular cylinder (V.C.), cortex $(C$.$) , and epidermis (e p$.$) , and the single meristematic cell (i$. from which the central cylinder arises.

derived from the growing point divide in all directions, and can be traced back into the central region of the stem, which latter consists of the vascular strands and pith (Fig. 8, V.C.). The middle layers of the meristem also segment in like manner, and develop into the tissue situated between the epidermis and the vascular strands, a part of the mature stem known as the cortex (Fig. $8, C$.). There are thus, at the growing point of the stem, three meristematic regions, known as the dermatogen, periblem, and plerome, which respectively give rise to the epidermis, cortex, and vascular cylinder of the adult. 
At the growing apex of the root four meristematic layers are often distinguishable. Three, the dermatogen, periblem, and plerome, serve the same purpose as in the stem; but external to the dermatogen is a fourth layer, the calyptrogen, which cuts off segments towards the outside only, and thus gives rise to the

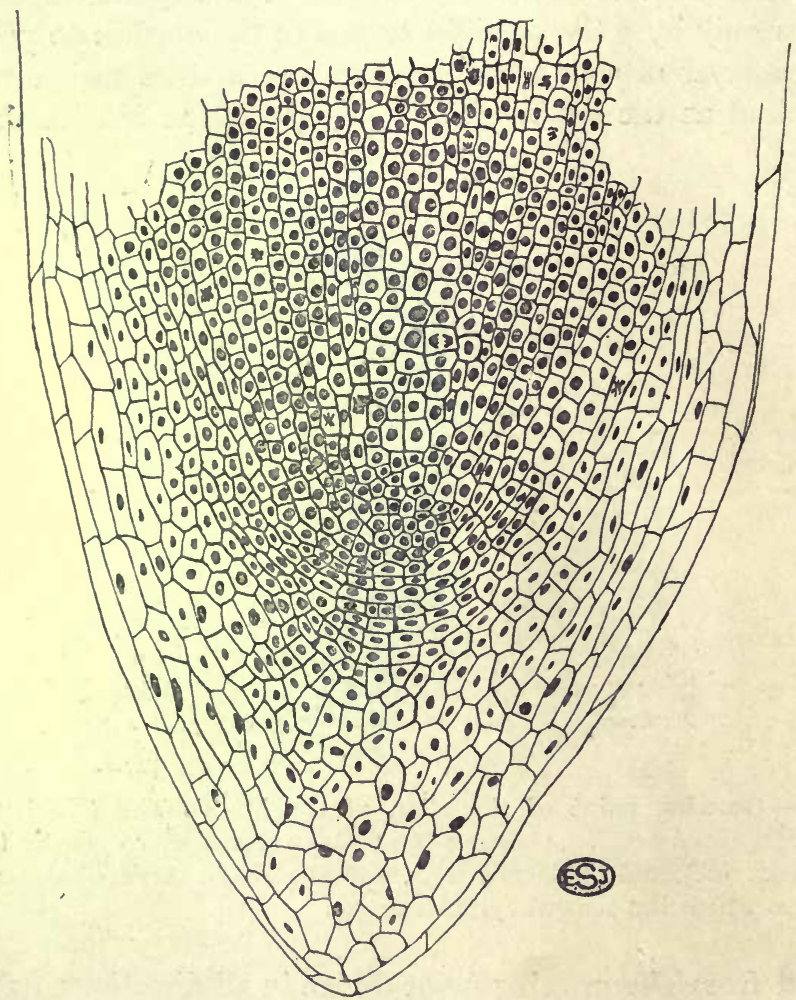

Fig. 9.-Root-tip of the Hyacinth (Scilla) in longitudinal section showing the growing point and the root-cap. Some of the nuclei (black) exhibit phases in nuclear division (cf. p. 22).

root-cap (Fig. 9). The latter is constantly replenished by the growing point, as the older cells in front become disorganised. In many roots, however, the epidermis and root-cap arise from a common meristematic layer, and there are other modifications of the growing point of the root which it is unnecessary to describe in detail. 
The intercalary meristems found at the base of the internode in Grasses, and at the top of the internode in Labiatæ, consist of a transverse plate of small meristematic cells in which no definite regions can be distinguished.

In the process of cell-division the nucleus always takes the lead, dividing into two parts, or daughter-nuclei, which become separated from one another by the development of an intervening
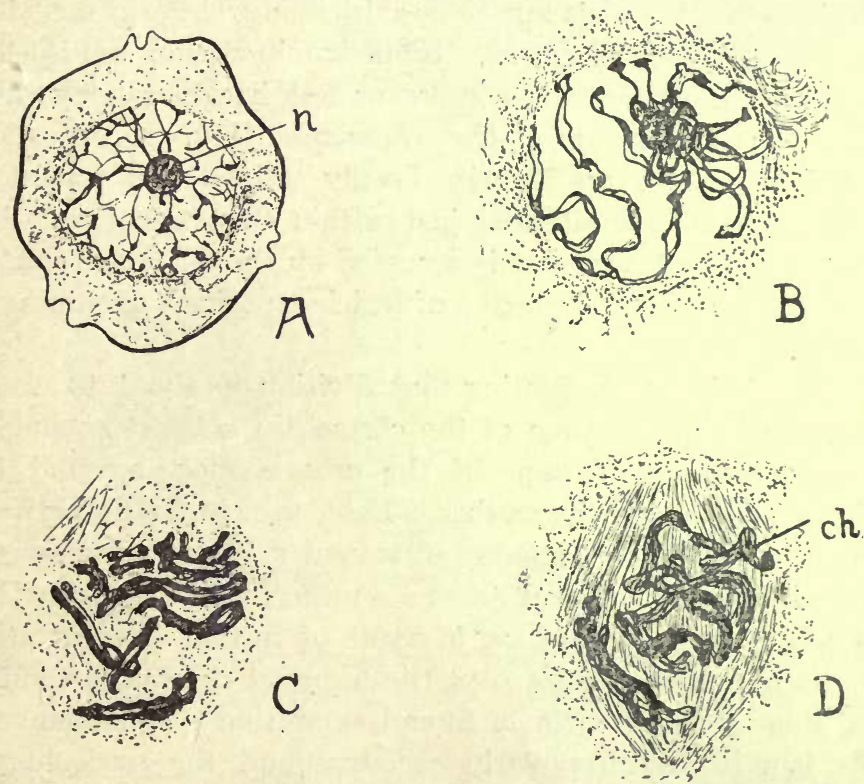

FIG. I0.-Early phases of mitosis (after Fraser and Snell). A, resting nucleus ; B, spireme ; C, formation of chromosomes ; D, establishment of nuclear spindle. ch., chromosomes ; $n$, nucleolus.

wall. Thus two new cells are established, each with its own nucleus similar in all respects to that of the cell from which they originated. Amongst some of the lower plants, and as a rare phenomenon in the higher, the nucleus merely divides into two portions by a median constriction. This is spoken of as direct nuclear division (amitosis).

More usually, however, there is a sequence of complex changes in the nucleus preparatory to and during division, and this whole 
process is designated mitosis or indirect nuclear division. ${ }^{1}$ All stages can be seen in a thin longitudinal section through the growing point of a higher plant (cf. Fig. 9); but in order to observe them readily, it is necessary to employ material which has been carefully preserved and suitably stained (see Appen$\operatorname{dix}$ VI).

The nucleus is usually oval in form (Figs. I, $\mathrm{N}$; IO, A), and, when not actually dividing, is spoken of as a resting nucleus, a term, however, which is apt to be misleading, since in this state it is probably just as active. Immediately within the nuclear membrane (p. 3) there is a more or less irregular network of deeply-stained substance, the chromatin (Fig. IO, A). The nucleoli $(n)$, which are likewise deeply stained and consist of similar material, are situated just within this reticulum. The whole of the central region is occupied by the unstained nuclear $s a p$, a denser peripheral portion of which contains the chromatin network.

The first change, indicating that division is about to occur, is a gradual simplification of the chromatin network, owing to the disappearance of some of the cross-connections and the closing up of some of the meshes. Later the chromatin network appears to consist of a number of irregular, ill-defined segments still exhibiting attachment to one another, especially near the ends. In the next stage, as a result of further concentration and disappearance of all except the terminal chromatin-connections, there remains a single thread exhibiting a split along its whole length, and irregularly coiled around the nucleolus or nucleoli (Fig. Io, B). This is known as the spireme stage.

Soon after this the nucleoli disappear, their chromatin probably having been absorbed into the thread. The latter now contracts somewhat, thus becoming thicker, and breaks up into segments or chromosomes (Fig. Io, C and D, ch.). The number of these has been found to be practically constant for the vegetative cells of any particular species.

Meanwhile the cytoplasm surrounding the nucleus becomes denser, and numerous strcaks become apparent, radiating from each end of the cell towards the nucleus (Fig. Io, C, D), whose membrane has by this time practically disappeared. The radia-

1 The antiquated term karyokinesis is now rarely employed. 
ting lines gradually extend into the central part of the nucleus and meet in its middle. They thus form a spindle-shaped group, termed the nuclear spindle (Figs. Io, D, and II), the two points from which the individual streaks or fibres originate being called the poles. It is debatable whether the spindle fibres represent specialised strands of cytoplasm, or are merely the expression of physical forces operating within the cell.

Since the chromosomes result from the breaking up of a double thread, each is split longitudinally, but this feature is often unrecognisable at this stage. The chromosomes now become grouped in the equatorial region of the nuclear spindle, and
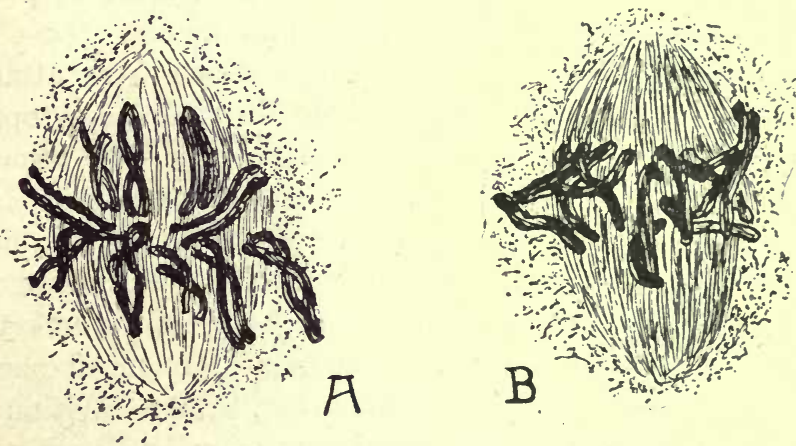

FIG. II.-Mitosis (after Fraser and Snell). A, splitting of chromosomes. B, equatorial grouping of same. Chromosomes are shaded.

seem to become attached to some of its fibres (Fig. II, A). In the next stage these apparently shorten, and the two halves of each chromosome become separated (Fig. II, B), and begin to move towards the opposite poles, which they ultimately reach (Fig. I2, A). The diverging pairs of chromosomes often form loops or V's, according to the mode of separation of the two halves, and during their passage to the poles each frequently exhibits a longitudinal split comparable to that present in the parent-chromosome. This split probably forms the plane of separation of the two halves at the next nuclear division.

On reaching the poles the chromosomes give rise to two daughter-nuclei (Fig. I2, B), which pass into the so-called resting- 
stage by a series of changes broadly the reverse of those taking place at the commencement of nuclear division. That is, the individuality of the chromosomes becomes obscured, owing to the formation of numerous cross-connections between them, and through the separation of the two halves of each chromosome, which only remain joined at the ends and by fine processes in between. With the appearance of nucleoli and a new nuclear membrane, the resting nucleus with its chromatin-reticulum is again established.

From the original nucleus two daughter-nuclei are hence formed, either of which contains a half of each of the chromosomes of the parent-nucleus. As the process is repeated at every division, all the vegetative cells ${ }^{1}$ of the plant come to possess nuclei with the same number of chromatin-masses.

In addition to the contractile fibres which were attached to the chromosomes, other fibres extend through the cytoplasm from pole to pole. These persist for some little time after the establishment of the daughter-nuclei, and develop thickenings in the equatorial plane, an appearance probably due to a modification of the cytoplasm in this region. These thickenings become more conspicuous as they extend horizontally across the parent-cell, till finally they join to form a complete diaphragm of modified cytoplasm, the cell-plate (Fig. I2, B). Within the latter there is formed a thin membrane of cell-wall substance which separates the two daughter-cells, and is traversed by the fine cytoplasmic connections representing the fibres. The latter persist, even in the adult condition, so that the protoplasts of adjacent cells of most plants are connected by living matter. Subsequently further layers, consisting largely of cellulose, are deposited on either side of the original membrane, which is the only part of this separating wall common to the two daughtercells. These additional layers frequently become chemically changed in various ways, but the original separating wall, termed the middle lamella (Figs. I4, I5, m.l.), does not usually undergo such profound modification, so that it is readily distinguished by its different density, and often stains more deeply. ${ }^{2}$

1 For details of nuclear division in reproductive cells, see p.p. 305, 306,

2 The separating wall in many Algæ arises as an ingrowing diaphragm (Fig. $10_{1}$, E, p. I96), and is not always directly related to nuclear division. 
The further modification of the cell-v,all will be dealt with in the next chapter.

From the foregoing it is evident that, when cell-division takes place, the nuclei of the daughter-cells are derived from that of the parent-cell. This fact holds generally throughout the Vegetable and Animal Kingdoms, the nucleus of any given cell always being the product of a pre-existing one. The origin of a new nucleus from the cytoplasm is unknown. In like manner it is probable that all chloroplasts (and other kinds of plastids) are derived by division from pre-existing specialised cytoplasmic
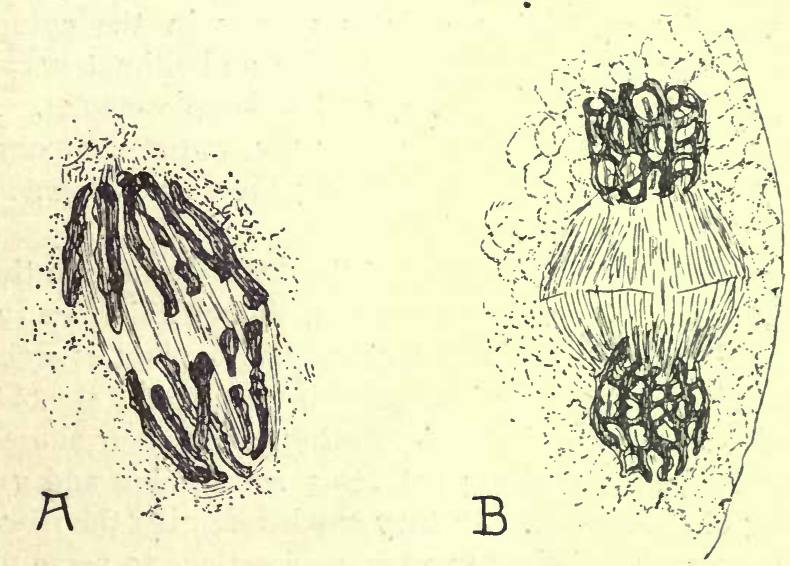

FIG. 12.-Mitosis (after Fraser and Snell). A, chromosomes have separated and reached the poles. $B$, establishment of daughter-nuclei and development of cell-plate.

bodies. During the enlargement usually following upon celldivision considerable increase of the cytoplasm may take place; but it should be emphasised that new protoplasm is only formed in connection with a pre-existing protoplast, the independent origin of living matter being unknown. In other words, both in the Animal and Vegetable Kingdoms, one generation is merely a continuation of the previous one, the reproductive cells of any one dividing and enlarging to form the body of the next.

We can now consider how the cells produced at the growing point gradually pass over into the mature condition. Just below the apex they have dense cytoplasmic contents, are more or less 
rectangular or polygonal, and fit closely together, without any interspaces. A little further back, representing an older phase, they increase appreciably in size and often become more or less rounded off, as a result of which small spaces, the intercellular spaces (Fig. 27, i.p.), appear between them, particularly at the angles.

The increase in volume of the cells is almost entirely due to the intake of water which results from the formation of substances that bring about an increase in osmotic pressure. These substances are produced during the living processes carried on in the cells, and, as a consequence of their solution, a number of small vacuoles containing cell-sap appear in the cytoplasm. These vacuoles gradually increase in size, and ultimately coalesce to form one (e.g. Onion, Fig. I) or few large vacuoles. Apart from its presence in the vacuoles, however, water also permeates the protoplasm and cell-wall, forming the so-called imbibitionwater.

With the gradual assumption of the mature condition the cells generally lose their power of division, though this faculty may be again stimulated into activity, as when a plant is wounded. The growth of any organ of the plant is thus mainly the outcome of multiplication of cells at its growing point, their subsequent increase in volume leading both to a lengthening and gradual widening of the tissues. As they reach maturity the new units mostly become specialised in various directions to serve diverse needs. Those, however, which have not undergone profound alteration, but have retained their juvenile character, often retain also their power of division and capacity to develop in different ways as the demands of the organism may dictate. It is the visible enlargement of plants that is popularly spoken of as growth, but in reality this involves the three phases of cell-division, enlargement, and the final differentiation which will form the subject-matter of the next chapter. 


\section{CHAPTER III}

\section{The Differentiation of Plant-cells}

Four principal types of element ${ }^{1}$ are found in vegetable tissues. A large part of the plant-body is composed of cells which usually appear rounded or polygonal in transverse sections, and in most cases contain a living protoplast. In longitudinal sections they are often rectangular in form, seldom more than two or three times as long as broad, and provided with square or rounded ends (cf. Fig. 9). Such cells, described as parenchymatous, frequently have thin walls, and often form an extensive tissue known as parenchyma.

In contrast to this type are other cells, termed prosenchymatous, in which the length greatly exceeds the breadth, such elements being found more particularly in the mechanical and conducting tissues. These cells usually have pointed ends and thickened walls (Fig. I6, C, D), and the width, as seen in transverse section, is commonly small as compared with that of a parenchymatous element (Fig. I6, B). Occasionally forms of cells are encountered which are transitional between these two types.

The elements of the third type are of quite a different character, and are termed vessels (Fig. I7). They are the principal water-conducting structures in the wood of Flowering Plants, possess no living contents when mature, and their longitudinal walls are thickened in the various ways to be described below

1 These, and the various modifications described in the present chapter, are most easily studied by teasing out (i.e. tearing to pieces with a pair of needles) small portions of a Celery-stalk or Marrow-stem that have been previously boiled for some time in water; the middle lamellæ are thereby dissolved, so that the individual cells readily separate from one another. 
(p. 36). In the mature condition they appear as long wide tubes or cylinders, but they are actually derived from vertical rows of cells by the more or less complete breaking down of the cross-walls. Perforated septa thus occur at intervals in the course of the vessel, and in woody plants are often obliquely radial with reference to the organ as a whole. The cross-walls may almost entirely disappear (as in the Maple and Oak, Fig. I7, F), but most commonly a distinct rim persists, only the central part being absorbed (e.g. the Lime or the Poplar, Fig. I7, E). In still other cases the walls break down in such a way that a number of cross-bars remain (e.g. the Alder, Fig. I7, D).

The elements of the fourth type likewise serve for conduction, in this case of elaborated food-materials. They are known as sieve-tubes, ${ }^{1}$ and are located in the bast or phloem, a tissue that in most stems lies just outside the wood. The sieve-tubes, like the vessels, are derived from vertical rows of cells whose cross-walls are perforated by a large number of fine pores through which the protoplasts of the adjacent units become connected. They retain their living contents in the mature condition, and the membranes remain relatively thin (Fig. I3). When the cross-walls are seen from the surface they present the appearance of a sieve, the meshes of which constitute the pores ; these walls are spoken of as sieve-plates (Fig. I3, s.p.).

Before the sieve-plates are fully developed each cell undergoes longitudinal division into two unequal portions. Of these the larger constitutes a segment of the sieve-tube whose nucleus degenerates; whilst the smaller, which retains its nucleus, forms a so-called companion cell (Fig. I3, c.c.). In some cases two, or even three, companion cells may be produced before the nucleus of the sieve-tube segment dies away. The narrow companion cells have dense granular contents in contrast to the wider sieve-tubes, which possess but a thin lining layer of cytoplasm and a large central vacuole, features by which they are often readily recognised in transverse sections.

Behind the growing point the cells have been seen to exhibit a steady increase in size until they attain their full dimensions.

1 See also p. 82. Sicve-tubes of a different type occur in Ferns and Gymnosperms (pp. 293, 339). 
During the phase of enlargement the walls undergo no appreciable thickening, but surface growth takes place. This is probably accomplished by stretching of the elastic membrane and intercalation of new particles of cell-wall substance between those already present. When fully grown more or less marked thickening of the wall occurs, successive layers formed by the agency of the living protoplast being deposited on the inner surface. Subsequently the wall often undergoes considerable chemical changes, as a result of which it may become more or less im-

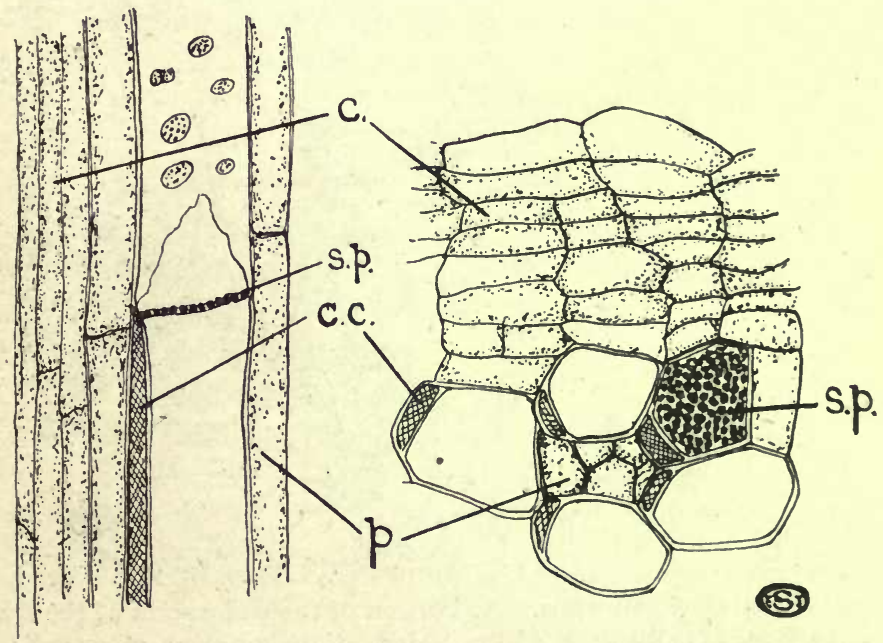

FIG. 13.-Phloem-structure of the Vegetable Marrow (Cucurbita) in longitudinal (left) and transverse (right) sections. c., cambium ; c.c., companion cells; $p$, phloem parenchyma; s.p., sieve-plate.

permeable to water, so that the contained protoplast dies. When the thickening is at all conspicuous, the successive layers generally appear more or less distinct (Fig. I4, D; I6, B). Thisstratification is due to the fact that the layers adjacent to one another are of unequal density, so that one is more transparent and consequently brighter than another.

As a general rule the thickening is not uniform over the whole inner surface of the wall, small areas commonly remaining thin (Fig. I4, B). The intimate relation between adjacent cells is evidenced by the fact that such thin areas, interrupting the 
layers formed by the protoplast of one cell, coincide with the thin areas on the outer sides of its walls, where the thickening has been deposited by the protoplasts of the surrounding cells. At these points, known as simple pits, the original middle lamella (the pit-membrane, Fig. I4, A, pm.) alone separates the adjacent cells. In these cases the bulk of the protoplasmic connections (cf. p. 24) pass through the pit-membrane.

Good examples of simple pits are seen in sections of Elder pith (Fig. I4, B). In surface view they appear as irregularly scattered oval or elliptical areas which are more transparent

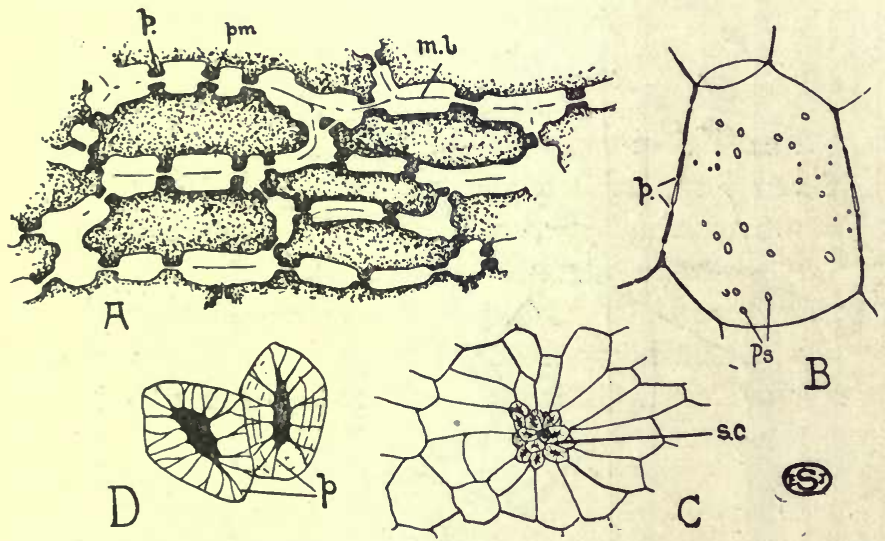

FIG. I 4.-Thickened cell-walls. A, Portion of the endosperm of the Date in section. B, Pith-cell of the Elder. C, A group of stone-cells of the Pear, together with a small part of the adjacent flesh. D, Two isolated stone-cells. m.l., middle lamella; $p_{\text {., }}$ pits seen in section ; pm., pit-membrane ; Ps., pits in surface view ; s.c., stone-cells.

than the rest (Ps.). In section ( $p$.$) the walls appear broken at$ sundry points; but careful focussing shows that the apparent gaps are really bridged by a thin line, the pit-membrane. In certain cases [e.g. the endosperm of the Date (Fig. I4, A), the cotyledon-walls of the Lupine and many other Leguminosæ, and cells of the cortex of the Mistletoe] the thickening is so extreme that the cavity of the cell becomes much reduced, and the pits then appear as deep depressions within the wall. It is in such tissues that the protoplasmic connections between cell and cell can most readily be demonstrated. 
The thick-walled elements of the plant are of considerable mechanical importance, since they form skeletal tissues which are mostly devoid of intercellular spaces. When such mechanical elements occur in young growing organs, where too great a rigidity is disadvantageous, they take the form of collenchyma, which differs from other strengthening tissues in the fact that its cells are living. Collenchyma is frequent immediately beneath the epidermis in young stems (Fig. 34, s), in the midribs of young leaves (Fig. 5I, M), and in the adult stems of some herbs (e.g. Hogweed). In its most typical form it is characterised by extreme thickening of the corners of the cells, as seen in transverse section (Fig. I5), the thickened angles appearing bright

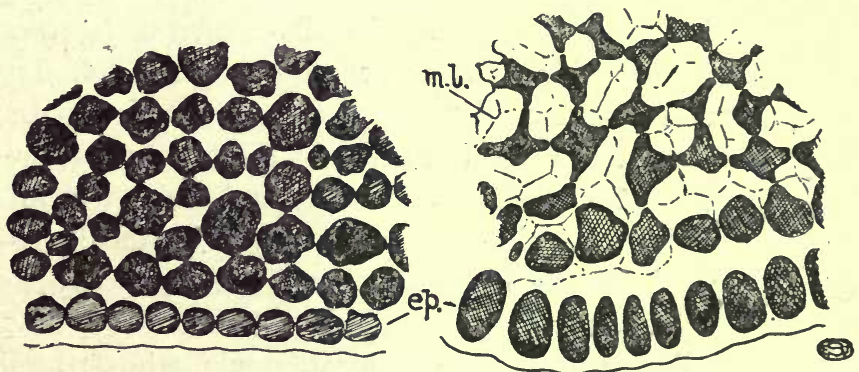

FIG. I5.-Collenchymatous tissue from the stems of the Burdock (Arctium) (left) and Dead Nettle (Lamium) (right). ep., epidermis ; m.l., middle lamella. Both in transverse section. The cell-contents are shaded.

and shining owing to their highly refractive character. Sometimes, however, collenchyma exhibits uniformly thickened walls, interrupted only by pits. The thickening, unlike that of most mechanical elements, is practically unaltered cellulose, which possesses considerable stretching power, so that collenchyma is well suited to act as a supporting tissue in young growing organs.

All cellulose-walls ${ }^{1}$ exhibit this character, and, moreover, are readily permeable to water. Cellulose is one of the more complex carbohydrates, belonging to the series of the polysaccharides, and composed of a large number of $\mathrm{C}_{6} \mathrm{H}_{10} \mathrm{O}_{5}$ groups. It is scarcely coloured by iodine solution, but is stained blue

1 Mingled with the cellulose in the walls of many plant-cells, especially those of succulent fruits (e.g. Apples, Gooseberries) and storage roots (e.g. Beetroot), are so-called pectic substances which are soluble in water. 
if the application of iodine is followed by that of strong sulphuric acid. The acid causes swelling of the wall, and this is followed by complete solution, the blue colour with iodine being due to one of the products. Cellulose-walls are likewise dissolved by ammoniated copper oxide (cuprammonia, see Appendix II). A blue colouration and swelling also results from the application of chlor-zinc-iodide (Schultze's solution, Appendix II), but it is necessary to use the freshly made compound, as it soon decomposes. For general purposes useful stains for cellulose walls are methyl blue and hæmatoxylin (Appendix VI), the latter giving a deep purple colour.

The remaining mechanical elements form tissues spoken of as sclerenchyma. They are distinguished from collenchyma by a chemical change of the thickened walls known as lignification, in consequence of which the cell-contents die. Such lignified walls are much harder and stronger than those consisting of cellulose, features which are the cause of the toughness of wood. They possess relatively little elasticity, and when wet are not readily permeable to water and practically impermeable to air. The exact nature of the chemical change is not known, but it is sufficiently profound to lead to marked differences in reaction. Thus such walls are insoluble in cuprammonia, whilst the lignin is dissolved by Eau de Javelle (Appendix II). Iodine solution stains lignified walls brown, and a similar colouration is obtained with chlor-zinc-iodide. Aniline chloride, or sulphate, which does not affect cellulose-walls, yields a brilliant yellow colouration and, as it does not stain starch, is particularly useful when that substance is present in quantity. An alcoholic solution of phloroglucin, followed by strong hydrochloric acid, produces a scarlet colouration. Lignified walls stain yellow or brown with hæmatoxylin, and are deeply stained by gentian violet. The latter is most effective in conjunction with Bismarck brown, the wood and other sclerenchyma becoming violet and the ordinary cell-walls brown.

There are two principal types of sclerenchymatous elements, viz. stone-cells and fibres; of these the former are more or less parenchymatous, the latter prosenchymatous in shape. The gritty character of the flesh of the Pear is due to stone-cells which, in a thin section, will be found to occur as little clusters 
(Fig. I4, C) irregularly scattered through the thin-walled parenchyma forming the flesh. The cavity of each stone-cell is very much reduced and inconspicuous owing to the strong thickening of the wall (Fig. I4, C and D, s.c.). This shows distinct stratification, and is traversed by a number of dark lines $\left(p_{.}\right)$ radiating from the centre and branching as they approach the surface. These are the pits (spoken of as pit-canals when they exhibit this narrow elongated form) which serve for the transference of nourishment to the protoplast during the process of thickening, on the completion of which the living matter dies.

The fibre is a narrow, very much elongated, cell with tapering pointed ends, and in the adult condition its protoplast is frequently dead. The walls are in general strongly thickened and lignified (Fig. I6, B-D), and usually bear a number of oblique slit-shaped pits. In the mature plant fibres are generally the most important form of mechanical element, and compose a large proportion of the wood of thicker branches (cf. P. I2I); these wood-fibres sometimes bear a modified type of bordered pit (see below). The cortex in the young stems of many woody plants exhibits a continuous mechanical ring composed of alternating groups of fibres and stone-cells.

Plant-fibres can undergo considerable elongation and can bear very heavy strains without losing the power of again contracting to their original length; as a matter of fact, loads varying from I4 to 25 kilogrammes are required to produce permanent lengthening. The powers of fibres in this respect may be compared with those of metal rods (e.g. of wrought iron and steel) which, whilst they become permanently stretched under similar strains, exhibit far less extension before the limit of their elasticity is reached. Fibres will thus permit bending on the part of a plant-organ (under the influence of the wind, for example), and will not give way, even under considerable strain; moreover, their elasticity will bring about a return to the normal position as soon as the strain is relieved. Whilst the breaking strain (Io-I2 kilogrammes) for collenchyma is not much less than that for many fibres, it exhibits permanent elongation under quite low tension, so that it is especially suited to the mechanical needs of a growing organ where constant extension is taking place. 
Owing to their great strength, fibres are of considerable economic importance, being used for the manufacture of textiles, rope, etc. They are usually prepared from herbaceous plants by removing the softer tissues or allowing them to rot

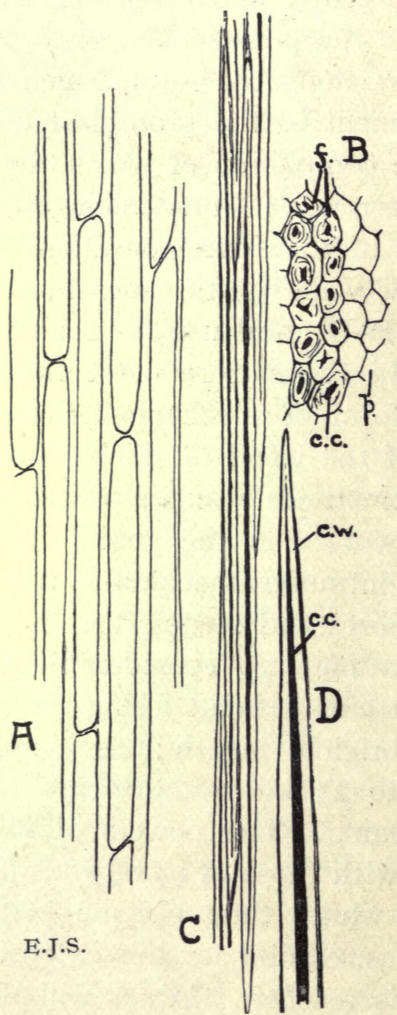

FIG. I6.-Collenchyma of Dead Nettle stem in longitudinal section (A), and fibres from the stem of the Hop in transverse (B) and longitudinal sections (C, D). c.c., cell cavity; c.w., cell-wall ; $f$, fibres ; $p$, parenchyma.

away. Important fibre-yielding plants are the Flax (Linum usitatissimum), New Zealand Flax (Phormium tenax), Hemp (Cannabis sativa), Jute (species of Corchorus, mainly cultivated in Bengal), whilst Raffia-tape or bast is obtained from the Palm 
Raphia.' Moreover, fibres treated in various ways are the source of wood-pulp and other paper-making materials.

The main function of vessels (Fig. I7) is water-conduction, but they also are elements of mechanical importance on account of their thick walls. These are necessitated by the frequent existence of negative pressures in the water-conducting tracts,
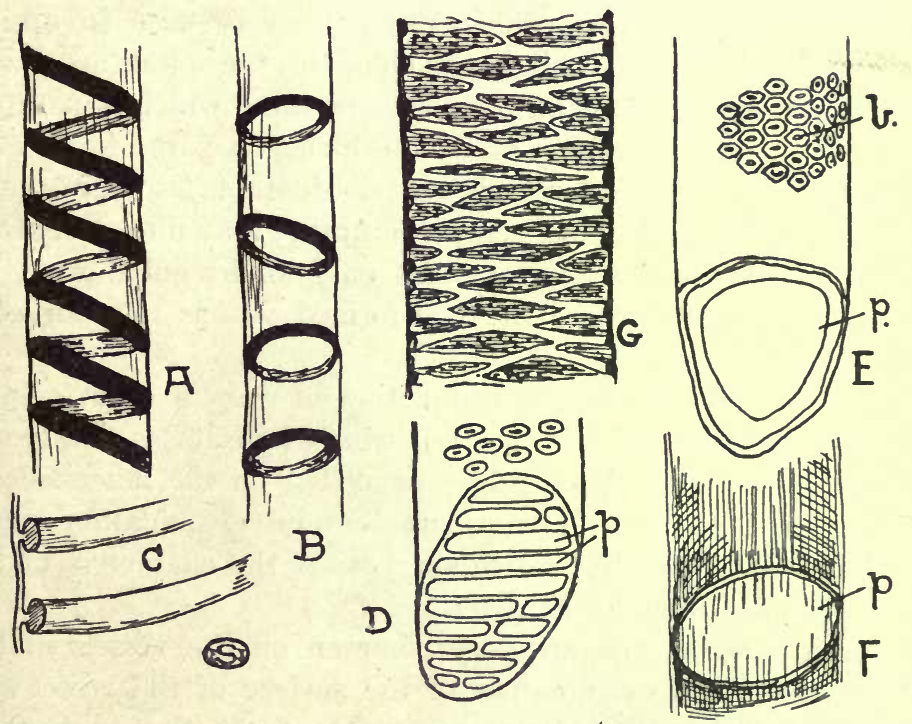

FIG. I7.-Vessels showing various types of thickening and perforation. A, spiral ; B, annular ; $\mathrm{C}$, portion of $\mathrm{A}$ in longitudinal section showing attachment of thickening to wall; $\mathrm{D}$, end of vessel of Alder (Alnus) showing ladder-like perforation; E, vessel of Poplar (Populus); F, vessel of Oak (Quercus); G, reticulate vessel of Marrow (Cucurbita). b., bordered pit; $p$., perforations between segments of vessel.

such as obtain, for instance, when transpiration is active. At these times collapse of the vessels, under the positive pressure exerted by the surrounding elements, is prevented by the rigidity of their walls. The type of thickening varies greatly, depending largely on whether the vessel is formed in a part of the plant that is still growing or in one that has reached maturity. In the former case the thickening must be of such a nature as to

1 Regarding cotton see p. IO3. 
admit of stretching of the wall, which would otherwise be ruptured.

The first-formed vessels of the wood, which differentiate a short distance behind the growing point, exhibit continuous spirals or disconnected rings of thickening (spiral and annular vessels, Fig. I7, A, B). These are deposited on the inner side of the original vessel wall, to which, however, they are only attached by a narrow connection, as can be seen in optical section (Fig. I7, C). As the organ elongates the spirals are drawn out like a spring, and the rings become more widely separated, by the gradual stretching of the unthickened part of the wall. If, however, growth in length is very considerable (as, for instance, in many Monocotyledons), complete rupture may ultimately take place, so that in the mature plant only an irregular canal remains to indicate where the first-formed vessels were situated (Fig. 40, p.c.).

The vessels formed after completion of growth often exhibit reticulate thickening (Fig. I7, G), which appears as a more or less irregular network of ridges deposited on the inner side of the original wall. In many cases careful examination shows that the meshes of this reticulum possess the characters of the bordered pits about to be described.

Such bordered pits are very common on the vessels in the older wood. An examination of the surface of the vessel wall under the high power of the microscope shows that each pit is provided with a broad border (Fig. I7, E, b. ; also Fig. I8, B, b). This is either circular in outline, or more commonly polygonal as a result of dense crowding, the actual aperture of the pit appearing round (Fig. I8, B, $p$ ) or more or less slit-shaped. In section the border is recognised as a dome-shaped cover formed by the thickening of the wall, which around the pit arches away from the middle lamella (i.e. pit-membrane); the top of this low dome is perforated by the aperture leading into the pit (cf. also Fig. 197, D, p. 34I). Where two vessels adjoin one another, such a perforated dome occurs on either side of the common wall, so that these bordered pits coincide. The pitmembrane possesses a central thickened area, the torus, which, owing to its modified character, is impervious alike to air and water.

When the pit-membrane, which is easily displaced, is forced 
to one side or the other, the torus closes up the aperture of the dome, and thereby any passage of water or of air from one vessel to the other is prevented. Such displacement will occur when the pressure in one vessel differs from that in the adjacent one, so that the bordered pits operate as safety-valves whereby differences of pressure in the wood become localised.

The conduction of water in the higher plants is mainly effected by means of vessels, but another kind of conducting element,
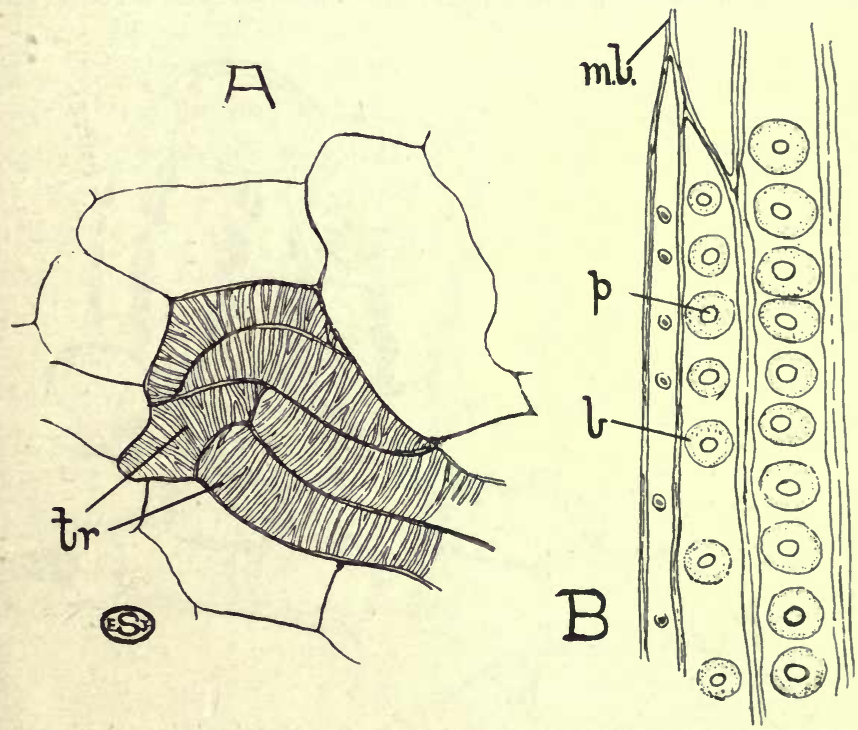

FIG. I 8.-Tracheids. A, Spiral type, from the leaf of Dog's Mercury Mercurialis). B, Bordered-pitted, from the stem of the Scotch Fir (Pinus). b, border of bordered pit; m.l., middle lamella ; $p$, aperture of bordered pit; $t r$. , tracheids.

the tracheid, which is likewise dead, is also found in the wood of thicker branches and, more commonly, in leaves. Fach tracheid is formed from a single cell which is more or less elongated in form (i.e. prosenchymatous), and usually bears bordered pits (Fig. I8, B) or spiral thickening (Fig. I8, A). The wood of the Fir and other Conifers is entirely made up of long tracheids, similar in appearance to fibres, but bearing pronounced bordered pits (Fig. I8, B). Very narrow bordered pits appearing as oblique slits are not uncommon in fibres proper. 
The walls of mature cells often undergo chemical changes other than lignification, some of which (e.g. the change affecting the cells of the cork, p. I36) will be described later, but a few special cases may conclude the present chapter. In a number of plants the thickening of the walls of certain cells takes the form of layers of mucilage, whose exact chemical constitution is not known. These, in the dry condition, are hard and horny, but in the presence of moisture they soften and swell up considerably. Indeed, such mucilaginous walls possess a remarkable

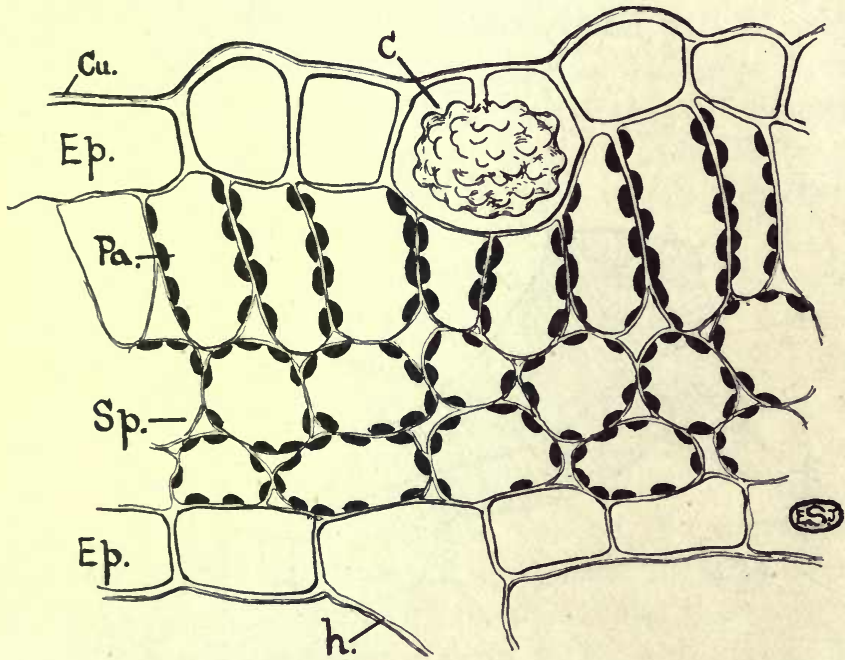

FIg. 19.-Transverse section of the leaf of a Nettle (Urtica) showing a cystolith $(C$.). $C u$., Cuticle; Ep., epidermis ; $h$, hair; $P a$., palisade tissue ; $S p$., spongy parenchyma.

power of absorbing and holding water, and are consequently particularly prevalent in plants characteristic of dry situations. The slimy character of many Seaweeds (e.g. the common Bladderwrack) is due to the mucilaginous nature of nearly all the cellwalls, and here the change in bulk, as between the dry and wet condition, is especially evident. Mucilaginous walls stain deeply and rapidly with aniline blue.

The gums formed by certain plants are probably very similar chemically to the mucilages, and, like them, appear, in many cases at least, to arise as a result of chemical alteration of the 
cell-wall. Diverse species of Acacia yield gums of economic importance, e.g. gum arabic (from $A$. senegal), catechu used in tanning (from $A$. catechu), etc.; gum tragacanth, which is far less soluble, is obtained from species of Astragalus.

Another frequent modification consists in the deposition of mineral substances within the cell-wall. For example, in the Horsetails and most Grasses the membranes of the outer cells are impregnated with silica, to which many Grass-leaves owe their sharp cutting edges. This silicification increases the stability of the plant and also serves as a protection against snails, etc. A siliceous envelope is very characteristic of certain minute unicellular Algæ known as Diatoms (cf. p. 206), which often occur in countless numbers in fresh and salt water.

In a few cases the surface of the plant is encrusted with carbonate of lime, as in some Stoneworts (Chara) and certain other lowly members of the Vegetable Kingdom (cf. p. 204). Local deposits of lime on special ingrowths of the walls are not uncommon in the epidermal cells in certain groups of Flowering Plants. The resulting structures are known as cystoliths, and are well seen in the leaves of the Stinging Nettle (Fig. I9, C.) and of various kinds of Fig (e.g. in Ficus elastica). If sections of the leaves are placed in weak acetic acid, the carbonate of lime dissolves with effervescence and the framework of the cystolith becomes visible. 


\section{CHAPTER IV}

\section{The Non-living Contents of Cells}

APART from the living constituents, cells usually contain numerous substances which are either dissolved in the sap or, when insoluble, occur as solid bodies or suspended drops. These substances can for the most part be grouped as food-bodies or as by-products, according as they are known to be employed in the nutrition of the plant or are believed to be of no further nutritive value.

Among the commoner food-substances are various carbohydrates (e.g. sugars, starch, etc.), oils, and proteins, all of which are built up by the plant from simple inorganic compounds by a series of complex changes. During the earlier part of the season such food-substances are used directly to supply the necessary materials for growth, but subsequently, with decreasing demands, a large proportion are stored up for future use. In perennial plants they accumulate in those organs which persist from year to year (e.g. bulbs, tubers, etc., and, in the case of woody perennials, the stem- and root-systems). ${ }^{1}$ Similar food-substances are, moreover, laid up within the cotyledons or endosperm of all seeds.

One of the most important food-substances is starch, which is insoluble in the cell-sap. It is often the first easily recognisable product of photosynthesis in a green leaf which has been exposed to light. On microscopic examination the starch appears as very small shining grains, mainly within the chloroplasts (Fig. 3, St.). These grains gradually increase in amount during the day, but generally disappear over-night, and are consequently spoken of as transitory starch. Their gradual accumulation on a bright day 
is due to the conversion of the soluble carbohydrates (e.g. sugars), which are produced more rapidly than they can be removed, into insoluble starch. In darkness, when carbon dioxide assimilation ceases, the accumulated starch is changed back into sugar and transferred to other parts of the plant.

In contradistinction to this transitory starch, that which accumulates in storage-organs usually takes the form of rather large grains which originate within colourless plastids, known as leucoplasts. The latter occur in those cells which are not exposed to light, and differ from chloroplasts only in the absence of chlorophyll, which is generally not produced in darkness. Leucoplasts, however, readily change to chloroplasts. When a Potato-tuber, for instance, is exposed to light, it turns green through the formation of chlorophyll within the leucoplasts. In a few cases large starch-grains are actually produced within green plastids (e.g. in a small greenhouse plant known as Pellionia), and these provide particularly appropriate material for studying the mode of formation of the grains.

Thus, in a transverse section of the stem of Pellionia the outermost cells (Fig. 20,a) are seen to contain chloroplasts (ch.), in some of which there is a bright shining dot, the transitory starch-grain $(s)$. The larger starch-grains, seen nearer the centre in various stages of development, may be supposed to have originated likewise, as small bodies within the chloroplasts there situated (Fig. 20, b). But in the mature condition these grains have enlarged to such an extent that the enveloping chloroplasts appear merely as green caps to one side of them (Fig. 20, c). On staining with dilute iodine both small and large grains take on the blue colouration typical of starch.

The large starch-grains that can be scraped out of a Potato are more suitable for the study of details of structure. Examined in a drop of water, each shows a number of asymmetrical layers arranged concentrically around the darker hilum, or point of origin (Fig. 20, d). This stratification indicates the manner of growth of the starch-grain, the successive layers being distinguished, presumably, by differences either in chemical or physical structure. In the grains of the Potato (Fig. 20, $d$ ) and Pellionia (Fig. 20,c) the point of origin is towards one end (excentric type), On the other hand, the hilum, in those from the cotyledons of 
the Pea or Bean, lies in the centre (centric type, Fig. 20, $f$ and $g$ ), and the layers are arranged symmetrically around it. The dry grains of Pea, Bean, etc., exhibit a number of radiating
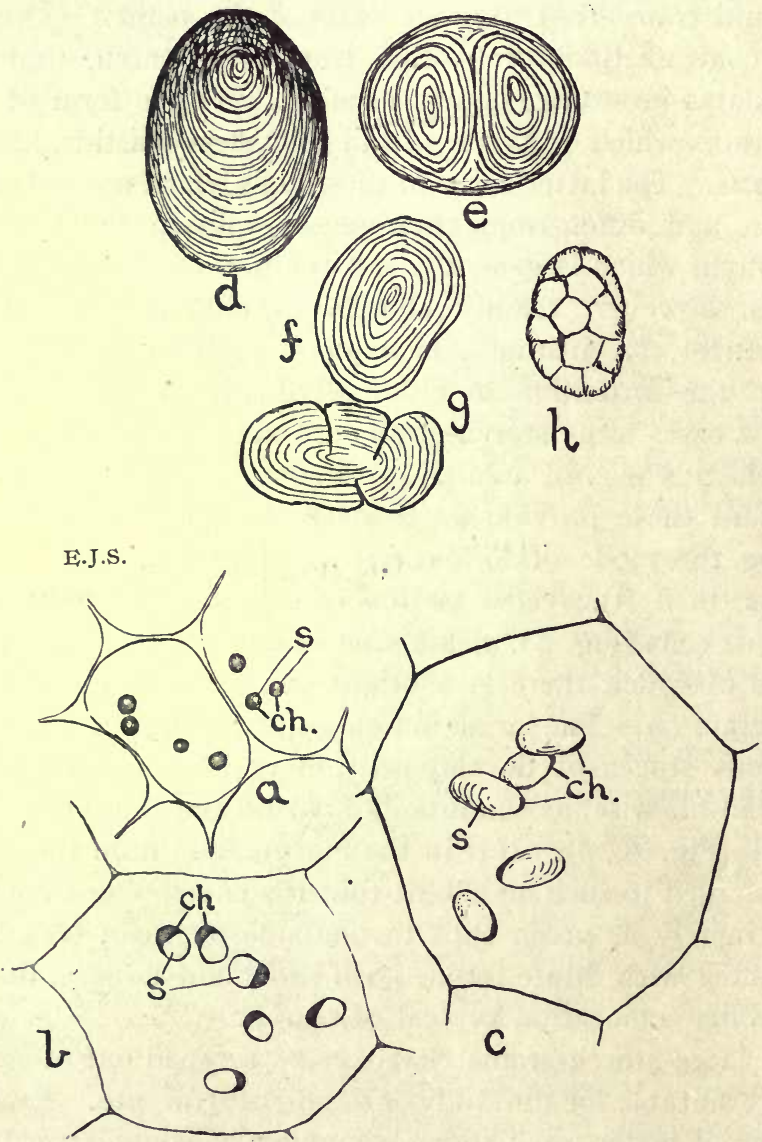

FIG. 20.-Starch-grains. $a-c$, stages in the development of grains within the chloroplasts of Pellionia; chloroplasts shown black. ch., chloroplasts; $s$, starch-grains ; $d$ and $e$, Potato starch; $f$ and $g$, Pea starch ; $h$, compound starch-grain of Rice.

cracks, which appear as dark irregular lines (Fig. 20, g); these seem to be due to the drying up of some of the imbibed water which permeates the substance of all starch-grains. 
Whilst most of the grains of Potato-starch show but a single hilum, an occasional one will be found to possess two or three, each with its own system of layers (Fig. 20,e). This results from the development of several grains within the same leucoplast, growth of each occurring independently until they meet; in some cases deposition of starch continues with the formation of layers common to the whole group (Fig. 20,e). Such structures are called compound grains. In some plants (e.g. in many cereals) numerous grains arise in each leucoplast, so that the compound structure may consist of a thousand or more units. Thus a starch-grain of the Rice (Fig. 20, $h$ ) or Oat is marked out into a number of small areas, each representing a constituent unit.

Starch is a carbohydrate belonging to the group known as polysaccharides - that is to say, its highly complex molecule is built up of $\mathrm{C}_{6} \mathrm{H}_{10} \mathrm{O}_{5}$ groups present in larger numbers than in the case of cellulose (cf. p. 3I), but here as there the actual number is unknown. The grains are regarded by some as consisting of a number of alternating amorphous layers, while others believe them to possess a crystalline structure. Under polarised light they exhibit a black cross with the hilum as its centre. When warmed in a drop of water starch-grains swell, lose their pronounced stratification, and ultimately pass into a " solution" similar to that obtained when a thin starch-paste is treated with an excess of boiling water. This "solution" is, however, colloidal in character, and therefore will not diffuse through an organic membrane. A similar result is obtained when starch is treated with caustic potash. Comparatively few dyes colour starch, although gentian violet and eosin are notable exceptions.

The accumulation of starch-reserves by plants is a feature of the greatest economic importance, constituting as they do a very important article of human diet. The cereals, the pulses, and Potatoes, all of which contain a high percentage of starch (cf. p. 53), furnish a sufficient illustration. Rice, which perhaps constitutes the most widely consumed food in the world, is the grain of Oryza sativa, a Grass cultivated in most parts of the Tropics where the necessary water for submerging the rice-fields is available. Tapioca is obtained from the root-tubers of the Cassava or Mandioc (Manihot utilissima, widely grown in the Tropics), whilst sago is the starch found in the pith of various 
Palms (mainly species of Metroxylon, cultivated in the Malay region), from which it is extracted after the appearance of the inflorescence.

The wide distribution of starch as a form of storage of carbohydrate material can probably be related to its insoluble character, in consequence of which it exerts no osmotic pressure. The small amount of moisture in seeds renders them unsuited to the storage of soluble carbohydrates, and doubtless explains the frequent occurrence of starch in the endosperm and cotyledons. In succulent storage organs, on the other hand, soluble carbohydrates often occur. One of the most important is inulin, another of the complex polysaccharides, though with a molecular weight smaller than that of starch. As a result, in spite of its solubility and frequent high concentration, inulin has but little effect on the osmotic pressure of the cells containing it (cf. p. II).

Inulin is found especially in the Compositæ and the allied family of the Campanulaceæ, but also in the bulb of the Wild Hyacinth (Scilla nutans) and in other Monocotyledons. Soluble carbohydrates of a similar chemical constitution are, moreover, encountered in many members of the latter group (e.g. the graminin of Grasses, the irisin of the Iris, etc.).

In sections of a fresh Dahlia-tuber, Salsify-root, or Jerusalem Artichoke, the intact parenchymatous cells will be seen to contain a faintly yellow oil-like liquid, the inulin. On placing the sections in spirit, the inulin is deposited as a finely granular precipitate. In sections of material kept for some weeks in spirit, so that the latter has only penetrated slowly into the tissues, the inulin will be found' as big spherical or lobed masses deposited on the cell-walls. These sphere-crystals (Fig. 2I, In.) usually show concentric layers, whilst radial lines traversing them indicate the numerous needle-like units of which they are built up. On warming in water, the precipitates and sphere-crystals of inulin readily dissolve. Iodine gives a brown colouration with inulin which is but little different from that of the reagent itself.

Sugars are among the most important of the soluble carbohydrates present in plants. They possess much simpler molecules than the polysaccharides just considered, being either monosaccharides with the general formula $\mathrm{C}_{6} \mathrm{H}_{12} \mathrm{O}_{6}$, or disaccharides with the formula $\mathrm{C}_{12} \mathrm{H}_{22} \mathrm{O}_{11}$. Disaccharides and polysaccharides 
are so called since they respectively split up, under certain circumstances, into two or many molecules of monosaccharides.

Among the monosaccharides found in plants, the commonest are dextrose or glucose (popularly known as grape-sugar) and fructose or levulose (fruit-sugar), whilst of the disaccharides sucrose or saccharose (cane-sugar) and maltose (malt-sugar) deserve mention. Owing to their simple molecules they produce a relatively high osmotic pressure, although for solutions of equal strength this is greater in the case of the monosaccharides

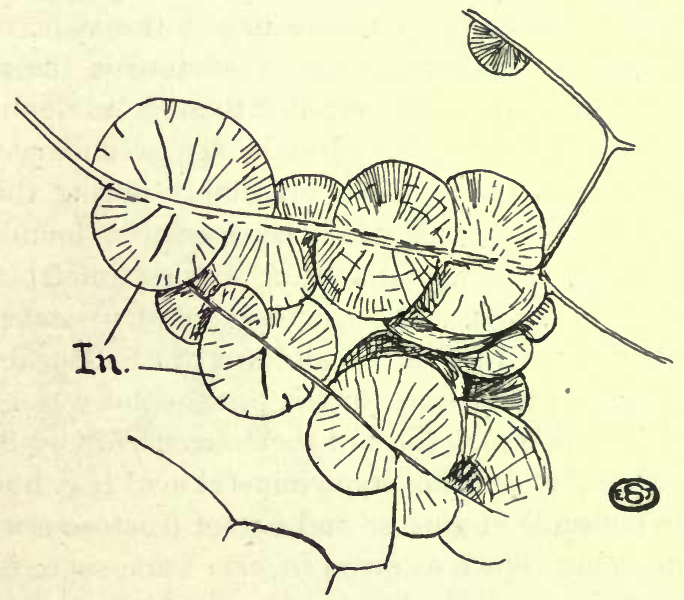

FIG. 2I.-Sphere-crystals of inulin (In.) in the cells of the tuberous root of a Dahlia.

than in that of the disaccharides. For this reason no doubt grape-sugar and cane-sugar, the two which function as foodreserves, are only found in very minute quantities in seeds (except for the cane-sugar in the Sweet Corn, a variety of Maize). On the other hand, grape-sugar is one of the principal carbohydrate-reserves in the bulb of the Onion, whilst cane-sugar occurs in the Sugar Beet (Beta), in the pith of the stem of the Sugar Cane (Saccharum officinarum), and in the Sugar Maple (Acer saccharinum) (see p. I24). The sugar is extracted from the sliced Beet with the aid, of warm water, whilst in the case 
of the Sugar Cane the juice is crushed out of the canes with the help of rollers. In all cases the crude sugar is subjected to subsequent processes of refinement.

Fructose is most abundant in succulent fruits, and is an important constituent of honey; in both, however, it is mixed with, usually smaller amounts of, grape and cane sugars. In such cases the sugars are, of course, not of the nature of foodreserves, but serve a biological purpose in connection with seeddispersal and cross-pollination.

All the four sugars above mentioned are found in foliageleaves, though in proportions that vary greatly both during the day and night and at different seasons of the year. It is still an open question whether glucose or sucrose is the first sugar to be formed in photosynthesis; but there is no doubt that the other two, like the transitory starch, are secondary products. Maltose appears to be produced invariably during the solution of starch within the plant, and is consequently found not only in foliage-leaves, but in germinating Barley (malt) and other starch-containing seeds. The fructose found in leaves, on the other hand, is formed by the breakdown of cane-sugar.

The latter is readily split (in the presence of water, so-called hydrolysis) into two molecules of monosaccharide by boiling the solution with a few drops of some mineral acid (e.g. hydrochloric acid) ; one molecule of glucose and one of fructose are obtained, the mixture being known as invert sugar. Cane-sugar is similarly converted into invert sugar by the agency of an enzyme invertase found in most plants. In the same way the polysaccharides above discussed can be split up with the formation of disaccharides or monosaccharides, as the case may be. For instance, a starch "solution" boiled with a few drops of a mineral acid becomes clearer, and the ordinary reaction to iodine gradually disappears ; the colour assumed with this reagent is now reddish, owing to the presence of simpler polysaccharides known as dextrins. If the boiling be continued, the whole of the starch " solution" ultimately breaks down into simple glucose. Similarly inulin gives rise to fructose. In the plant starch and inulin are acted upon by ferments, diastase and inulase respectively, which effect like changes, except that diastase breaks down starch into the disaccharide maltose, which in its turn is acted upon by 
an enzyme maltase with the production of two molecules of glucose.

The sugars are readily distinguished from one another by certain characteristic reactions. Thus glucose, fructose, and maltose all reduce Fehling's solution (which contains cupric oxide, Appendix II) with the formation of a red precipitate of cuprous oxide, and are consequently known as reducing sugars. Sucrose, on the other hand, is a non-reducing sugar, giving no precipitate with Fehling's solution, until it has been inverted by boiling or enzyme-action. It may be added that neither inulin nor the dextrins effect reduction of this reagent.

For microchemical purposes, especially when but small quantities of sugars are present, the following procedure is more advisable. The sections are mounted in a drop of a solution
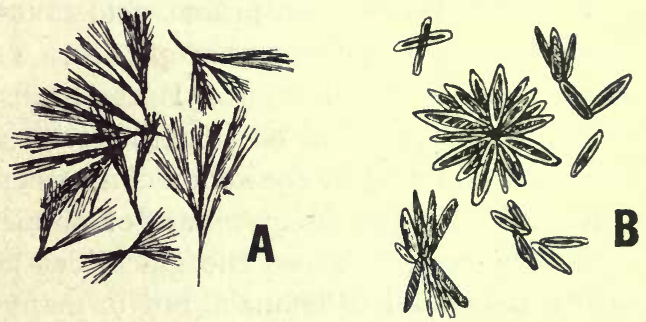

FIG. 22.-Osazones. A, of glucose; B, of maltose (after Plimmer).

of phenylhydrazine hydrochloride in glycerine with which a drop of a solution of sodium acetate in glycerine (Appendix II) is thoroughly mixed. The preparation is heated for about half an hour (although a longer period is often necessary) and allowed to cool. The phenylhydrazine reacts with many of the sugars to form insoluble yellow crystalline compounds, known as osazones. Glucose and fructose produce the same osazone, whose crystals appear as long needles arranged in sheaves (Fig. 22, A); that of maltose forms rosettes or plates of broad needles (Fig. 22, B), whilst in the case of cane-sugar no osazone is produced.

Another method of storage of carbohydrate-material takes the form of strongly thickened cell-walls (cf. p. 30 and Fig. I4, A), as in many seeds (Date, Lupine, Coffee, etc.). Such walls consist 
of so-called reserve-celluloses, polysaccharides which differ somewhat from ordinary cellulose and break down more readily into simple sugars.

Of very common occurrence in plants are complex compounds known as glucosides, which, in the majority of cases, consist of glucose combined with one or more aromatic substances or other organic residue. On boiling with dilute mineral acids they split up into their constituents (hydrolysis). The decomposition of the glucoside within the plant is effected by special ferments which generally occur in distinct cells, so that the chemical process is not initiated until, for some reason (e.g. injury), ferment and glucoside come into contact. Thus, a glucoside amygdalin occurs in the seeds of the Bitter Almond (but not in the cultivated form), whilst the appropriate ferment emulsin is situated in the skin ; on crushing the seeds decomposition of the amygdalin into glucose, benzaldehyde, and prussic acid takes place, this last being responsible for the poisonous properties. The hot taste of many members of the Cruciferæ (e.g. Horse Radish, Cress) is due to the formation of mustard oil (together with glucose and potassium hydrogen sulphate) by the action of a ferment (myrosin) on another glucoside (sinigrin or myronate of potash).

In the two instances just given the glucosides undoubtedly render the plant distasteful to animals, but in many cases they seem to serve as a means of storing glucose in a form which does not diffuse readily. Thus the leaves of many Willows contain a glucoside salicin which, during the night, is split up by the enzyme salicase into glucose and saligenin; the former is removed, whilst the latter combines with the new sugar formed the next day. To the glucosides also belong:- the saponins found in the Soapwort (Saponaria), Quillaia-bark, and many other plants, and easily recognised by the formation of a froth when shaken up with water; the active principles of the Foxglove (Digitalis), the most important being digitalin, which has a profound effect on the action of the heart; and the indican of the Woad (Isatis tinctoria) and of the Indigo-plant (Indigofera), the latter being the source of natural indigo. ${ }^{\mathbb{1}}$

1 Vanillin, the cause of the aroma of Vanilla (obtained from the pod of Vanilla planifolia, a tropical Orchidaceous climber), is an aldehyde, similar to those often combined with glucose to form glucosides. 
Oils or fats form another important group of reserve-substances found especially in those seeds in which carbohydrates are either scanty or absent (e.g. Castor Oil, Sunflower, etc.). Drops of oil are, however, not uncommon in the ordinary vegetative cells, and are particularly abundant in some lower plants [e.g.in the Alga Vaucheria (cf. p. 208), and in the Ergot (Claviceps)]. The vegetable fats are compounds (esters) of glycerine with various fatty acids (palmitic acid, oleic acid, etc.), and are decomposed by ferments known as lipases into these two constituents. In most cases they occur as fluids within the plant, although the fat of the Cocoa (Theobroma cacao), the so-called Cocoa-butter, forms an exception to this.

The oil appears in the cell-sap or protoplasm as shining globular drops of varying size which are readily soluble in ether, benzene, etc. ; some are even soluble in alcohol (e.g. those in the seeds of the Castor Oil). When a considerable quantity of fat is present (e.g. in the Brazil-nut), it can be squeezed out by pressure on to a piece of filter-paper, producing a greasy mark. The oil-globules turn pink or red on treatment with Scharlach Red (Appendix II), and assume a blackish colouration with osmic acid, which, however, also stains proteins and tannins in the same way. If sections of oil-containing material be placed in a solution of concentrated potash and ammonia in equal proportions, the globules after some time lose their sharply defined outline, and often become replaced by needle-shaped crystals. The change, spoken of as saponification, is due to the breaking up of the oil into glycerine and the fatty acid, the latter uniting with the alkali to form the corresponding salt.

Many plant-fats are of considerable economic importance ; thus, olive oil is obtained from the fleshy fruit-wall of the Olive (Olea europaa, mainly cultivated in the Mediterranean region); coconut oil, used in the preparation of margarine, from the ripe seeds of the Coconut Palm (Cocos nucifera) $^{\mathbf{1}}$; and Castor Oil from the seeds of the Castor Oil plant (Ricinus communis). Other important commercial sources are Linseed (Linum usitatissimum), Cotton-seed (Gossypium spp.), Pea-nuts (Arachis hypogaa, with $38-50$ per cent. of oil), and Soja-beans (Glycine spp.). In most cases the oil is extracted after crushing, the ultimate

1 Copra is the commercial name for the dried kernel of the Coconut. 
residue forming so-called “ oil-cake," which is extensively used for the feeding of cattle. Various vegetable oils are, moreover, employed in the manufacture of soap.

In the plant the fats are formed from carbohydrates and, since they contain a much smaller amount of oxygen than the latter, the volume of carbon dioxide evolved during their formation is considerably greater than that of the oxygen taken in. Conversely, when fats undergo change into carbohydrates during the germination of a seed, a large quantity of oxygen is absorbed in proportion to the carbon dioxide evolved. In correspondence with their low specific gravity, fats are a frequent form of nonnitrogenous food-reserve in seeds depending on wind-dispersal.

The carbohydrates or fats found in the various storage-organs are always accompanied by nitrogenous food-reserves, the most important and widespread of which are the proteins. We have already seen that very complex combinations of proteins are organised to form the living protoplasm, but simpler proteins often occur as non-living constituents of the ordinary vegetative cells, and are especially abundant in the diverse storage-organs. In the former case they may either be dissolved in the cell-sap or appear as crystal-like bodies, termed crystalloids, which may even be lodged in the plastids or nuclei. Succulent storageorgans, such as tubers, often likewise contain dissolved proteins, or these may take the form of crystalloids, as in the outer layers of a Potato; but not uncommonly a considerable part of the nitrogenous matter in these cases is a mixture of simpler compounds known as amides (e.g. asparagin in the Potato and glutamin in the Beetroot).

In seeds proteins generally occur as small grains which are well seen in the cotyledons of a Pea or the endosperm of the Castor Oil. If a section of the former be treated with iodine, the minute protein granules take on a brown colouration, in sharp contrast to the blue or blackish starch-grains with which they are intermingled. On warming a section in a few drops of Millon's reagent (Appendix II), the whole assumes a brick-red colour which microscopic examination shows to be due to the proteins. Heating with concentrated nitric acid gives a yellow colouration which, on addition of ammonia, changes to orange xanthoprotein reaction). 
The proteins of oil-containing seeds (e.g. Castor Oil, Brazilnut) occur in the form of especially large granules, known as aleurone grains. These appear to arise, as the seed dries during ripening, from the entire contents of vacuoles rich in proteinsubstance. Although the structure of aleurone grains is relatively complicated, they exhibit the characteristic protein-reactions mentioned above. If a thin section of the Castor Oil or Brazilnut (from which the fat has been removed by soaking in alcohol and benzene respectively) be stained with iodine, the brown-

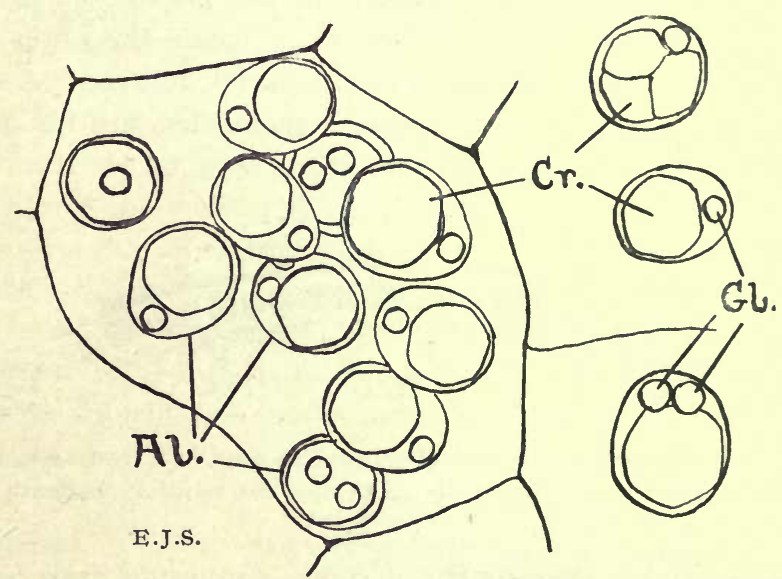

FIG. 23.-Aleurone grains ( $A l$.) from the endosperm of the Castor Oil plant (Ricinus) showing the globoid $(G l$.$) and crystalloid (C r$.$) .$

coloured grains are readily seen under the microscope. Each shows a bounding membrane, the original membrane of the vacuole, enclosing amorphous protein in which two or more bodies are embedded. One of these, the crystalloid (Fig. 23, Cr.), is large and more or less angular, whilst the other, the globoid, is smaller and rounded (Fig. 23, Gl.) ; both consist of protein, but in the globoid, of which more than one may be present, this is combined with a double phosphate of calcium and magnesium. By mounting sections in water the amorphous groundmass of the aleurone grain may be dissolved, and the bounding membrane rendered clearly visible ; on the other hand, treatment 
with dilute potash causes both bounding membrane and crystalloid to swell and disappear.

The protein-granules of the Pea, Bean, etc., are often regarded as small aleurone grains devoid of crystalloid and globoid. Similar structures occur in abundance in the so-called aleurone layer found at the periphery of the endosperm of Grasses (Wheat, Maize, etc.), just within the coat of the grain (Fig. 24). When the latter is detached, the protein-containing layer generally comes away with it, hence the greater nourishing properties of wholemeal bread as compared with that manufactured from white flour. For the same reason peeled potatoes are not so nutritious as those boiled in their skins, since the outer layers of the tuber contain protein-crystalloids (cf. above).

Proteins, owing to their complex molecules, are not readily

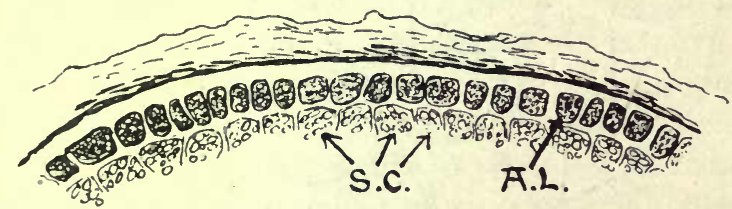

FIG. 24.-Aleurone layer (A.L.) of Wheat as seen in a transverse section. The starch-containing cells (S.C.) lie immediately beneath.

diffusible, and consequently become converted into simpler, freely-diffusing compounds before they are transferred to parts where growth is occurring (e.g. in a germinating seed). This conversion is brought about by so-called proteolytic enzymes (e.g. pepsin, erepsin), which are in general similar to those occurring in the digestive tracts of animals, although some uncertainty exists as to the exact nature of those present in plants. The chief compounds produced, by the action of these enzymes on proteins, are firstly peptones and subsequently amino-acids. The former still exhibit the general characteristics of proteins, although they diffuse more easily, while the latter no longer possess protein-properties. Amino-acids are exceedingly common in growing and other parts of the plant (e.g. leucin in the buds of the Horse Chestnut, tyrosin in seedlings of the Lupine, etc.). 
Approximate Food-content of Various Plant-products

(In percentages of the fresh weight)

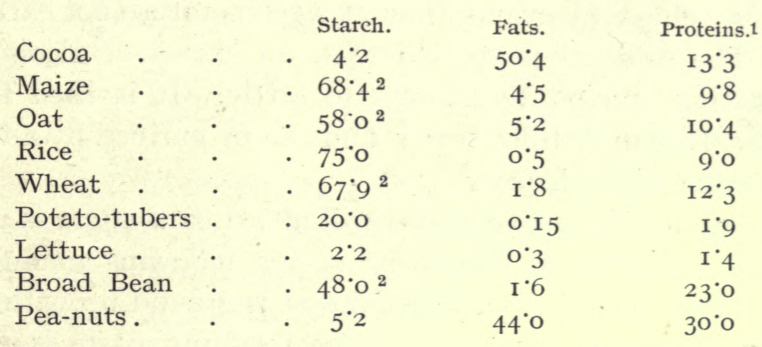

In view of the great importance of enzymes in the activities of the plant, the present chapter may be concluded with a brief consideration of their mode of action. It should be realised that such substances are probably of universal occurrence in living cells. A very large number of enzymes are now known, and others are continually being discovered, but only a few can be mentioned here. The following table gives an epitome of some of the principal enzymes found in plants :

Enzyme. Substance acted upon.

Diastase.

Maltase.

Invertase.

Inulase.

Cytase.

Emulsin, Myrosin, etc.

Lipases.

Proteases (proteolytic en-

$\begin{array}{cl}\text { zymes). } & \text { Proteins. } \\ \text { Zymase (in Yeast, p. 256). } & \text { Monosaccharides. }\end{array}$
Starch.

Maltose.

Cane-sugar.

Inulin.

Reserve-cellulose.

Glucosides.

Oils.

Proteins.
Products.

Dextrin and Maltose.

Glucose.

Glucose and Fructose.

Fructose.

Mannose and Galactose.

Glucose, etc.

Glycerine and Fatty Acids.

Peptones and Amino-acids. Alcohol and Carbon Dioxide.

The method of extraction of diastase described on p. 169 of our Introduction to the Study of Plants is applicable to many

1 In most cases the percentages given are calculated for the entire nitrogenous organic matter.

2 Total carbohydrates. 
enzymes. The material should always be ground to a fine powder, from which an extract is made, usually with cold water ; if the filtered solution is at all bulky, it will be convenient to reduce its volume by evaporation at a temperature not exceeding $50^{\circ} \mathrm{C}$. The solution is treated with an excess of alcohol and the resulting precipitate allowed to settle. It is then filtered off, redissolved in distilled water, and can be purified by a further precipitation with alcohol.

A solution of invertase which will invert cane-sugar is readily obtained, according to Plimmer, ${ }^{1}$ in the following manner. A quantity (Ioo grams) of ordinary Yeast is ground up with about 6 grams of calcium carbonate. The resulting paste is treated with 5 c.c. of chloroform or ether (to kill the Yeast cells), and allowed to stand exposed to the air for three or four days, after which the enzyme is precipitated from the filtrate with an equal volume of alcohol. An impure solution of invertase, which will demonstrate the inversion of cane-sugar, can, however, be obtained by simply mashing Yeast in water with the addition of a little ether, and filtering off the solid matter (preferably through an asbestos filter).

Lipase can be prepared from the seeds of the Castor Oil by cutting up the endosperm into small pieces, and soaking these for a short time in a small quantity of ether in order to remove the oil. The material is then ground into a pulp with a very dilute ( 0.5 per cent.) solution of acetic acid, which sets free the enzyme. The insoluble matter is filtered off, washed till the filtrate gives no acid reaction, and the residue is shaken up with a small amount of water. If some of this suspension be added to a little olive oil, an acid reaction will soon be obtained due to the formation of fatty acids consequent upon the decomposition of the oil.

The preparation of proteolytic enzymes from plant-material is a matter of some difficulty and beyond the scope of this book. The action of such ferments is, however, well illustrated by the use of peptonising powders (containing the enzyme pepsin) in rendering milk more digestible for invalids.

The action of enzymes is very often a hydrolytic one, that

1 Practifal Organ.ic and Biochemistry, p. 399. 
is to say, the compound is broken. down with the addition of water, thus :

$$
\begin{gathered}
\mathrm{C}_{12} \mathrm{H}_{22} \mathrm{O}_{11}+\mathrm{H}_{2} \mathrm{O}=\mathrm{C}_{6} \mathrm{H}_{12} \mathrm{O}_{6}+\mathrm{C}_{6} \mathrm{H}_{12} \mathrm{O}_{6} 1 \\
\text { cane-sugar water glucose fructose } \\
\left(\mathrm{C}_{6} \mathrm{H}_{10} \mathrm{O}_{5}\right)_{n}+n \mathrm{H}_{2} \mathrm{O}=n \mathrm{C}_{6} \mathrm{H}_{12} \mathrm{O}_{6} \\
\text { inulin water fructose. }
\end{gathered}
$$

In other cases, however, the action appears to be different, as, for instance, that of the fermenting enzymes (cf. p. 256) and of the oxidising enzymes or oxidases which are in part responsible for the change in colour of the cut surface of an Apple, and of many Fungi, when exposed to air. Evidence is accumulating in support of the view that all the chemical processes depending on enzyme-action are reversible, taking place in one direction or the other according to the prevailing conditions. Thus, for example, it is believed that the building up of starch from sugar and the reverse process are both dependent on the same set of enzymes. Little is known as to the chemical nature of enzymes, but all of them are colloids, and as a consequence the rate of diffusion through parchment and similar membranes is either very slow or practically nil.

Our knowledge of this class of substances is almost entirely confined to their mode of action. The reactions influenced by enzymes are all such as require an appreciable interval of time for their completion, so that it is possible to measure their rate under any given set of conditions. One of the most important aspects of enzyme-activity is the small quantity of the enzyme necessary to bring about a pronounced chemical change; thus invertase is stated to invert 200,000 times its weight of canesugar. Moreover, at the end of the reaction the enzyme appears to be unaltered both as regards amount and characteristics. In both these respects enzymes resemble the so-called catalytic agents employed in certain chemical processes. As examples we may mention the use of small quantities of manganese dioxide to accelerate the liberation of oxygen from chlorate of potash,

1 Although the formulæ for glucose and fructose are the same, these two compounds differ in the arrangement of the atoms within their molecules. 
and the effect of traces of colloidal (finely divided) platinum, known as platinum black, in causing the explosive combination of oxygen and hydrogen at ordinary temperatures. In either case the reaction is one that would also take place in the absence of the catalytic agent, but with this difference, that the rate would be very much slower. Similarly, there is reason to believe that the changes brought about by enzymes would also occur in their absence, but at so slow a rate as not generally to be capable of recognition. In this connection it is well to recall that much the same effect as is produced by the enzyme can often be attained by the use of other catalytic agents (e.g. boiling with small quantities of mineral acids).

Although the enzyme appears unaltered at the end of the reaction, it is almost certain that combination of some kind with the substances undergoing change takes place while the process is proceeding. In view of the large surface which is presented by colloids, it is very probable that this combination is a physical one (adsorption), and it is thought by many that, in the hydrolytic enzymes, for instance, the water and the compound undergoing hydrolysis are brought into intimate contact at the surface of the ultramicroscopic ferment particles.

Enzymes differ from most other catalysers in that each is usually only effective in accelerating one or few particular reactions (cf. p. 53), and they are, moreover, very sensitive to heat and light. The rate of the reaction is doubled or trebled with every rise of $10^{\circ} \mathrm{C}$., but soon a temperature is reached (usually about $60^{\circ} \mathrm{C}$.) at which most enzymes are destroyed. It may be added that heating which suffices to kill the protoplasm leaves ferments unharmed. Strong light destroys them very quickly, an effect for which the violet end of the spectrum appears to be mainly responsible. Many chemical compounds (e.g. sulphuretted hydrogen, prussic acid, chloroform, etc.) arrest enzyme-action to a more or less marked degree according to their concentration. If the products of enzyme activity are not removed, a retardation of the process is at once manifest, continued action of diastase, for example, being dependent upon the removal of the maltose. In some cases the accumulation of the products of the reaction actually exerts a poisonous effect, as, for instance, that of the alcohol produced by Yeast. Usually, however, the substances 
resulting from enzyme-action are removed by the plant as soon as formed.

Enzymes frequently act only in the presence of another substance, an activator, which is commonly a salt, acid, or alkali, although sometimes more complex. These activators differ from the enzymes in being able to diffuse through a parchment membrane, and can consequently be separated from the latter by dialysis. ${ }^{1}$ Examples are afforded by the small quantity of acid requisite for the action of pepsin, and the necessity of the presence of certain complex phosphatic compounds for the action of the zymase of Yeast.

Just as these substances have the effect of accelerating enzyme action, so, too, there are others which exert a retarding influence, and there is every reason to believe that either the one or the other can be produced as may be required by the living cell. Moreover, the rate of the reaction depends on the amount of the enzyme present, and this latter is regulated by the cell's activity. It is clear, then, that the character and vigour of catalytic activity is subject to considerable modification, and is indeed intimately related to the momentary requirements of the organism.

1 That is to say, placing the mixture of enzyme and activator in a parchment tray floating on a large bulk of water. 


\section{CHAPTER V}

\section{Cell-Contents (By-PRoducts, ETC.)}

THE by-products comprise chemical compounds formed during the metabolism of the plant which do not, as far as our present knowledge goes, appear to play any further part in the elaboration of food-substances. This does not, however, mean that they fulfil no functions in the living organism, for they may be of importance in warding off the attacks of preying animals, in the creation of attractive mechanisms, in the reduction of transpiration, etc.

One of the most widespread of the by-products is oxalate of lime, which may occur in practically every organ and tissue of the plant. It is produced by the neutralisation of the oxalic acid formed during metabolism and, being insoluble, appears in the shape of crystals which assume diverse forms. Large solitary crystals (Fig. 25, C), each occupying the greater part of the cell-contents, are very common; but most frequent are clusters, or rosettes, of crystals radiating from a dark centre (Fig. 25, A). Another widespread type takes the form of bundles of needle-shaped crystals (raphides), generally situated in enlarged cells containing mucilage (Fig. 25, B). This last type is particularly characteristic of the Monocotyledons, though by no means lacking in Dicotyledons (e.g. Enchanter's Nightshade). In some few cases, especially in the Solanaceæ, the oxalate of lime is deposited in the form of a powder-like mass of numerous very minute crystals (so-called crystal-sand).

Crystals of calcium oxalate are always found in quantity where active metabolism is going on. They are often very abundant in the tissues adjoining actively secreting organs 
(cf. p. I44); also in the leaves of deciduous plants, just prior to leaf-fall, features which respectively emphasise that this substance is a by-product, and that it is not generally useful to the organism.

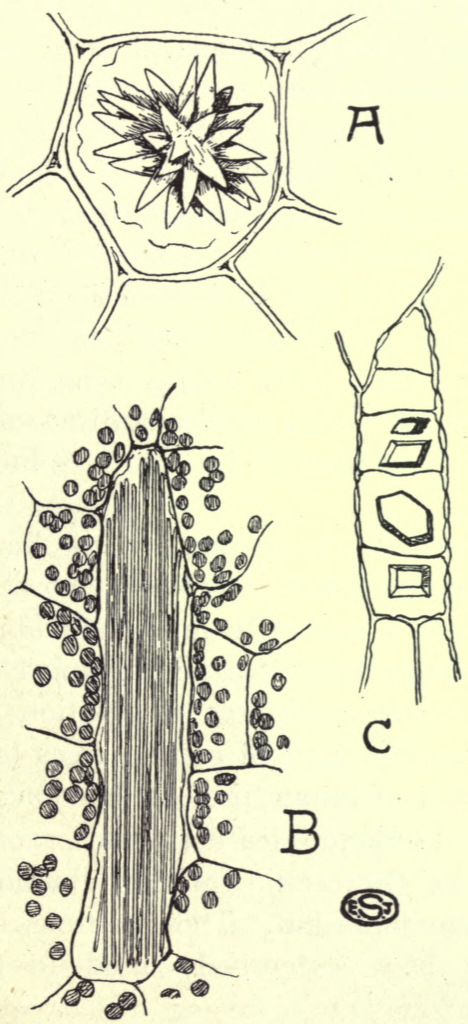

Fig. 25.-Crystals.' A, Cluster crystals from leaf of Dog's Mercury (Mercurialis). B, Raphides from leaf of Enchanter's Nightshade (Circaa). C, Solitary crystals from phloem of Horse Chestnut (ÆIsculus).

The crystals of calcium oxalate are not soluble in acetic acid, but readily dissolve in sulphuric acid, with the production of calcium sulphate. The latter, being itself insoluble, becomes deposited promiscuously in the form of needle-like crystals. These tests serve to verify the presence of oxalate of lime, but in most 
cases their application is unnecessary, since crystals of other inorganic compounds are very rare.

Many by-products occur in solution in the cell-sap, and of these the commonest are diverse organic acids and bodies known as tannins. The former are responsible for the frequently acid reaction of the sap, and are especially abundant in unripe fruits. As examples mention may be made of malic acid (in Apples), citric acid (in Lemons), tartaric acid (in Grapes), etc. The name of tannins is given to diverse organic substances, whose chemical constitution is not fully established, but all of which possess an astringent taste and are characterised by the following reactions: They reduce Fehling's solution, are precipitated by the salts of many metals (e.g. basic lead acetate), and take on a blue-black or greenish colour with ferric chloride. Dilute iodine solution, together with a little ro per cent. ammonia, gives a brilliant red colour even with small quantities of tannins, whilst they are readily precipitated by dilute solutions of caffein (cf. p. I3).

Tannins are particularly common in the bark of trees (e.g. Oak, Alder), in unripe fruits (e.g. Pear, Acorn), in leaves (e.g. Bracken), and occur abundantly in certain abnormal growths (e.g. Oak-galls). They are often accompanied by a yellow or reddish pigment which facilitates recognition of the cells in which they occur. The bark of certain trees (e.g. Oak), owing to the large quantity of tannin present, has long been employed in the conversion of hide into leather, which process depends on the coagulation, by the tannin, of the albuminous substances contained in the animal skin. The reactions of tannins with ferric salts have been extensively used in the preparation of ink.

Another group of by-products, encountered particularly in certain families of Flowering Plants (e.g. Ranunculaceæ, Scrophulariaceæ, Solanaceæ, Umbelliferæ), are the alkaloids. These are complex basic organic compounds containing nitrogen, which are either dissolved in the cell-sap or present in the solid state; in the plant they are often combined with organic acids. The alkaloids are of such importance, owing to their poisonous and medicinal properties, that the following list of some of the more familiar is given: 
Alkaloid.

Aconitine

Atropine

Cephaeline

Cocaine

Coniine

Hyoscyamine. Poisonous (seda-

- Emetic (active principle of ipecacuanha).

- Local anæsthetic.

- Poisonous (paralytic effect) . tive)

Morphine

Nicotine .

- Narcotic (active principle of opium

Quinine . Febrifuge .

- Poisonous

Strychnine
- Poisonous, and respiratory stimulant.
Source, etc.

Leaves, root, etc., of Monkshood (Aconitum napellus).

All organs of Deadly Nightshade or Belladonna (Atropa belladonna), seeds especially of Thornapple ( $D a$ tura stramonium, Fig. 26) (Solan. aceæ).

Root of Psychotria ipecacuanha (Fam. Rubiaceæ, Brazil).

Leaves of Erythroxylon coca (Fam. Linaceæ, Bolivia and Peru).

Seeds of Hemlock (Conium maculatum, Umbelliferæ).

Henbane (Hvoscyamus niger), Atropa belladonna, Datura stramonium (Fig. 26).

Young fruits of Opium Poppy (Papaver somniferum).

- Leaves of Tobacco (Nicotiana tabacum, Solanaceæ).

Bark of Cinchona spp. (Rubiaceæ).

Seeds of Strychnos nux-vomica (Fam. Loganiaceæ).

The alkaloids as a whole are not characterised by any very specific reactions, but they are precipitated from solution by many different reagents (e.g. iodine in potassium iodide, tannic acid). They give very marked colour-reactions with various substances; thus a section of the rhizome of the Monkshood treated with a little 50 per cent. sulphuric acid shows a bright red colouration in the parenchyma adjoining the vascular strands, as a result of the presence of aconitine.

The ptomaines, which are basic in character, are compounds produced during the decomposition of flesh, etc., by the agency of Moulds and Bacteria, but it is not altogether certain that the effects of so-called "ptomaine-poisoning" are solely due to these substances. Such stimulants as the caffeine of tea-leaves, coffee-beans, cocoa-beans, and Kola (Cola acuminata), and the theobromine present in the Cocoa, are derivatives of purine and very similar to the alkaloids.

The characteristic and often pleasing odour of many Labiatæ (e.g. Lavender, Mint, etc.) and Umbelliferæ (e.g. Fennel), as well 
as of flowers, is due to the presence of so-called volatile or ethereal oils, which are composed of mixtures of hydrocarbons (i.e. compounds containing carbon and hydrogen only), known as terpenes, and of their oxygen derivatives. Examples are: lavender oil from the flowers of the Lavender (Lavendula); peppermint oil, which contains the antiseptic menthol, from the Peppermint (Mentha piperita) ; bergamot oil, used in the manu-

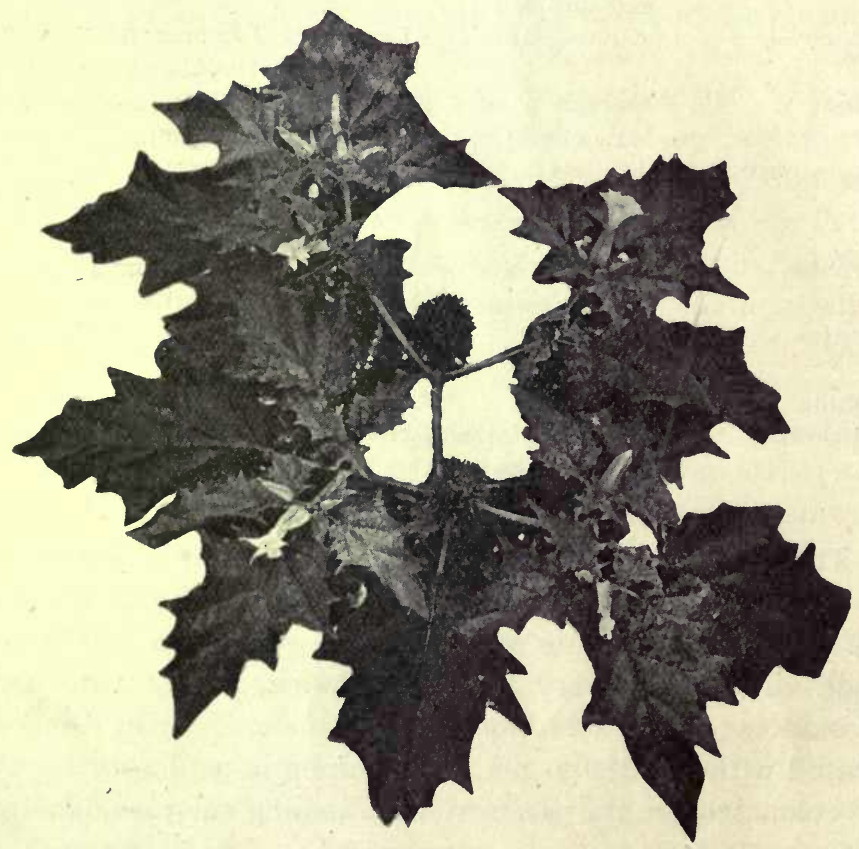

Fig. 26.-Photograph of part of the shoot, including two fruits, of the Thornapple (Datura stramonium), which contains the alkaloids atropine and hyoscyamine. [Photo E. J. S.]

facture of Eau de Cologne and other perfumes, from the Bergamot Orange (Citrus aurantium var. bergamia), a variety of the ordinary Orange ; oil of aniseed from the Aniseed (Pimpinella anisum); and the numerous oils from the many species of Eucalyptus (Myrtaceæ). Many of these are used commercially in the preparation of perfumes. Similar oils are the essential principles of such spices as Cloves (the flower-buds of Eugenia caryophyllata) 
Cinnamon (the bark of young twigs of Cinnamomum zeylanicum), Ginger (the rhizome of Zingiber officinale), Pepper (the berries of Piper nigrum), etc. Moreover, the active principle of the Hop (Humulus lupulus), which is contained in special hairs (cf. p. I05) borne on the bracts of the female catkins, and that causing the odour of Tea, likewise belong to the ethereal oils.

Camphor is a solid terpene-derivative obtained from the wood of the Camphor-tree (Cinnamomum camphora), whilst turpentine is a mixture of terpenes which flows from the resin-passages (cf. p. 339) in the trunks of various species of Pines (especially Pinus pinaster) and of the Spruce Fir (Picea excelsa), when cuts are made in the surface. After the oil of turpentine has been distilled off, the solid residue left is rosin.

Most of the terpenes are colourless, highly refractive liquids, which evaporate completely if sections containing them are heated on a slide for about ten minutes. They are readily soluble in alcohol, chloral hydrate, glacial acetic acid, etc. Compounds of this nature, often together with other substances, commonly occur in specially differentiated cells or intercellular spaces within the tissues of the plant (cf. pp. I5I, I52), or in glandular hairs (p. I05). The ethereal oils are sometimes combined with glucose, etc., in the form of glucosides (e.g. the mustard oil of Cruciferæ, cf. p. 48), and become liberated only after coming in contact with the appropriate enzyme.

The colours of flowers are due in many cases to pigments, which are classed as anthocyanins, dissolved in the cell-sap of the petals, the colour being red, blue, or violet according to the acid or alkaline reaction of the sap. Such pigments are also frequently present in the vegetative organs, as, for instance, in the Beetroot and in the leaves of the Mother-of-Thousands (Saxifraga sarmentosa). Their development appears to be stimulated by excessive transpiration and intense illumination, conditions which are realised in alpine and arctic regions where high colouration is a conspicuous feature.

The yellow and red colouration of many flowers (e.g. Garden Nasturtium) and fruits (e.g. Tomato) is, however, not due to substances in the cell-sap, but to the presence of pigments (carotin, etc.) in special plastids, termed chromoplasts. In many cases the pigment occurs in the latter in a crystalline form. 
Carotin is present in considerable quantity in the root of the Carrot, to which it imparts the orange colour.

A considerable number of plants are still used as the source of dyes; thus the dried stigmata of Crocus sativus yield saffron, and the rhizome of Curcuma longa (tropical Asia) the yellow dye turmeric.

It is doubtful whether any of the substances dealt with in this chapter are produced for their intrinsic utility to the plant. But it is quite possible that the poisonous alkaloids, the astringent tannins, or the pungent resins may render the plants containing them distasteful to herbivorous animals, and so prove indirectly beneficial. Similarly the antiseptic character of resin may well be of service in protecting a wound over which it has congealed. Tyndall showed that the vapour of ethereal oils tends to diminish the passage of heat through air, so that their common occurrence in plants of dry situations may be a means of checking evaporation by retarding the heating effect of sunshine. It may be, however, that some of the substances here referred to constitute a means of storage of food-material, and this applies especially to some of the tannins which seem to have the structure of glucosides. 


\section{CHAPTER VI}

\section{The Structure of Roots}

IN the mature plant the different types of elements are grouped together to form tissues subserving diverse functions. Thus, for instance, one tissue, which is always superficial and has the general function of protecting the underlying parts, constitutes the epidermis. The vascular tissues, comprising the wood or xylem and the bast or phloem, form the respective conducting systems for water and mineral salts, and for elaborated food-substances, whilst others-largely parenchymatous in character-go to form the general matrix or ground tissue. It must not be supposed, however, that, because there is one general function, the component elements of these and other tissues are necessarily uniform in character, a fact that will be best realised by the study of concrete examples.

In every young root we can distinguish a number of regions, beginning with the root-cap, covering the growing point (Figs. 9 and $30, r . c$.$) , and followed successively by the zone of elongation,$ the zone bearing root-hairs, and the older part where the roothairs have withered and the lateral roots (Fig. 30, l.r.) are seen emerging through the surface-layers, commonly in four or five vertical rows. In a longitudinal section through the tip of the root the cap is found to consist of a number of concentric layers of thin-walled parenchymatous cells, whose arrangement becomes less regular towards the outside (Fig. 9). The outermost cells gradually become mucilaginous and break down, whilst the substance of the cap is constantly renewed from the underlying meristem. A little way behind the growing point (p. 20) the middle of the root is occupied by a continuous, rather denser strand of elongated elements, which develop into the vascular tissue, and which offer a marked contrast to the thin-walled parenchymatous cortex around. 
The general structure of the root can best be studied in a cross-section through the mature region, the Creeping Buttercup (Ranunculus repens) furnishing suitable material for a first example. Under the low power of the microscope the broad parenchymatous cortex (Fig. 27, C.), whose cells contain numerous starch-grains $(s$.$) , and the central conducting strand, are again$

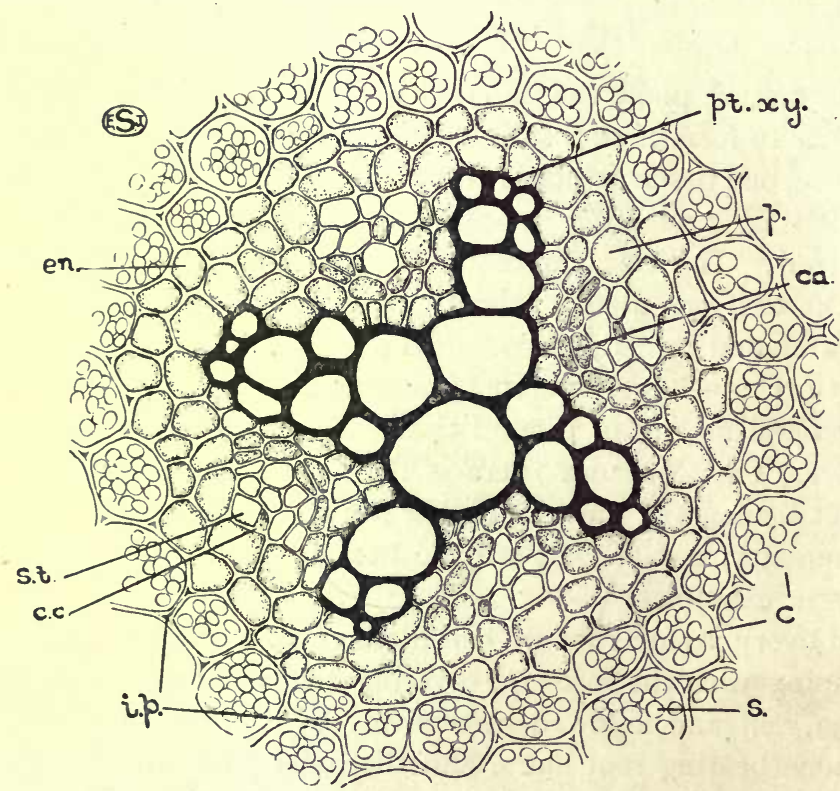

FIG. 27.-Transverse section of the central part of the root of the Creeping Buttercup (Ranunculus repens). The walls of the xylem elements are shown black. C., inner part of cortex ; ca., cambium ; c.c., companion cell ; en., endodermis; i.p., intercellular spaces; $p$. , pericycle ; pt.xy., protoxylem ; s., starch ; s.t., sieve-tube.

sharply contrasted. At the edge of the section is a layer of shrunken cells (Fig. 28, r.), some of which are prolonged into shrivelled root-hairs. This epidermis withers above the zone of root-hairs and, since its chief function is their production, it is more usually termed the piliferous layer. It is only in sections cut nearer the growing tip that its cells are as yet uncontracted, and can be seen to form a single layer.

The root-hairs, each arising from a separate cell (Fig. 28, r.), 
appear as tubular unbranched outgrowths with bluntly rounded tips. The greater part of any root-hair is occupied by a large vacuole continuous with that of the epidermal cell and filled with sap. There is consequently only a thin lining layer of cytoplasm, which is best seen near the tip where the single nucleus usually lies embedded.

Beneath the withered piliferous layer, in the older part of the root, lies the exodermis (Fig. 28, Ex.), a layer of protective cells which are on the whole rather smaller than the adjacent cells of the cortex. Their brownish, slightly thickened walls are chemically changed (i.e. suberised, cf. p. I36) in such a way that

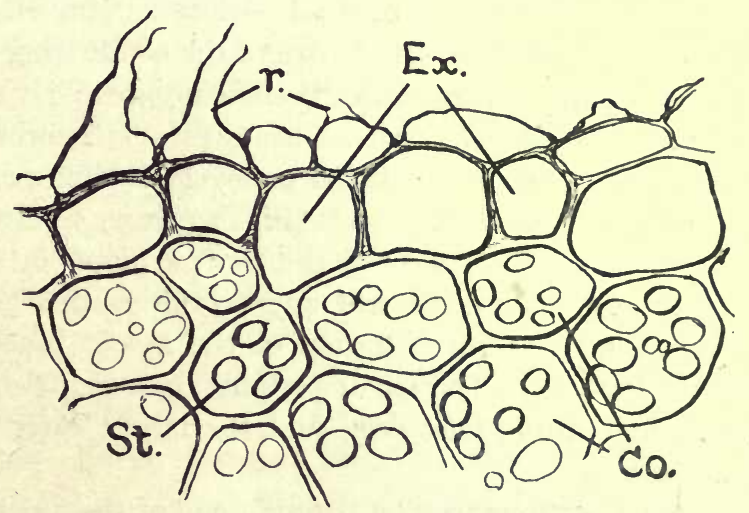

FIG. 28.-Piliferous layer (in part withered) and exodermis of the root of the Creeping Buttercup (Ranunculus repens). Co., cortex ; Ex., exodermis ; $\boldsymbol{r}_{\text {, }}$, root-hairs arising from cells of piliferous layer ; St., starch.

they are almost impermeable to water; but this alteration does not usually take place till the root-hairs begin to wither, so that the water they absorb can readily pass inwards to the vascular cylinder. In some cases, however (e.g. in most Monocotyledons), where the exodermis differentiates at an early stage, thin-walled passage-cells (Fig. 33 P.), through which the water travels, occur at regular intervals.

The cell-walls of the cortex become thinner towards the centre, and intercellular spaces (Fig. 27, i.p.) are abundant. The latter form a continuous system which permits of gaseous exchange with the aerial parts of the plant. The vascular strand is de- 
limited from the cortex by two well-defined layers of cells, an outer, the endodermis (en.), and an inner, the pericycle (p.) (cf. below).

In sections stained with aniline chloride a four-rayed group of yellow elements, composing the lignified wood or xylem (Fig. 27), is seen to occupy the greater part of the conducting strand. In the bays between the four arms of the xylem are oval groups of small-celled unstained tissue, the phloem, in which the wide and empty-looking sieve-tubes (s.t.) are plainly distinguished from the narrower companion-cells (c.c.) with their dense contents (cf. p. 28). Each phloem-group is separated from the adjacent xylem by one or two layers of parenchyma (ca.).

The xylem consists chiefly of dead, empty-looking elements, the vessels, of which those at the centre of the conducting strand are the largest, whilst the remainder become progressively smaller in passing outwards along any one of the rays. The end of each xylem-arm, immediately beneath the pericycle, is thus occupied, by a strand of the narrowest vessels (Fig. 27, pt.xy.). In crosssections, through younger parts of the root, a larger or smaller number of the central vessels will appear thin-walled and unlignified, showing that differentiation of the xylem takes place from without inwards. The small peripheral elements are consequently spoken of as protoxylem, and the larger, later-formed ones, as metaxylem.

Longitudinal sections passing through one of the xylem-arms will show that the vessels of the protoxylem are spirally thickened, whilst those of the metaxylem bear bordered pits. The walls between the larger vessels, in the transverse section, exhibit a thin dark line down the middle (the middle lamella), with the thickening layers on either side. These latter are not homogeneous, however, but appear to consist of short dark lengths alternating with lighter and narrower portions, where the pits are seen in optical section.

The cells of the endodermis (Fig. 27, en.) are distinctly smaller than the adjacent cortical cells and somewhat flattened. Their radial walls are thicker and look darker than the others, although when sharply focussed in optical section they appear bright owing to their highly refractive character. On treatment of a section with strong sulphuric acid, the membrane swells and dissolves, 
except for the radial walls, which persist unaltered, implying that they are chemically different from the other walls of the endodermal cells. These features of the radial walls are commonly exhibited by the endodermis of roots.

The purpose of the endodermis is still obscure, but such a layer, showing the characteristic thickening of the radial walls, is found in the roots of many Flowering Plants, as well as in the stems of aquatics (cf. p. I $7 \mathrm{r}$ ). The firm lateral connection of the cells due to the thickening renders this layer an efficient sheath to the vascular system. The considerable thickening of the entire endodermal wall which obtains in some cases (cf. below), suggests a probable mechanical value. In the unthickened state the chief function of this layer may perhaps be to cut off the water-conducting strand from the air-spaces of the cortex, a delimitation rendered necessary by the frequent negative pressure ${ }^{1}$ within the vascular system. Not uncommonly the layer of cortical cells next to the endodermis develops characteristic thickenings on the transverse and radial walls, a feature well seen in the roots of many Cruciferæ (e.g. White Mustard), and no doubt of mechanical value.

The pericycle whick lies immediately within the endodermis is another continuous layer not characterised by any structural peculiarities (Fig. 27, p.).

Many of the features just described are typical of roots generally, viz. the aggregation of the vascular tissue near the centre; the alternation of phloem and xylem resulting in a radial structure; the peripheral location of the protoxylem; the wide cortex ; the differentiation of the endodermis ; and the occurrence of exodermis and piliferous layer. Roots differ among one another in two principal respects-namely, as to the number of phloem-and xylem-strands, and in the presence or absence of parenchyma (pith) in the centre of the conducting tissue. As regards the former feature, there may be two or more of such strands, roots being described as diarch, triarch, tetrarch (Fig. 27), pentarch (Fig. 30), etc., according as the number of alternating xylem- and phloem-groups is two, three, four, or five, etc., whilst when they are numerous the structure is said to be polyarch (Fig. 29). Thus the root just examined is tetrarch, that of the 
Wallflower diarch, and that of most Monocotyledons polyarch. A pith composed of parenchyma, which is sometimes thickwalled, is frequent in the roots of herbaceous Dicotyledons (Fig. 30) and in Monocotyledons (Fig. 29), . but in woody Dicotyledons and Conifers the xylem-groups often meet at the centre. Roots also vary in the manner of thickening of the mature cells of the endodermis.

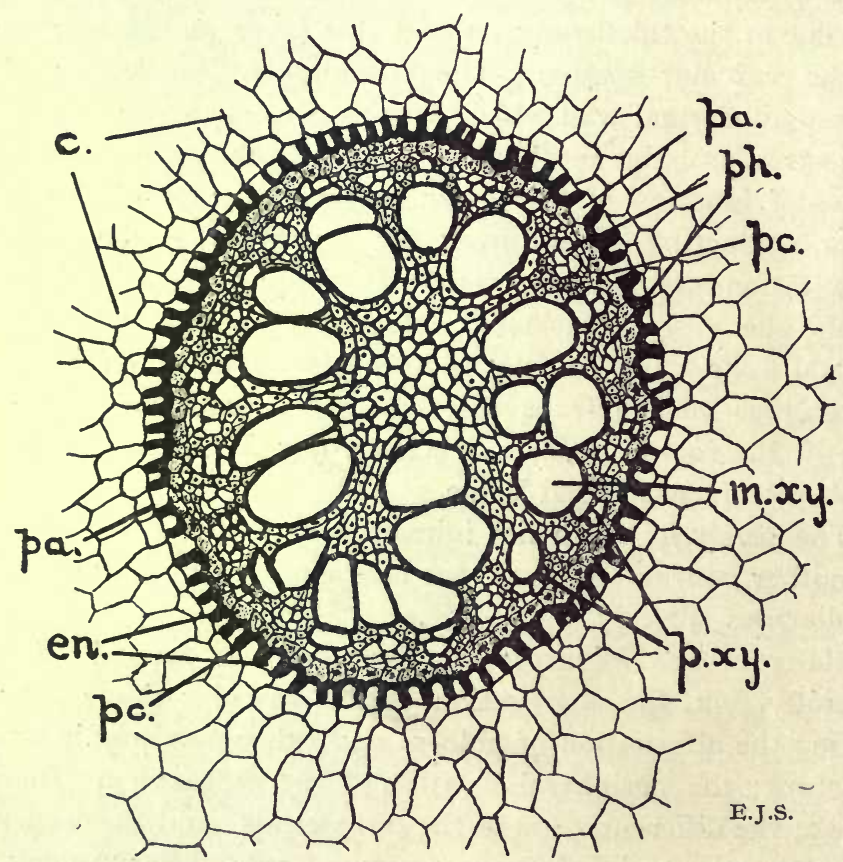

FIG. 29.-Transverse section of the central part of the root of the Iris. $C .$, cortex ; en., endodermis ; m.x.y., metaxylem ; pa., passage cells ; pc., pericycle ; ph., phloem ; p.xy., protoxylem.

A transverse section of the root of the Iris illustrates these features. It exhibits the typical Monocotyledonous structure, viz. a central pith and numerous alternating groups of xylem and phloem (Fig. 29). The endodermis (en.) is conspicuous owing to the marked thickening of all but the outer walls of most of its cells. Opposite the protoxylem groups ( $p . x y$.), however, the endodermal cells are often thin-walled, and such passage-cells ( $p a$.) 
serve for the transference of water through the endodermis, which is otherwise impermeable. A thickened endodermis interrupted by thin-walled passage-cells is found particularly amongst Monocotyledons (see also Fig. 33, $P$ a.).

The mode of origin of the laterals can be readily studied in longitudinal sections through a Bean root. The lateral roots arise by division of pericyclic cells, either opposite the protoxylem

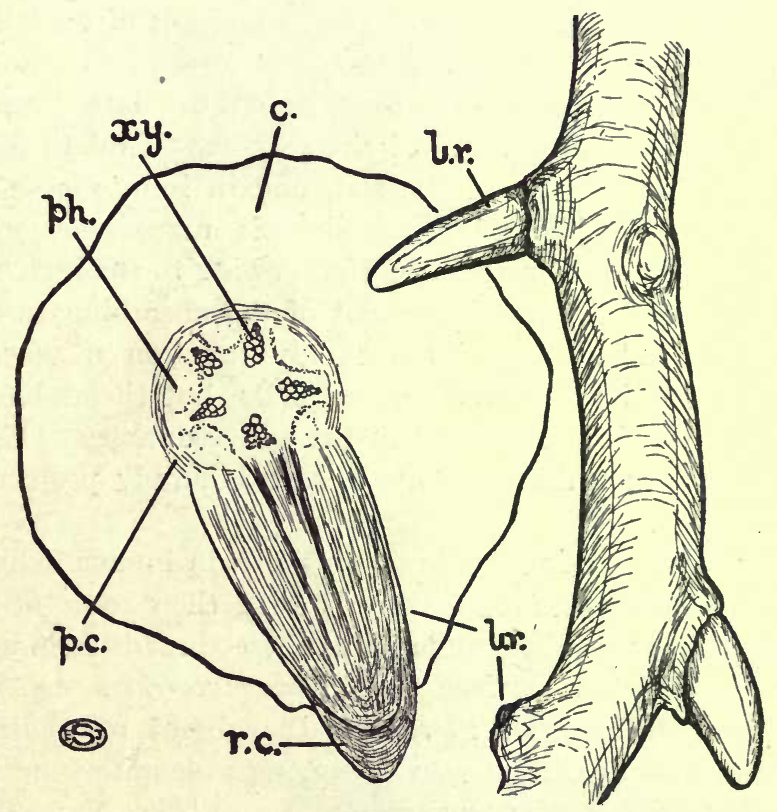

FIG. 30.-The root of a Bean seedling in surface-view and in transverse section showing the origin of the lateral roots (i.r.). C., cortex; ph., phloem ; p.c., pericycle ; r.c., root-cap; $x y$., xylem.

strands (Fig. 30, l.r.), or between these and the phloem, so that in Dicotyledons they form vertical series equal to or double the number of xylem groups. In Monocotyledons, owing to the numerous protoxylem-strands, and the early decay of the taproot, this arrangement is often obscured. In the course of their further development, the side-roots push their way through the cortex, so that, by the time they emerge at the surface, the protective root-cap is fully formed (Fig. 30, r.c.). This so-called 
endogenous origin contrasts with the superficial origin of the branches of the stem, and can be related to a need for protection, until the young root has developed sufficiently to withstand the resistance of the soil.

It is now possible to inquire how the structure of the root is related to its two principal functions, viz. absorption of water from the soil and the anchorage of the plant. Absorption of water takes place through those regions in which the piliferous layer is still intact and living. The outgrowth of its cells into root-hairs, which are the chief organs of absorption, affords an enormously increased surface over which the latter can take place. The size and number of the root-hairs tend to decrease with increasing wetness of the soil, and in some marsh-plants (e.g. Marsh Marigold) and a considerable number of aquatics they may be altogether absent. Here, owing to the high watercontent of the soil, an enlargement of the absorbing surface is unnecessary. The root-hairs not only function in absorbing the water with its dissolved mineral salts, but themselves play a part in rendering substances in the soil available. The older part of the root which does not absorb is amply protected by $\checkmark$ the exodermis.

Many plants characteristic of soils rich in humus exhibit an intimate relation of fungal threads with their roots or other underground organs. In some cases these threads form a dense weft over the whole surface (ectotrophic mycorrhiza, e.g. Beech, Birch, etc.), and appear to replace the absent root-hairs. In others the Fungus can be seen occupying a definite zone within the cortex [endotrophic mycorrhiza, e.g. Bird's Nest Orchid, (Fig. 3r, M.), Heather, etc.]. The advantage of association with the Fungus would appear to depend mainly on the power of the latter to break down and absorb the organic material which is then in part utilised by the Flowering Plant.

$\checkmark$ The root is suited to its anchoring function by its more or less extensive branching, and the central location of the mechanical elements which enable it to withstand the pulling strain to which it is subjected when the shoot sways to and fro in the wind. In most roots the mechanical tissue is constituted merely by the xylem, as well as by the pith when the latter is thickwalled, but in the Pea, Bean, and other Leguminosæ groups of 
fibres are developed in relation to the phloem. When roots serve not only for anchoring, but also for the support of the plant, a modified structure may obtain. Thus, a cross-section of the prop-root of the Maize (Fig. 32) contrasts with one of an ordinary root of this plant in the presence of a special cortical ring of mechanical elements and the larger size of the central cylinder,

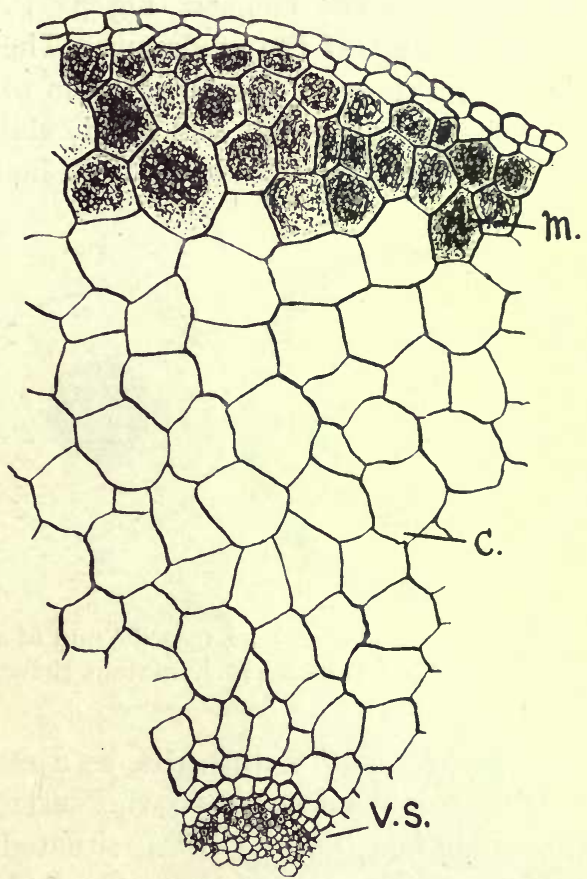

FIG. 31.-Transverse section of the outer part of one of the underground branches of the Bird's Nest Orchid (Neottia nidus-avis), showing the vascular strand (V.S.), the cortex $(C$.$) and the mycorrhizal zone (M.).$

so that the xylem also is more peripheral. In both these respects the mechanical construction of such a prop-root ap- proaches that of a stem (cf. p. 87).

The aerial roots of many tropical epiphytes (e.g. Orchids) also exhibit a modification of structure in correspondence with their special method of absorption and their function as assimilatory organs. The cortical cells frequently contain chlorophyll, 
a feature doubtless merely related to their growth in the light, since ordinary terrestrial roots (e.g. those of the Pea) will often become green when exposed to illumination. In extreme cases (e.g. Taniophyllum) the entire carbon dioxide assimilation is carried out by means of the aerial roots, which assume a leaf-like appearance, whilst the true leaves are reduced to mere scales. The absorption of water in such roots is accomplished with the aid of a tissue known as the velamen (Fig. 33, V.), formed by a remarkable development of the epidermis. The dermatogen usually divides to form several, or many, layers of cells, which ultimately lose their living contents and often exhibit a peculiar spiral or reticulate thickening. Moreover, large holes frequently

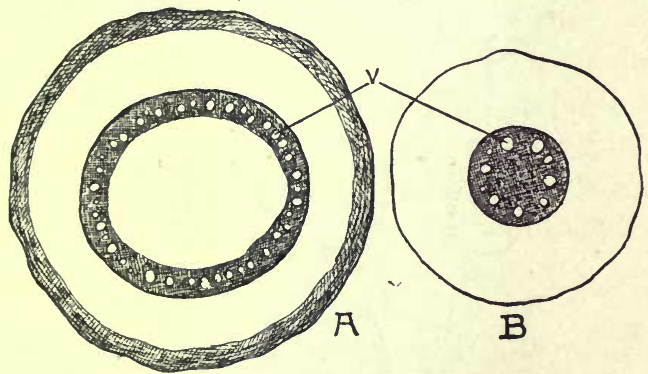

FIG. 32.-Diagrams of a normal absorbing root (B) and of a prop-root (A) of the Maize (Zea mais). The sclerenchymatous tissues are indicated by shading. $V$., vessels.

develop in the walls of many of the cells, as a result of which any moisture falling or condensing on the surface is rapidly absorbed. , The exodermis (Fig. 33, Ex.), situated at the inner edge of the velamen, is interrupted by thin-walled passage-cells $(P$.$) for the inward transference of the water absorbed. In$ dry weather air fills the cells of the velamen, so that they appear white and opaque; but when occupied by moisture they become translucent and the green colour of the cells beneath is visible.

The cells in the central region of the root-cap in many roots contain numerous large starch-grains, which readily move from one part of the cell to another, when the direction of the root is altered. Thus, in the normal erect position they lie against the cytoplasm lining the lower walls, but if the root be placed 
horizontally they rest on the side walls. Similar readily movable bodies (not always consisting of starch, however) have been found in herbaceous stems which are sensitive to gravity. Since detailed experiments have established that the stimulus of gravity is most readily perceived in the root-tip, ${ }^{1}$ some Botanists

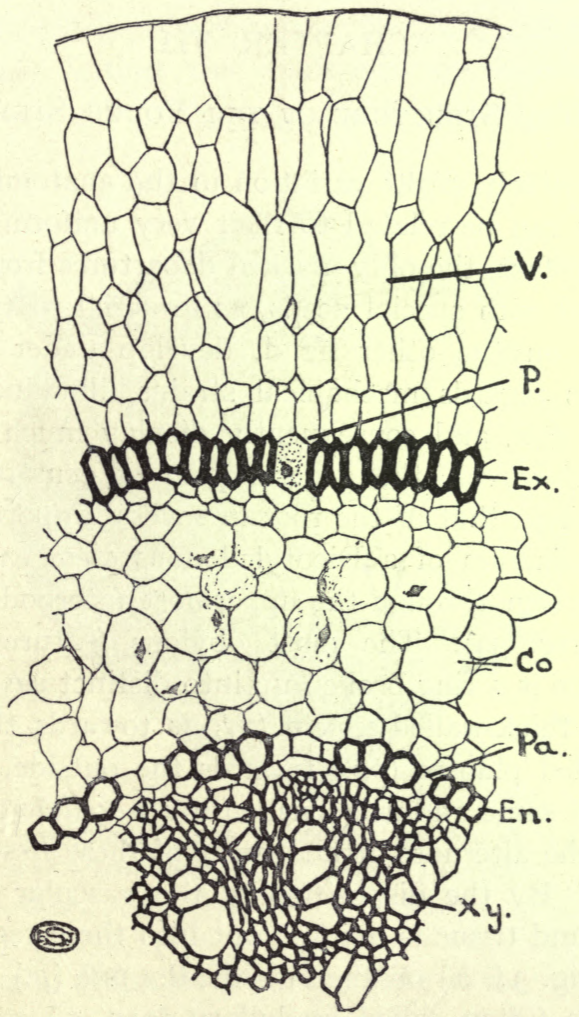

FIG. 33.-Transverse section through part of a root of an epiphytic Orchid (Dendrobium), showing the velamen $(V$.$) . Co., cortex ; En., endo-$ dermis; Ex., exodermis ; $P$. and $P a$., passage cells ; $X y_{\text {., }}$ xylem.

regard the impact of these falling starch-grains as the mechanism for perceiving orientation. No adequate proof of this hypothesis has, however, yet been given, the mechanism of gravity-perception being still unsolved. 


\section{CHAPTER VII}

\section{The Structure of the Young Stem}

THE comparatively slight variation in the anatomical construction of roots can be related to their very uniform environment (the soil) ; in fact, the only marked departures from the normal are associated with special needs, as in aerial roots, prop-roots, etc. Stems, on the other hand, develop under very diverse conditions as regards mechanical strains, illumination, supply of moisture, etc., and consequently display much more varied structure. Moreover, the general plan of construction of the stem differs from that of the root in several important respects.

The organisation of a Dicotyledonous stem can be studied in a cross-section through the uppermost internode of a young Sunflower (Fig. 34). The most obvious feature is that the vascular tissue is a ring broken up into distinct yascular bundles. In each the thick-walled xylem $(x y$.$) is towards the inside and$ the thin-walled phloem ( $p h$.) towards the outside, the two thus lying on the same radius (constituting a collateral bundle), in contrast to the alternate arrangement of these tissues obtaining in the root. By the disposition of the vascular bundles in a ring the ground tissue is marked out into three regions, namely the cortex (Fig. $34, c$.) on the outside, the pith ( $p$.) in the centre, and the rays (often called medullary rays, $r$.) connecting the two and separating adjacent bundles. The terms pith, cortex, and rays are, however, purely topographical, and do not necessarily imply any differentiation between the component cells, which in fact, in most cases, are largely thin-walled and parenchymatous in all three regions (cf. Fig. 36, Co. and p.). The edge of the section is bounded by an epidermis ${ }^{1}$ which differs

1 For a detailed consideration of epidermis and stomata, see the next chapter. 
from that of the root in having strongly thickened outer walls. It forms a continuous layer except where interrupted by the presence of stomata ${ }^{1}$ or lenticels (p. I39). Its function is to protect the more delicate internal tissues, and in correspondence with this it does not wither at an early stage like the piliferous layer. Occasional cells grow out into hairs (Fig. 34, h.), but these are quite different in character from the root-hairs.

Immediately within the epidermis are several layers of collenchymatous cells (Fig. 34, s.), thickened mainly on the

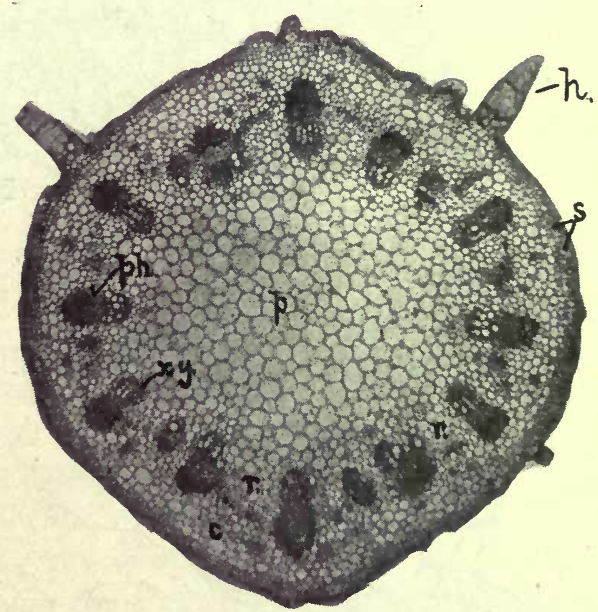

FIG. 34.-Photomicrograph of a transverse section of the young stem of the Sunflower (Helianthus). c., cortex; $h_{.}$, hair ; $p_{.}$, pith ; $p h$. , phloem; $r$, rays ; $s$, collenchyma ; $x y$., xylem.

tangential walls, whilst between the cells of the inner layers are relatively large intercellular spaces. Such spaces are also abundant in the remainder of the ground tissue, and form small triangular cavities between the polygonal cells of the pith.

At the inner limit of the cortex there is a single layer of cells distinguished. by the frequent presence of large starch-grains (Fig. 36 , sh.), which can be rendered more prominent by staining with iodine. The layer in question bends outwards around each

1 For a detailed consideration of epidermis and stomata, see the next chapter. 
vascular bundle, and so presents a sinuous outline. Such a starch-sheath is not uncommon in herbaceous Dicotyledonous stems (cf. also Fig. 57, S.s.), and its readily movable starch-grains have been regarded as fulfilling a similar function in perceiving geotropic stimuli as that attributed to the starch-grains in the cells of the root-tip (cf. p. 75). The central cylinder, which is separated off from the cortex by the starch-sheath, is termed

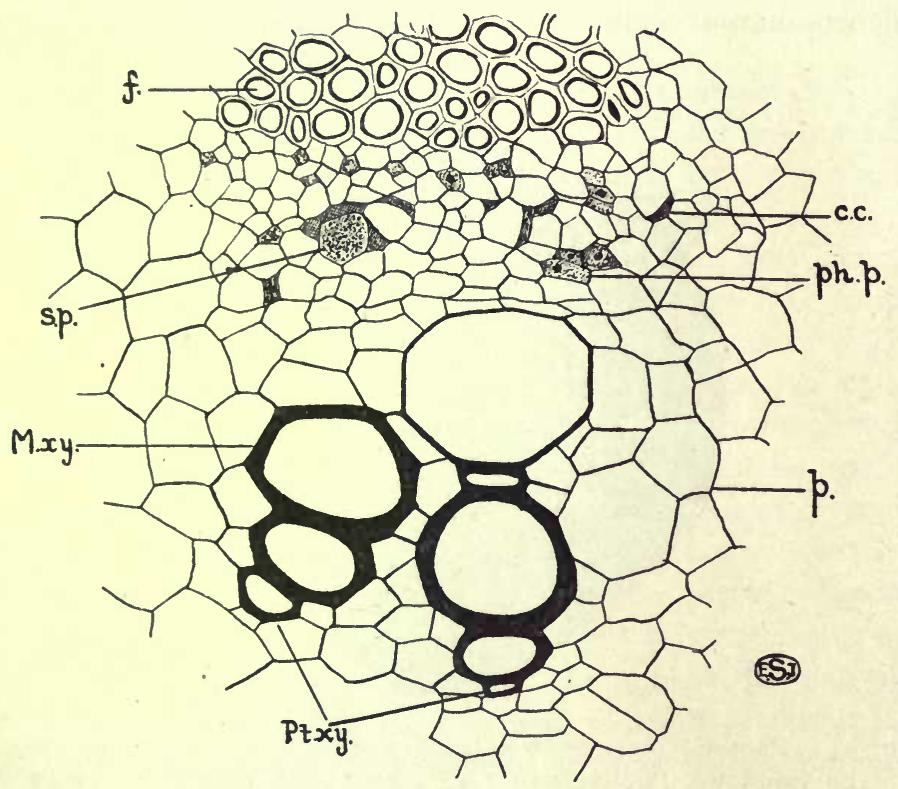

Fig. 35.-A single vascular bundle from the stem of the Sunflower (Helianthus) in transverse section. c.c., companion cells (shaded) ; $f$., fibres of the pericycle; $M . x y$. , metaxylem ; $p$. , parenchyma of the rays; ph.p., phloem-parenchyma ; Pt.xy., protoxylem ; s.p., sieve-plate.

the stele, and comprises the vascular bundles and the accompanying ground tissue.

The zone of tissue between the starch-sheath and the phloem constitutes the pericycle, which, in the Sunflower, is composed of groups of fibres (as yet not fully thickened) opposite the bundles (Fig. 35, f. ; Fig. 57, F), and elsewhere of parenchyma (Fig. 34). In other plants the pericycle is often parenchymatous throughout, consisting of one or more layers of cells. The term, 
as in the case of the root, is a purely topographical one, being applied to the region situated between the conducting strands and the starch-sheath or a similar continuous layer of cells.

In the individual bundles the phloem can be distinguished by the shining appearance of the cell-walls, which resemble those of the young fibres, as well as by the unequal size of its component elements (Fig. 35). The large, empty-looking sieve-tubes, exhibiting an occasional sieve-plate $(s . p .)^{\mathbf{1}}$ and associated with small companion cells (c.c.), which have dense contents, are intermingled with ordinary parenchyma-cells (phloem-parenchyma, ph.p.). Two or three layers of flattened thin-walled cells, situated between the phloem and the xylem, constitute the cambium (cf. Fig. 57, C, p. II8), a meristematic region which becomes active in the older stem and forms additional vascular tissue (cf. Chapter X).

The wood consists of radial files of vessels separated by rows of smaller parenchymatous cells (the wood-parenchyma, Fig. 35). The smallest vessels $(P t . x y$.), which represent the protoxylem (cf. p. 68), are situated nearest the pith, while the larger metaxylem-elements (M.xy.) are towards the outside, a further point of contrast to the root. The wood-parenchyma cells have more or less thickened walls, which are lignified like those of the vessels, but they nevertheless retain their living contents. Small cells resembling the wood-parenchyma, but not lignified, form a sheath (the medullary sheath, Fig. 34) at the inner edge of each bundle.

A longitudinal section of a Sunflower-stem passing radially (i.e. parallel to a radius) through one of the bundles will show the same succession of tissues and enable us to complete our picture of the various elements (Fig. 36). The short and somewhat bulging epidermal cells are succeeded by those of the collenchyma, which are many times longer than broad, and have strongly thickened longitudinal walls (cf. Fig. I6, A, of Dead-nettle). The thin-walled rectangular cells of the cortex (Fig. 36, Co.) are much shorter. Next come the starch-sheath $(s h$.$) , recognisable by its large starch-grains, and the typical fibres$ of the pericycle $(f$.$) . The detailed structure of phloem and cam-$ bium $(c$.) will be studied in another stem, but in the former the

1 The sieve-plates are not readily recognised in the Sunflower. 
long, apparently empty, sieve-tubes (s.) and the narrow, densely granular, companion cells (c.c.) are readily distinguished. The vessels of the metaxylem (p.v.) bear very dense spirals or occasionally bordered pits, whilst in the protoxylem the thickening takes the form of rings or a loose spiral $(p x$.). The narrow elongated elements sometimes seen between are the woodparenchyma cells.

Further insight into the structure of the Dicotyledonous stem will be afforded by a study of that of the Vegetable Marrow

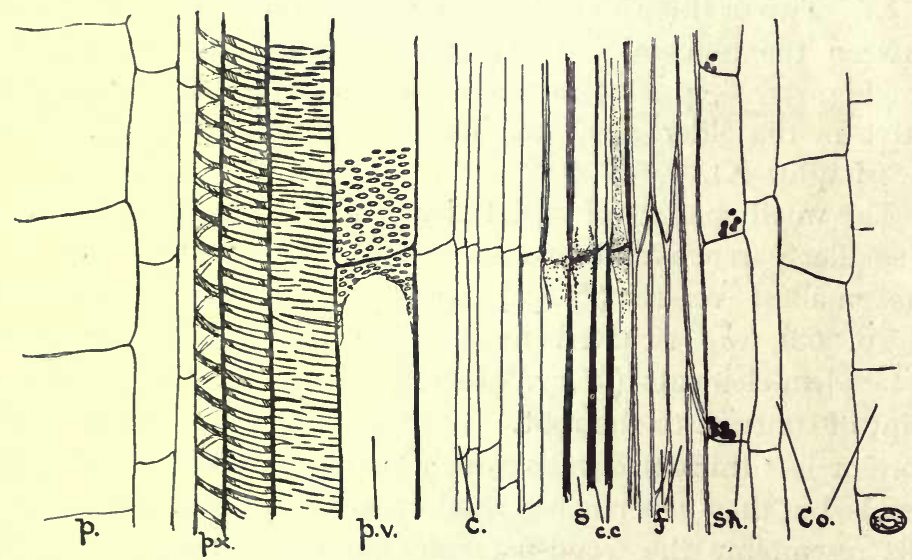

FIG. 36.-Longitudinal section through a vascular bundle of the Sunflower (Helianthus). c., cambium; c.c., companion cells ; Co., cortex ; $f$., fibres of pericycle ; $p$. , pith-parenchyma ; $p . v$., pitted vessel of metaxylem; $p x$., spiral vessel of protoxylem; $s_{\text {., }}$ sieve-tube ; sh., starchsheath.

(Cucurbita) (Fig. 37). The transverse section differs from that of the Sunflower in the hollow pith, in the presence of a broad ring of sclerenchyma $(S c l$.) some little way beneath the epidermis, and in the arrangement and construction of the vascular bundles. A pith-cavity is a frequent feature in the stems of herbs (e.g. Labiatæ, Umbelliferæ, Gramineæ, etc.), and can be related to cessation in the growth of the pith-cells, so that rupture occurs as a result of the continued enlargement of the stem.

In the stem of the Marrow there are two rings of vascular bundles, of which the larger and inner alternate with the smaller 
and outer (Fig. 37). Two groups of phloem occur in each bundle, one external $\left(P h .^{\prime}\right)$, separated from the xylem by the cambium $(C a$.$) , the other internal \left(P h_{.}^{\prime \prime}\right)$, separated from the protoxylem $(P . x y$.$) by ordinary parenchyma. Such bundles, termed bicol-$ lateral, are not uncommon in certain families of Flowering Plants,

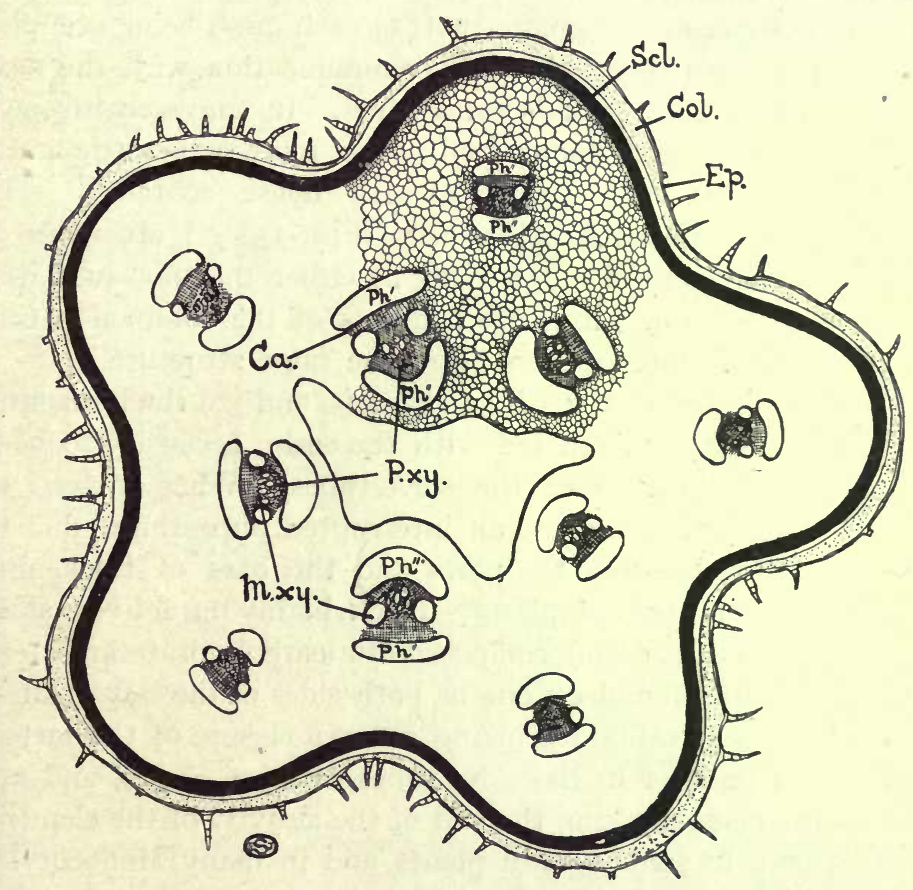

FIG. 37.-Diagram of a transverse section of the stem of the Vegetable Marrow (Cucurbita). Ca., cambium ; Col., collenchyma ; Ep., epidermis ; M.xy., metaxylem ; $P h .^{\prime}$, outer phloem ; $P h .^{\prime \prime}$, inner phloem ; $P . x y$., protoxylem ; $S c l$., sclerenchyma (black).

such as the Cucumber-family or Cucurbitaceæ (to which the Marrow belongs), and the Potato-family or Solanaceæ, and offer a marked contrast to the normal collateral type seen in the Sunflower. The elements of both xylem and phloem are remarkably large, and are therefore especially suited for a study of their detailed structure, which can be rendered more distinct by staining with eosin (see Appendix VI). 
In the phloem many of the large somewhat thick-walled sieve-tubes appear empty; but here and there, where the plane of section coincides with a separating wall, there is a deeply stained sieve-plate (Fig. I3, s.p.), recognisable by its dotted structure. Under the high power the sieve-plate exhibits an irregular network of thickening, completely covered by a thin layer of cytoplasm, the greater part of each mesh being occupied by an open pore through which communication with the next segment of the sieve-tube is established. In contact with each sieve-tube are one or more small, often more or less triangular companion cells (Fig. I3, c.c.), with dense contents. The numerous phloem-parenchyma cells (Fig. I3, p.) are more or less intermediate in size, but without either the thickened wall of the sieve-tube or the dense contents of the companion cell. Both inner and outer phloem show the same structure.

In longitudinal sections (Figs. I3, left, and 38) the horizontal sieve-plates (s.p.), stained red with the eosin, occur at frequent intervals in the course of the sieve-tubes. When viewed in optical section, they present an interrupted appearance, due to the alternation between the pores and the bars of thickening with their covering of cytoplasm. Thick, highly refractive masses (stained red by the eosin), composed of a carbohydrate known as callose, are often found on one or both sides of the sieve-plates. Such deposits of callose, bringing about a closure of the latter, arise sooner or later in the sieve-tubes of most plants, and are often permanent, marking the end of the activity of the element in question ; in some woody plants and in many Monocotyledonous rhizomes this is, however, a periodic phenomenon, the callose being formed in the autumn and becoming redissolved at the advent of spring. Callose is distinguished by being insoluble in ammoniated copper oxide, but soluble in a I per cent. solution of caustic potash, and by its reddish-brown colouration with chlor-zinc-iodide; it becomes deeply stained by a dilute aqueous solution of aniline blue, which should be allowed to act for half an hour.

Here and there the plane of the section passes through a companion cell (Fig. I3, c.c.) in contact with its sieve-tube. The former tapers off towards the sieve-plates above and below, so that its length coincides with that of the sieve-tube segment 
from which it was cut off. The large nucleus can generally be seen embedded in the dense cytoplasm which completely fills the cell. The phloem-parenchyma cells (Fig. $13, p$.) are readily distinguished by their shape from the other elements. The cells of the cambium (Fig. 38, Ca.) appear much elongated in the longitudinal section and have abruptly pointed ends.

In the transverse section of the wood one can recognise, as in the Sunflower, small protoxylem-elements towards the interior (Fig. 37, P.xy.), large metaxylem-vessels beyond (M.xy.),
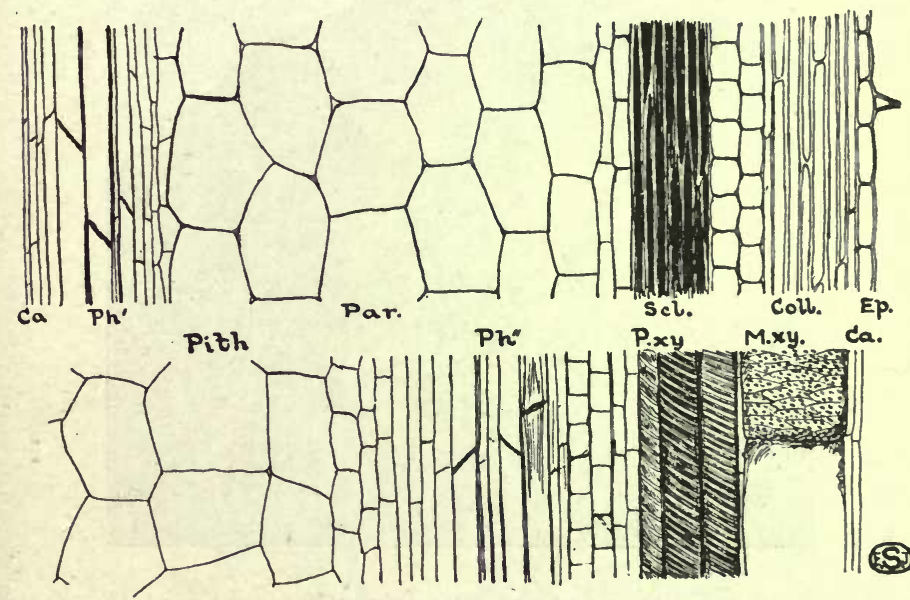

FIG. 38.-Longitudinal section through the stem of the Vegetable Marrow (Cucurbita). Ca., cambium; Coll., collenchyma; Ep., epidermis ; M.xy., metaxylem ; Par., parenchymatous cortex; $P h_{.}^{\prime}$ and $P h . "$, outer and inner phloem ; P.xy., protoxylem ; Scl., sclerenchyma.

and numerous intermingled wood-parenchyma cells. Where two vessels are in contact, the middle lamella between the pitted thickening layers of the common wall can be clearly made out. In longitudinal sections the broad metaxylem-vessels (Fig. 38, $M . x y$.) show reticulate thickenings which are in marked contrast to the spiral thickenings of the protoxylem-vessels (P.xy.); the slight constrictions occurring at short intervals mark the limits of the cells from which the vessel was built up, and, in thick sections, the ring-like remnants of the original septa can often 
be distinguished. The exceptionally large vessels are a marked feature of many other climbers besides the Vegetable Marrow.

Stems of Monocotyledons (of which the Maize furnishes a typical instance) usually exhibit a large number of bundles which frequently appear scattered throughout the whole of the ground tissue (Fig. 39), so that a definite cortex and pith cannot be distinguished, i.e. there is often no sharply circumscribed stele as in the ordinary Dicotyledon. The bundles, though differing in certain details, show the same general structure, being col-

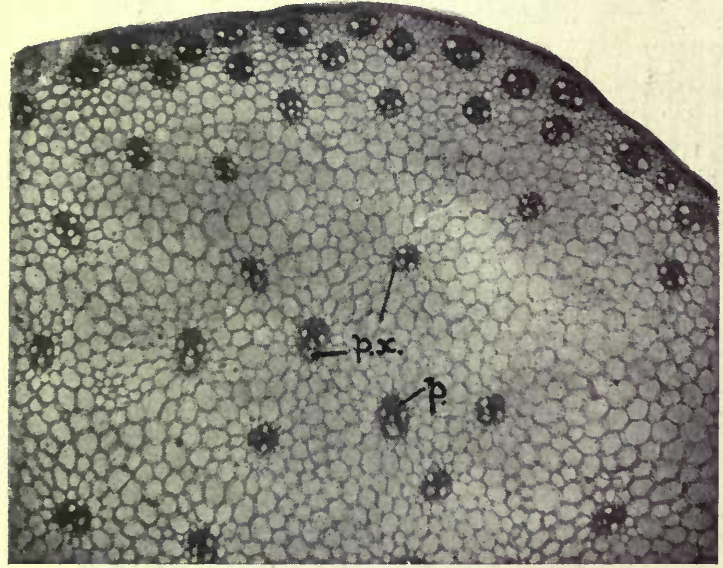

FIG. 39.-Photomicrograph of part of a transverse section of the stem of the Maize (Zea mais). p., phloem ; p.x., protoxylem.

lateral with xylem and phloem on the same radius and with the protoxylems $(p . x$.$) directed inwards.$

The phloem (Fig. 39, p.), which often has similar shining cellwalls to that of the Sunflower, is an oval or rounded group of tissue composed of sieve-tubes (Fig. 40, A, S.t.) and small companion cells $\left(C_{\text {. }}\right)$. The latter are more rounded than is usual in Dicotyledons, and this, combined with the absence of parenchyma," leads to the remarkably uniform appearance of the phloem. There is no cambium between the xylem and the phloem, an important respect in which the Monocotyledonous bundle usually differs from that of the Dicotyledon. The shape

1 Phloem-parenchyma is present in some monocotyledons. 


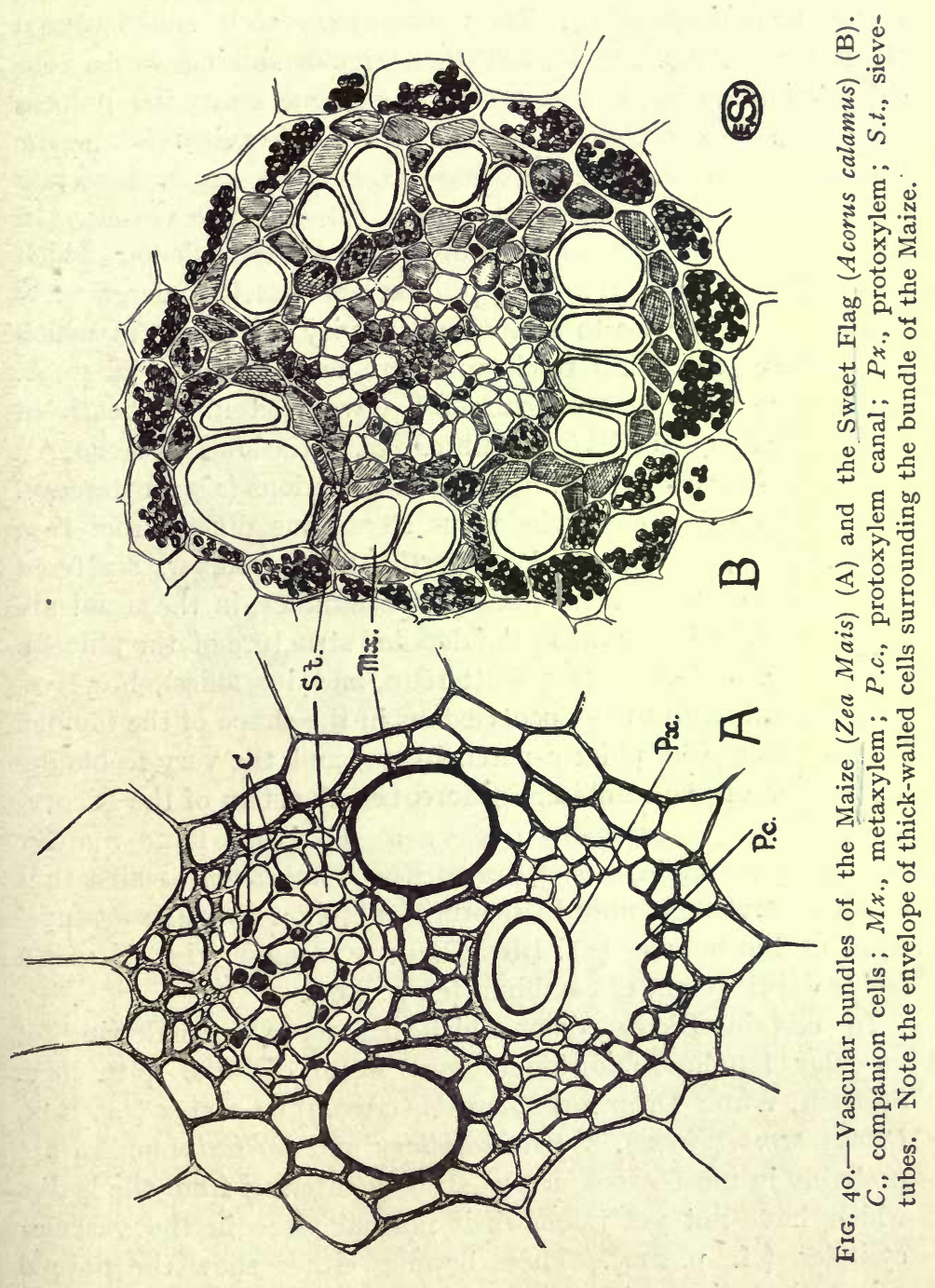


of the xylem as a whole is often roughly that of a Y, the stem of which is occupied by a radial row of protoxylern-vessels (Fig. 40, A, $P x_{\text {. }}$ ), whilst the arms are formed by a pair of large metaxylem-vessels $(M x$.). The phloem tends to be sunk between the latter (cf. Fig. 40, A), but the degree of sinking varies considerably in different stems, and in extreme cases the phloem may even be completely surrounded by the xylem (as in the Sweet Flag, Acorus calamus, Fig. 40, B). As usual, a certain amount of wood-parenchyma occurs between the vessels. In the stems of the Maize and many other Monocotyledons which exhibit rapid elongation, the protoxylem-vessels undergo early rupture and give rise to an irregular cavity (cf. p. 36) in which remnants of the spiral thickenings are recognisable (Fig. 40, A, $P . c$.$) . The bundles are commonly enveloped in a sheath of$ small, thick-walled cells (often fibres, e.g. in the Maize, Fig. 40, A), a feature also encountered in some Dicotyledons (e.g. Buttercup).

The typical Monocotyledonous stem thus differs from that characteristic of Dicotyledons in the large number, scattered arrangement, and general form of the bundles, in the usual absence of a cambium, and in the detailed structure of the phloem. Certain Dicotyledons (e.g. Buttercup and its allies), however, show resemblance to Monocotyledons in the shape of the bundle, in the absence of phloem-parenchyma, and the very feeble development of the cambium. Moreover, the stem of the Dicotyledon may exceptionally possess a relatively large number of scattered bundles (e.g. Meadow Rue, Thalictrum), whilst that of Monocotyledons may in its turn exhibit a ring-like arrangement of the bundles (e.g. Black Bryony, Tamus, Fig. 4I) and a feeble development of cambium (cf. p. II7).

In certain Dicotyledons which possess a normal ring of vascular bundles additional ones occur in the pith (e.g. Spinach, Water Dropwort, Oenanthe crocata) or cortex (e.g. Box, Buxus sempervirens). Such medullary and cortical bundles are probably in most cases merely strands entering from the leaves which have not yet taken their normal place in the vascular cylinder (cf. p. II4). These bundles either show the normal orientation (i.e. with the xylem towards the inside), or they exhibit the reverse arrangement ; in many plants, moreover, they consist of phloem only. 
The functions of the stem are manifold, but among the most important are those of bearing leaves and flowers, and of constituting a connecting-link between the root-system and the foliage. The shoot of the ordinary erect plant is most liable to bending, under the influence of the wind, etc., and thus contrasts with the root, which is exposed chiefly to pulling strains. Related to this the mechanical elements of the stem are more or less symmetrically arranged near the periphery. In the young stem the upright position is maintained by the frequent subepidermal collenchyma (Figs. I5, 34, and 37), by the xylem of

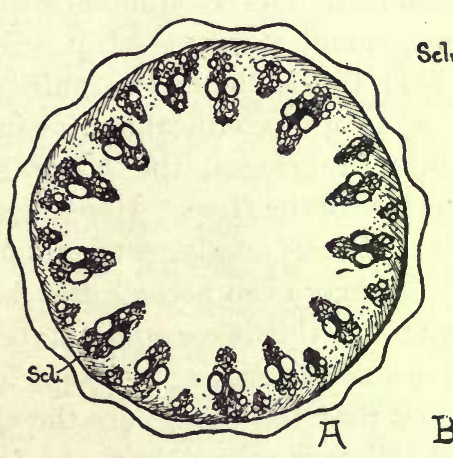

E.J.S.

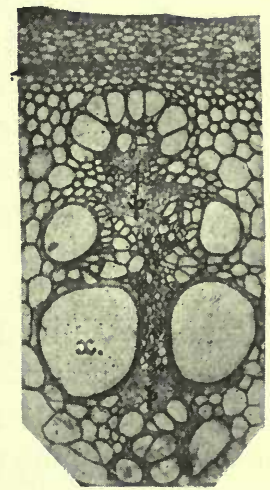

FIG. 4I.-Stem of the Black Bryony (Tamus communis). A, Diagram of transverse section. $B$, Photograph of a small portion on a larger scale. P., phloem; Scl., sclerenchyma-ring; $x$., metaxylem vessel.

the bundles, and by the turgidity of the living cells combined with tissue-tensions. ${ }^{1}$ As the stem matures, additional mechanical tissue is often furnished by the development of pericyclic fibres (e.g. Sunflower, Fig. 35, f.), of a thick-walled sheath to the bundles (Monocotyledons, Fig. 40, A, Buttercup), or of a zone of sclerenchyma in the cortex (e.g. Vegetable Marrow, Fig. 37, $\mathrm{Scl}$.). Even in the ordinary Monocotyledonous stem the bundles are far more numerous near the edge (cf. Fig. 39); moreover, Monocotyledons frequently exhibit a copious development of peripheral sclerenchyma, to which, for instance, the hardness of a

$$
1 \text { Cf. F. \& S., p. } 103 .
$$


Bamboo is largely due. In slender underground stems aiding in the attachment of the plant, and therefore chiefly exposed to tension, a much more centralised disposition of the mechanical elements is observed than in the upright shoot. This is well seen in many Sedges and Grasses. An extreme condition is reached in water-plants, where, owing to the prevalent pulling strain, such mechanical tissue as is present becomes concentrated at the centre of the stem (cf. p. I7o).

Most young stems exhibit chloroplasts in the cortical cells (even when these are collenchymatous, as in the Campion), the carbon dioxide requisite for photosynthesis being obtained from the intercellularspaces between them, which communicate with the atmosphere by means of occasional stomata (cf. p. 96) in the epidermis. In some cases (e.g. Umbelliferæ) the outer cortical zone consists of alternating bands of mechanical and assimilatory tissues, the former occupying the ridges, the latter situated beneath the furrows on the surface of the stem. Here the stomata are restricted to the strips of epidermis overlying the assimilatory tissue. The cortex of the stem may even become the chief seat of the assimilatory function; but this is exceptional, occurring mainly in plants inhabiting dry situations (e.g. Broom).

Various experiments ${ }^{1}$ show that the vessels are the channels by which water and mineral salts are passed from the root into the leaves. The phloem, on the other hand, serves to conduct elaborated food-substances, the proteins appearing to travel mainly by way of the sieve-tubes. If, for instance, the stem of the Vegetable Marrow be dipped into boiling water so as to coagulate the contents of the sieve-tubes, the abundant proteins can be demonstrated by heating longitudinal sections with Millon's reagent. Opinions differ as to whether the carbohydrates are transferred by the phloem, or, as some Botanists believe, mainly by way of the cortical- and phloem-parenchyma. The storage of food-substances in perennial organs (e.g. rhizomes, tubers, etc.) is effected chiefly by the ground tissue, which in these cases is thin-walled.

Whilst it has been seen that the arrangement of the tissues differs materially in root and stem, the ground tissues in the two organs pass over imperceptibly into one another. The 1 Cf. F. \& S., pp. IIo, III. 
protoxylem-strands are directly continuous, whilst the metaxylem is developed towards the exterior in the stem and towards the interior in the root, and occupies a more or less intermediate position in the region (i.e. the hypocotyl) where the transition from the one to the other type of structure takes place. The phloem is similarly continuous, and the transition from root to stem mainly involves a lateral and outward displacement accompanying the enlargement of the vascular cylinder (Fig. 42). The transition between the two organs is, however, often very complex, being accompanied by splitting or union of conducting strands, as a result of which the number of protoxylem-groups in stem and root do not necessarily correspond.

The development of the different regions of the axis from the growing points has already been described in Chapter II, but some additional details as to the manner of origin of the vascular tissues will serve to amplify the picture. These first appear in the plerome a short distance behind the tip as so-called procambial strands, composed

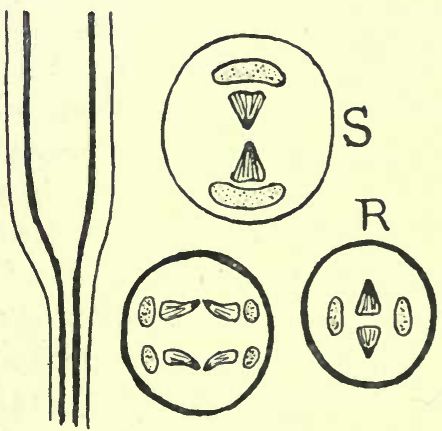

FIG. 42.-Diagrams showing one type of transition from the vascular structure of the root to that of the stem. The dotted areas represent phloem and the shaded xylem ; protoxylem shown black. $R$., root; $S$., stem; the remaining cross-section, hypocotyl. of very narrow, elongated, thin-walled cells with pointed ends, dense protoplasmic contents, and prominent nuclei. They develop by repeated longitudinal divisions in the cells of the plerome, in which transverse division almost ceases at an early stage, the narrow segments thus formed subsequently elongating.

In the stem each procambial strand gives rise to a vascular bundle, whilst in the root there is usually a single strand from which the whole vascular cylinder is differentiated. The innermost elements, in each of the procambial strands of the stem, become the spirally thickened protoxylem-vessels, whilst simultaneously the outermost elements develop into the first-formed 
phloem (protophloem). As the strands are traced further and further from the apex (i.e. from younger to older stages) the differentiation of xylem and phloem proceeds towards the middle of each. Ultimately all that remains is a narrow strip of thin-walled cells between xylem and phloem, which in the case of Dicotyledons gives rise to the cambium. In the root alternating groups of protoxylem and protophloem arise at the periphery of the procambial strand, whilst the later-formed elements, and the pith if present, develop from the central region.

Both leaves and branches originate close behind the growing point of the stem as superficial outgrowths (i.e. are exogenous, Figs. 7, 8), a mode of development contrasting markedly with the internal (endogenous) origin of lateral roots. Leaf-rudiments, at first, consist merely of a mass of periblem enveloped by a protrusion of the epidermis (Fig. 8), but as they enlarge procambial strands are formed within them by division of certain cells. The differentiation of these strands gradually extends inwards till they ultimately join those of the stem. As a result the vascular bundles of leaf and stem are continuous in the mature condition (p. II4, Fig. 55). When vascular strands occur in the stipules they are branches of those in the leaf-base. The strands arising in the young branches also become connected with those in the stem. 


\section{CHAPTER VIII}

\section{The Epidermis}

THE surface of the shoot is protected by a skin, or epidermis, composed of one layer of living cells which possess certain marked characteristics. In transverse sections the cells usually appear somewhat flattened with slightly convex outer walls, whilst the lateral and inner ones are generally straight; moreover, they fit closely together without intercellular spaces (Figs. I9 and $43, E p$.). The epidermal cells have living contents, usually contain plastids, and possess large vacuoles filled with watery, generally colourless, sap (Fig. 43, Ep.). Chloroplasts are not developed in the cells in well-illuminated situations, but are often present in the epidermis of submerged aquatics or of land-plants when growing in the shade (cf. p. I69).

The outer epidermal walls in most cases are more strongly thickened than the others, and so changed (cuticularised) as to render them more or less impermeable to water vapour and gases. The exact nature of the modification is not known, but it appears to consist essentially in impregnation with substances of a fatty or waxy character; these are most abundant in the outermost region of the external walls, which forms a continuous, relatively impermeable, layer over the whole epidermis (except for the stomata) known as the cuticle (Figs. 43 and 46, Cu.). Where the walls are strongly thickened an intervening zone (the cuticularised layers), in which cuticularisation has not progressed to the same extent, can sometimes be distinguished between the cuticle and the unaltered cellulose on the inside. The cuticle is often yellowish in colour, whilst the cuticularised layers, when present, appear faintly yellow and less transparent than the colourless cellulose. The cuticle may be quite smooth, but in some cases (e.g. Helleborus fatidus) it is provided with numerous minute 
ridges which appear as a faint striation in surface sections. Cuticularisation may sometimes extend to the lateral walls, which in these cases are generally thickened, so that in section they appear as pegs projecting inwards.

The cuticle is insoluble in cuprammonia and concentrated sulphuric acid, being the only part of the epidermis that does not dissolve in the latter reagent. With iodine and sulphuric acid it yields a brown colouration, and it is easily stained with

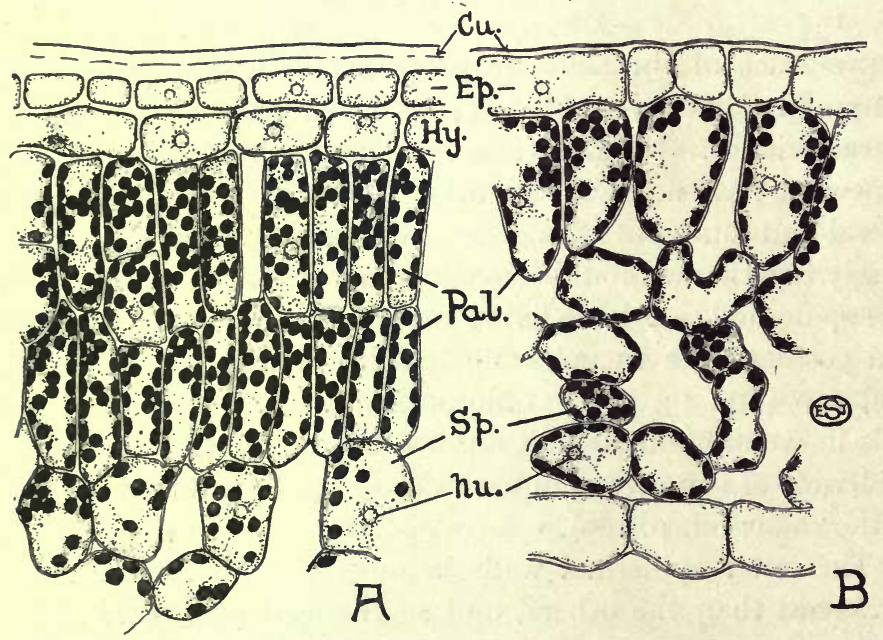

FIG. 43.-Transverse sections through the leaves of the Holly (A) and Ranunculus auricomus (B). In the former only half of the vertical extent of the leaf is shown. Cu., cuticle ; Ep., epidermis ; $H y$., hypodermis; $N u$., nucleus; Pal., palisade layers; $S p$., spongy parenchyma. The chloroplasts are shown black.

Scharlach red, probably as a result of the presence of fatty substances.

Owing to its toughness, the cuticle, especially when strongly developed, renders the epidermis more efficient as a protection against mechanical injury; but its main function is certainly the restriction of transpiration to the stomata. In correspondence with this its thickness depends mainly on the nature of the habitat; thus the cuticle is most strongly developed in plants of dry situations (e.g. Gorse, Fig. 8I, B, Psamma, Fig. 85, A, etc.), whilst it is extremely thin in submerged aquatics (Fig. 89), 
where absorption of water takes place over the whole surface. Most young organs, before they have attained their full size, possess but a very thin cuticle and exhibit considerable cuticular transpiration; hence the necessity for other devices for the reduction of transpiration (e.g. hairs, folding of leaves, etc.). Even a very thick cuticle is, however, not completely impermeable, and its efficiency in reducing transpiration is sometimes augmented by a covering of wax secreted by the epidermal cells ; when present in any considerable quantity (e.g. leaves of the Sea Holly, fruit of the Plum, etc.), this gives the surface a bluish tinge. Such coverings of wax also prevent the collection of moisture on the surface of the plant. They are readily rubbed off, but may be subsequently renewed.

Not uncommonly the epidermis can be easily stripped off and so examined in surface view, when it will again be seen to form a continuous layer (Fig. 44, B-D) without intercellular spaces, the only gaps being constituted by the stomata to be described below. The shape of the cells in such surface sections is very varied. In stems (Fig. 44, D) and in the leaves of most Monocotyledons (Fig. 44, C) they are usually considerably elongated parallel to the longitudinal axis, whilst in Dicotyledonous leaves they are in most cases roughly isodiametric (Fig. $44, \mathrm{~B})$. Where the lamina is thin the lateral walls of the epidermal cells are often undulated (Fig. 44, B). Through this wavy outline the cells interlock, so that the surface of contact between them is increased, and the risk of tearing by the wind is proportionally diminished. It is significant that the lower epidermis, which has numerous points of weakness constituted by the stomatal perforations, generally exhibits this undulation to a more marked degree than the upper. This feature is also more pronounced in the leaves of certain aquatics than in those of the corresponding land forms.

The epidermis keeps pace with the increasing size of the underlying tissues by the division of its cells, but usually all the septa are at right-angles to the surface, so that it remains a single layer. This subdivision is plainly recognisable in the stem of the Runner Bean, where the spindle-shaped epidermal cells, after reaching a certain size, become divided by transverse septa (Fig. 44, D). 
In plants of dry habitats the epidermal cells may attain a considerable size (e.g. in the Sea Purslane, Arenaria peploides,
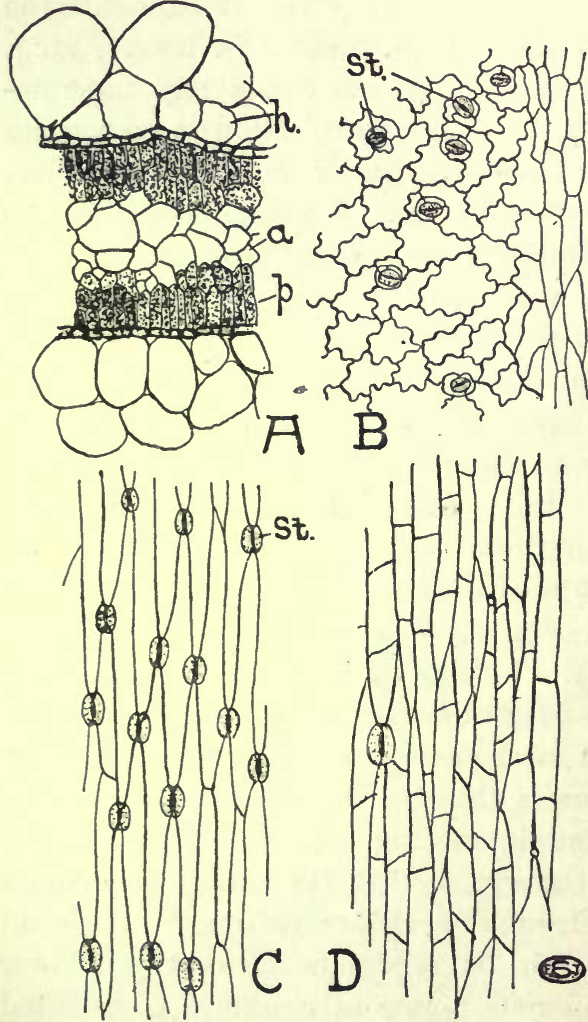

FIG. 44.-Structure of the epidermis. A, Transverse section of leaf of Silver Goosefoot (Obione portulacoides) showing the bladder-like hairs (h.), the aqueous tissue $(a$.$) , and the palisade layer (p.) B, Sur-$ face section of leaf of Ground Ivy (Nepeta glechoma). C, Surface section of leaf of Onion. D, Surface section of stem of Runner Bean (Phaseolus multiflorus). St., stomata. and in the Prickly Saltwort, Salsola kali), and serve for the storage of water, which is possibly always a function of this layer, though here to a much greater extent than is normally the rule. In extreme cases water may be stored in localised enlargements, which often project as watercontaining hairs or bladders (e.g. the Iceplant, Mesembryanthemum crystallinum, and the Silver Goosefoot, Obione portulacoides, Fig. 44, A, h.). In times of drought the adjacent assimilatory tissues withdraw moisture from these cells, which consequently contract, the side walls becoming undulated; when water again becomes plentiful, the cells fill and the walls gradually straighten out. The epidermis

is not uncommonly aided in the storage of water by the development of one or more additional layers, which may be 
formed by the division of the dermatogen (Ficus elastica), or by differentiation from the underlying periblem (as in the Holly, Fig. 43, A, Hy.). The cells of such a hypoderm (cf. also p. III) resemble the epidermal cells in the possession of large vacuoles and the absence of chloroplasts, but usually have thinner walls. It will be realised that in all cases leaf and stem are surrounded by a water-jacket tending to diminish the heating effect of the sun.

Another device serving for water-storage consists in the development of thick mucilaginous internal walls (cf. p. 38) by the epidermal cells (e.g. Hollyhock, Sycamore, etc.). Thesc are inconspicuous in sections mounted dry, but swell up considerably, often projecting deeply into the assimilatory tissues (e.g. Hollyhock), when placed in water. This device tends to retard the evaporation of water during periods of drought.

The pronounced thickening and cuticularisation of the outer walls of the epidermal cells, and the absence of intercellular spaces between them, endues the epidermis as a whole with considerable strength. It hence serves as a mechanical protection, but also (in combination with the hypoderm, when present) as a light-screen for the underlying chlorophyll. Moreover, the polished cuticle, especially characteristic of tropical plants, serves to reflect a large part of the light and heat rays falling upon it. Most important of all, it checks evaporation from the general surface of the plant, and indeed, where the cuticle is thick, transpiration is almost entirely restricted to the special apertures or stomata which will be considered below.

Certain modifications of the epidermis are associated with special physiological functions. Thus in many shade-loving plants the outer walls of the epidermal cells are markedly convex (e.g. Wood Sorrel; Moschatel, Adoxa, Fig. 45). Where this is the case, each cell functions as a plano-convex lens focussing a localised patch of bright light at its base. It has been suggested that this acts as a mechanism for the orientation of the leaf, since, when the direction of illumination is altered, the position of the patch of light likewise changes. The protoplasm upon which this light falls is assumed to be sensitive, so that an adjustment of the leaf follows until the patch of light is again located in the normal position. But quite apart from any value 
that this may possibly have in enabling the plant to place its leaves at an appropriate angle to the light, ${ }^{1}$ the concentration of the latter may well be of value in connection with carbon dioxide assimilation.

The general characteristics of stomata are readily studied by stripping off a piece of the epidermis from a fresh leaf of the Iris. Scattered among the colourless elongated cells are oval stomata, each consisting of two bean-shaped guard-cells (Fig. 46, C, g.c. ; see also Fig. 44, C) surrounding the narrow elliptical pore by means of which the intercellular spaces of the leaf communicate with the atmosphere. The guard-cells contrast with the ordinary epidermal cells in containing numerous chloroplasts and starchgrains, and are especially distin-

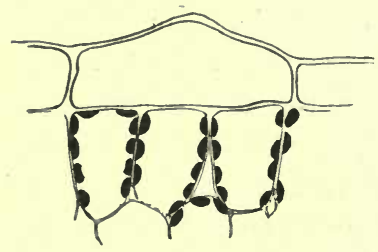

Fig. 45.-Transverse section through part of the upper epidermis and palisade layer of the leaf of the Moschatel (Adoxa moschatellina). The chloroplasts are shown black. guished by the uneven thickening of their walls, which are thin on the side away from the pore, but markedly thickened around the latter (Fig. 46, C). Each guard-cell has a well-developed protoplast and a prominent nucleus. The ordinary epidermal cells almost meet above the guard-cells, so that the latter are only plainly visible on focussing to a lower level (Fig. 46, C) ; in this way the pore comes to be situated at the bottom of a miniature hollow (vestibule) constituted by the surrounding cells, and the stoma consequently occupies a sheltered position. This feature is especially marked in plants of dry situations, but in those growing in damp, shady habitats the guard-cells are often level with or raised above the adjacent epidermis (Fig. 8r, C, D).

The overarching of the guard-cells by the adjoining epidermal cells is equally obvious in a transverse section (Fig. 46, D) of the Iris-leaf. Here each guard-cell appears approximately oval in form and provided with thick walls, the thickening being especially marked adjacent to the pore, and giving rise to a slight upwardly projecting ridge at the outer edge of the aperture. In many plants, however, the walls of the guard-cells are not so 
uniformly thickened, those remote from the pore being relatively thin, whilst those towards the aperture show a marked decrease in thickness opposite the middle of the pore (e.g. Onion, Fig. 46, E); in such cases the outer ridges are well marked, and there is frequently a second pair of ridges on the inner side. As a result of this unequal distribution of the thickening, the cavities of the guard-cells taper abruptly in the direction of the

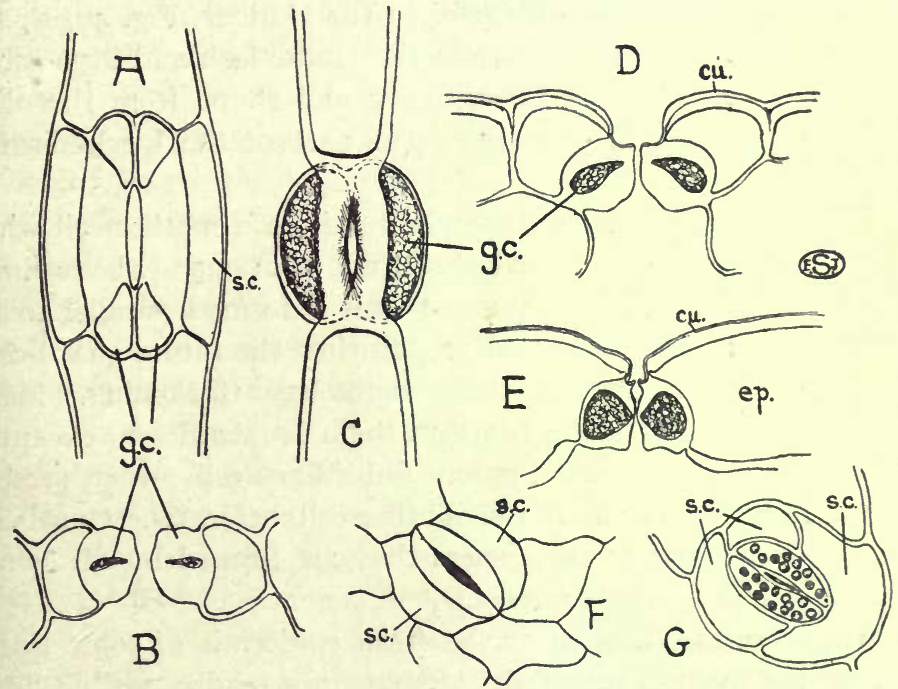

Fig. 46.-Structure of stomata. A, C, F, and G, in surface view ; B, D, and $\mathrm{E}$, in transverse section. A, B, Millet-Grass (Millium effusum). C, D, Iris germanica. E, Onion (Allium cepa). F, Madder (Rubia peregrina). G, Sedum spectabile. cu., cuticle ; ep., epidermal cell ; g.c., guard-cells ; s.c., subsidiary cells.

pore, so that a maximum thickness is obtained towards the upper and lower sides (Fig. 46, E); in some plants this is so marked that the whole cavity merely appears as a slit (e.g. Grass, Fig. 46, B). Beneath the stoma lies an air-space, the respiratory cavity (Fig. 5I, R.), serving for the immediate interchange of gases and water-vapour between the intercellular system of the plant and the air around.

The vertical leaf of the Iris, in which both surfaces are alike, bears almost the same number of stomata on each. Moreover, 
the latter are all placed parallel to the axis, as is usual for the elongated leaves of Monocotyledons (Fig. 44, C) and the epidermis of young stems; in the latter case, however, the stomata are often few in number (Fig. 44, D). Those of horizontal dorsiventral leaves (e.g. of most Dicotyledons) are chiefly situated on the lower surface, being sometimes altogether absent from the upper (e.g. most trees), and, moreover, exhibit an irregular arrangement. (Fig. 44, B). In some families the guard-cells are accompanied by so-called subsidiary cells (two in the Madder, Fig. 46, F, s.c., and Bedstraws, three or more in the House-leek and Stone-crop, Fig. 46, G, s.c.) which differ in size and shape from the other epidermal cells, and probably form part of the mechanism of the stoma.

The stomatal apparatus originates from a mother-cell which is cut off by means of a curved septum from one of the ordinary epidermal cells. Soon a vertical wall is formed parallel to the long axis of the mother-cell, separating the future guard-cells, which gradually acquire their distinctive thickening. Meanwhile the middle lamella between them breaks down, except at the two ends, to form the pore. Subsidiary cells, when present, are usually cut off from the mother-cell before the guard-cells are produced, but in some cases they are formed by division of the surrounding epidermis.

On mounting a strip of the fresh epidermis of some leaf in water, the open pores of the stomata are readily visible, being usually occupied by air. On transference to glycerine or a 5 per cent. sugar solution, which will reduce the turgor of the guard-cells, the width of the pores decreases appreciably (i.e. they " close "), but when returned to water the turgor is restored and they again open. Measurement shows that, though there is usually no marked change in length, there is an appreciable increase in width, when the stoma opens (cf. Fig. 47). The alteration in form of the guard-cells, to which the variation in the size of the stomatal aperture is due, is thus primarily determined by changes in turgescence.

The mechanism is, however, directly dependent on the unequal distribution of thickening which, in transverse section, has been seen to be mainly localised around the upper and lower edges of the pore; on the other hand, the middle of the convex 
wall next to the pore, as well as the curved wall remote from the pore, remain comparatively thin (Fig. 46, E). When the guard-cells are turgid and the thin walls stretch, the pull exerted separates the thick walls, thus opening the pore (Fig. 47,op.). As seen in section, opening of the stoma is accompanied by a flattening of the convex walls on each side of the pore and an increased convexity of the unthickened part. As a result there is movement of the guard-cells at the lines of junction with the adjacent epidermis both above and below, due to the bulging of the walls away from the pore.

Where both the outer and the inner walls of the epidermal cells are thickened, there is a sudden thinning at the points of junction with the guard-cells to admit of their movement (e.g. Onion, Fig. 46, E) ; but when the external wall alone is thickened, such a hinge occurs only on the outside (e.g. Iris, Fig. 46, D). The flattening of the convex walls adjacent to the pore is facilitated by the thinner middle portion, which constitutes a similar hinge. A good illustration of the action of a stoma, depending likewise on unequal distribution of thickening, is afforded by the change in form of the two halves of a split Daffodil-scape, tied

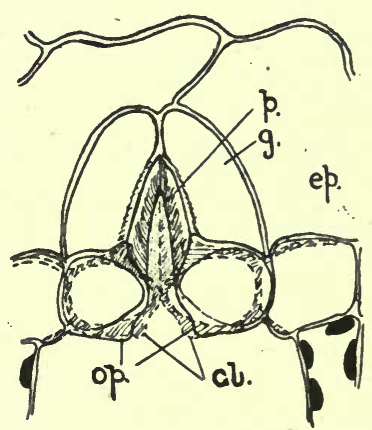

FIG. 47.- Half of a stoma, seen in perspective, showing the positions of the guard-cells in the open (op., firm lines) and closed (cl., dotted lines) conditions. ep., epidermis ; g., guard-cell; $p .$, pore. together at their ends with the outer sides in contact, and placed alternately in salt solution and water. ${ }^{1}$

The influence of external conditions on the size of the stomatal pore can be indirectly studied by means of the porometer, ${ }^{2}$ or by the following method adopted by Lloyd: Leaves, growing in the particular conditions to be studied, are detached from the plant and immediately placed in strong alcohol, which fixes the guard-cells in their momentary condition. A strip of epidermis can now be removed without alteration, and the average

1 C.f. F. \& S., p. I 17.

2 F. \& S., p. 369. 
size of the stomatal apertures directly measured under the microscope (cf. Appendix VII).

Either of these methods will serve to demonstrate the tendency of the stomata (except in many shade and marsh plants, e.g. the Water Plantain, Alisma plantago) to "close" when the shoot becomes flaccid owing to deficiency of water, although, as shown especially by the porometer, they often open more widely at the first commencement of wilting. "Closure" is also brought about by a change from light to darkness, which emphasises the fact that alteration in turgidity of the plant as a whole is not necessarily involved. It may be noted that Fungi, which have no stomata, also exhibit diminished transpiration in darkness. The sensitiveness of the stomatal mechanism is so great that even the effect of temporary shading, as by a big cloud, can be observed with the help of the porometer. Moreover, shaking of a leaf may cause a more or less marked temporary "closure," and for this reason it is best to allow a short interval to elapse, after fixing the porometer, before readings are taken.

We thus realise that the stomatal mechanism furnishes an automatic control on the escape of water-vapour from the leaf. The rate of transpiration has been shown to decrease as the humidity increases, though it becomes zero only when the air is slightly supersaturated.

The stomata are therefore highly irritable, and the ultimate cause for their response to different conditions must, at all events in part, reside in the living protoplasm of the guard-cells. It would seem that conditions causing "closure" of the stoma must lead to a reduction in the permeability of the protoplasm, and vice versa. In the case of the stomata present on the capsule of Mosses (cf. p. 284), it has been found that the guard-cells, when open, have nearly five times the osmotic pressure of the surrounding epidermal cells, but when "closed " have the same osmotic pressure.

A much modified type of stoma is found in the Graminex and Cyperaceæ (Sedge-family), though the mechanism is essentially similar. The much elongated guard-cells surround a large pore having the form of a flattened hexagon (Fig. 46, A). In the middle portion of each guard-cell the outer and inner walls 
are so strongly thickened as to leave only a small slit-shaped cavity (Fig. 46, B), but the end-portions are thin-walled and somewhat enlarged (Fig. 46, A, g.c.). On either side of the stoma is a thin-walled subsidiary cell (s.c.). These latter, together with the terminal portions of the guard-cells, function in much the same way as the thin-walled part of an ordinary guard-cell, whilst the thick median portions correspond to the thickened walls of the latter.

Submerged leaves of water-plants usually bear no stomata, whilst in floating leaves they are restricted to the upper surface, and exhibit marked differences from the ordinary type. The guard-cells, in transverse section, are here roughly triangular through the inner walls being bevelled off towards the outer edge of the pore, where the thickening is most pronounced. As the upper surface of such floating leaves is usually covered by a fine waxy bloom (e.g. Water Lily, Nymphaa), this side of the leaf is not readily wetted, and hence the formation of waterfilms across the stomatal apertures is prevented.

Not uncommonly a few, or even many, of the epidermal cells (cf. Fig. 37) grow out into more or less elongated, often branched, processes called hairs, which are especially common on leaves and, when numerous, are very obvious to the naked eye. They may remain unicellular (Fig. 48, B, C), or become multicellular (Fig. 48, D) by the formation of septa whose development is probably related to mechanical requirements. Nearly every type of hair, whether branched or not, may be uni- or multi-cellular without any appreciable difference in outward form; but the presence or absence of septa, as well as the structure of the hair, are often characteristic of whole groups of plants. The walls of the hairs are of varying thickness, and either consist of cellulose or have undergone chemical alteration.

One of the most important functions of hairs, when numerous, is to bring about a decrease in the rate of transpiration. The moist air entangled between these hairs is sheltered from the wind, so that it is not readily removed by air-currents, nor does it diffuse rapidly into the dry atmosphere around. As a result of the presence of this moisture-laden air in the immediate neighbourhood of the leaf-surface, transpiration of water-vapour from the interior through the stomata is retarded. These cover- 
ing hairs are usually dead and, at maturity, occupied only by air; such dead hairs often appear white, showing that a large proportion of the light falling upon them is totally reflected, hence they also afford protection against the heating effect of the sun and against excessive illumination. Hairs thus serve to reduce transpiration and act as a screen to the underlying chloroplasts. An
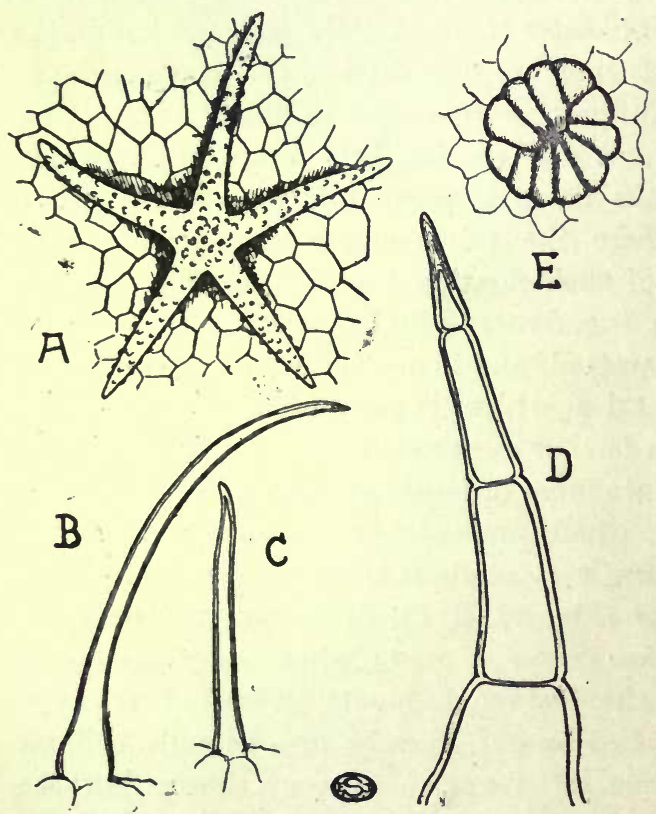

FIG. 40.-Hairs. A, of Deutzia ; B, of Cynoglossum; C, of Shepherd's Purse (Capsella) (unbranched type); D, of Vegetable Marrow (Cucurbita): E, of Hop (Humulus). analogy could be drawn with the interior of a woodland, where beneath the trees the air is cool, shady, and damp, just as it is beneath the hairs on a leaf. Not uncommonly hairs are only present on the young leaf, falling off as the latter matures (e.g. Plane, Horse Chestnut, etc.).

The simplest type of covering hair is unbranched and usually tapers towards the tip (Fig. 48, B-D). Short stiff hairs of this kind are

found in some Boraginaceæ (e.g. Forget-me-not, etc.), but they are often much longer, and either interwoven to form a woolly tangle (e.g. Coltsfoot, Thistles, young leaves of the Horse Chestnut, etc.) or all disposed in the same direction, giving a silky appearance to the surface of the leaf (e.g. Silverweed). Long unbranched hairs from the seed-coat of the Cotton-plant (Gossypium spp.), a member of the Mallow-family (Malvaceæ), 
are the source of cotton. ${ }^{1}$ They are unicellular and may reach a length of 2-5 centimetres and, in the plant's economy, serve for the dispersal of the seeds by the wind. Similar hairs, with the same function, occur on the seeds of the Willow, Willowherb, and many other plants. ${ }^{2}$

The epidermal cells of many petals are drawn out into very short processes, or papilla, which are the cause of the velvety surface and prevent wetting. A similar production of papillæ is not uncommon on the stomatal surface of the leaves of tropical plants growing in damp situations (e.g. tropical rain-forest).

Branched hairs assume very diverse forms, only a few of which can be mentioned. In the Mullein and Plane they are tree-like, and consist of an erect multicellular axis from which numerous tapering branches radiate at intervals. More frequent are so-called stellate hairs, in which there is but one set of radiating branches terminating a short stalk which, however, is sometimes practically absent (e.g. Deutzia). Multicellular hairs of this kind are typical of the Lime-family (Tiliaceæ) and Mallow-family (Malvaceæ), whilst unicellular ones are exemplified in Deutzia (Fig. 48, A). Stellate hairs are

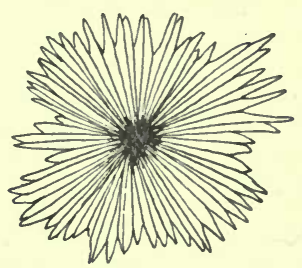

Fig. 49.-Peltate hair of Sea Buckthorn (Hifpophe rhamnoides). also found in the Cruciferæ, but here they are accompanied by simpler forms with only two or three branches and by unbranched hairs.

A very efficient transpiration-check is afforded by the peltate hairs, which are well seen in the Sea Buckthorn (Hippophe, Fig. 49), and in Elaagnus, where, owing to the large number of these scale-like structures, the under-surface of the leaf has a characteristic silvery appearance. The short stalk of these hairs is surmounted by a horizontal expansion, consisting of a large

1 The walls of these hairs are practically pure cellulose. Many hairs, which from their length might be used for the manufacture of textiles, are slightly lignified, and consequently too brittle to spin. Kapok of commerce is obtained from the slightly cuticularised hairs lining the fruit of the Silk-Cotton tree (Eriodendron anfractuosum), which is common in the Tropics.

2 See F, \& S., p. 286 , 
number of unicellular rays which are joined together at their base but free at their tips.

Certain climbers are materially aided in clinging to their support by the possession of stiff hairs. Thus the ridges on the stem of the Goosegrass (Galium aparine) bear numerous reflexed unicellular hairs, shaped like a hook and having a stout base and a very strongly thickened tip. In the Hop the ridges are similarly beset by stiff two-armed hairs seated on a small elevation of the epidermis; the two arms are situated in the vertical plane, and the downwardly directed one is considerably longer than the other. Extreme types of multicellular climbing hairs are furnished by the prickles of the Rose and Bramble.

Whilst the walls of the ordinary covering hairs are generally not appreciably thickened, both branched and unbranched hairs may possess thick walls which are frequently silicified or calcified ; when thus stiffened, they constitute a chevaux-de-frise against small animals (e.g. slugs). Good examples are afforded by the unbranched bristle-hairs of many Boraginaceæ (e.g. Borage, Comfrey, etc.) and the branched types found on the Stocks and other Cruciferæ. Their effect is often accentuated by the presence of numerous minute teeth on their surface (Fig. 48, A).

One of the most striking examples of hairs acting as a deterrent to animal attacks is, however, furnished by the unicellular stinging hairs of the Nettle (Urtica) (Fig. 50, D). Each is borne on a multicellular stalk in which is embedded the thinwalled swollen base of the actual stinging hair. The upper part of the latter is comparatively thick-walled and tapers gradually to near the apex, where it suddenly enlarges to form a tiny bead-like tip (Fig. 5o, E). The lower part of the wall is calcified, the upper part silicified. The living protoplasmic contents often show distinct streaming movements, and include a large vacuole filled with acrid sap. When an animal brushes against one of these hairs, the little tip breaks off, leaving exposed a fine needlelike point formed by the upper tapering part of the hair. As a result of the pressure of contact, this fine tube penetrates the skin, and the compression of the bladder-like base injects the contained fluid into the wound.

The hairs of many plants produce secretions ${ }^{1}$ which are often

1 Water- and sugar-secreting hairs are considẹred in Chapter XII. 
of the nature of ethereal oils (cf. p. 62). Such glandular hairs are multicellular and generally consist of a basal cell, which is usually sunk in the epidermis, a projecting stalk, and a glandular head (cf. Fig. 7I, g.h.), but are otherwise of very diverse form. In the case of the Chinese Primrose (Primula sinensis, Fig. 5o, $\mathrm{A}-\mathrm{C}$ ) and the Garden Geranium (Pelargonium), the head is formed by a single cell and the stalk by a varying number of cells. In the Labiatæ (e.g. White Deadnettle) the head is com-

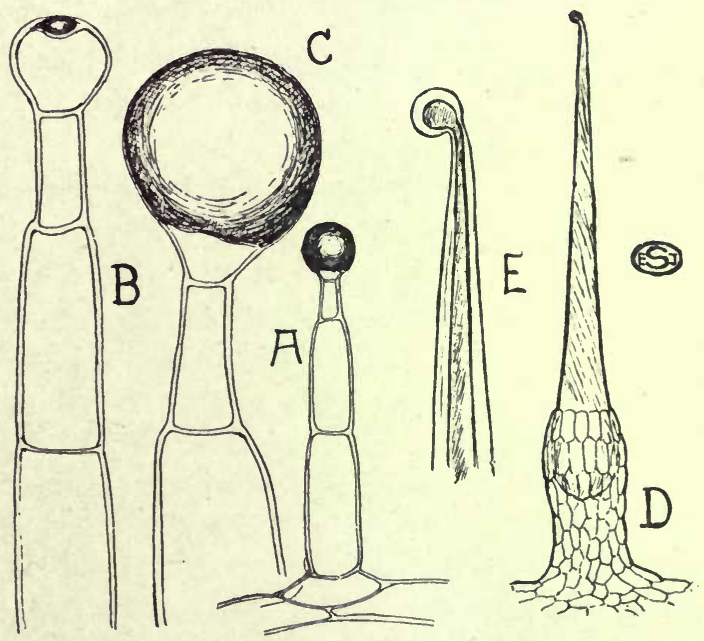

FIG. 50.-Glandular hairs of Chinese Primrose (Primula sinensis, A-C) and Stinging Hair of Nettle (Urtica, D, E). In A-C the secretion is shaded, and in $\mathrm{C}$ and $\mathrm{E}$ only the greatly magnified tip of the hair is shown.

posed of four or more cells disposed in a plane parallel to the surface of the leaf. Extended division of the head leads to the peltate type of glandular hair, such as is seen in the Hop (Fig. 48, E) and the Black Currant.

All the cells of such glandular hairs are living, those of the head which are concerned in active secretion being specially characterised by dense protoplasmic contents and large nuclei. Small droplets of secretion can often be recognised within the young glandular cells, but in the mature condition the ethereal oil is found deposited between the cuticle and the cellulose-layer 
of the outer wall (Fig. 50, B), so that after solution of the oil by means of alcohol a space is evident beneath the cuticle. The volatile oils produced by these glands are the cause of the fragrant perfume of many herbs (e.g. Lavender), and in some cases play a part in the reduction of transpiration (cf. p. 64) or render the plants distasteful to animals. 


\section{CHAPTER IX}

\section{The Structure of the Leaf}

THE blade of a dorsiventral foliage leaf exhibits the following general structure in transverse section. Beneath the colourless epidermis (Fig. 5I, Ep.) of the upper side are one or more layers of vertically elongated cells constituting the palisade tissue (Pa.), which is especially concerned with carbon dioxide assimilation; its cells are deep green owing to the numerous chloroplasts. Between the palisade layer and the lower epidermis lies the loose "spongy" tissue (Sp.), which is composed of irregular cells separated by many and often conspicuous intercellular spaces $(I n$.$) . This tissue contains fewer chloroplasts and communicates$ with the external atmosphere by way of the stomata, its chief function being to facilitate gaseous exchange. Palisade and spongy tissues together constitute the thin-walled ground-tissue, or mesophyll, of the leaf. Here and there the section will pass through veins, some cut transversely, others obliquely or longitudinally; the veins include the vascular tissue with xylem towards the upper and phloem towards the lower side, and each is surrounded by a well-defined layer of cells, the bundle-sheath (Sh.).

Good material for a detailed study of the structure of an ordinary leaf is furnished by the Fuchsia. The features of the epidermis have been fully described in the previous chapter and require no further mention. The palisade cells are four to six times as long as broad and form a single layer (Fig. $5 \mathrm{I}, \mathrm{Pa}$.). They are attached on the one hand to the upper epidermis, and on the other to the rounded cells constituting the uppermost layer of the spongy parenchyma. Narrow intercellular spaces, extending the whole depth of the palisade layer, occur at intervals between the cells, but these spaces are only apparent here and there 


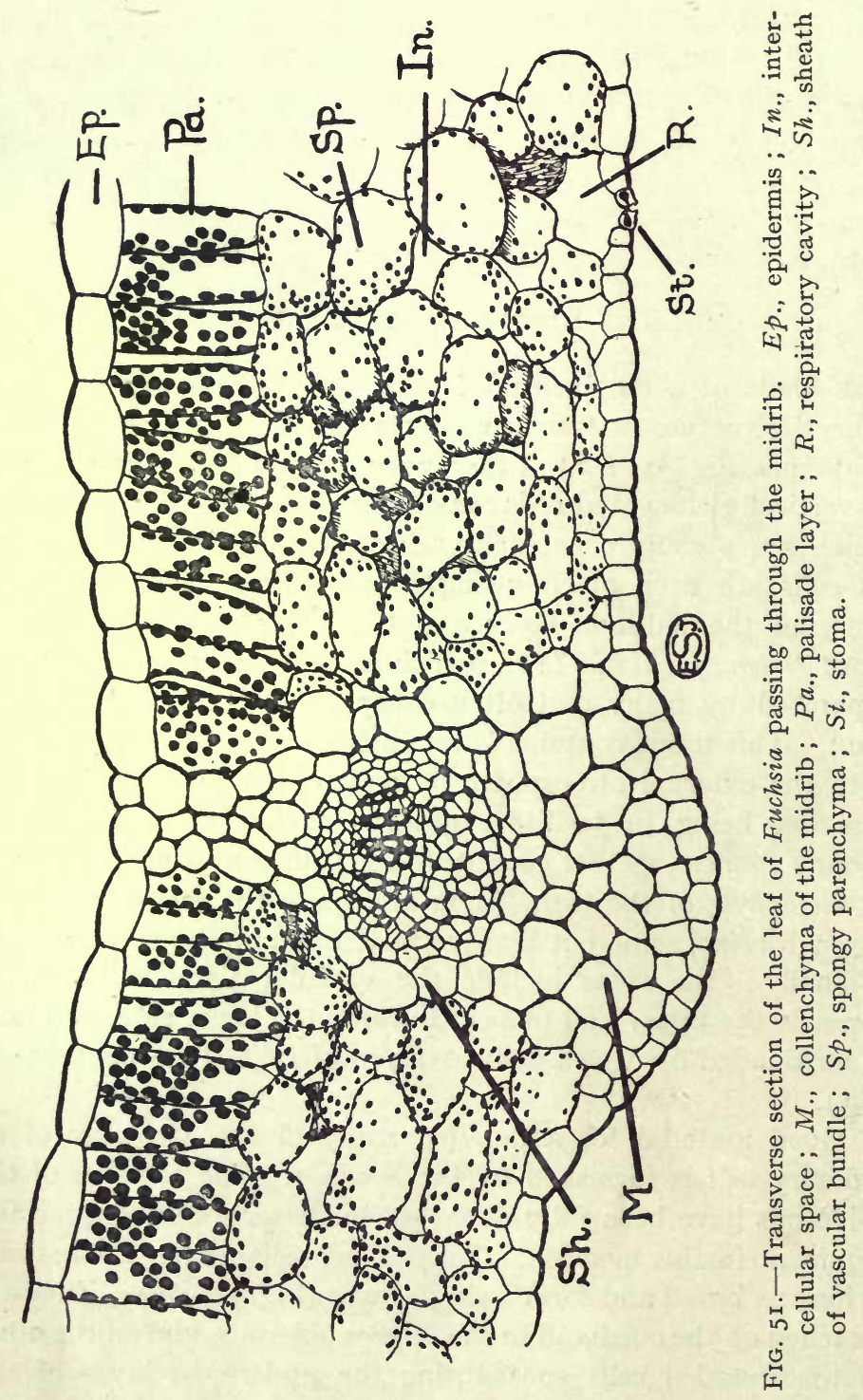


in the transverse section (cf. Fig. 5I, $P a$.). In sections parallel to the surface of the leaf the palisade cells appear rounded (being cut transversely, Fig. 52, A), so that they have the form of a number of closely packed cylinders placed side by side and interspersed with regularly disposed vertical spaces (i.p.), where the curved surfaces are not in contact.

The numerous lenticular chloroplasts ${ }^{1}$ form an almost continuous layer in the cytoplasm lining the vertical walls, a feature well seen in

both transverse and surface sections (Fig. 5I, $\mathrm{Pa}$.; Fig. 52, A ; see also Fig. 43). This peripheral position is clearly favourable to the rapid absorption of carbon dioxide from the adjacent intercellular spaces. Moreover the chloroplasts,
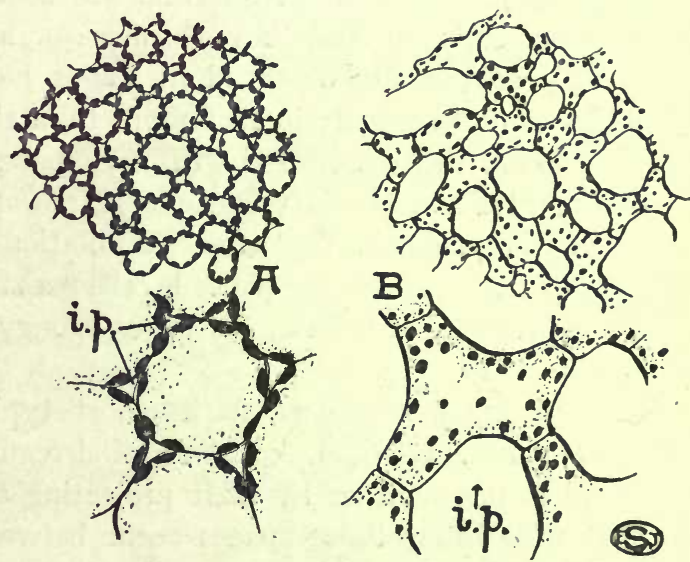

FIG. 52.-Sections parallel to the surfaces of a Fuchsia leaf, cut respectively through the palisade layer (A) and through the spongy parenchyma (B). In each case the lower figure shows a small portion on an enlarged scale. i.p., intercellular spaces.

as a result, present their edges to the light so that the chlorophyll

1 For the general properties of chlorophyll, see F. \& S., p. Izc. It is now known that the chloroplasts of all plants contain two green (chlorophyll $a$ and chlorophyll $b$ ) and two yellow pigments (carotin and xanthophyll), the former being present in considerably greater quantity than the latter. The chlorophylls, which differ but slightly from each other, are complex compounds of Carbon, Hydrogen, Oxygen, Nitrogen, and Magnesium, whilst the yellow pigments are of simpler composition ; carotin has the formula $\mathrm{C}_{40} \mathrm{H}_{56}$, and xanthophyll the formula $\mathrm{C}_{40} \mathrm{H}_{56} \mathrm{O}_{2}$. A rough separation of the green and yellow pigments may be effected by shaking up an alcoholic extract with benzene and allowing the liquids to settle; the alcohol then contains the yellow, the benzol floating above it the green, pigments. 
is protected from the injurious effects of intense illumination. Owing to the considerable length of the palisade cells, there is accommodation for a large number of chloroplasts in each. In some plants, however, the chloroplast-bearing surface is increased by the development of special infoldings of the wall [arm-palisade, e.g. in the leaves of the Elder (Fig. 53, a.p.) and White Lily].

In the transverse section of the leaf of the Fuchsia two or three palisade cells frequently join by their lower ends on to one and the same cell of the spongy parenchyma (Fig. 5I ; see also Fig. 53, c.c.). The cells in question are usually broadened at their upper ends, so that they are more or less funnel-shaped. Such cells are usually observed in leaves having the kind of structure here described; in fact more than three palisade cells may be found thus connected with one spongy cell (e.g. Indiarubber plant, Ficus elastica; Oleander, Nerium oleander, Fig. 82, Col.). It is probable that the assimilation-products (carbohydrates, etc.) formed in the palisade cells pass into these collecting cells and from them diffuse, via other spongy elements, to the veins.

The spongy parenchyma, in its most typical form (e.g. in Euphorbia amygdaloides), consists of irregularly lobed cells attached to one another by their projecting arms (cf. Fig. 82), so that wide intercellular spaces occur between them. In the leaf of the Fuchsia and in many other cases, however, the cells are more rounded and the interspaces consequently smaller (Fig. 5I, In. ; Fig. 52, B). The rather few chloroplasts in the spongy, as compared with the palisade, parenchyma may be related to the fact that the former tissue receives relatively little light. The layer in contact with the lower epidermis is not uncommonly continuous (except for the gaps constituted by the often large respiratory cavities beneath the stomata, Fig. $5 I, R$.), and its cells may even show a palisade-like form; in this case they generally contain rather numerous chloroplasts (e.g. Fuchsia, Corn Cockle), and serve to utilise the light reaching the under-side of the leaf.

The chief function of the spongy tissue is to constitute an extensive intercellular system communicating on the one hand with the atmosphere by way of the stomata, and on the other hand with the entire aerating system of the rest of the plant. 
The spongy cells also serve to conduct elaborated food-materials in various directions to the adjacent veins, a function to which they are well suited by virtue of their irregular shape.

The structure of the mesophyll just described is characteristic of dorsiventral leaves generally, the following being the chief modifications. The palisade tissue not uncommonly consists of several layers (e.g. Wallflower, Holly, Fig. 43, A), a feature especially encountered in leaves exposed to strong illumination, whilst leaves developed in dull light may have little or no palisade $\mathrm{t}$ is sue. In

some cases

(e.g. the House-leek) the assimilatory cells have quite a differentform, being elong a t e d parallel to the midrib, whilst in transverse section they appearmore or less rounded. Plants growing in dry sunny situations often ex-

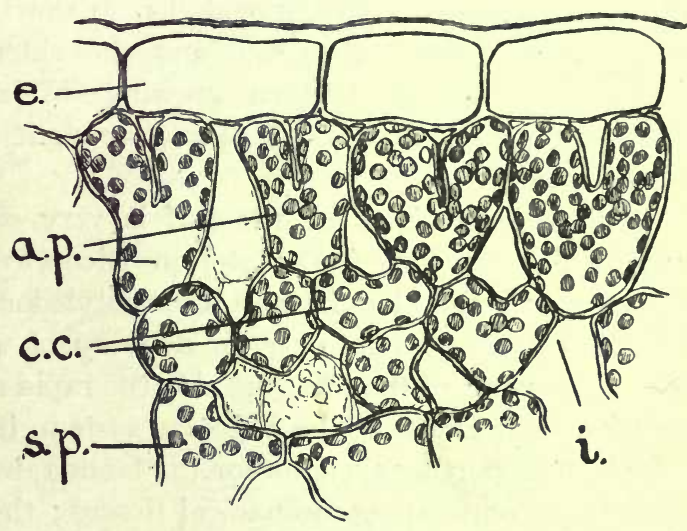

FIG. 53.-Transverse section through part of the leaf of the Elder (Sambucus) showing the upper epidermis (e.); the arm-palisade cells (a.p.); the collecting cells (c.c.); and the spongy parenchyma (s.p.). i., intercellular space.

hibit a second layer of colourless cells beneath the upper epidermis. The cells of this hypoderm (p. 95, Fig. 43, Hy.) are usually thick-walled, and consequently form with the epidermis a strong surface skin, besides further screening the underlying chloroplasts from excessive illumination.

Variegated leaves usually exhibit a similar structure to that of the normal foliage of the same species, except that localised areas show no development of chlorophyll; these areas in consequence appear white or yellow. It need hardly be said that the structure of reduced foliage-leaves (e.g. scale-leaves of rhizomes, bud-scales, etc.) is of a much simpler character. Such are 
usually colourless with a homogeneous mesophyll, and often possess no veins.

Movement of chloroplasts in conformity with the intensity of the illumination is well seen in a few plants (e.g. Duckweed, Lemna; Moss-leaves, etc.). In these cases the chloroplasts occupy a profile position on the vertical walls when the light is intense, whilst when weak the chloroplasts pass to the horizontal walls, so that their full surface is presented towards the source of illumination. Similarly the leaves of some plants (e.g. Bracken ; Yorkshire Fog, Holcus mollis) take up fixed light-positions, at an oblique angle to strong sunlight, so that much of this is reflected from the leaf-surface and the chloroplasts are consequently protected. When growing in shady situations, however, the leaf-blades are placed at right-angles to the incident rays.

The vascular tissue of the leaf is very extensive, forming an irregular network (reticulate) in Dicotyledons and a very regular (parallel) system in most Monocotyledons. ${ }^{1}$ The repeated branching facilitates not only the delivery of water and mineral salts to all parts of the leaf, but also the rapid removal of elaborated food-substances. The vascular system, however, also constitutes a supporting skeleton for the lamina, in which it is often aided by accompanying mechanical tissues; the latter are found especially in the larger veins, and consist of strands of collenchyma (Fig. 5I, M.) or sclerenchyma, which run both above and below the vascular bundles or sometimes on the lower side only.

A transverse section through one of the principal veins of the Fuchsia-leaf shows a single collateral bundle (cf. p. 76) enveloped in a sheath of one or more layers of large transparent thin-walled parenchyma-cells (Fig. 5I, Sh. ; cf. also Fig. 82, V.) ; the accompanying collenchyma $\left(M_{\text {. }}\right)$ is developed especially on the lower side. The xylem, which is adjacent to the palisade tissue, consists of rows of vessels alternating with wood-parenchyma, the protoxylem being directed towards the upper epidermis; the phloem is of the normal type and lies towards the lower side. Between xylem and phloem a cambium can often be recognised (Fig. 5I) ; this is especially the case in evergreen

1 The Cuckoo-pint (Arum maculatum) and Black Bryony (Tamus communis), for instance, have a venation similar to that of Dicotyledons. 
leaves (e.g. Holly) which remain on the plant for more than one year.

In passing to the finer and finer ramifications of the vascular system a gradual simplification in structure is apparent. The differentiation of the phloem becomes less and less distinct, its place being taken by a more or less uniform tissue of thin-walled elongated cells, whilst at the ultimate terminations of the bundles it often disappears completely. Similarly the xylemvessels gradually give place to relatively short spiral or reticulate tracheids (Fig. I8, A, p. 37), the amount of wood-parenchyma diminishing till it dies out. Thus the bundleends usually consist only of tracheids surrounded by the sing le-la yered parenchym a sheath.

In larger leaves (e.g. Sunflower) the midrib often contains several bundles, and ex-

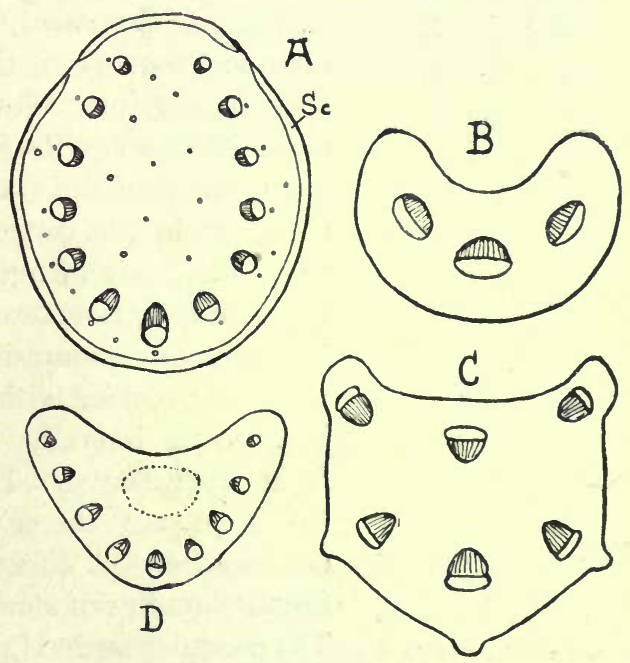

FIG. 54.-Diagrams showing the petiolar structure of A, Sea Holly (Eryngium maritimum); B, Dog's Mercury (Mercurialis perennis) ; C, Black Bindweed (Polygonum convolvulus); D, Bishop's Weed (Egopodium podagraria). The shaded part of the bundles represents xylem, the unshaded part phloem. $S c$., sclerenchyma.

treme cases are seen in the Docks (Rumex) and the Rhubarb (Rheum rhaponticum), where quite a large number of strands occur. On the whole the vascular supply is proportional to the size of the leaf, and this is true also of that of the petiole. In small leaves (e.g. Mouse-ear Chickweed, Cerastium, Fig. 55) the latter frequently contains but a single vascular strand orientated as in the lamina, whilst in larger ones there are several bundles usually grouped in the form of a $C$ with the 
opening towards the concave or flattened upper surface and with the protoxylems directed inwards (Fig. 54, A-D). The part of the ground-tissue included within the group of strands is spoken of as pith, and that outside as cortex, the peripheral layers of the latter not uncommonly consisting of mechanical tissue (Fig. 54, Sc.).

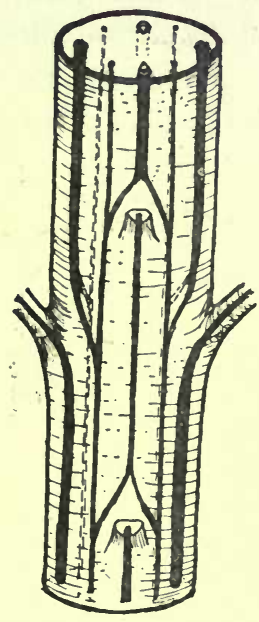

Fig. 55.-Diagrammatic representation of the vascular system in a small portion of the stem of the Mouse-ear Chickweed (Cerastium) (modified from Prantl). Only the bases of the leaves, with a single vascular strand, are shown.

The bundles of the petiole can be traced backwards some little distance into the cortex of the stem, following a slightly oblique course, so that, in sections cut transversely just below the nodes, the one or more bundles (leaf-trace bundles) passing from the leaf into the stem appear cut obliquely in the cortex. After penetrating some little way into the latter the bundles turn abruptly downwards and run vertically through one or more internodes, ultimately, with (Fig. 55) or without previous branching, fusing laterally with strands derived from other leaves. The bundles traversing the stem are therefore merely downward continuations of those found in the leaves, i.e. the bundles of stem and leaf are common. The vascular strands passing to the axillary buds (cf. Fig. 69, p. I42) are branches of those serving the leaves, whilst the vascular supply of stipules originates in a similar manner.

In Monocotyledons, where the sheathing leaf-bases completely encircle the axis, numerous strands pass from each leaf into the stem. The median vascular bundles pass almost to the centre of the stem before they bend downwards; subsequently they pursue a longitudinal direction, at the same time returning obliquely towards the periphery. The more laterally placed strands follow a similar course, but do not penetrate to the same depth. As a result the vascular bundles from the successive leaves usually appear irregularly scattered through the cross- 
section (cf. p. 84), but tend to be more densely crowded near the outside (cf. Fig. 39, p. 84). The bundles are thus again common to stem and leaf.

The customary disposition of the mechanical elements in petiole and midrib in the form of an arc admits of considerable flexibility under the influence of wind, etc., but at the same time is well suited to withstand the vertical strain caused by the weight of the leaf-blade. In peltate leaves (e.g. Marsh Pennywort, Hydrocotyle; Garden Nasturtium, Tropaolum, etc.), where the strain is equally distributed, the petiole, however, exhibits a corresponding radial construction, as in stems. The broadly expanded lamina of the ordinary leaf is exposed to risk of injury under the tearing action of wind and hail, but this is to a large extent obviated by the strengthening network formed by the veins, and especially by the occurrence of marginal mechanical elements. The latter either form arched connections running parallel to the leaf margin, between the finer vascular strands (e.g. Red Currant), or bundles of fibres (e.g. Iris) occupying the same position. Moreover, the epidermal cells at the edge of the leaf are usually especially thickened and have a pronounced cuticle.

In leaves which normally exhibit sleep-movements ${ }^{1}$ (e.g. False Acacia, Robinia pseudacacia; Runner Bean, Phaseolus multiflorus; Sensitive Plant, Mimosa pudica), and in which the petiole takes up different positions in darkness and in light, the swollen leaf-base, or pulvinus (Fig. 56, p.), is the region of movement. This is facilitated by a flattening of the vascular tissue in a plane at right angles to the direction of curvature (Fig. 56, Pu.), the necessary rigidity being attained by a greater development of the cortex, which consists of large, turgid, thinwalled parenchyma-cells. The fall of the leaflet at dusk is accompanied by a decrease in the turgidity of the cortical cells on the lower side of the pulvinus, whilst at the same time some of their watery sap escapes into the intercellular spaces. This is due to a change in the permeability of the protoplasm and, as a result of the infiltration of the intercellular spaces, the whole pulvinus acquires a more transparent, deeper green appearance. At dawn the cells of the pulvinus once more become turgid and 1 Cf. F. \& S. p. 22I. 
the leaf assumes the day-position. In the case of the Sensitive Plant (Mimosa pudica, Fig. 56, B) a similar but very sudden fall in turgidity results when the leaf is touched or injured.

The leaves so far considered exhibit a marked difference of structure and appearance between the upper and lower surfaces (dorsiventral type), but this is not always the case. In the Iris

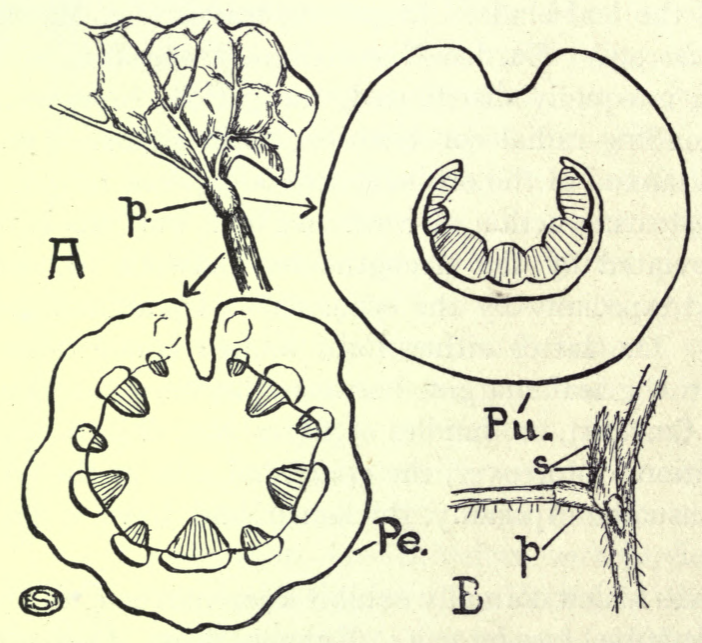

FIg. 56.-A, Pulvinus of Runner Bean (Phaseolus) and diagrams of transverse sections of the pulvinus $(P u$.$) and the petiole (P e$.$) . B, Pulvinus$ of Sensitive Plant (Mimosa pudica). p., pulvinus ; s., stipules.

and other Monocotyledons, where the leaf-blades stand vertically, the structure is identical on the two sides (isobilateral type). In still other cases, particularly in succulents among British plants (e.g. White Stonecrop, Sedum album), the leaves are more or less cylindrical, and, apart from the dorsiventral arrangement of the vascular bundles, exhibit radial organisation (Fig. 86, p. I65), with a palisade layer extending uniformly round the periphery. 


\section{CHAPTER $\mathrm{X}$}

\section{SECONDARY ThICKENING}

IT was pointed out in the preceding chapter that the vascular supply of the leaf is roughly proportional to its size (p. II3), and in the same way the vascular system of the stem is correlated with the area of leaf-surface which it bears. With the annual increase of foliage exhibited by all woody perennials, a need for additional conducting elements arises, and this want is supplied through the activity of a meristem (the cambium, p. 79) situated between the xylem and phloem of the bundles. Cambium is found in this position in all Dicotyledons and Conifers (see p. 340), but in Monocotyledons is present only in certain cases and as a rudimentary vestige (e.g. in the leaf-sheaths of the Maize and in the leaves of many other Grasses). The division of the cells of the cambium leads to the formation of additional conducting elements, accompanied by a gradual increase in the size of the stem, spoken of as secondary thickening. Enlargement does not, however, always imply cell-division, since in Palms, where no active cambium occurs, there is increase in girth mainly due to growth of the cells already present.

The cambium may be regarded as arising from an unaltered remnant of the procambial strand (cf. p. 9o), which has retained its powers of division, but until it becomes active it is difficult to recognise. The actual cambium is established by the appearance of two parallel tangential walls in the persisting procambial elements. There is thus cut out a single layer of radially flattened cells (Fig. 57, C.) which, as seen in longitudinal section (cf. Fig. 38, Ca., p. 83), have an elongated form and pointed ends. Subsequent division of these cells takes place parallel to the two tangential faces whereby files of segments are produced, both on the outer and inner side, those adjacent to the phloem be- 
coming differentiated as additional (secondary) phloem, those adjacent to the xylem as additional (secondary) xylem.

Subsequent to the development of the cambium within the bundles a similar division by two parallel tangential walls takes place in certain of the cells of the medullary rays, and, where a

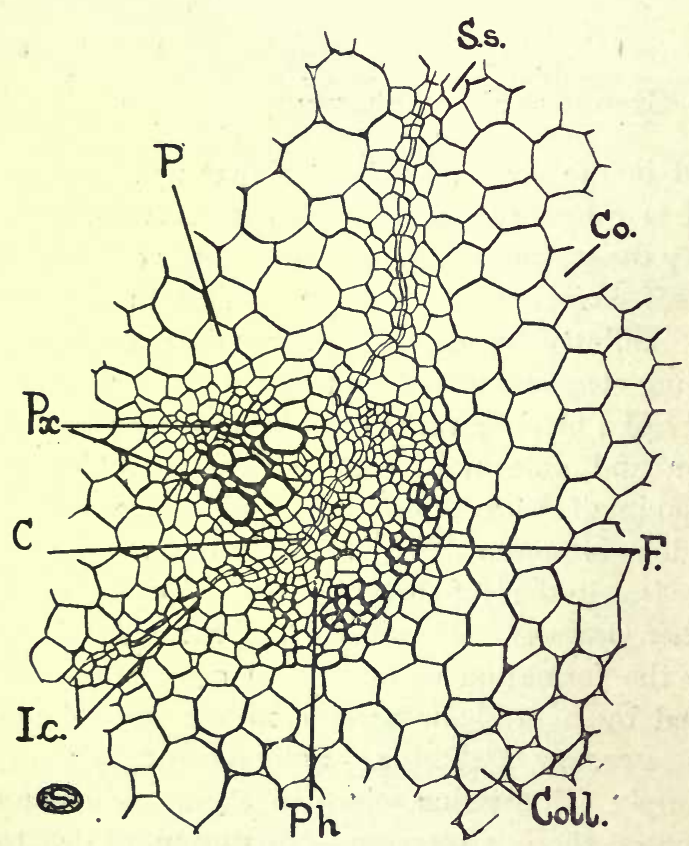

Fig. 57. -Transverse section of a small portion of the hypocotyl of the Castor Oil plant (Ricinus communis). C., intrafascicular cambium; Co., cortex; Coll., collenchyma; $F$., pericyclic fibres ; 1.c., interfascicular cambium ; $P$., parenchyma ; $P h$., phloem ; $P x$., protoxylem; S.s., starch sheath. starch-sheath is present, such divisions will be recognised as occurring in the pericyclic layer (e.g. hypocotyl of the Castor Oil plant, Fig. 57, I.c.). In this way a cambium between the bundles (interfascicular, Fig. 57, I.c.) links up with that within the bundles (intrafascicular, Fig. $57, C$.) to form a complete cambial ring. In some plants, however, the formation of inter-and intrafascicular cambia takes place almost simultaneously. The subsequent division of the interfascicular cambium is like that of the intrafascicular, so that the whole cambial cylinder cuts off segments on both sides. In woody perennials the cambium resumes its function each year, although division is arrested during the winter months (cf. below). 
The secondary wood thus added on the inside forms a larger and larger core each year (Fig. 58). Since it is composed of hard persistent tissue, there is practically no compression of the wood, which progressively accumulates, so that the increase in girth of the stem serves as a rough measure of the amount of tissue added. To this enlargement the secondary phloem con-

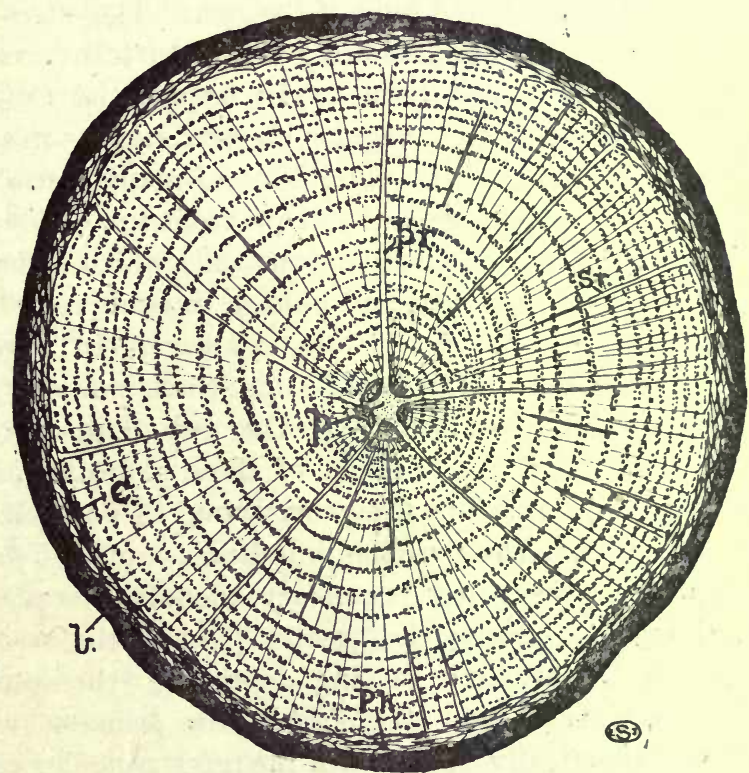

Fig. 58.-Diagram showing the arrangement of tissues in a transverse section of a woody trunk about twenty-four years old. The large vessels of the spring wood of each annual ring are shown as black dots. $b_{.}$, bark; $c_{\text {., }}$ region of cambium; $p_{\text {., }}$ pith ; $P h_{\text {., }}$ secondary phloem; $p r$., primary ray; $S r$., secondary ray.

tributes but little, since this tissue is mainly thin-walled, and the outer earlier-formed elements become compressed more and more, as a consequence of the increasing pressure resulting from the growth of the wood and the annual formation of intercalated phloem. The cambium keeps pace with the enlargement of the circumference of the secondary wood by tangential stretching and occasional radial divisions in its cells.

The pressure on the outer tissues, due to the interpolalion 
of secondary vascular elements between the primary xylem and phloem, becomes more and more marked as the years go by and its effects are most pronounced in the cortex. Moreover, the steady increase in size of the woody core results in a gradual enlargement of its circumference, so that the softer tissues beyond become tangentially stretched. This tension can be readily demonstrated by making an extended vertical incision through the cortex of a three-year-old twig of the Ash. The edges of the cut are seen to separate immediately owing to transverse contraction of the thin-walled tissues. In nature the tangential tension is exemplified by the irregular longitudinal fissures which are so marked a feature of the older bark of many trees.

At certain points the segments cut off from the cambium on either side develop into parenchymatous cells which differ from the other elements of the wood and phloem in being radially and not longitudinally elongated (Fig. 60, A, m.r.). In transverse sections of a secondarily thickened stem these medullary rays appear as a number of radiating streaks, one or more cells in width (Fig. 58, pr. and $S r$.). Some of these rays extend from cortex to pith $(p r$.$) and, since they correspond in position to the$ original rays between the vascular bundles, are called primary medullary rays; others (the secondary medullary rays, $S r$. ), however, though traversing the greater part of the secondary phloem, penetrate only to a varying depth into the secondary wood. In some plants (e.g. Aristolochia) the primary rays are very wide, and practically the whole of the interfascicular cambial strips participate in their formation.

The secondary wood is composed of four main types of elements, viz. vessels, tracheids, wood-fibres, and wood-parenchyma, but transitions between the different types are not infrequent. The component elements often exhibit a distinct radial arrangement (e.g. Horse Chestnut), though this may be somewhat obscured when the vessels are large and numerous (e.g. Lime, Elm). The vessels of the secondary wood usually bear densely crowded bordered pits (Fig. 60, V; Fig. I7, E, b, p. 35), often arranged in distinct vertical series, and not uncommonly show additional reticulate or spiral thickenings deposited on the inner surface of the wall. The septa are frequently placed obliquely (Fig. 6o, C, V) with reference to the radial plane, a feature which 
can be related to the peculiar form of the cambial segments from which the vessels are derived. The perforations are varied (cf. p. 35 and Fig. I7, D-F), but the type in which a number of cross-bars remain is commoner than in the primary wood. Tracheids (cf. p. 37) differ from vessels in being derived from single segments of the cambium, which show no open perforations in the end-walls ; they are usually much shorter than the vessels, but of about the same width. The vessels and tracheids are the water-conducting elements of the secondary wood, and the former are generally much more numerous than the latter; in fact in some cases there are practically no tracheids (e.g. Willow).

The typical wood-fibres closely resemble those described on p. 33, having pointed ends and more or less thickened walls, which are provided with oblique slit-shaped simple pits (Fig. $60, f)$. Such fibres are connected by transitions with others which bear bordered pits with oblique slits (e.g. Beech), and differ

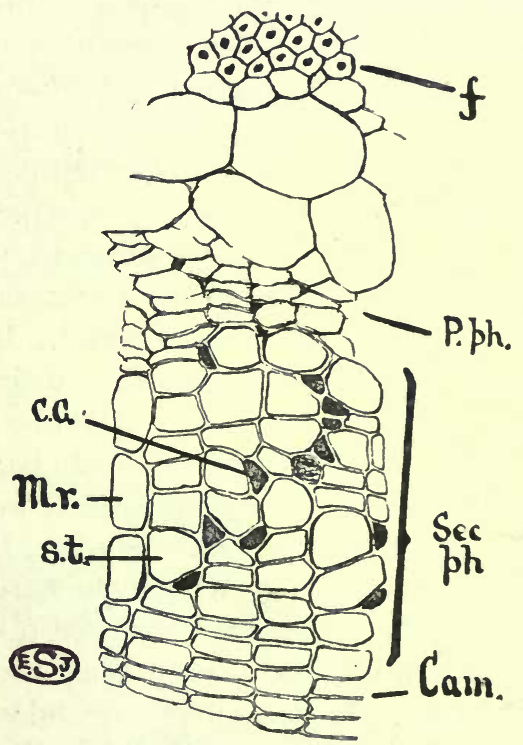

FIG. 59.-Transverse section of the outer part of the stem of the Horse Chestnut (Esculus) showing the secondary phloem (Sec. ph.) and the cambium (Cam.). c.c., companion cells; $f$, fibres of pericycle; $M . v$. medullary ray; P.ph., primary phloem; s.t., sieve-tube.

but little from the fibrous tracheids of the Conifers (see p. 340). In the typical wood-fibres the walls are lignified and the contents dead; but /fibre-like cells with living protoplasts occur in the secondary wood of the Sycamore and of many herbs. Such fibres are occasionally septate (e.g. Vine) and constitute transitions to wood-parenchyma. Where vessels are in contact with the ordinary mechanical fibres, pits are not developed on the walls. 
The wood-parenchyma cells of the secondary xylem resemble those of the primary xylem, in form and in the possession of a living protoplast; the walls are, however, commonly thicker and often lignified, the horizontal ones showing prominent pitting (Fig. 6o, p). Wood-parenchyma cells may be generally distributed throughout the wood (e.g. Birch, Beech), or more especially confined to the autumn-formed wood and the immediate vicinity of the vessels, sometimes completely ensheathing them (e.g. Ash). The parenchyma in contact with the vessels bears simple pits corresponding in position to pits of the vessel wall, which latter are here either simple (e.g. Oak) or bordered (onesided bordered pits), like the remaining pits of the vessel wall.

The structure of the medullary rays can only be fully appreciated by a study of their appearance in transverse, radial, and tangential, longitudinal sections. In the transverse section the cells, except for the fact that their long axis is placed radially and not vertically, are very similar to those of the wood-parenchyma, though not uncommonly having somewhat thinner walls. The latter bear simple pits which are often particularly numerous on the tangential walls (Fig. 6o, B; Fig. 62, m.). The simple pits of the radial walls and the bordered pits of the vessels combine to form one-sided bordered pits like those described above.

Attention has already been drawn to the variable width of the rays. In general the secondary medullary rays are narrow and often only one cell wide; the primary ones in most trees do not greatly exceed the secondary in width, but in many herbs the contrast is extremely marked. Each ray is a plate of cells of which the full vertical extent is seen in tangential longitudinal sections (Fig. 60, C, m.r.). The secondary medullary rays are very limited in this direction, rarely exceeding ten or twelve cells in height, whilst the primary rays usually extend through an entire internode. Each ray, as seen in tangential section, is somewhat spindle-shaped as a result of the tapering of the cells at the upper and lower margins (Fig. 60, C, m.r.).

In radial longitudinal sections the secondary rays are cut parallel to their flat faces, and appear as so many narrow parenchymatous strips passing at right-angles across the longitudinal grain of the wood (Fig. 60, B). The detailed structure of the ray here somewhat resembles that of a brick wall, the alternation 


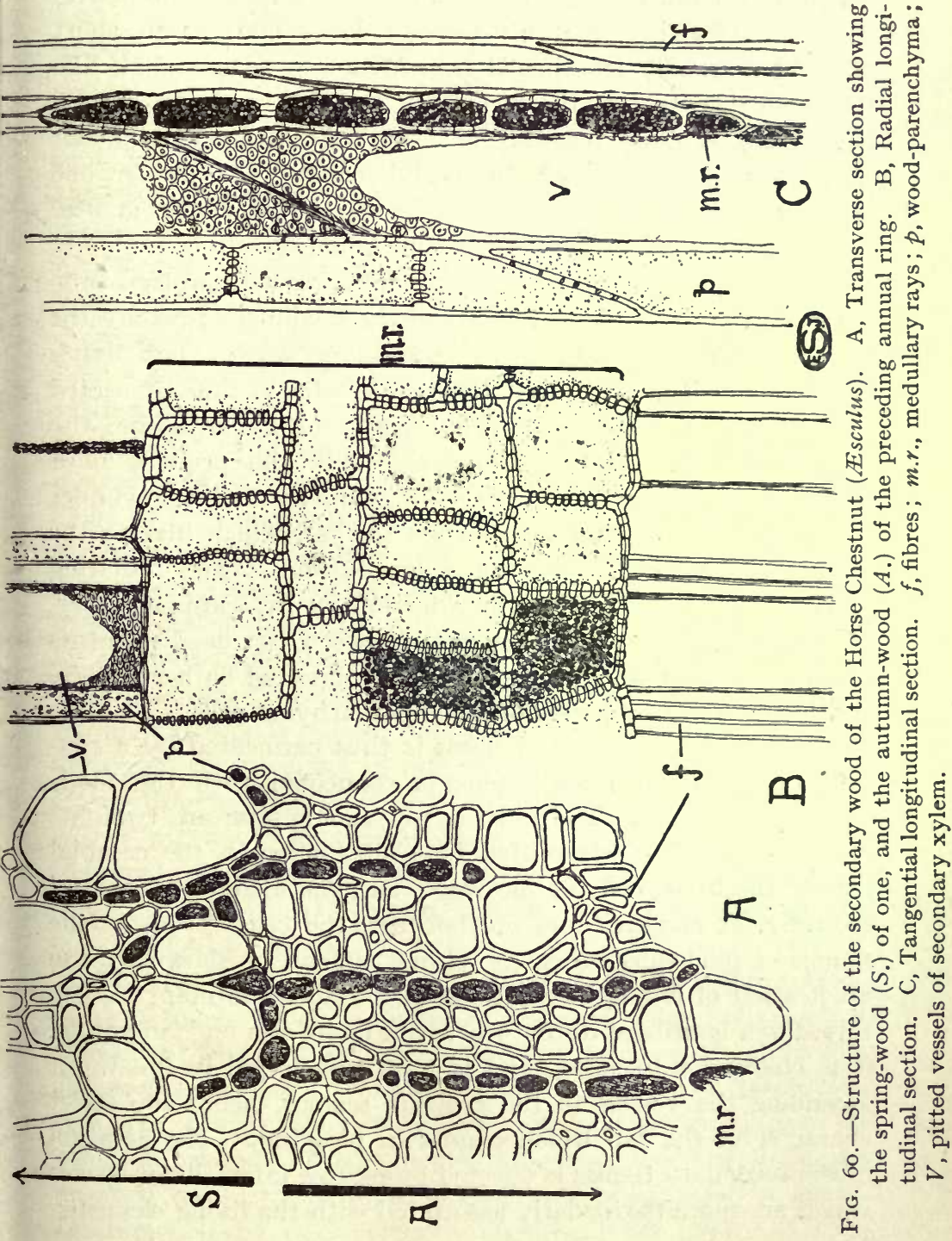




\section{I24 LIVING ELEMENTS OF SECONDARY WOOD}

of the cells simulating the "bond" of the bricks. The component cells show plainly the radial elongation referred to above, but sometimes those at the upper and lower margins are short and not markedly lengthened (e.g. Willows). The radial, like the vertical, extent of the secondary rays is seen to be limited (cf. p. I20), the rays penetrating inwards from the cambium for varying distances. Should the radial section pass through one of the primary rays, the latter will be seen to show a far greater development, both in the vertical and radial directions.

Apart from the occasional presence of fibres with protoplasmic contents, the wood-parenchyma and the medullary rays are the only living constituents of the secondary wood. The living cylinder constituted by the phloem and cortex is thus connected with numerous inwardly directed plates of living tissue, the medullary rays. Of these, however, only the primary ones extend to the pith, where they are linked up by a second cylinder of living cells, the medullary sheath (p. 79), which invests the inner. margins of the primary xylem strands. The radiating plates formed by the secondary medullary rays are, however, not isolated, even where they pass between the dead elements of the secondary wood, since they are connected both vertically and horizontally by bands of wood-parenchyma cells.

The mass of vessels and fibres is thus permeated by a continuous system of living elements connected with the foodconducting tissues. The functions of this system are twofold: firstly, to conduct elaborated food-substances to the cambial region, the living cells of the wood, and the medullary sheath; secondly, at certain times of the year, the cells serve for the storage of food-substances, e.g. starch, as can be shown by the application of iodine to a section of a twig in autumn. When this starch is utilised during the sprouting of the buds in spring, it is changed into sugar, and is then transferred in the water ascending the vessels to the growing regions, hence the sweet character of the sap which exudes in bleeding. ${ }^{1}$ The aeration of the secondary tissues is effected by narrow intercellular spaces which are more particularly associated with the living elements.

1 Cf. F. \& S., pp. 109, 169. Maple-sugar is derived from the evaporated sap of Acer saccharinum (United States), obtained by tapping the trees in spring. 
The major part of each annual addition to the secondary wood usually consists of vessels and fibres, but the proportion of these latter varies with the kind of plant and during each season's growth. The wood formed in the spring (spring-wood, Fig. 60 A, S.) mostly contains a much larger percentage of vessels than that produced later (autumn-wood, A.); its vessels, moreover, are often larger and have thinner walls, and the same may be true of the fibres. This difference can be related to the sudden demand on the water-supply in the spring on the part of the newly-expanding leaves, whilst later in the season provision can be made for the growing mechanical requirements ${ }^{1}$ of the plant by an increased proportion of fibres. As a consequence there is a sharp boundary between the dense small-celled autumn-wood of one season and the wide-celled spring-wood of the next (Fig. 60), and this leads to the marking out of the secondary wood into a succession of annual rings (Fig. 58), by means of which the approximate age of a trunk can be estimated. Occasionally, however-as, for example, when a new set of leaves is produced to replace a first crop killed by frost or devoured by caterpillars - their expansion is accompanied by the formation of a second zone of spring-wood, so that two "annual rings" are formed in a single season. The width of the annual ring is mainly determined by nutrition, though the variations in thickness in one and the same ring are probably the result of mechanical strain. Such asymmetrical annual rings, with a maximum development on the upper or lower side, are commonly found in horizontal branches. An extreme condition is seen in the "buttress-roots" observed in many tropical trees (e.g. species of Ficus).

The constant addition of new wood is probably mainly necessitated by changes in the central earlier-formed xylem, as a result of which it becomes useless for purposes of conduction, although such changes often increase its value as a mechanical support ; it is then spoken of as heart-wood in contrast to the active $s a p$-wood beyond. The elements of the heart-wood often become impregnated with tannins, resins, etc., which are frequently accompanied by dark-coloured pigments; in some cases the

1 A feature that is probably also connected with the growing mechanical strain is the increased length of the fibres in the later-formed annual rings. 
latter are extracted and afford useful dyes, e.g. the logwood (hæmatoxylin) obtained from Hamatoxylon campechianum (Tropical America). The employment of mahogany, walnut, etc., in cabinet-work is largely due to the rich colouration of the heartwood and the high polish which its hard character enables it to take. In the Ebony-tree (Diospyros) the living sap-wood is white and even soft, the ebony of commerce being the mature, very hard, and jet-black heart-wood. In some cases (e.g. Beech) little heart-wood is formed, most of the xylem remaining functional.

The impregnating substances are often antiseptic, and prevent decay by inhibiting the development of Fungi and Bacteria, thus increasing the durability of the wood. Teak (Tectona grandis) owes its great value as a tropical timber to the presence of an oil which renders it immune from the depredations of wood-boring insects ; it is also the cause of its peculiar scent. The liability of many Willows to develop hollow trunks at an early stage may be a.tributed to the absence of antiseptic substances from the heart-wood.

The cavities of the water-conducting elements in the heartwood are frequently blocked in various ways, most commonly by the ingrowth of structures known as tyloses (Fig. 6I). These are bladder-like intrusions through the pits, from the woodparenchyma cells, into the vessels, and are sometimes so numerous as to fill the latter completely with a false tissue resembling parenchyma. They are bounded by the extended thin-walled pit-membrane, which undergoes a certain amount of surface growth, and occasionally becomes thickened and lignified (e.g. False Acacia, Robinia pseudacacia). Each of the young tyloses is living, containing cytoplasm, cell-sap, and sometimes also a nucleus; but when they have reached their full size both the tyloses and the wood-parenchyma cells ${ }^{1}$ of the heart-wood die, so that the whole of the latter consists of dead elements. Tyloses are also produced in herbaceous stems (e.g. Vegetable Marrow, Fig. 6I), but here their function is obscure. The plugging of the vessels of the heart-wood is, however, not always effected in this way, since in other cases mineral deposits (lime in the Elm) may take their place.

1 The latter are invariably dead in the mature heart-wood, even when no tyloses are formed. 
The mechanical properties of timbers depend largely on the proportion of fibres, on the number and size of the vessels, and on the thickness of the fibre walls. Thus, so-called close-grained woods (e.g. Box, Holly, etc.) are characterised by having abundant fibres and small vessels, whilst the coarse or open grain of wood (seen in the Lime, Horse Chestnut, etc.) is due to the

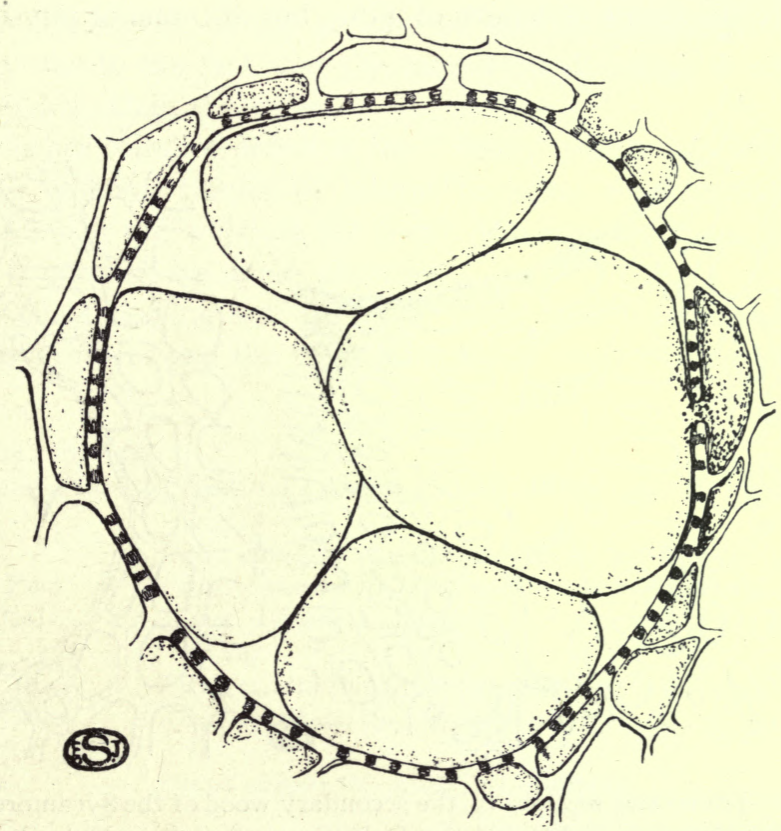

FIG. 6r.-Tyloses in a vessel of the Vegetable Marrow (Cucurbita) as seen in transverse section. On the right the connection with the woodparenchyma is shown.

large number of wide vessels and often to the thin character of the fibres as well. The "soft woods" of commerce are mostly obtained from Conifers whose wood is solely composed of long narrow tracheids (cf. pp. 340, 34I), having thinner walls than the fibres of hard-wood trees.

The employment of timber for commercial purposes is determined by such qualities as closeness of grain, ease of working, elasticity, toughness, durability, colour, figure, etc. Thus, the 
resilience of the wood of certain types of Willow is responsible for their use in the manufacture of cricket-bats, whilst the ancient esteem of Yew (Taxus baccata) for bows, and the modern use of Ash for aeroplane-construction, are the outcome of the elasticity and toughness of these woods. The elastic limit of Ash is only reached under a strain of about 5,000 lb. per square inch as compared with 3,500 lb. for Douglas Fir. Such qualities are probably not only the result of length of fibre, but also one of physical or
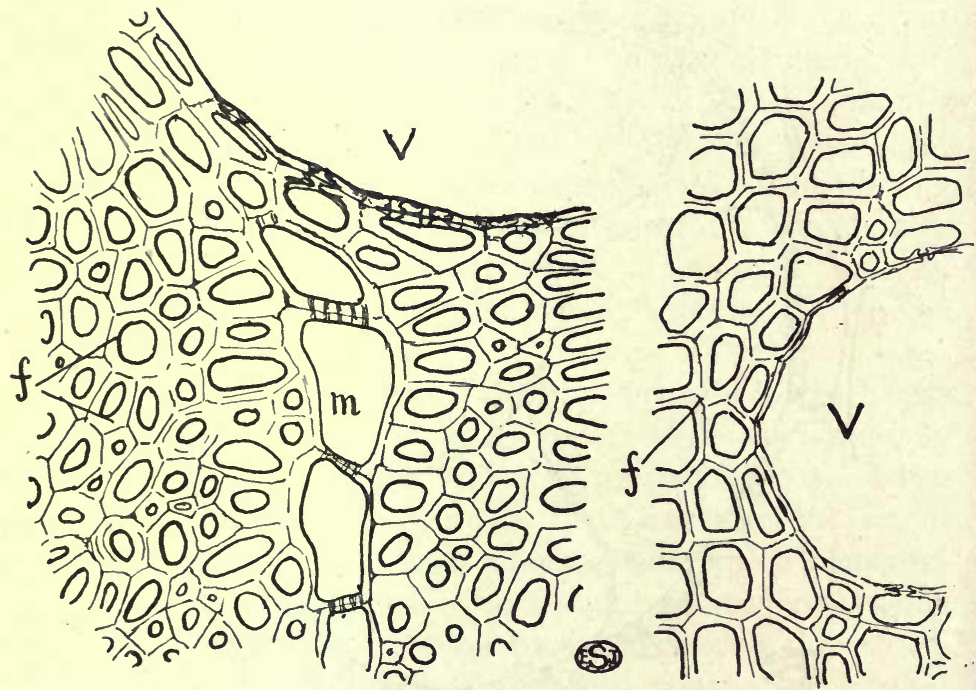

FIG. 62.-Transverse sections of the secondary wood of the Sycamore (Acer pseudoplatanus) (right) and the Oak (Quercus) (left). Note the fibres $(f)$, which are much thicker-walled in the latter than in the former. $m$, medullary ray ; $V$, vessels.

chemical constitution of the xylem walls. Liability to splitting is often due to a large size of the constituent elements, and it is the reverse condition which renders the timber of the Hornbeam (Carpinus) so suitable for cog-wheels, etc. The "figure" of timber, to which many woods owe their value in cabinet-work, is dependent on the direction of the fibres and the prominence of the medullary rays when cut in certain planes.

To render timber suitable for use, the cut log needs to undergo a process of seasoning, which involves the drying out of the 
sap and water, a process that in the open occupies from six months to a year or even longer in the case of hard woods, and about half the time for Coniferous timber. Artificial means (e.g. heating in special sheds or by electricity) are now frequently employed in order to shorten this period. The greatest care is necessary in seasoning to avoid undue stresses and strains, which bring about warping and splitting of the trunk, defects that commonly develop if the process be unduly hastened. This is mainly due to the fact that the tangential shrinkage is considerably greater than the radial. ${ }^{1}$

Another common defect is the " knots," which are constituted by the vascular tissues of lateral branches that have become embedded in the wood of the trunk ; in forestry their development is checked by dense planting.

In the following list are enumerated some of the more important timbers and the trees from which they are derived:

Douglas Fir or

\section{A. Coniferous.}

Oregon Pine . Pseudotsuga douglasii (Rocky Mts.) (woodwork in buildings). ${ }^{2}$

Larch . Larix europaa (Europe) (sleepers, pit-props, etc.).

Pitch Pine . Pinus palustris (United States) (constructional work).

Red Deal. . Pinus sylvestris (Britain and Scandinavia) (building work).

White Deal . Picea excelsa (Norway) (paper-pulp, floor-boards, etc.).

White Pine . Abies pectinata (S. Europe) (joinery).

White or Yellow

Pine . . Pinus strobus (N. America) (joinery).

\section{B. Dicotyledonous.}

Ash . . . Fraxinus excelsior (Europe) (wheelwright's work, too!handles, aeroplanes, etc.).

Beech . . Fagus sylvatica (Europe) (furniture and tools).

Black Walnut . Juglans nigra (N. America) (furniture).

Blue Gum . Eucalyptus globulus (Australia) (constructional work, piers).

Birch . . Betula spp. (N. Europe) (furniture, cabinet-work, bobCedar Wood . Cedrela spp. (America) (cigar-boxes).

1 The tangential shrinkage is often nearly double, and for this reason radial cracks, that open wider and wider as the trunk dries, are not uncommon.

2 A few only of the more important uses are mentioned in each case. 


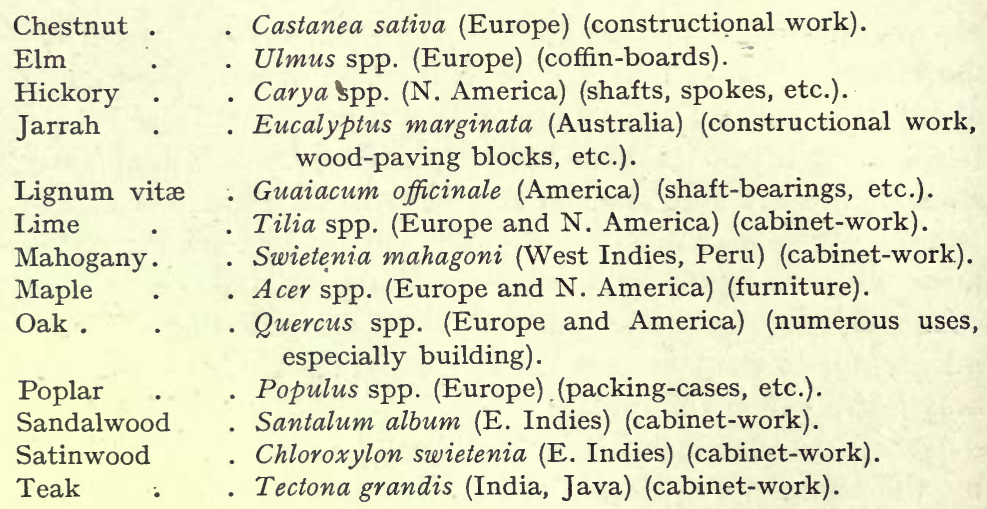

The secondary phloem, like the primary, is composed of sievetubes, companion cells, and phloem-parenchyma, but these are often accompanied by fibres. The septa in the sieve-tubes are not uncommonly oblique and provided with several perforated areas separated by bar-like thickenings (compound sieve-plates). In many herbaceous plants, however (e.g. Vegetable Marrow, Cucurbita), the septum is horizontal and bears but one perforated area. Functionless sieve-like areas are sometimes present as isolated patches on the longitudinal walls of the sieve-tubes (cf. Fig. I3, left). In some cases the phloem-parenchyma and sievetubes, with their companion cells, are produced in such regular sequence as to form alternating tangential bands. The fibres, which show no distinctive features, are likewise often disposed in layers separating the zones of thin-walled elements (e.g. Lime, Mallow, etc.). Vertical series of phloem-parenchyma cells, occupied by crystals of oxalate of lime, and elongated secretory elements (e.g. tannin-sacs, cf. p. I5I) are not uncommon. The older secondary phloem which has passed out of use is, in the case of woody plants, not infrequently shed with the bark (p. I40).

The parenchyma rays of the secondary phloem are continuous with those of the xylem and exhibit the same general structure, except that the component cells remain thin-walled. In certain Flowering Plants (e.g. Lime, etc.) the outer ends of the primary medullary rays exhibit a marked V-shaped enlargement, whereby the secondary phloem becomes divided up into a number of wedge-like groups. 
A transverse section of any unthickened Dicotyledonous root will show a narrow band of two or three layers of parenchymatous cells between each phloem-group and the adjacent xylem. Prior to the commencement of secondary growth tangential divisionwalls arise in these cells, leading to the production of a cambium (Fig. 27, ca., p. 66) like that of the stem. In this way there originate as many cambial strips (Fig. 63, A, $\mathrm{B}, \mathrm{C}$.) as there are groups of phloem, and, by the active division of the former, secondary xylem is produced on the inside and secondary phloem on the outside. Differentiation of the cambium is at first confined to the inner surface of each phloemgroup, but by slow degrees it extends along the sides of the xylem-arms till finally, by the development of tangential division-walls in the

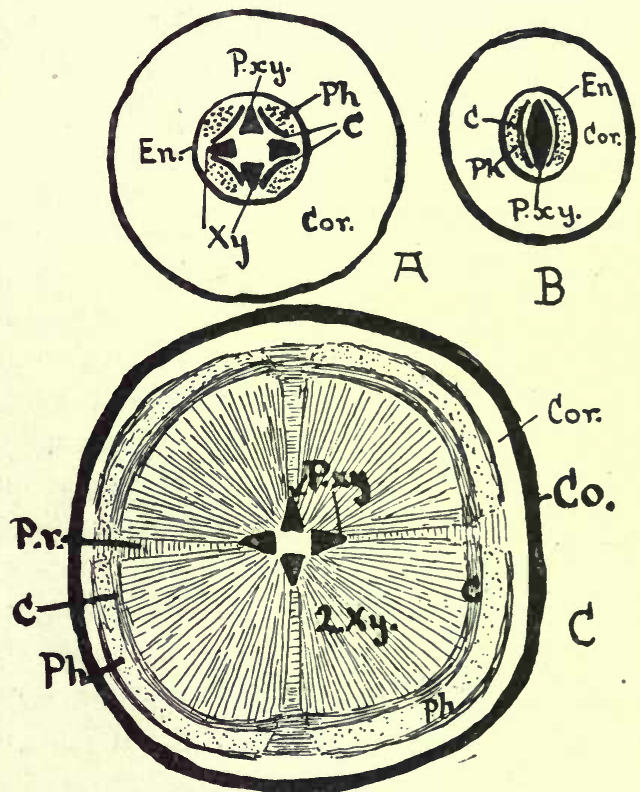

FIG. 63.-Diagrams showing secondary thickening in the root. A, a tetrarch, and B, a diarch root, before thickening, showing the position of the cambium $(C$.$) . \mathrm{C}$, a tetrarch root after secondary thickening has been going on for some time. Co., cork ; Cor., cortex; En., endodermis; $P h .$, phloem ; P.r., primary ray; $P . x y$., protoxylem ; $X y$., primary xylem ; $2 X y$., secondary xylem.

cells of the pericycle opposite the protoxylems, a complete lobed cylinder of cambium is established. Owing to the late development of the cambium opposite the protoxylem-groups, secondary thickening is at first more extensive in the bays, so that the outline of the cambium, at first lobed, gradually becomes circular. 
The root soon comes to possess a broad ring of secondary wood and phloem (Fig. 63, C), similar to that of the stem, and, as in the latter, traversed by primary (P.r.) and secondary rays. The former, which, especially in herbs, often attain a considerable width, are situated along the same radii as, and are equal in number to, the primary xylem-groups $(P . x y$.). When these primary rays are broad and consist only of parenchyma, separate wedges of secondary vascular tissues result. The radial extension of the parenchymatous rays is not so marked as that of the wood, so that certain diarch roots, after secondary thickening, sometimes exhibit a band-like structure (e.g. Nettle). The secondary rays, like those of the stem, are generally narrow.

The vessels and wood-parenchyma of the secondary wood of the root are relatively more numerous and more evenly distributed than in the stem, so that the annual rings are usually less conspicuous. In its detailed structure the secondary phloem is similar to that of the stem, and, except for the points already mentioned, the same is true of the secondary wood. In the root, as in the aerial axis, the production of secondary tissues forces the primary phloem progressively farther from the centre. Old; secondarily thickened roots resemble stems very closely, but, when the central tissues are preserved, the original rootstructure can be traced by following down the primary rays and locating the protoxylem-groups at the periphery of the metaxylem.

The storage of food in fleshy roots is effected by thin-walled parenchyma, which is often copiously developed both in the secondary phloem and in the secondary xylem. The vessels of the latter then form isolated groups, which are either scattered or arranged in radial files (e.g. Salsify, Tragopogon, Fig. 78, B) in the storage tissues, a distribution facilitating rapid transference from the storage cells when growth is resumed. In such roots the limits of secondary xylem and phloem are often difficult to distinguish, unless the cambium be first located.

The process of secondary thickening so far described is that normally found in the vast majority of Dicotyledons and Conifers, but a few so-called anomalous types merit a brief consideration. 
In many members of the Spinach-family (Chenopodiaceæ) the normal cambium functions only for a short time and, after forming a narrow strip of secondary xylem and phloem, ceases to divide. Thereupon another cambium arises in the inner part of the cortex, and a new strip of xylem and phloem is produced till this cambium in its turn ceases to be active. This process is repeated again and again, each successive cambium forming xylem on the inner and phloem on the outer side. In the root of the Beet (Beta) the successive cambia form continuous rings of xylem and phloem, its fleshy character being mainly due to the extensive development of the latter tissue. As a general rule, however, xylem and phloem are produced only at certain points, and appear as secondary bundles separated by the thick-walled groundtissue, developed from the remaining parts of each cambium (e.g. Silver Goosefoot, Obione portulacoides).

Another uncommon method, shown by, certain Monocotyledons which exhibit marked secondary increase in girth, is well illustrated by the Dragon-tree of Teneriffe (Dracana), the stems

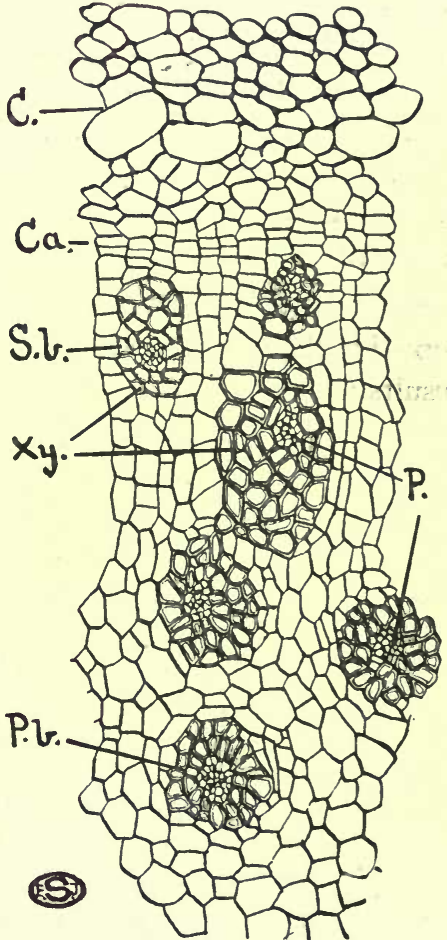

FIG. 64.-Transverse section of a portion of an old stem of Dracana showing secondary thickening. C., cortex ; $C a$., cambium ; $P$., phloem; P.b., primary bundle; S.b., secondary bundle ; $X y_{\text {., }}$ xylem.

of which may attain a diameter of ten feet or more. The bundles of the young stem here exhibit the usual scattered arrangement; but in the cortex, immediately beyond the vascular region, there arises a cambium (Fig. 64, Ca.) which cuts off segments mainly 
towards the inside, the small number cut off on the outside simply forming an addition to the cortex $(C$.). Some of the inner segments divide and undergo gradual differentiation to form secondary bundles (S.b.), whilst the remainder, retaining a somewhat radial arrangement, become thickened and lignified. The secondary bundles embedded in this thick-walled tissue are concentric with central phloem $(P$.$) , but the latter is often very$ scanty in amount; the xylem contains no vessels consisting of fibrous tracheids only.

Very complicated types of anomalous thickening are exhibited by the woody climbers (lianes) of tropical forests. In many of these the old trunks develop cambial rings about several centres, each such cambium producing a separate xylem-core, so that a rope-like structure composed of several intertwined woody strands results. 


\section{CHAPTER XI}

\section{CORK-FORMATION, ETC.}

ONE result of secondary thickening is a marked enlargement of the periphery of stem or root, in consequence of which the outer tissues are subjected to increasing tension (cf. p. I20). These, the epidermis and cortex, ordinarily have but a limited power of stretching, and, as soon as this limit is reached, they rupture and no longer form an effective covering for the underlying tissues. This function is henceforth fulfilled by a secondary protective tissue, the cork, formed by the active division of another cambium, the phellogen or cork-cambium, which arises somewhere in the cortex. In a few cases (e.g. in some cultivated Maples, viz. Acer striatum) the epidermal and cortical cells are capable of limited growth and division, and here the formation of a cork-cambium is correspondingly delayed.

Cork-formation takes place in essentially the same way in both stem and root. The phellogen invariably arises by the development of two successive tangential walls, in the case of the stem, most commonly in the cells of the subepidermal layer (Fig. 65). The cells are thus each divided into three segments, of which the central constitute the actual cork-cambium (c.c.), whilst the outer form the first layer of cork and the inner the first, and often the only, layer of a tissue known as phelloderm $(P h$.$) . Whilst the outer$ and inner segments undergo no further division, the cells of the phellogen divide again and again, one of the two products of each division becoming differentiated as cork or phelloderm, while the other remains as the cell of the cork-cambium. In the majority of cases, however, these divisions of the phellogen lead to the cutting-off of cells on the outside only, so that no further formation of phelloderm takes place.

The activity of the cambium results in the development of 
a continuous tissue consisting of numerous radial files of cells, each file (Fig. 65, I-6) representing the product of one corkcambium cell. This tissue is the cork and, apart from the absence of intercellular spaces between its cells, it is especially characterised by a chemical modification of the cell-walls spoken of as suberisation. This latter renders them practically impervious alike to gases and to liquids, features to which cork owes its utilisation in closing bottles.

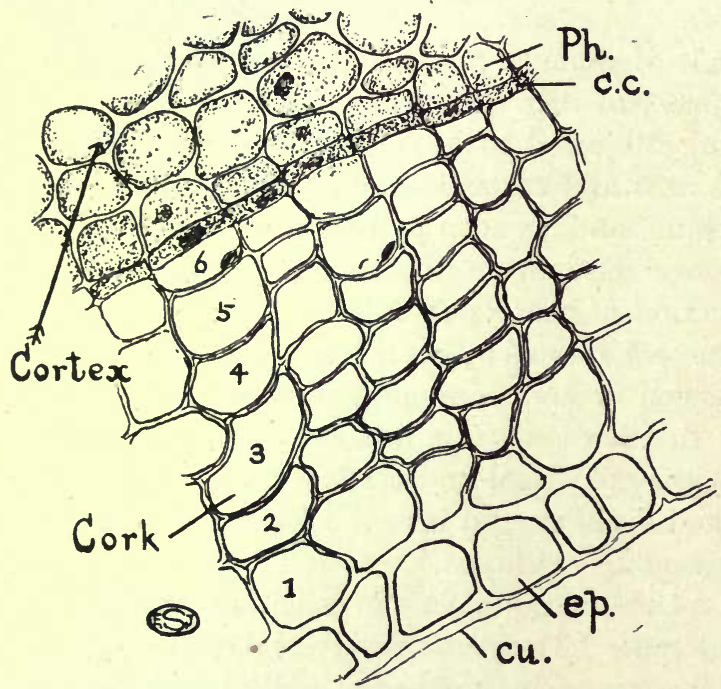

FIG. 65.-Transverse section through the cork layer in the stem of the Elder (Sambucus). c.c., cork-cambium ; cu., cuticle ; ep., epidermis ; $P h$., phelloderm ; I-6, successive segments of the cork in order of production.

Suberisation is known to be due to the presence of various fatty compounds, and hence the walls of the cork are coloured by the same reagents (Scharlach red, etc.) as are employed for staining fats. A yellow-brown colouration is assumed with chlorzinc-iodide, and a yellow one with strong potash. A marked green colouration is obtained by treating cork-cells with a freshly prepared alcoholic solution of chlorophyll, which is allowed to act for about a quarter of an hour in the dark. On boiling with concentrated potash, large yellow globules often escape from the 
walls. Suberised membranes, moreover, are highly resistant, being insoluble both in cuprammonia and concentrated sulphuric acid. Suberisation of the walls ensues soon after the cork-cells are cut off from the cambium, and it is almost unnecessary to add that, as a result, the cells die, their contents ultimately consisting of air or more rarely of pigmented bodies (often tannins and their derivatives). The characteristic white appearance of the surface of the Birch (Betula) is due to the presence in the cells of the cork of solid granules of a substance known as betulin.

The cells of the cork vary considerably in shape, although very commonly flattened. The walls are often relatively thin. The radial walls are frequently thrown into folds, whilst the tangential ones are often almost straight (cf. Fig. 65), facts that can be related to the tangential tension and radial compression set up by the increasing girth of the enclosed axis.

In a few cases (e.g. Currant, Fig. 66, Laburnum, etc.) cells are likewise cut off by the phellogen towards the inside, so that a more or less extensive phelloderm $(P h$.$) is formed. The cells$ of this tissue also are arranged in radial files, but the walls remain unsuberised. The phelloderm consists of living cells, and thus merely serves to augment the primary cortex, although where the walls are thickened it has an additional mechanical value.

It has already been mentioned above that the cork-cambium most commonly develops in the subepidermal layer of cells, but not infrequently it arises in deeper-seated cortical layers (Fig. 66) or even in the pericycle. A good instance is furnished by the Barberry (Fig. 67), where the phellogen (C.C.) develops just inside the ring of mechanical tissue $(S c$.) occupying the inner part of the cortex. In roots, too, it almost invariably arises in cortical cells in the immediate neighbourhood of the pericycle (Fig. 63, C, Co.). The origin of the cork-cambium from the epidermis is seen in Willows, as well as in the Rose, Apple, Hawthorn, and other members of the Rosaceæ.

The cork not only prevents the excessive transpiration which would ensue from rupture of the epidermis consequent upon secondary thickening, but also takes over other protective functions of that tissue. Suberised walls possess considerable strength, though their elasticity is slight, and the cork consequently forms a mechanical envelope whose efficiency is heightened by the 
close connection between its cells. The frequent presence of air in the latter retards excessive heating by day or excessive cooling by night. Moreover, the waste substances commonly encountered in the walls or cavities of the cork-cells are antiseptic, excluding access of various parasites to the living tissues

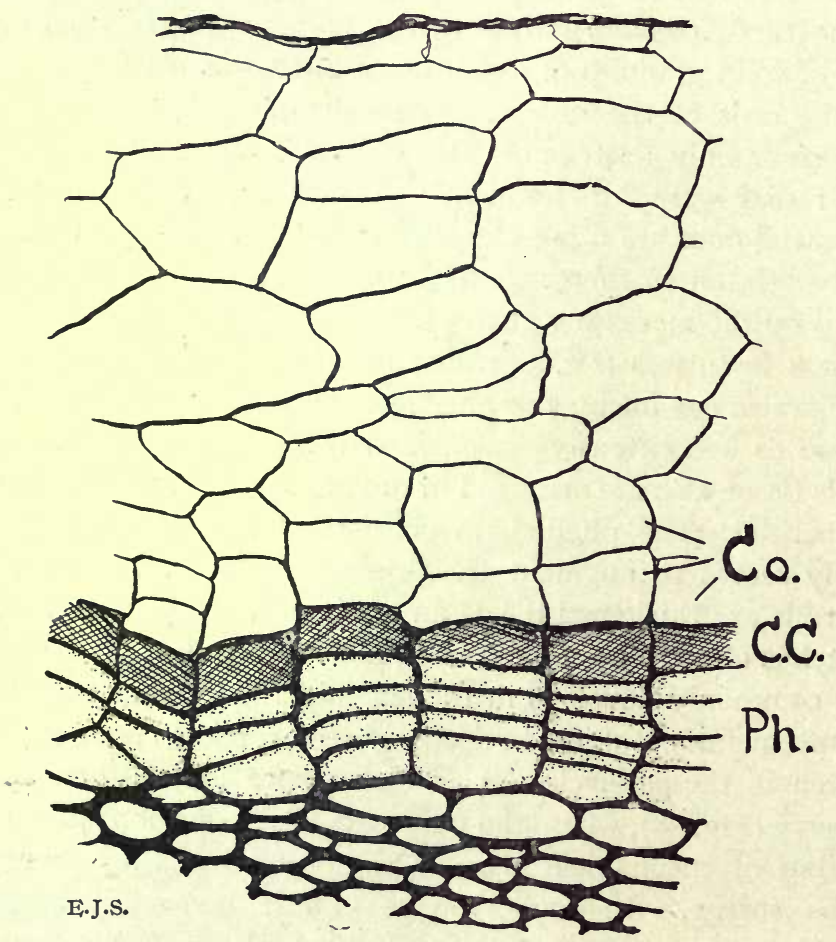

FIG. 66.- Transverse section through the peripheral part of the stem of the Currant (Ribes), showing the cork (Co.), the cork-cambium (C.C.), and the extensive phelloderm $(P h$.$) , of four to six layers of cells.$ Beyond the cork are seen cortex and epidermis.

within. As-soon as cork-development commences, therefore, the parts concerned become ensheathed in an almost impermeable protective layer which would practically sever all direct communication between the internal tissues and the atmosphere, but for the formation of localised patches of loose tissue, the 
lenticels, whose development often commences slightly before that of the cork.

The first lenticels usually arise beneath the stomata of the young stem, where strips of cambium are formed in the subepidermal layer by the customary tangential divisions. These cambial strips divide very actively, cutting off segments on both sides. Those on the inner give rise to radial rows of phelloderm, whilst those on the outer remain thinwalled and unsuberised, but sooner or later round off and lose all connection with one another (Fig. 68, l.). This loose tissue $^{1}$ is formed in considerable bulk, so that it leads to a gradual elevation and ultimate rupture of the overlying epidermis. The tissue of the lenticel is thus exposed, and air from the surrounding atmosphere can freely circulate between its cells and, by way of the narrow air-spaces between the cells of the phellogen and phelloderm, into the inter-

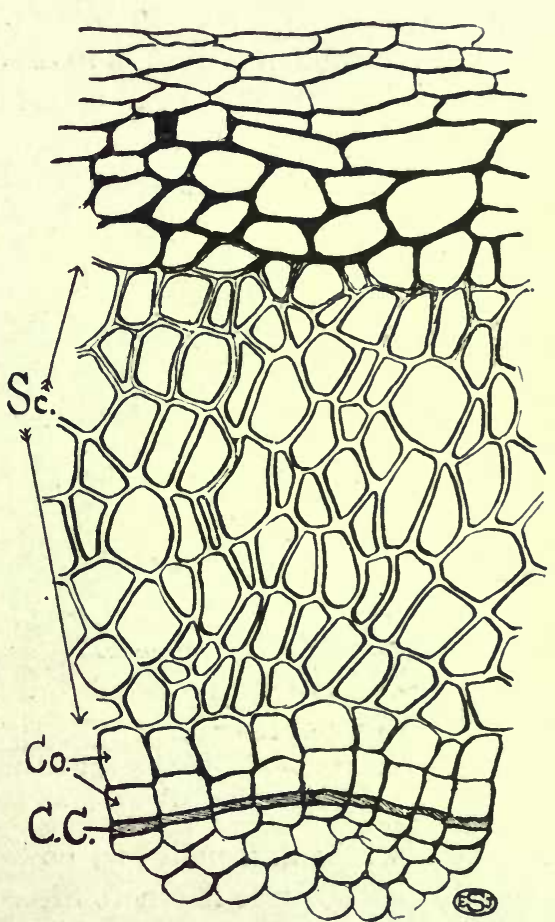

FIG. 67.-Transverse section through the deep-seated cork in the stem of the Barberry (Berberis) showing two layers of cork-cells $(\mathrm{Co}$.) situated between the cork-cambium (C.C.) and the zone of sclerenchyma $(S c$.). cellular spaces of the cortex. The rounding-off of the constituent cells and consequent development of the intercellular spaces vary considerably, so that the lenticel may be spongy (e.g. Elm, Birch) or relatively compact (e.g. Willow, Spindle-tree). The

1. Forming the so-called " complementary tissue." 
strips of cambium giving rise to the lenticels subsequently become continuous with the cork-cambium (Fig. 68).

Where the cork is deep-seated, the tissues external to it are practically cut off from all sources of food and all connection with the interior, and therefore die away. These dead tissues, on the outside of the cork, are shed sooner or later, leaving the latter exposed as bark. In some plants (e.g. Beech, Oak) the same cork-cambium continues to divide year after year, although inactive during the winter, so that a thick mass of cork is formed. This is also the case in the Cork Oak (Quercus suber), which is the main source of the commercial article. The first cork, which

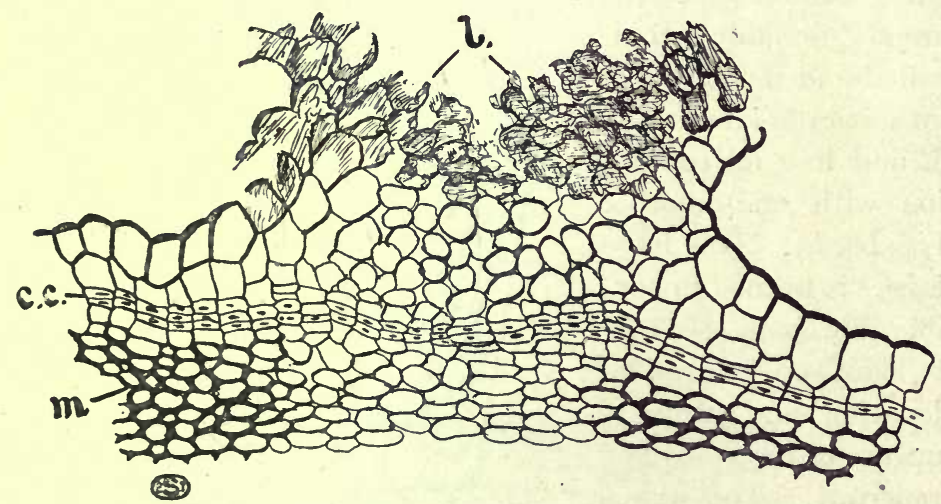

FIG. 68. - Transverse section through a lenticel (l.) of the Elder (Sambucus). c.c., cork-cambium ; $m$, mechanical tissue.

here arises subepidermally, is, however, of no value, being removed when the tree is ten to fifteen years old, the cortex thus exposed forming a new phellogen which gives rise to the thin-walled cork of commerce. This is peeled off every eight to twelve years.

In most woody plants the first-formed phellogen ceases to divide, and indeed itself becomes changed into a layer of cork, at a comparatively early stage. A new cambium then arises at a deeper level in the cortex, produces a fresh zone of cork, and then in its turn passes out of action, to be succeeded by another situated still deeper. The bark formed in this way consists of alternating layers of cork and dead cortex, and comprises all the tissues beyond the most recently established phellogen. When 
the original cork-cambium is deep-seated, the subsequent cambia are continuous cylinders; whilst when the first is superficial, the later ones are often merely vertical strips whose margins are in contact with one another. As the bark gets thicker and thicker the outer portions are subjected to a growing tension, so that, being dead tissue, fissures appear at the surface as irregular longitudinal furrows well seen in the Oak and Elm. In many cases, however, the oldest bark is shed, and this takes place either as rings (ring-bark, e.g. Birch, Cherry) or as scales (scalebark, e.g. Plane, Scotch Fir), according as the successive corkcambia are continuous cylinders or separate strips. ${ }^{1}$

After the shedding of the first-formed cork, lenticels may develop from any part of an active phellogen. As a result of subsequent stretching they assume various forms, which are often characteristic of individual species. Thus on the bark of the Birch and Cherry they appear as slightly raised horizontal lines, whilst in the Poplar they are usually lozenge-shaped. The cork-wings developed in many varieties of woody plants (e.g. Cork Elm, Cork Maple, etc.) are due to the copious formation of cork which, consisting as it does of dead cells, necessarily splits at the surface ; the regularity of the fissures may, however, depend in part on excessive local activity of the cork-cambium.

It will have become apparent that cork serves to protect surfaces which would otherwise be exposed, and indeed it is even found covering wounds and the scars left by the shedding of leaves, flowers, branches, etc. The fall of leaves is preceded by the differentiation of a definite separating layer (abscission layer) which is produced, with or without division, from the cells at the base of the leaf-stalk (Fig. 69, S.). The layer in question is generally recognisable by the smaller size of its cells, and its position is often indicated externally by a slight constriction of the petiole. Subsequently the middle lamellæ between the cells of this layer become mucilaginous and break down, so that for a short time the leaf is connected with the stem solely by the epidermis and vascular bundles. Beneath the abscission layer a protective layer of cork, which subsequently becomes continuous with that of the stem, is developed, either before or after the detachment of the leaf. The smooth layer of cork

1 Regarding commercial uses of bark, see pp. 48, 6o, 62 . 
covering the leaf-scar is interrupted only where the vascular bundles are broken across, and here the vessels are usually plugged by tyloses. The detachment of flowers and the phenomenon of self-pruning, by which small branches of certain trees (e.g. Poplar) are regularly shed, is due to a similar development

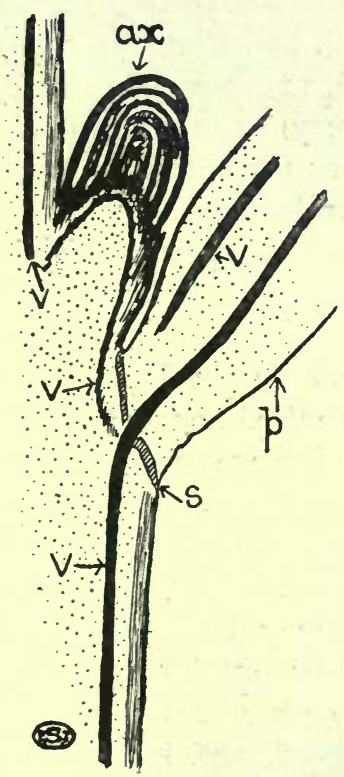

FIG. 69. - Diagrammatic longitudinal section through part of a node of the Sycamore (Acer pseudoplatanus), showing the abscission layer (S.). ax., axillary bud; $p$., petiole; $V$., vascular bundles. of a definite abscission-layer.

When a branch is artificially removed, the living cells, and especially those of the exposed cambium, give rise to a large-celled, thin-walled tissue which at first forms a ring, but often gradually becomes broader till the whole wounded surface is completely covered, the outermost cells usually becoming suberised. The tissue thus formed is called a callus, and may subsequently produce adventitious shoots (e.g. pollarded trees) or adventitious roots (e.g. cuttings). Regions of injury in the parenchymatous tissues (e.g. of cortex or leaf) are commonly isolated from the healthy surrounding tissue by the formation of cork from a cambium produced by tangential divisions in the uninjured cells nearest the wound. In this way the attack of a parasitic Fungus may often be localised.

The cut surfaces of scion and stock, brought into contact in the process of grafting, become intimately associated owing to fusion of the intact cells, which is usually accompanied by some cell-division. Renewed activity in the tissues of a plant may likewise result from wounding by various organisms, either vegetable or animal. The local enlargements produced are termed galls (Fig. 70), and in their formation the, probably chemically, stimulated cells may either enlarge or undergo division.

Little or no tissue differentiation exists in galls caused by 
plants (e.g. those of the Finger and Toe disease, Fig. 135, or those produced by many Rust Fungi), and externally they commonly present an irregular and very variable form. Many animal galls offer a striking contrast in these respects, not only exhibiting a definite external structure, but, as in many of those produced by gall-flies, a complex internal differentiation. In

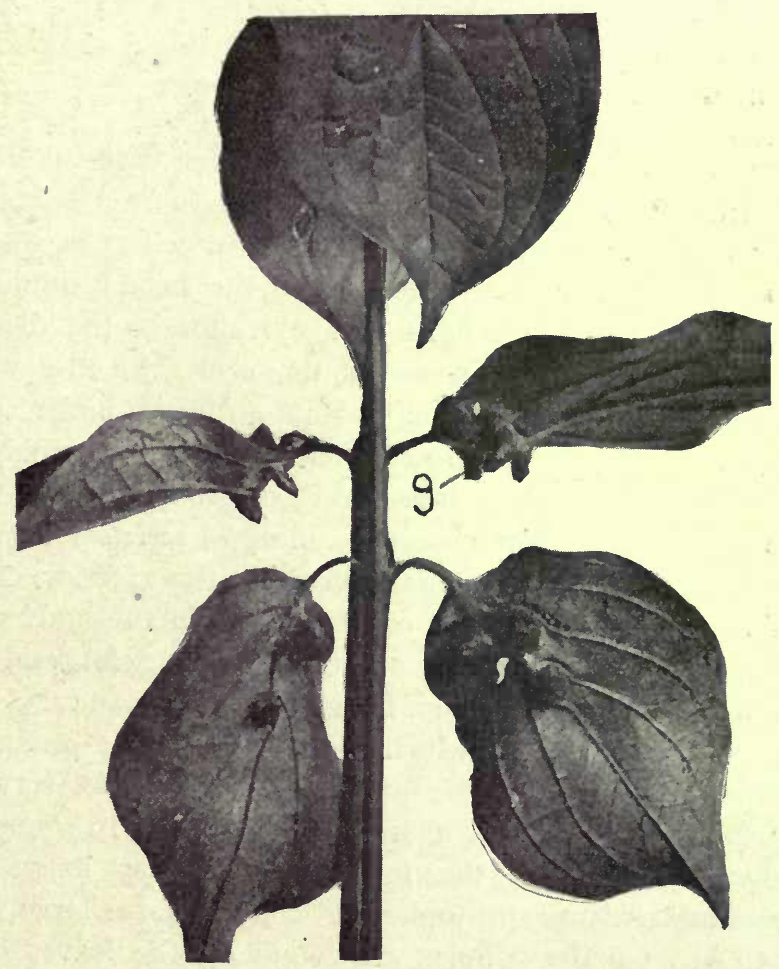

Fig. 70.-Photograph of galls (g.) on leaves of Dogwood

(Cornus sanguinea). [Photo E. J. S.]

some of these cases the central region, where the larva of the gall-insect resides, consists of nutritive tissue composed of cells with dense cytoplasm, rich in protein material, and serving for the animal's nourishment. This region is in some cases surrounded by a layer of sclerenchyma, whilst the outermost zone consists of parenchymatous tissue. The latter is usually well supplied with intercellular spaces, contains little or no chlorophyll, and is often traversed by vascular tissue. 


\section{CHAPTER XII}

\section{SECRETORY ORGANS}

REFERENCE has already been made in Chapter $\mathrm{V}$ to the various by-products which are formed during the constructive and destructive processes in plants and which often collect as so-called secretions. This term is also applied to the sugary liquid produced by nectaries (both floral and extrafloral), the digestive juices formed by insectivorous plants, and even the watery exudations appearing at different points on leaves under certain conditions. These diverse secretions are very commonly the result of the activity of specialised cells or groups of cells, which may be classed under the general heading of secretory organs or glands. Their products may pass direct to the exterior, or may be retained in special cavities or canals within the body of the plant. Many of the secretory organs are superficial, and some of these are developed as hairs, for instance the glandular hairs, already described (p. I05), which secrete various by-products.

Another widespread type, known as a hydathode, serves to remove excess water, either as liquid or vapour. In some cases these hydathodes take the form of glandular hairs, their cells possessing dense protoplasmic contents and large nuclei (Fig. 7I, A). Good examples are found on the leaves of the Runner Bean, where they are bent, club-shaped structures situated near the veins and consisting of a row of thin-walled cells, the terminal cell often being divided into two by a vertical wall (Fig. 7I, A). Many semi-parasites (e.g. Yellow Rattle, Bartsia, etc.) bear numerous water-secreting hairs situated chiefly along the veins of the leaves. These hydathodes (Fig. 7I, B) have the form of small domes consisting of living cells, viz. a large basal cell (b.c.) embedded in the epidermis, a short stalk-cell (s.c.), and a head composed of two to four cells lying side by side 
(Fig. 7I, B, left-hand figure) ; in the middle of the head there is a small space between the cells, and above this the overlying cuticle is pierced by a minute hole $(p$.$) through which the water$ is secreted. Both here and within the leaf cavities of the Toothwort (Lathraa), where similar hydathodes occur (Fig. 7I, C, h.), glandular hairs (g.h.) of another type are present, which may take part in the secretion of water.

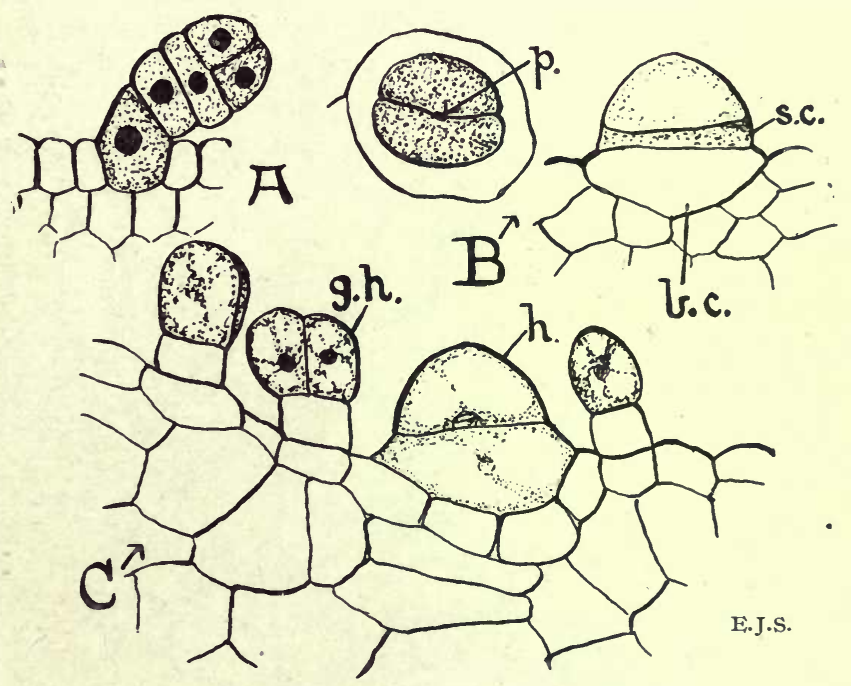

FIG. 71.-Water-secreting hairs. A, Runner Bean (Phaseolus multiflorus). B, Yellow Rattle (Rhinanthus). C, Toothwort (Lathraa squamaria). The left-hand figure of B from the surface, the others in vertical section. b.c., basal cell; g.h., glandular hair; $h .$, hydathode; $p .$, pore ; s.c., stalk cell.

Another kind of hydathode, exhibiting quite a different mechanism, is much commoner among British plants. These hydathodes are not hair-like, but occur generally above the bundle-endings, being especially located at the leaf-apex (Grasses) or on the tips of the leaf-teeth (Lesser Celandine). In all these cases the water escapes from so-called water-pores, which are situated in the epidermis of the hydathode (Fig. 73, St.) and resemble ordinary stomata, except that they are often larger and that their pore remains permanently open in correspondence 
with the absence of the characteristic thickenings in the guardcells ; in surface sections they chiefly differ in their more rounded form (Fig. 72). In some plants but a single water-pore is associated with each hydathode (e.g. Enchanter's Nightshade, Fuchsia), but in others they are numerous (e.g. Wild Strawberry), and occasionally grouped in shallow depressions that can be recognised with the unaided eye (e.g. Marsh Marigold).

In a longitudinal section through an entire hydathode of this

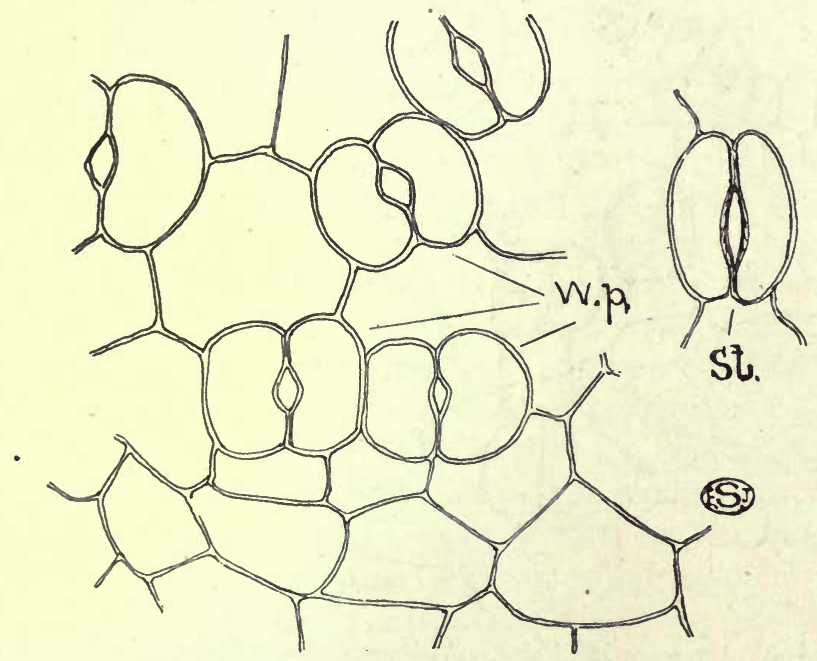

FIg. 72.-Water-pores (W.p.) of the Lesser Celandine (Ficaria verna) seen from the surface. St., a normal stoma on the same scale for comparison.

type (Fig. 73) the end of the vascular bundle, which is here seen to consist of tracheids only, usually enlarges somewhat, often in a cup-shaped manner. Between the tracheids and the overlying water-pores there is commonly a small-celled tissue, the epithem (ep.), composed of cells with prominent nuclei and dense cytoplasmic contents ; there is often, however, a space immediately beneath the epidermis. The epithem is traversed by a system of fine intercellular spaces through which the water passes from the tracheids to the water-pores. In many Monocotyledonous water-plants (e.g. Water Plantain, Alisma plantago) the tissue. 
above the bundle-end breaks down completely, so that the escaping water passes directly to the exterior.

Hydathodes can often be recognised at a very early stage, and are probably most active in the young leaf, which develops in an almost saturated atmosphere within the expanding bud. Active secretion of water takes place when the hydrostatic pressure within the plant becomes excessive, that is when transpiration is slow and absorption considerable, a condition that is most frequently attained at night, but which can be artificially produced by placing a well-watered plant under a bell-jar in a warm room. ${ }^{1}$ The hydathodes can therefore be regarded as safety-valves which avert blocking of the intercellular spaces

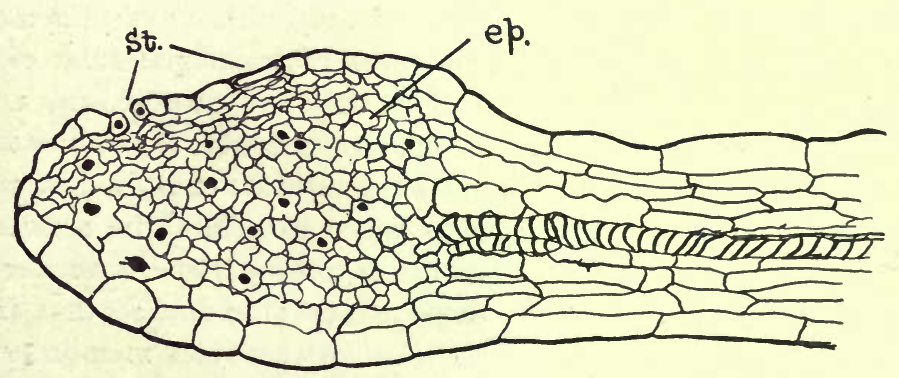

FIG. 73.-Hydathode of Lesser Celandine (Ficaria verna) in vertical section (after Salisbury). ep., epithem tissue ; $S t$., water-pores.

with water under the conditions just mentioned. In the case of the semi-parasites they probably serve to get rid of the water which is absorbed in excess from the host. The liquid exuded from hydathodes is not pure water, but usually contains a very small percentage of dissolved salts. In some cases, however, the amount of the latter may be so considerable that they remain behind as an incrustation when the water evaporates. Thus in the Saxifrages (Saxifraga) a little white scale consisting of carbonate of lime forms on the leaf-teeth over each of the hydathodes.

The exudation of water from the hair-like hydathodes of semi-parasites and the Runner Bean is due to active secretion on the part of the protoplasts of the constituent cells. As a 1 Cf. F. \& S., p. I2I. 
consequence secretion of water ceases, if the cells be killed by painting the surface of the leaf with a solution of corrosive sublimate or other poison. Hydathodes possessing water-pores are, however, mainly passive in their action, the water being forced out by hydrostatic pressure through the intercellular spaces of the epithem, and not by active secretion on the part of the living protoplasm.

Insects are most commonly attracted to flowers by honey formed in special structures termed nectaries. The honey is produced by the active secretion of cells belonging either to the modified epidermis (usually palisade- or papilla-like, the latter in the Wood Anemone, Anemone nemorosa) or to the underlying tissue. The nectary of the Hogweed or other common member of Umbelliferæ, forming the disc on the top of the ovary, is seen in a cross-section to consist of a mass of small glandular cells having the customary thin walls, abundant protoplasm, and large nuclei, and covered by an epidermis containing numerous stomata. The prominent cuticular ridges often exhibited by the epidermis of these nectaries may serve to retain the secreted fluid in situ. In the Buttercup the secreting tissue is similarly composed of small cells, but there are no stomata, so that the nectar only escapes by filtration through the outer membrane.

Extrafloral nectaries on the vegetative organs occur in quite a number of plants, e.g. on the under-surfaces of the stipules of the Broad Bean (Vicia faba), on the leaf-bases of the Black Bindweed (Polygonum convolvulus), at the forks of the fronds of the Bracken (Pteris aquilina, Fig. 74, A), and on the upper part of the petioles of the Guelder Rose (Viburnum opulus). In the firstnamed plant the nectary appears as a dark depression, which when cut across is found to be composed of a palisade-like.layer of secreting hairs, each consisting of an oblong head of several cells, borne on a short stalk. In the Guelder Rose the nectaries take the form of short cup-like projections, each supplied with a vascular bundle, the actual secreting surface being situated in the depression at the tip, and being similar in structure to that of the floral nectary of the Hogweed. The structure of the nectaries of the Bracken will be apparent from a reference to Fig. 74, C.

In all nectaries it is primarily the secretion of osmotic sub- 
stances on the surface (largely sugars) which initiates the exudation of liquid, and the mechanism can be well imitated by placing sugar in a hollow scooped out of a Patato. ${ }^{1}$ The osmotic substances secreted by extrafloral nectaries tend to withdraw water from the plant when it is turgid, and these organs are indeed most active in a moist warm atmosphere. They may well serve primarily, therefore, to fulfil the same purpose as hydathodes. Floral nectaries, however, have another more important function, namely the attraction of inserts.

Glands whose liquid secretion is a digestive fluid containing proteolytic and other enzymes are present on the leaves of most insectivorous plants. By their agency the nitrogenous materials of the captured insects are gradually dissolved and rendered available for absorption. In the Butterwort (Pinguicula) the digestive glands are found on the upper surface of the leaf and take the form of small

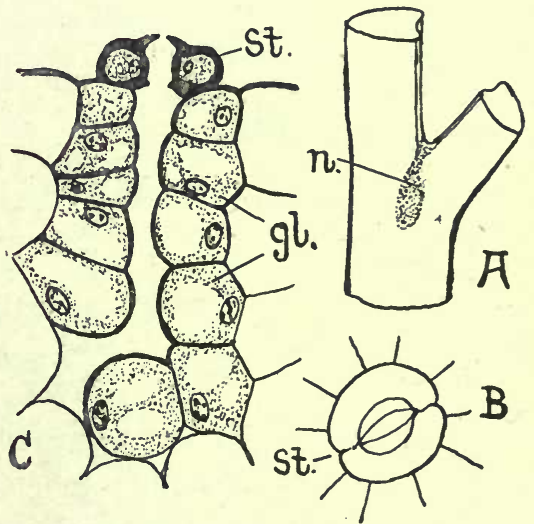

FIG. 74.-Extrafloral nectary of the Bracken (Pteris aquilina) (after Lloyd). A, The fork of a frond showing the nectary $(n$.). $\mathrm{B}, \mathrm{A}$ stoma from the nectary in surface view. C, The nectary in section. gl., glandular cells ; St., stoma.

hairs (Fig. 75, d.) composed of a basal cell, a short stalk-cell, and a usually eight-celled head. Similar glands occur also on the under-surface, but these are probably mainly concerned in the removal of water. The digestive glands are accompanied by other long-stalked glands $(m$.), whose umbrella-like heads secrete the sticky mucilaginous matter to which the insects adhere. In the Pitcher-plant (Nepenthes) the glands occur on the inner surface of the pitcher; they are short-stalked structures, with a very robust oval head of palisade-like secreting cells. 
The tentacles of the Sundew (Drosera) are much more elaborate, being supplied with a vascular bundle which extends into the slightly enlarged tip. In the latter the bundle is enveloped by three distinct layers of cells, of which the innermost is thickened after the manner of an endodermis, while the two outer layers which contain the crimson pigment are those actually concerned in secretion; the superficial one has a palisade-like structure. The tentacles of the Sundew, like many other digestive glands, serve not only for the secretion of the digestive fluid,

but also to

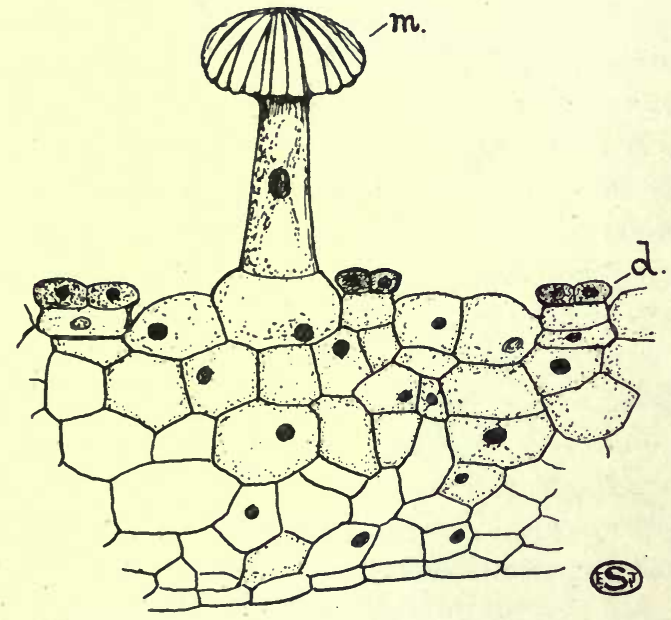

Fig. 75.-Transverse section through a small part of the leaf of the Butterwort (Pinguicula), showing the short digestive glands $(d$.$) and a mucilage-$ secreting hair $(m$.$) .$ absorb the products of its action; in addition they produce the adhesive mucilage which forms a thick glistening layer over the head of the tentacle.

The Bladderwort (Utricularia) is stated to secrete no digestive fluid, the four-armed hairs which occur on the inner surface of the bladders serving solely for the absorption of the products of decay of the minute bodies of the entrapped animals.

Various digestive ferments are likewise secreted by the surface of the cotyledons of albuminous seeds whereby the foodsubstances in the endosperm are changed into a diffusible form. In general there is no differentiation of a special secreting layer, although in Grasses the palisade-like epidermal cells of the scutellum, in contact with the endosperm, are glandular in character. These same cells are also concerned in the absorption of the digested food-substances. 
Secretions of the nature of by-products (ethereal oils, resins, etc.), ${ }^{1}$ although often deposited in glandular hairs, are frequently lodged within the body of the plant. In some plants (e.g. Bay Laurel, Laurus nobilis) such secretions are found in isolated cells (secretory cells), often differing, apart from their contents, in shape and in their larger size from the cells of the surrounding parenchymatous tissues in which they usually occur. Typical instances are furnished by the so-called tannin-sacs, which are generally characterised by a slightly elongated form ; extreme examples are found in the cortex and pith of the Elder (Sambucus).

More striking are the secretory cavities, i.e. large intercellular spaces, approximately isodiametric in form, and again usually lodged in the parenchymatous tissues of the plant. In many cases they appear as transparent dots when leaves containing them are held up to the light, a phenomenon well seen in the St. John's Wort (Hypericum perforatum) and in the Rue (Ruta graveolens). The cavities are filled with an oily secretion, to which these plants owe their peculiar odour. In cross-sections of the leaves of the St. John's Wort the cavities appear more or less circular, each being lined with a layer of thin-walled, somewhat flattened cells (the epithelium, cf. Fig. 76, B, S.), which discharge the secretion into the central space. The cavities of this plant originate by a gradual separation of the cells, a type of development spoken of as schizogenous, and recognisable even at maturity by the presence of a well-defined epithelium ; similar schizogenous cavities are encountered in the leaves of the Myrtle (Myrtus communis).

In those of the Rue, on the other hand, the cavities arise by a disorganisation of the secreting cells whose remains (cf. Fig. 76, A) persist at the periphery, this mode of origin being described as lysigenous; thus at maturity no epithelial layer is present. Similar lysigenous cavities are encountered in the heads of the Clove (Eugenia caryophyllata, Fig. 76, A) and in the skin of the Orange. The secretory cavities of the Rue immediately adjoin the upper epidermis of the leaf, and the secretion in this instance gradually escapes to the exterior through a special pore, somewhat resembling a stoma, except that the slit is zigzagged and surrounded by four cells in place of two; bending of the leaves

1 Regarding the nature and function of these secretions see Chapter V. 
(e.g. by the wind) leads to momentary distortion of the secretory space and consequent emission of part of the secretion. In the Mallows (Malva spp.) many parts of the plant contain irregular lysigenous cavities due to the confluence of cells with mucilaginous walls.

In many plants, and especially in the stems and roots, the secretions are present in elongated structures, the secretory canals, which are generally schizogenous in origin, the bounding epithelium being developed throughout their entire length. In cross-

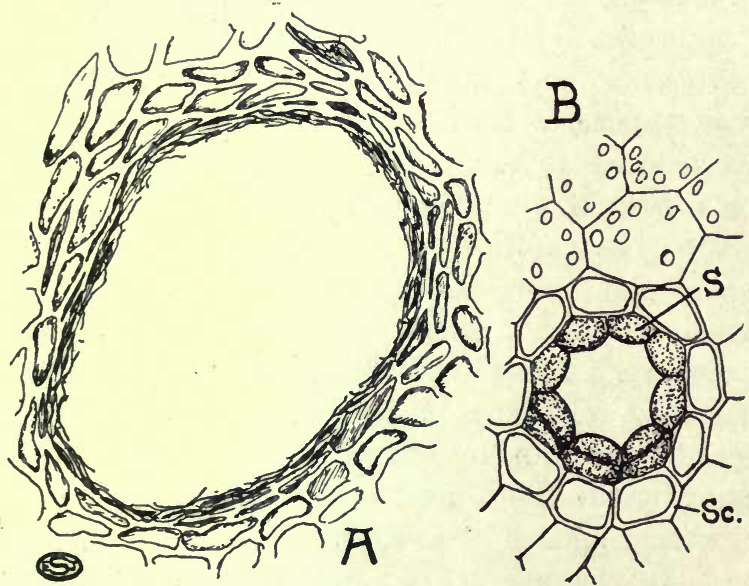

FIG. 76.-Secretory organs. A, Lysigenous secretory cavity of the Clove (Eugenia caryophyllata). B, Schizogenous secretory canal of the Ivy (Hedeva helix) in transverse section. S., secretory epithelium; Sc., sclerenchyma sheath.

sections these canals appear as rounded (Fig. $76, \mathrm{~B}$ ) or oval cavities, whilst in longitudinal sections they are seen to be extensive sinuous tubes which frequently branch and fuse, thus forming a system often pervading all the parenchymatous tissues of the plant. The secretory canals are commonly (e.g. leaf of the Scotch Fir, Pinus sylvestris; petiole of the Ivy) enveloped by a sheath of thick-walled cells (Fig. $76 \mathrm{~B}, S c$.), which prevents compression or collapse through turgor of the surrounding tissue. They often occur in the immediate neighbourhood of the phloem of the vascular bundles, as in the Ivy (Hedera helix) and the 
Umbelliferæ. In the St. John's Wort and certain other cases the secretory cavities of the leaf are replaced by canals in the stem, but it may be pointed out that the difference is one of shape, not of kind.

It is a familiar fact that in some plants a milky, though sometimes coloured; juice (brilliant orange in the Greater Celandine, Chelidonium majus) issues from every cut or broken surface. This latex is especially found amongst British plants in members of the Poppy-family(Papaveraceæ),Spurge-family(Euphorbiaceæ), Harebell-family (Campanulaceæ), the tribe Ligalifloræ of Compositæ, and in the White Convolvulus (Convolvulus sepium); but it is still more characteristic of certain tropical genera. The latex is contained in much elongated tubes which constitute a branched system throughout the thin-walled tissues of the plant and which conform to one of two types.

In the Spurges (e.g. Wood Spurge, Euphorbia amygdaloides) the laticiferous tubes can be recognised already in the embryo as several isolated cells, situated just outside the rudimentary vascular system of the cotyledonary node. Each of these laticiferous cells elongates considerably as the seedling develops, insinuating itself between the surrounding parenchymatous cells, and this process of growth continues throughout the life of the plant. Thus, even in the adult condition, the number of laticiferous cells remains the same as in the embryo. In the course of their elongation the laticiferous cells develop frequent branches which follow a more or less longitudinal course into all the organs of the plant, including the different parts of the flower, but the branches do not fuse with one another. In spite of this extensive growth, which leads to the penetration of the latex-tubes even into the ultimate branches-in tropical Spurges as much as 50 feet above the ground-no cross-walls arise in these elements. On the other hand, as elongation and branching occur, repeated nuclear division takes place, the numerous minute nuclei in the adult laticiferous cell being embedded in the lining layer of cytoplasm which envelops a continuous vacuole occupied by the latex.

In transverse sections through the mature stem of a Spurge, the branches of the laticiferous cells (Fig. 77,l.) will be seen at the outer limit of the phloem $(p h$.$) as a number of large circular elements$ 
with thick white walls. Longitudinal sections, cut tangentially to the phloem, show the characteristic form of the tubes, and branching can often be recognised (Fig. 77, B). The granular latex, which has been coagulated by the preservative (spirit), contains curious starch-grains somewhat resembling minute knuckle-bones. These, and the thick walls of the tubes, are peculiar to the Spurges, but in other respects the features just described are applicable to all laticiferous cells.

By contrast laticiferous vessels, which are characteristic of Papaveraceæ, Campanulaceæ, and Compositæ, are formed from rows of cells (which may run in any direction, though prevalently longitudinal)

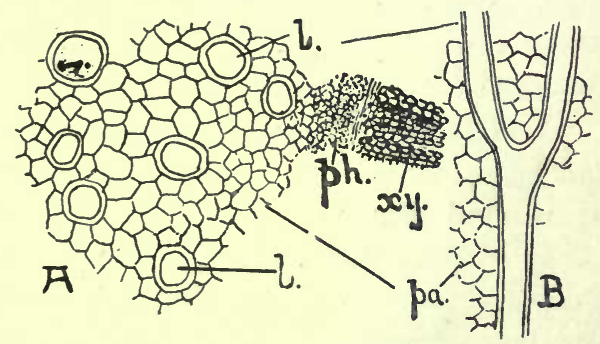

FIG. 77.-Laticiferous cells $(l)$ in the stem of a Spurge (Euphorbia) in transverse (A) and longitudinal (B) sections. pa., parenchyma of cortex; ph., secondary phloem ; $x y$., secondary xylem. by the partial, or usually complete, breaking down of the cross-walls. Laticiferous vessels are usually not recognisable in the embryo, but arise at a later stage in development. They too form an extensive system in all parts of the plant, most commonly near or within the phloem.

They are readily distinguished from the laticiferous cells, however, by' the occurrence of frequent fusions between their branches, as a result of which they form a highly irregular network (Fig. 78, C). The mode of origin of these elements can seldom be recognised in the adult condition, but in the Greater Celandine (Chelidonium majus) longitudinal sections show remains of the partially absorbed transverse septa quite clearly.

Laticiferous vessels are abundant in the secondary phloem of the fleshy roots of the Dandelion (Taraxacum) or Salsify (Tragopogon, Fig. 78, A) ; in transverse sections of preserved material they are plainly recognisable by their brown contents. They have comparatively thin walls and present a very irregular shape (Fig. $78, \mathrm{~B}, l$.), which is due to the plane of section often 
more or less coinciding with that of a cross-connection between the vertical components of the system; moreover, owing to the thin walls of the laticiferous vessels, the pressure of the surrounding elements leads to distortion. The dense irregular network resulting from the numerous cross-connections is a very prominent feature in a radial longitudinal section (Fig. $78, \mathrm{C}, l$.).

Latex, like milk, is an emulsion, the fluid basis of which is a solution of diverse substances (mineral salts, sugars, proteins, tannins, etc.). In some cases it includes an important active principle of the plant; for example, in the Opium Poppy $(\mathrm{Pa}$ paver somniferum) the alkaloid morphine. The suspended particles include on the one hand oil-drops, on the other granules of resin, gum, protein, and caoutchouc, whilst, as already noted, starch-grains occur, in the latex of the Spurges. On exposure to air latex as a general rule congeals rapidly, a change often accompanied by discolouration" ; the " set-
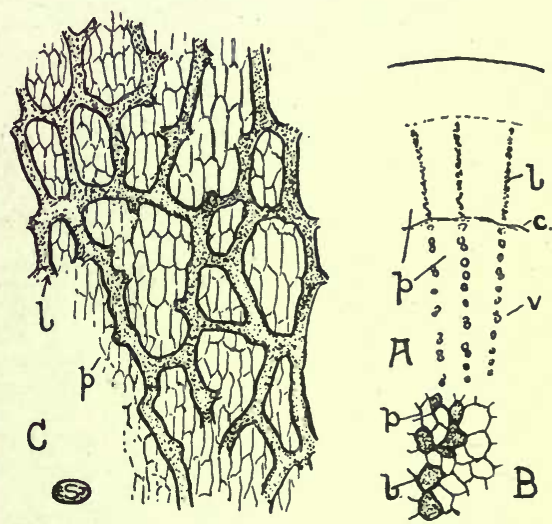

FIG. 78.-Laticiferous vessels in the root of the Salsify (Tragopogon). A, Diagrammatic representation of a small part of a transverse section, showing the distribution of the laticiferous vessels $(l$.$) , in relation to the cam-$ bium (c.), and the vessels of the xylem $(V$.$) . B, a small part of the$ secondary phloem enlarged. C, Longitudinal section. p., parenchyma. ting " is partly due to evaporation of water, but mainly to a confluence of the oil-globules and suspended particles. The coagulation of the latex, like that of blood, is of advantage in protecting and rapidly covering a wounded surface; moreover, the "dressing" in this case is even antiseptic. The laticiferous elements further serve as food-reservoirs, and in this connection

1 This feature is especially marked in the case of the latex of the Lacquer-tree (Rhus vermicifera), where the action is due to an oxidising enzyme (cf. p. 55) which converts the white juice into a dark shining varnish. 
it may be noted that the latex of starved plants becomes thin and watery. Moreover, the frequent association of these

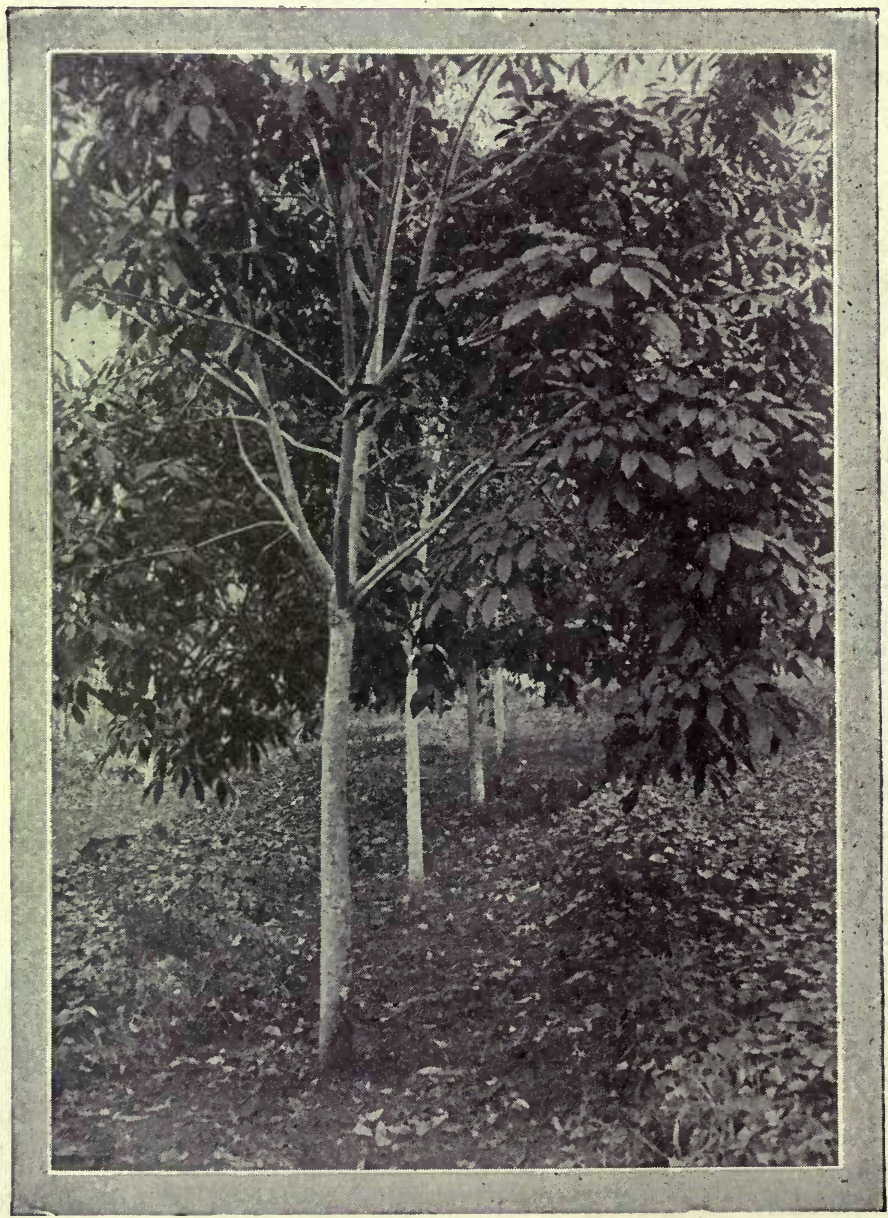

FIG. 79.-Row of Para-rubber trees (Hevea brasiliensis) on Galley Beach Estates, Ceylon. (Reproduced by permission of the proprietors of the Indiarubber Journal.)

elements with the phloem, and the often intimate contact between them and the assimilatory tissues, seem to indicate a rôle in the transport of elaborated food-material. The by- 
products, which are not uncommonly present in considerable quantity in the latex, probably render these plants distasteful to animals.

Those plants, whose latex contains a considerable percentage of caoutchouc-particles, are of great economic importance, since they are the source of the rubber and gutta-percha of commerce. Para-rubber is obtained from a member of the Spurge-family (Hevea brasiliensis) in which, however, the latex is contained in laticiferous vessels. Other kinds are Ceara-rubber (from Manihot glazinvii, a member of Euphorbiaceæ), African rubber (from species of Landolphia, which belong to the family Apocynaceæ), and those obtained from the Indiarubber plant (Ficus elastica) and from Castilloa elastica (Central America). Gutta-percha is derived from diverse members of a tropical family, the Sapotacex, but here the latex is contained in vertical rows of cells.

The latex is obtained by making V-shaped or herring-bone cuts in the bark of the tree and collecting the exuding juice in a small cup. The flow is maintained by paring off thin slices from the lower edges of the sloping cuts, so that the latex-tubes are kept open. After the latex has been artificially coagulated it is washed, and thereupon the raw product is vulcanised. The process of vulcanisation or curing involves a combination with sulphur in varying proportions, according to whether soft rubber or vulcanite is required. 


\section{CHAPTER XIII}

\section{Anatomy in Relation to the Habitat}

THE fundamental organisation of the plant is essentially the same for that of the desert as for that of the lake or mountaintop, but the detailed structure is nevertheless subject to considerable modifications in harmony with the differing conditions of the environment. The most striking of these modifications are related to the conditions of water-supply in the varied habitats in which vegetation occurs, but light and other factors may also play a part in moulding the structure of the plant. The effect of diverse conditions is most patent when the self-same species occupies two different habitats, as in the case of the sun- and shade-forms of many common plants, and the land- and waterforms of aquatics.

The anatomical features exhibited by plants that have to economise their water-supply ${ }^{1}$ may be taken first. Such economy may be necessitated by diverse factors, the most important of which are deficiency of water in the soil (e.g. sand-dunes), extreme transpiration (as on a heath), or conditions which retard absorption by the roots (e.g. moorlands) ; these factors may either act separately or several may operate simultaneously. Many of the structural peculiarities, associated with environments in which such factors prevail, are of the nature of transpiration-checks, whilst others are connected with the storage of water during times of plenty to be gradually utilised during periods of drought. Among the former the most important are : development of a thick cuticle, depression of the stomata below the general surface (cf. p. 96) and other modifications of the stomata, restriction of the latter to grooves or pits, copious production of hairs (p. IOI), and reduction of the leaf-surface. ${ }^{2}$

1 Such plants are frequently spoken of as xerophytes.

2 See also F. \& S., chapter xiii. 
Several of these features can be observed in a cross-section of the cladode of the Butcher's Broom (Ruscus aculeatus, Fig. 80). The epidermis (ep.) has thick outer walls furnished with a pronounced cuticle, but there is no appreciable depression of the stomata. The latter (St.) are protected by the development, on the external portions of the contiguous faces of the guard-cells, of two pairs of ridges which extend upwards towards the pore ; the outer pair are the more prominent, whilst the smaller inner

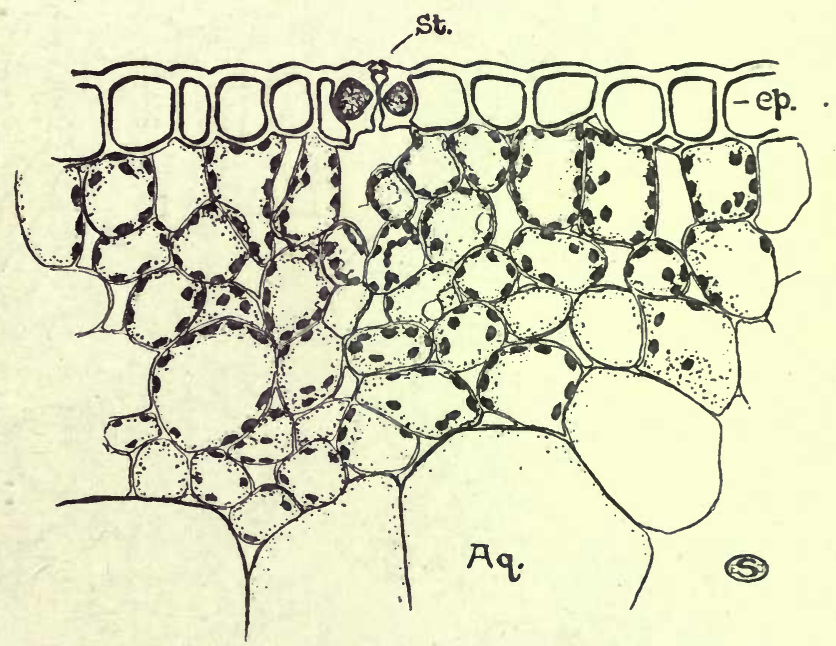

FIG. 8o.- Transverse section through part of a cladode of the Butcher's Broom (Ruscus aculeatus), showing a single stoma (St.), the thickwalled epidermis (ep.), and the aqueous tissue $(A q$.$) below the assimi-$ latory zone. The chloroplasts are shown black.

ones, which correspond to those present in most stomata, almost meet in the middle line. In consequence two antechambers are interposed between the actual pore of the stoma and the outside air, the larger and outer being known as the vestibule. The upper and lower epidermis are alike as regards thickening of the walls and distribution of stomata, and this, as well as the absence of a marked palisade layer, can be related to the "edge-on" position which this modified branch assumes. The assimilatory tissue, which is almost uniformly developed, exhibits rounded 
cells having slightly thickened walls with simple pits and separated by narrow intercellular spaces (Fig. 80). The central region of the cladode is occupied by large water-storing cells (aqueous tissue, $A q$., cf. below, p. I66) which are enveloped by the assimilatory tissue. The arrangement of the vascular system is like that of a leaf.

The development of a vestibule impedes the escape of the water-vapour exhaled from the stoma, and consequently

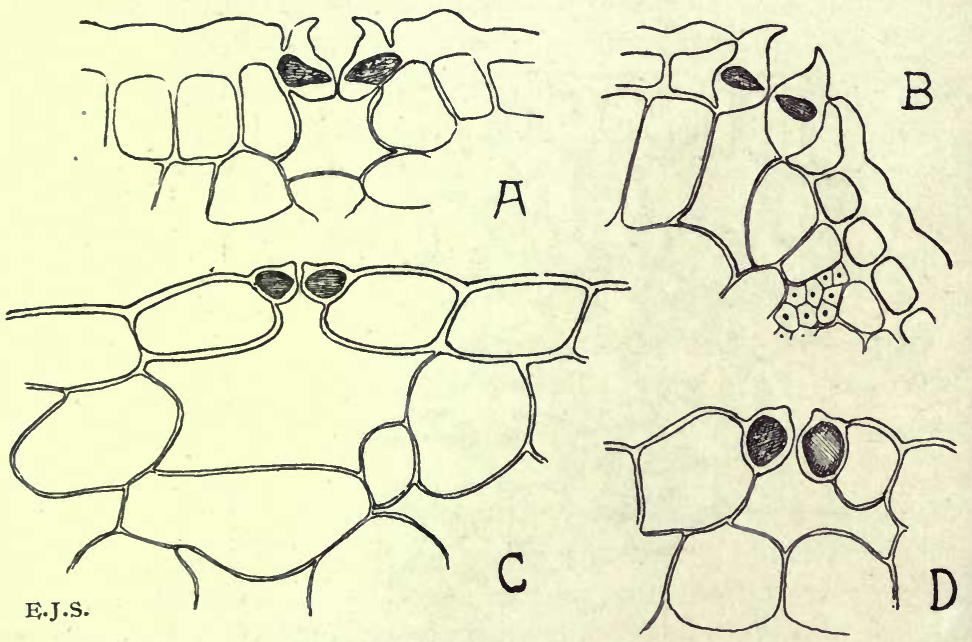

Fig. 8I.-Stomata. A, Holly (Ilex aquifolium). B, Gorse (Ulex europaus). C, Brooklime (Veronica beccabunga). D, Yellow Pimpernel (Lysimachia, nemorum).

effects reduced transpiration. Protection is more commonly attained by the location of the stoma at the base of a depression, a condition already noted in the leaf of the Iris (Fig. 46, D, p. 97). More pronounced examples are afforded by many plants with leathery leaves. In the Holly (Fig. 8I, A) each stoma is situated at the base of a canal which is formed mainly as a result of the great thickening of the cuticle. Similarly protected stomata are well seen in sections through the stem-spines of the Gorse (Fig. 8I, B), where the cuticle also reaches an extreme development. Plants of damp situations offer a marked contrast in these respects, 
the cuticle being relatively thin and the stomata often slightly raised above the general surface (Fig. $8 \mathrm{I}, \mathrm{C}, \mathrm{D}) .^{1}$

Retarded transpiration is also very effectively attained bÿ restriction of the stomata to grooves or hollowes whose communication with the exterior is often partially occluded by an outgrowth of hairs. A good example is furnished by the common Oleander (Nerium oleander). If the lower surface of a leaf of

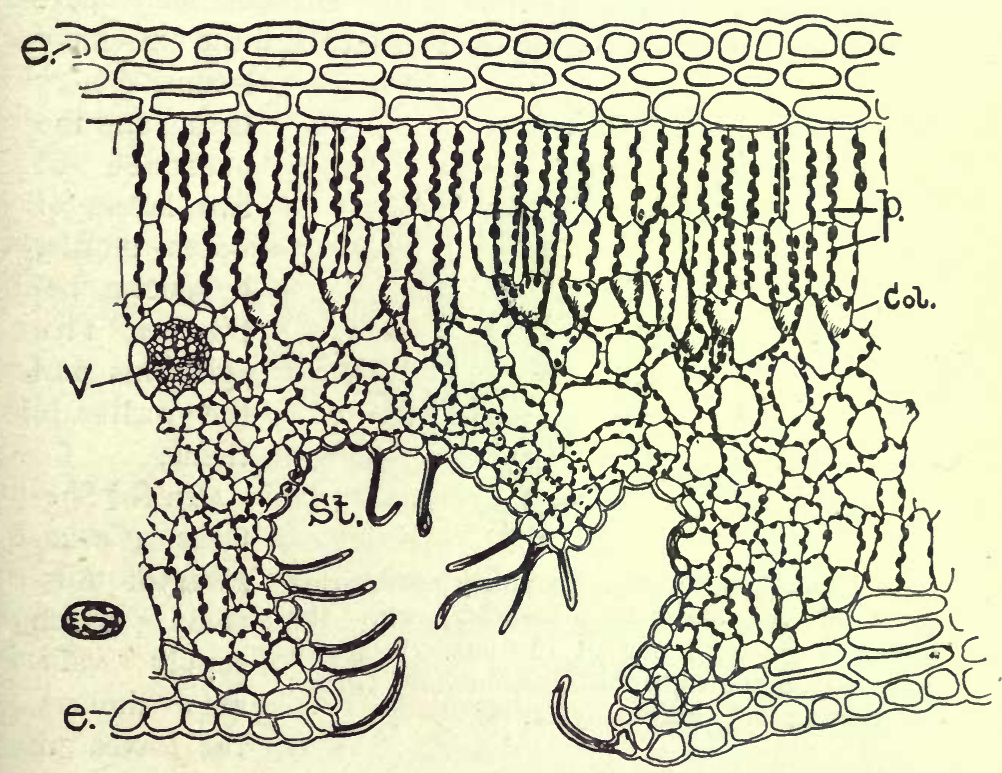

FIG. 82.-Transverse section of part of the leaf of the Oleander (Nerium oleander) showing a stomatal chamber. Col., collecting cells; e., epidermis ; ., palisade layers ; $S t$., stoma ; $V$, vascular bundle.

this plant be examined with a lens, a large number of lightcoloured patches appear dotted between the principal veins, each patch being due to a tuft of hairs arising from one of the numerous hollows. In transverse sections (Fig. 82) the latter are seen to extend inwards for slightly more than one-third the

1 Plugging of the stomatal apertures with particles of wax is observed in some plants (e.g. certain Conifers), which thereby impede the escape of water-vapour and consequently check transpiration, which is almost entirely cuticular. 
thickness of the leaf. The stomata (St.) are confined to the portions of the epidermis within these depressions, and interspersed among them are numerous thick-walled unicellular hairs. Each stoma is raised, on a papilla-like ring of cells, above the level of the epidermis lining the hollows, a fact which is not surprising when it is realised that, since the depressions contain a damp atmosphere, the stomata within them develop under the same conditions as those of ordinary leaves growing in moist situations. Other striking features of the Oleander-leaf, apart from the thick cuticle on the exposed surface, are the extremely

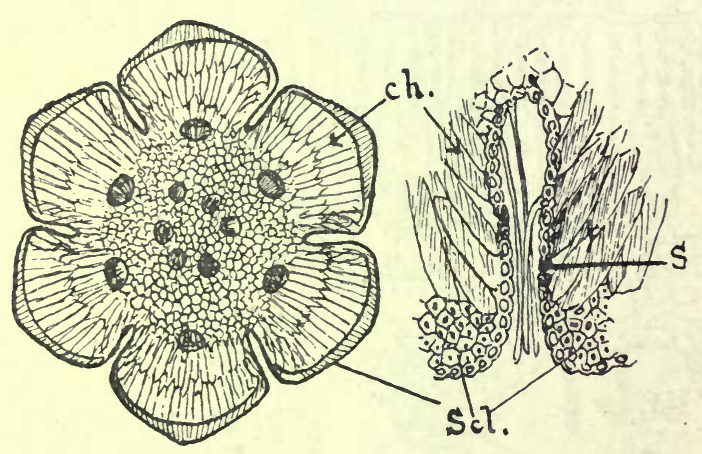

FIG. 83.-Transverse section of a young assimilating stem of the She-Oak (Casuarina) with the stomatal grooves; one of the latter is shown enlarged on the right. ch., assimilatory tissue ; S., stoma ; Scl., sclerenchyma.

lacunar spongy tissue, and the presence of two layers of water - storing hypoderm between the epidermis and the palisade tissue.

In the SheOaks (Casuarina) of Australia, which supply valuable timbers, the leaves are scale-like and carbon dioxide assimilation is carried on by the green twigs, which possess longitudinal grooves (Fig. 83) to which the stomata $(S$.$) , protected by hairs, are restricted. The$ stomata are located at the sides of each furrow, and are situated close to the assimilatory tissue, which is developed in the same position as in other green stems (cf. below, p. I67).

In the leaves of the Heather (Calluna, Fig. 84, B) the stomata are confined to a single groove (stomatal chamber, St.) situated on the under-surface of the leaf. The upper or outer surface is protected by a thick cuticle, and the aperture of the groove is closed by interlacing hairs (Fig. 84, B). The form of the transverse section is roughly that of an inverted triangle, with 
the groove occupying a small area in the lower angle and surrounded by the very lacunar mesophyll. The rolled leaves of the Crowberry (Empetrum) have a similar organisation, except that the stomatal chamber is much larger (Fig. 84, A).

More extreme types of this kind are found in Grasses, where, moreover, the leaf is often capable of rolling and unrolling in response to changes in humidity. An excellent example is afforded by the leaves of the Marram Grass (Psamma arenaria), which clothes many young dunes in great profusion. A section across the rolled-up leaf is more or less circular in outline (Fig. 85, A), and is bounded on the outer (i.e. under) surface by an epidermis provided with a thick cuticle $(C u$. $)$ and devoid of stomata; within are several layers of rounded thick-walled cells (Fig. 85, B, m.). The inner (i.e. upper) surface has a corrugated appearance, being produced into a number of longitudinal ridges, each of which is traversed by a vascular bundle $(V . b$.$) . The intervening grooves are$ flanked by assimilatory tissue consisting of more or less rounded cells (A.t.). The scattered stomata $(S$.) correspond in position with this tissue and are consequently confined to the furrows, where additional protection is furnished by numerous stiff interlocking hairs (Fig. 85, A).
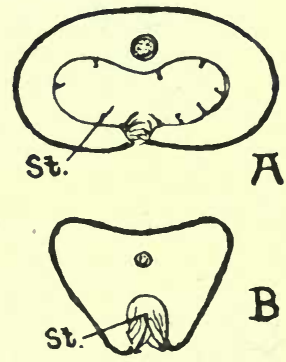

FIG. 84.-Diagrams of transverse sections of the leaves of the Crowberry (Empetrum nigrum) (A) and Heather (Calluna vulgaris) (B). St., stomatal chamber.

The epidermal cells at the base of each groove are exceptionally large (hinge-cells, Fig. 85, h. and h.c.) and, being relatively thinwalled, are the first to lose water and shrink when transpiration is excessive. As a result the inner surface of the leaf contracts in width (i.e. transversely), so that the flat edges formed by the marginal ridges are brought together and the leaf as a whole becomes tubular. When the water-supply is plentiful, the reverse action takes place.

The preceding paragraphs will have indicated the manifold devices that are employed to restrict transpiration from the stomata, and such are particularly characteristic of plants of dry situations. The efficacy of the sunken stoma and of pro- 


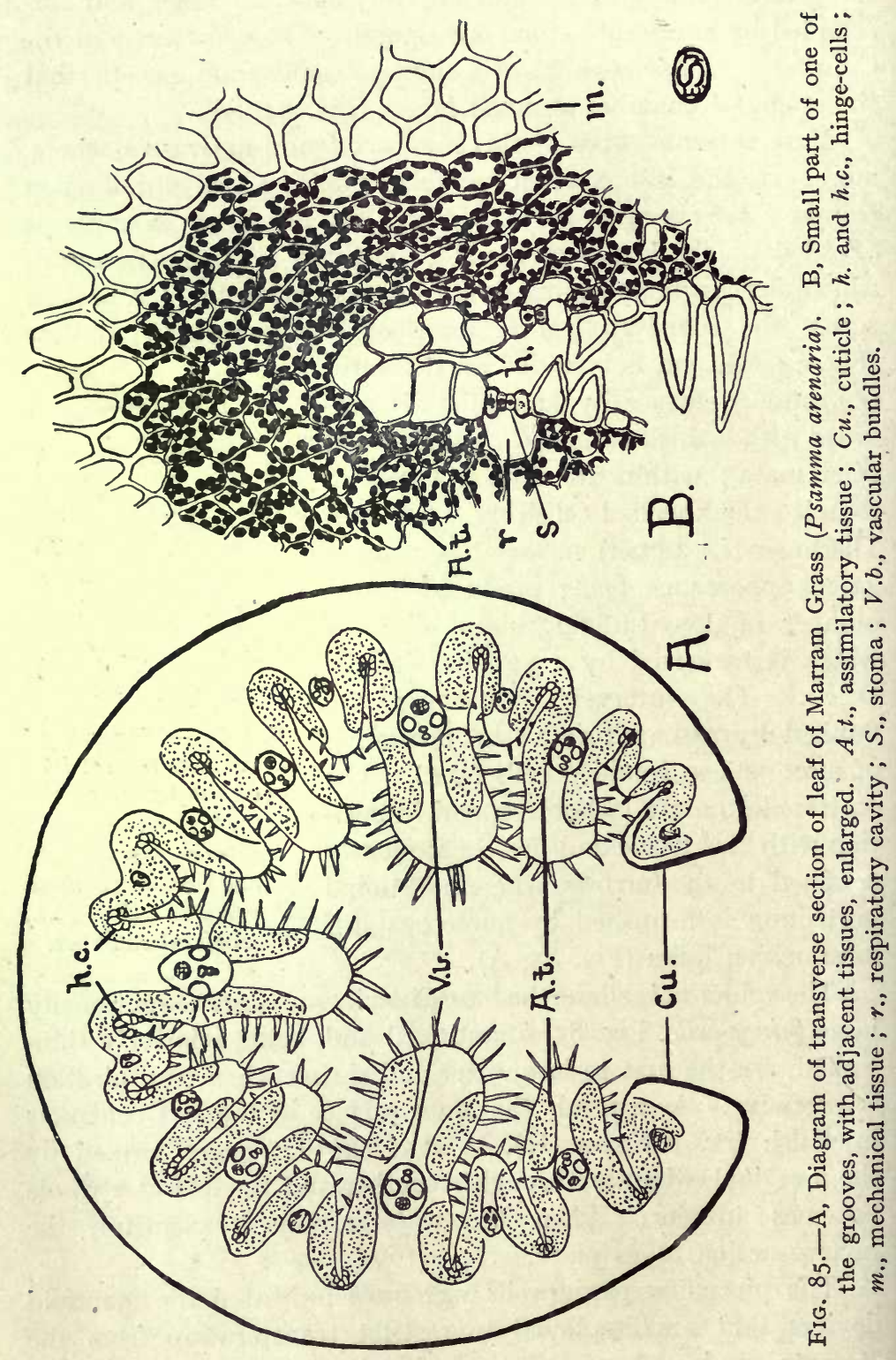


tective hairs can be gauged by comparing the rates of evaporation of water from three similar wide-mouthed bottles (about $8 \mathrm{oz}$. capacity), the neck of one being protected by a cardboard collar about $I_{2}^{\frac{1}{2}}$ inches high, and that of a second by a similar collar filled with thistle-down or cotton-wool. Each bottle should be filled originally with the same volume of water, and this should again be measured at the end of the experiment. After a few days' exposure in the open air, preferably when it is windy, it will be found that the bottle with thistledown has lost least water, whilst that un protected by a collar has lost most. There are, however, many other peculiarities that are associated with plants exhibiting a structure that tends to reduce transpiration. A feature
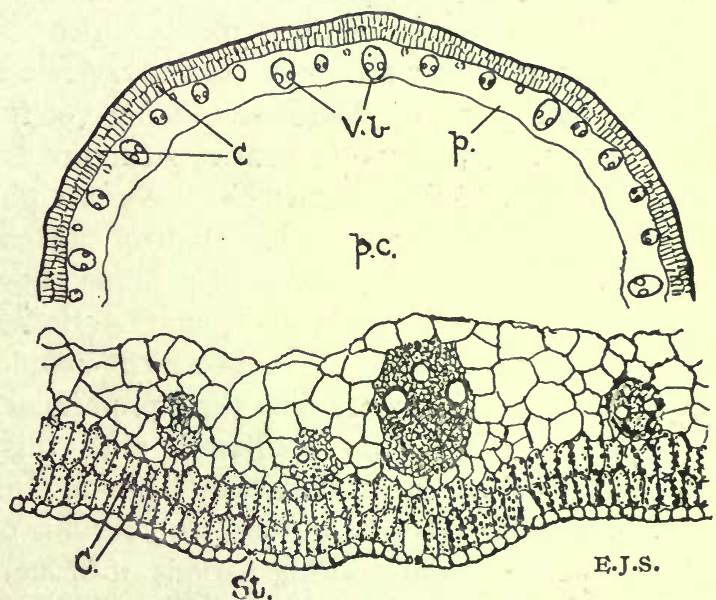

FIG. 86.-Transverse section of the leaf of the Jointed Rush (Juncus articulatus). The upper figure shows a diagram of about half the section, the lower a small segment on a larger scale. $C$., assimilatory tissue ; $p$. , parenchyma ; p.c., central cavity; St., stoma; V.b., vascular bundles.

which often accompanies a reduction of leaf-surface is the absence of markedly dorsiventral structure. In its, extreme form this results in the leaf acquiring radial organisation, i.e. it becomes centric, and it may then closely resemble a stem. Such is the leaf of the Jointed Rush (Juncus articulatus), whose sheathing base passes over into an almost cylindrical lamina with a slight concavity on the upper side which faces the stem. The transverse section (Fig. 86) presents an epidermis with thick outer walls and a pronounced cuticle. The sunken stomata (St.) are distributed at intervals 
around the whole periphery, but in surface sections are seen to be arranged in approximately longitudinal series. Beneath the epidermis is a typical palisade tissue $(C$.) of two to four layers of cells, interrupted by large gaps (the respiratory cavities) below the stomata. Within the assimilatory tissue follows an irregular ring of typical Monocotyledonous bundles ( $V . b$.$) accompanied by$ sclerenchyma. The large central cavity (p.c.), which is bounded by parenchyma $(p$.$) , is interrupted at intervals by transverse$ septa containing vascular elements. The Jointed Rush affords an excellent example of those plants which, though growing in wet places, exhibit a structure that suggests a need for economy of water (cf. p. I77). The reason is not yet fully understood.

A less extreme type of centric structure is found in Hakea, a native of Australia, in which the leaves are pinnately branched, the cylindrical segments being slightly flattened on the upper surface. A transverse section of a pinna shows the customary thick cuticle and deeply sunk stomata. Beneath the epidermis are two layers of palisade cells which completely encircle the central parenchyma containing three prominent vascular bundles. Stretched between the epidermis and this central region are occasional mechanical elements having thick whitish walls and slightly dilated ends, and probably serving to prevent collapse of the palisade tissue during periods of drought. The vascular bundles are accompanied, both on their upper and lower sides, by strands of sclerenchyma. The central region in which the bundles are embedded consists of colourless water-storing (aqueous), tissue in which are scattered occasional large tannincells having deep brown contents. A similar type of centric leaf is seen in the Scotch Fir (Pinus, cf. p. 343).

Aqueous tissue, already observed in the Butcher's Broom (Fig. 80, Aq.) and Hakea, is a most prominent feature of succulents. In the leaves of the Stonecrop (Sedum), the Prickly Saltwort (Salsola kali), and the Sea-blite (Suceda), the large colourless and thin-walled cells in which water is stored up form the bulk of the leaf-tissue. The aqueous tissue occupies the centre of the leaf, with the assimilatory tissue towards the periphery; in the Prickly Saltwort these tissues are sharply marked off from one another (cf. also Fig. 44, $A$, p. 94), but in the other two cases there is quite a gradual transition between them. Similar 
water-storing tissue is encountered in stem-succulents (e.g. Cactus). Through the loss of water, by transpiration and absorption by the adjacent assimilatory cells during periods of drought, the cells of the aqueous tissue shrink, and this results in the walls becoming thrown into small folds which disappear, during the wet season, as the plant regains turgidity. Loss of water from such aqueous tissue is often retarded by the presence of thin mucilage in the cell-contents which exudes from a broken surface as a slimy fluid (e.g. in the Ice-plant, Mesembryanthemum).

Attention has already been drawn to the presence of chloroplasts in the outer cortical cells of young stems (cf. p. 88); but, where the leaf-surface is small, the stem may retain its assimilatory powers for some years after the inception of secondary thickening, and may even become enlarged by winglike outgrowths whereby its efficiency is increased. The Broom (Cytisus scoparius), the Whortleberry (Vaccinium myrtillus, Fig. 87), and the Gorse (Ulex) furnish examples of such assimilatory stems. ${ }^{1}$ In the first-named downward prolongations from the margins of the leaf-bases give the stem a ridged appearance. A cross-section shows the usual thick-walled epidermis beneath which, in each ridge, there is a strand of fibres; but, except for these, the whole periphery of the cortex consists of a layer of palisade tissue succeeded by four or five layers of closely packed cells, also containing chloroplasts. The stomata occur at intervals throughout the parts of the epidermis overlying the assimilatory tissue. In other respects the stem exhibits the normal structure of a secondarily thickened axis.

The leaves of the Whortleberry, though not greatly reduced, are usually deciduous, so that during the winter the plant only assimilates by means of its winged stem. Beneath the epidermis is a continuous zone of assimilatory tissue (Fig. 87, as.), consisting of rounded or polygonal cells, uninterrupted by fibres. The inner cortex is formed by a network of chlorophyll-containing cells in which the meshes are occupied by large aqueous elements (aq.).

It has been possible, in the preceding pages, to recognise a more or less intimate relation between the construction of the plant and the circumstances under which it lives. The effect 
of environment upon structure is, however, best illustrated by the changes exhibited in one and the same organ when growing under diverse conditions, and leaves are particularly plastic in this respect. A comparison of leaves of the same species growing under different conditions as regards light-intensity and degree of humidity, factors which often go hand in hand, helps to emphasise the significance of some of the features above considered. Thus, the structure of the leaves of the Ground Ivy (Nepeta glechoma), growing in an exposed sunny situation, is quite distinct from that of the leaves of the same species, de-

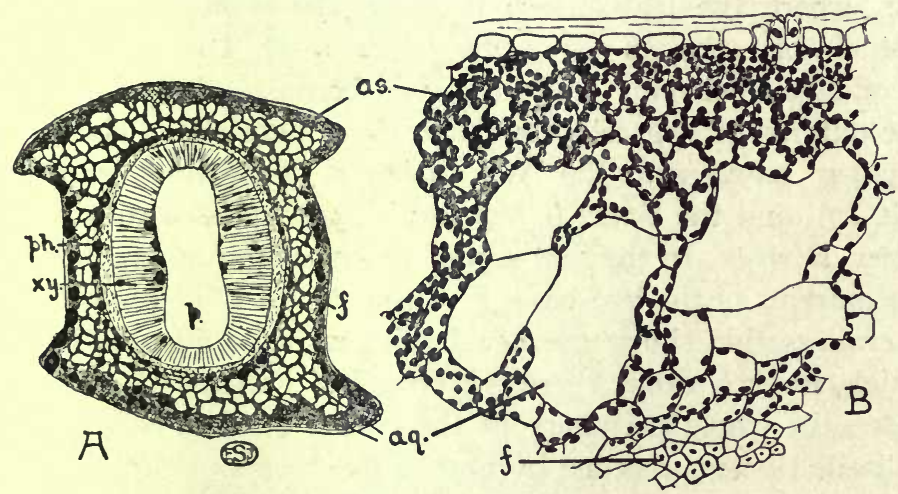

FIG. 87.-Diagram of transverse section (A), and detail of small portion (B), of the assimilating stem of the Whortleberry (Vaccinium myrtillus). $a q$. , aqueous tissue ; as., assimilatory tissue ; $f_{.,}$fibres ; $p_{.}$, pith ; ph., phloem ; xy., xylem.

veloped in the shade of a wood, and a similar contrast is found between the sun- and shade-forms of many other plants ${ }^{1}$ (e.g. Enchanter's Nightshade, Circaa lutetiana; Dog's Mercury, Mercurialis perennis; Yellow Deadnettle, Lamium Galeobdolon, Fig. 88). Comparable anatomical differences are encountered, in trees, between the leaves on the outside of the canopy and those growing in the shade of the interior.

In general shade-leaves are larger, thinner (cf. Fig. 88), and very commonly, if the leaf be lobed or compound, not so deeply cut as the corresponding sun-forms. The colour is usually a 
fresher green, owing to little decomposition of the chlorophyll (through the absence of strong light) and the greater translucency of the leaf; moreover, chloroplasts are not infrequent in the epidermal cells (e.g. Bracken). In the case of hairy leaves (e.g. Yellow Deadnettle, Dog's Mercury) the production of hairs is usually much reduced in the shade-form. A similar difference, between the exposed and sheltered parts of the same plant, is exemplified by the more numerous hairs on the stem-leaves as compared with the radical leaves of species having rosettes. In the Meadowsweet (Spircea ulmaria) the lower leaves, protected
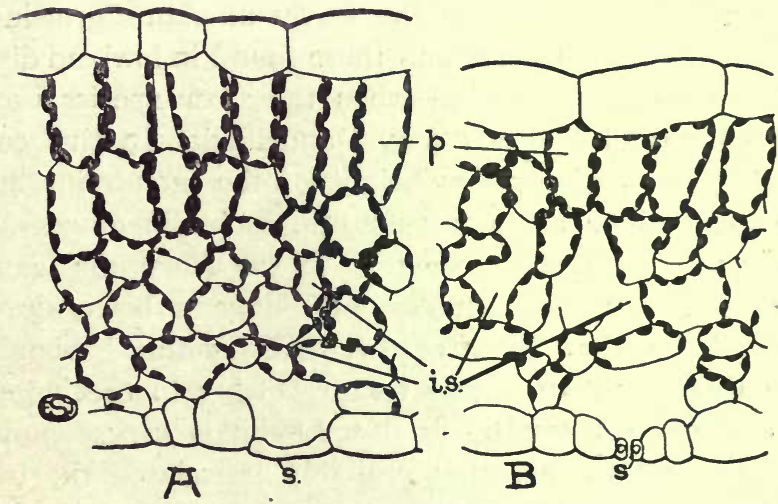

FIG. 88.-Transverse sections of the sun- (A) and shade- (B) leaves of the Yellow Deadnettle (Lamium Galeobdolon), on the same scale. i.s., intercellular spaces ; $p$. , palisade tissue ; $s$., stoma (in A, cut longitudinally).

among the herbage, are smooth and green on the underside, whilst the corresponding surface in the exposed upper leaves is of a greyish tinge owing to the downy felt of hairs.

The cuticle and outer epidermal walls of the shade-forms are thinner than in the sun-forms, a feature which can be related to the greater humidity of the air. A comparison of strips of epidermis from the two kinds of leaves shows that the vertical walls of the epidermal cells tend to be straighter in the sun-form where the leaves are thicker (cf. p. 93). The palisade cells (Fig. 88, p.) are shorter and the number of palisade layers, as compared with leaves growing in bright light, exhibits reduc- 
tion (e.g. Beech), accompanying which there is a relative increase of spongy tissue, whose intercellular spaces (i.s.) become much more conspicuous. The last-named feature may probably facilitate transpiration in a humid atmosphere. As a general rule the stomata tend to be level with, or even raised above, the epidermis in the shade-form, whilst very commonly more or less depressed in the sun-form. On the other hand, they are generally more numerous in an equivalent area of the latter, the fewer stomata in the shade-form being possibly related to the greater humidity of the surroundings.

Analogous differences to those exhibited in sun- and shadeleaves are observed between the leaves of plants growing near the tops of high mountains and those found in lowland districts ; this is especially exemplified when the same species occurs in the two kinds of habitats (e.g. Dandelion). In this country, owing to the relatively low height of the mountains and the humid atmosphere at their summits, such differences are not well marked. But elsewhere (e.g. in the Alps) the leaves are commonly thicker and smaller, and have a better-developed palisade tissue, than those of the corresponding lowland form, though they possess a looser texture owing to the large intercellular spaces. A rosette- or dwarf-habit is very common (cf. Fig. 22I) ; and to this may probably be related the frequent presence of more numerous stomata on the upper, as compared with the under, surfaces of the leaves, since the latter are closely adpressed to the humid soil.

The relation between anatomical structure and habitat is nowhere more plainly shown than in the aquatics ${ }^{1}$ among Flowering Plants, which, however, in many cases betray distinct evidence of their origin from terrestrial ancestors. All the submerged organs of such plants are modified to suit the exceptional conditions of the environment. This is well exemplified by a study of the transverse section of the stem of an aquatic like the Hornwort (Ceratophyllum demersum, Fig. 89), which grows completely under water. The vascular system forms a single central strand, as in a root, in correspondence with the fact that here, as there, the main strain is a pulling one. In fact, such a completely submerged plant is everywhere supported by the sur1 Cf, F, \& S., pp. 33i et seq. 
rounding water, so that protection is alone required against the longitudinal tension due to the force of the current. A small intercellular space $(c$.), surrounded by three or four layers of thin-walled parenchyma-cells, occupies the centre of the strand. This space is actually a longitudinal canal (the xylem-canal) formed by the breaking down of elements of the procambial strand which, in less extreme aquatics (e.g. Water Milfoil, Myriophyllum), give rise to xylem. Beyond the enveloping parenchyma lies the phloem, which can be recognised by its large, emptylooking sieve-tubes (Fig. 89, s.t.). The absence of xylem, whilst
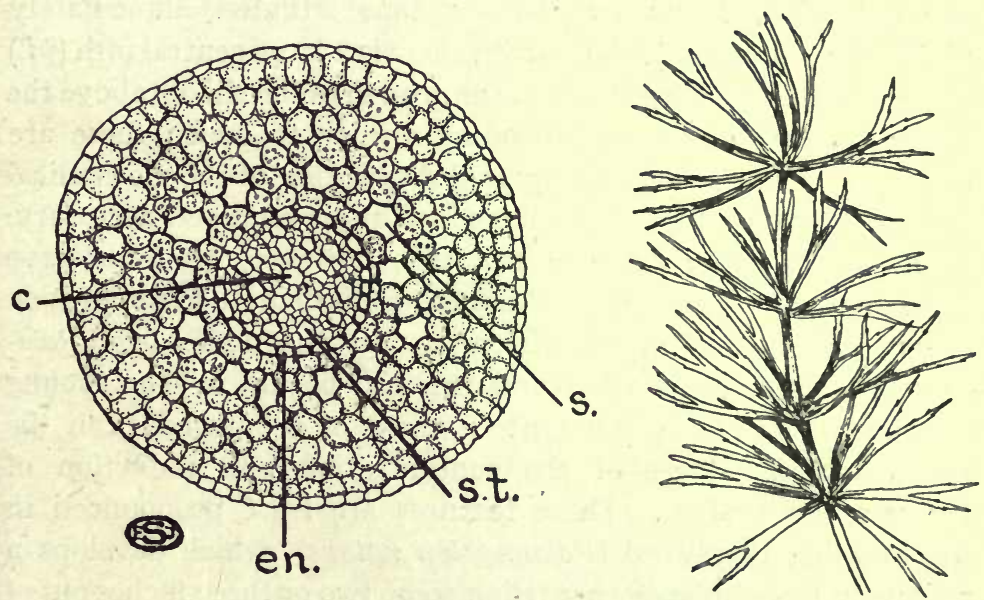

FIG. 89.-Habit, and transverse section of the stem, of the Hornwort (Ceratophyllum demersum). c., xylem-canal ; en., endodermis ; s., intercellular space of cortex ; s.t., sieve-tube.

the phloem is well represented, can be related to the fact that the aquatic absorbs water over its whole surface, whilst conduction of elaborated food-materials remains as necessary as in a terrestrial plant.

The vascular strand is sharply bounded towards the cortex by a well-defined endodermis (Figs. 89 and 9o, en.), showing suberised thickenings on the radial walls, and immediately within is a pericycle, just as in many young roots. The wide cortex beyond consists of thin-walled parenchyma in which a ring of large intercellular spaces (s.) surrounds the central strand. The 
epidermal cells have thin outer walls with a very thin cuticle, and there are no stomata.

The structure of the leaf, as seen in cross-section, is essentially similar to that of the stem. Its centric character may perhaps be related to the absence of illumination from any particular direction, owing to the diffuse character of the light and the constant movement of the leaves by the water. ${ }^{1}$

Those aquatics, whose lower parts only are submerged, approach more nearly to land-plants in their internal structure. In the Mare's-tail (Hippuris, Fig. 90), for instance, the xylem $(X y$.), which is necessary to supply water to the aerial parts, though not extensive, forms an obvious zone situated immediately within the phloem $(p$.$) . Moreover, there is a large central pith ( p i$.) whose presence can be related to the growth of the shoots above the water and the consequent bending strains to which these are exposed. Hence a more peripheral disposition of the mechanical elements than occurs in submerged plants is rendered necessary.

Even the more extreme aquatics, however, often preserve indications of their terrestrial ancestry in their vascular system. Thus spiral vessels commonly persist at the nodes (e.g. Potamogeton), and transient spiral vessels occur in the young internodes. In the different species of Pondweed can be found various stages of the concentration and reduction of the vascular system. These features are least pronounced in the Floating Pondweed (Potamogeton natans), which develops a relatively large inflorescence rising some two or three inches out of the water, and further possesses floating leaves. The large central cylinder (Fig. 9I) encloses eight to ten fairly well-defined vascular bundles, each of which is separated from its neighbours by two to three layers of thin-walled parenchyma, and consists of a large xylem-canal $(C$.) with accompanying phloem $(P$.$) . The$ likewise broad-leaved, but completely submerged, $P$. lucens presents a more marked concentration of the bundles, whose individuality is consequently less pronounced. Lastly, the narrowleaved $P$. pectinatus, which is also totally submerged, exhibits but a single xylem-canal surrounded by phloem, as in Cerato-

1 A similar reduced structure is encountered in the Fennel-leaved Pondweed (Potamogeton pectinatus) and in completely submerged marine aquatics (e.g. the Grasswrack, Zostera). 
phyllum. It may be noted that the leaf-traces exhibit a similar reduction, one bundle passing to each leaf in the last-named species, whilst in the two former the trace consists of three

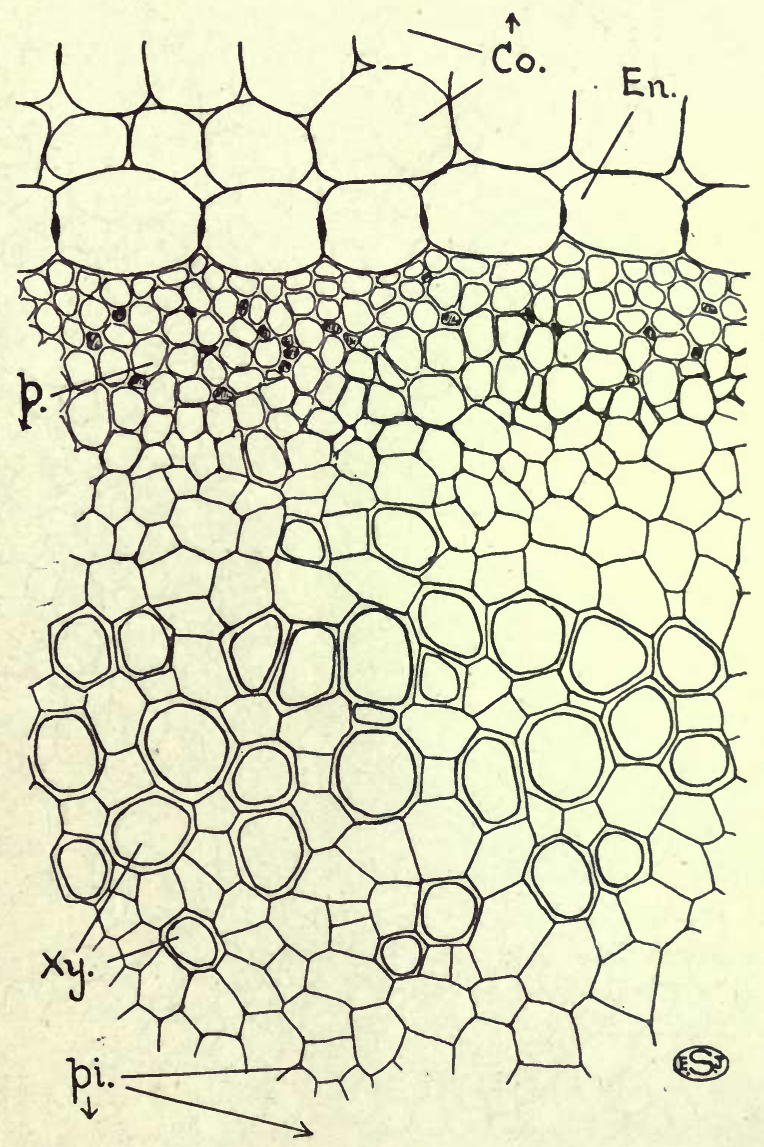

FIG. 90.-Portion of transverse section of the stem of the Mare's-tail (Hippuris). Co., lacunar cortex; En., endodermis; $p$. phloem ; pi., pith ; $X y$., xylem.

bundles. In view of the reduction which water-plants show in respect to the vascular tissue, it is scarcely surprising that they rarely exhibit cambial activity. A trace of cambium can, however be detected in the stem of the Mare's-tail (Hippuris). 
Other examples of adaptation to the habitat-conditions in aquatics are:-restriction of the stomata to the upper surface of floating leaves, development of a covering of wax on the

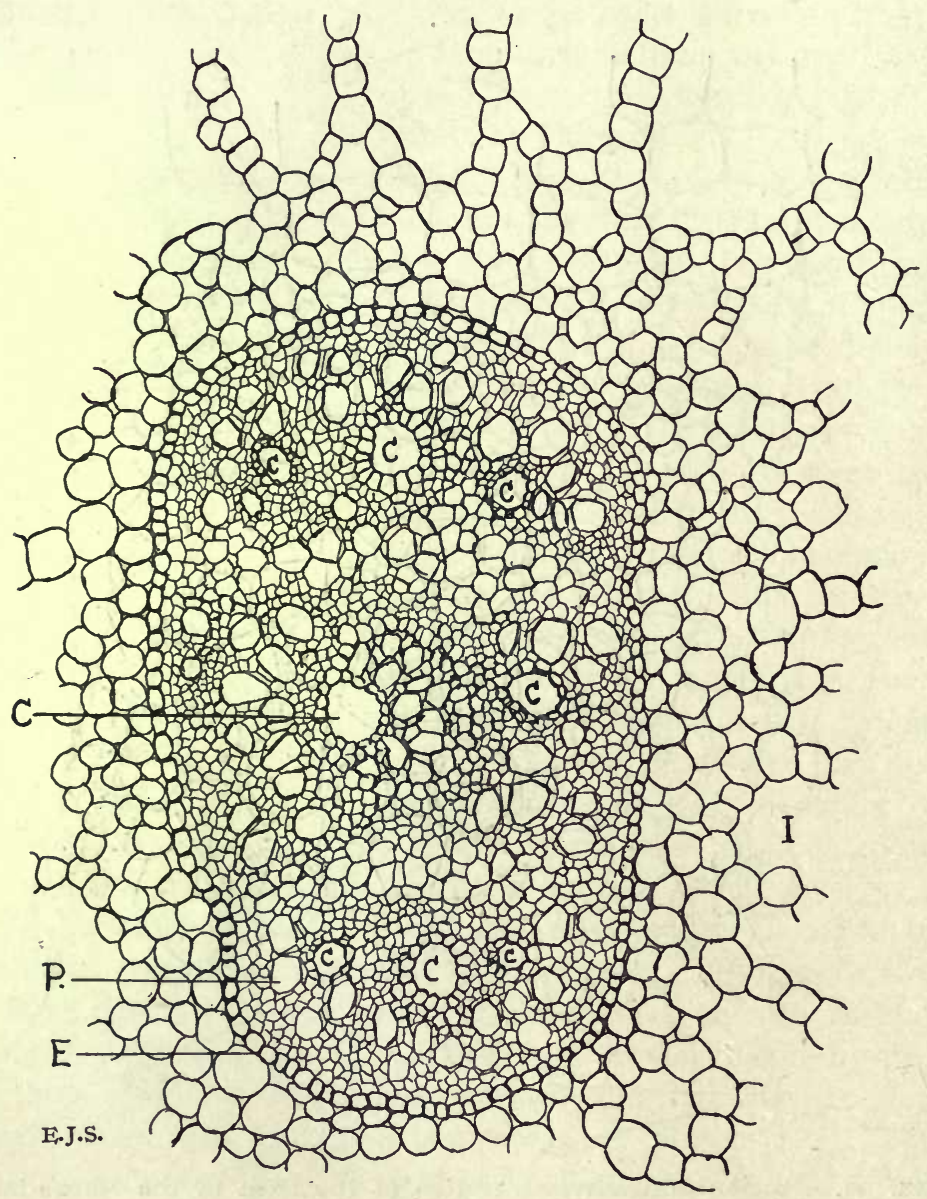

FIG. 91.-Transverse section of the vascular strand of the Floating Pondweed (Potamogeton natans). C., xylem-canals; E., endodermis ; $I$., air-canal ; $P$., sieve-tube.

latter, and the aerenchyma encountered in certain marsh-plants (e.g. Purple Loosestrife, Lythrum salicaria; Gipsywort, Lycopus eurofaus; Hairy Willow-herb, Epilobium hirsutum). Aeren- 
chyma is a very lacunar secondary tissue, formed in place of cork by the phellogen, which cuts off cells only on the outside. These remain thin-walled and living and, as they enlarge, partially separate, and so produce a system of wide air-spaces (often concentric in their arrangement) to which the spongy character is due. The function of this tissue is to supply air to the submerged parts, and it is particularly well developed in plants growing in water-logged soil.

The numerous large air-canals of aquatics are often segmented by platelike septa or diaphragms composed of many small cells separated by minute intercellular perforations. These latter are too small to admit of the passage of water, and thus prevent the injection of the air-canals, when fragments of water-plants become detached, as normally occurs in vegetative reproduction; owing to the perforations the flow of air is not obstructed.

Aquatics display a remarkable plasticity of structure, particularly in

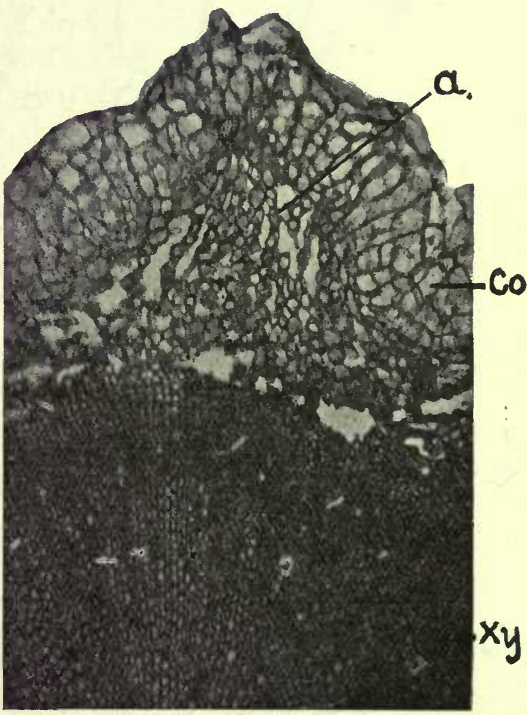

FIG. 92.-Photomicrograph of a small portion of the aerenchyma $(a)$ of the Marsh Samphire (Salicornia.) Co., cortex; $X y$., xylem. (Photo E. J. S.) relation to their anatomy, as a result of which many can grow either completely submerged or on the mud near the edge of the water. A comparison of such land-and water-forms, belonging to the same species, clearly shows the adaptational significance of most of the characters of aquatics. Thus, in a cross-section of the stem of the land-form of the Water Starwort (Callitriche, Fig. 93, a), the cortex consists of closely packed rounded cells with small intercellular spaces between them, whilst that of the water-form (Fig. 93, d) is mainly occupied by 
two large air-canals separated only by narrow strips of tissue. Thicker outer walls and a distinct cuticle characterise the epidermal cells of the land-form (compare Fig. 93, $b$ and $e$ ). The vascular strand of the latter has an almost continuous ring of
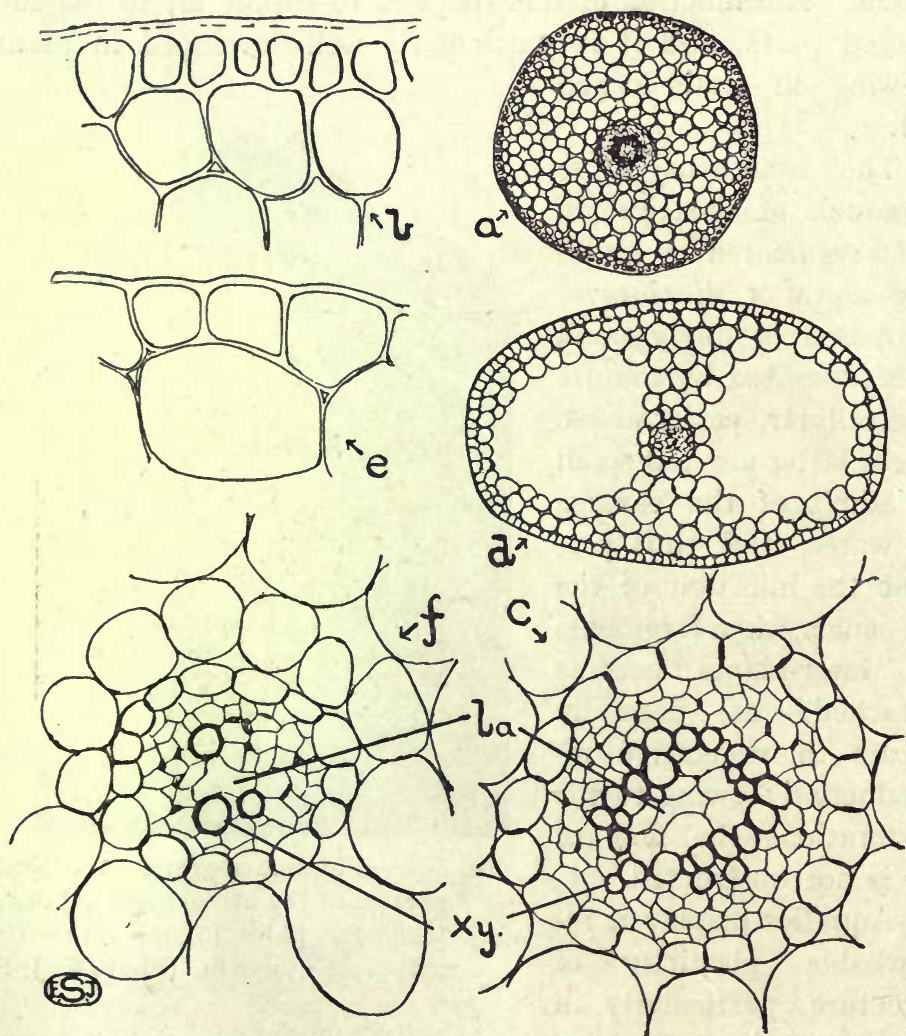

Fig. 93.-Transverse sections of the stems of the land- $(a-c)$ and waterforms $(d-f)$ of the Starwort (Callitriche stagnalis). $a$ and $d$, entire sections ; $b$ and $e$, epidermis ; $c$ and $f$, vascular strands. la., lacuna ; $X y$., xylem-vessels.

xylem (Fig. 93, c) in contrast to the one or two xylem elements bordering the central canal ( $l a$.) in the water-form, whose vascular strand is slightly smaller (Fig. 93, $f$ ). Similar differences are encountered between the structure of the stems of the two forms in other aquatics. 
The leaves of the Starwort do not differ appreciably in the two forms, since the blades are thin and broad in both, but there is a distinct cuticle and a better developed vascular system in that of the land-plant. Since stomata are in this case present even on (the upper sides of) the submerged leaves, the only difference with respect to them is that they remain closed in the water-form. A marked contrast is, however, presented by the leaves of the two forms of the common Water Buttercup (Ranunculus aquatilis, Fig. 94). Those of the landform (L) have a definite palisade layer, which occupies the bulk of the mesophyll, and the epidermis is devoid of chloroplasts and provided with stomata through which gaseous exchange
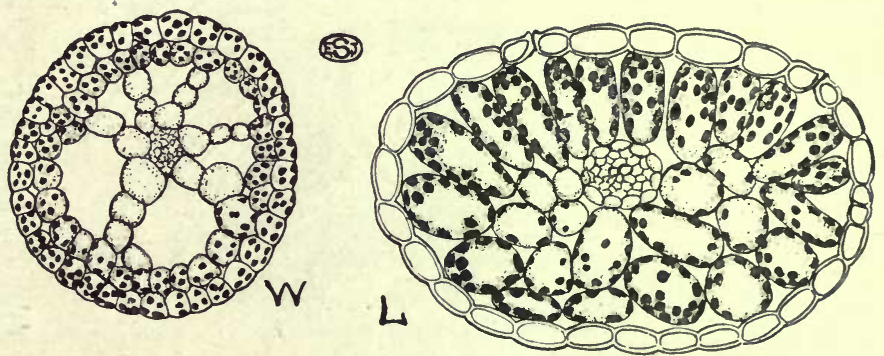

FIG. 94--Transverse sections of the leaves of the land (L) and water (W) forms of the Water Buttercup (Ranunculus a Iutilis). $^{2}$

takes place. In the leaves of the water-form (W), on the other hand, palisade tissue is absent and there are large and conspicuous intercellular spaces. There are no stomata in the epidermis whose cells contain chloroplasts. Similar differences can be observed between the floating and submerged leaves of this plant, but the contrast is more striking in the Mare's-tail (Hippuris), where the leaves borne above and below water are of the same form.

A combination of aquatic characteristics with others, usually encountered in the vegetation of dry habitats, is not uncommonly exhibited by plants rooted in boggy ground, but whose shoots are exposed to conditions tending to encourage excessive transpiration. These conditions may in part explain the phenomenon, 
which is, however, probably an outcome of complex causes. Excellent examples are furnished by the Cotton Grass (Eriophorum), the Rush (cf. p. I65), and the Bulrush (Scirpus), all typical of such localities. The principal feature reminiscent of aquatics is the vast system of intercellular air-canals which serves to supply the underground organs with oxygen.

The numerous examples cited in this chapter have shown that specialised structure and habitat often go hand in hand. Thus the plants of a salt-marsh are mostly succulents, those of a desert often have small leaves or assimilating stems, many heath-plants have rolled or hairy leaves, whilst those of a wood

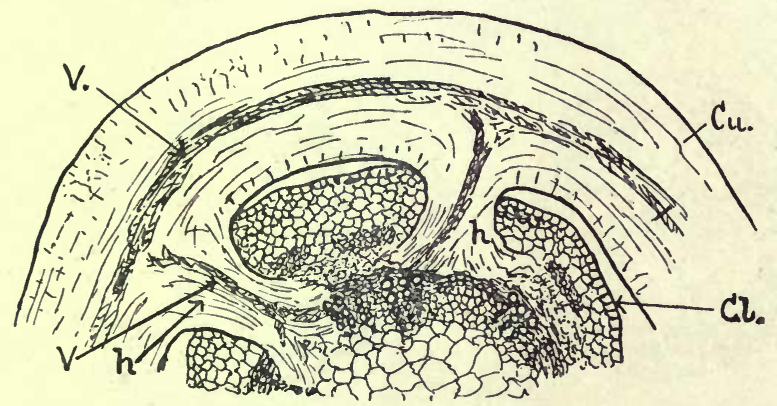

FIG. 95.-Transverse section of Clover-stem (Cl.) showing penetrating haustoria (h.) of Dodder (Cuscuta) (Cu.), whose stem is cut longitudinally. $\quad V$., vascular tissue of Dodder.

mostly possess large thin leaf-blades without marked checks to transpiration. Certain types of structure are, moreover, associated with certain families, as evidenced by the succulence of most members of the Stonecrop-family (Crassulaceæ) and Cactusfamily (Cactaceæ), the small-leaved habit of most plants of the Heather-family (Ericacex), and the aquatic character of all members of the Pondweed-family (Potamogetonaceæ).

Modifications of structure in relation to the special mode of life are also exhibited by the parasites and saprophytes amongst Flowering Plants. ${ }^{1}$ A striking feature of both is the feeble development of the xylem, no doubt in relation to the reduction of the leaves (e.g. in the parasitic Dodder and in the 
saprophytic Bird's-nest Orchid). The haustoria of parasites arise from the stem (Dodder, Cuscuta, Fig. 95) or root (Cowwheat, Melampyrum) as outgrowths (h.), which flatten out in a sucker-like manner in contact with the surface of the host. The centre of the sucker grows out as a peg-like process, which penetrates the cortex and ultimately reaches the vascular tissue of the host, where it often widens out in a fan-shaped manner (Fig. 95, h.). At first this process consists of undifferentiated cells, but later those adjacent to the xylem become tracheids, whilst those in contact with the phloem develop as phloem-like elements.

By the connection thus established between the conducting elements of host and parasite, the assimilatory products and absorptive system of the former become available for the parasite, which regulates any excess of water thus obtained by means of the hydathodes already described (cf. p. I44). The parasite, being relieved of those functions which necessitate elaboration of the vegetative structure, is enabled to utilise almost its entire energies for the purpose of reproduction. Simplification of structure, in every other direction but that which tends towards an increase in the output of seeds, is a marked characteristic of these organisms. Even the ovules (cf. p. 365) and embryos are simpler in construction than those of most green plants, the material economised in this way presumably making possible a quantitative increase. 


\section{PART II}

\section{THE LIFE-HISTORIES AND REPRODUCTION OF PLANTS}

\section{CHAPTER XIV}

\section{Simple Vegetable Organisms}

Plants exhibit a great variety of external forms, but nevertheless can be collected into groups having many features, both of structure and life-history, in common. In the preceding portion of this book the highly elaborated structure of the Flowering Plant has been almost exclusively considered, but very many vegetable organisms are, of course, far simpler in every way. For example, whole groups of lower plants lack true roots and possess no vascular system. A specialised conducting tissue consisting of xylem and phloem is only encountered in the Flowering Plants (Angiosperms), in the Gymnosperms (e.g. Fir-tree and other Conifers), and in the Ferns and their allies (Horsetails, Clubmosses, etc.), whilst it is lacking in Mosses, Liverworts, Fungi, and Seaweeds.

The Vegetable Kingdom can therefore be conveniently divided into vascular and non-vascular plants. ${ }^{1}$ This difference may perhaps be related to the fact that a considerable percentage of the latter flourish in wet or damp habitats, for it is only amongst some of the larger and definitely terrestrial Mosses that anything simulating the vascular tissues of higher plants is developed. The majority of non-vascular plants possess a type of body called a thallus which exhibits no definite stem and leaves, in the sense in which these terms are used among higher plants, and is in fact in many cases a mere flattened

- Since many so-called vascular plants possess no true vessels, these terms are apt to be misleading, but long use justifies their retention. 
cellular expansion (Figs. I08, I09). The further classification of the Vegetable Kingdom (cf. Appendix, IX) is based mainly on the methods of reproduction and the structure of the reproductive organs, as will become apparent in the course of the subsequent chapters.

A considerable number of the simplest types of plants consist of a single cell, and such minute organisms, by their abundance, often cause the green colouration of small stagnant pools. Some of the forms commonly responsible for this phenomenon belong to the genus Chlamydomonas, which will serve to illustrate the structure of these plants, one of whose most striking characteristics is a power of movement from place to place, usually associated with animals.

If a drop of water containing species of this genus be examined under the microscope, the individuals will be seen to move rapidly across the field of view. Each consists of a spherical or oval cell, about one-fiftieth of a millimetre in diameter. The cell is bounded by a thin wall which is often produced into a slight colourless papilla at the front end (Fig. 96, d), i.e. that which is foremost during movement. There is a single large chloroplast, having the shape of a deep cup with a very thick base and with the opening directed forwards (Fig. 96, d, ch.). Within the thickened part is embedded a pyrenoid (cf. p. 7), surrounded by a sheath of small starch-grains (Fig. 96. p.), but after active assimilation starch may also be found stored in other parts of the chloroplast. Adjacent to the rim of the latter lies a small red speck or streak of modified cytoplasm known as the eye-spot (stigma) (Fig. 96, s.), which is believed to be concerned with the perception of light intensity (cf. below). A single nucleus $(n$.) lies in front of the pyrenoid suspended by cytoplasmic strands within the cavity of the chloroplast.

If a stationary individual be observed under the high power of the microscope, a pair of small circular highly refractive vacuoles will be recognised in the clear cytoplasm at the front end (Fig. 96, C.V.) ; when these are watched closely they will be seen, alternately, to enlarge gradually and then suddenly to collapse. Similar contractile vacuoles occur in many unicellular plants, as well as among the lower animals, and they probably serve for the excretion of waste products to the exterior. 
The movement of the Chlamydomonas-individuals is accomplished by means of two delicate thread-like outgrowths, the cilia (Fig. 96, $a$ and $d$ ), which can be detected arising close together at the front end, and are usually as long, or longer than, the body of the cell. They are recognisable under the high power in stationary individuals, but are more readily seen after adding a drop of iodine, which has the effect of killing
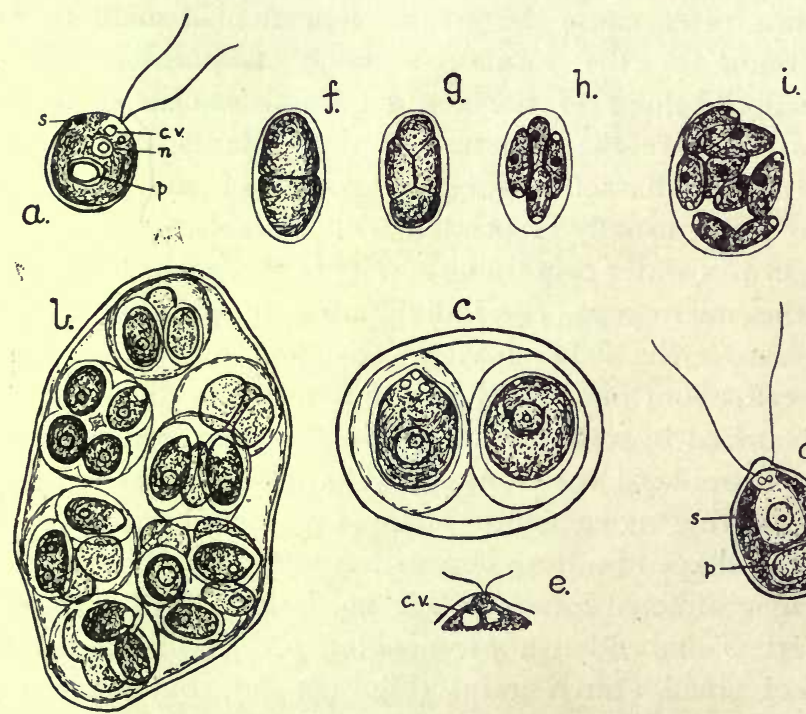

FIG. 96.-Individuals and vegetative reproduction of Chlamydomonas. $a$, single individual of $C$. reinhardii ; $d$, ditto of $C$. angulos $a ; b$ and c, Palmella-stage of $C$. monadina; $e$, front end of a cell of $C$. reinhardii, greatly magnified ; $f, g, h$, and $i$, stages in the vegetative propagation of C. media. ch., chloroplast; C.V., contractile vacuole ; n., nucleus ; p., pyrenoid ; s., eye-spot. $\quad(a-c$ and $e$ after Goroschankin, $d$ after Dill, $f-i$ after Klebs.)

the organisms without much change, and not only brings out the cilia, but also makes the nucleus more distinct. The cilia, as a matter of fact, are whip-like prolongations of the cytoplasm which, by their rapid backward strokes, pull the plant through the water, the movement being accompanied by a rapid rotation of the organism upon its axis. In returning to the front position, the cilia are not stretched out, and thus do not counteract the effect of the back stroke, the movements being thus comparable 
to those of the arms in swimming. The action is of essentially the same type wherever cilia are the organs of traction. The rate of travel of these organisms is very slow as measured by inches, but is rapid relative to their size. Thus, a Chlamydomonas occupies a mere fraction of a second in traversing a distance equal to its own length, whilst in the case of a liner this evolution requires several seconds.

The direction of movement is influenced by various external stimuli, such as light, distribution of chemical substances, etc. If some water containing Chlamydomonas be placed in a glass bottle covered, except for a small aperture on one side, with brown paper, after exposure to illumination for some hours, the organisms will be found to concentrate in a dense cluster at the spot where the beam of light penetrates. If, however, the latter is very intense, the plants swim away from the illuminated region, which consequently becomes colourless. The influence of the direction and intensity of the light on such movements is spoken of as phototaxis. Just as in the case of the movements of the protoplasts within the cells of higher plants (cf. p. 4), the movement of these unicellular organisms can be temporarily arrested by slight traces of anæsthetics.

The Chlamydomonas-individual, with the help of its chloroplast, is able to manufacture food from simple inorganic substances like any other green plant, and as a result the cell grows. After attaining a certain size it comes to rest, draws in its cilia, and begins to form daughter-individuals (Fig. 96, f-i). The protoplast contracts slightly away from the wall and, after nuclear division has taken place, gradually constricts into two equal portions $(f)$, each containing half the nucleus, chloroplast, etc. The resulting segments may divide again $(g-h)$, and these even for a third time $(i)$, the successive divisions taking place in planes at right-angles to one another. Each segment develops a cell-wall and two cilia, and thus 2, 4, or 8 new individuals are constituted which, apart from size, resemble the parent in every respect.

This process of vegetative reproduction is completed by the rupture or dissolution of the membrane of the parent-cell, with consequent liberation of the daughter-individuals. Since, under favourable circumstances, the succession of events just 
described recurs about every twenty-four hours, one individual would in the course of a week give rise to $2,097, I_{52}$ ! Hence the often rather sudden appearance of such organisms in huge numbers in small pieces of water. It is to be noticed that the protoplasm of the parent is entirely incorporated in the bodies of its offspring, the dead cell-membrane alone remaining behind; but for the destruction of a large proportion, such organisms might be regarded as potentially immortal, in the sense that death from senile decay is unknown.

Under certain undetermined conditions the daughter-individuals fail to produce cilia and remain at rest within the parent cell-membrane, which gradually becomes mucilaginous (Fig. 96, c). The daughter-individuals assimilate and grow and sooner or later divide again, their membranes in their turn becoming mucilaginous. This may be repeated indefinitely until large gelatinous masses, enclosing numerous cells and known as Palmella-stages (Fig. 96, b), are produced. In the temporary adoption of this sedentary mode of life, Chlamydomonas and similar organisms exhibit a more marked resemblance to the majority of plants. On the return of favourable conditions, the individual cells acquire cilia and, escaping from the enveloping mucilage, resume the motile condition.

For a long time multiplication may be purely vegetative, but sooner or later-usually when growth is checked by a deficiency in nutritive salts-another method of reproduction sets in. This too is accompanied by division, but the resulting segments are more numerous, 16 or even 32 being formed; these are liberated as sexual cells or gametes (Fig. 97, a), which only differ from ordinary individuals in being considerably smaller and, in most species of Chlamydomonas, naked (i.e. devoid of a cell-wall). They move for a short time, but soon meet in pairs, whose cilia become entangled, and thereafter a gradual fusion (Fig. 97, $b$ and $c$ ) of the two protoplasts and of their nuclei (Fig. 97, $j, k$ ) takes place. There results a single cell (termed a zygote) which moves for a brief period with the aid of its four cilia (Fig. 97, d) and then comes to rest. The cilia are withdrawn, the protoplast assumes a spherical shape and secretes a thick stratified membrane, and large quantities of a reddish-yellow oil appear in the cytoplasm. The resulting 
body (Fig. 97,e,f), known as a zygospore, sinks to the bottom and enters upon a resting condition.

The process of reproduction just described is known a a sexual one since, as in all such processes, the fusing together of two distinct individuals is involved. Despite the fact that the gametes are outwardly all alike, there is evidently some internal (probably chemical) difference between them, for it

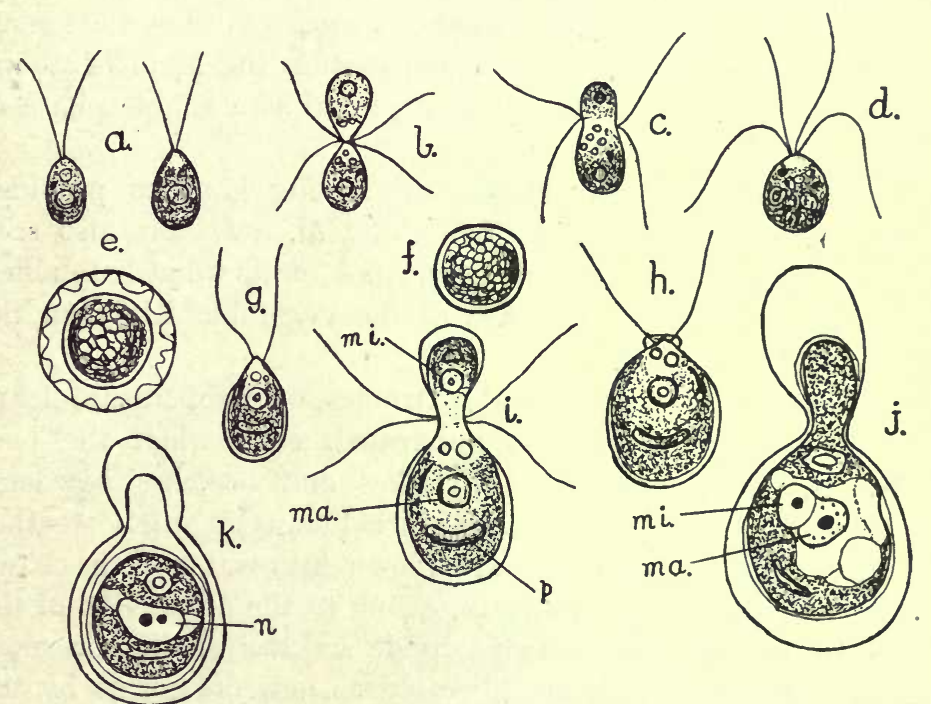

FIG. 97.-Sexual reproduction of Chlamydomonas. $a$, two gametes of $C$. perty $i ; b$ and $c$, stages in the fusion of the same; $d$, zygote, and $e$, zygospore of $C$. perty $i ; f$, zygospore of $C$. veinhardii; $g$, microgamete, and $h$, macrogamete of $C$. monadina; $i-k$, stages in fusion of gametes of the same. ma., nucleus of macrogamete; mi., ditto of microgamete; $n$., fusion-nucleus (in $k$ ) ; $p$., pyrenoid. (All after Goroschankin.)

has occasionally been observed that only gametes derived from distinct parent-individuals are attracted towards one another and fuse together. In one species of Chlamydomonas (C. monadina), however, sexual union always takes place between gametes which differ both in size and behaviour. Some, produced by few divisions of an individual, are large (macrogametes, Fig. 97, $h$ ), relatively sluggish in their movements, and soon come to rest (although the cilia persist), whilst others, formed by numorous, 


\section{I86 CHLAMYDOMONAS, SEXUAL REPRODUCTION}

divisions in the parent-cells, are small and quick-moving microgametes (Fig. 97, g). The zygote is produced by one of the latter approaching a passive macrogamete and fusing with it (Fig. 97, $i-k$ ).

This kind of sexual union in which the two gametes are unlike is described as anisogamous, in contrast to the isogamous process, with fusion of similar gametes, found in the majority of species of Chlamydomonas. Since in higher forms of plants, where the differentiation between the gametes is more extreme, the female are motionless and the male alone motile, the state of affairs obtaining in $C$. monadina can be regarded as a simple phase of differentiation of sex.

C. monadina is also peculiar in having gametes provided with a cell-membrane (Fig. 97, $g$ and $h$ ), a feature also seen in a few other species of this genus and of its immediate allies, although in all other members of the Vegetable Kingdom the sexual cells are naked.

Prolonged desiccation and extremes of temperature leave the thick-walled zygospores unharmed, even when the pool dries up. As the caked mud flakes and becomes powdery, both it and the zygospores are whirled away in windy weather as dust. If the zygospores are blown into water, their colour sooner or later changes to green, owing to the absorption of the yellowish oil, and the contents divide successively into a small number of parts which are liberated as new organisms by the bursting of the thick membrane. It is in this way that Chlamydomonas and similar forms reach, and develop in, almost any suitable piéce of water.

The genus Carteria, individuals of which are not infrequent in fresh-water, resembles Chlamydomonas in all essential respects, except for the possession of four cilia (Fig. 98, F). Another motile unicellular form, commonly found in small pools of water, is variously known by the generic names Homatococcus, Sphaerella, and sometimes Protococcus; the last name is, however, altogether antiquated. The individuals (Fig. 98, A and C), though moving with the aid of two cilia and of much the same shape and size as those of Chlamydomonas, differ somewhat in structure. The protoplast is separated from the firm bounding membrane by a wide transparent layer of mucilage 
which forms the inner part of the thick wall. This mucilage is traversed by a number of, usually branched, thread-like prolongations of the protoplast extending up to the surface layer (Fig. 98, A, C), and comparable to the cytoplasmic strands in the pit-canals of higher plants. Practically the whole of the
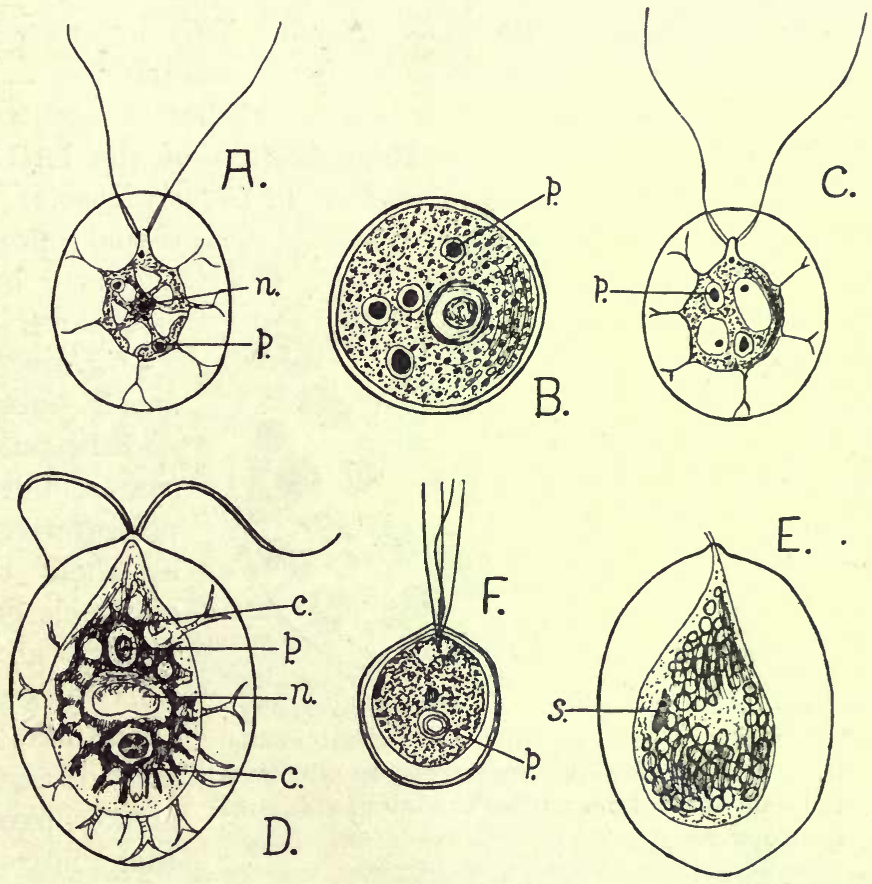

FIG 98.-Hamatococcus (Sphaevella). A and C, Hamatococcus pluvialis, motile individuals (after Schmidle). B, D, and E, H. droebakensis (after Wollenweber). B, Resting cell ; D, single individual in optical section, showing the chloroplast (c.) ; $\mathrm{E}$, the same, in surface view, showing the numerous contractile vacuoles; $F$, an individual of Carteria (after Takeda). $n$., nucleus ; $p$. , pyrenoid ; S., eye-spot.

peripheral region of the cytoplasm is occupied by an ill-defined reticulate chloroplast (Fig. 98, D, c.), containing a number (2-8) of scattered pyrenoids $(p$.$) , and bearing an eye-spot (S$.) near the front end. The contractile vacuoles (Fig. 98, E) are numerous and irregularly distributed. 
The normal green colour of the individuals of Hamatococcus is often obscured by the accumulation of a bright red pigment (hæmatochrome) which appears particularly at certain times, when, with the withdrawal of the cilia and thickening of the cell-membrane, the individuals round off to form characteristic resting-stages (Fig. 98, B). If these occur in quantity they may lend a deep red colour to the water or mud. This simple method of entering upon a resting-stage is also encountered in a few species of Chlamydomonas (e.g. in red snow), but is much rarer there than in Hamatococcus. Reproduction of the latter is effected in almost the same manner as in Chlamydomonas, the
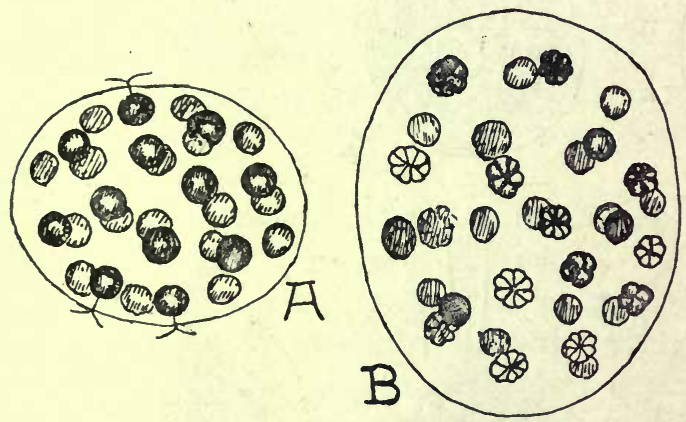

FIG. 99.-Eudorina elegans. A, Vegetative colony ; the cilia are seen on three of the individuals. $\mathrm{B}, \mathrm{A}$ colony showing vegetative reproduction, the daughter-colonies being in various stages of development. sexual process always being isogamous.

Chlam ydomonas, Carteria, and $H$ amatococcus are but representatives of a whole class of simple green plants, all of which swim about like animals during the greater part of their life, ${ }^{1}$ and mist of which are inhabitants of fresh-water. In some genera the unicellular individuals are combined in different ways to form definite groups or colonies. Such is Eudorina (Fig. 99), which is commonly to be found in small pieces of water among the slimy growth covering submerged parts of higher aquatics. The colony here consists of 16 , or more commonly 32 , globular cells embedded at regular intervals in the peripheral portion of a spherical or oval mass of mucilage. Each individual cell is almost identical in structure with a Chlamydomonasplant, the two cilia projecting well beyond the mucilage-invest-

1 In their mode of nutrition, however, they are altogether plant-like, and there is no justification for placing them in the Animal Kingdom. 
ment, and often being easily recognisable where they traverse the latter (Fig. 99, A). By the united action of the cilia of all the individuals, the whole colony moves rapidly through the water, exhibiting a simultaneous rotation upon its axis. Reproduction is usually effected by the subdivision of each of the constituent cells to form as many small daughter-colonies (Fig. 99, B), which are subsequently liberated by the breaking down of the mucilage-investment of the parent. Occasional

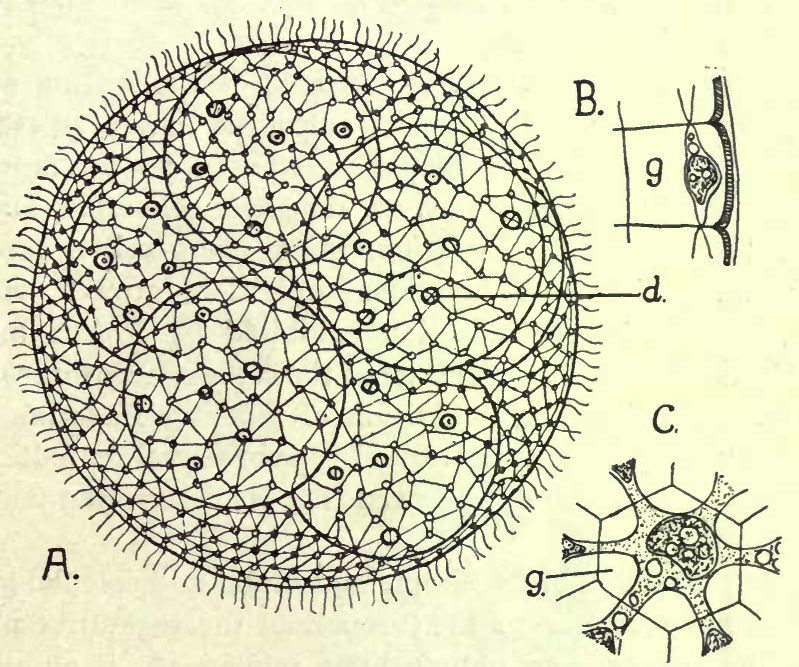

FIG. 100.-Volvox. A, Colony of $V$. aureus (after Klein) enclosing five daughter-colonies, the latter already exhibiting the large cells (d.) from which another generation will be developed. B, Section, and $\mathrm{C}$, surface view of single cells of $\mathrm{V}$.globator (after Meyer). g., mucilagelayer of cell-wall.

specimens of Gonium (Fig. IOI, E), in which I6 Chlamydomonaslike cells are combined to form a flat plate, are not uncommonly associated with Eudorina.

The spherical colonies of Volvox (the globe animalcule), sometimes found in large quantities in fresh-water, are far more elaborate. Since they are composed of several thousand cells, they are of quite appreciable dimensions (usually about the size of a pin's head) and readily visible to the naked eye (Fig. I00, A). The constituent cells (Fig. Ioo, B, C) show more resemblance to 
Hamatococcus than to Chlamydomonas, since they have thick mucilaginous walls (g.) traversed by protoplasmic processes, which correspond in adjacent individuals. Movement is effected, as in Eudorina, by the combined lashing of the pairs of cilia borne by all except a few large cells (Fig. Ico, A, d.) located in that part of the colony which is always directed backward during movement. It is these large cells alone that divide to form daughter-colonies, the latter being at first liberated into the hollow interior of the parent (Fig. Ioo, A), where they grow until set free by its rupture.

Volvox thus affords a simple example of the setting apart of certain cells for special purposes ; most are purely vegetative and perish with the death of the parent, whilst a few are destined to undergo division and persist as daughter-colonies. This is in marked contrast with Eudorina, where every cell is capable of performing all the life-functions (nutrition, growth, reproduction, etc.). The specialisation exhibited by Volvox brings with it the death of the greater part of the colony, a feature which is generally associated with division of labour. Eudorina and Volvox also exhibit a rather advanced type of sexual reproduction which is, however, so rarely observed that its description may be dispensed with.

A large proportion of the simple unicellular and colonial green plants are, however, non-motile throughout the vegetative phase of their life-history, and only exhibit movement, if at all, in connection with reproduction. They thus recall the Palmellastages of Chlamydomonas, to which some, indeed, show considerable resemblance, e.g. the bright green gelatinous masses of Tetraspora, commonly found in spring in small ponds, and containing numerous cells arranged in groups of four (Fig. IOI, D).

As an example of a motionless unicellular plant, the very widely distributed Pleurococcus, which commonly forms the green powdery covering on tree-trunks, palings, etc., may be studied. Under the microscope the green powder is seen to consist of small groups of 2,4 , or rarely more, cells, intermingled with which are more or less numerous rounded individuals (Fig. I02, A), all representing different stages of Pleurococcus. Each cell has a moderately thick wall, and contains a single nucleus and a lobed chloroplast (Fig. I02, B). The isolated cells constitute 
the adult form, and sooner or later divide, commonly by two successive walls at right-angles to one another, to form fourcelled packets. Each daughter-cell is thus the quadrant of a sphere, but with subsequent growth it rounds off and separates from its neighbours, giving rise again to the adult form. This process of vegetative propagation is, when conditions are favourable, repeated at frequent intervals, and in this way Pleurococcus rapidly covers large areas. ${ }^{1}$

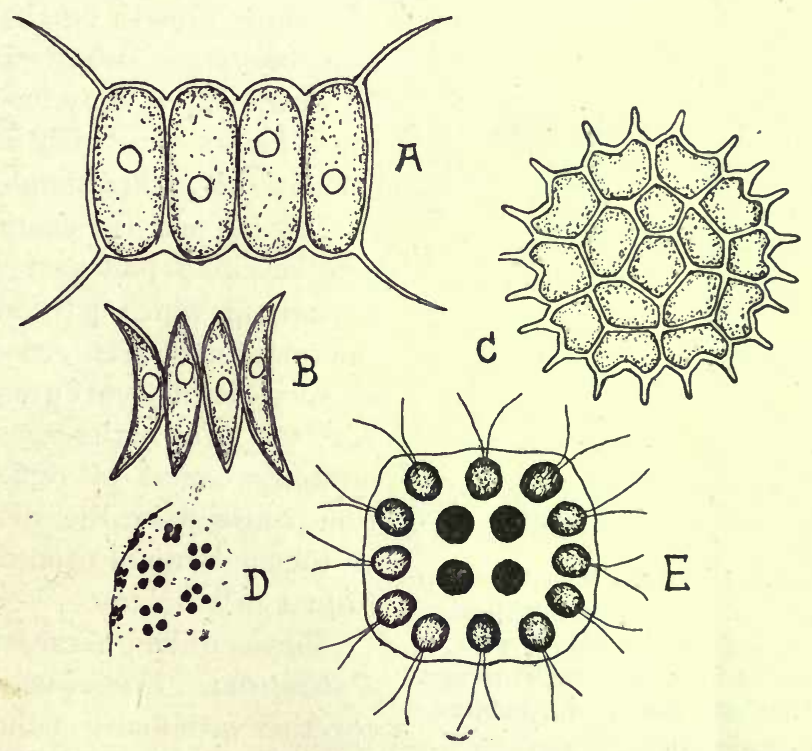

FIG. Ior.-Various colonial Green Algæ. A, Scenedesmus quadricauda. B, S. obliquus, var. dimorphus. C, Pediastrum. D, Small part of a colony of Tetraspora (the cells are shown black). E, Gonium.

The ordinary cells of Pleurococcus possess a remarkable power of withstanding drought, almost comparable to that of the zygospores of Chlamydomonas and other simple forms

1 In wet weather, when the cells are covered with a surface film of moisture, the protoplasmic contents sometimes divide to form a number of masses which are liberated by the breaking open of the cell-wall as naked biciliate motile elements, known as zoospores (cf. p. 214); the latter sooner or later come to rest and, withdrawing their cilia, secrete a membrane and form new Pleurococcus-individuals. Gametes exhibiting an isogamous sexual process are also stated to be produced at times. 
in which the vegetative individuals are extremely susceptible to adverse conditions. It is this property which enables Pleurococcus to exist, and even flourish, in the exposed dry habitats that it usually frequents. Although retaining its normal green appearance throughout the year, its activities are more or less completely arrested during prolonged periods of drought, when its only source of moisture is dew.

Many of the motionless colonial green forms are common in freshwater pools where they occur entangled among the filaments of the pond-scums or in the slimy growth on the sur-

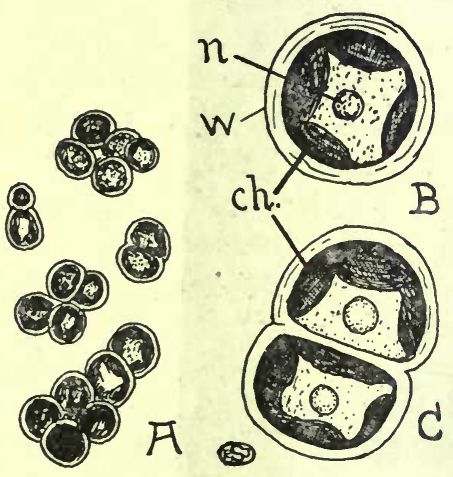

Fig. 102.-Pleurococcus. A, Group of cells, under the low power. $\mathrm{B}$, Single cell, and $\mathrm{C}$, pair of cells, under the high power. ch., chloroplast ; $n$. , nucleus ; w., cell-wall. face of larger water-plants. As examples we may mention the four- or eight-celled colonies of Scenedesmus (Fig. Ior, $\mathrm{A}, \mathrm{B})$, and the characteristic disc-like plates of Pediastrum, in which particularly the marginal cells are often of very distinctive form (Fig. Ior, C). In both cases the ordinary course of reproduction consists in the division of the contents of each cell to form a new colony.

Forms like Scenedesmus, Pediastrum, Eudorina, etc., together with many of the unicellular Diatoms and Desmids

to be mentioned in the next chapter (pp. 206, 209), very commonly occur in considerable numbers floating freely in the surface-layers of lakes, rivers, etc. (Fig. I03). These floating microscopic plants or vegetable Plankton form the food for many aquatic animals, and may at times occur in such prodigious quantity as to lend a visible colouration even to large pieces of water, a phenomenon known popularly as "water-bloom," and for which the Blue-green Algæ (p.234) are most frequently responsible. It may be added that the character of the Plankton usually. differs markedly in different seasons of the year.

The Vegetable Kingdom taken as a whole comprises sedentary 
organisms, and consequently the capacity for movement has come to be regarded as an essentially animal characteristic. But the motile habit has been seen to occur in quite a considerable number of unicellular and colonial aquatic plants, and is by no means confined to them, being observable also among higher forms (e.g. Mosses, Liverworts, Ferns, etc.), although here restricted to certain reproductive cells. The forms studied in this chapter force us to recognise that the power of movement cannot serve as an invariable distinction between the two Kingdoms.

Nevertheless, viewing them as a whole, the Vegetable Kingdom may be described as essentially sedentary, and the Animal Kingdom as essentially motile. This distinction may be related to the necessity for animals to move from place to place in search of food, whilst plants, depending as they do almost solely on simple chemical compounds, can best obtain these by being stationary. It will, for instance, be clear that, for the terrestrial plant,

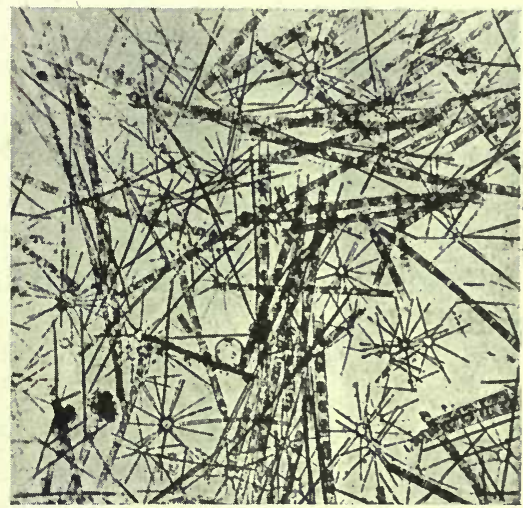

FIG. I03.-Photograph of freshwater Diatom-Plankton; the two principal forms present are the filamentous Melosira, and the star-shaped colonies of Asterionella (from Wesenberg-Lund).

the intimate contact which is necessary between root and soil is totally inconsistent with a motile habit. Another distinction between higher plants and animals is the possession by the latter of highly developed sense-organs. This too can probably be related to the motile habit, with the concomitant necessity for rapid response to the everchanging conditions of the environment. It is significant that in animals like the Hydrazoon Obelia, which have a motile and sedentary phase in their lifehistory, the more specialised organs of sense occur in the former.

Contractile vacuoles and eye-spots are found alike in many lowly plants and animals, and cannot be said to be characteristic 
of either. In fact, the only satisfactory criterion as to the inclusion of an organism in the one Kingdom or the other is the method of nutrition. Plants absorb their food in liquid or gaseous form, and, with the exception of those lacking chlorophyll, build up their bodies from simple inorganic compounds; animals feed on complex organic substances, and take up a large part of their food in the solid form.

f The most important distinction between the two Kingdoms thus depends on the presence or absence of chlorophyll; but even this fails in some cases, as, for example, in certain unicellular motile forms which may or may not possess a green pigment according to the conditions under which they live. Just as it is amongst the simple unicellular plants and animals that we find the closest resemblances, so too it is the complex organisms of the highest groups, as exemplified by Flowering Plants and Mammals, that exhibit the greatest distinction and portray the salient plant and animal characters in the most marked degree. 


\section{CHAPTER XV}

\section{Pond-scums, Seaweeds, etc. (Alg $æ$ )}

THE organisms considered in the last chapter are simple representatives of a lowly group of the Vegetable Kingdom termed the Alga, to which also belong the Pond-scums and the Seaweeds. The Algre, with very few exceptions, possess chlorophyll and feed in the same way as other green plants. They are distinguished mainly by the relatively simple construction of their body, which varies from a single cell to a multicellular thallus of some complexity, and by the simple character of their reproductive organs.

The simplest type of multicellular thallus is a filament or row of cylindrical cells, all nearly identical, both in structure and function. Examples are furnished by Ulothrix (Fig. II5, c), Spirogyra, and Edogonium (Fig. II7, h), genera whose species commonly occur as floating tangled masses in ponds and stagnant ditches, although some are found in flowing water. In most such forms every cell is capable of division, but in EEdogonium this is restricted to occasional cells, recognisable by the presence, at one end of the cell, of a succession of fine rings (known as caps), formed singly at each division (Fig. II $7, h, c$.).

The habit of the plant becomes slightly more complex when the filaments are branched, as in Cladophora (Fig. ro4, A) and Ectocarpus (Fig. II9, f). Species of the latter commonly occur as brown tufts or tresses attached to diverse substrata in the rock-pools on the seashore, whilst those of the former, though also found in similar situations, are commoner in well aerated fresh-water. Each branch of a Cladophora terminates in a pointed apical cell, with specially dense green contents. These cells are the growing points, by whose enlargement and division the branches gradually lengthen, the segments cut off 
from them not uncommonly undergoing no further division. Branching originates by the outgrowth from the upper ends of the cells, just beneath the septa, of small protrusions which are cut off to form the apical cells of the new branches
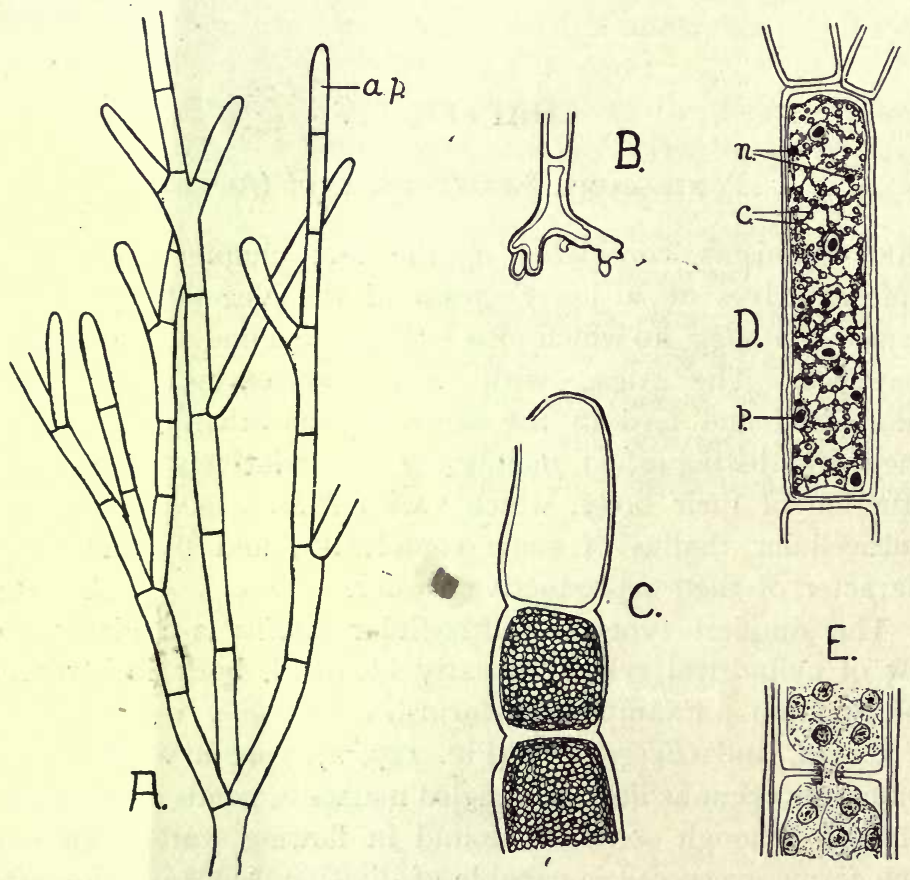

Fig. I04.-Cladophora. A, Small part of a plant of C. glomerata (after Migula). B, Attaching cell (after Brand). C, Apex of a branch with three zoosporangia, the top one having liberated its contents through the aperture seen on one side (after Oltmanns). D, Single cell stained to show nuclei $(n$.$) , pyrenoids (p$.$) , and the net-like chloroplast (c$. (after Wettstein). E, Small part of a cell showing the manner of ingrowth of the septum (cf. p. 24) separating two daughter-cells (after Strasburger).

(Fig. I04, A); the degree of branching varies greatly in the different species and even in different individuals.

The lowest cell of the main Cladophora-filament is modified as an organ of attachment, has scanty contents, and is often considerably branched (Fig. I04, B). Basal attaching cells 
are found in young stages of most filamentous Algæ (Figs. II5, $a$; II7, g), but in forms like Spirogyra, Edogonium, and Ulothrix, the filaments usually break off and become free-floating as they grow older, except in the case of species inhabiting flowing water. The lobes of the attaching cells grow into all the irregularities of the substratum (Fig. I04, B), to which they cling after the manner of a sucker, adhesion being often increased by the secretion of a cementing substance. The great efficiency of the attaching cells is very evident in those species of Cladophora which grow on wavebeaten rocks or in rapidly flowing streams.

The restriction of the power of division to the terminal cells of Cladophora marks a considerable step in the direction of division of labour as compared with Ulothrix, and this is also seen in the formation of reproductive units, which is almost con-

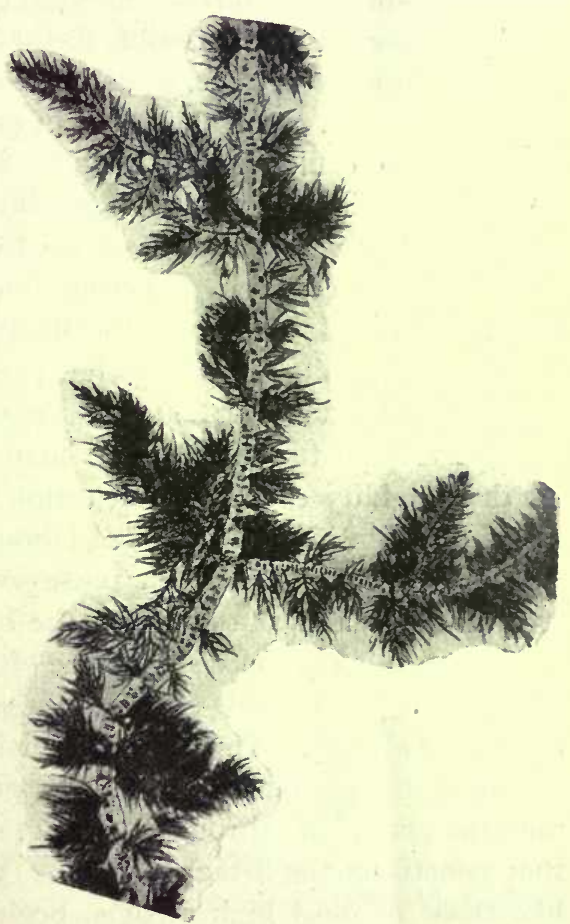

FIG. 105.-Photograph of part of a plant o the Green Alga Draparnaldia, seen under the low power of the microscope. [Photo: E. J. S.]

fined to the cells of the lateral branches (cf. p. 215). A more extreme condition is seen in Draparnaldia (Fig. 105), which is not uncommon in slowly flowing water. Here the main axes, which serve almost solely for support, consist of large cells with small chloroplasts, whilst assimilation and reproduction are relegated to the densely branched laterals. 
Division of labour is also well marked in Ectocarpus, where the thallus usually exhibits a differentiation into upright and prostrate portions, both of which are branched and filamentous, the latter acting as the organ of attachment. The growth of the erect portion takes place by the division of certain cells near the ends of the branches, beyond which the latter usually consist of almost colourless tapering cells (Fig. II9, $b$ ).

An unusual type of thallus is that of Vaucheria, the species of which form rather coarse dark green wefts on damp soil (e.g. by streams or in greenhouses) or in pools of fresh or salt water. The branched filaments, though of considerable width and length, are uninterrupted by septa (Fig. II8, A), and might as a consequence be regarded as consisting of single cells. Since, however, each contains numerous nuclei (Fig. II8, D, n.), it is better compared with a multicellular organism where too the cytoplasm exhibits continuity (cf. p. 24), but where mechanical support is afforded by the walls separating the uninucleate portions. As a matter of fact the filaments of Vaucheria depend for their rigidity entirely upon turgor, and very readily collapse. Vaucheria displays a division of labour similar to that of Cladophora, inasmuch as it often possesses an attaching organ and growth is localised at the tips of the branches.

At low tide on rocky shores one often sees thin crinkled slimy sheets of a vivid green colour; these belong to the Alga Ulva (the Sea Lettuce). The thallus, which may reach a diameter of a foot or more, consists of two superposed layers of cells of a uniform character throughout (Fig. II6, E, F) except where they constitute the attaching base. Very young plants of Ulva, like those of most higher Algæ, begin as a simple unbranched filament, whose cells, however, soon undergo division in several directions to produce the flat thallus.

Most of the genera hitherto studied are so-called Green Algæ (Chlorophycea), ${ }^{1}$ the majority of which inhabit fresh-water. The bulk of the Seaweeds, however, are brown or red owing to the presence of special pigments in the chloroplasts side by side with the chlorophyll. The colouring matters are readily extracted with

1 To this group also belong the Stoneworts (Chara), which, however, exhibit much greater elaboration in their vegetative and reproductive structures than other Green Algæ. 
water from dead specimens, which then assume a green colour. It is possible that these pigments serve to screen the chlorophyll against the strong light to which Seaweeds are at times exposed, although other functions have been attributed to them.

Many Brown Seaweeds (Phreophycea), of which Ectocarpus (Fig. II9, f) has already furnished a relatively simple example, attain to very considerable dimensions, are far bulkier than any Algæ yet noticed, and evince marked division of labour. Thus in Laminaria, which occurs in the zone just below low-tide level, the thallus consists of three distinct regions (Fig. I06) : viz. a richly branched holdfast, by which the Alga is anchored to rocks, a stout cylindrical stalk which may, in some species, be several feet in length and as much as an inch in diameter, and a correspondingly large leathery blade. The latter either takes the form of a broad ribbon (L. saccharina) or of a deeply divided frond like the palm of a hand ( $L$. digitata, Fig. I06). Whilst the stalk and attaching organ

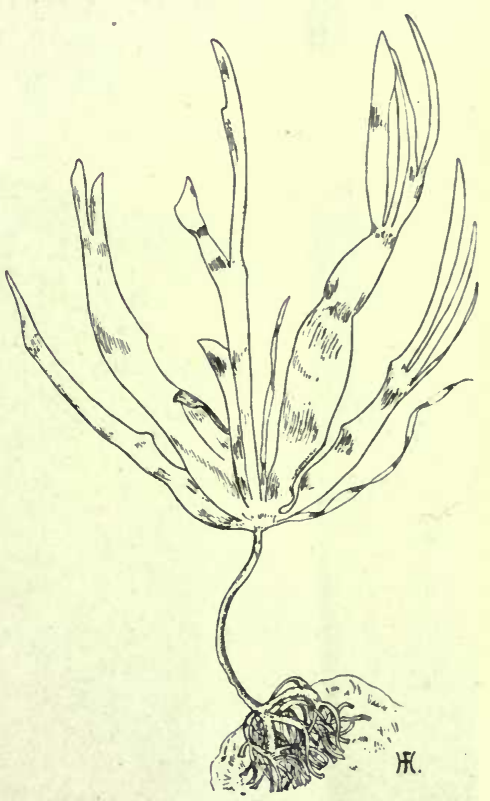

FIG. 106.-Young plant of Laminaria digitata, about one-fifth natural size. are perennial, the blade is renewed, usually in the spring of each year, by means of a small-celled meristem situated at the top of the stalk; the new frond therefore appears at the base of the old one, which eventually becomes detached by the action of the waves. The holdfast is composed of numerous cells with strongly thickened walls, and develops additional branches as the plant grows older, whilst simultaneously the stalk slowly increases in thickness. ${ }^{1}$

1 This Alga not infrequently develops on rock-fragments that are too small to anchor the adult, in consequence of which the thalli and attached 
The Seaweed Macrocj'stis, which is a close ally of Laminaria and particularly common in the Southern Hemisphere, attains to enormous dimensions, often measuring as much as 400 feet from end to end.

Another large Brown Alga is the common Bladder Wrack (Fucus vesiculosus, Fig. I08), which, however, is found on rocks between low and high tide-levels, so that it is uncovered for several hours at a time (Fig. I07). An entire plant often attains a length

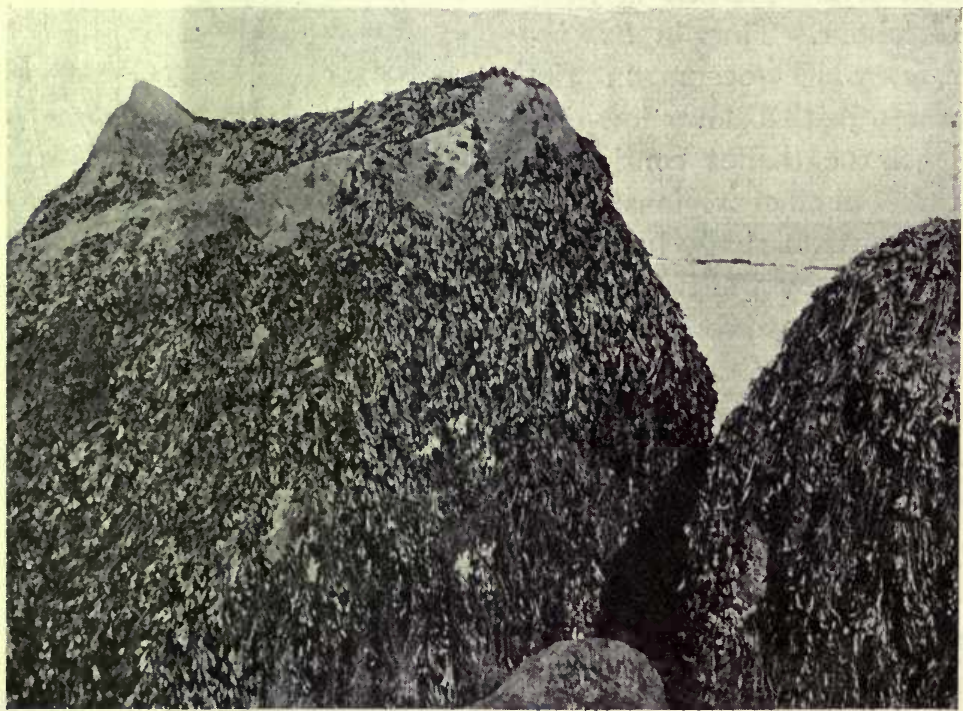

FIG. 107.- Photograph of Fucus vesiculosus on rocks between tide-levels. [Photo: E. J. S.]

of from one and a half to three feet, and exhibits three regions similar to those of Laminaria, but the stalk is short and the frond repeatedly forked (Fig. I08). Each portion possesses an obvious midrib $(m$.), which is thicker and more pronounced in the older parts where it gradually passes over into the stalk. The latter is indeed nothing else than the persistent midrib of the firstformed part of the thallus. The holdfast is similar to that of Laminaria.

rocks get carried out to sea. This may sometimes take place on a considerable scale. 
The slight notch at the tip of each branch of the frond harbours an apical cell by whose divisions growth is effected. The paired air-bladders (Fig. I08, a.b.), which appear as occasional large swellings on either side of the midrib, and to which the specific

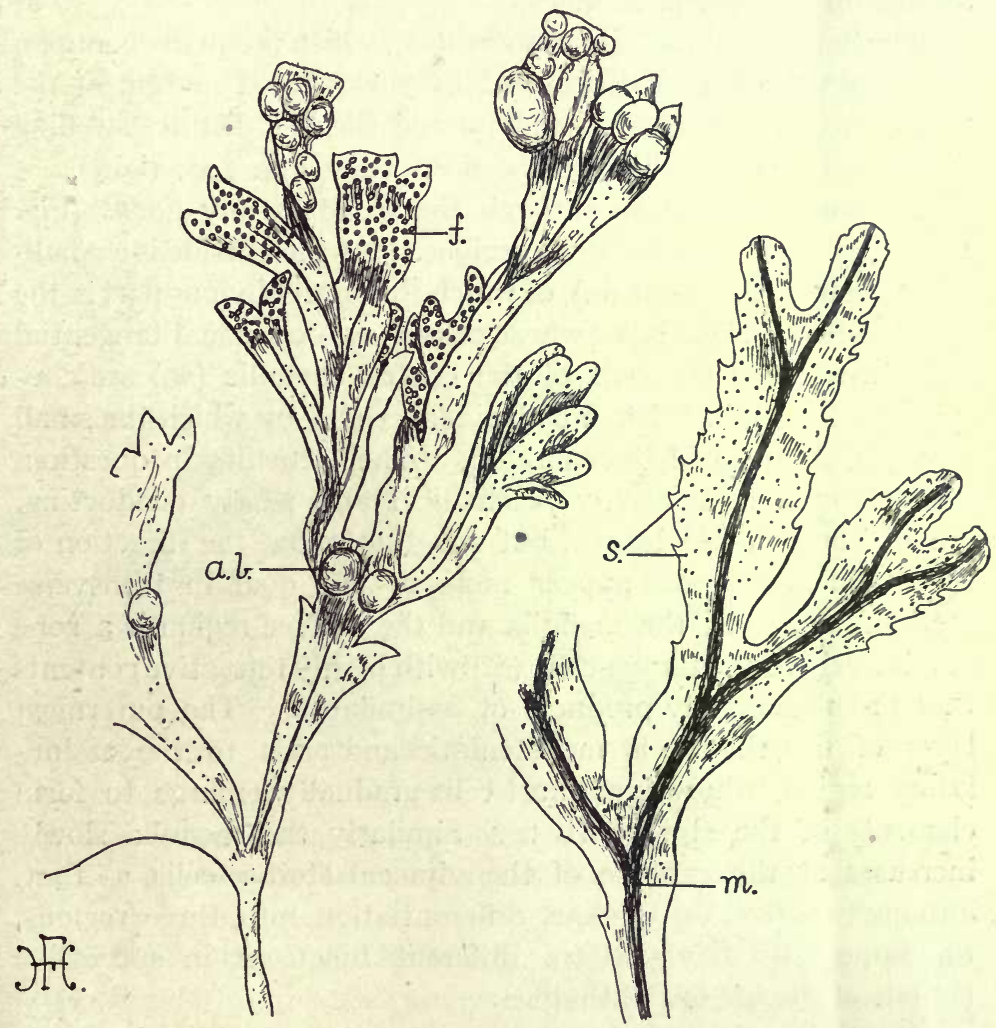

Fig. I08.-Fucus. On the left, part of a thallus of the Bladder Wrack ( $F$. vesiculosus); on the right, of the Serrated Wrack ( $F$. serratus). $a . b$, , air-bladder ; $f$., fertile conceptacles ; $m$., midrib of thallus; s., sterile conceptacles.

name is due, increase the buoyancy of the plant when submerged, and may also, like the air-canals of aquatic Phanerogams, serve for purposes of respiration.

Scattered irregularly over the whole surface of the frond are slightly protruding dots (Fig. I08, s.) marking the positions of 
small cavities (conceptacles) in the thallus. These are lined with hairs which often project as a minute tuft through the tiny aperture leading to the exterior. Usually some of the branches of the frond have swollen ends (Fig. I08) provided with much more conspicuous (fertile) conceptacles enclosing the sexual reproductive organs (cf. p. 224).

The Serrated Wrack (Fucus serratus), which is equally common on rocky shores, is distinguished by the toothed margin of the frond, the absence of air-bladders, and the less distinct swelling of the ends bearing the fertile conceptacles (Fig. Io8, right).

A transverse section through the frond of any Fucus (Fig. I2I, B, p. 225) presents three regions. At the outside is a smallcelled assimilating zone $(a$. $)$, of which the most obvious part is the palisade-like surface-layer whose cells show occasional tangential division-walls. The cells of the central medulla $(m$. $)$ are conspicuous for their thick mucilaginous walls, by which the small protoplasts are widely separated. The elements in question, whose function is partly mechanical and partly conducting, are of considerable length, but roughly follow the direction of the thallus, and hence appear more or less oval in transverse section. Between the medulla and the surface region is a zone of relatively large storage-cells $(S$.) with highly refractive contents that are presumably products of assimilation. The outermost layer of the thallus is meristematic and adds to the assimilatory region, whose innermost cells gradually enlarge to form elements of the storage tissue. Similarly the medulla slowly increases at the expense of the adjacent storage-cells, so that, although there is an obvious differentiation into three regions, the same cells may perform different functions in successive periods of the life of the thallus.

A cross-section of the stalk or frond of Laminaria shows essentially the same construction, and here the high specialisation of the Brown Algæ is evidenced by the presence of sieve-like areas, analogous to those of sieve-plates, on the cross-walls of many of the elongated elements of the medulla. Similar structures occur in Fucus, but are not so easily recognised.

Clothing the rocks, which for the greater part of the season are only reached by spray, one finds the Seaweed Pelvetia, which, though closely allied to Fucus, is much smaller, and in which the 
swollen fertile ends are a particularly conspicuous feature (Fig. I09). The structure of the thallus shows no important differences, but the branches of the frond are much narrower and channelled on their upper surface, features which aid in the retention of moisture ; moreover, the thallus is thus mainly illuminated by oblique light, so that the heating effect of the sun, and therefore transpiration, is presumably diminished during the hot hours of the day. The principal differences exhibited by Pelvetia can therefore be related to the special conditions of its habitat.

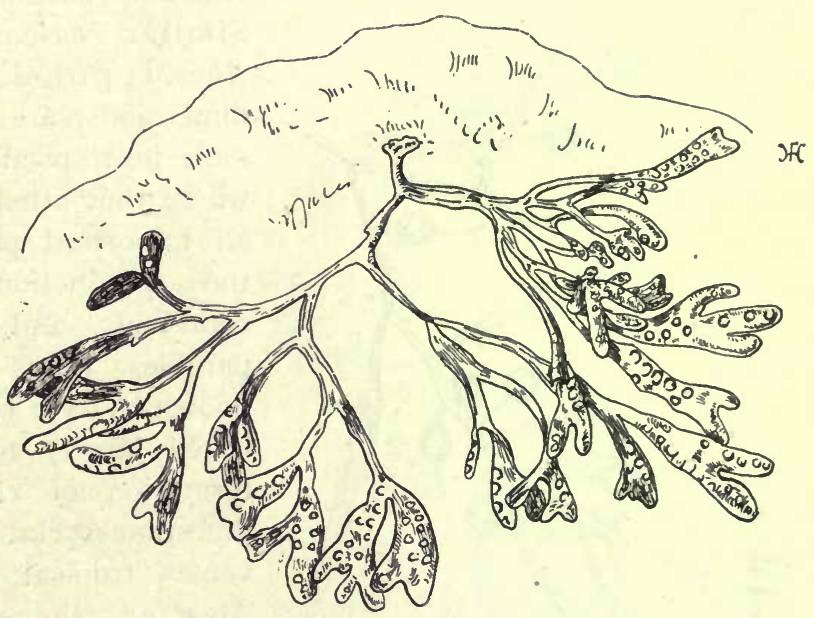

Fig. I09.-Plant of Pelvetia canaliculata, about natural size, showing the basal attaching disc and the prominent fertile conceptacles.

On rocky shores the Brown Algæ Pelvetia, Fucus, and Laminaria often form three zones at successively lower levels, occurring in the order named. Other members of the group are free-floating, as, for example, Sargassum bacciferum (Fig. IIO), huge stretches of which characterise the Sargasso Sea in the Mid-Atlantic. Many of the larger Brown Algæ are edible, and indeed extensively cultivated in Japan, whilst in the West of Scotland Fucus and similar forms are abundantly used as manure.

Most of the Red Algæ (Rhodophycea) are much smaller plants preferring weakly illuminated habitats, so that they either grow at considerable depths below low-tide level or in shady rock-pools. 
In many the thallus is branched and thread-like (e.g. Callithamnion, Ceramium, Fig. III, C), whilst in others it is flattened (Plocamium, Chondrus, Fig. III, A). In the former the larger branches usually consist of several rows of cells, whilst in the latter a number of distinct tissue-regions can often be distinguished. An interesting form, often very abundant in the rock-pools, is Corallina (Fig. III, B), whose branched thallus is composed of numerous pinkish-white segments loosely jointed to one another, and densely encrusted

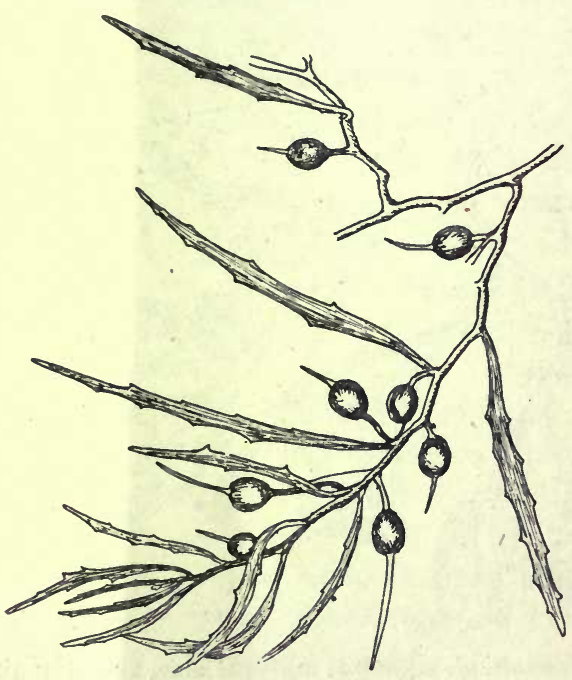

FIG. Iro.-Part of a plant of Sargassum bacciferum, about natural size, showing the numerous air-bladders to which the specific name is due. with carbonate of lime. Similar calcareous $A \lg e$, in part of larger dimensions, are common in tropical seas, where they often play an important part in the production of coral-reefs, and comparable forms are known to have contributed largely to the formation of certain limestone rocks. Diverse tropical Red Algæ are the source of agar-agar, a substitute for gelatine extensively used for bacterial cultures, whilst Carrageen (Chondrus crispus, Fig. III, A) furnishes a valuable invalid diet.

There is still another large group of Algæ (Cyanophycea) named after the prevailing colour, which in this case is blue-green. The forms in question, though relatively rare in the sea, are common in fresh-water, and often particularly characteristic of damp terrestrial habitats, such as rocks exposed to a constant trickle and the muddy sides of watercourses. Their great adaptability to varied conditions is also shown by their playing the leading rôle alike in the vegetation of hot springs and in the frigid lakes 
of the Antarctic continent. The Blue-green Algæ are either unicellular (Chroococcus, Fig. II2, A), colonial (Glococapsa, Fig. II2,C), or filamentous (Oscillatoria; Lyngbya, Fig. II2, F; Nostoc, Fig. II2, B). The cells possess either no nucleus in the ordinary sense, or one of very simple construction, and are in nearly every case devoid of a definite chloroplast. The cells or filaments, as the case may be, are often contained in large numbers in mucilaginous envelopes (e.g. Glcoocapsa, Nostoc), to which may be partly

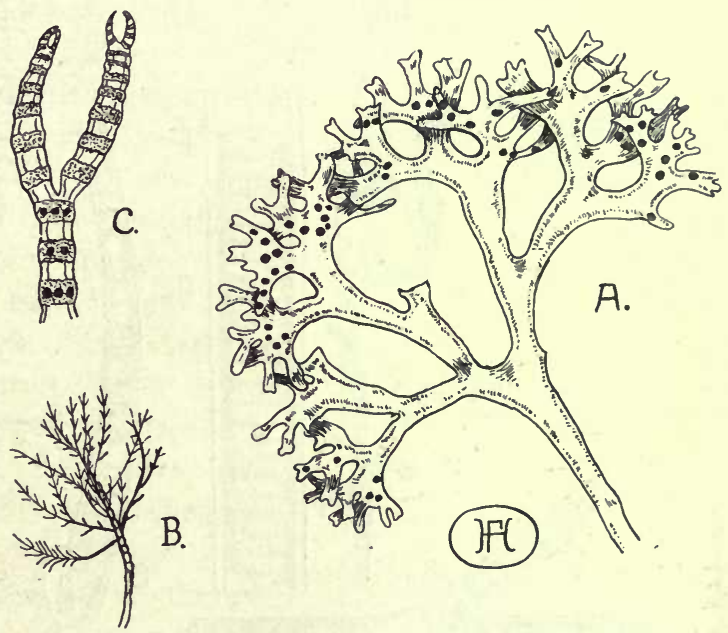

Fig. III.-Red Algæ. A, Carrageen (Chondrus crispus), the dark patches being due to groups of carpospores (p. 228) . B, Corallina. C, Apex of a branch of Ceramium (after Kützing), with groups of tetraspores (shown black).

attributed the capacity of many species to survive considerable periods of drought.

Common members of the group are: Glococapsa forming extensive gelatinous coverings on damp substrata and, under the microscope, resembling a blue-green Palmella-stage (Fig. II2, C) ; Oscillatoria and Lyngbya, whose undifferentiated and unbranched filaments of discoid cells (Fig. II2, F) occur as sheets or bundles in freshwater pools, on moist stonework, etc. ; Tolypothrix, a branched filamentous form, usually aquatic (Fig. II2, E); and the mainly terrestrial Nostoc, the species of which take the form 
of variously shaped gelatinous clumps (Fig. II2, B) in which are embedded numerous tortuous chains of rounded cells, interrupted here and there by slightly larger colourless ones (heterocysts, seen also in Tolypothrix, Fig. II2, E, h.) of uncertain function. The Cyanophyceæ often play a very important part in nature as the first plants to colonise bare ground.

In every habitat where other Algæ occur there can usually be found unicellular, or more rarely colonial, forms known as Diatoms .(Fig. II3) which possess quite special characteristics of

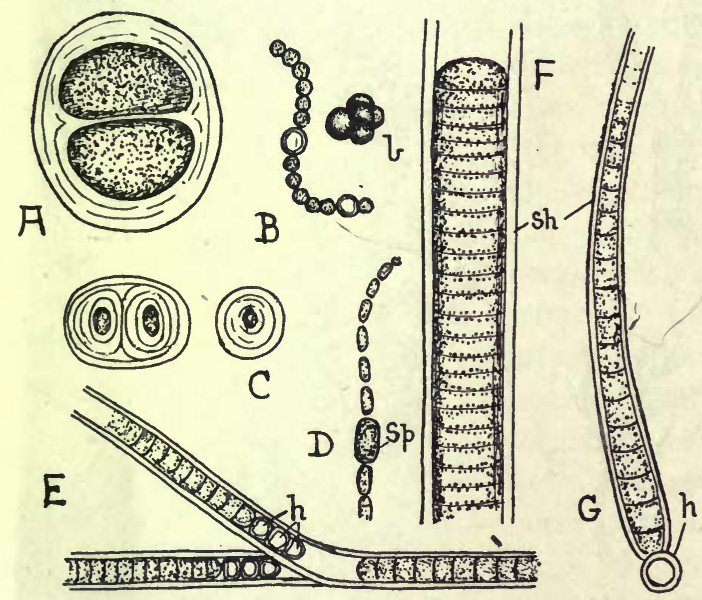

FIG. I I2.-Diverse Blue-green Algæ. A, Chroococcus. B, Single thread from colony of Nostoc (shown natural size in b). C, Gloocapsa. D, Anabana (with spore, Sp.). E, Tolypothrix. F, Lyngbya. G, Rivulavia. $h .$, heterocysts ; sh., sheath.

their own, and whose exact relationship to the other groups is not clear. The individuals are either unattached, and in that case often endowed with a power of fairly rapid movement, or else fixed to the surface of larger Algæ and other aquatics. The Diatoms are brown or greenish in colour, though a few species are colourless and saprophytic, and each plant is provided with a cell-wall richly impregnated with silica, and usually bearing a symmetrical and often highly elaborate pattern of sculptured markings (Fig. II3). With the death of the organisms the practically unaltered siliceous shells sink, so that, where Diatoms are plentiful, deposits 
of almost pure silica slowly accumulate at the bottom of the water. Instances are afforded by the extensive beds of " diatomaceous earth " found at Dolgelly in Wales, at Bilin in Bohemia, and elsewhere, some being of marine, others of freshwater origin. Such deposits are utilised commercially in the preparation of dentifrices and, owing to the very small size of the individual particles, for mixing with nitroglycerine in the manufacture of dynamite. The extensive vegetable Plankton of the sea at times consists almost entirely of Diatoms.

Although several groups of Algæ are designated according to the prevailing colour of their chloroplasts, they are characterised by many more important features, and especially by the nature of their reproductive processes (see next chapter). The chloroplasts, in general, assume the most complex forms among the Green Algæ, where there is often but a single one in each cell, a condition already noticed in Chlamydomonas and its allies (cf. p. I8I). Moreover, the chloroplasts of the Green

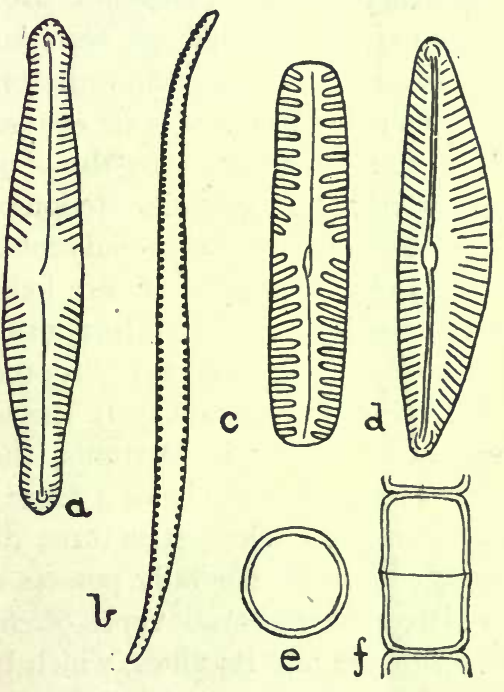

FIG. II3.-Various Diatoms (only the siliceous shells are shown). $a$ and $c$, Navicula ; b, Nitzschia ; $d$, Cymbella; $e$, end-view, and $f$, side-view of cells of the colonial Melosira.

Algæ commonly possess one or more pyrenoids and, during active assimilation, starch is formed as a reserve-product, first around the pyrenoids, and then in the general substance of the plastid. Whilst most of the colonial (e.g. Scenedesmus) and less differentiated filamentous forms (e.g. Ulothrix) have a relatively simple undivided chloroplast, greater complexity is met with in many of the more highly organised Green Algæ.

The chloroplast of Ulothrix is a curved band having the form of an incomplete cylinder (Fig. II5, $a, c$ ), which occupies the 
lining layer of cytoplasm within the thin cell-wall; it contains one or more pyrenoids. ${ }^{1}$ In Cladophora and Edogonium, on the other hand, the chloroplast is perforated and becomes a network with numerous scattered pyrenoids. In Edogonium (Fig. II7, a) the meshes are elongated and more or less parallel to one another, whilst in Cladophora (Fig. I04, D) the network is irregular and often consists of numerous separate disc-shaped portions, some of which possess pyrenoids. Other peculiarities of the cell-structure of Cladophora are the numerous small nuclei (Fig. I04, D, n.), that are recognisable after careful staining, on the inner side of the chloroplasts, and the thick stratified wall to which this Alga owes its coarse texture; the former feature is in marked contrast to the single nucleus found in the cells of Ulothrix and especially obvious in $E$ dogonium (Fig. II7, $a, n$.).

The non-septate threads of Vaucheria possess numerous discoid chloroplasts which are lodged in the lining layer of cytoplasm and lack pyrenoids (Fig. II8, D, c) ; this Alga also contrasts with other Chlorophyceæ in producing no starch, the excess food being stored as oil. In the Red and Brown Algæ (Fig. II9, $d$, ch.), as well as in Diatoms, the cells contain several chloroplasts which usually have a more or less lobed outline, and not uncommonly enclose structures resembling pyrenoids; in these groups the cells generally possess one or two nuclei.

The most elaborate types of chloroplasts are, however, found in Spirogyra and its allies, which belong to a group of the Green Algæ known as the Conjugate, practically confined to fresh-water. Some of these are filamentous like Spirogyra (Fig. 4), whose spiral chloroplasts, one or more to each cell, have already been described (p. 7), and Zygnema (Fig. I22, F), where the cells contain two star-shaped chloroplasts with a conspicuous pyrenoid $(p$.$) at the centre of each. A large number of the Conjugatæ$ are, however, unicellular forms, named Desmids (Fig. II4), which resemble Spirogyra and Zygnema in their methods of reproduction, but in many cases have even more elaborate chloroplasts.

The Desmid-cell usually exhibits two symmetrical halves,

1 In the Alga Hormidium, which occurs very commonly on damp soil, and in which much the same type of chloroplast is encountered (Fig. I I 6, D), the character of the latter is often very readily recognised. 
each containing one or two chloroplasts, and not uncommonly separated by a median constriction, where the single nucleus is situated (e.g. Cosmarium, Fig. II4, D, E). The wall is often richly sculptured or provided with spinous outgrowths (Fig. II4, A, D). In Closterium (Fig. II4, G), species of which are
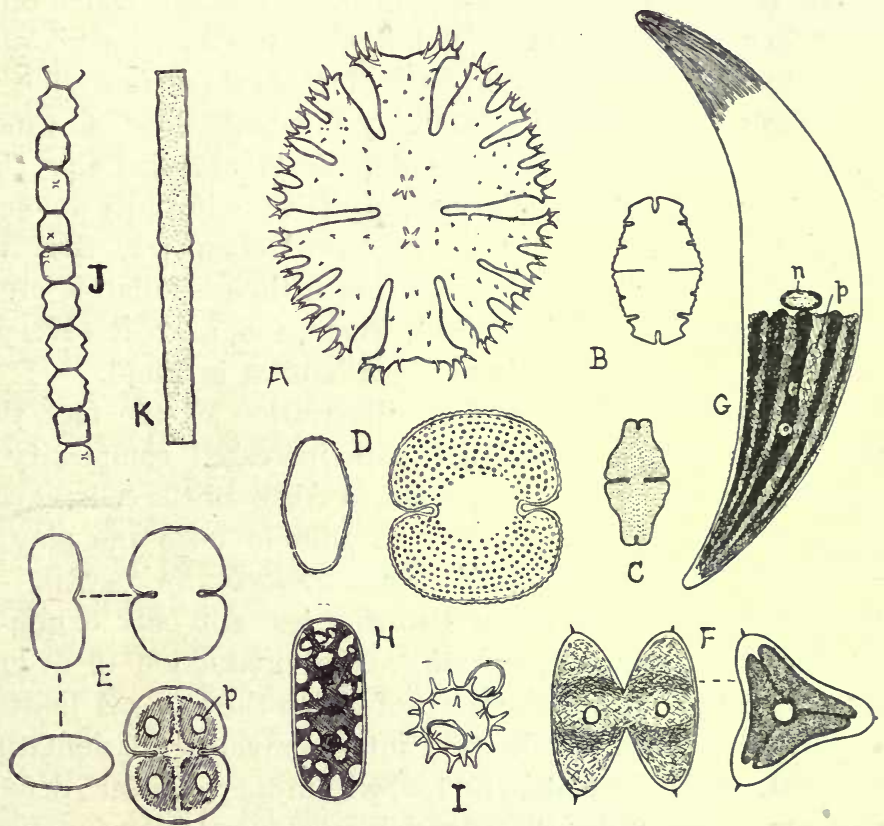

FIG. II4.-Diverse Desmids. A, Micrasterias. B and C, Euastrum. $\mathrm{D}$ and $\mathrm{E}$, Cosmarium (in $\mathrm{E}$ the top left-hand figure shows the cell in side-view; the lower left-hand figure the cell in end-view). F, Staurastrum (the right-hand figure shows the end-view) (after Ostenfeld). G, Closterium. H, Cylindrocystis. I, zygospore of Cosmarium meneghini (after West). J, Desmidium (filamentous). $\mathrm{K}$, Pleurotanium. The cell-contents are shown only in $\mathrm{E}-\mathrm{H}$. $n$. , nucleus ; $p$. , pyrenoids.

very frequent, each half of the, usually semilunar, cell is occupied by a chloroplast consisting of a central rod which contains a row of pyrenoids $(p$.$) and bears a number of radiating longi-$ tudinal plates ; the latter appear as dark green streaks when the cell is viewed from the surface. The small vacuoles, commonly seen at either end of the cell, enclose minute crystals of gypsum 
exhibiting Brownian movement, and are a peculiar feature of this genus. Similar elaborate chloroplasts are found in Cosmarium (Fig. II4, E), where the two halves of the cell are usually rounded or oblong, and Micrasterias (Fig. II4, A), where the outline of the cell as a whole is often very complex. This last feature reaches a climax in those Desmids which often abound in the surface-water of lakes, and where, as in other unicellular floating organisms, the increased surface due to their complicated outline considerably augments their buoyancy.

The extreme variety of the chloroplasts amongst the Algæ is in striking contrast with their comparative uniformity amongst Flowering Plants. It may be remarked, however, that the chloroplast of the simple Alga is as much the assimilating organ as is the leaf in the higher plant, where, too, a great diversity of structure in an apparently uniform habitat is found.

The Algæ afford an excellent illustration of the fact that division of labour is associated with increased complexity of structure. In many filamentous Green Algæ all the cells, except that serving for attachment, may be alike in form and play an equal part in growth and division (e.g. Ulothrix, Spirogyra). At the other extreme the larger Brown Algæ not only exhibit a relegation of attachment, growth, and reproduction to definite parts of the thallus, but the units of which the latter is built up also show a certain specialisation into conducting, assimilating, and meristematic elements. So too, within the cell itself, definite bodies are organised for the carrying on of particular functions. Whilst in the simply constructed Blue-green Algæ it is difficult, or even impossible, to recognise either chloroplast or nucleus, such division of labour is well marked within the cell in the majority of plants. The complexity which the chloroplast sometimes attains merely affords an extreme illustration of this principle.

It is probable that the complex type of cell arose from a much simpler one, and that similarly the multicellular organism had its origin in the unicellular, as is usually the case in the course of the individual development (cf. next chapter). High efficiency for particular conditions of life demand complexity of structure which, however, like all specialisation, tends to diminish the adaptability of the organism, to reduce its capacity for meeting 
changed conditions. It is in harmony with this that only a small part (viz. the relatively unspecialised gametes, see next chapter) of highly specialised organisms persists from one generation to the next, whereas in the case of a simple organism, the whole may survive in the bodies of its offspring. We may see in this, in the case of the more highly differentiated forms, a provision whereby the next generation is temporarily relieved of the trammels of the specialisation of its parents, and thus probably becomes better fitted to meet the extremely varied conditions to which the different individuals are subjected during their development.

The diverse freshwater Algæ are not found in equal quantity all the year round, many disappearing more or less completely during the warmer months or during the winter. A maximum abundance is usually attained during the spring (the time at which sexual reproduction occurs in many forms), whilst renewed development may take place in the autumn. The different genera and species frequently succeed one another to a more or less marked extent, so that a number of phases can be distinguished in the algal vegetation of small ponds, etc. This periodicity is probably conditioned by a multiplicity of factors, among which varying temperature and gascontent of the water play an important rôle. Some forms (e.g. Cladophora, several Diatoms), however, are encountered all the year round, although in varying abundance.

Many marine Algæ appear to persist throughout the year, although their development is often retarded during the summer or winter, as the case may be. The late winter is often the period at which sexual reproduction occurs. The power of withstanding low temperatures, which is so manifest in the case of the Seaweeds, also appertains to many freshwater Algæ, even such delicate forms as Desmids, for instance, often occurring as healthy individuals beneath the ice-sheet in the depth of winter. 


\section{CHAPTER XVI}

\section{Reproduction Among the Alge}

JUST as the vegetative structure of the Algæ shows progressive stages in complexity, so also do the processes of reproduction, although specialisation in the one respect does not always go hand in hand with specialisation in the other. Thus Cladophora, with its markedly differentiated vegetative system, shows the same simple reproductive processes as does Ulothrix, and Ectocarpus is scarcely more advanced. Our consideration of the life-history of Chlamydomonas and its allies (Chapter XIV) has already shown that new individuals may be formed in various ways, and, if we survey the Algæ as a whole, it is especially in relation to the sexual method of reproduction that progressive differentiation becomes most apparent. It will be convenient at the outset to study an Alga like Ulothrix, which, while exemplifying the various types of reproductive processes customary in the group, shows them in their simplest form.

At times of active growth this Alga reproduces vegetatively by the mere splitting or fragmentation of its filaments into short lengths which develop into new threads. Not infrequently, however, a more specialised mode of vegetative multiplication obtains, spoken of as asexual reproduction owing to its general resemblance to the sexual method except for the absence of fusion. Both the sexual and asexual reproductive cells are motile, although the ordinary Ulothrix-filament is without any power of movement.

Asexual reproduction may take place in some or all of the cells of a filament. At its commencement, the protoplasts round off slightly and thereupon usually divide, along successive planes at right-angles to one another, into 2,4 , or even 8 separate parts, the number depending upon the size of the cell (Fig. II5, b). 
The products of division ( $s p$.$) , each of which has a chloroplast$ and pyrenoid of its own, are liberated through a small round hole formed in the side-wall, but remain enveloped for a few seconds in a thin bladder of mucilage (Fig. II5, b). Meanwhile

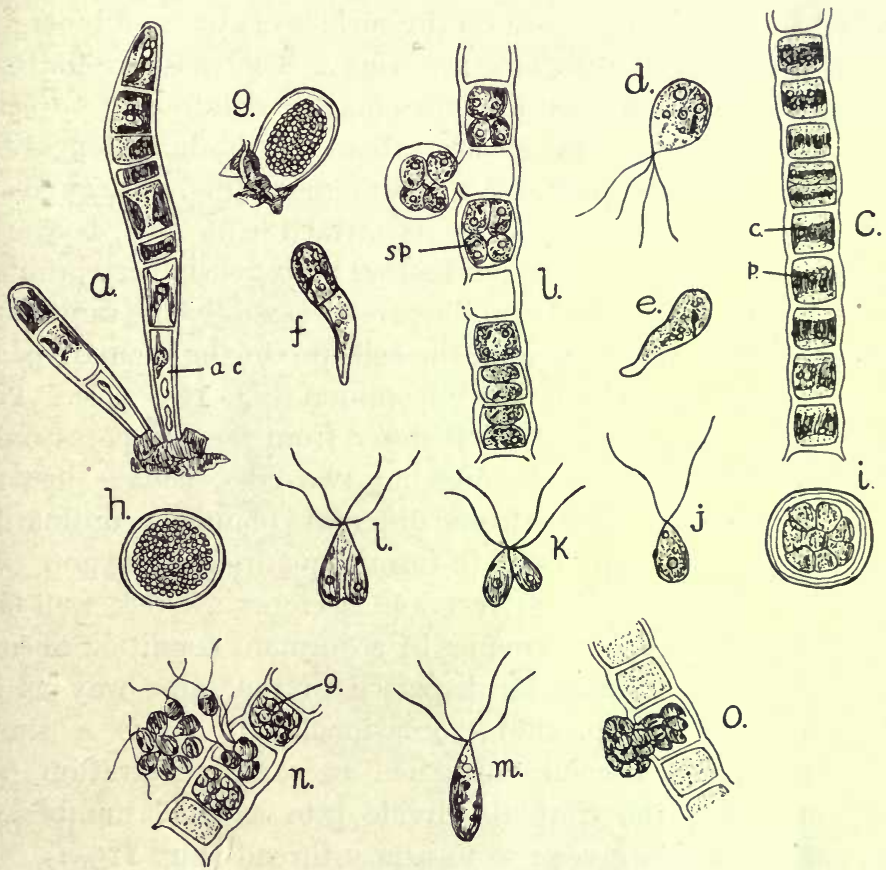

FIG. II5.-Ulothrix zonata. a, two young plants recently developed from zoospores; $b$, thread showing stages in the development of zoospores $(s p) ;$.$c , short length of vegetative filament showing$ chloroplasts $(c)$ with pyrenoids $(p) ;$.$d , zoospore ; e$ and $f$, young plants developed from zoospores; $g$ and $h$, two forms of zygospores ; $i$, germination of same; $j$, gamete; $k$ and $l$, stages in fusion of gametes; $m$, the resulting zygote ; $n$ and $o$, stages in development and liberation of gametes (g.). a.c., attaching cell. ( $b$ and $c, j-m$, after Klebs, all the rest after Dodel-Port.)

each portion has developed four cilia, two contractile vacuoles, and an eye-spot, so that in all essential respects it resembles a naked Chlamydomonas.

The pear-shaped motile elements thus formed are the 
asexual reproductive cells or zoospores (Fig. II5, $d$ ), which can swim after the manner of a Chlamydomonas for several hours, and thus travel some distance away from the parent-filament. During most of this time they seek out regions of the water which are well, though not excessively, illuminated, but ultimately their sensitiveness to light alters and they move towards darker spots, where they come to rest on the surface of stones, submerged parts of Flowering Plants, or other Algæ. The zoospore flattens out against the substratum and the cilia are withdrawn; a cellwall is secreted (Fig. II5,e) and then, by gradual elongation and division, there is produced a new filament (Fig. II5, $f$ and $a$ ), which soon breaks away from its attachment and becomes free-floating. Such asexual reproduction is obviously very prolific.

The gametes are formed and liberated in exactly the same way as the zoospores, except that in the cells producing them division into 16 or even 32 parts is not uncommon (Fig. II5, $n, 0$ ). The isogamous sexual cells $(j)$, which differ from the zoospores only in their small size and in having but two cilia, behave just as in Chlamydomonas, those from different filaments ordinarily fusing together in pairs $(k, l)$ to form a quadriciliate zygote $(m)$ which, soon after, comes to rest and secretes a thick wall $(h)$. The resulting zygospore remains in a dormant condition during the hot season, and may be dispersed in the same way as in Chlamydomonas (cf. p. I86); occasionally it forms a short outgrowth which becomes attached to some substratum $(g)$. On germination, the contents divide into a small number of parts each of which gives rise to a new thread (Fig. II5, i). It appears that under certain conditions the gametes lose the tendency to fuse with one another, and round off singly to produce spores which in all respects resemble the zygospores, an indication that their sexual character is not very pronounced.

At certain times the cells of the Ulothrix-filament develop mucilaginous walls and commence to divide along successive planes at right-angles to one another. Since the products become rounded off and themselves secrete mucilaginous walls, a condition is assumed closely resembling the Palmella-stages. of Chlamydomonas (Fig. II6, A). These may persist for some considerable time, but sooner or later the cells are liberated as. zoospores which produce new filaments. At times of extreme 
drought, the cells of the ordinary Ulothrix-threads, after thickening their walls and becoming laden with food-reserves, often fall apart and form as many separate resting spores (Fig. II6, B).

During the greater part of its life-history Ulothrix is thus sedentary like the majority of plants, but motility is definitely associated with the customary methods of reproduction. At such times a form is assumed resembling that of the unicellular
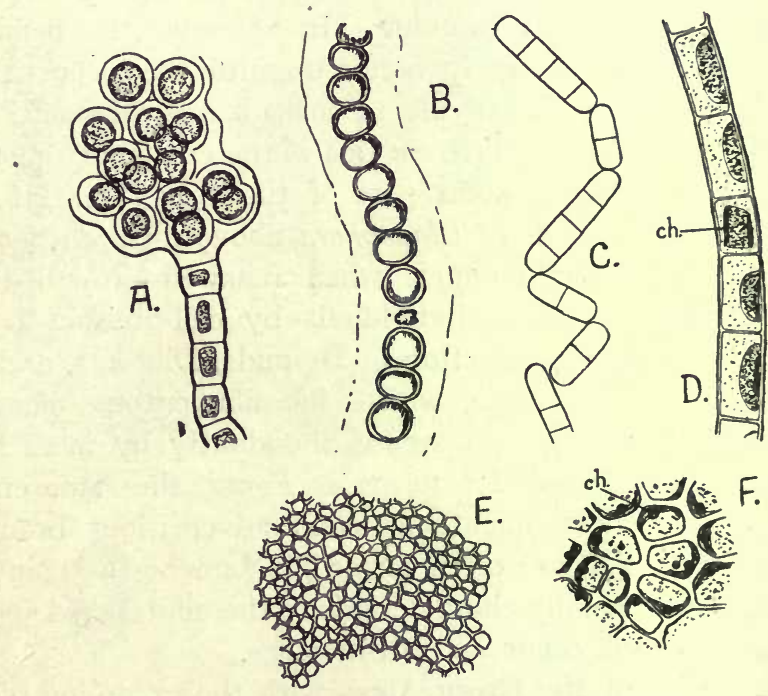

FIG. Ir6.-A and B, Ulothrix. A, Thread passing into Palmella-stage (after Cienkowski). B, Thread showing formation of resting spores (after Fritch). C and D, Hormidium (after Klebs). C, Thread undergoing fragmentation. D, Short length of filament. $\mathrm{E}$ and F, Ulva lactuca (original). E, A small part of the thallus, seen from the surface under the low power. F, A few cells, more highly magnified. ch., chloroplast.

organisms which are motile throughout their existence. The reproductive cells in question are differentiated into asexual zoospores and sexual gametes, but it will be gathered that this distinction is here no very sharp one.

The reproduction of Cladophora is essentially similar to that of Ulothrix, but the zoospores and gametes are produced in large numbers (Fig. I04, C), and their formation is usually restricted to the cells of the finer branches. During periods that are 
unfavourable to vegetative growth (e.g. the cold months of the winter), the cells of this Alga often become laden with foodreserves and develop exceptionally thick walls.

All the higher Algæ resemble Ulothrix and Cladophora in the restriction of motility to the reproductive phase, which, however, exhibits a varying degree of specialisation in the different forms. Vegetative propagation by fragmentation (i.e. cellseparation without preparatory division), as among aquatic Flowering Plants, is very common. In Spirogyra, for instance, the threads not uncommonly break up into their constituent cells, each of which can divide to form a new filament. In the common soil Alga Hormidium this is the customary method of propagation during a great part of the year (Fig. II6, C). The thick-walled threads of Cladophora above mentioned often fragment in a similar manner, when renewed growth takes place. Formation of new individuals by cell-division is the commonest form of reproduction in Desmids, Diatoms, and the unicellular Blue-green Algæ, whilst the filamentous members of the last-named group propagate abundantly by mere fragmentation. In such massive forms as Fucus, the same end is attained by the detachment of small adventitious branches of the thallus, which are often formed in bunches at points of injury, and are especially characteristic of the unattached species of this genus which occur on salt-marshes.

The majority of the Green Algæ, with the exception of the Conjugatæ, reproduce asexually by means of zoospores, but these often possess a more elaborate structure than those of Ulothrix. . For example, in Edogonium, where they are produced singly from the ordinary cells, they are much larger and bear a ring of cilia a little way behind the colourless front end (Fig. II7, e). If filaments of this Alga are brought indoors, zoospores are usually formed within a few hours, and, with a little patience, their development and liberation can be observed.

In the first place the protoplast contracts slightly away from the wall, and soon after this a colourless area, marking the future front end of the zoospore, arises on one side of the cell (Fig. II $7, b$ ). Around the edge of this area the numerous short cilia sprout out, appearing as so many fine lines. Thereupon the wall breaks across, near one end of the cell, and the shorter piece 
hinges back to form an aperture through which the contents slowly glide (c). At the moment of liberation the almost spherical zoospore is surrounded by a thin bladder of extruded mucilage (d), but it almost immediately commences to move away with the help of its cilia, though rather more slowly than in the case of Ulothrix or Cladophora. Sooner or later the zoospore becomes attached to some submerged object by its colourless front end
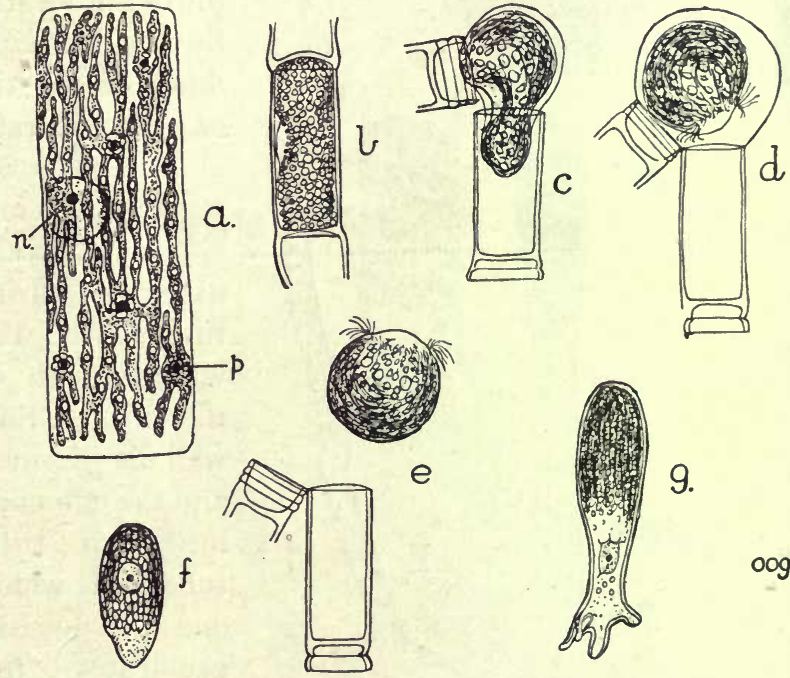

9.

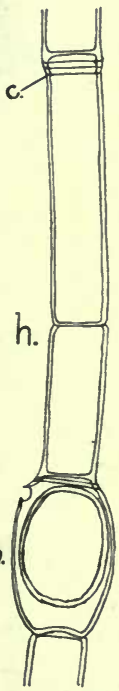

FIG. II7.-Edogonium. a, single cell, highly magnified (after Schmitz), showing the net-like chloroplast with pyrenoids $(p$.$) , and$ the single nucleus $(n.) ; b-g, E$ Edogonium concatenatum (after Hirn); $b-d$, stages in formation of zoospores ; $e$, liberation of ditto; $f$ and $g$, germination of zoospores (in $g$ the characteristic attaching cell is seen) ; $h$, Edogonium pachyandrium (after Hirn), showing cells with caps $(c$.$) , and an oogonium (oog.).$

(Fig. II $7, f$ ), and the cilia are withdrawn; then a cell-wall is secreted and division takes place to form a new filament, whilst the end in contact with the substratum grows out into the branched holdfast (Fig. II $7, g$ ).

In the case of Vaucheria, zoospore-production commences with the swelling of the tips of the branches, which become filled with abundant cytoplasm, chloroplasts, etc., and are finally 
cut off by separating walls to form so-called zoosporangia (Fig. II8, B). Within each the contents round off and the numerous nuclei take up a position outside the chloroplasts. A pair of cilia arise opposite each nucleus, so that a large multi-

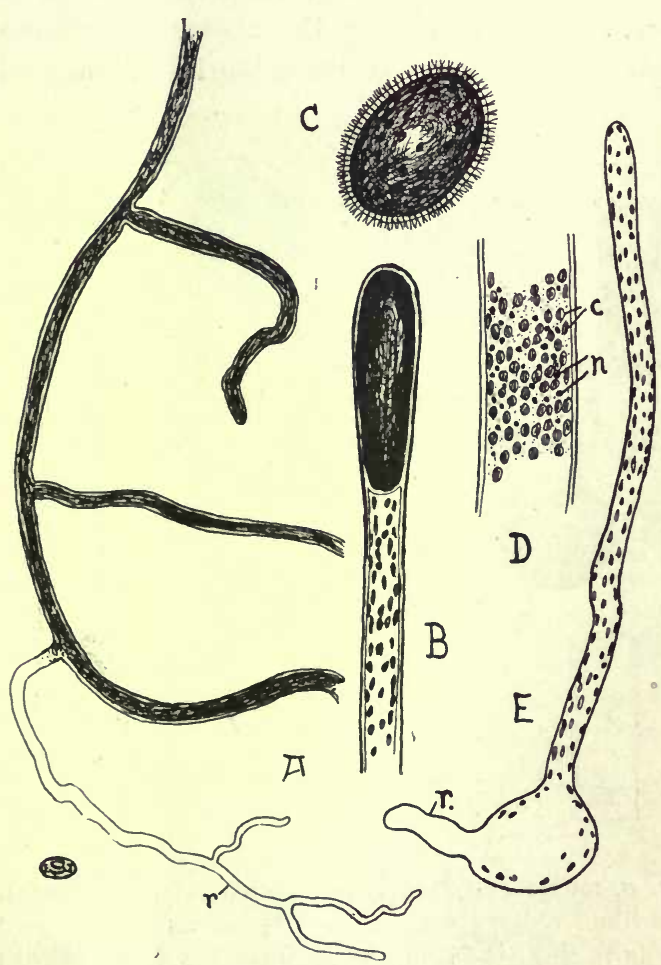

FIG. II8. Vaucheria. A, Portion of a plant showing the branched non-septate thallus and the colourless rhizoid-like attaching organ $(i$.). B, Zoosporangium. C, Zoospore. D, Small part of thallus, showing the numerous chloroplasts $(c$.$) , and nuclei (n$.$) . E, Germinated$ zoospore. (C after Oltmanns ; rest original.) ciliate oval zoospore (Fig. II8, C) is formed, which escapes into the water by the breaking down of the tip of the sporangium. Its movements are slow and usually cease after a short time with the withdrawal of the cilia; a thin wall is formed and the two ends lengthen into tubes, of which one frequently penetrates the soil or mud and becomes a colourless attaching organ (Fig. II8, $\mathrm{E}, r$.).

Zoospores are also encountered in some of the Brown Algæ, e.g. in Ectocarpus and Laminaria. In the former they develop in considerable numbers in oval sporangia, each borne laterally on a branch of the filament (Fig. II9, c), and liberating its contents by rupture of the apex. The pear-shaped zoospores have two 
cilia, one directed forwards and the other backwards during movement, and these are attached to one side adjacent to the chloroplast and the prominent eye-spot (Fig, II9, g).

The production of zoospores serves as a rapid means of
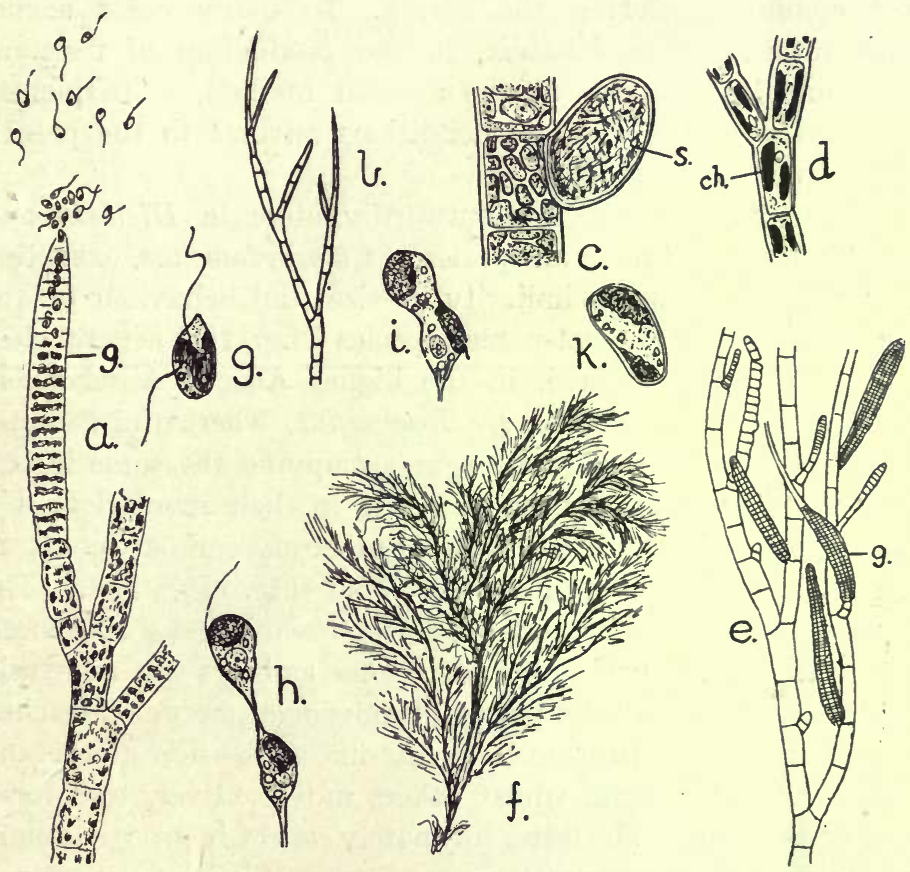

FIG. II9.-Ectocarpus. a, small part of a thread showing a gametangium (g.), liberating gametes ; $b$, tip of a branch showing hair-like terminations; $c$, small part of a thread bearing a zoosporangium (s.); $d$, part of thread showing cell-contents and chloroplasts (ch.); $e$, branched filament bearing several gametangia $(g) ;$.$f , plant of$ $E$. littoralis, natural size; $g$, zoospore; $h$ and $i$, stages in fusion of gametes ; $k$, zygospore, still showing two chloroplasts and two eyespots. ( $a$, after Thuret; $b$ and $d-f$ after Migula; $c$ and $g$ after Reinke; $h-k$ after Berthold.)

multiplication and dispersal at times when vegetative activity is at its height, but this method lacks the stimulus which sexual fusion appears to provide. As a matter of fact continued asexual reproduction has, in certain cases (e.g. some Diatoms), 
proved to be detrimental to the organism. On the other hand, there are quite a number of Algæ in which a sexual process is unknown, as in the whole group of the Cyanophyceæ and in many Desmids. Apart from these, however, most Algæ reproduce sexually at some time or other in the course of the year, most commonly during the spring. In many cases sexual fusion results, as in Ulothrix, in the production of resistant spores and, in contrast to the asexual method, is frequently associated with the onset of conditions adverse to the plant's growth and nutrition.

The fusing gametes are outwardly alike in Ulothrix and Cladophora, as well as in all species of Chlamydomonas, excepting C. monadina. The dissimilarity in size and behaviour of the sexual cells seen in the last-named species (cf. p. I85) is paralleled, or even more emphasised, in the higher Algæ. A relatively simple instance is afforded by Ectocarpus, where the gametes are produced in special gametangia, occupying the same lateral position as the sporangia, but differing in their more elongated shape and in being divided into numerous compartments in each of which a single gamete is formed (Fig. II9, $a, g$.). The sexual cells are smaller, but otherwise resemble the zoospores. Despite their structural uniformity, some gametes are relatively sluggish, and, after a brief period of movement, become attached to any suitable substratum by a disc-like expansion at the end of their forward cilium, whilst others move actively and for a much longer time. The latter ultimately collect in groups around the others and, sooner or later, an active gamete fuses with a resting one (Fig. II9, $h$ and $i$ ). There is thus a marked difference in behaviour between the two fusing cells, but the differentiation into active males and passive females is here purely physiological, although in a few species of Ectocarpus the two gametes differ in size and other respects.

The distinction between the two sexes is much more marked in forms like Edogonium, Vaucheria, and Fucus, where one sexual cell (the female or egg) is large, motionless, and provided with plentiful food-material, whilst the other (the male or spermatozoid) is small, actively motile, and possessed of very scanty cytoplasm. The two kinds of gametes are usually formed in special sexual organs differentiated from ordinary vegetative 

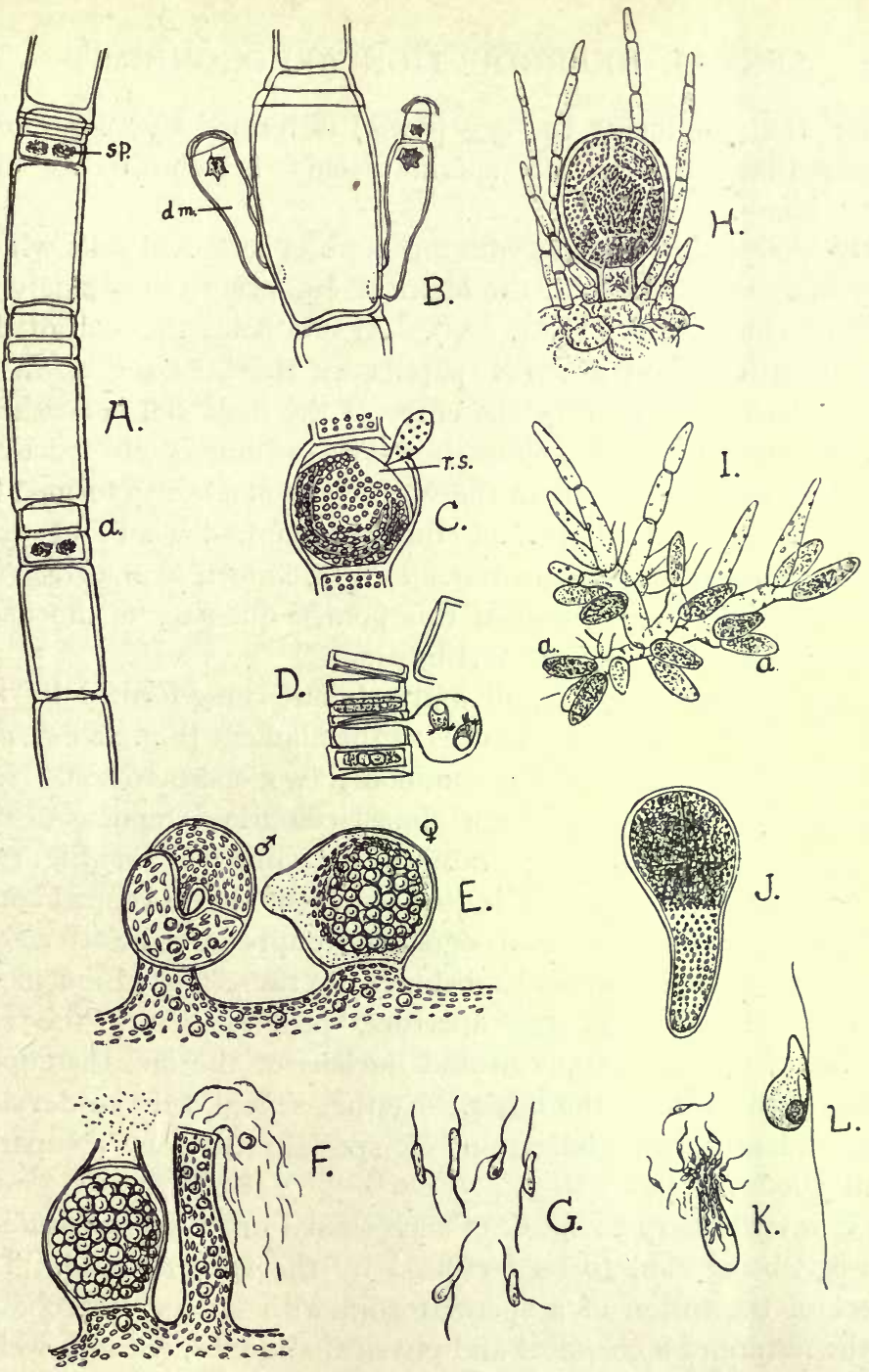

J.

FIG. I20.--Oogamous sexual reproduction among the Algæ. A-D, $E$ dogonium. A, Part of thread of $O$. pachyandrium showing antheridia (a.) with spermatozoids (sp.) ; $\mathrm{B}$, oogonium of $O$. concatenatum, with two dwarf-males (d.m.) ; C, oogonium of $O$. diplandrum showing the spermatozoid penetrating into the egg at the receptive spot (r.s.); $\mathrm{D}$, antheridia of $O$. landsboroughi, with escaping spermatozoids. E-G, Vaucheria sessilis. E, Oogonium (O) and antheridium (ô); F, oogonium with oospore and dehisced antheridium; $G$, spermatozoids. H-L, Fucus. H, Oogonium with surrounding hairs; I, branched hair bearing numerous antheridia $(a$.$) ; J, young plant;$ $\mathrm{K}$, liberation of spermatozoids ; L, spermatozoid. (A, B, and D after Hirn ; C after Juranyi ; E-G after Sachs ; H-K after Thuret ; L after Guignard.) 
cells; that producing the egg (ovum) is termed the oogonium, whilst that forming the spermatozoids is known as the antheridium.

In $C$ Edogonium the oogonia are more or less oval cells which may arise in any part of the filament and occur either singly or in short chains (Fig. II7, $h$, oog.). At one point the wall of the oogonium develops a small papilla by the breaking down of whose tip an aperture for the entry of the male cell is created; in some species of $C E$ dogonium, however, opening is effected by a complete transverse split in the wall. The single egg, formed by the contracted protoplast of the oogonium, develops a small colourless area, adjacent to the aperture, known as the receptive spot (Fig. I20, C, r.s.), and at this point a quantity of mucilage is extruded shortly before fertilisation.

The antheridia are small and tabular, being formed by repeated transverse division of cells of the filament (Fig. I20, A, a.). Each produces one, or more commonly two, spermatozoids ( $s p$.) which are diminutive, though almost colourless, replicas of the zoospores, and which are liberated in the same manner (Fig. I20, D). Should a spermatozoid in the course of its movement come into the neighbourhood of an oogonium, it appears to be attracted, probably by some chemical substance ${ }^{1}$ in the extruded mucilage, and, passing through the aperture, penetrates into the egg (Fig. I2O, C), the cytoplasm and nucleus of the one thereupon fusing with those of the other. Neither sexual cell can develop independently, an indication of specialisation as compared with Ulothrix.

It is customary to speak of such sexual union as fertilisation, the egg being said to be fertilised by the spermatozoid. The effect of the fusion of a spermatozoid with an egg is probably of the nature of a chemical and physical stimulus, without which further development of the ovum is impossible. This view is supported by the fact that the eggs of Sea-urchins have been induced to develop into embryos by mere immersion in suitable solutions, whilst those of the Frog have been caused to undergo

1 The influence exerted by chemical substances on the direction of movement of motile elements is spoken of as chemotaxis, and the positive chemotaxis evident in sexual union is only one of many examples of such chemical stimulation (cf. p. 232). 
the first stages of development by mere pricking. Moreover, in plants, fertilisation sometimes stimulates other cells near the egg to develop into embryos (cf. p. 372).

The fertilised eggs, or oospores, develop thick protective walls and fatty pigmented contents and, as the filaments containing them die away, sink to the bottom of the water. Here they pass through a prolonged resting period, and, should the pond dry up, may be dispersed by the wind. In the few cases in which their germination has been observed, they gave rise to three or four ordinary zoospores, which were set free by the bursting of the thick membrane.

The sexual organs are arranged in various ways in the different species of Edogonium, male and female sometimes occurring in the same filament (monœcious forms), sometimes in different filaments (diœcious forms); in the latter case the male plants often consist of only a few cells (dwarf males, Fig. I20, B, d.m.), and arise from special smaller zoospores which become attached to the female plant, on or near an oogonium.

It will be evident that Gidogonium exhibits considerable specialisation in its methods of multiplication, and not the least conspicuous feature is the division of labour manifest in the oogamous sexual reproduction. Owing to its stationary character, the egg can possess the greater bulk which a more adequate provision of food-material for the benefit of the next generation necessarily entails (cf. p. 220). Since the spermatozoids contribute nothing to this food-supply, they can be correspondingly smaller, and therefore, without additional strain on the organism, produced in larger numbers, whereby the chance of fertilisation occurring is greatly increased. The probability of fusion between the two gametes is, moreover, doubled by one of them remaining stationary. The greater certainty of sexual union admits of a corresponding decrease in the production of eggs, which will afford as many offspring as would a larger number of motile female gametes. These remarks apply with equal force to all plants in which oogamy occurs.

The oogonia and antheridia of Vaucheria are produced near one another as outgrowths of the main filament or of short lateral branches, from which in either case they become separated by a septum (Fig. I20, E). Their relative positions are very 
diverse, but the adjacent sexual organs usually mature almost simultaneously, so that self-fertilisation is probably the rule. The more or less oval oogonium (ㅇ) develops a protrusion on one side, whose tip becomes mucilaginous, and breaks down to form the aperture through which the male cell enters. The contents are rich in chloroplasts and at first multinucleate, but in the mature egg, which possesses a pronounced receptive spot opposite the opening, only one large nucleus persists.

The antheridium is a coiled tube tapering slightly near the apex, and giving rise, by division of its contents, to numerous minute spermatozoids (Fig. I20, E, o); the latter are pearshaped bodies with two laterally attached cilia, a very small yellowish chloroplast, and a prominent nucleus (Fig. I2O, G), and are liberated by a breaking open of the tip of the antheridium (Fig. I20, F). The attraction of the spermatozoid towards the egg is probably again connected with the extrusion of mucilaginous matter by the latter. After fusion, the oospore becomes enveloped by a thick wall and accumulates large stores of reserve oil (Fig. I2O, F); it then enters on the usual resting period, which is ultimately terminated by the direct development of a new plant.

Apart from the vegetative propagation above described, Fucus exhibits only sexual reproduction, the antheridia and oogonia being developed in the large fertile conceptacles occupying the swollen tips of the thallus (Fig. Io8, $f$ ). In some species (e.g. F. platycarpus) the two kinds of sexual organs occur in the same conceptacle, but in $F$. vesiculosus and $F$. serratus there are distinct male and female plants. The globular cavities of the conceptacles (Fig. I2I, A) are separated from the rest of the thallus by a wall (w.) composed of several layers of flattened cells, from whose inner surface arise numerous unbranched multicellular hairs which bend towards, and indeed often protrude from, the small aperture ; in the fertile conceptacles the sexual organs are interspersed among these hairs (Fig. I2I, A).

The oval oogonia possess a thick transparent several-layered membrane, and are seated on a short stalk which arises directly from the wall of the conceptacle (Fig. I20, H; I2I, A). At maturity the contents are divided into eight uninucleate eggs, containing abundant chloroplasts and separated by delicate 
septa. The antheridia are oval cells, which likewise possess relatively thick walls and occupy the ends of most of the short lower segments of richly branched hairs (Fig. I20, I). ${ }^{1}$ In each are formed numerous minute biciliate spermatozoids (Fig. I2O, L), containing a well-marked nucleus, but only traces of a chloroplast. The mature antheridia have a yellowish colour, which they impart to the entire conceptacle, and by this means, in the diøcious species, the male plants can be distinguished.
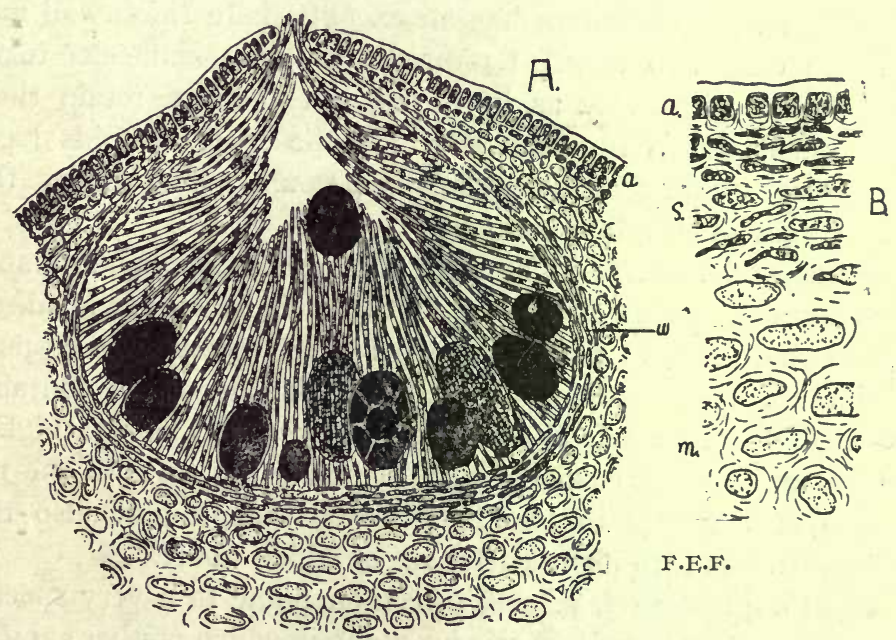

F.E.F.

FIG. 12I. - The Bladder Wrack (Fucus vesiculosus). A, Transverse section through a fertile conceptacle containing oogonia, in different stages of development. B, Small part of a transverse section through the thallus, more highly magnified. a., assimilating layer ; m., medulla ; s., storage cells; w., wall of conceptacle.

When the sexual cells are ripe, the outermost layer of the antheridium or oogonium, as the case may be, breaks open and sets free the contents which remain enclosed in the inner part of the wall. The gradual extrusion of the packets of ova, or spermatozoids, from the opening of the conceptacle often occurs between the tides, and is probably largely brought about by expansion of the mucilage, secreted by the hairs, combined with

1 These antheridial hairs are best examined by teasing out the contents of a male conceptacle in a drop of water. 
desiccation and contraction. The sea-water dissolves the membranes still enveloping the sexual cells (Fig. I20, K), and the ova, which have now assumed a spherical form, become fertilised by the actively moving spermatozoids. The oospore secretes a thin membrane and immediately, without a resting period, develops into a new Fucus-thallus. The young plant is at first spherical, but at an early stage produces the basal holdfast (Fig. I20, J) and acquires a strap-shaped form, and this is soon followed by branching.

In Pelvetia, where both sexual organs occur in the same conceptacle, the oogonium has an exceptionally thick wall and produces only two eggs. Extrusion of the sexual cells takes place in the same way as in Fucus, but the ova retain their thick mucilaginous investment which the spermatozoids have to penetrate, and which envelops the young plant during the early stages of development.

Fucus and Pelvetia differ from most other oogamous plants in the number of eggs and in the fact that the latter are fertilised outside the plant, in both of which respects these Algæ appear relatively unspecialised. Normally the female organ contains but a single ovum (cf. Edogonium, Vaucheria, and the higher plants), and in this connection it is interesting to note the reduction of the eggs to two in Pelvetia, though here also the nucleus divides into eight parts, six of which abort.

The Conjugatæ (cf. p. 208) owe their name to a very special type of sexual reproduction (conjugation), in which neither gamete is free-swimming. In the filamentous forms, such as Spirogyra and Zygnema, two threads become ranged parallel to one another, and their' opposing cells develop finger-like protrusions which grow towards each other till they meet and fuse (Fig. I22, B); after this the separating wall breaks down, so that an open tube (the conjugation canal, Fig. I22, c.c.) is established. In Spirogyra and many species of Zygnema the development of processes always commences a little sooner on the one filament than on the other, and a similar difference is observed with respect to the contraction of the protoplasts which now ensues. The cells of the filament that first put out processes, in these cases, act as males, since their contents commence to glide over, through the conjugation canals, into the opposite cells (Fig. I22, B, b), with whose passive (female) protoplasts they fuse. 
In some species of Zygnema (e.g. Z. pectinatum), however, the events leading up to conjugation take place simultaneously in the two filaments, and the fusing protoplasts meet in the middle of the conjugation canal; in such forms there is no outward differentiation of sex, the gametes being isogamous as in Chlamydomonas and Ulothrix (Fig. I22, D). But even in the case first

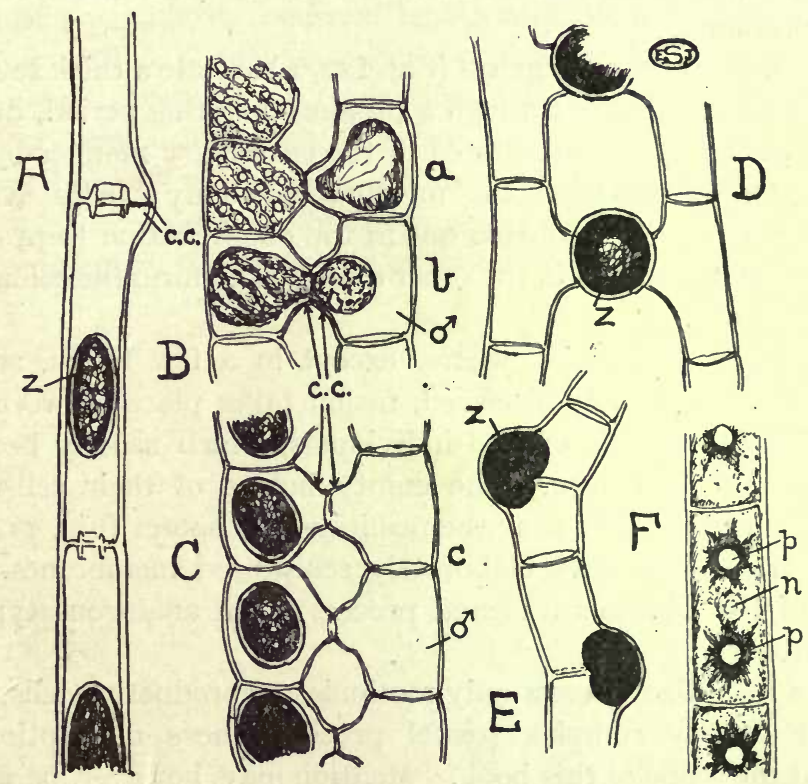

FIG. 122.- Sexual reproduction in various Conjugatæ. A, Spirogyra weberi, showing lateral conjugation (after Petit). B and C, Spirogyra bellis. B, successive stages $(a$ and $b$ ) in conjugation; C, completed conjugation. D, ladder-like, and E, lateral conjugation in Zygnema pectinatum. F, small part of filament of same. c.c., conjugation canal ; $n$. , nucleus ; $p$. , pyrenoid ; $z$., zygospore ; $\hat{\sigma}=$ male cells.

described the gametes are merely distinguished physiologically, although a difference of size occurs in one of the related genera. The sexual process of the Conjugatæ therefore, whilst peculiar in itself, shows the same analogous series of stages to that observed in other Algæ.

In some species, both of Spirogyra and Zygnema, sexual union may take place between adjacent cells of the samê filament 
(Fig. I22, A and E), the conjugation canals (c.c.) forming looplike connections between their contiguous ends. Here the threads must be regarded as including cells of both sexes, the zygospores as before being formed either in the conjugation canal or in one of the two cells. The fact that both methods of conjugation may occur simultaneously in the same mass of Spirogyra or Zygnema indicates that sexual differentiation between the filaments is not very profound.

In all cases the zygospores (Fig. I22, z.) secrete a thick severallayered wall and pass through a prolonged resting period, during which they may be distributed in the customary manner by the wind. On germination the membrane usually bursts at two places, the contents growing out in the one direction to produce the new filament, and in the other direction to form the colourless attaching cell.

Among the Desmids where, except in a few forms, sexual reproduction is rarely observed, fusion takes place between the liberated protoplasts of two individuals, which usually become enveloped in mucilage. The empty halves of their cell-walls are often recognisable near the resulting zygospores (Fig. II4, I), which frequently have elaborately sculptured membranes. In some of the Diatoms a sexual process of an analogous type is encountered.

The Red Algæ possess only motionless reproductive cells, and exhibit a very complex sexual process whose description is beyond the scope of this book. Mention may, however, be made of the copious production of filamentous outgrowths from the female organ after fertilisation, the ends of these threads giving rise to special asexual reproductive cells known as carpospores. The dense clusters thus produced are often conspicuous as minute oval patches of a darker colour (Fig. III, A). The ordinary asexual cells, so-called tetraspores, are produced in fours in small usually spherical sporangia, and are readily recognised on the thalli under the microscope (Fig. III, C).

The examples of reproductive processes among the Algæ might be multiplied considerably, but sufficient have been described to show the diversity of methods by which the same end, namely the multiplication and perpetuation of the species, is attained. It is the result rather than the means which must 
be regarded as the more important biological phenomenon. The methods of reproduction have been seen to vary, not only amongst closely related forms, but even in one and the same species. But, with all this variety, the outcome is a cell, or cells, each capable of giving rise to a new plant.

Why reproductive bodies, capable of resisting adverse conditions and of remaining dormant for considerable periods, should be more particularly produced by sexual fusion, is a question as yet unanswered. It is, however, easy to recognise the importance of a second type of multiplication, by cells which, being unprovided with either food-reserves or resistant walls, can be formed rapidly and in large numbers. The association of this type with asexual reproduction may perhaps be related to the greater facility of responding to favourable conditions. 


\section{CHAPTER XVII}

The Structure and Reproduction of the Fungi

THE Algæ are not the only plants in which the body is a thallus of relatively simple construction, but the remaining representatives of the Thallophyta, the lowest class of the Vegetable Kingdom, are characterised by the absence of chlorophyll. They are, consequently, like the colourless saprophytes and parasites among higher plants, dependent upon organic material elaborated by other organisms. The plants in question are grouped as Fungi and show many peculiarities, both in vegetative structure and the nature of their reproductive processes.

A considerable number derive all their nourishment from other living plants or animals, such parasites, exemplified by the Smut of Wheat, the Gooseberry Mildew, the Potato Blight, the Salmon and Silkworm diseases, often doing serious harm to their host. Numerous Fungi, however, live upon decaying organic matter (e.g. many Moulds and Toadstools), and these saprophytes play an important part in nature in connection with processes of decay.

The plant-body is of a peculiar type, consisting generally of a loose weft, the mycelium (Fig. I25, a), composed of very delicate branched threads or hyphe, which are usually colourless, and which may or may not be septate (Fig. I29, a). The narrow diameter of the hyphæ facilitates their penetration either into the interior of a host (parasites), or between the particles of decaying organic material (saprophytes). The hyphæ, moreover, secrete at their tips various enzymes (cf. p. 53), which bring about solution of the obstructing cell-walls and also convert the organic material into a readily assimilated form, a single species of Fungus producing a number of different enzymes, 
according to the substratum upon which it occurs. The extreme simplicity of the vegetative structure may well be compared with that of parasitic Flowering Plants (cf. p. I79), some of which-encountered only in the Tropics-have as a matter of fact a plant-body so reduced that it resembles a mycelium.

In some Fungi the wall of the hyphæ consists of cellulose, but much more commonly of a complex nitrogenous compound similar to the chitin found in animals, together with other substances such as pectose, callose, etc. Embedded in the lining layer of cytoplasm in the lower forms are numerous minute nuclei, but in the septate hyphæ of the higher types there are usually only one or two in each cell ; neither plastids nor starchgrains are ever present, but there are often small oil-drops and sometimes crystalline albuminous bodies. The central vacuole is prominently developed. Where abundant food-storage occurs, as, for instance, in the reproductive cells, it is customary to find the polysaccharide glycogen, which can be recognised by the deep brown colouration assumed with iodine. In coloured hyphæ, such as occur in species of Peziza, etc., the pigment is generally confined to the cell-wall.

The Fungi are classified in three groups-Phycomycetes, Ascomycetes, and Basidiomycetes - each of which has so many characteristic features that it will be convenient to consider them separately. The Phycomycetes, which are not modified to so marked an extent as the other two groups, include forms which usually show a well-marked sexual process, and which, in this and other respects, resemble Algæ such as Vaucheria. The hyphæ, for example, contain numerous nuclei, and often only exhibit transverse walls in relation to the formation of reproductive bodies. The group includes many common parasites, such as Cystopus (the White Rust of Cruciferæ, Fig. I23, A), Pythium debaryanum (the cause of the "damping off " of seedlings, Fig. I24, B), Phytophthora infestans (the Potato Blight), Empusa (responsible for a disease of house-flies), as well as the saprophytes Mucor (the black Mould appearing on jam, bread, etc.), Saprolegnia, and Achlya (the last two frequent on decaying water-plants).

Cystopus, a species of which often attacks the Shepherd's Purse, furnishes a typical example, whose life-history can easily 
be studied. The parts affected by the Fungus, most commonly situated in the region of the inflorescence, are swollen and contorted (Fig. I23, A), and exhibit a white surface which looks as though it had been whitewashed. Such enlargement, or hypertrophy, is a frequent symptom of fungal attack, and is an outcome of the abnormal development of the diseased tissue, whose cells undergo increase in size with, or without, division. A longitudinal section through such a blister (best stained with eosin) shows the hyphæ of the parasite ramifying in all directions within the intercellular spaces and middle lamellæ of the host (Fig. I23, B, h.). Here and there, however, small club-shaped branchlets (the haustoria, S.) will be observed penetrating into the actual cellcavities, and by this means the Fungus absorbs food-material elaborated by the host.

Near the surface of the stem the hyphæ are more densely packed, and their almost parallel branches form a pile-like felt (the hymenium, Fig. I23, C) which ruptures the overlying epidermis and causes the white appearance above mentioned. The slightly swollen ends of the hyphæ of the hymenium exhibit various stages of constriction, resulting in the gradual formation of chains of spherical structures called gonidia (Sp.), the oldest of which is farthest away from the point of origin. As the short fragile stalks connecting the gonidia with one another get broken across, the latter are removed by the wind, and sometimes travel many yards before reaching the ground.

When rain or heavy dew causes a sufficient accumulation of moisture, the contents of the gonidia divide into several parts, which are liberated as minute colourless zoospores (Fig. I23, G), swimming by means of a pair of cilia. Many doubtless perish before reaching a suitable host, but should they encounter seedlings of a Cruciferous plant, they come to rest on the surface, secrete a membrane, and elongate into a short hypha which penetrates into the interior by way of a stoma. The stimulus directing the movement of the zoospore towards the host-plant is probably a chemotactic one (p. 222), whilst the growth of the hypha into the interior affords an example of positive chemotropism. For some weeks further development of the Fungus consists in the ramification and gradual spread of the hyphæ through the tissues of the host, until a sufficiently large haustorial 


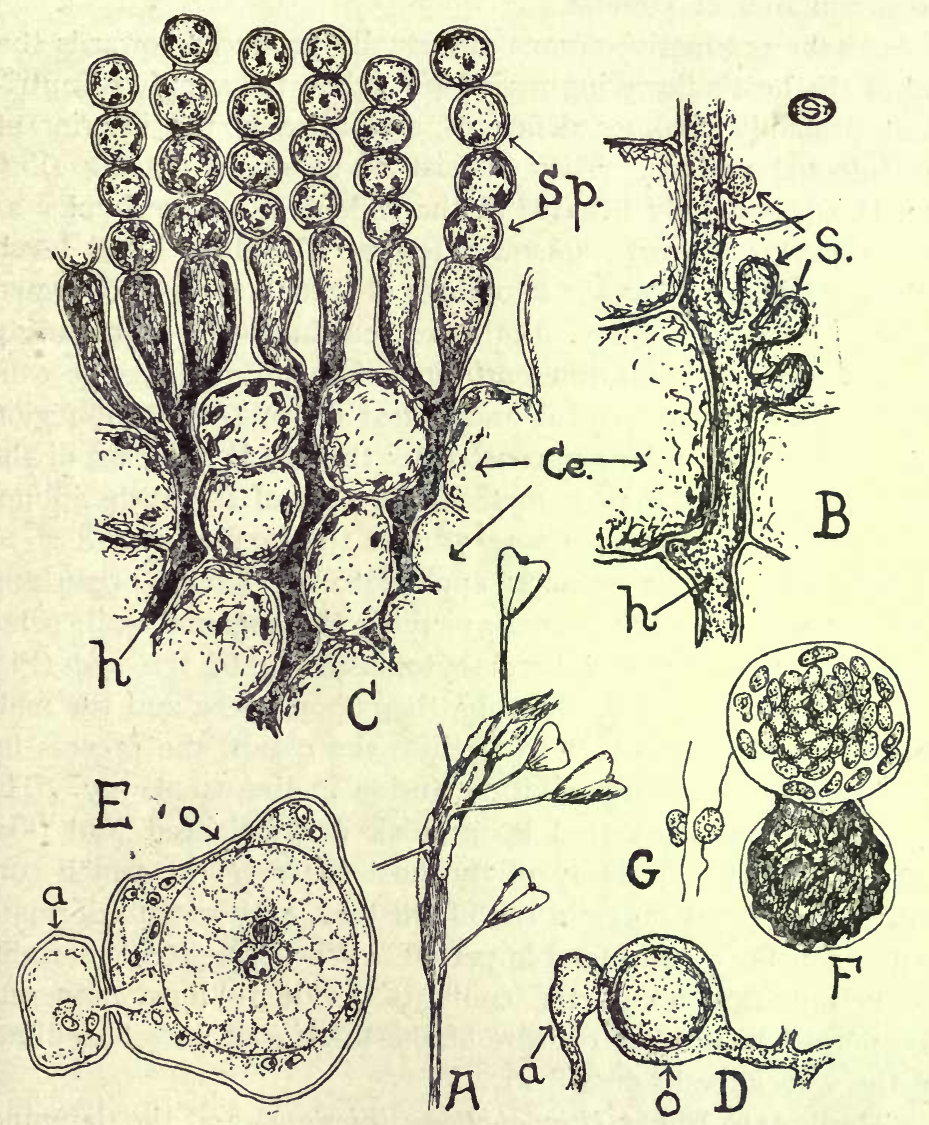

FIG. I23.-The White Rust of Cruciferæ (Cystopus candidus). A, Diseased inflorescence of Shepherd's Purse, showing the white patches where the gonidia of the Fungus are being formed. B, Hypha (h.) with haustoria $(S$.$) , as seen in a longitudinal section between the cells (\mathrm{Ce}$.) of the host. C, Transverse 'section near surface of host, showing hyphæ $(h$.) and gonidia (Sp.). D, Antheridium (a.) and oogonium (o.) (after De Bary). E, The same in section at the time of fertilisation (after Stevens). F, Germination of oospore (after De Bary). G, Zoospores (after De Bary). (Figs. A-C, original.) 
system has been created to supply the material necessary for the production of gonidia.

Sexual reproductive organs are usually produced towards the end of the host's flowering period (i.e. when the supply of nutriment probably becomes deficient), and arise in the interior of the infected regions. They consist of spherical oogonia (Fig. I23, D, o.), generally situated at the ends of the same hyphæ as bear the club-shaped antheridia (a.) at a slightly lower level, although in some cases the two kinds of sexual organs are formed on neighbouring hyphæ. Both are multinucleate and, during development, undergo differentiation of their protoplasmic contents into a denser central and a less dense peripheral region (Fig. I23, E) ; the former constitutes the egg in the case of the oogonium and the male gamete in the case of the antheridium, while the outer region plays no part in the sexual fusion.

The antheridium becomes applied to the female organ and puts out a slender tube which, piercing the oogonial wall, penetrates through the peripheral cytoplasm up to the egg (Fig. I23, D, E). The tip of the tube thereupon opens and the male gamete passes through it to fertilise the ovum, the process involving nuclear and cytoplasmic fusion in the usual way. The product becomes invested by a thick dark-coloured wall (Fig. I23, F). After the decay of the host the oospores, which constitute the resting-stage in the life-history, may remain dormant in the soil for a considerable period. When conditions suitable for germination occur, the contents divide to form numerous zoospores which, after rupture of the thick wall, infect seedlings in the way already described.

The Potato Blight (Phytophthora infestans) and the dampingoff Fungus (Pythium debaryanum) have life-histories very similar to that of Cystopus, except that their gonidia can, under certain circumstances, germinate direct into a new plant (i.e. without forming zoospores). In both cases the mycelium is intercellular, and the asexual reproductive organs alone appear on the surface of the host. In the Potato Blight the oval or elliptical gonidia are formed singly at the ends of branched hyphæ, which emerge through the stomata of the diseased leaves (Fig. I24, A). If blown on to the leaves of another Potato-plant, the gonidia grow out direct into an infecting hypha; whilst, if they fall on 
the ground, they can, in the presence of moisture, produce zoospores, as in Cystopus. The first signs of disease are discoloured spots exhibiting a dark central region surrounded by successive zones of greyish and pale green tissue, which rapidly become brown or even blackish; closer inspection discloses the white
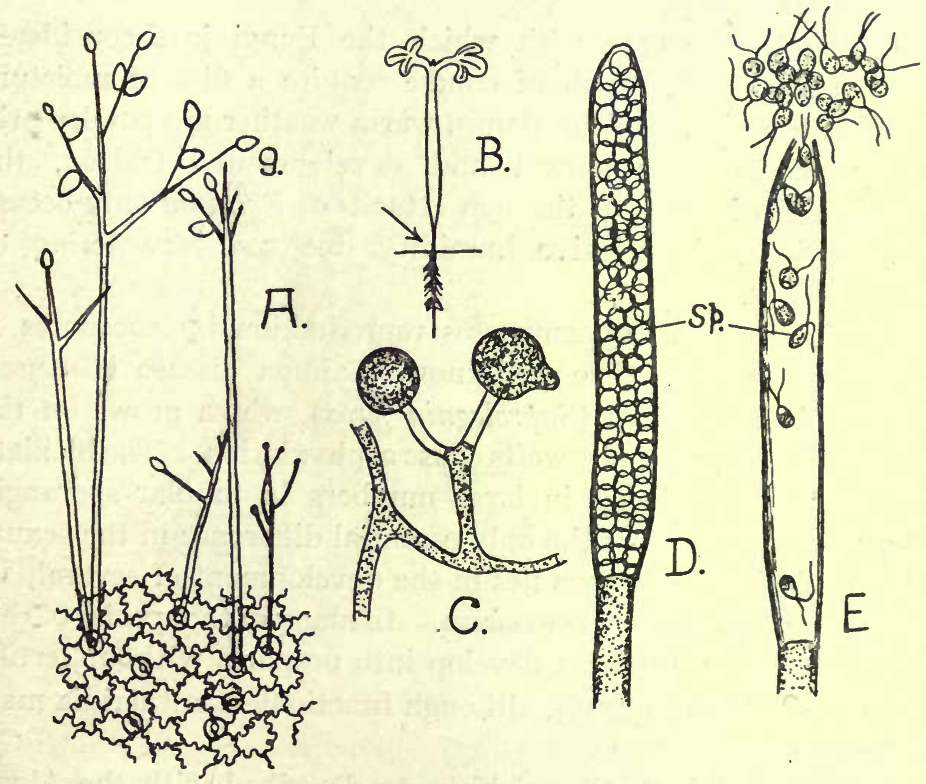

FIG. 124.-Asexual reproduction in various Oomycetes. A, Small part of epidermis of Potato-leaf, infected with Blight (Phytophthora infestans), showing branched hyphæ bearing gonidia ( $g$.) emerging from the stomata. B, Seedling of Cress which is "damping off," due to an attack of Pythium debaryanum; the point at which the hypocotyl is giving way is indicated by an arrow. C, Hypha with sporangia of the same. D, Young, and E older, sporangia of Saprolegnia, showing numerous zoospores (Sp.). (A after Strasburger; B after Miyake ; $\mathrm{C}$ after Hesse ; D and $\mathrm{E}$ after Thuret.)

tufts of hyphæ bearing the gonidia, especially on the lower surface of the infected leaf. The hyphæ of the parasite gradually spread to the underground parts, thus infecting the tubers, so that early removal of diseased shoots is advisable.

An attack of Pythium results in a rapid softening of the 
hypocotyls of the diseased seedlings (Fig. I24, B), which soon give way at this point and collapse. The rounded gonidia, which are borne on simple or forked hyphæ (Fig. I24, C), usually give rise to zoospores without becoming detached, so that the disease rapidly spreads from one seedling to another. The sexual reproduction of Pythium and Phytophthora is practically identical with that of Cystopus.

Owing to the ease with which the Fungi just considered produce zoospores, which of course require a film of moisture in which to swim, spells of damp, warm weather are particularly favourable to their spread and development. Indeed, the damping-off of seedlings through attacks of Pythium only occurs in conditions of excessive humidity due to overwatering or overcrowding.

In the aquatic Phycomycetes reproduction by zoospores is naturally the rule. The well-known Salmon disease is caused by one of these Fungi (Saprolegnia ferox), which grows on the gills of the fish, where its wefts cause asphyxiation. The biciliate zoospores are produced in large numbers in tubular sporangia (Fig. I24, D, E), whilst the only essential difference in the sexual reproduction of this genus lies in the development of several, or even many, eggs in each oogonium. In many of the species of this genus, moreover, the eggs develop into oospores without fertilisation (so-called apogamy), although functionless antheridia may be formed.

In contrasting the Fungi hitherto described with the Algæ, one of the most striking peculiarities, apart from the absence of chlorophyll, is the non-motile character of the male gamete. Spermatozoids, as a matter of fact, are known to occur only in one small group of the Phycomycetes. This feature may be related to the fact that the Fungi as a whole are a terrestrial group, living under conditions (e.g. in the interior of a host-plant) in which the necessary moisture for the movement of spermatozoids is not available.

The saprophytes among Phycomycetes are well exemplified by Mucor, which thrives on all kinds of decaying substrata (especially horse manure), upon which its mycelium forms a white weft (Fig. I25, a). Numerous absorptive branches penetrate downwards into the source of nourishment, and sooner or 
later conspicuous, dark brown or black, spherical sporangia $(s p$.$) appear at the ends of relatively thick upright hyphæ,$ which in some species are branched. An ally of Mucor (Rhizopus stolonifer), that occurs very commonly on stale bread and horse dung, spreads very rapidly by hyphæ resembling
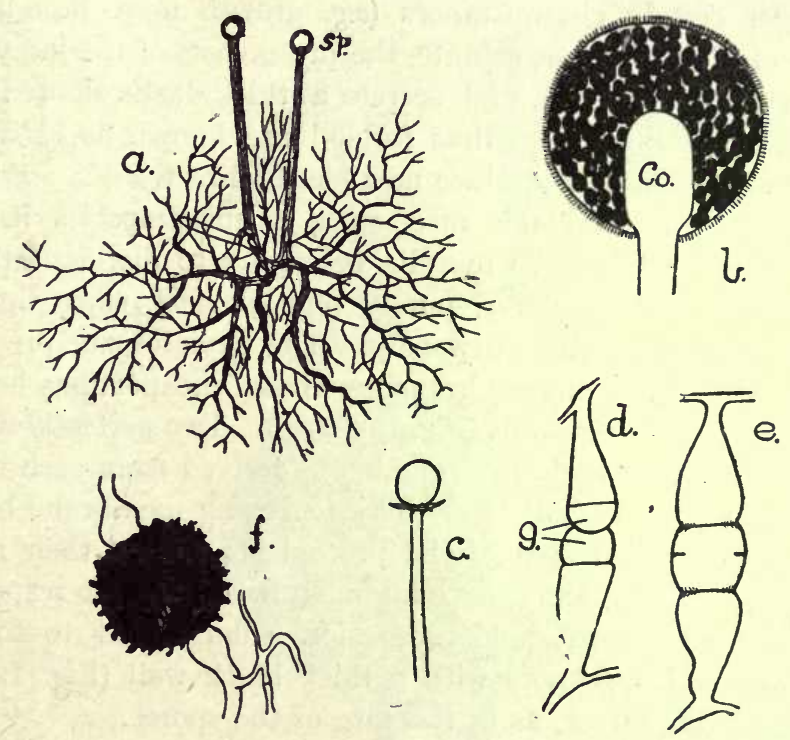

FIG. I25.-Mucor. a, mycelium, slightly magnified, showing two of the long-stalked sporangia $(s p) ;$.$b , sporangium, much enlarged, in$ optical section, showing the numerous spores and the central column (Co.); $c$, dehisced sporangium in which only the column and a small part of the wall remains ; $d$ and $e$, conjugation of gametes $(g$.$) ;$ $f$, mature zygospore. $(a, b$, and $f$ after Brefeld ; $c$ after Sachs ; $d$ and $e$ after De Bary.)

minute Strawberry runners, at the end of each of which a tuft of absorptive threads and sporangia is produced.

The wall of each sporangium (Fig. I25, b) is beset with numerous minute needles of oxalate of lime, whilst the swollen end of the hypha below projects into the cavity as a central column (Co.); between this and the wall are many small thickwalled spores embedded in'a mucilaginous substance. The latter swells in the presence of moisture, and thus contributes to the 
bursting of the sporangium. It is also responsible for the adhesive nature of the spores, which are so widely disseminated by the wind that they are almost ubiquitous. They are extremely resistant, and are capable of remaining dormant for long periods. On germination they grow direct into a new plant without the production of zoospores.

Under certain circumstances (e.g. growth in a liquid) the hyphæ of Mucor become septate, the protoplasts of the individual compartments contract, and secrete a thick dark-coloured wall. The special resting spores thus formed are known as chlamydospores, which likewise produce new plants direct.

Sexual reproduction is rare, since in most species it only takes place between two mycelia belonging to distinct strains. The difference is, however, purely physiological, since the two are not distinguishable outwardly. The gametes are produced within swollen club-shaped branches whose end-portions become separated off by cross-walls (Fig. I25, d, g.). Two perfectly similar branches meet by their tips, one being derived from each of the two plants (Fig. I25, $d$ ), and the intervening membrane breaks down, whereupon fusion of the protoplasts and of their nuclei ensues $(e)$. The product, deriving nutriment from the respective mycelia, subsequently undergoes slow enlargement to form a large spherical zygospore with a thick black wall (Fig. I25, $f$ ). Germination is direct, as in the case of the spores.

The sexual process of Mucor is thus isogamous and analogous to that of the Conjugatæ, where likewise an entire plant is often of one sex or the other (cf. p. 227). In the parasitic Fungus which attacks house-flies (Empusa musca) the fusing cells are, however, of unequal size, and thus show a structural distinction.

Mucor and Empusa belong to a subdivision of the Phycomycetes, known as the Zygomycetes, all characterised by this type of sexual process. The other forms previously considered are oogamous and classed as Oomycetes, but they are also distinguished by the ease with which they produce zoospores. The Zygomycetes are much more markedly adapted to terrestrial conditions from this point of view, since all of them reproduce by means of motionless spores.

The second great group of Fungi, the Ascomycetes, are characterised by their method of spore-formation and by the absence. 
of a true sexual process in the vast majority of cases. Common parasites belonging to this group are the Mildews (Erysiphacea), the Ergot of Rye (Claviceps purpurea, Fig. I28, A), and the Vegetable Caterpillar (Cordyceps) ; but there are numerous saprophytes, such as the ubiquitous Blue Mould (Penicillium), the $\mathrm{C} u \mathrm{p}$ - $\mathrm{f}$ u n $\mathrm{g} \mathrm{i}$ (species of Peziza, Fig. I26, C), the Stag's Horn Fungus (Xylaria, Fig. I26, A), the Morel (Morchella, Fig. I26, D), and Nectria, which is the cause of the bright red pustules so common on decaying branches and sticks.

A general idea of the Ascomycetes can be obtained from an examination of a species of Peziza. The septate mycelium of this Fungus is perennial and ramifies in the decaying

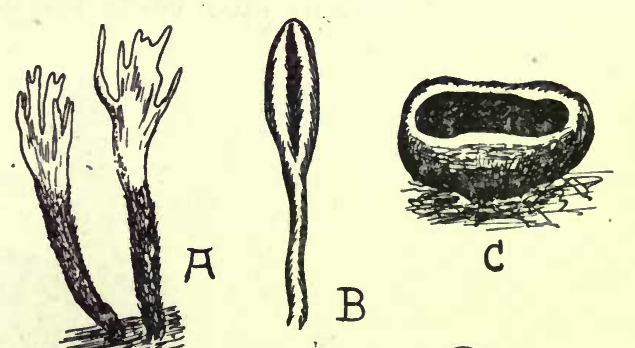
substratum (e.g. dead trunks and branches, soil rich in humus), its presence only becoming apparent in autumn, when conspicuous, and often brightly coloured, cup-shaped fruit-bodies (apothecia, Fig. I26, C) are produced at the surface. In a vertical section through one of these (Fig. I27, B) the hyphæ are seen to be so densely compacted as to produce a false tissue, the elements of which are quite irregularly arranged, except for those lining the 
inner surface of the cup. These form a palisade-like layer (the hymenium, h.) composed of numerous elongated sporangia or asci (Fig. 127, A, $a-f)$, interspersed with the slender hair-like ends of barren hyphæ $(p$.$) . Each ascus contains eight ellipsoidal$ ascospores $(e, f)$, which are liberated when mature/ through a terminal aperture on contact with moist air. Mere breathing on a ripe fruit-body may often cause the liberation of a cloud of spores.
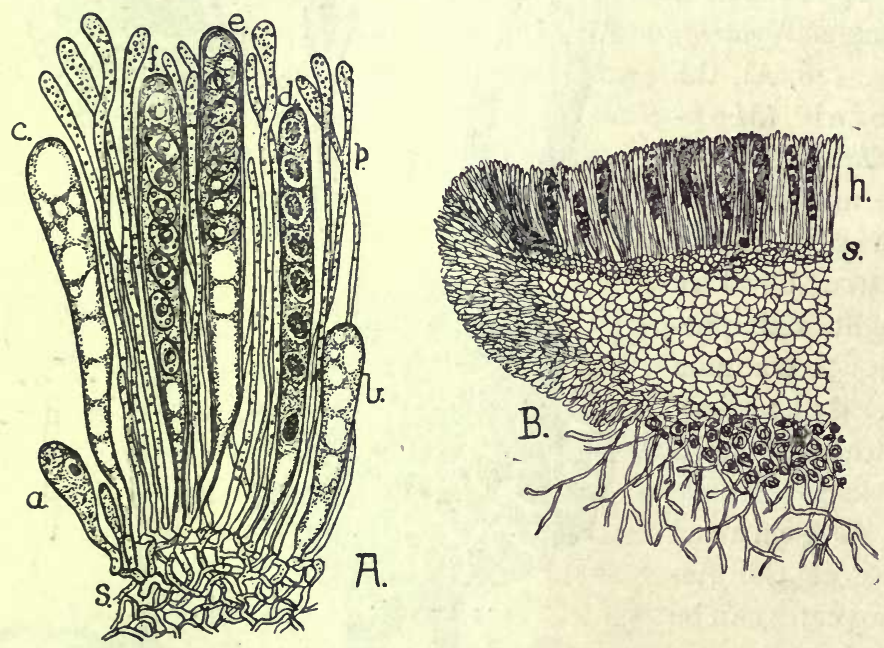

FIG. I27.-Peziza vesiculosa. B, Section of half an apothecium (diagrammatic), and A, Small part of the hymenium enlarged, showing asci in progressive stages of development $(a-f)$. $h_{.}$, hymenium ; $p_{\text {., barren }}$ hyphæ, of same ; s., small-celled subhymenium. (After Goebel.)

The ascus is typical of the Ascomycetes as a whole, and constitutes one of the chief characteristics. For, by contrast with the Basidiomycetes (cf. p. 245), the spores are produced within the mother-cell, whilst in contradistinction to the Phycomycetes they are nearly always only eight in number, though sometimes fewer (e.g. two or four, as in the Truffle), or more numerous (sixteen or thirty-two). In many cases the asci, as in Peziza, are grouped together in compact and often large fruit-bodies, the hymenium either covering a great part of the exposed surface (as in the Morel, Morchella, Fig. I26, D, and Geoglossum, Fig. I26, B) or being completely enveloped within sterile hyphæ; 
the last is the case in the Truffles (Tuber), whose fruit-bodies are, moreover, subterranean.

Ergot (Claviceps) infests the ovaries of Rye, Oats, and other Grasses, becoming very conspicuous at the time of harvest, as a result of the gradual replacement of the grains by a black banana-shaped mass (about half an inch long) of closely inter-
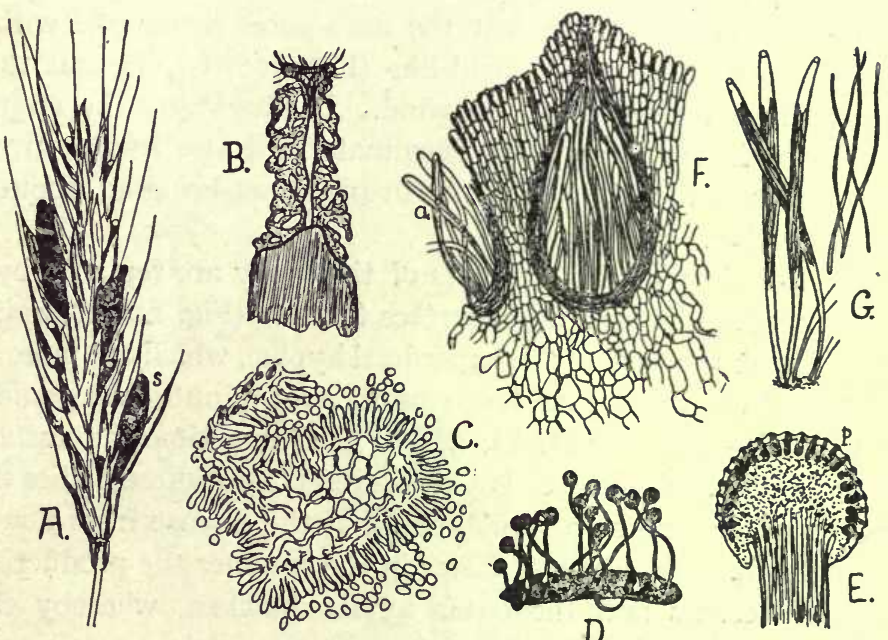

FIG. I28.-Ergot of Rye (Claviceps purpurea). A, Head of Rye, with a number of black sclerotia (s.). B, Longitudinal section of gynæcium of Rye-flower, showing the dense hyphæ forming the sclerotium in the lower part, and the looser mass of hyphæ producing gonidia in the upper. C, A small part of the latter in section, highly magnified, showing the budding off of gonidia. D, Germinating sclerotium. $E$, Vertical section through one of the swellings arising from the latter, showing numerous perithecia ( $p$.$) . F, Part of same, highly$ magnified, to show perithecia with asci $(a$.$) . G, Three asci and (on$ the right) four of the thread-like ascospores. (A and D after Wettstein ; the remainder after Tulasne.)

woven hyphæ (Fig. I28, A, s.). This constitutes a resting-stage of the Fungus, and is so hard that the term sclerotium ${ }^{1}$ is applied to it. In transverse section all the hyphæ, and especially those at the periphery, are seen to have very thick walls, whilst the

1 Similar sclerotia occur, as resting-stages, in the life-cycle of several other Ascomycetes, e.g. Sclerotinia (Fig. I26, E), a close ally of Peziza, whose cup-shaped apothecia arise from the sclerotia. 
more central ones, forming the lighter-coloured region, are laden with food-reserves. The sclerotia drop off in the autumn and remain dormant in the soil until the following spring. Then they send up one or more stalked swellings (Fig. I28, D), in which are embedded numerous flask-shaped cavities (perithecia, Fig. I28, E, p.) communicating with the exterior by small pores (Fig. I28, F). Each perithecium is lined with a hymenium similar to that of Peziza, but the ascospores developed within the asci are in this case thread-like (Fig. I28, G), so that they are readily distributed by the wind. If caught by the stigma of a Grass-flower the spores germinate and the hypha grows down through the style into the ovary, thus bringing about a fresh infection.

By slow degrees the contents of the ovary are replaced by a dense hyphal mass with deep surface furrows (Fig. I28, B, upper part). From the ends of the superficial hyphæ, which are more or less parallel to one another, large numbers of minute oval gonidia are budded off (Fig. I28, C), and at the same time the surface secretes a sugary liquid. This attracts insects, to whose bodies the gonidia adhere, and so a rapid spread of the disease from flower to flower is brought about. Later in the summer the production of gonidia ceases and the outer hyphæ blacken, whereby the resting sclerotium is formed.

The bright red pustules of Nectria and the branched sclerotia of the Stag's Horn Fungus (Fig. I26, A) harbour flask-shaped perithecia similar to those of the Ergot.

In some Ascomycetes reproduction by gonidia is far more frequent than the formation of asci, as, for instance, in the two common Moulds Penicillium (Fig. I29, $a, b$ ) and Eurotium (Aspergillus, Fig. I29, $f$ ). Here the gonidia are budded off in chains from the terminal branchlets of erect hyphæ which, in the case of Eurotium (Fig. I29, f), are strongly swollen at their apices. Both Fungi also occasionally produce spherical ascusfruits (Fig. I29, d), which arise from special sexual organs (Fig. I29, c), although it is doubtful whether any actual fusion of cell-contents occurs.

In the White Mildews (Erysiphacea) ${ }^{1}$ formation of gonidia

1 The Mildews are the cause of many familiar diseases of cultivated plants, as instances of which may be mentioned the Gooseberry Milde w 
is again relatively common. The mycelium in these parasites develops externally, on the surface of the leaf (Fig. I30, b), the haustoria alone penetrating into the epidermal cells. The mildewed appearance is usually due to the extensive production of chains of gonidia from the ends of unbranched upright hyphæ (Fig. I30, c), such chains being very striking in the Mildew
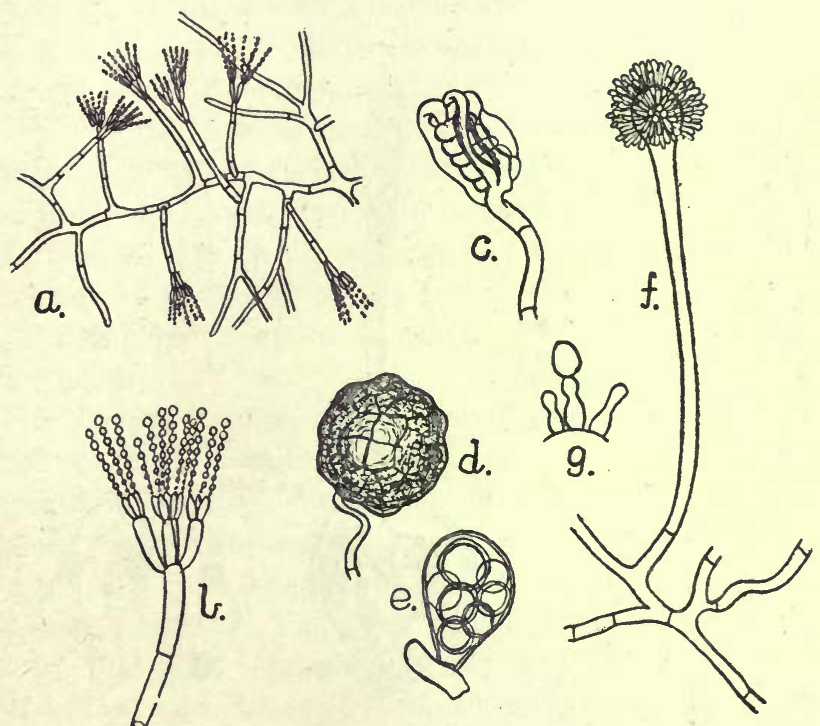

FIG. I29.- $a-b$, Penicillium. $a$, small part of mycelium with gonidiophores ; $b$, one of the latter enlarged. c-g, Eurotium (Aspergillus). $c$, very early stage of fruit-formation, showing the coiled hypha (female organ) from which the asci arise ; $d$, mature fruit; $e$, an ascus from the interior of the same; $f$, gonidiophore; $g$, small part of apex of same, showing the way in which the gonidia are budded off. ( $a$ and $b$ after Brefeld ; the remainder after De Bary.)

commonly found on Forget-me-not leaves (due to a species of Oidium). Later in the season many of these forms develop

(Sphaerotheca mors-uva), the Rose Mildew (S. pannosa), Erysiphe polygoni (on Field Peas and Cucumber), and E. graminis (on Wheat). Many. so-called Mildews do not, however, belong to the Ascomycetes, but are Phycomycetes, whose richly branched gonidial-bearing hyphæ give a. whitish appearance to the leaves; such are the Cabbage Mildew (Peronospora parasitica) and Grape Mildew (Plasmopora viticola). 
numerous small dark specks (Fig. I30, a), the ascus-fruits, on the greyish-white mycelium. Under the microscope they are seen to be almost spherical structures, provided with very diverse hair-like appendages and without an aperture (Fig. I30, b.). The hard black wall ruptures irregularly, exposing one or more small asci (Fig. I30, e).

In the Hop Mildew (Sphaerotheca castagnei) and one or two

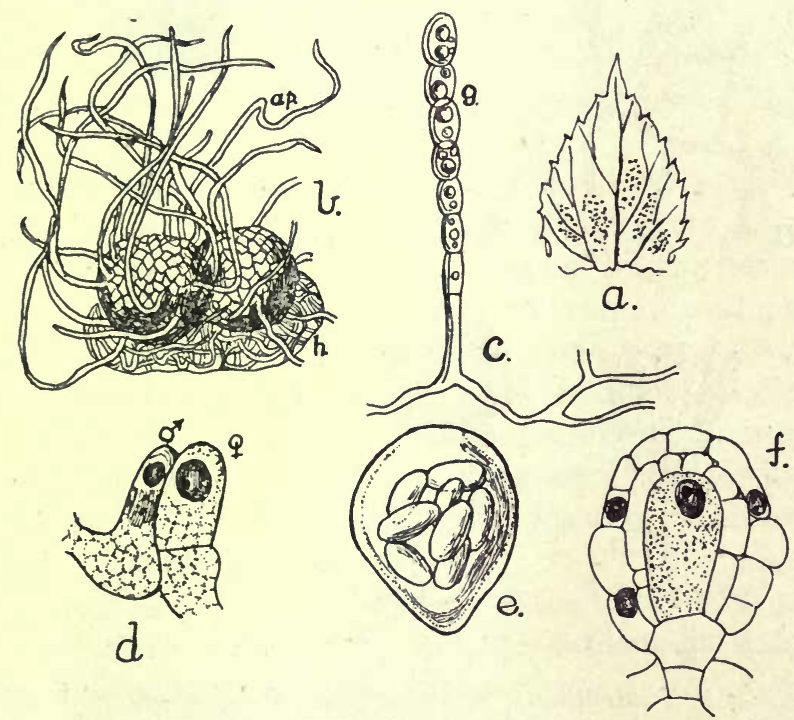

Fig. I30.-The Hop Mildew (Sphaerotheca castagnei). a, part of the leaf of the Hop, with the ascus-fruits ; $b$, small part of the surface, greatly magnified, showing the superficial hyphæ $(h$.$) and two ascus-fruits,$ each with numerous long appendages (ap.) ; $c$, production of a chain of gonidia $(g) ;$.$d , sex organs in apposition ; e$, ascus ; $f$, young fruit. ( $a$ after Wettstein ; $b, c$, and $e$ after Tulasne ; $d$ and $f$ after Harper.)

other cases it has been established that the fruit arises from club-shaped sexual organs of unequal size, the larger functioning as the female (Fig. I30, d). Their tips become closely adpressed, but, owing to the great difficulty of establishing such facts; there exists a marked difference of opinion as to whether or not there is a fusion analogous to that above described for the Phycomycetes. The same doubt attaches to all cases of sexual fusion 
that have been investigated in the Ascomycetes. Since, however, a production of sexual organs has been observed, in one case or another, prior to the formation of all the different kinds of ascus-fruits encountered in this group, the view is generally held that its members are to be regarded as being descended from Fungi which exhibited a sexual process, now functionless, at least in the vast majority of cases. In fact, in most Ascomycetes no traces of sexual organs are to be found.

The young asci in all cases arise from binucleate cells, the first step in their development consisting in the fusion of the two nuclei, and this is regarded by many as giving the same stimulus as a sexual fusion. The single nucleus thus produced undergoes successive division into eight, whereupon membranes are formed independently around each nucleus and the adjacent cytoplasm, so that eight ascospores are cut out ; a small portion of the cytoplasm remains, however, which is not incorporated in the latter.

The Basidiomycetes, which are likewise characterised by a special mode of spore-formation in which a definite number (4) of spores is usually constricted off from the mother-cell, are altogether devoid of sexual organs. The group includes many diverse Fungi, mostly saprophytes, familiar examples being the Mushroom (Agaricus, Fig. I32), various Toadstools (e.g. Coprinus, Boletus), Puff-balls (Lycoperdon, Fig. I34, C), etc. The Smuts (Ustilaginea) and Rusts (Uredinea) are usually also included in the Basidiomycetes, but exhibit many peculiar features of their own.

The Rusts are of special importance as being the cause of many serious diseases of crops, and of these the Rust of Wheat (Puccinia graminis) is, unfortunately, all too common. Like many other members of the group, it possesses a very complicated life-history, whose phases, in this species, occur on two different hosts. In summer the parasite attacks the leaves and stems of various Grasses, and betrays its presence by the development of orange-coloured streaks upon them. These are due to clusters of unicellular thick-walled spores (summer- or uredo-spores) of an orange colour and beset with numerous minute spines; each spore arises at the end of a projecting hypha (Fig. I3I, $b, u$.). After detachment they may be blown by the wind on to another 
appropriate Grass, whereupon hyphæ grow out through special thin areas of the wall (Fig. I3I, f), to start a fresh generation of the Rust. In this way the disease rapidly spreads during the summer months.

In rare cases the Fungus may persist through the winter by
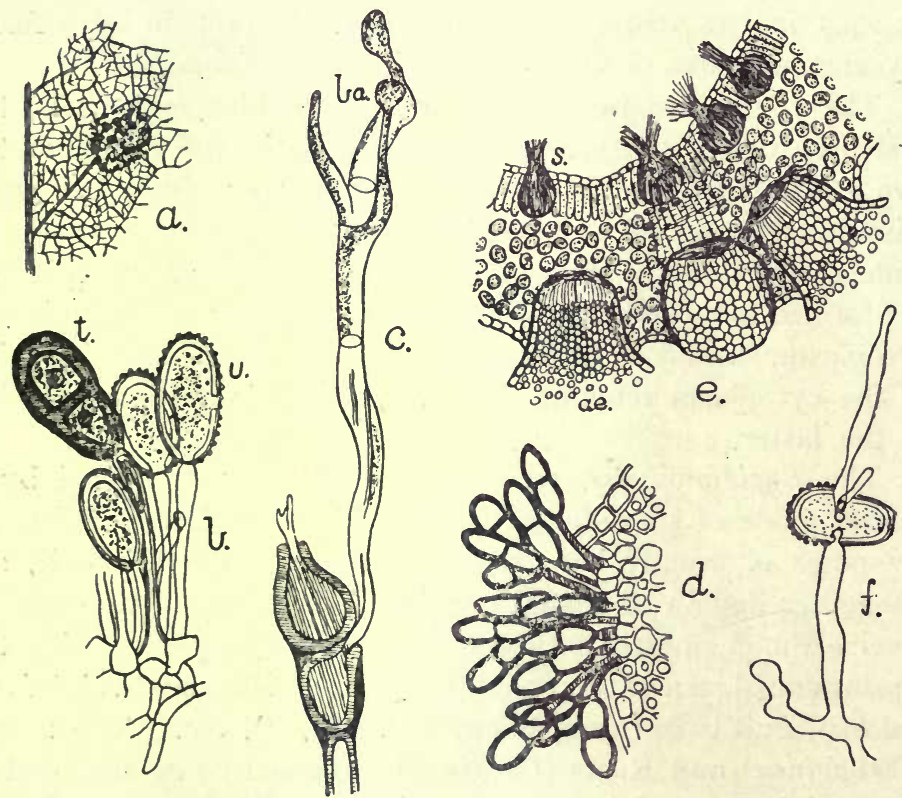

FIG. 131.-Rust of Wheat (Puccinia graminis). a, small part of leaf of Barberry, showing a group of æcidia; $b$, group of uredospores $(u$.) and one, teleutospore $(t)$; $c$, germinating teleutospore (only the hypha growing from one of the two cells is shown at its full length), with developing basidiospores $(b a) ;$.$d , group of teleutospores, seen in$ section of leaf of Wheat ; $e$, section of Barberry-leaf, showing clustercups or æcidia (ae.) in two stages of development, and spermogonia (s.) ; $f$, germinating uredospore. ( $b, d$, and $f$ after De Bary ; $c$ after Tulasne; $e$ after Sachs.)

means of these spores, and possibly also as a parasite on wild Grasses. As a general rule, however, special winter- or teleutospores are produced towards autumn, and these remain dormant in the soil until the following spring. The formation of teleutospores is evidenced by a darkening of the spots on the Grassleaves. Microscopic examination shows that they are now caused 
by clusters of bicellular spores (Fig. I3I, $d$ ), again borne singly at the ends of projecting hyphæ, and provided with a thick dark brown membrane (Fig. I3I, b,t.) which has a thin germinal pore in each cell.

With the advent of spring both cells of the teleutospore put out a short hypha composed of four cells (Fig. I3I, c), each of which gives rise to a short process bearing a small spherical gonidium (basidiospore, ba.). The latter is only capable of further development if carried by the wind to a plant of the Wild Barberry (Berberis vulgaris). In that case a mycelium is produced within the new host, and the presence of the disease is soon manifested by the appearance, usually on the under-surface of the leaf, of groups of small orange-coloured cups (the clustercups or acidia, Fig. I3I, a). The minute specks, recognisable on the upper surface of the leaf, are caused by small flask-shaped cavities (spermogonia, Fig. I3I, e, s.) containing reproductive cells of unknown function.

In a vertical section (Fig. I3I, e) the hyphæ of the æcidium (ae.) are seen to form a compact bounding wall and a dense interwoven mass at the base, whilst occupying the floor of the cup is a palisade-like hymenial layer whose vertical hyphæ bud off rows of orange-coloured acidiospores. The latter, if carried by the wind to Wheat or other Grasses, give rise to a new uredo-form.

Whilst the cells of the ordinary mycelium in the Barberry-leaf are uninucleate, they become binucleate in the young æcidium, and the resulting spores are similarly provided with two nuclei. The binucleate condition persists throughout all the uredo-forms, and the cells of the young teleutospores show the same feature, but fusion of the nuclei occurs as the latter mature.

The existence of the Fungus on different host-plants, at different stages of its life-cycle, is paralleled among animal parasites (e.g. Malarial Parasite, Tapeworm), and affords one means for the extermination of the disease, viz. by the eradication of one host. This is, however, only partially successful, since Wheat Rust occasionally appears in successive years, even where the Barberry does not grow (e.g. Australia), which is probably due to the persistence of uredospores through the winter. In some Rusts there is no uredospore phase (e.g. Puccinia anemones), 


\section{SMUTS (USTILAGINEÆ)-MUSHROOM (AGARICUS)}

whilst in others (e.g. Puccinia malvacearum) only teleutospores are known. ${ }^{1}$

Some of the Rusts exhibit very extreme specialisation in relation to definite host-plants, possessing biologic strains which can only develop on one particular species. Others, however, can attack a variety of related hosts, and, in the continuance of a disease of cultivated crops, wild plants may often play an important part in bridging the interval of a rotation.

The Smuts (Ustilaginea) are characterised by the sooty black mass of spores which are formed by the breaking up of the hyphæ into unicellular chlamydospores (cf. p. 238) with a thick pigmented wall. They occur extensively as diseases of various Cereals (e.g. Oats, Wheat, Maize), infesting either the leaves (Fig. I34, G) or the ovaries.

The common Mushroom (Agaricus campestris) affords a typical example of the Basidiomycetes proper. The mycelium, which inhabits soil rich in humus, and is present in considerable amount in so-called Mushroom spawn, is composed of binucleate cells. The hyphæ, as in many other Basidiomycetes, tend to be interwoven in bundles, so that the mycelium appears thicker and coarser than in other Fungi. The overground edible portion is the reproductive body which first appears on the mycelium as a knob-like swelling (Fig. I32, b) composed of densely interwoven hyphæ, but later, as it gradually enlarges, broadens out at the top (Fig. I32, $c, a$ ). In the mature condition it consists of a stalk and an umbrella-shaped cap (cf. also Figs. I37, I38), with a large number of radiating plates or gills, which bear the hymenium, protruding from the under surface. A little way below the cap the stalk is surrounded by a membranous ring of broken tissue (the annulus, Fig. 138) which, before the expansion of the cap, extended continuously from the edge of the latter to the stalk, thus constituting a protection for the developing gills (Fig. I32,a).

In a vertical section through the cap (Fig. I32, $a, e$ ) the middle of each gill $(d, f)$ is seen to consist of longitudinally arranged hyphæ. These are composed of rather large cells $(t$.), and diverge

1 Other well-known and widespread Rusts are those occurring on the Hollyhock (Puccinia malvacearum), on the Wood Anemone (P.anemones), and that causing purple spots on the leaves of the Blackberry (Phragmidium bulbosum). 
at their ends to form the superficial palisade-like hymenium (h.) and a round-celled subhymenium (s.). The former comprises two kinds of club-shaped hyphal terminations: some, the basidia (ba.), bear at their apex two, or four, short processes, from the end of each of which a basidiospore is formed, whilst the others are purely sterile, and probably play a part in the
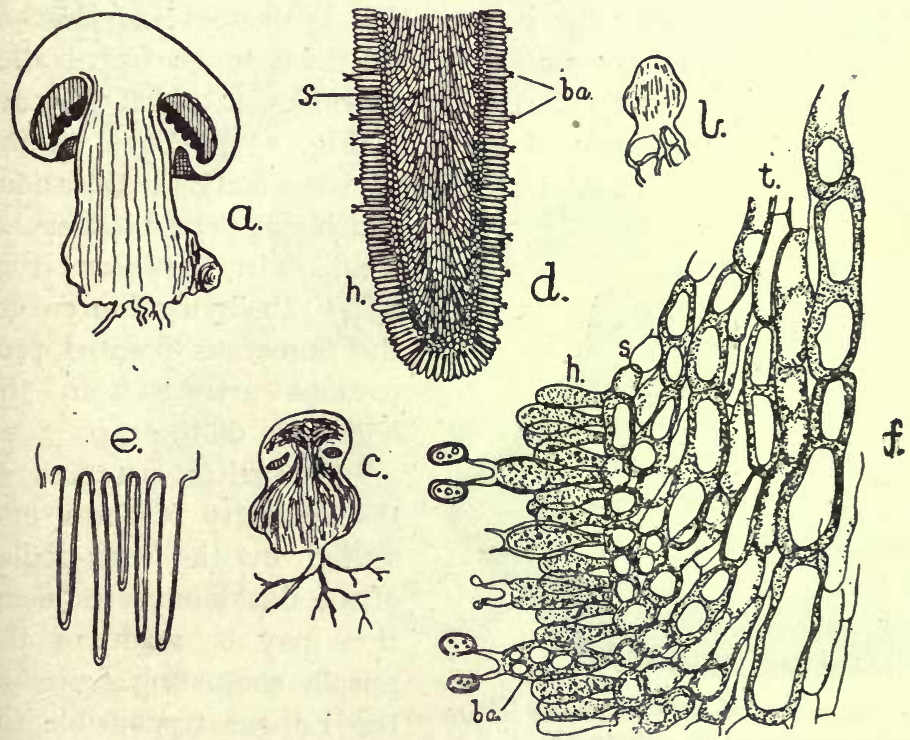

Fig. 132.-The common Mushroom (Agaricus campestris). $b,{ }^{\circ} c$, and $a$, successive stages in the development of the fructification (in $a$ the annulus is distinct, but yet unruptured) ; $e$, transverse section through small part of cap, showing gills ; $d$, one of the latter enlarged ; $f$, surface of a gill, in section, highly magnified. ba., basidium ; $h$., hymenium ; s., subhymenium ; $t$., large cells of middle of gill. (After Sachs.)

detachment of the spores. This method of spore-formation is that characteristic of Basidiomycetes generally, four being the usual number produced. The colour of the spores varies considerably in different forms.

The enormous production of spores in this and other similar Fungi can be gauged by placing the mature caps, with the gills downwards, on a sheet of white paper, when, after a short time, the outline of each gill will be marked by the spores which have 
been shed. It has been estimated that a moderate-sized specimen will produce some I,800,000,000 spores, and other allied species form spores in even greater profusion!

In the genus Boletus, whose fruit-body has the same general form as that of the Mushroom, the underside of the cap presents the structure of a honeycomb, consisting of a multitude of vertical tubes, the inner surfaces of which are lined with hymenium (Fig. I34, E). A similar construction is seen in the Bracket Fungus (Polyporus squamosus), whose thick tough fruit-bodies are commonly found on decaying tree-trunks, to which they are attached along one side of the cap (Fig. I33). Some of the related Fungi (e.g. Dadalea quercina) have woody fructifications

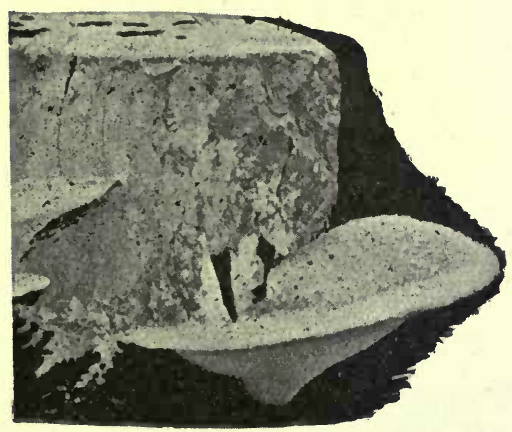

Fig. 133.-The Bracket Fungus (Polyporus squamosus). [Photo: E. J. S.] which may persist for several years. In Hydnum (Fig. I34, F) the hymenium covers the numerous pointed projections arising from the underside of the cap.

As further instances of the diversity of form presented by the fruit-bodies of the Basidiomycetes, mention may be made of the purple encrusting fruits of the Fungus responsible for the Silver-leaf disease of the Plum (Stereum purpureum), of the Puff-balls (Lycoperdon, Fig. I34, C), whose spores form a powdery mass within the pearshaped fructification, and of the Coral Fungus (Clavaria, Fig. I34, B), where the fruit-body is richly branched and bears the hymenium over its entire surface.

In conclusion, a brief reference must be made to the Slime Fungi (Myxomycetes), whose relation to other Thallophyta is exceedingly obscure; in some respects they show decided resemblances to Protozoa, although the methods of multiplication recall those habitual among lower plants. The Slime Fungi are most evident in damp weather, when the large naked protoplasmic masses (plasmodia), constituting the vegetative phase, creep out from the crevices of the decaying tree stumps, humus, or other 
substratum. Small, often rounded sporangia, containing numerous spores, are formed, especially in the autumn, and are sometimes very conspicuous owing to their brilliant colouration (e.g. the yellow-coloured Flowers of Tan, common on tanner's

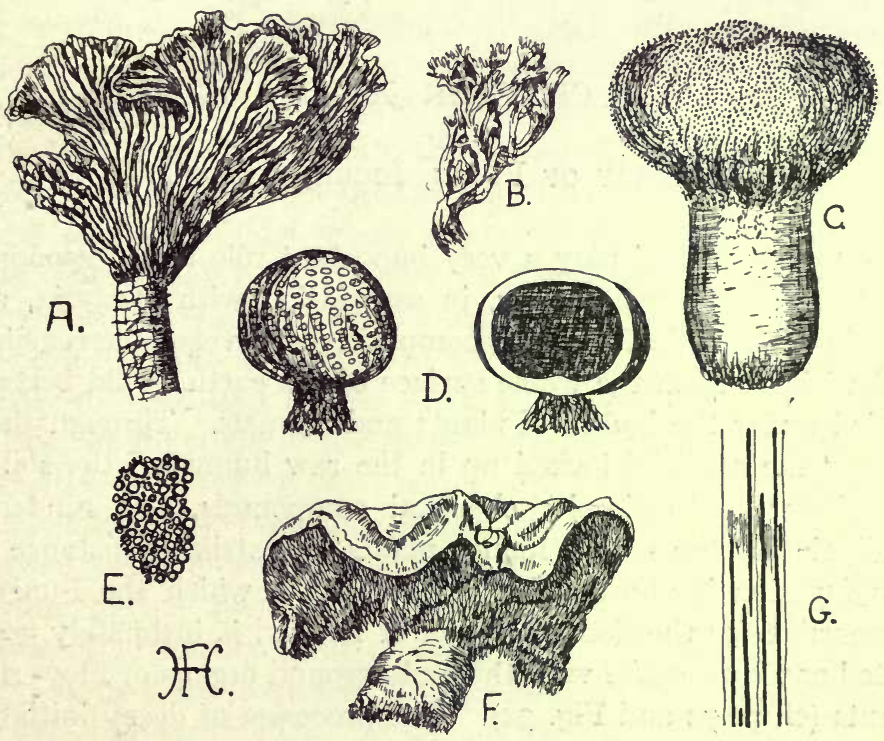

FIg. I34.-Various Basidiomycetes. A, Cantharellus. B, Clavaria cinerea. C, Lycoperdon. D, Scleroderma vulgare (on the left entire fructification, on the right the latter in vertical section, showing the wall and the dark mass of contained spores). E, Surface-view of small part of hymenium of Boletus. F, Hydnum repandum. G, Ustilago longissima (on leaf of Glyceria aquatica).

bark). The group also includes some parasites, one of the most noteworthy (Plasmodiophora brassica) being that responsible for the disease "known as "Finger and Toe" in Cabbages, etc. (Fig. I35). 


\section{CHAPTER XVIII}

\section{Physiology of Fungi, Lichens, Bacteria}

MANY of the Fungi play a very important rôle in the economy of nature. The saprophytes, in association with Bacteria, are largely responsible for the decomposition of vegetable remains, and without them the whole surface of the earth would become buried under the bodies of plants and animals. Through their agency the material locked up in the raw humus of the soil is transformed into simpler chemical compounds, and rendered available for the use of higher plants. A striking instance of this function is afforded by those cases in which the Fungus, responsible for the decomposition of humus, is intimately associated as a mycorrhiza with the underground organs of Flowering Plants (cf. p. 72 and Fig. 3I). The processes of decay initiated by saprophytes are, however, often detrimental to the interests of man, as in the case of the Basidiomycete (Merulius lacrymans) responsible for so-called "dry rot" of timber, and the diverse and almost ubiquitous Moulds which all too readily develop upon articles of food.

The parasitic species frequently do great damage to cultivated plants and to animals. In some cases the parasite sooner or later brings about the death of its host (e.g. Silver-leaf Disease of Plum), but more commonly (e.g. Mildew, Rust) the diseased plant, though injured, continues to live as an unhealthy individual and to maintain the parasite which grows at its expense. A considerable number of parasites can also exist as saprophytes, so that they remain alive after the host has died (e.g. Pythium and many Smuts). Conversely, certain saprophytes (e.g. Mucor) can occasionally act as parasites, generally after access has been obtained at a point of injury. The so-called wound-parasites 
(e.g. Nectria, a species of which causes Coral-spot Disease of various trees) belong to this category, though their saprophytic phase is of short duration. The majority of Fungi, however, are either strictly saprophytic or strictly parasitic.

In many cases special conditions, such as excess of moisture (e.g. Pythium), the general state of health of the host, or accidental injuries to the latter, may be instrumental in bringing about the attacks of parasitic Fungi. Epidemics of such widespread diseases as the Potato Blight and the Gooseberry Mildew have, for instance, often been associated with particularly damp warm seasons. Fungi proper are rarely the cause of disease in man, but it may be mentioned that various skin diseases (e.g. Ringworm, Favus) are due to Fungi.

Owing to the small size of the spores, Fungi often become widely disseminated by the wind, but so far as can be gauged by the careful study of the spread of plant diseases due to these parasites, infection by wind-borne spores seldom occurs beyond a few miles. Long-distance carriage is generally to be attributed to transport in infected plants or plant-fragments, through human or other agency, hence the value of careful inspection and control of imported horticultural produce. It is owing to such introduction that the parasitic Fungus-flora of Botanic Gardens is so extraordinarily rich.

In dealing with the Rust Fungi, mention was made of the fact that a particular species or strain of these parasites may be so specialised as to be able to attack only one particular kind of host, and the same is true of the Mildews. There is thus often a difference, with regard to susceptibility to a certain disease, between the various races of a cultivated plant; for instance, some varieties of Potato and Wheat are immune to Blight and Rust respectively, and would tend to be grown in regions in which these Fungi were known to be prevalent. Much has also been done by the production of immune hybrids (cf. p. 384) between immune and non-immune races. The ravages of a disease may decrease in intensity after it has been rampant for some years, the host presumably becoming adapted to the presence of the parasite; thus the Hollyhock Rust (Puccinia malvacearum), when first introduced into Europe about 1870 , played great havoc with its host, but now, though Hollyhocks 
are still commonly attacked, they do not appear to suffer appreciably.

Artificial control of fungal diseases is accomplished by diverse means, but in each case success depends upon a knowledge of the life-history of the parasite. The method of treatment varies according as the parasite attacks the overground or underground organs of the host. Several of the Fungi considered in the last chapter (e.g. Potato Blight)

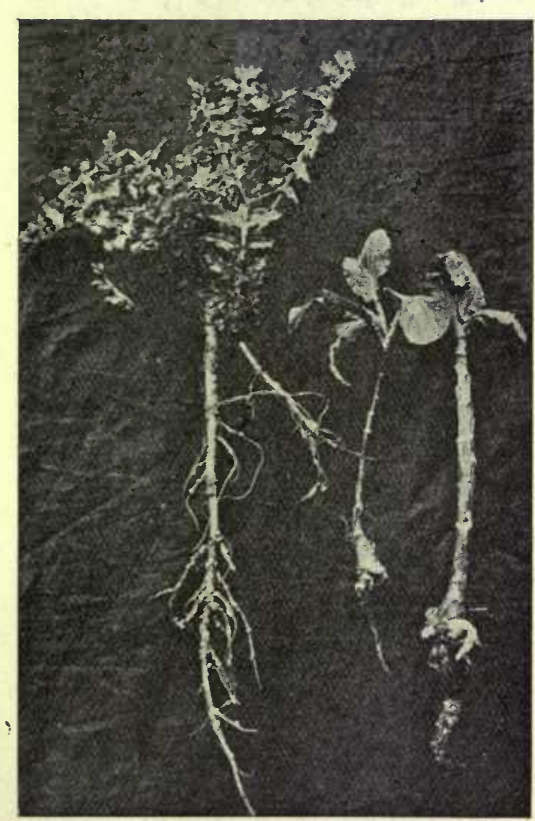

FIG. I35--Brussels Sprouts attacked by "Finger and Toe" (Plasmodiophora brassica.) [Photo: E. J. S.] afford instances of the former mode of attack. Diseases of the subterranean parts are exemplified by the pernicious Wart Disease of the Potato, due to a lowly Fungus of a peculiar kind, and that known as Finger and Toe (Fig. I35 and p. 25I), which attacks Turnips, Cabbages, etc., causing irregular swellings upon the rootsystem.

One of the remedies most commonly employed against Fungi infesting the overground parts is spraying with a fungicide which, while deleterious to the parasite, leaves the host practically unharmed. For this purpose Bordeaux mixture; consisting of a solution of copper sulphate and slaked lime, is one of the most popular. Where the disease is subterranean, application of unslaked lime is often successful, as, for instance, in the case of Finger and Toe. Such diseases are, however, far more difficult to eradicate than those which develop overground. They are often best counteracted by growing only such crops, in the infected soil, for several seasons in succession, as are not attacked by the parasite in question. 
PLATE II
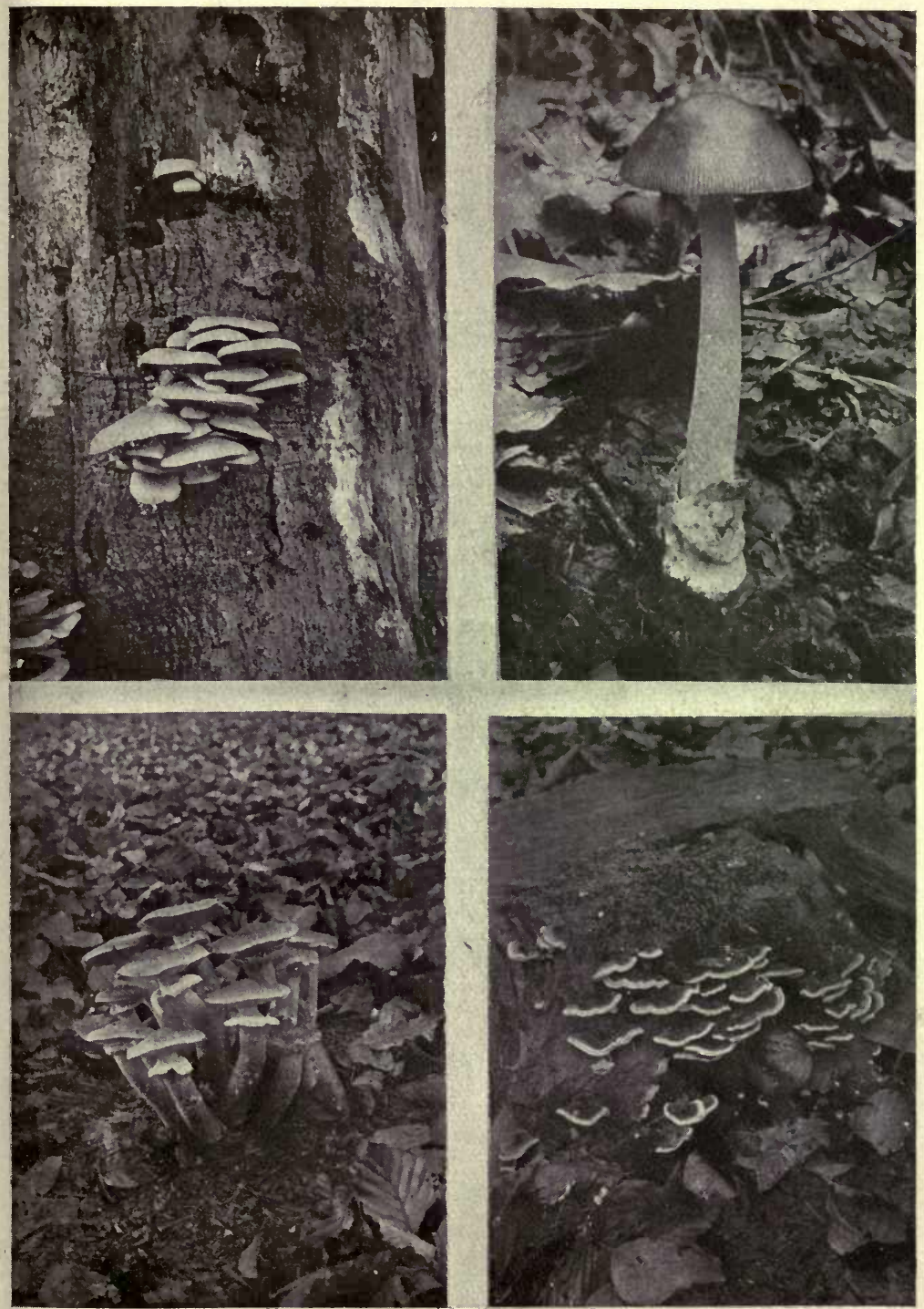

FIG. I36.-Pleurotus ostrextus.

FIG. I38.-Armillaria mellea.

FIG. 137.-A manitopsis vaginata. FIG. 139.-Polystictis versicolor. [All after photos by Mr. E. M. Cutting, M.A.] 

In many cases, unfortunately, no adequate remedy has yet been discovered, and the only advisable procedure, in the event of an outbreak of disease, is to burn all infected plants, so as to prevent the spread of the parasite.

Fungi are, however, not only of importance in causing decay and disease, but also afford several greatly prized articles of diet (e.g. Mushrooms, Truffles, Morels, etc.), although their actual food-value is probably small. The great majority of the British Basidiomycetes are innocuous, but there are a certain number of species, some very widely distributed, which harbour deadly poisons (alkaloids, etc.), and attention may be drawn to the fact that such Fungi are by no means always highly coloured (Fig. I37). As examples of poisonous forms we may mention the Fly Toadstool (Amanita muscaria) and the Death Cap (Amanita phalloides). The sclerotia of an Australian species of Polyporus, which may attain the size of a football, are eaten by the natives. An edible Fungus of another kind is seen in the so-called Vegetable Caterpillar (Cordyceps), where a dense mass of hyphæ or sclerotium completely replaces the internal organs of the animal; this parasite is extensively cultivated in parts of the East and used as a condiment. Fungi are not often employed in medicine, except for the powdered sclerotium of the Ergot (Claviceps) which contains a nitrogen base having the property of causing muscular contraction.

The production of most alcoholic beverages is due to the activity of Yeasts (Saccharomyces), whose exact relationship to other Fungi is not quite clear. The Yeast-plant (Fig. I40) consists of oval cells, which are either isolated $(a)$ or adhere together in short chains $(c)$, each the product of a peculiar method of division of a single individual. The thin-walled cells contain a large central vacuole (Fig. I $40, e, v a$.), and, in contact with the latter at one point, a nucleolus with surrounding chromatin (n.), which become apparent on staining the living cells with a dilute aqueous solution of methylene blue; vacuole, nucleolus, etc., together probably represent the nucleus. The Yeast-cells often contain large glycogen-vacuoles $(g$.$) , as well as small bodies (v.),$ stained deeply by methylene blue, and known as volutin-granules, which appear to constitute another kind of reserve.

When a cell has reached a certain size, it gives rise to a small 
outgrowth (Fig. I40, b) which slowly enlarges and assumes the form of the parent, from which it becomes separated by gradual constriction; if this process of budding takes place rapidly, the cells do not immediately separate, and thus the chains (Fig. I40, c) above mentioned are formed. A stage, capable of a prolonged resting period, can also be obtained, for instance by growing Yeast on the surface of a raw Potato; under these circumstances the cell-contents undergo division into four, and each part becomes surrounded by a thick wall (Fig. I40, $d$ ).

There are a number of different species of Yeast which
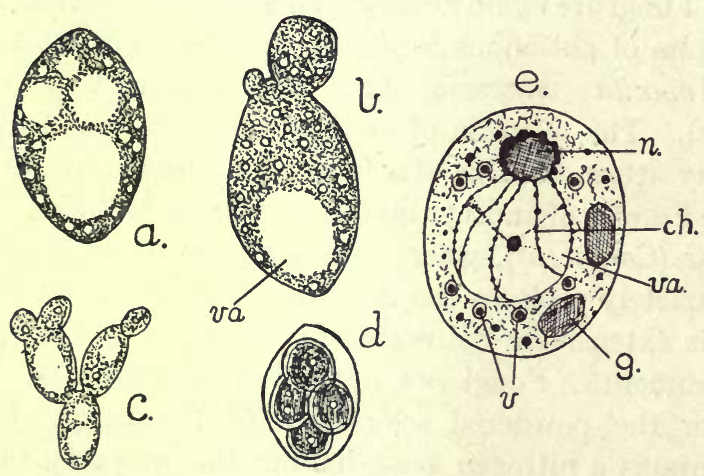

FIG. I40.-Yeast (Saccharomyces). $a-c$, various individuals, showing general form and multiplication by bud'ding ; $d$, individual containing resting spores ; $e$., cell-structure. ch., chromatin threads ; g., glycogen vacuole; $n$., nucleolus with surrounding chromatin; $v$., volutin granules ; va., nuclear vacuole. ( $a-d$ after Wettstein; $e$ after Wager.)

ferment various sugars and split them up into alcohols (mainly ethyl alcohol) and carbon dioxide. The chemical change is brought about by an enzyme zymase (cf. pp. 53, 55) which can, with some difficulty, be extracted from the cells; in addition the latter contain invertase and other ferments. The mode of action of zymase is complicated and not yet fully understood, but it is known that fermentation depends on the contemporary presence of phosphates (cf. p. 57). The alcohol present in beer, wine, etc., is formed by the action of Yeasts, while the carbon dioxide simultaneously evolved is compressed into cylinders and sold as a by-product. In the manufacture of beer, malt (i.e. germi- 
nated Barley) is treated with hot water, and supplies part of the sugar, various other sugars being used according to local practice. Hops are added to the liquid to provide the peculiar flavour, and the whole of this wort is then fermented in vats. ${ }^{1}$ The success of brewing depends upon the employment of pure races of Yeast and the realisation of the right temperatures at the different stages of the process; a small supply of oxygen has also been shown to be favourable to active fermentation.

In the fermentation of sugars a considerable quantity of energy is liberated, and herein possibly lies the advantage to the Yeast-plant. It appears likely that some of this energy is utilised in synthesis. The process of alcoholic fermentation shows much resemblance to anaerobic respiration, but there is this difference, that the compounds broken down are outside and not within the organism, so that no loss of weight is involved. It may be added that Yeasts are not alone among Fungi in fermenting sugars, since spores of Mucor, for example, if placed in a sugary liquid, will reproduce by budding and cause alcoholic fermentation, forming so-called Mucor-yeast.

We have already noted that a parasitic Fungus may sometimes do very little harm to the host, and such cases are but a step removed from those in which the balance, between the Fungus and the organism with which it is associated, is so perfect that both are mutually benefited. Such a condition is found in the Lichens, whose body is composed of algal cells embedded in a weft of fungal hyphæ. Lichens, with but few exceptions, are subaerial, being commonly found on peaty soils, rocks, treetrunks, old walls, etc. In conjunction with various Algæ (commonly Cyanophyceæ) and Mosses, Lichens play an exceedingly important part in the primary colonisation of rock surfaces and of other ground laid bare of vegetation, being conspicuous, for instance, among the early vegetation of burnt heaths. The blackcoloured blotches, which are such a familiar sight on the pebbles

1 If all the starch in the malt is allowed to undergo conversion into sugar, and the fermented product is distilled, whisky results ; brandy is similarly obtained by distilling the alcohol from fermented grape-juice. The Japanese sake is obtained by the fermentation of rice. 
of a shingle-beach, are due to various Lichens (e.g. Rhizocarpon confervoides, Fig. I4I, C), which are here the first colonisers.

The shape of the thallus is very diverse, and, as a general rule, almost entirely determined by the Fungus. It most commonly takes the form of flat, lobed expansions which are often

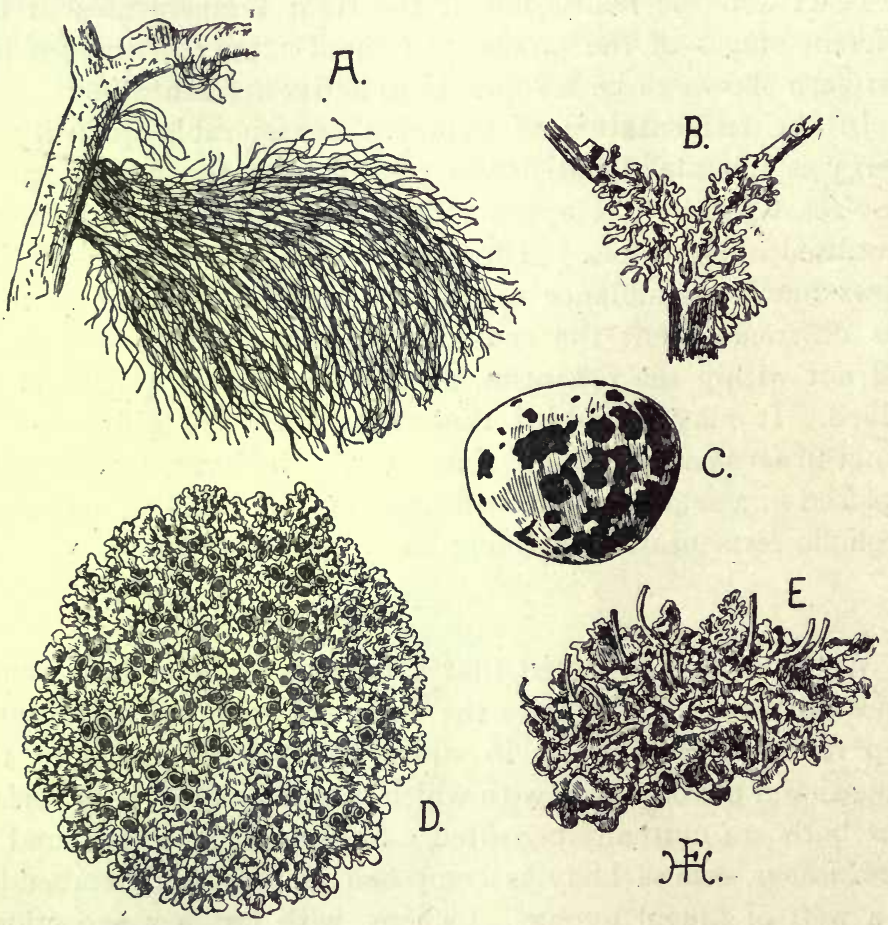

FIg. I4I.-Various common Lichens. A, Usnea barbata. B, Parmelia physodes, on twig. C, Rhizocarpon confervoides, on pebble from shinglebeach at Pevensey. D, Xanthoria parietina (showing numerous apothecia). E, Cladonia sp., showing the upgrowths on which the apothecia are borne.

almost circular (e.g. Parmelia, Xanthoria, Fig. I4I, D). Other frequent types are those exhibiting repeated branching (Fig. I4I, B) ; in such the segments may be upright (e.g. Iceland Moss, Cetraria islandica, Fig. I43) or hanging (e.g. Old Man's Beard, Usnea, Fig. I4I, A). In some cases the thallus is highly gelatinous, so that it is much more conspicuous in wet than in dry weather (e g Collema). 
The Algæ found within the thalli of Lichens are forms which can also grow independently in the terrestrial habitats in which the latter occur; examples are afforded by the unicellular green Cystococcus (found in the Lichen Cladonia) and the filamentous blue-green Nostoc (found in Peltigera). The Fungi concerned are, on the other hand, unable to lead an independent existence in nature, and are only capable of growth when associated with their appropriate Alga. A single species of Alga may be coupled with several different Fungi to form as many distinct Lichens, but each Fungus has only one algal associate. It has even proved possible to produce a Lichen artificially by sowing the spores of its fungal constituent among the appropriate algal cells. The vast majority of Lichen Fungi belong to the Ascomycetes, as shown by their fructifications, which closely resemble small rather flattened Peziza-cups (Fig. I4I, D). In Cladonia (Fig. I4I, E), where the thallus itself is often inconspicuous, the apothecia are borne on the edges of variously shaped upgrowths.

Since the hyphæ at the two surfaces of the thallus are usually densely compacted and thick-walled (Fig. I42, C), the Alga (al.) embedded in the interior is well protected during times of drought. As a general rule, moreover, tufts of hyphæ project from the underside of the thallus into the substratum, absorbing moisture which is held there by capillarity, and thus the algal cells are kept supplied during dry periods. In the case of Lichens growing on rocks, the penetration of these hyphal tufts is facilitated by the secretion of solvents, and it is to this property that such Lichens owe their important rôle in the disintegration and colonisation of bare rock-surfaces.

Whilst the Alga thus obtains protection and a supply of moisture, the Fungus no doubt profits by absorbing part of the photosynthetic products of the former, with whose cells some of its hyphæ come into intimate contact (Fig. I42, B). In some cases, in fact, the hyphæ are stated actually to penetrate the algal cells, so that the partnership borders closely on parasitism. Despite this intimate relation, the enveloping hyphæ are usually loosely arranged, so that a well-marked system of intercellular spaces admits of the inward diffusion of carbon dioxide (Fig. I42, C, hy.). In transverse sections the algal cells are seen as patches 
scattered throughout the thallus (e.g. Collema) or occupying definite zones (e.g. Xanthoria parietina, Fig. I42, C).

The association between Alga and Fungus is paralleled by the so-called "green cells" found in several aquatic animals (e.g. freshwater Sponges, Hydra viridis) ; these are due to unicellular Algæ (species of Chlorella, etc.), and in all such cases the partnership between plant and animal appears to be of mutual benefit. In this connection it may be noted that various Algæ are found commonly inhabiting the in te rcellular spaces of certain higher plants (e.g. Duckweed, the Liverwort Anthoceros), but in these cases the Algæ appear to be nothing more than "space-parasites" seeking protection.

The ordinary cup-like fructifications of Lichens originate from the Fungus, and the resulting ascospores reproduce the latter only, so that germination of the spores must take place in the presence of the appropriate Alga, if a fresh Lichen is to be formed. But multiplication involving the dual organism also occurs; in this case algal cells surrounded by interlacing hyphæ become detached, and when, 
as usually, this takes place on a large scale, the surface of the thallus acquires a powdery appearance. The individual granules (so-called soredia, Fig. I42, A), being distributed by the wind, afford a rapid means of propagation of the Lichen.

The bright colours of many Lichens are due to the presence of pigments which are remarkable for their " fast " character, and have been employed in dyeing. The well-known chemical indicator litmus is obtained from species of Roccella. Iceland Moss (Cetraria islandica, Fig. I43), which forms a slimy fluid when boiled with water, is used as an invalid food.

The Bacteria constitute an extremely important group of Thallophyta whose relationships are very obscure. The majority of species are colourless unicellular plants of extremely small size. The larger specimens are not more than I/Iooth $\mathrm{mm}$. in length and about onetenth as wide, whilst

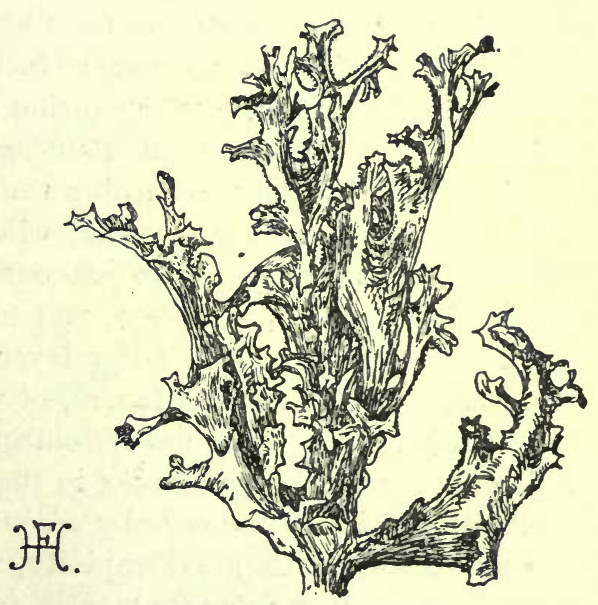

FIG. 143.-Iceland Moss (Cetraria islandica), part of a large specimen, about natural size.

the globular forms average I/Ioooth $\mathrm{mm}$. in diameter. Some are so small as to be almost, if not quite, invisible under the highest powers of the microscope. They occur in almost every possible situation, and live under the most varied conditions. They are not killed by cold, and some can survive for many months at the temperature of liquid air. Whilst most die if heated to about $50^{\circ} \mathrm{C}$., a few (thermophilic Bacteria) live in fermenting hay and manure heaps which, owing to their activity, may attain a temperature of $70^{\circ} \mathrm{C}^{1} \cdot$ Many Bacteria also inhabit the waters of hot springs.

1 Hence the employment of manure for hot-beds, etc, 
Bacteria are rod-shaped (Bacillus, Fig. I44, $d, f$, Bacterium), spherical (Micrococcus), or curved (Spirillum, Fig. I44, $k$ ). The rod-shaped individuals may be joined end to end to form long filaments (Fig. I44, d), whilst the Coccus-forms may occur in chains (Streptococcus, Fig. I44, b), in cubical packets (Sarcina), or in irregular masses (Staphylococcus, Fig. I44, a). The individuals may be capable of movement owing to the possession of cilia, which are only visible after careful staining, or they may be devoid of these, and merely exhibit Brownian movement in correspondence with their minute dimensions. In the elongated forms the cilia are either situated in tufts at the two ends (Spirillum, Fig. I44, $k$ ), or else arise all over the body (Bacillus, Fig. I44, $e, f$ ), but there are some genera possessing only one or two cilia restricted to one end of the cell (Pseudomonas, Fig. I44, l).

Very little is known regarding the internal structure of the cells, and it is a matter of doubt whether or not they possess a true nucleus; plastids are of course absent. The cell-wall appears to consist of a protein, and may probably be regarded merely as a differentiated outer layer of the cytoplasm. The latter contains various substances, of which the commonest are glycogen (cf. p. 23I), granules of volutin (p. 255), and fat-globules which appear as brighter specks in the cytoplasm.

Multiplication of the cells by division, under normal circumstances, ensues, with great rapidity, often taking place once every hour, or even more frequently. In some of the elongated forms the daughter-individuals are separated off by a mere constriction of the cell (e.g. Bacterium spp.), whilst in other cases the latter is subdivided by a transverse septum which subsequently splits down the middle. In certain Bacteria inhabiting water or other fluids, the daughter-individuals develop thick mucilaginous walls, and in consequence adhere together in vast numbers to form an often iridescent pellicle on the surface, or thick pale-coloured Palmella-like floating masses (zoogloea-stages).

Bacteria survive unfavourable conditions by the formation of resting spores, produced within the cells by a localised concentration of the greater part of the protoplasm, which then stains differently to the peripheral portion. This central region eventually becomes surrounded by a thick wall, and at maturity the remainder of the cell is often empty (Fig. I45, b, c). The 
spores are frequently located in special parts of the cell, giving the spore-forming individuals a characteristic appearance, as in Bacillus tetani, where they occupy a dilation of one end (Fig. I45, a). In certain forms (e.g. Bacillus amylobacter) more than one spore may be developed by each individual. Bacterial spores are often very resistant to extremes of temperature, and can indeed in some cases (e.g. Bacillus subtilis) withstand prolonged boiling.

Most sorts of Bacteria live a free life in water, earth, etc. Many exert a very beneficial action in promoting decay and main-

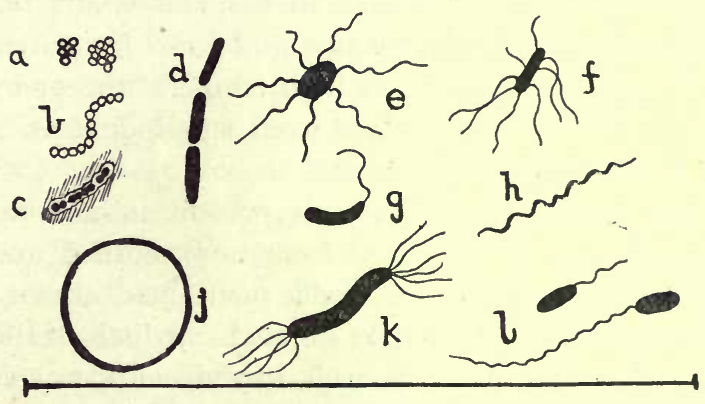

FIG. I44.-Various Bacteria (magnified about I, ooo times). a, Staphylococcus aureus ; $b$, Streptococcus pyogenes ; $c$, Pneumococcus ; $d$, Bacillus anthracis ; e, B.typhosus ; $f, B$. tetani ; g, Microspora comma; $h$, Spirillum of relapsing fever (possibly Protozoal); $k$, Spirillum rubrum; $l$, Pseudomonas spp. $j$ represents a blood corpuscle on the same scale, and the line below, the width of a fine human hair, or about half the thickness of a sheet of newspaper. $\quad(a-h$ from Muir and Ritchie ; $k-l$ after Engler and Prantl.)

taining the circulation of nitrogen and carbon dioxide in nature, whilst a small number are extremely harmful, living as parasites on higher animals and plants, and causing such diseases as typhoid, cholera, plague, etc. Whilst some, such as Bacillus subtilis, which is found in infusions of hay, etc., require a free supply of oxygen (aerobic species), others, such as the organism responsible for lock-jaw (Bacillus tetani), will grow only in the almost complete absence of oxygen (anaerobic species). Hence the value of oxidising agents (e.g. peroxide of hydrogen) for the clẹansing of woundș, 
It will be realised that the identification of the pathogenic forms (i.e. those causing disease), in particular, is of great importance. Owing, however, to the small dimensions of the individuals, it is as a rule impossible to identify the species by microscopic examination alone. Resource is therefore had to the various reactions of Bacteria under conditions of culture.

Bacteria can be grown in the laboratory on various artificial media, such as extract of meat, decoctions of fruit, etc. Under such conditions, and at temperatures suitable for each species, growth is very rapid, and a single organism soon multiplies to such an extent that the mass of its offspring is visible to the naked eye. In making cultures all the vessels and instruments employed are scrupulously cleaned and freed from live Bacteria (sterilised), either by heating at high temperatures or by washing with special disinfectants, since even small quantities of dust are replete with bacterial spores.

The different kinds of Bacteria present in any material to be examined can be separated from one another and isolated by using the various culture media mentioned above, with the addition of gelatine or agar-agar (p. 204). A little of the material containing the organisms is well mixed with a considerable quantity of such a medium, liquefied by warming, so that the individual bacilli are widely separated from one another. On allowing the mixture to cool the Bacteria are immobilised; they soon grow, and each of the widely separated individuals gives rise to a small group (colony), visible to the naked eye, which can be transferred to another lot of culture medium. In this way cultures can be obtained which have arisen from single organisms and consist of one species only (pure cultures); in them the form of the Bacteria, their mode of growth, the formation of pigment (usually outside the cells) and other chemical substances, can be studied and the species identified.

Some Bacteria are entirely parasitic (e.g. the Micrococcus which causes spotted fever) and cannot live apart from their host; whilst some are semi-parasitic (e.g. Bacillus tetani) and can live either in earth, etc., or in the body of a Mammal. Each parasitic species can generally use only one or two species of Mammal as hosts ; children, for example, do not have distemper, and dogs and cats do not have measles and chicken-pox. Simi- 
larly Bacillus carotovorus causes "soft rot" of Carrot, but does not attack Parsnips.

The evil effects of parasitic Bacteria are due to poisons (toxins) which they produce; these are proteins in nature, and those which have been isolated are the most poisonous chemical substances known. The infected animal resists the invading organism, partly by the amœboid cells of the blood (leucocytes) which devour them, and partly by producing chemical substances which neutralise the toxins. The power of the body to make a successful resistance is much improved by practice. Hence one attack of an infectious disease often enables a person to destroy that particular bacillus at once if it gets into the body a second time, and so another attack of the disease is avoided.

A large number of Bacteria obtain energy ${ }^{1}$ by bringing about processes of fermentation. Thus the Vinegar Bacteria (Bacterium aceticum, etc.) convert alcohol into acetic acid, ${ }^{2}$ whilst the lactic acid Bacteria cause the souring of milk, changing the milk sugar (lactose) into lactic acid. Another product of bacterial activity is butyric acid, which is the chief cause of the rancid character of bad butter. The putrefaction of meat is likewise due to the agency of Bacteria, which in this case decompose protein substances. Each type of cheese is the product of a definite bacterial and Fungus flora. In many cases fermentation is due to a group of organisms ; for instance, the so-called ginger-beer plant, used in the manufacture of the beverage of that name, consists of a Yeast (p. 255) associated with certain Bacteria.

It is to Bacteria, moreover, that we owe the decomposition of the cellulose in dead leaves, etc., whereby undue accumulation is prevented, and the carbon is again brought into circulation as carbon dioxide. The processes of decay involved in the "retting " of fibres (e.g. Flax, Hemp, Jute), the " curing " of tobacco, and the conversion of sewage are likewise brought about by members of this group.

The nitrates of the soil, upon which plants are dependent

1 In some cases part of this energy is dissipated as heat (cf. p. 26r ) or light (e.g. the phosphorescence of bad meat).

2 In the commercial production of vinegar either wine or spirit is used as the raw material, and to these vinegar is added in order to introduce the necessary Bacteria. 
for their supply of nitrogen, would, owing to their ready solubility, soon become depleted, were it not that they are continually being reinforced by the action of Bacteria. The decay of plant and animal bodies, which is likewise due to bacterial agency, leads to the production of a large number of waste products, of which one of the most important is ammonia. The latter combines with the calcium carbonate to form ammonium carbonate, and this is oxidised to a nitrite by the so-called NitriteBacteria which belong to the genus Nitrosomonas. The nitrites in their turn are converted into nitrates by the Nitrate-Bacteria (Nitrobacter), and in this way the ammonia, liberated by the decay of dead organisms, again becomes available to living plants.

By means of these oxidative processes the Bacteria in question gain the energy necessary for their vital activities. It has been found possible to cultivate them only in the absence of organic matter, but in nature the presence of the latter in the soil seems even to be beneficial. Nevertheless these organisms appear to be capable of building up organic substance from simple compounds, utilising for this purpose the energy obtained in the oxidative processes which they carry on. These nitrifying Bacteria cannot flourish in acid soils, or in such as contain an appreciable amount of free ammonia. This probably explains their paucity in many soils which are rich in humus (e.g. moorlands) or poor in lime. Where the decay of organic matter takes place on a large scale, as in the guano-fields of Chile, so large a quantity of nitrates may be formed that they accumulate as an efflorescence on the surface of the soil. This is the mode of origin of 'Chile saltpetre (potassium nitrate).

The beneficial action of these organisms is to some extent countered by the breaking down of nitrates with the evolution of nitrogen by such Bacteria as Bacterium denitrificans (denitrification). There are, however, others (the nitrogen-fixing Bacteria), which actually have the power of fixing the free nitrogen of the air, with the formation of organic nitrogen compounds, although the chemical processes involved are obscure. The most important of the organisms concerned is Azotobacter (Fig. I45, d), an aerobic form which obtains its energy by the breakdown of carbohydrates, a process setting free a considerable amount of carbon dioxide. The amount of nitrogen fixed is proportional 
to the amount of organic material decomposed. Another of the nitrogen-fixing Bacteria, Clostridium pasteurianum (Fig. I45,e), is anaerobic, the principal product of its activity, apart from nitrogenous compounds, being butyric acid. It is the organisms mentioned that are largely responsible for the gradual increase in the nitrogen-content of unmanured grassland.

Even in the time of the Romans the inclusion of Leguminous plants in a rotation of crops was recognised as beneficial. Experience has shown that cultivation of Clovers, Sainfoin, Lucerne, etc., materially increases the nitrogencontent of the soil, especially if the crop is subsequently ploughed into the field (socalled green manuring). This phenomenon remained unexplained until it was discovered that the swellings upon the roots of Leguminous plants are inhabited by Bacteria (Bacillus radicicola, Fig. I45, $f$ ), capable of fixing free nitrogen, and present in every healthy soil. Infection of the root takes place through the root-hairs, probably when the organism is in the motile phase ; having penetrated the roothairs, the Bacteria pass into the adjoining cells, which are thereby caused to divide, so that a gall-like structure arises. Within the cells of this nodule rapid multiplication of the Bacteria ensues, probably at the expense of carbohydrates furnished by the Leguminous plant. At the same time, however, the latter profits by the nitro-

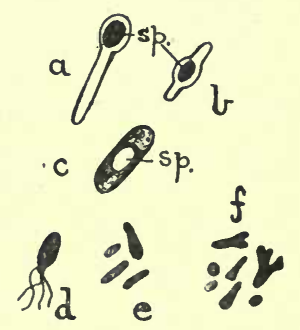

FIG. I 45.- $a-c$, sporeformation in Bacteria. $a$, Bacillus tetani; $b$, Bacillus of malignant œdema ; c, Bacillus cedamitis. $d-f$, nitrogen-fixing Bacteria. d,Azotobacter; $e$, Clostridium pasteurianum sp.; $f$, Bacillus radicicola. (After Engler and Prantl, and Ellis.) genous material formed by the Bacteria, the removal of which is indeed necessary for their continued activity. In the mature condition large numbers of the Bacteria, within the cells of the nodules, assume an irregular form, and become digested by the action of the host. A limited number persist unaltered, and return to the soil as the roots decay away.

The relation between the nodules and these nitrogen-fixing Bacteria is shown by the fact that Leguminous plants, grown from seed in soil which has been thoroughly sterilised by heating, 
fail to develop any nodules, and are just as dependent on a supply of soil-nitrates as other green plants. It appears that different strains of these Bacteria infect different Leguminous plants, and that normally those of a particular strain only attack other individuals of the same species. Similar nodules of a larger size occur on the roots of the Alder and the Bog Myrtle (Myrica gale).

Brief reference may be made to the so-called Thread-Bacteria, whose exact relation to the other forms is, however, dubious. In many respects they show a closer approximation to the Bluegreen Algæ than to the Bacteria proper. A common form, Beggiatoa, which is like a colourless Oscillatoria, is frequent in waters rich in sulphuretted hydrogen; the organism in question obtains the energy necessary for the building up of organic substances by the oxidation of the sulphuretted hydrogen to sulphur. The Iron-Bacteria (Leptothrix, Crenothrix, etc.), whose branched threads are abundant in ferruginous waters, and are often the cause of the brown deposit of ferric hydroxide, are further examples of these forms. 


\section{CHAPTER XIX}

\section{LIVERWORTS AND MOSSES}

THE plants so far considered all agree in possessing relatively simple sexual organs, consisting of more or less modified cells, whose protoplasmic contents, with or without division, give rise to the gametes. The sexual organs, moreover, are usually, except in such forms as Fucus and Pelvetia, distributed over the entire thallus. On the other hand, in Liverworts (Hepaticæ) and Mosses (Musci), which are grouped as Bryophyta, the second class of the Vegetable Kingdom, the sexual organs are not only much more elaborate, but are commonly restricted to definite portions of the plant-body. The latter usually also exhibits greater complexity of structure, which may be related to the fact that the Bryophyta are on the whole terrestrial plants in contrast, for instance, to the essentially aquatic Algæ. Other distinctive features of the Bryophyta will become apparent in the subsequent pages.

The majority of Liverworts are damp-loving plants, many of them growing in situations that are covered with moisture in the wetter seasons of the year (e.g. along the sides of watercourses), whilst some are even truly aquatic. Many Mosses, on the other hand, can flourish in habitats that are comparatively dry for a great part of the year, extreme examples being furnished by the Hair Moss (Polytrichum juniperinum), found on dry heaths, and the Wall Moss (Tortula muralis, Fig. I50, D), common on old walls, rocks, etc. In relation to this difference of habitat, Mosses generally display a structure which is more elaborate, and better suited to resist drought, than that of Liverworts.

The body of the Liverwort in its simplest form is a small flat green ribbon-like structure, often repeatedly forked, and growing 
in close contact with the soil (e.g. the common Liverworts Pellia, Fig. I46, B, and Marchantia, Fig. I46, E). At the base of the notch situated at the tip of each lobe of the thallus lies the growing point (Fig. I46, D, g.p.), which commonly consists of a marginal row of cells. The middle part of each segment is

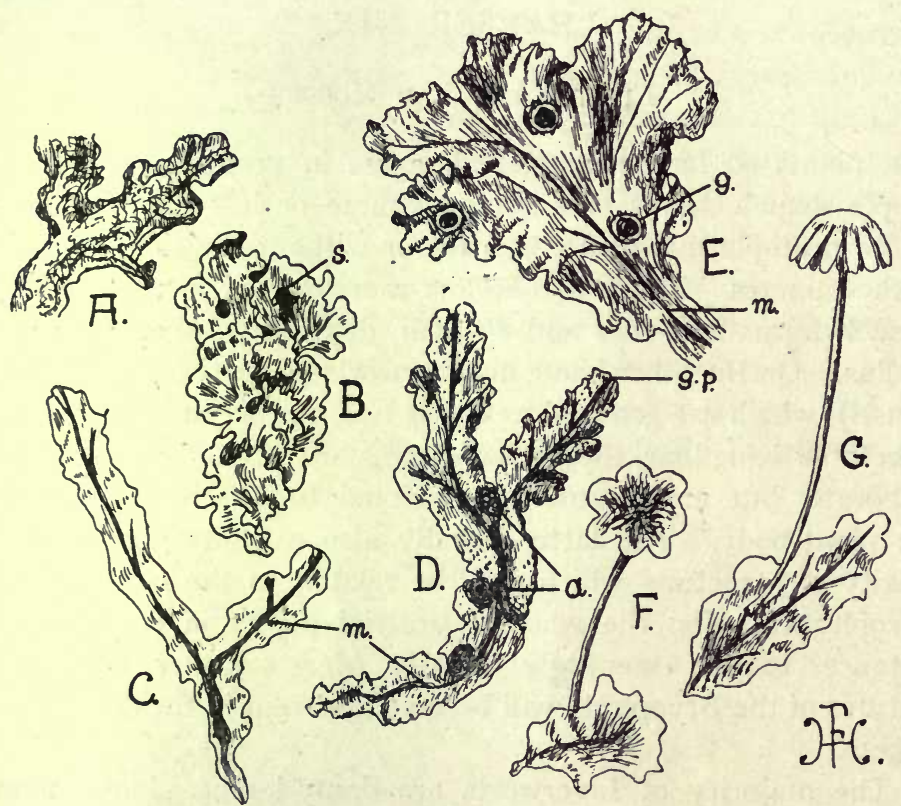

FIG. I46.-Various thalloid Liverworts. A, Small part of the thallus of Fegatella, seen from the under-surface, showing the dense weft of rhizoids. B, Group of thalli of Pellia (ordinary form), one with a young sporogonium (s.). C, Pellia, form characteristic of very wet habitats. D, Thallus of Fegatella from the upper surface, showing the antheridial discs (a.). E-G, Marchantia, showing respectively gemma-cups (g.), antheridial head, F, and archegonial head, G. g.p., growing point ; $m$., midrib. (All figures approximately natural size.)

generally somewhat thickened like a midrib (Fig. I46, m.), and projects to a more or less marked extent on the lower side of the thallus. Water and nutrient salts are absorbed by numerous fine thin-walled unicellular hairs (rhizoids, Fig. I46, A ; Fig. $152, \boldsymbol{r}$.), which grow out into the soil from the lower surface 
often mainly from the region of the midrib; these rhizoids also function as organs of attachment.

The underside of the thallus, in many Liverworts, also bears one or more rows of flat overlapping scales (Fig. I49, s.), which are one cell thick, and not uncommonly purplish in colour. Some forms (e.g. Marchantia) exhibit, in the axils of these scales,

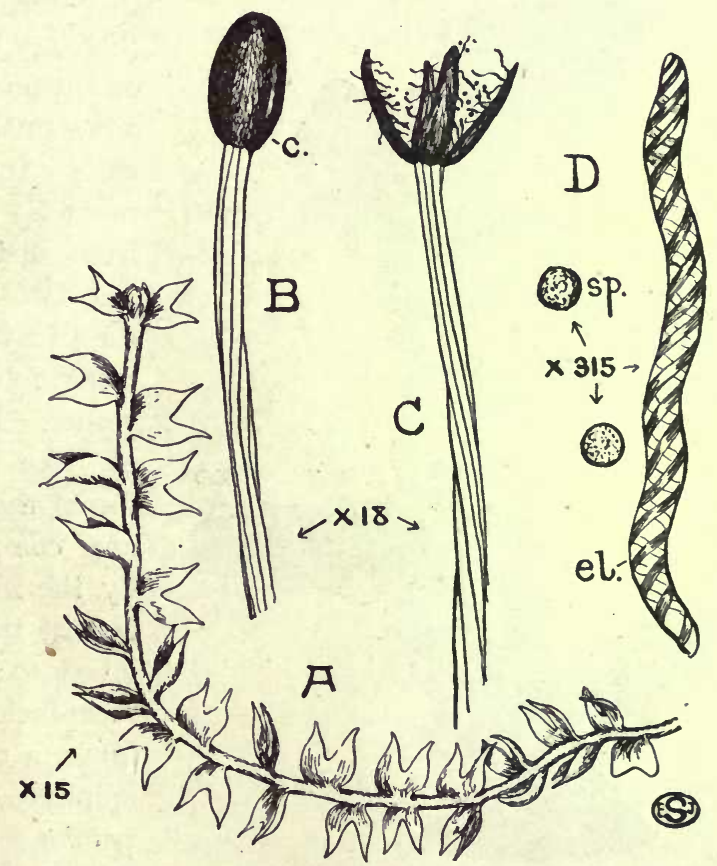

FIG. 147.-A leafy Liverwort (Cephalozia bicuspidata). A, Portion of a plant. B, Sporogonium showing capsule $(c$. ) before dehiscence. C, The same with dehiscent capsule, showing elaters. D, Elaters (el.) and spores $(s p$.$) :$

a second type of rhizoid, characterised by possessing a wall with numerous peg-like internal thickenings (Fig. I49, A, B) The capillary channels, between the dense weft of rhizoids and the overlapping scales, are no doubt of importance in the retention of water for use during periods of drought.

The Liverworts, however, also include leafy types which somewhat resemble Mosses in habit, and, in general, grow in 
rather drier situations than do the simple thalloid forms; the leaves, unlike those of Mosses, are usually lobed (Fig. I47, A), and sometimes even deeply divided. Some of the commonest of the foliose Liverworts belong to the genera Lophocolea and Cephalozia, whose structure is typical of these forms. Here there is a prostrate stem, bearing on either flank a row of over-

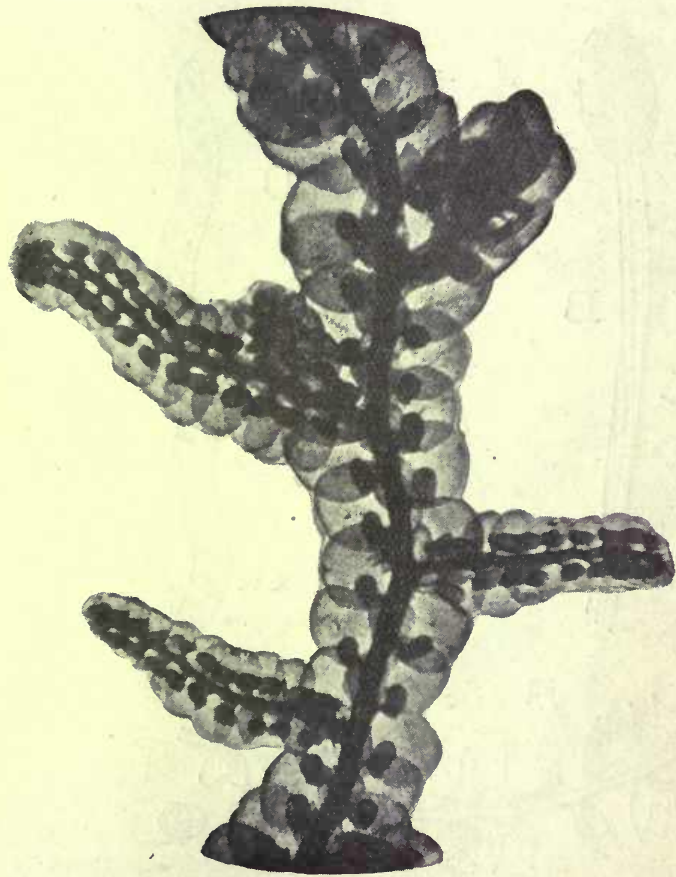

FIG. I 48.-Photomicrograph of a small portion of the epiphytic Liverwort Frullania tamariscini, showing the pitcher-like lower lobes of the leaves. [Photo, E. J. S.] lapping, sessile, two-lobed leaves (Fig. I47, A) and, on the underside, a few small scales with tufts of rhizoids arising from their base. The two lobes of the leaves are often folded together. In some of the foliose forms the leaves are complicated by the modification of the lower lobe into a waterreceptacle. Frullania, a common epiphyte on treetrunks, affords an extreme example, in which this lobe is developed as a

small, helmet-shaped pitcher (Fig. I48). In the leafy Liverworts the growing point of the stem is invariably a single cell.

The thallus of most Liverworts exhibits little anatomical differentiation. In such an one as Pellia, for example, all the cells, apart from their elongated form in the region of the midrib, are similar in shape, and most of them contain the numerous small discoid chloroplasts which are typical of Bryophyta 
(Fig. I52, A). An equally simple structure is displayed by most of the foliose forms, the leaves being invariably only one cell thick, and usually devoid of a midrib. In Marchantia and some of its allies, however, the upper part of the thallus, which is always the principal assimilating region, shows considerable complexity (Fig. I49, A). It is subdivided into a large number of shallow polygonal chambers, each of which is roofed over by an epidermis, and communicates with the exterior through a

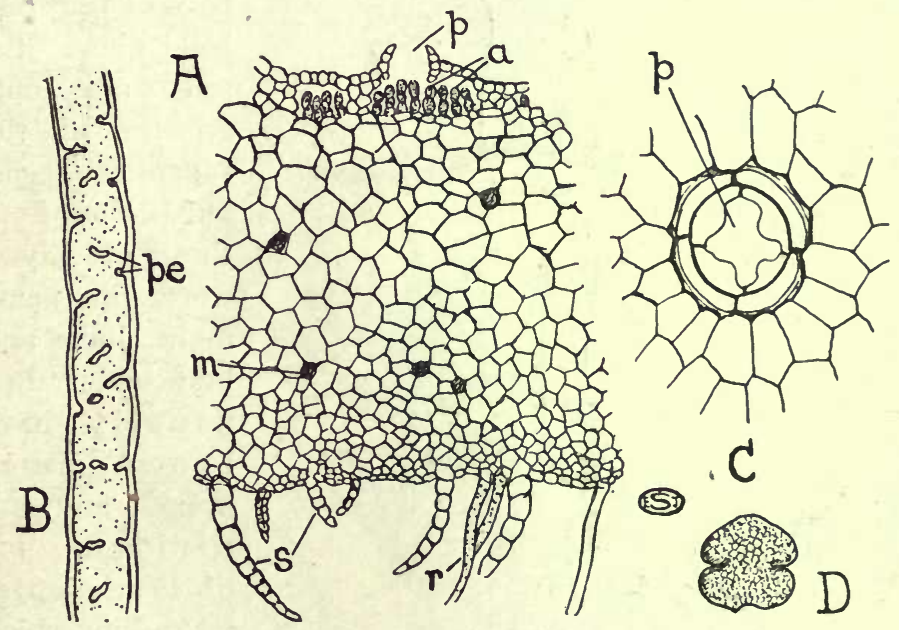

FIG. 149.-Structure of Marchantia. A, Small part of a transverse section through the thallus. B, Part of a peg-rhizoid, much enlarged, showing the thickenings (pe.). C, Surface-view, showing a single pore. D, Gemma, seen from the surface. $a$., assimilatory filaments ; $m$., mucilage-cell ; $p$., pores ; $r$., rhizoid ; $s$., scales.

central barrel-shaped pore $(p$.$) . From the floor of each chamber$ arise numerous short filaments of green cells $(a$.$) , which form$ the assimilatory system of the thallus. The whole of the lower portion of the latter consists of large colourless cells, serving in the main for the storage of food-reserves, and in part showing reticulate thickenings. On the surface of the thallus the assimilatory chambers appear to the naked eye as a number of small diamond-shaped areas, each with a minute central dot corresponding to the pore. 
In the Mosses there is a definite stem, bearing three or more rows of alternate sessile leaves. The plants either have a prostrate habit (e.g. Hypnum, Fig. I50, C), like that of most leafy Liverworts, or grow erect, as in the Hair Mosses (Polytrichum, Fig. I50, A, Funaria, etc.). The lower part of the stem, which is buried in the soil, forms a kind of rhizome bearing numerous rhizoids, and sometimes small scales as well. The rhizoids,
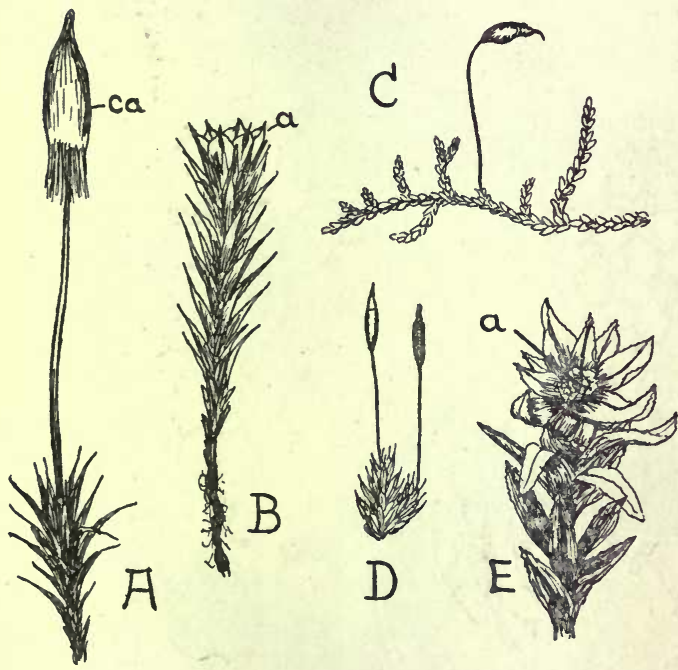

FIG. I 50.-Various Mosses. A, Plant of Polytrichum, bearing a sporogonium whose capsule is covered by the calyptra (ca.). B, Male plant of the same, showing the antheridial head $(a$.$) . C,$ Hypnum, with sporogonium. D, Tortula muralis. E, Male plant of Mnium, with antheridial head (a.). which may also develop from the part of the stem just above the soil, are outgrowths of the superficial cells, but differ from those of Liverworts in being multicellular and branched ; they usually have brown mem branes, and are divided by oblique septa, just behind which the branches originate.

The leaves of Mosses are never lobed, are attached to the stem by a broad cushion-like base, and usually, except in the region of the midrib, consist of a single layer of cells (Fig. I5I, B). The midrib $(m$.$) is generally well differentiated, and its presence$ forms a point of contrast with the leafy Liverworts. The branches of the stem arise from below the leaves, and, in some of the erect forms, are produced in such large numbers from the base that the plants exhibit a densely tufted habit (e.g. Tortula, Fig. I50, D). Many of the Bog-mosses (Sphagnum) are likewise 
richly branched. The peculiar habit of these Mosses is partly due to the fact that some of the branches elongate considerably, and hang down alongside the main stem (Fig. I5I, F). The

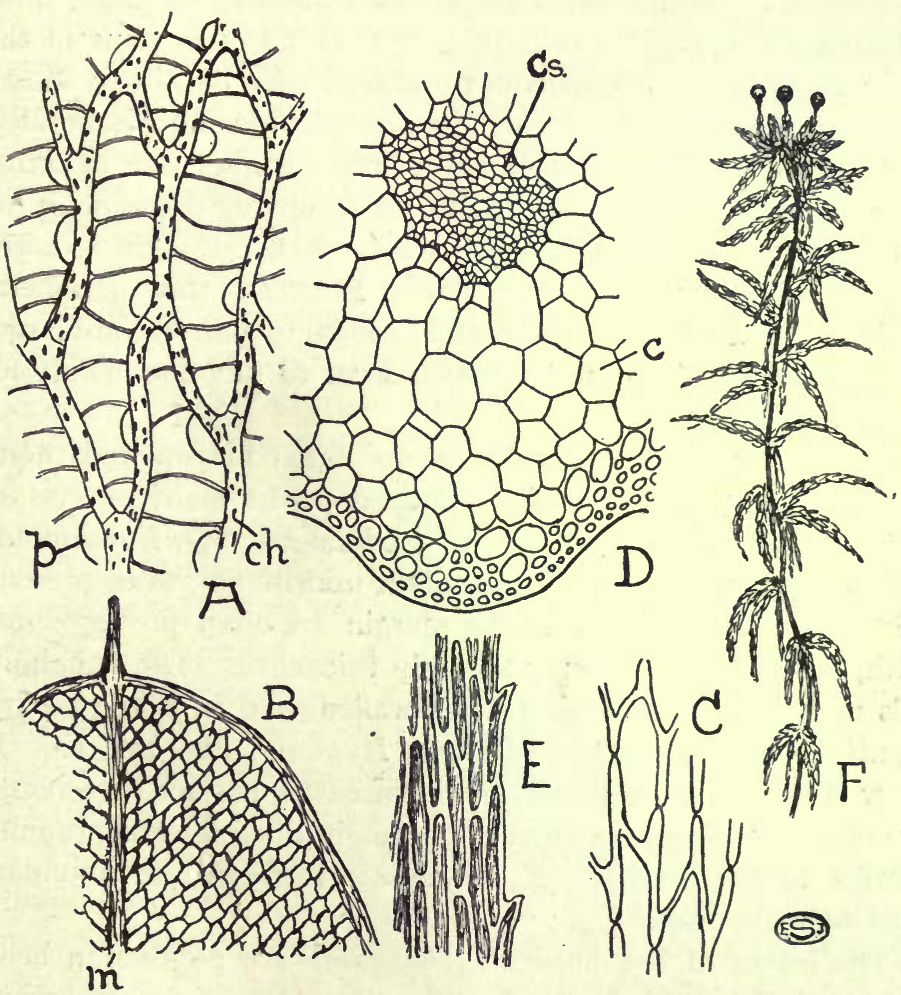

FIG. I5I.-Structure of Mosses. A, Portion of a leaf of the Bog-moss (Sphagnum), showing cells with chloroplasts $(c h$.$) and the large empty$ cells with their thickenings and pores (p.). B, Leaf-apex of Mnium, with the midrib $(m$.$) . C, Ieaf-cells of Hypnum, from the surface,$ showing thickened walls and pits. D, Transverse section of the stem of Mnium hornum (after Bastit), showing conducting strand (Cs.) and storage-cells $(C$.$) . E, Leaf-cells of Hylocomium, from the surface.$ F, Habit of Sphagnum (after Schimper), showing three sporogonia.

branching of many prostrate Mosses takes place in a pinnate manner (e.g. Hypnum, Fig. I50), whilst that of the erect forms is usually forked. Growth of the stem and its branches is 
effected by means of three-sided apical cells, similar to those of Equisetum (cf. p. I8).

Mosses show a greater degree of anatomical complexity than Liverworts, as is well illustrated by the invariable presence in the stem of a distinct conducting strand, consisting of small, thinwalled, much elongated cells (Fig. I5I, D, Cs.) ; in some of the larger Mosses (e.g. Polytrichum) these cells may be of two kinds, serving respectively for the conduction of water and elaborated food-materials. The outer tissues of the stem, as seen in transverse section, consist, except in Mosses occupying damp habitats, of cells with strongly thickened walls which are often reddishbrown in colour (Fig. I5I, D). Between this peripheral mechanical cylinder and the central conducting strand are larger cells $(C$.) with thinner walls, which seem to function mainly for food-storage.

The cells composing the leaves are either narrow and rhomboidal (Fig. I5I, E), or spindle-shaped (as in many species of Hypnum, Fig. I5I, C), or almost isodiametric (e.g. Funaria and Mnium, Fig. I5I, B), whilst those of the midrib $(m$.$) , when present,$ are elongated; the cells at the margin are often produced into teeth, and not uncommonly strongly thickened. The remaining cells may also be somewhat thick-walled, and in that case frequently bear well-marked pits (e.g. Hypnum, Fig. I5I, C). In a few Mosses (e.g. Thuidium) the assimilating surface is increased by outgrowths from the stem or by longitudinal lamellæ, running parallel to the midrib (e.g. Catharinea undulata, an abundant Moss in many woods).

The leaves of Bog-mosses (Sphagnum) are peculiar in being composed of two kinds of cells (Fig. I5I, A). The green assimilating cells (ch.) are of narrow form, and are arranged as a reticulum whose meshes are occupied by large spirally thickened transparent cells which are dead and empty. The latter communicate with the exterior by means of one or more holes $(p$. in their walls. These large colourless cells readily fill with water, and it is owing to this that so much liquid can be squeezed out of a handful of Bog-moss. Similarly, dry Sphagnum can suck up a great deal of moisture, hence its employment as an absorbent in surgery, or for molasses in the manufacture of Molassine Meal. In the dry condition, the air in the dead cells 
obscures the green colour of the living ones, and causes the whole plant to appear whitish. The Moss Leucobryum, common on wet heaths, possesses leaves with a somewhat similar structure.

Both Liverworts and Mosses propagate abundantly by vegetative means, most frequently by fragmentation of the thallus. In many Liverworts, and some few Mosses, the thallus forms special bodies called gemma, which consist of a varying number of cells and are often of characteristic shape (Fig. I49, D). In Marchantia they are formed in special cup-like outgrowths on the upper surface of the thallus (Fig. I46, E, g.), but in the leafy Liverworts they are usually budded off from the tips of the shoots. An abundant production of gemmæ is likewise seen in the Moss Aulacomnion androgynum, where they arise in a spherical cluster at the top of the stem.

The ordinary Moss or Liverwort plant, however, also reproduces by sexual means, the sexual organs developing especially in the early spring. Their general character, in the case of Liverworts, will be gathered from an examination of Pellia. The male organs are found in the region of the midrib, and are visible to the naked eye as a number of dark pimples, each of which, in a vertical section (Fig. I52, A), is seen to correspond to a single more or less spherical antheridium. The latter (Fig. I52, B) is borne on a very short stalk, and almost fills a flask-shaped depression in the thallus which communicates with the exterior by a narrow pore.

The antheridium possesses a wall (Fig. I52, B,w.) which is composed of a single layer of cells, and at maturity encloses numerous small, colourless, closely packed spermatozoid mother. cells, each producing a single spermatozoid (Fig. I52, D). Through vigorous absorption of water, in wet weather, by the cells of the wall, the apex of the antheridium is ruptured, and the mucilaginous mass of mother-cells is discharged into the surrounding moisture. Here occurs the final liberation of the spermatozoids, each of which possesses a spirally coiled body (formed mainly from the nucleus of the mother-cell), bearing two long cilia at the slightly tapering front end (Fig. I52, C) ; a small vesicle, representing the remaining cytoplasm of the mother-cell, is generally seen at the opposite end, but is shed during movement. 


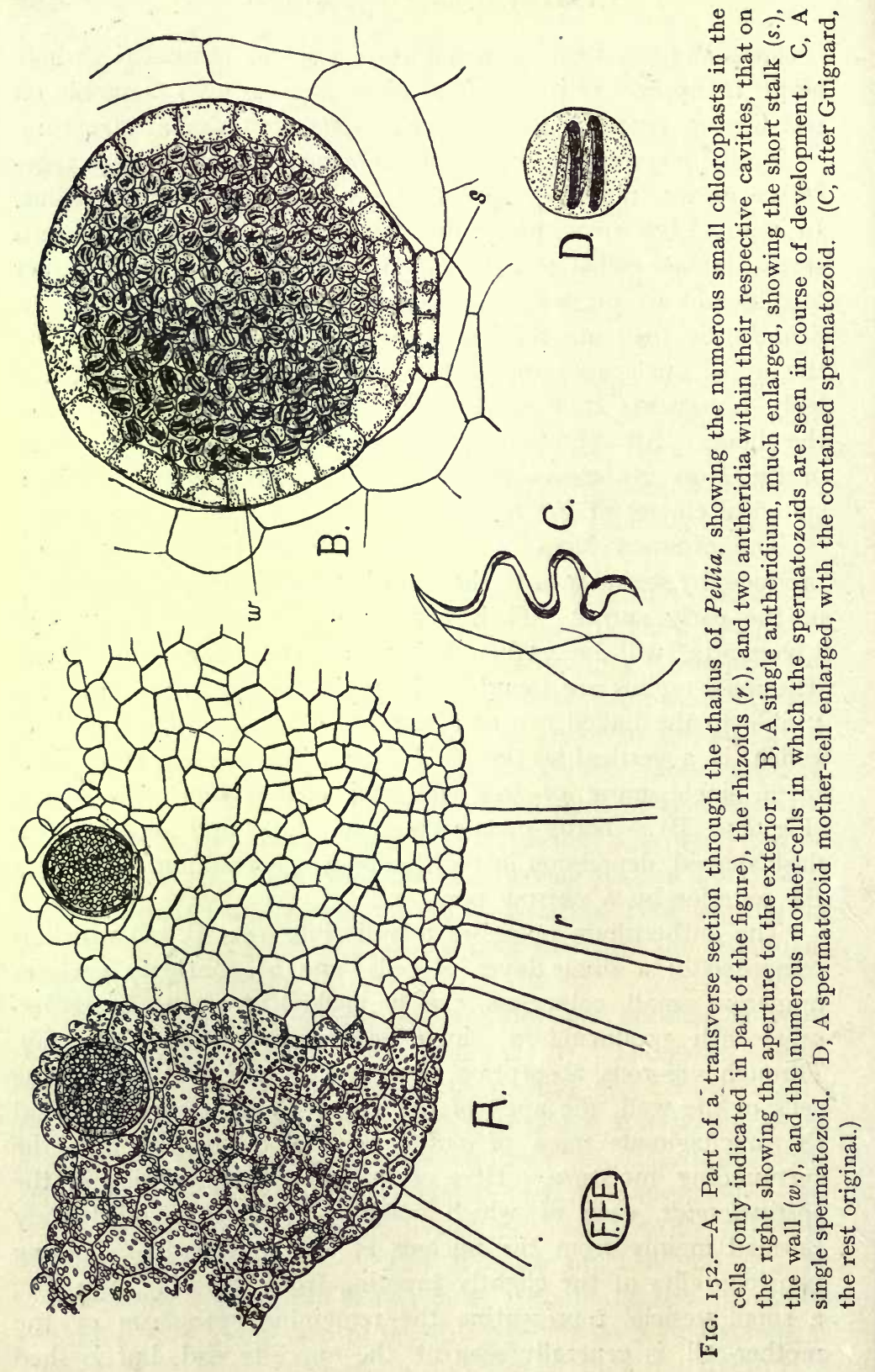


In some Liverworts (e.g. Fegatella) the groups of antheridia are embedded in slightly thickened patches on the upper side of the thallus (Fig. I46, D, a.). A rather exceptional condition obtains in Marchantia, where the thallus-lobe, in which the male organs are sunk, is raised above the general surface on a definite stalk (Fig. I46, F). Amongst the leafy forms the antheridia are situated, either singly or in groups, in the axils of the leaves.

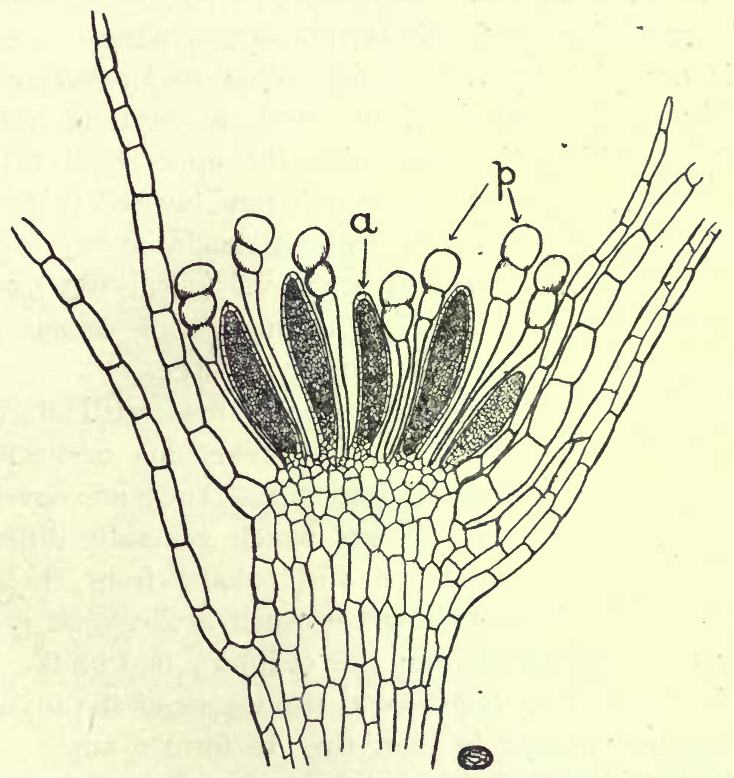

FIG. I53.-Vertical section through the antheridial head of the Moss Funaria, showing the antheridia (a.) and interspersed sterile hairs ( $p$.$) .$

The antheridia of Mosses show essentially the same structuie as those of Liverworts, except that they are elongated and possess a broader stalk (Fig. I53, a.). They occur in groups, interspersed with multicellular sterile hairs $(p$.$) , at the top of$ the stem or its branches. Each antheridial group is surrounded by a number of spreading protective leaves (involucre), which are not infrequently arranged to form a kind of cup, and usually differ in shape and size, and sometimes also in colour, from the ordinary leaves. The male plants of Mosses are thus easily 
recognised (Fig. I50, B, E). The spermatozoids show the same general structure as do those of Liverworts, and are liberated in much the same way during wet weather, the mass of mothercells often being ejected to some slight distance.

The female organs, or archegonia, which are essentially similar in the two groups, differ markedly from those of Thallophyta. They are flask-shaped structures (Fig. I54, B), composed of a lower swollen part, or venter (v.), and a long neck $(n$.), which, in Mosses, is often twisted. The neck is a tube consisting of a single layer of elongated cells surrounding a narrow canal. The latter is occupied by a row of naked neck-canal cells which ultimately become mucilaginous, and, in swelling, push apart four lid-cells which, till then, close the upper end of the neckcanal. The venter possesses a wall (one-layered in Liverworts, Fig. I54, B, two-layered in Mosses), enclosing a large naked ovum (e.), with a small ventral canal cell $(v$.) at the base of the neck. The venter is borne on a short stalk which is rather more massive in Mosses than in Liverworts.

In Mosses, where they are interspersed with sterile hairs, and in most leafy Liverworts, the archegonia occur in groups at the top of the stem or its branches. They are enveloped by a number of " involucral" leaves which generally differ in size, and sometimes also in shape and colour, from the ordinary leaves; in most Mosses, however, such archegonial groups are scarcely distinguishable from the ordinary leaf-buds. In some leafy Liverworts (e.g. Lophocolea) the leaves of the involucre are fused together, almost to their tips, to form a cup.

In some of the thalloid Liverworts (e.g. Pellia) the archegonia are found at the front end of the thallus, where they occupy depressions roofed over by a scale-like involucre (Fig. I54, A, i.). In other cases (e.g. Marchantia, Fig. I46, G) they are situated on special stalked star-like upgrowths which are divided into 4-Io lobes; the archegonia in these cases form groups on the lower surface, between the lobes, each group being enclosed between a pair of involucral scales.

The diverse forms of involucre surrounding the archegonia not only protect them from desiccation, but form capillary chambers tending to retain water. This facilitates the movement of the sperms in fertilisation, which only takes place when 
the thallus is covered with a film of moisture. In forms like Marchantia, the stalk bearing the lobed archegonial disc does not lengthen till after fertilisation, so that the necks of the archegonia remain in close contact with the moist upper surface of the thallus. In the same way the branches bearing the archegonial groups of Mosses are at first usually short and adjacent to the soil, so that they are readily covered with moisture during rain.

After escaping from the antheridia, the spermatozoids, swimming in the surface films of moisture, are chemotactically attracted to the open necks of the archegonia by substances

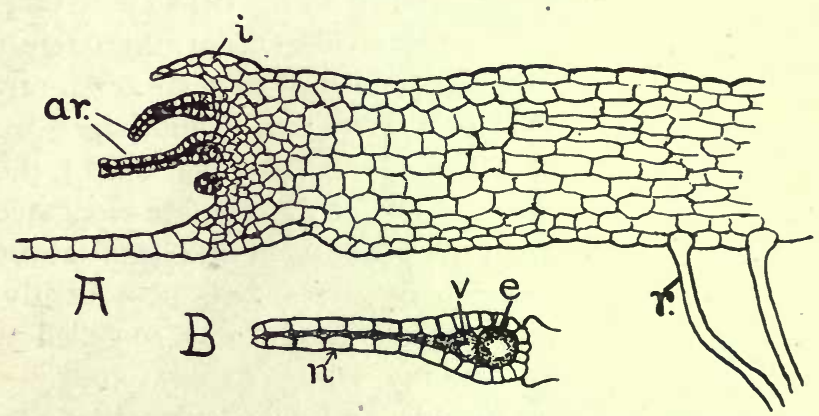

FIG. I 54.-A, Longitudinal section through the apex of the thallus of Pellia, showing a group of archegonia $\left(a r_{.}\right)$and the involucre $(i.) . \quad r_{.}$, rhizoid. B, Single archegonium of Marchantia, on a larger scale. e., egg ; $n$., neck ; $v$., ventral canal cell.

(cane-sugar in Mosses) contained in the mucilage formed by the breaking down of the canal cells. The spermatozoid passes down the neck-canal and enters the egg, after which nuclear fusion occurs.

The fertilised ovum secretes a cell-wall and, without any resting-stage, commences to divide and grow into a new Moss or Liverwort plant. This, however, differs fundamentally from the parent, especially in being parasitic upon the latter, and at maturity constitutes an organism (the sporogonium, Fig. I50; Fig. I55, A) that is almost solely concerned in the production and dispersal of spores. This simplicity of structure can possibly be related to the parasitic habit, thus affording an interesting 
analogy to parasitic Angiosperms. As the embryo enlarges, the venter of the archegonium stretches and grows into a protective covering, the calyptra, which can often be recognised around the young sporogonium (Fig. I55, B ; and Fig. I56, I, c.).

In Liverworts the dividing ovum first forms a spherical mass of cells which later elongates and gradually becomes differentiated into three regions. The end adjacent to the thallus penetrates into the underlying tissue as a cone-shaped sucker, the foot (Fig. I55, B, f.). This consists of parenchymatous cells, and serves to absorb and transfer food to the developing sporogonium. The opposite end swells into a, usually, spherical capsule (ca.) with a wall of one or more layers enclosing a mass (the archesporium) of spore-forming cells. Up to a certain stage all the latter are alike, but the archesporium ultimately differentiates into two kinds of cells, which at first are often arranged in radiating series. One type is rounded, and, since each of these cells divides to form four spores (Fig. I55, C, sp.), they are known as spore-mother cells. The other cells are elongated and at first thin-walled, when they assist in the translocation of food-materials to the developing spores, but subsequently they acquire spiral thickening bands, and form so-called elaters (Fig. I55, C, e. ; see also Fig. I47, D).

The region between capsule and foot, consisting of small cells, often densely crowded with starch-grains, constitutes the seta or stalk (Fig. I55, B, s.), which always remains short until the spores are mature. At this stage, however, in Pellia and most leafy Liverworts, the stalk elongates rapidly, owing to a great increase in the length of its cells at the expense of the starch-reserves, and bursts through the calyptra, which can be recognised as a torn sheath around its base (Fig. I55, A, c.). The capsule is thus raised above the damp soil into the less humid air, where dispersal of the spores by air-currents is more readily attained. Where, as in Marchantia, the archegonia are borne on a special receptacle, the stalk of the latter elongates after fertilisation (cf. above), and serves the same purpose as the seta of other Liverworts, whilst the sporogonium retains the short stalk of the embryonic phase.

In Pellia and most leafy Liverworts the capsule-wall splits into four petal-like valves (Fig. I47, C), although in Marchantia 
the apex ruptures irregularly. Owing to their unequal thickening, the elaters tend to coil and bend as the contents of the capsule become drier. This movement is, however, prevented until rupture of the capsule takes place, when the tensions set

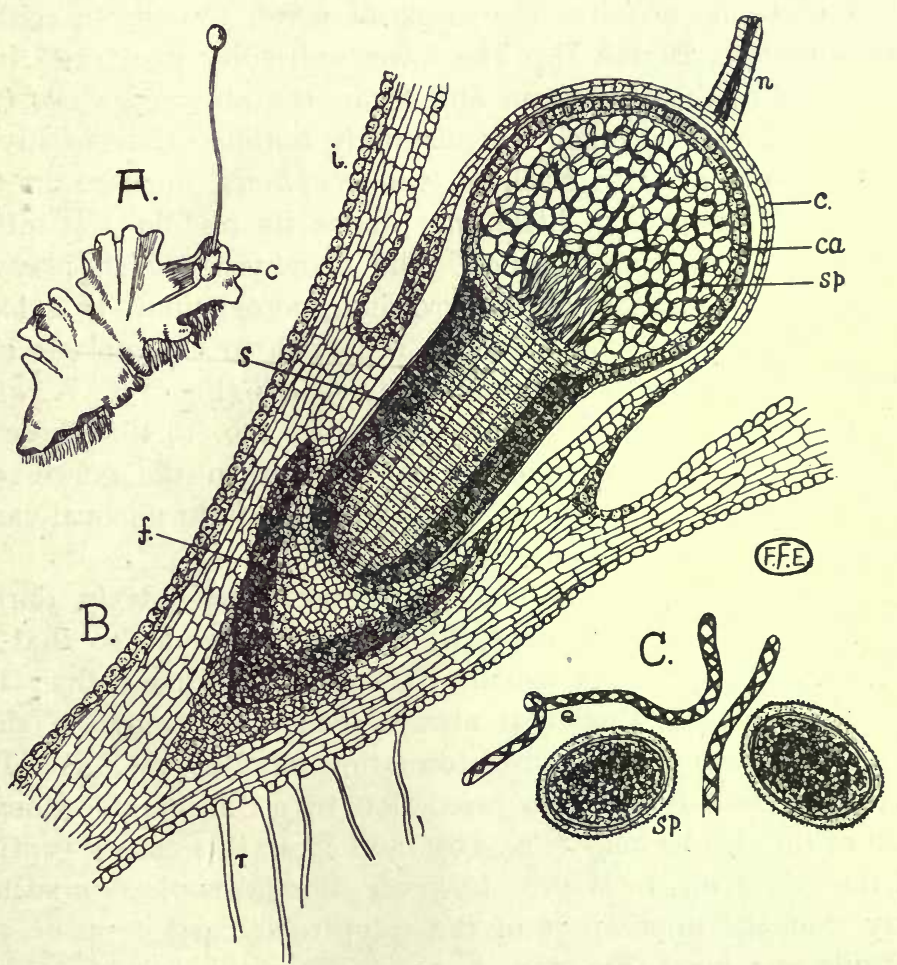

FIG. 155.-The sporogonium of Pellia. A, Small part of a thallus, showing a mature sporogonium, with the ruptured calyptra $(c$.$) at the base$ (after Leunis). B, Longitudinal section through an almost mature sporogonium, still enclosed within the calyptra $(c$.$) ; the elaters are$ shown as dark lines among the much larger spores. ca., wall of capsule ; $f$., foot ; $i$., involucre ; $n$., neck of archegonium ; $r$., rhizoid ; $s .$, seta; $s p$. , spore. $\mathrm{C}, \mathrm{A}$ group of spores $(s p$.$) and elaters (e$.$) .$

up in the elaters are manifested in a wriggling movement which loosens the mass of spores and flicks them into the air. Not uncommonly tufts of elaters remain adhering to the top of the stalk (e.g. Pellia) or to the valves (Fig. I47, C). 
In a few Liverworts belonging to the genus Riccia, the sporogonium, which is here embedded in the thallus, is of much simpler construction, consisting merely of a spherical capsule within whose one-layered wall only spores are produced.

In Mosses the embryo elongates considerably at an early stage, and soon acquires the shape of a rod tapering at either end (Fig. I56, H and I). The lower extremity penetrates into the tissue of the Moss-stem and forms the absorptive foot $(f$.$) ,$ which is, however, much less distinctly outlined than in Liverworts. Soon after, a swelling, the apophysis, appears on the rod-shaped embryo, a little way above its middle, separating the future seta and capsule. The apophysis, which plays a part in the nutrition of the growing sporogonium, is a local assimilatory region composed of cells rich in chloroplasts and provided with abundant intercellular spaces (Fig. I56, A, ap.). The epidermis here contains stomata (Fig. I56, F) like those of higher plants, except that the septa between the guard-cells break down at maturity, so that the latter form an oval canal surrounding the pore (Fig. I56, G).

The upper part of the embryo enlarges progressively, during the subsequent development, to form the capsule, so that at maturity the latter is usually far more prominent than the apophysis, which, though it always remains recognisable, does not grow much after its first formation (cf. Fig. I56, A). The mature capsule is here also raised aloft by a considerable elongation of the slender stalk (Fig. I50), and again this causes rupture of the calyptra. In Mosses, however, this takes place in such a way that the upper part of the calyptra is carried up on the capsule as a hood (Fig. I50, A, ca.). When this is removed, the apex of the capsule is seen to be separated from the rest by a slight constriction, and it is this part that becomes detached as a lid to allow of dispersal of the spores.

The internal structure is best studied in a longitudinal section of a nearly ripe capsule (Fig. I56, A). Here the axis is occupied by a rather broad column of thin-walled parenchyma (the columella, Co.) passing below into the tissue of the apophysis (ap.) and above into that of the lid $\left(l_{0}\right)$. Surrounding the columella are two concentric cylinders of cells, separated by the granular archesporium (a.), each mother-cell of which, as in Liverworts, 


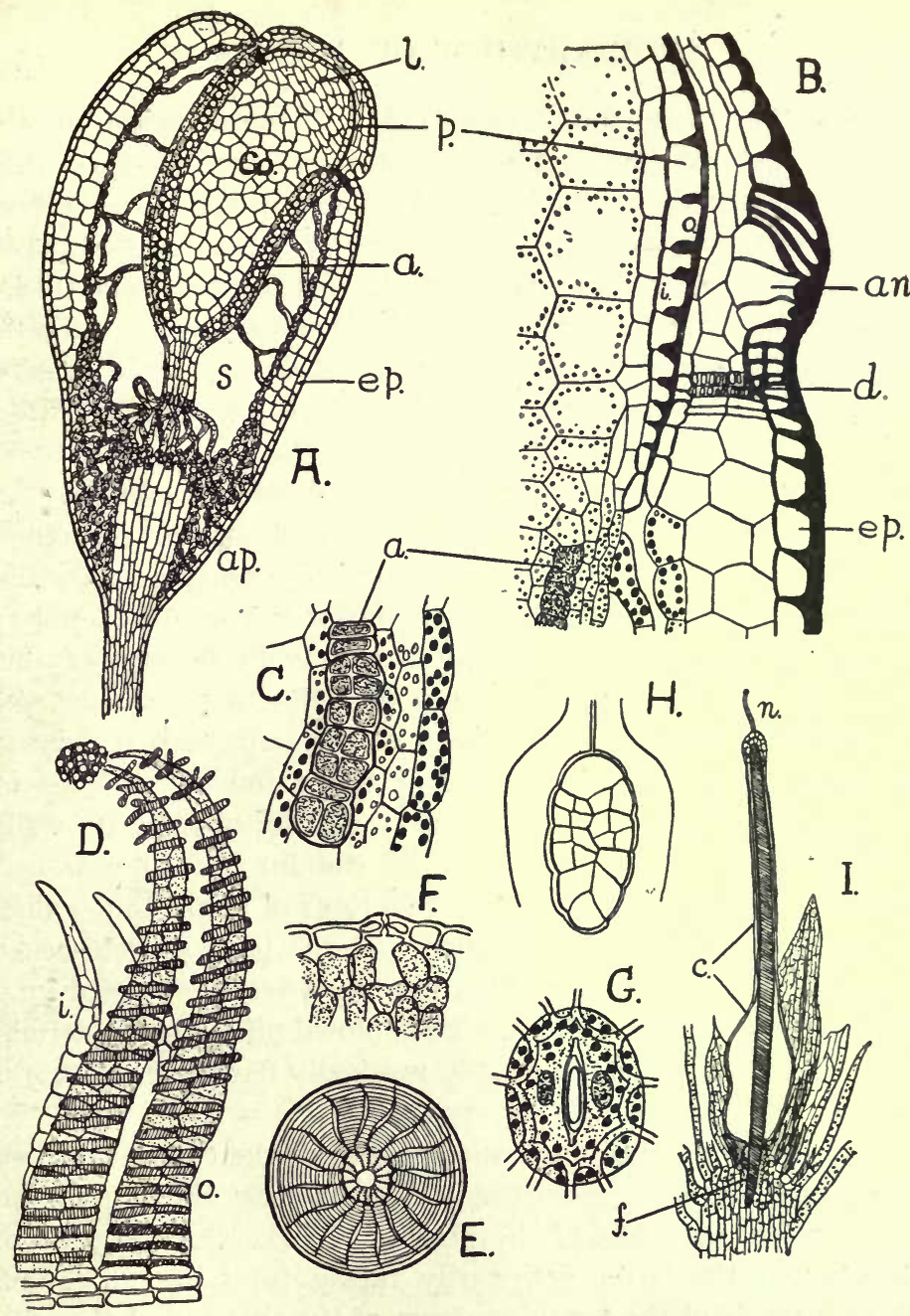

FIG. I 56.-Structure and development of the Moss-sporogonium (all figures, except F, represent Funaria hygrometrica). A, Longitudinal section through young capsule, apophysis $(a p$.$) , and the top of the stalk.$ B, Part of the capsule in the region of the lid, much enlarged. C, Small part of archesporium and spore sack, enlarged. D, Two pairs of peristome-teeth (inner and outer). E, Aperture of dehisced capsule, showing arrangement of peristome-teeth. F, Section of small part of apophysis of Bryum argenteum. G, Single stoma, in surface view. $\mathrm{H}$ and $\mathrm{I}$, Stages in development of the sporogonium. a., archesporium; an., annulus ; c., calyptra ; $C_{o}$., columella ; $d$., diaphragm ; ep., epidermis ; $f$., foot ; $i$., inner peristome tooth ; $l$., lid ; $n$., neck of archegonium; o., outer peristome tooth; $p$., peristome-layer; $S$., air-space. (D after Ruhland ; E after Braithwaite ; F and $G$ after Haberlandt ; the remainder after Sachs.) 
divides to form four spores (Fig. $\mathrm{I}_{56} 6, \mathrm{C}$ ). Between the sporesack so formed and the capsule-wall is a conspicuous air-space $(S$.) bridged by. occasional fine threads of green cells; in a few Mosses (e.g. Polytrichum) a similar air-space separates the sporesack from the columella. The capsule is protected by a thickwalled epidermis $(e p$.$) beneath which are several layers of cells$ containing chloroplasts. The features just described are equally recognisable in a transverse section through the middle of the capsule.

Just below the constriction (Fig. $\mathrm{I} 56, \mathrm{~A}$ and $\mathrm{B}$ ) marking the commencement of the lid, a diaphragm $(d$.) composed of thickened cells, and having the form of a circular ledge perforated by the central thin-walled tissue, stretches inwards from the epidermis $(e p$.$) of the capsule. Arising from the inner edge of this$ diaphragm is a dome-shaped layer of cells (the.peristome, p.) characterised by marked thickening of the tangential (i.e. inner and outer) walls ( $i$. and 0.$)$, and of the adjoining portions of the horizontal walls, although the radial (i.e. vertical) walls remain thin. At the lower edge of the lid the thick-walled epidermis is interrupted by one or two rings of larger thin-walled cells which constitute the so-called annulus (an.). Dehiscence eventually takes place along this line, as a result of the drying of the capsule, and soon after the lid is blown off. The epidermal cells above the annulus are usually markedly flattened, and form the lower edge of the lid (Fig. $\mathrm{I}_{56} 6, \mathrm{~B}$ ).

At maturity all the thin-walled tissues of the capsule shrivel, leaving, apart from the spores, only the epidermis, the diaphragm, and the thickened walls of the peristome. Each of the rows of cells forming the latter necessarily tapers from base to apex, and, as a result of the breaking down of the thin radial (i.e. vertical) walls between some or all of the rows, a number of separate plate-like peristome-tecth (Fig. I56, D), attached below to the edge of the diaphragm, are formed. When both inner and outer tangential walls of the peristome are thickened, a double set of teeth (o. and $i$.) of course arises as the thin portions of the horizontal walls shrivel. The peristome varies greatly in different Mosses, and, together with the characters of the leaves, constitutes one of the chief means for distinguishing the different genera and species. 
The peristome-teeth are extremely sensitive to changes in the moisture-content of the air, curling outwards when the latter is dry, and inwards when it is damp. In dry weather, therefore, the powdery mass of spores inside the capsule is readily dispersed, whilst in wet weather the dome is reconstituted (Fig. I56, E), and the aperture, left by the shedding of the lid, closed. The actual dispersal, in which the elasticity of the seta plays a considerable part, is effected by a catapult-mechanism similar to that of the Cowslip and Poppy fruits.

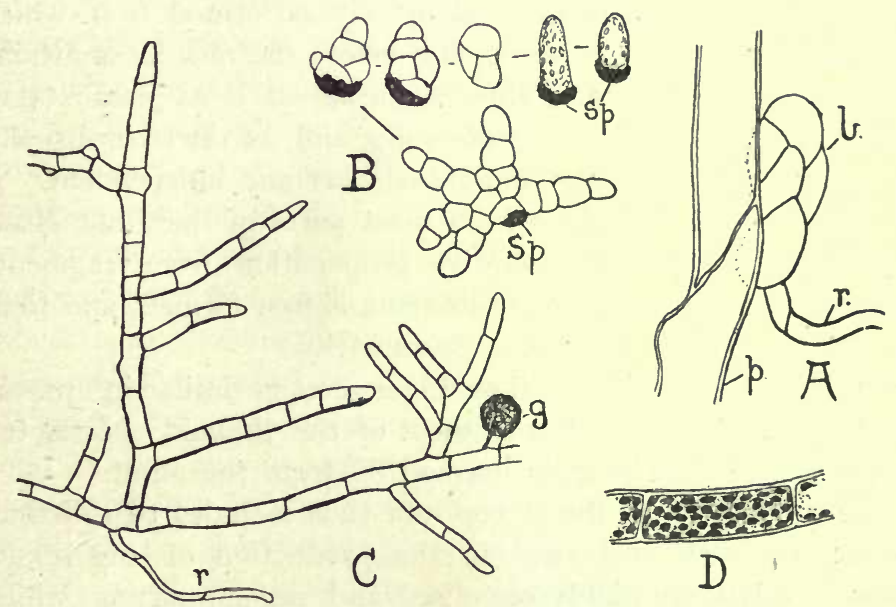

Fig. 157.-Moss-protonema. A, Development of young Funaria-plant (b.) from the protonema $(p$.$) (after Campbell). B, Stages in germination$ of spores, and C, protonema of Dicranella heteromella (after Servettaz). g., gemma on protonema ; $r$., rhizoids ; sp., spore-membrane. D, Single cell of overground part of protonema.

The spores ultimately give rise to a new Liverwort or Moss, but the adult plant is not produced directly on germination. This is especially obvious in Mosses, where the spores in most cases develop into a branched multicellular filamentous structure, the protonema, which exists partly above and partly below ground (Fig. I57, C). The overground portion appears as a green weft on the surface of the soil, and its cells contain numerous discoid chloroplasts (Fig. I57, D), whilst the underground portion closely resembles the rhizoids of the ordinary Moss (Fig. I57, C, r.). 
In fact, the latter readily grow out into protonema, if exposed to the light, and can thus bring about the development of new plants.

Vegetative multiplication of the protonema, by the detachment of branches or of small terminal groups of cells (Fig. I57, C, g.), is often prolific, and may set in at a comparatively early stage. Sooner or later, however, some of the short lateral branches become pear-shaped and undergo segmentation by three oblique walls, which cut out the characteristic tetrahedral apical cell of the stem of the adult Moss (Fig. I57, A, b.). By its continued divisions a small mass of cells is formed from which leaves soon arise, and it is not long before the new Moss attains recognisable dimensions. This formation of Moss-plants takes place at many points on the protonema, and, as the latter usually dies away soon after, the individuals become independent. A protonema readily develops from most parts of the adult Moss, in fact, in all cases of vegetative propagation from fragments of the thallus (cf. p. 277), a protonema is first formed, and from this the new plants only arise secondarily.

The protonema of the foliose Liverworts is similar in appearance to that of Mosses, but in most of the thalloid species the early stages are not sharply marked off from the adult.

The life-history of the Bryophyta thus includes two distinct phases: the one, concerned in the production of the sexual organs, is relatively highly organised and self-supporting, whilst the other, concerned solely with the production of asexual spores, is always dependent, and relies for its sustenance, either entirely (most Liverworts) or partly (Mosses), upon food obtained from its host. These two phases normally alternate with one another and arise from one another, and a similar alternation is encountered in all the higher groups of plants. For convenience of designation the spore-producing phase is spoken of as the sporophyte, and the sexual phase as the gametophyte. 


\section{CHAPTER XX}

\section{FERNS}

THE Bryophyta, though exhibiting a marked advance in organisation as compared with the Thallophyta, differ from the remaining flowerless plants, comprised in the third class of the Vegetable Kingdom, or Pteridophyta, in lacking roots and true vascular tissue. In the Pteridophyta, moreover, the gametophyte is usually thalloid in form and short-lived, whilst the spore-producing phase in the life-history is far more conspicuous and more permanent, and is quite independent of the gametophyte except in its very earliest stages. In the sexual process and the general structure of the sexual organs, however, there is considerable correspondence between the two classes, the Pteridophyta, like the Bryophyta, possessing motile male cells, and thus depending on the presence of liquid water at the time of fertilisation. The Pteridophyta, as a matter of fact, in general favour moist habitats, although quite a considerable number can grow under relatively dry conditions. Included in this class are the Ferns (Filicales), the Horsetails (Equisetales), and the Clubmosses (Lycopodiales), of which the first-named will be considered in the present chapter.

In many common Ferns the stem is an underground rhizome, which is relatively insignificant in comparison with the conspicuous, usually deeply divided, pinnate fronds which alone appear above the surface; a notable exception is, however, afforded by the Tree Ferns of subtropical regions, which have well-marked trunks. The subterranean stem is either horizontal (as in the Bracken, Pteris aquilina, Fig. I58, B), or compact and more or less erect (e.g. Lady Fern, Athyrium filix-fomina; Royal Fern, Osmunda regalis). It rarely shows branching, though this occurs at intervals where the rhizome is elongated 
(e.g. Bracken). In the creeping forms, the leaves usually arise in a row, on either side, and are separated by well-marked internodes (Fig. I58, B). In short-stemmed species, however, whether the rhizome be erect or inclined (e.g. Male Shield Fern, Nephrodium filixmas $\left.^{1}\right)$, the leaves are densely crowded, and show an obvious spiral arrangement. In the more compact forms a conspicuous feature

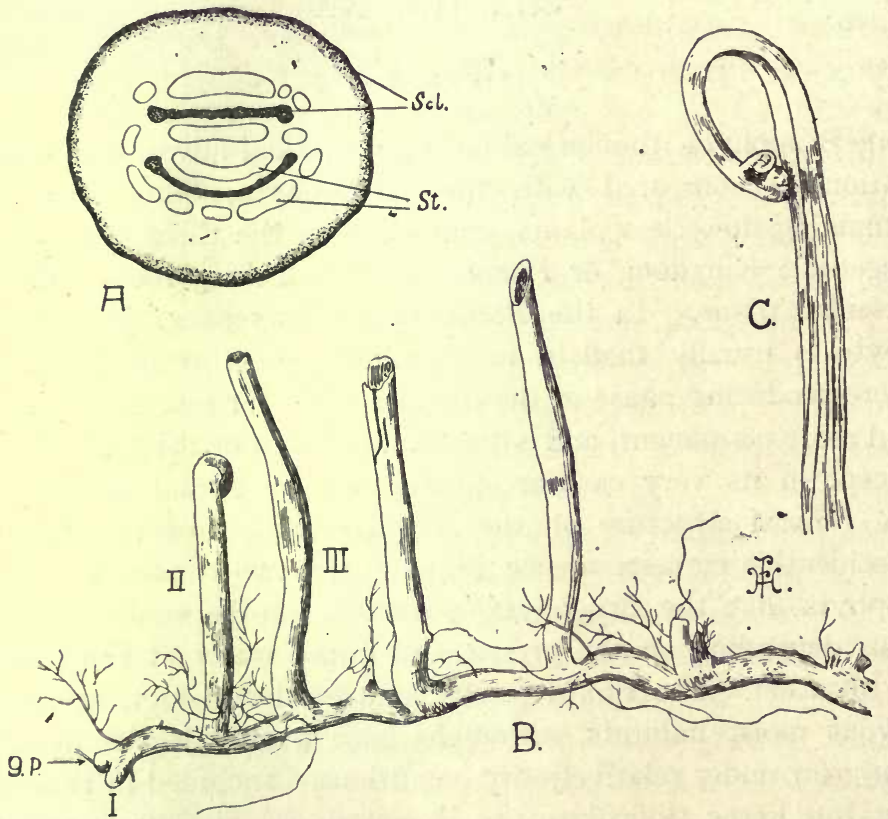

FIG. I58.-The Bracken (Pteris aquilina). A, Diagram of transverse section of rhizome. Scl., sclerenchyma; St., steles. B, Rhizome showing apex with growing point (g.p.), successively older leaves (I, II, III), and the bases of leaves of former seasons. C, Young frond.

of the rhizome, which adds appreciably to its apparent thickness, are the adhering bases of the leaves, which persist after lamina and petiole have died away. All the roots of the adult Fern are adventitious (Fig. I58, B) ; they usually arise in the neighbourhood of the leaf-bases, and are generally black in colour and forked.

The young leaf is mostly more or less densely clothed with

1 Also known as Aspidium filix-mas and Lastraa filix-mas. 
brown scaly hairs or ramenta, many of which are shed as the blade unfolds ; they usually persist, however, upon the petioles (Fig. I59, A, r.) and on the leaf-bases clothing the older parts of the stem. As the leaf-rudiment produced behind the growing point gradually develops, the axis of the lamina becomes coiled after the manner of a watch-spring, and, in the case of compound leaves, the individual portions become inrolled in a similar way (Fig. I58, C). Each segment of the blade grows by means of an apical cell, which thus occupies a protected position within the spiral formed by the maturer parts. These features are readily observed in a young Bracken-frond, as it emerges from the soil in spring, and the prolonged growth of its tip is evidenced by the retention of the coiled character in the uppermost portion, long after the older basal portion is fully expanded. The development of Fernleaves is usually very slow, the rudiment being produced and undergoing gradual enlargement for two or more years before

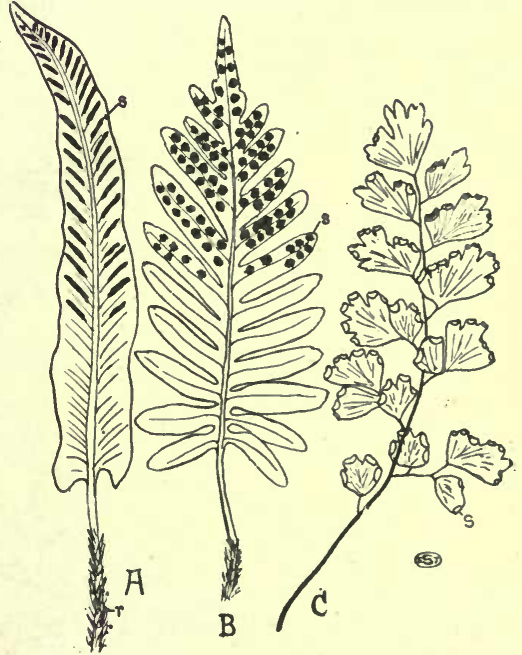

FIG. I59.-Entire fronds of A, Hart's Tongue Fern (Scolopendrium vulgare); B, Polypody (Polypodium vulgare); and $\mathrm{C}$, Maidenhair Fern (Adiantum). $\quad r_{\text {., }}$ ramenta ; s., sor1. (All three considerably reduced.)

the frond appears above the surface (cf. Fig. I58, B, I, II, III).

Not all Fern-leaves are as deeply compound as in the Bracken (Pteris aquilina) or Lady Fern (Athyrium filix-fomina). Those of the Polypody (Polypodium vulgare, Fig. I59, B) and Hard Fern (Blechnum spicant, Fig. I72), for instance, are simply pinnate, whilst those of the Hart's Tongue Fern (Scolopendrium vulgare, Fig. I59, A) are undivided. The venation is exceedingly characteristic, the midribs of the leaves or pinnæ, as the case may 
be, bearing numerous forked lateral veins which are not connected by cross-branches (Fig. I63, A, B).

A general idea of the more characteristic features in the internal structure of the stem can be gathered from a study of the Bracken rhizome. In transverse section (Fig. I58, A) this is seen to be traversed by two rings of vascular strands or steles $(S t$.). Separating the rings are two bands of dark brown sclerenchyma $(S c l$.$) , and the same tissue also forms a layer$ beneath the epidermis; its elements (Fig. I6I, Scl.) differ from

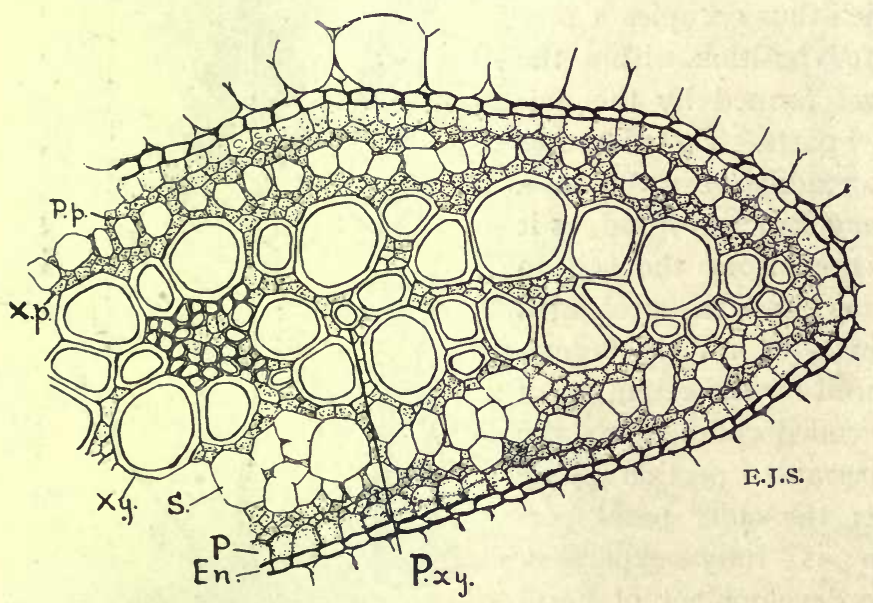

FIG. I6o.-Part of a stele from the rhizome of the Bracken (Pteris aquilina) in transverse section. En., endodermis ; P., pericycle; P.p., phloemparenchyma ; P.xy., protoxylem ; $S$., sieve-tube ; $X . p$. , xylemparenchyma ; $X y$. , xylem.

the fibres of higher plants in being short and relatively thinwalled. The remaining ground tissue is parenchymatous, and contains an abundance of starch.

The vascular strands are concentric (Fig. I60), with the component tissues more or less symmetrically arranged. The term stele is customarily applied to these concentric strands of Ferns, but this does not necessarily imply that they are comparable to the entire vascular cylinder of a higher plant. Each stele is bounded by an endodermis $(E n$.$) , with dark brown mem-$ branes, and a pericycle $(P$.$) , composed of rather larger thin-$ 
walled cells having comparatively few contents. Next follow one or two layers of much smaller cells, constituting the so-called phloem-parenchyma (P.p.). Immediately within is a conspicuous zone consisting chiefly of large, empty-looking, thin-walled elements, the sieve-tubes $(S$.$) , which are often absent towards$ the ends of the strands, and which are separated from the central xylem by a zone of small-celled wood-parenchyma (X.p.). The bulk of the xylem consists of large tracheids $(X y$. $)$, but near the ends and towards the centre of the strand, small elements, representing the protoxylem $(P . x y$.$) , can usually$ be recognised. There is generally a small central mass of wood-parenchyma.

In longitudinal sections, cut so as to pass radially through a stele (Fig. I6I), the sievetubes (s.) appear as elongated structures with tapering ends, and bearing the sieve areas on their sloping radial faces. Under the high

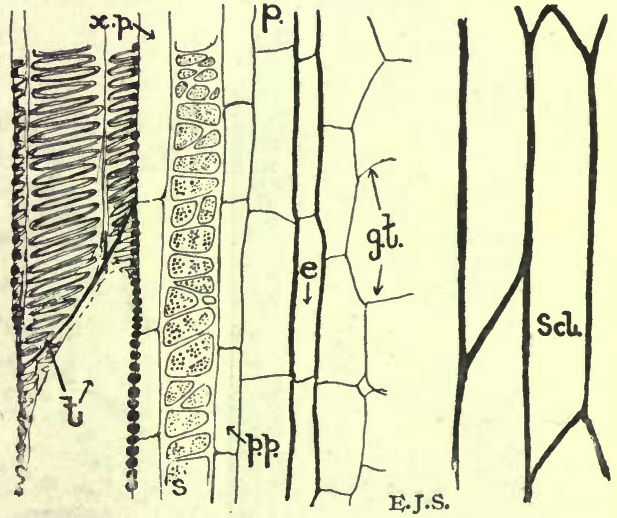

FIG. I6r.-On the left, part of a radial longitudinal section through one of the steles of the Bracken-rhizome. e., endodermis ; g.t., ground tissue; $p$., pericycle; $p . p .$, phloempa renchyma ; $s$. , sieve-tube ; $t$., tracheid of the metaxylem; x.p., xylem-parenchyma. On the right, a little of the sclerenchyma $(S c l$.$) in longitudinal section.$

power these sieve areas exhibit a rather irregular ladder-like (scalariform) thickening of shining bars, with intervening darker zones exhibiting a fine dotting and bearing a number of highly refractive adhering granules. The longitudinal walls of the metaxylem tracheids $(t$.) exhibit several rows of closely arranged oblong bordered pits, producing an exceedingly characteristic type of scalariform thickening, whilst the protoxylem tracheids are spirally thickened in the usual way.

The number of vascular strands observed in a transverse 
section of the stem varies considerably in different Ferns. Some (e.g. Gleichenia, the Bracken of the Tropics) possess but a single central stele of comparatively large size, whilst many of the Tree Ferns exhibit a complex system of concentric rings of steles. In the Male Fern (Nephrodium filix-mas), again, the strands form but a single ring (Fig. I62, B, St.). The arrangement and histological structure of the vascular tissues within each stele is, however, essentially the same in all cases. The young Fern
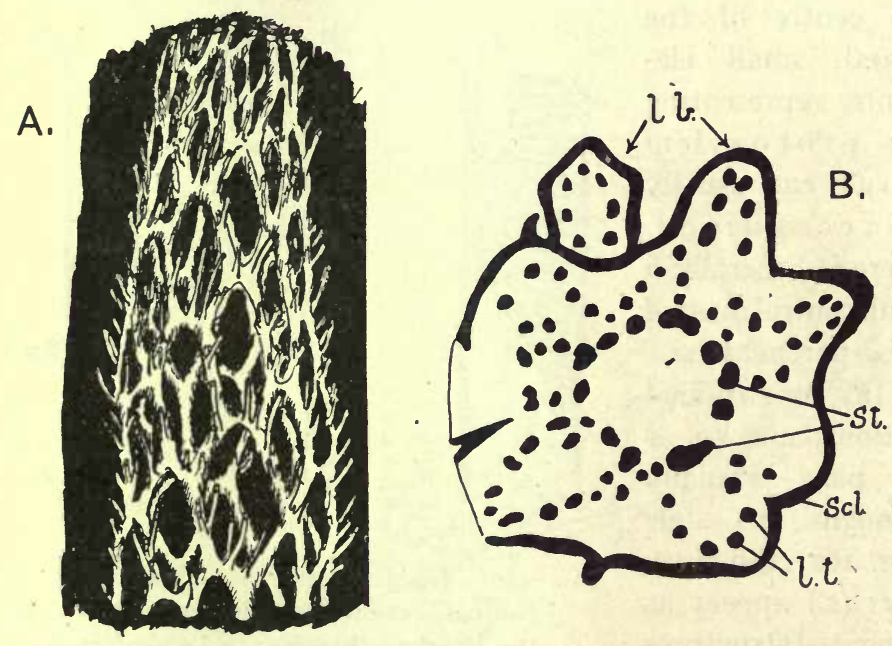

Ii'1G. I62.-Nephrodium filix-mas. A, Skeleton of vascular system showing the cylindrical network of steles, with numerous leaf-trace strands arising from the edges of the "leaf-gaps" (original). B, Transverse section of the rhizome (after De Bary). l.b., leaf-bases; l.t., leaftrace strands ; $S c l$., sclerenchyma; St., stem-steles.

seedling always possesses but a single central strand which, as the plants become older, enlarges and, in forms like Nephrodium and Pteris, gradually breaks up into the separate steles of the adult.

The relations between the vascular system of stem and leaf are particularly plain in the Male Fern (Nephrodium), and are easily deciphered, if its rhizome be allowed to rot, so that all but the hard vascular tissues decay. In the resulting skeleton (Fig. I62, A) the steles form a cylindrical network, in which each 
mesh or leaf-gap corresponds in position to the attachment of a leaf. The latter is supplied by the numerous fine strands passing out from the margin of the mesh. These leaf-traces can be recognised, in a transverse section of the rhizome (Fig. I62, B), as arcs of small strands (l.t.) occupying the lobes that represent the leaf-bases (l.b.) In the case of the Bracken (Pteris) a series of transverse sections, cut in the region of the node, shows that the leaf-base is supplied by several strands passing off from the outer ring. The gap thereby created is filled by steles from the inner series, which pass out, through a break in the sclerenchyma-ring, on the side towards the leaf-trace. The petiole in both these Ferns is thus traversed by a large number of strands, normally forming an arch, but in certain genera (e.g. Gleichenia) the leaf-stalk, like the stem, contains but a single stele.

In its general structure the leaf conforms to the ordinary dorsiventral type. Since many Ferns grow in shady situations, the epidermis often contains chloroplasts, and the palisade tissue is not uncommonly poorly developed or the mesophyll even spongy throughout (cf. Fig. I65). The ultimate branches of the concentric strands traversing the petioles become collateral within the pinnæ, owing to development of the phloem only on the lower side.

The roots of most Ferns are diarch, and owe their frequent wiry character to the development of an exceedingly thickwalled sclerenchymatous cortex, but otherwise do not differ appreciably from those of higher plants. The tetrahedral apical cell, by whose divisions the tissues of stem and root are formed, has already been described on p. I8.

In the ordinary course of events the Fern-plant sooner or later commences to produce spores. These are developed within small, stalked, usually brown-coloured sporangia, almost invariably borne on the under-surfaces of the fronds, which in some few cases are completely covered by them (e.g. RustyBack). Most commonly, however, they are arranged in numerous separate groups, or sori, each usually comprising a considerable number of sporangia arising from a slight central swelling of the leaf-tissue, known as the placenta (Fig. $x 65, p l$. .).

Such sori are well seen in the Common Polypody (Polypodium 
vulgare, Fig. I59, B, s.), where they appear as small circular brown patches situated over the lateral veins of the pinnæ. A similar arrangement obtains in the Male Fern (Nephrodium, Fig. I63, B), but here each sorus is protected by a kidney-shaped outgrowth (indusium, In.) of the placenta, which withers when the sporangia are mature. In the Maidenhair Fern (Adiantum, Fig. I59, C, s.) and the Wall Rue (Asplenium ruta muraria) the sori are near the edges of the pinnules, and are covered by indusia taking the form of little flaps projecting inwards from

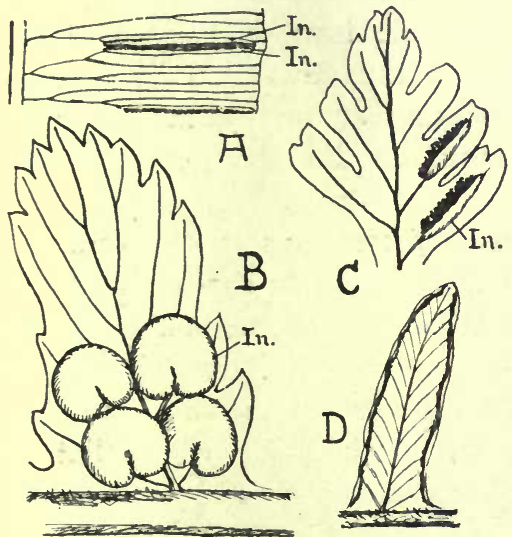

FIG. I63.-Sori of various Ferns. A, Hart's Tongue Fern (Scolopendrium). B, Male Fern (Nephrodium). C, Asplenium. D, Bracken (Pteris). In., indusium. the margin, whilst in the Bracken (Pteris, Fig. I63, D), where the sporangia form a continuous fringe all round the margin of the pinnule, the incurved edge of the latter functions as an indusium.

A peculiar type of sorus is found in the Filmy Ferns (Hymenophyllaceæ), which are so-called because of their delicate fronds, consisting of but a single layer of cells. Though mainly encountered in the Tropics, they are represented in Britain by one or two species (e.g. Hymenophyllum tunbridgense, Fig. I64) occurring in damp caves and other humid situations in rocky districts. Here the sporangia arise from rod-like placentæ (s.) projecting from the leaf-margin, and each sorus is enveloped at its base in a cup-shaped indusium (i.).

In transverse sections through a fertile pinnule of Nephrodium filix-mas (Fig. I65), the bulging placenta ( $p l$.) on the underside is seen to be supplied by tracheids $(t$.) from the overlying vascular bundle. The indusium (i.) appears as an umbrella-shaped outgrowth, one layer of cells thick, arising from the top of the placenta. On the sides of the latter are borne numerous sporangia in various stages of development. 
A mature sporangium (cf. also Fig. I66, B) consists of a stalk of usually three rows of elongated cells, terminated by a biconvex capsule which is more or less oval in side-view, and encloses the spores within a wall of a single layer of cells. The cells of the wall fit firmly together, and most of them are thin-walled and very flat, appearing more or less polygonal in surface-view, but tabular in optical section. The edge of the capsule, however, is occupied by a single row of specially differentiated cells. The greater part of this band, from the base of the capsule on one side to half-way down the other edge, is composed of cells which have all but their outer walls strongly thickened, and which constitute the annulus ( $a$. in Fig. I65 and an. in Fig. I66, B). The remainder, forming the so-called stomium, are much broader and thin-walled throughout (Fig. I66, B, s.), and it is here that the mature capsule ruptures.

Each sporangium arises as a papillate outgrowth from a single surface cell of the placenta, which is cut off by a crosswall, and undergoes division by three oblique septa to produce a tetrahedral apical cell (Fig.

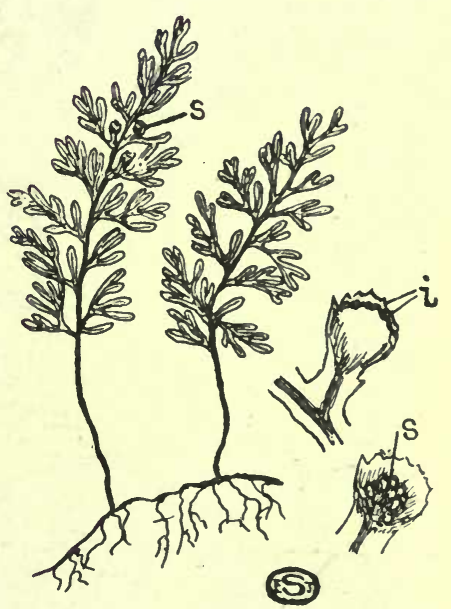

FIG. I64.-On the left part of a Filmy Fern (Hymenophyllum tunbridgense), bearing sori (s.). The two smaller figures on the right show a sorus, entire and in longitudinal section respectively. $i$., indusium; $s$., sorus. I66, C, I, II). The three rows of segments, primarily cut off parallel to the three flat faces of the latter (III), elongate and become the stalk. Next, walls are formed parallel to all four faces of the apical cell (IV), and give rise to the one-layered capsule-wall (Fig. I66, D and E, w.), an inner nutritive layer, or tapetum $\left(t_{0}\right)$, and a central cell, the archesporium $(a$.$) . The$ last-named divides to form the spore mother-cells, each of which gives rise to four spores (cf. p. 309). Their development takes place at the expense of the food-materials contained in the 


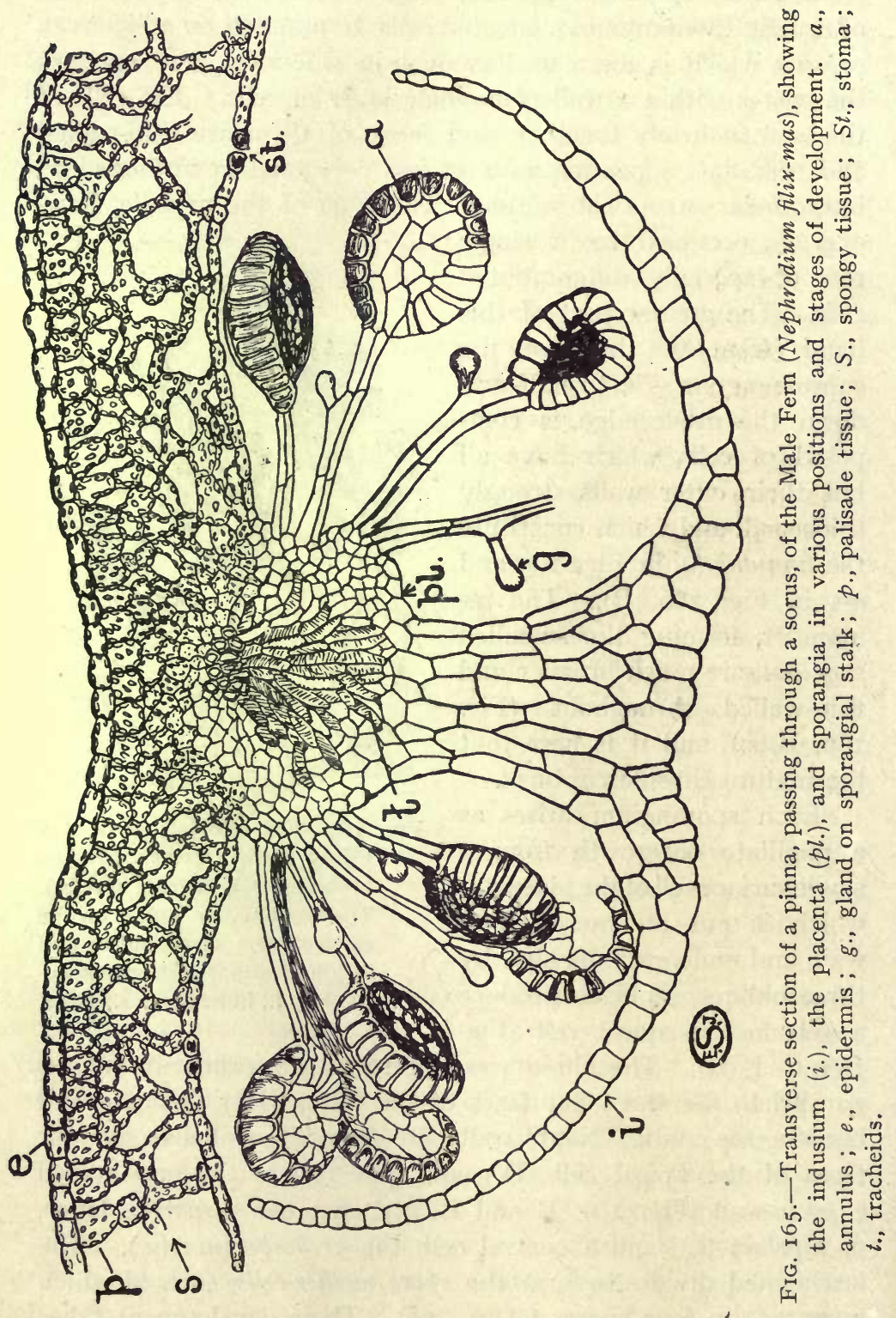


granular thin-walled cells of the tapetum, which thus disorganises before the sporangium matures.

With the ripening of the sorus the indusium dries and shrivels, exposing the sporangia, which likewise begin to lose moisture. As evaporation proceeds, the thin outer walls of the annulus
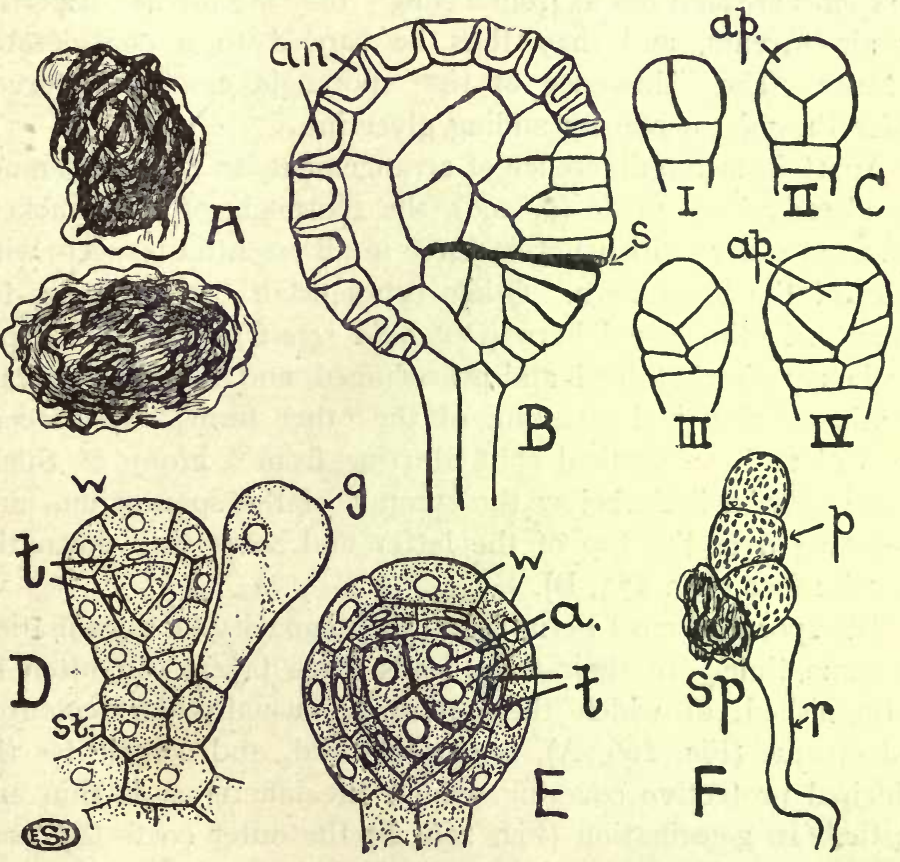

FIG. I66.-Nephrodium filix-mas. A, Mature spores, greatly magnified. B, A single sporangium, showing the annulus (an.) and stomium (s.). C, Early stages (I-IV) in the development of the sporangium (after Müller). $\mathrm{D}$ and $\mathrm{E}$, Successively later stages. $a_{\text {., }}$ archesporium; $a p$. , apical cell ; g., gland ; $S t .$, cells of stalk; $t$., tapetum ; $w_{\text {., wall. }}$ $\mathrm{F}$, Germinating spore (sp.). p., beginning of prothallus ; $\boldsymbol{r}$., rhizoid.

commence to bulge inwards (cf. Figs. I65 and I66, B), owing to the gradual reduction in the volume of their sap. Thus an increasing tension is set up in the annulus, ${ }^{1}$ which eventually

1 It will be realised that the side-walls of the annulus are much less thickened than the horizontal walls, so that the contraction is entirely in the longitudinal direction. 
leads to rupture of the capsule-wall along the plane of greatest weakness, viz. the junction between the transversely extended cells of the stomium (Fig. I66, B). On the sudden release of the tension the annulus, carrying with it a great part of the capsule-wall and many of the contained spores, flies back beyond the position of equilibrium, and, with the subsequent recoil, the spores are shot out as from a sling ; they are further dispersed by air-currents, and may thus be carried to a considerable distance. The dehiscence of ripe sporangia can be observed under the microscope by adding glycerine.

Apart from the difference of arrangement, to which reference has already been made (p. 296), the sporangia of the Bracken, and most other British Ferns, agree in all essential respects with those of the Male Fern. Other types occur, however, as, for instance, in the Royal Fern (Osmunda regalis). Here the sporangia are short-stalked and pear-shaped, and lack the characteristic annulus and stomium of the other forms. Dehiscence takes place by a vertical split starting from a group of thickwalled cells, a little below the summit of the sporangium, and extending over the top of the latter and some way down the opposite face (Fig. I73, B).

The spores of most Ferns retain their capacity for germination for some time. In their thick walls three layers can often be distinguished, of which the outermost, usually dark-coloured and opaque (Fig. I66, A), is cuticularised, and constitutes the principal protective covering, whilst the innermost is thin and elastic. In germination (Fig. I66, F) the outer coats $(S p$.) are burst, and the innermost is stretched to form a long, colourless, tubular outgrowth, which becomes separated off from the main body of the spore by a transverse wall, and penetrates the soil as the first rhizoid $(r$.$) of the future plant. The remainder$ of the spore-contents, enveloped by the innermost layer of the wall, lengthen into a short horizontal filament $(p$.$) whose few$ cells contain chloroplasts. Within the terminal cell two intersecting walls soon arise, and the apical cell thus formed cuts off segments on either side, so that the filament widens into a flat plate.

As the front margin broadens, two or more of its cells become meristematic (Fig. I67,g.p.) and development proceeds rapidly; 
at the same time horizontal divisions in the middle region of the plate form the cushion, which is several cells thick. The green thalloid plant thus established is known as a prothallus, and when mature is usually heart-shaped, sometimes attaining a diameter of over a centimetre (Fig. I67). All the cells are alike and contain chloroplasts. From the underside, especially

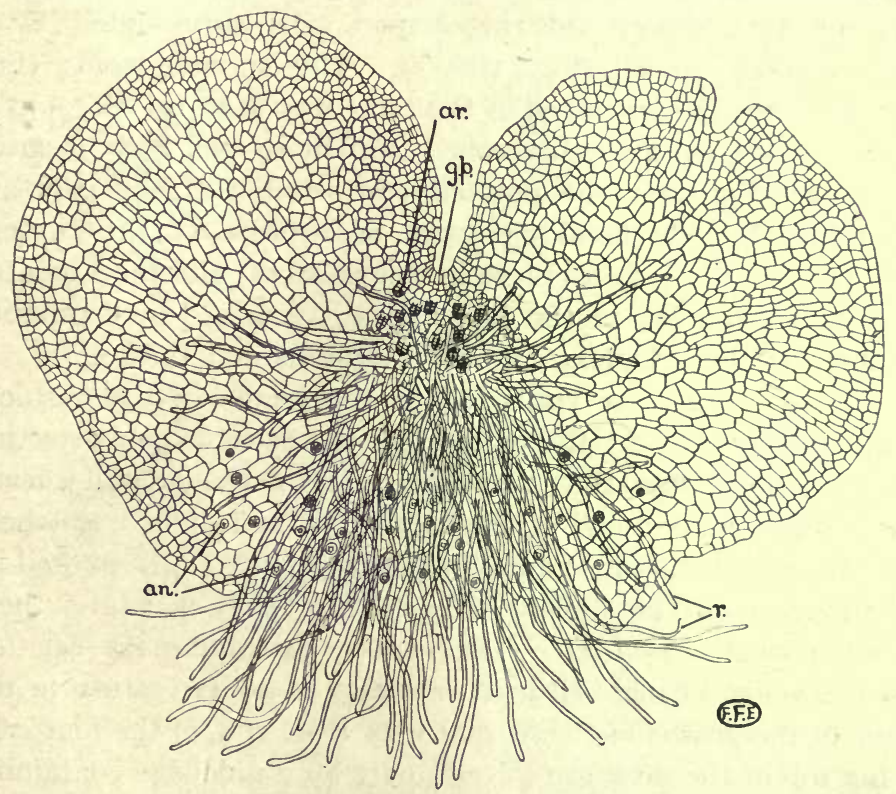

FIG. I67.-Fern-prothallus (magnified about thirty-five times), from the under-surface. A considerable number of antheridia (an.) are seen, on the older part, and the projecting necks of twelve archegonia (ar.), in the region of the cushion, from which also numerous rhizoids $(r$.) arise. g.p., growing point.

in the region of the cushion, scattered superficial cells grow out as colourless rhizoids $(\boldsymbol{r}$.$) , which serve for anchorage and the$ absorption of moisture.

When the prothalli grow densely crowded, they often remain more or less filamentous, a condition which is normal for most of the Filmy Ferns.

1. The prothallus is the Fern-plant (gametophyte) that bears the sexual organs. These are of the same general type as in 
Bryophyta, and are produced by the outgrowth of single surfacecells. They are situated on the lower surface, and both kinds usually occur on the same prothallus (Fig. I67). The antheridia (an.), found mainly on the thinner marginal wings and the less robust prothalli, are almost spherical structures (Fig. I68, g.) with a small number of relatively large spermatozoid mothercells (s.). The enveloping wall (w.) is peculiar in being composed only of two superposed ring-shaped cells surmounted by a dome-shaped cap-cell (Fig. I68, e). The spermatozoids (Fig. $I 68, d)$, which are developed in the same way as in the Bryophyta (cf. p. 277), have a spirally coiled, spindle-shaped body bearing numerous cilia near the pointed end. Dehiscence of the antheridium, by the breaking away or rupture of the cap-cell (Fig. I68, $e$ and $f$ ), takes place in wet weather, and the liberated spermatozoids swim in the film of water retained by capillarity between the lower surface of the prothallus and the soil.

The archegonia are restricted to the region of the cushion, in which they are partially embedded, the necks alone projecting (Fig. I67, ar. ; Fig. I68, $a$ and $b$ ). The necks are all curved towards the pointed end of the prothallus, and differ from those of a Bryophyte archegonium in being short and composed of only four longitudinal rows of cells (Fig. I68, c), whilst there is but a single canal cell. The venter, containing the egg (o.) and the ventral canal cell (v.c.), appears as a mere cavity in the tissue of the prothallus. At maturity (Fig. I68, $b$ ) the four cells at the top of the neck are forced apart by a mucilage containing malic acid, formed during the disorganisation of the canal cells, and an open passage is thus left leading down to the egg. It is apparently the malic acid that attracts the spermatozoids to the archegonia.

After fertilisation the ovum becomes enveloped by a thin membrane, and divides by three successive walls into octants of a sphere (Fig. I69, A). Their further segmentation leads to the differentiation of four apical cells, which are situated one in each quadrant, and which respectively give rise to the root, the stem, the first leaf, and the "foot" of the embryo. The foot develops as a large parenchymatous sucker which becomes firmly lodged in the tissue of the cushion, from whose cells it absorbs nourishment for the young Fern (Fig. I69, B, $f$ ). 
By this means rapid growth of the root (Fig. I69, B, r.) takes place, and it soon pierces the prothallus and penetrates into the soil. Simultaneously the first leaf $(l$.$) , carrying with it the still$ rudimentary stem (s.), emerges on the lower side, and, arching up through the notch at the front end of the heart-shaped prothallus, exposes its green blade to the light. The stem now grows more vigorously, giving rise to adventitious roots which
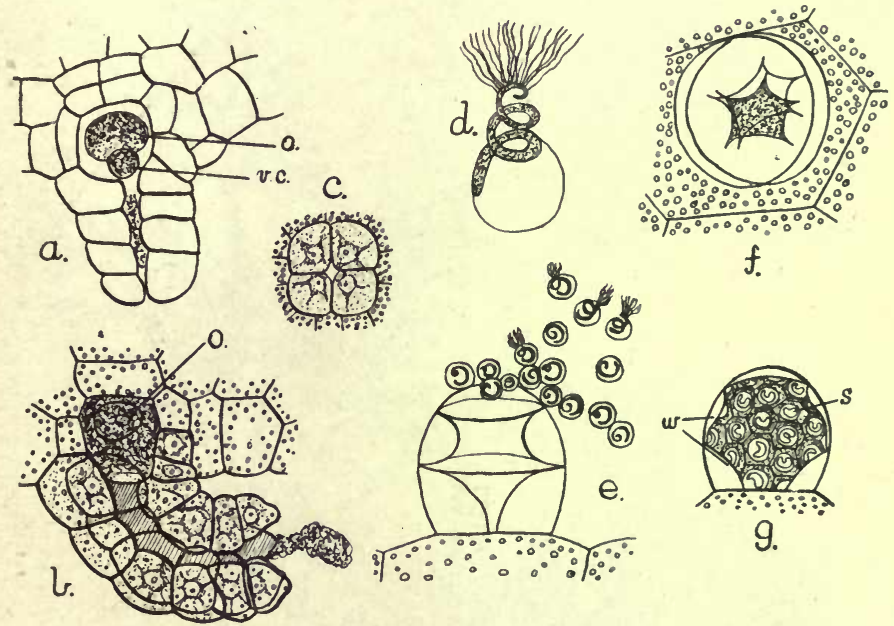

FIG. I68. - $a-c$, archcgonia, $e, f$, and $g$, antheridia, and $d$, single spermato zoid of the Fern. $a$, immature, and $b$, mature, archegonium in longitudinal section; $c$, neck in surface view. o., egg; v.c., ventral canal cell. $g$, almost mature antheridium in optical section, showing the wall $(w$.$) and the spermatozoid mother-cells (s$.$) ; e$, dehiscing antheridium with the cscaping spermatozoids ; $f$, dchisced antheridium from above. ( $a$ after Goebel ; the remainder after Kny.)

replace the short-lived primary root, and it is not long before further leaves develop (Fig. I69, C). The latter, however, show but a very gradual increase in complexity, from the first, often almost undivided leaf, to the normal compound foliage of the adult Fern, which is frequently only attained after the lapse of several years. Production of sporangia is generally deferred till this stage is reached.

The young Fern, like the sporophyte of Liverworts and Mosses, is thus for a time dependent for nourishment on the 
prothallus. But, as soon as the root has become established in the soil, and the first leaf has spread out its lamina to the light, this dependence ceases, and soon after that the prothallus withers away (cf. Fig. I69, C). As compared with Bryophyta, the relative importance of the two phases in the life-history is therefore reversed. The free-living sporophyte usually attains large dimen-
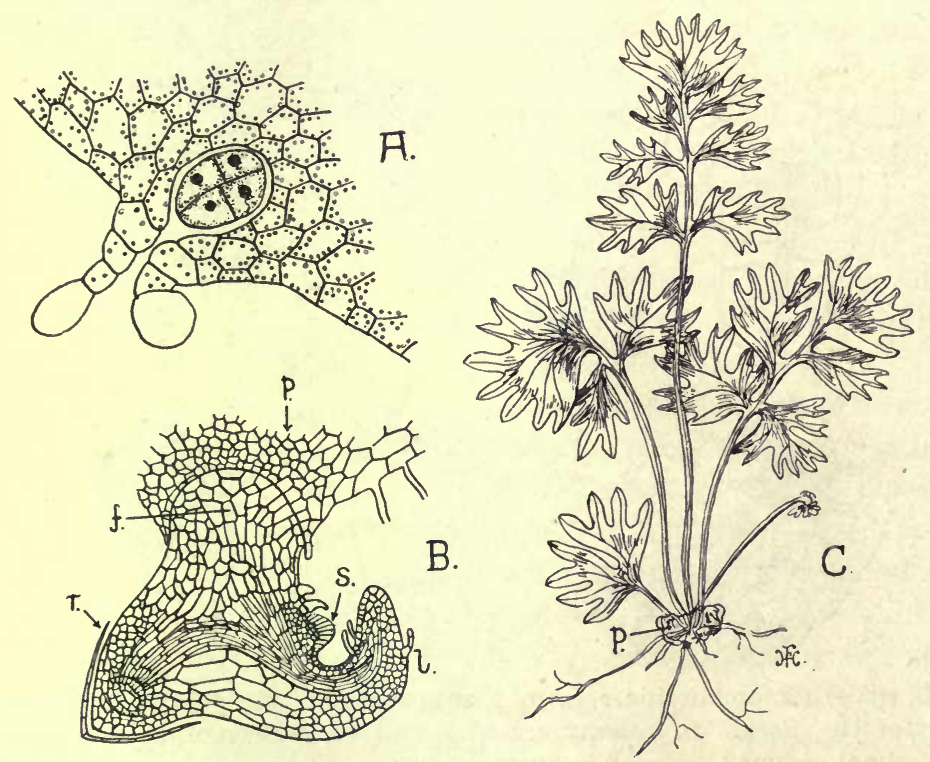

FIG. I69.-Embryology of the Fern. A, Longitudinal section through an archegonium, showing the octant-stage in the division of the fertilised egg (after Sadebeck). B, Young embryo escaping from the prothallus (p.) (after Hofmeister). $f$., foot; $l$., first leaf; $r$., first root ; $s .$, stem. C, Young plant, with the remains of the prothallus ( $p$.) at the base, four unfolded leaves of increasing complexity, and one leaf not yet unfolded (original).

sions and exhibits a complexity of structure which is only approached by the perennial gametophytes of some few Mosses. On the other hand, the sexual generation is as a general rule short-lived, though in a few Ferns (e.g. the Royal Fern, Osmunda) it persists for some years. Normally the two generations alternate regularly with one another, but occasional abnormalities are encountered. 
Thus, in certain Ferns the prothalli may arise by direct budding from the leaves or sporangia, without the formation of spores (apospory), a condition that has been experimentally induced in a variety of the Lady Fern (Athyrium filix-foemina) by pinning detached segments of the fronds on damp sand. In other cases the sporophyte develops vegetatively from the prothallus without the intervention of sexual organs (apogamy). ${ }^{1}$

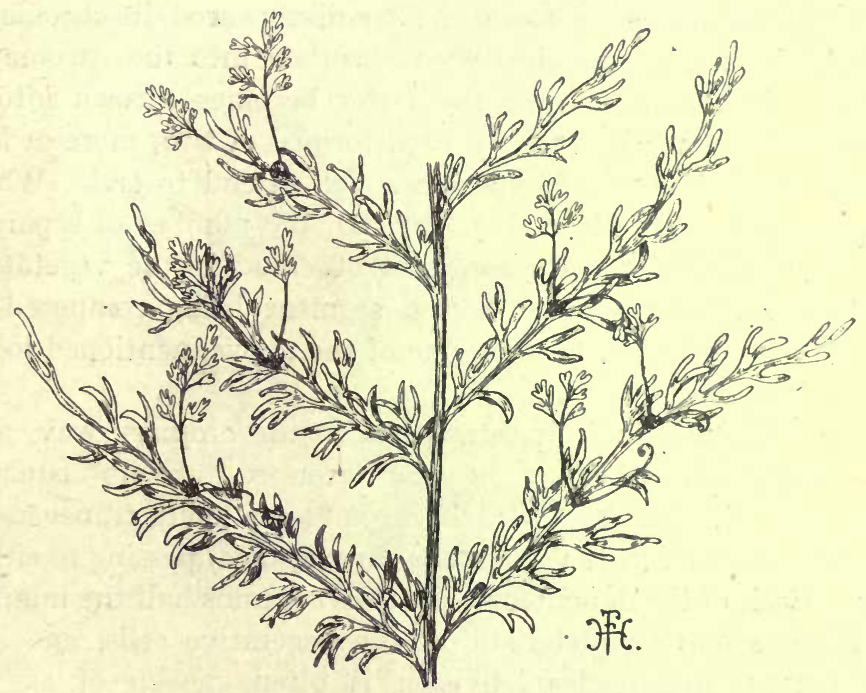

FIG. I70.- Small part of a frond of Asplenium bulbiferum, showing the vegetative production of new plants (about half natural size).

Vegetative multiplication of the sporophyte is not infrequent, new plants arising from buds formed on the surface of the fronds, as in the commonly cultivated Asplenium bulbiferum (Fig. I70).

In the normal life-cycle, of Bryophyta and Pteridophyta alike, the spore is the starting-point of the sexual, and the oospore of the asexual, generation. The spore mother-cells almost invariably give rise to four spores, after undergoing two successive nuclear divisions. The nuclear changes involved differ, however, in several important respects, from those observed in the ordinary vegetative divisions of the plant (cf. p. 2I et seq.).

1 This is analogous to the loss of sexuality observed among Fungi (cf. pp. 236, 245). 
Prior to the first division the chromatin reticulum concentrates into its constituent chromosomes, but these soon appear joined together to form a thread. The latter thereupon contracts in a tangled manner around the nucleolus, towards one side of the nuclear cavity (Fig. I7I, A) ; this stage, which is termed synapsis, has in other groups been recognised in living material, and is not therefore a contraction due to methods of fixation.

As the thread spreads out again, on emerging from this condition, the nucleolus is seen to have disappeared, its chromatic material having presumably been absorbed into the chromatin thread. At the same time the latter becomes thrown into a number of loops (Fig. I7I, A), each formed of two, more or less closely approximated, chromosomes, joined end to end. When segmentation takes place (Fig. I7I, B), the number of separate portions produced is only half that observed in the vegetative divisions of the same plant, each segment being composed of the two chromosomes forming one of the above-mentioned loops (Fig. I7I, C).

The nuclear spindle is established in the ordinary way, and the double chromosomes become grouped at its equator (Fig. I7I, D). Thereupon each chromosome splits transversely into two, one half (actually a whole chromosome) passing to either pole. Each of the daughter-nuclei thus contains half the number of chromosomes characteristic of the vegetative cells, and, for this reason, this nuclear division is often spoken of as the "reduction" division. The second nuclear division follows almost immediately on the first, and does not differ essentially from that described for the ordinary vegetative cells. ${ }^{1}$.

Each of the four resulting spores (Fig. I7I, E) thus possesses the halved number of chromosomes, and so do all the cells of the gametophyte arising from a spore. The normal number of chromosomes is restored in the fertilised egg, in consequence of the fusion of the male and female nuclei. It will be realised that the reduction division differs chiefly from the normal type in the occurrence of synapsis and in the passage of entire chromosomes to the respective poles of the nuclear-spindle. Moreover, there is good reason for believing that the associated chromosomes

1 The phenomena exhibited during the two nuclear divisions in the spore mother-cells are often referred to as meiosis. 
of each separating pair respectively represent the chromatic material of the two parents from which the given organism arose (cf. p. 390).

The importance of this phenomenon is also emphasised by the occurrence of an essentially similar method of reduction in animals. Reduction occurs in the formation of spores, not only in all Bryophyta and Pteridophyta, but also in the higher plants (Phanerogams). In the case of the Thallophyta the actual reduction has only been observed in relatively few cases, but it cannot

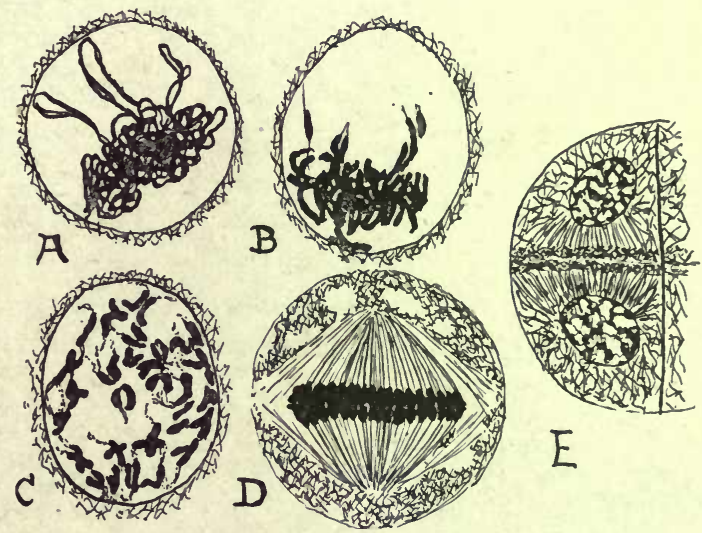

FIG. I7I.-Nuclear divisions in spore mother-cells of Nephrodium (after Yamanouchi). A, Late synapsis. B, Segmentation of chromosomes. C, Association of chromosomes. D, Equatorial plate. E, Part of a tetrad.

be doubted that it takes place in all forms exhibiting a sexual process. It would appear, however, that it may be effected at various stages in the life-history, sometimes (as in Spirogyra) during the first division in the zygospore, in other cases (e.g. Fucus) during the formation of the sexual cells. It may be noted that the latter is the customary stage for the occurrence of the reduction division amongst animals.

The four nuclei, produced in the spore mother-cell, usually become grouped so as to form a tetrahedral figure. Each, together with part of the cytoplasm, becomes surrounded by a separate wall, the whole of the contents of the mother-cell being 
thus used up to form four spores (Fig. I7I, E). Their faces of contact are more or less flattened and triangular, while the outer walls are convex, so that each spore, at its inception, has the form of a tetrahedron with a rounded base. In many cases, however, the spores round off after the mother-cell membrane has broken down.

In most Ferns there is only one kind of frond which fulfils the ordinary functions of a leaf, as well as those connected with

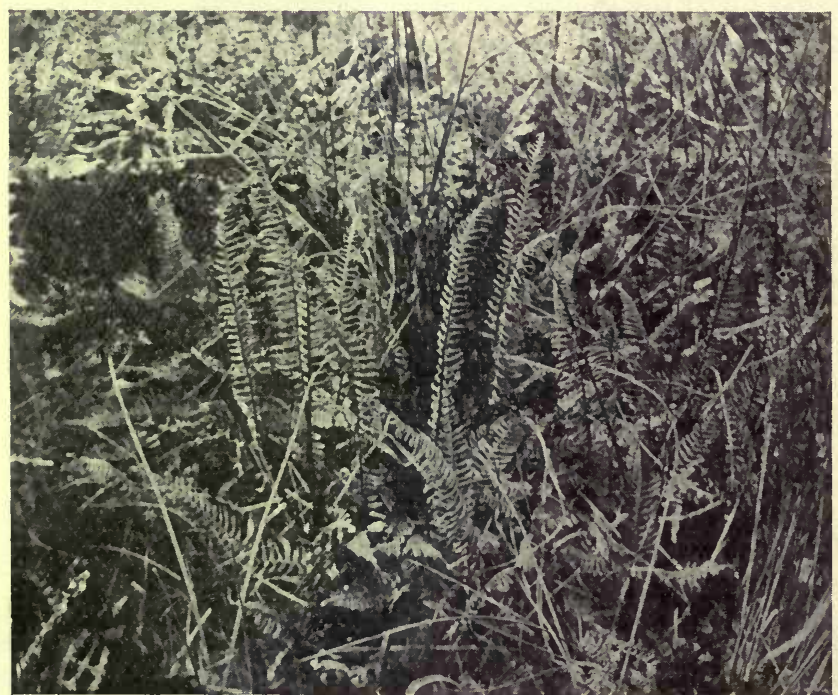

FIG. I72.-The Hard Fern (Blechnum spicant), showing foliage leaves and sporophylls. [Photo E. J. S.]

spore-production. In a few species, however, there is division of labour, as in the Hard Fern (Blechnum spicant, Fig. I72). Here some of the leaves, spreading out near the surface of the ground, have broad pinnæ and are purely vegetative, whilst others, which assume an erect position, have narrow lobes and are almost entirely concerned in the production of sporangia. Such fertile leaves are called sporophylls.

A less marked specialisation is seen in the Royal Fern (Osmunda regalis), where the lower part of the huge frond is sterile, whilst the upper pinnules, bearing the sporangia on both 
sides, exhibit practically no lamina (Fig. I73). In this connection it may be noted that in some tropical Ferns (e.g. Platycerium), which grow aloft on the branches of trees (a habit commonly seen

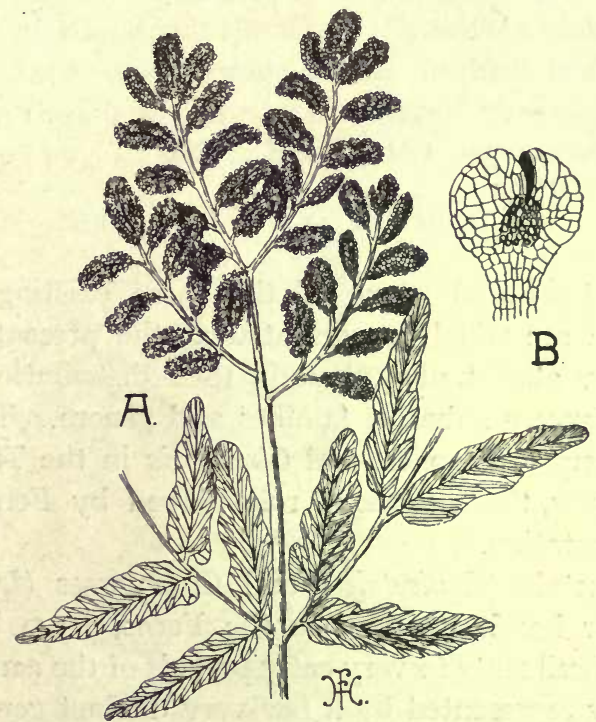

FIG. I73.-A, Small part of a frond of the Royal Fern (Osmunda regalis), showing sterile pinnules (below) and fertile pinnules (above). (About half natural size.) (Original.) B, Single sporangium (after Luersen).

in the Polypody in this country), the lowest leaves are modified to form an oblique bracket by which humus is retained.

A greater contrast between leaves and sporophylls is met with in other groups of Pteridophyta, some of which are considered in the next chapter. 


\section{CHAPTER XXI \\ Horsetails and Clubmosses}

THE Ferns (Filicales) alone of the three existing groups of Pteridophyta are widely represented at the present day. Not only are they almost ubiquitous in their distribution, but they comprise a large number of families and genera. The Bracken in North Temperate zones, and Gleichenia in the Tropics, illustrate, moreover, the important rôle played by Ferns in many types of vegetation.

The Horsetails (Equisetales) and Clubmosses (Lycopodiales), on the other hand, which, like the Ferns, have been traced back in the fossil state to very early periods of the earth's history, are now only represented by a few very distinct genera. These groups flourished vigorously, however, at the period when the Coal Measures were laid down; then they comprised woody plants which, in great part, attained to the dimensions of trees (Fig. I77), at least sixty feet high in the case of Calamites. These features have been lost by the living forms of the present day, which are herbaceous and of small dimensions. It is, indeed, probably correct to regard the remote past as the age of trees and the present rather as that of herbs.

By contrast with Ferns the leaves of Horsetails and Clubmosses are remarkably small and simple in form, so that the habit of the plant is here determined mainly by the character and extent of branching of the stem (Fig. I74, B and C; Fig. I80, A). The Horsetails (Equisetum) are switch-plants (Fig. I74, B and C) whose green, longitudinally furrowed, stems bear whorls of brownish scale-leaves fused to form a toothed sheath $(S l$.) around each node. The branches are likewise whorled (Fig. I74, B), and, since they arise relatively late, have to pierce their way through the bases of the fused leaves. The 
erect shoots are the upturned ends of the branches of horizontal underground rhizomes (Fig. I74, C), which bear similar whorls of scale-leaves, as well as numerous adventitious roots, at each node, the primary root dying away at an early stage, as in Ferns. The rhizome of the Field Horsetail (Equisetum arvense) bears, in addition, tuber-like storage-organs formed from modified branches. In the ancient Horsetails (Calamites) of the Palæozoic, leaves (cf. Fig. I75, B) and branches were likewise whorled.

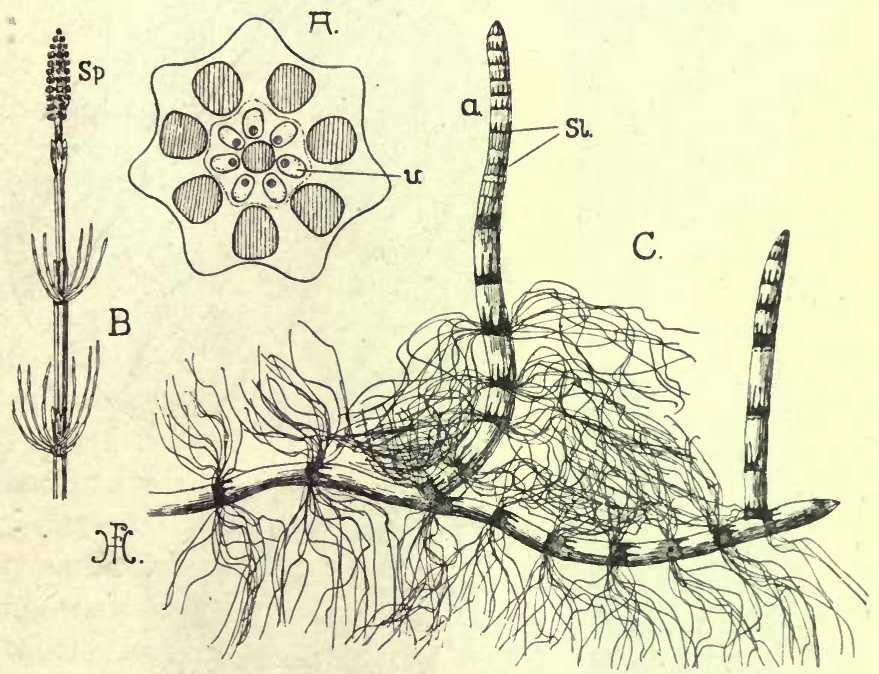

FIG. I 74--A, Section of stem of Equisetum palustve (after Pfitzer), showing the numerous air-spaces (shaded) and the ring of vascular strands $(v$.). B, Upright, fertile shoot of $E$. palustre, bearing a terminal cone (Sp.). C, Habit of E. limosum, showing the subterranean rhizome, with numerous adventitious roots arising from the nodes and two aerial shoots (a.). Sl., whorls of scale-leaves. (B and C original.)

The anatomy of the stem superficially resembles that of a Dicotyledon in the arrangement of the vascular tissues, and invariably shows great reduction of the xylem and a conspicuous system of air-canals in the cortex and pith (Fig. I74, A). These aquatic characteristics are to be expected in the case of partially submerged species like Equisetum limosum and $E$. palustre, but the fact that others (e.g. $E$. arvense), growing in relatively dry situations, nevertheless exhibit these features indicates that the 
genus as a whole is derived from aquatic ancestors. Indeed there is good evidence that the Calamites of the Coal Measures, which exhibited similar aquatic features combined with pronounced secondary thickening, were inhabitants of swamps.

The sporangia of Horsetails are borne on little mushroomshaped sporophylls (Fig. I76, B), differing widely from the
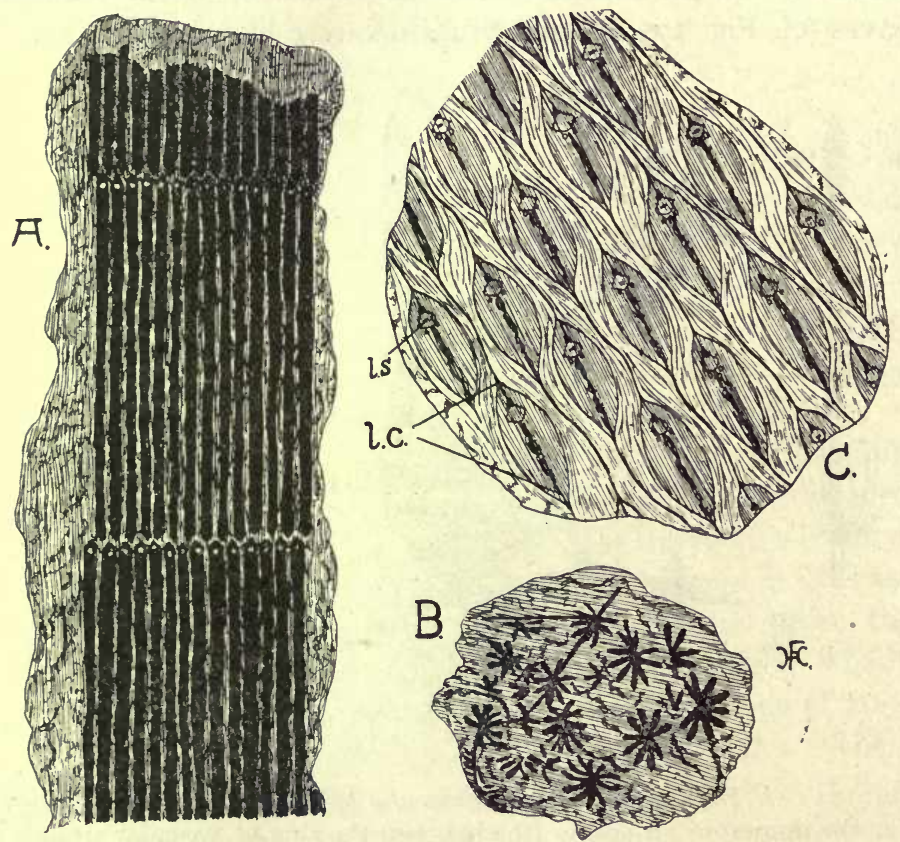

FIG. I75.-Common forms of preservation of the fossil Horsetails and Clubmosses. A, Pith-cast of Calamites. B, Annularia sphenophylloides, the foliage of Calamites. C, Small part of the surface of the stem of a Lepidodendron, showing the characteristic leaf-cushions (l.c.): l.s., leaf-scar.

ordinary leaves, and collected together at the ends of the stems to form cones or strobili (Fig. I74, B; Fig. I76, A). These usually terminate the ordinary vegetative shoots, though in the Common Field Horsetail (E. arvense) they are found on special fertile shoots, produced earlier in the season, and distinguished by their brown colour and the absence of branches. Each peltate sporophyll stands out at right-angles to the main axis, 
and bears on the inner face of its hexagonal lamina a ring of 5 to Io sporangia encircling the stalk (Fig. I76, B). In the young cone the heads of the sporophylls fit closely together, thus forming a compact protection for the sporangia; but as the latter mature the sporophylls separate, through elongation of the axis, and permit escape of the green spores. The individual sporangia are rather larger than those of Ferns, and have a several-layered wall. Many of the fossil Horsetails possessed cones of more elaborate structure.

At maturity the outermost coat of each spore is seen to consist of four extremely hygroscopic spiral strips (Fig. I76, C, D) which only remain attached at one point. Groups of spores consequently tend to cling together when the contents of a sporangium are scattered by the wind, and this may be of importance, since the archegonia and antheridia are usually produced on distinct prothalli. The spores are, however, all alike, the sex of the resulting

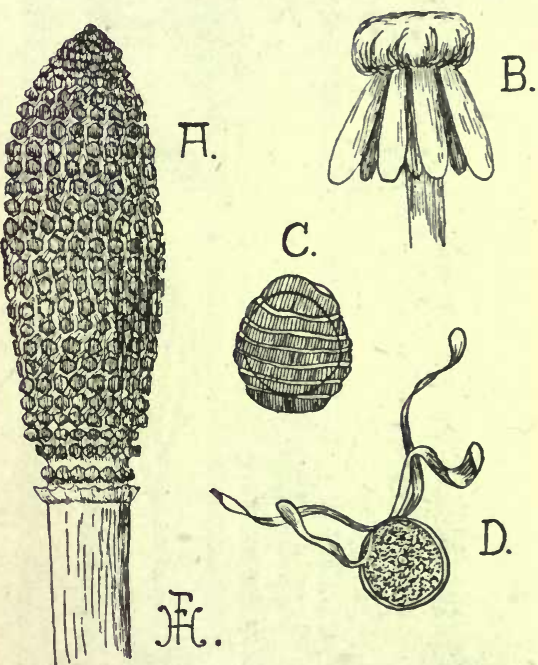

Fig. I76.-A, Entire cone, and B, single sporophyll of Equisetum maximum (original). C and D, Mature spores, showing the splitting of the outer coat (C after Sachs; D after Dodel-Port).

prothalli depending on the conditions of nutrition, those poorly nourished becoming male, whilst those well nourished become female. Both kinds are more or less richly branched, but the male are much smaller than the female. The sexual organs borne upon them do not differ essentially in construction from those of Ferns. It may be noted that, when Fern prothalli grow densely crowded, they often bear antheridia only, although unisexuality is here the exception rather than the rule:

The present-day Clubmosses are represented mainly by the 
genera Lycopodium (Fig. I79) and Selaginella (Fig. I80). The Quillwort (Isoetes lacustris, Fig. I78), which occurs submerged in mountain tarns, is a peculiar member of this group, many of whose features recall those of the fossil Clubmosses (Lepidodendron and Sigillaria, Fig. I77). Fragments of the stems of

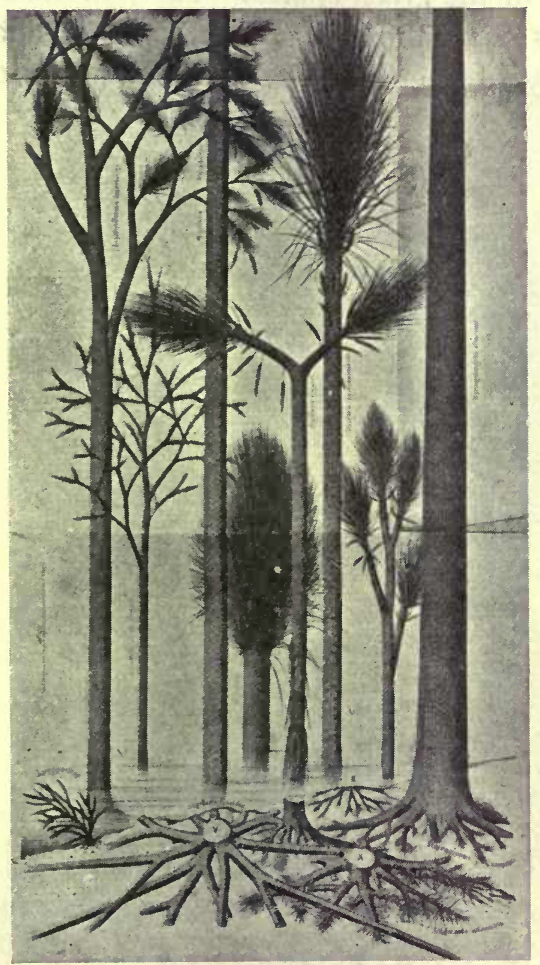

FIG. I77.-Restoration of various fossil Clubmosses (Lepidodendron and Sigillaria). (After Grand Eury.) the former with, or often without, the leaves, are very common in the Coal Measures and are readily identified by their characteristic markings (Fig. I75, C).

The small spirally arranged leaves of Lycopodium densely clothe the stems, which are either erect, as in the Fir Clubmoss (L. selago), or prostrate, except for the cone-bearing shoots, as in the Bear's Foot (L.clavatum, Fig. I79. A). The stems, as well as the occasional adventitious roots, exhibit forked branching, and never contain more than a single stele, ${ }^{1}$ whose detailed structure is often some. what complex. The sessile leaves are attached by a broad cushion-like base, and are traversed by but a single median vein (Fig. I79, B) ; the same was the case in Lepidodendron, the persistent leaf-cushions affording the distinctive markings above referred to. The British species of Lycopodium are found in moist upland pasture, except for L. inundatum, which occurs in lowland bogs.

1 A single stele was likewise found in Lepidodendron where, however, it became enveloped by a broad zone of secondary wood and phloem. 
The sporophylls (Fig. I79, B) are similar to the foliage-leaves, and are commonly in whorls; they are readily recognised by the single large, somewhat kidney-shaped, sporangium which each bears on its upper surface. In most species the sporophylls are aggregated in cones, as in Equisetum, although in L. selago, for example, the reproductive region is not clearly marked, zones of sporophylls usually alternating with vegetative leaves. Another peculiarity of this species is the development of large bulbils in the axils of the uppermost leaves ; these structures, which serve for vegetative reproduction, must not be mistaken for fertile leaves.

The cones, when present, occupy the ends of erect branches. which are of the ordinary type, except in L. clavatum (Fig. I79, A); here they bear minute leaves, at rather wide intervals, contrasting markedly with the densely arranged sporophylls of the cone. Each sporangium contains numerous spores (Fig. I79, D) which, after being shed, give rise to peculiar fleshy prothalli (Fig. I79, C). In most species these grow underground as saprophytes, obtaining their food with the aid of a mycorrhiza from

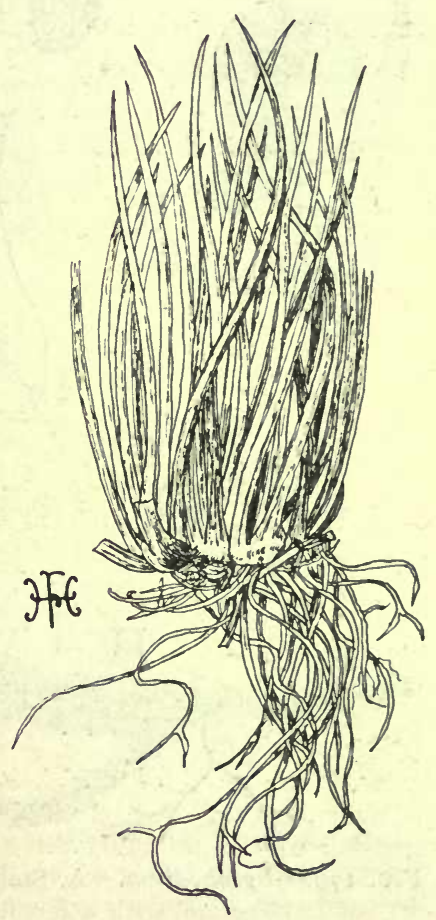

FIG. I78.-The Quillwort (Isoetes lacustris), somewhat reduced. the humus in which they are embedded. Both kinds of sexual organs are borne on the same prothallus.

Although Selaginella is represented in Britain only by S. spinosa (Fig. I80, C), which is found in similar habitats to those frequented by our species of Lycopodium, several members of the genus are commonly cultivated in greenhouses. The general habit is like that of Lycopodium, but in most 
species the leaves are arranged in four rows, two comprised of small leaves situated on the upper side of the stem, and two rows of large leaves towards the lower side (Fig. I8o, A and B); at

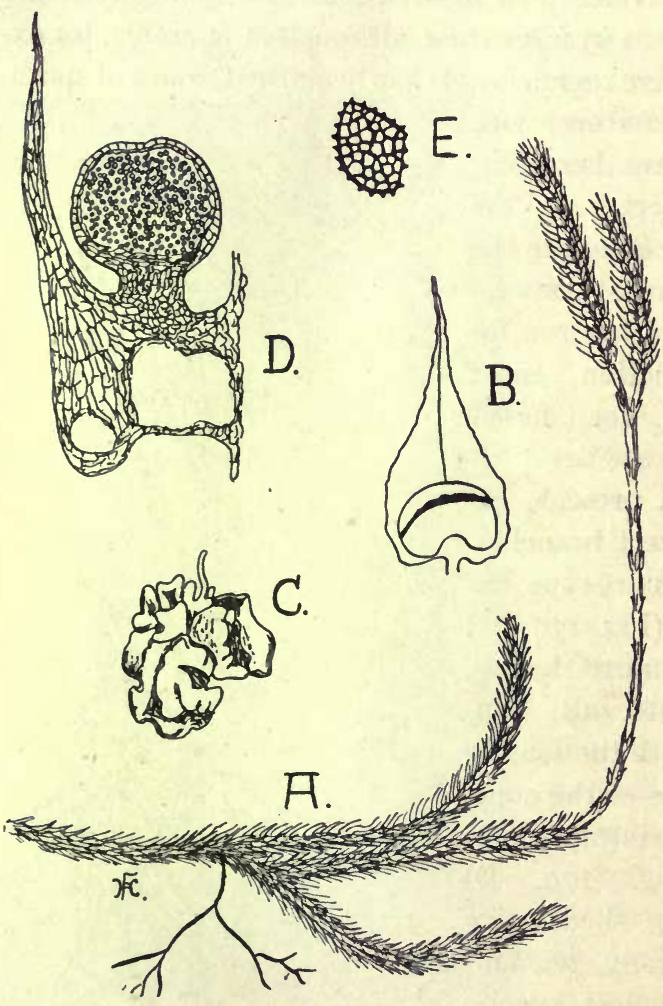

FIG. 179.-Lycopodium. A, Small part of a plant of $L$. clavatum, with a fertile shoot bearing two cones. B, Single sporophyll of same, with a dehiscent sporangium. C, Prothallus of $L$. clavatum. D, Longitudinal section of sporophyll with sporangium. E, Spore. (B after Strasburger; $\mathrm{C}$ after Bruchman; the rest original.) each node there is one large and one small leaf. The British $S$. spinosa, and other species in which the habit is erect, however; possess leaves that are all alike (Fig. I80, C).

In many $\mathrm{Se}$ laginellas the method of rooting is peculiar, the roots arising from special leafless branches known as rhizophores (Fig. I80, A, rh.). These are formed in pairs at the points of forking of the stems, but usually only one member of each pair develops. This grows downwards, generally forking repeatedly, and, on reaching the soil, roots originate from the swollen tips of the ultimate branches.

The stem is traversed by one or few steles, ${ }^{1}$ essentially like those of Ferns, except that each is surrounded by an air-space, 1 One in S. spinosa, three in S. kraussiana. 
which is bridged either by strands of cortical cells, or by radially elongated endodermal cells. The roots and rhizophores are peculiar in exhibiting but a single protoxylem group.

The cones, again situated at the ends of usually erect branches (Fig. I8o, C), exhibit four rows of sporophylls, all of the same

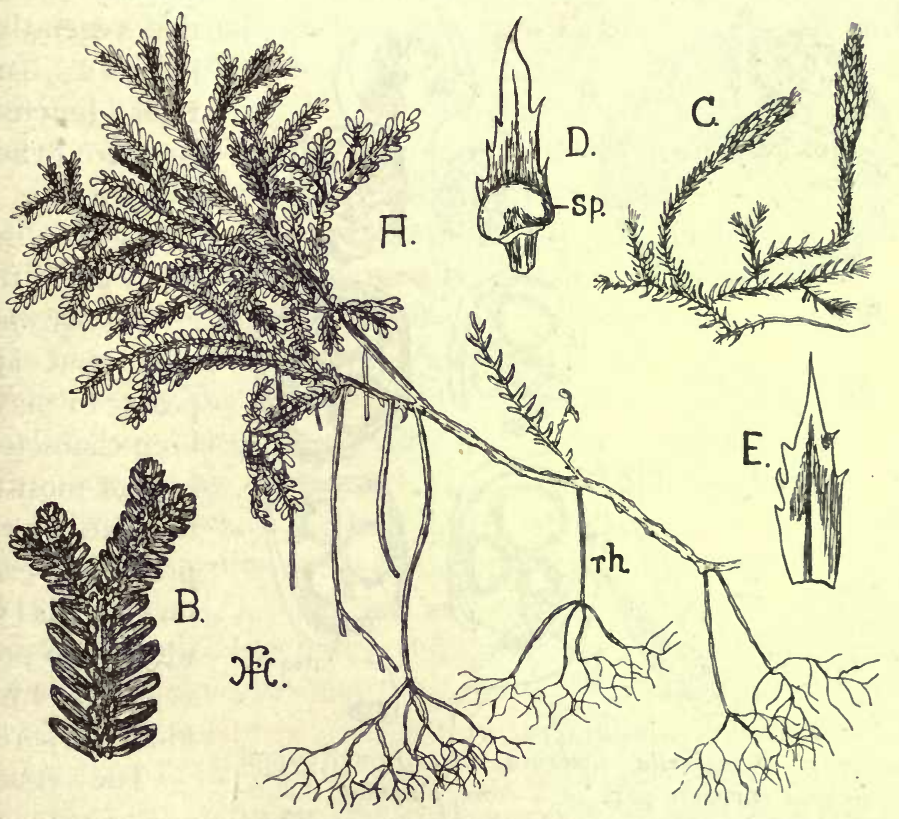

FIG. I 80.-A and B, Selaginella martensii (original). A, Part of a plant showing the leafy shoots, the rhizophores $(r h$.$) , and the roots arising$ from their ends. B, Small part of a branch enlarged, to show the two kinds of leaves. C, Plant of S. spinosa (after Wettstein), showing two cones. D, Sporophyll, and E, foliage leaf of S. spinosa (after Hieronymus). $S p .$, sporangium.

size and shape, and each with an axillary sporangium borne on a short stalk (Fig. I8o, D, Sp. ; Fig. I8I, A, E). The sporangia are of two kinds : the one kind (microsporangia), generally found towards the apex of the cone, are filled with numerous small microspores (Fig. I8I, A, mi.); the other kind (megasporangia) each contain only four large megaspores (Fig. I8I, A, mg.). 
These features are readily observed in longitudinal sections through the cones, which also show the presence of a small outgrowth (the ligule, Fig. I8I, l.) from the upper surface of each sporophyll, between its upturned tip and the sporangium. Such ligules, though most conspicuous on the

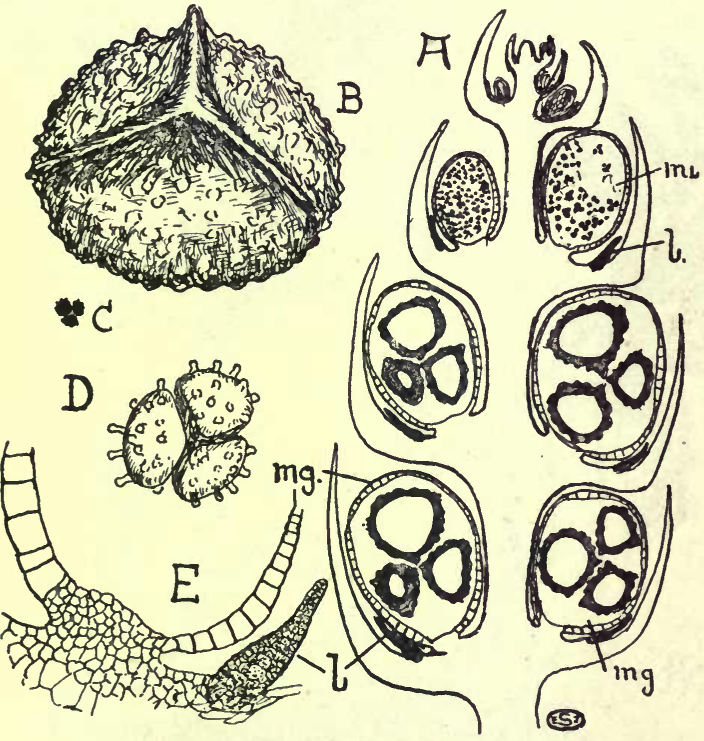

FIG. I81.-Selaginella umbrosa. A, Longitudinal section through part of a cone, showing microsporangia ( $m i$.) above and megasporangia ( $m g$.) below. E, Small part of megasporangium enlarged to show the stalk and wall. $l$., ligule. B, Single megaspore enlarged. C, Tetrad of microspores on the same scale of magnification as B. D, Tetrad of microspores enlarged.

sporophylls, occur also on all the vegetative leaves, but their function is altogether obscure. A ligule is not met with in the genus Lycopodium, but appears to have been characteristic of most of the extinct representativesof the family, which also possessed two kinds of spores.

The young sporangia of Selaginella have a "several-layered wall, enclosing a large number of spore mother-cells, and, up to this stage, all are alike. In the microsporangia each mother-cell gives rise to four small spores, but in the megasporangia only one develops further, enlarging rapidly at the expense of the others, and dividing to form the single tetrad (Fig. I8I, A). An American species (S. rupestris) exhibits an even greater reduction, since occasionally only one of the four megaspores reaches maturity, and, in this and 
certain other cases, the spores are retained within the sporangium until after the fertilisation of the archegonia produced in the resulting prothalli. Both kinds of sporangia dehisce by a wide slit, the spores ordinarily maturing their prothalli on the ground.

The contents of the microspore divide to form a few-celled, exceedingly reduced male prothallus, developing neither chloroplasts nor rhizoids, and consisting in the main of a small number of spermatozoid mother-cells (Fig. I82, $f$ ). The resulting biciliate spermatozoids (Fig. I82, d) are liberated, during wet weather, by the rupture of the coat's of the microspores.

The female prothallus begins to develop within the megaspore long before the latter is shed, and, like the male, exhibits considerable reduction and usually remains colourless. The early stages of its formation are characterised by repeated division of the megaspore-nucleus, separating walls only arising much later. When ready for fertilisation the prothallus consists of a smallcelled tissue, situated opposite the apex of the tetrahedral spore and exposed by the rupture of its coats at this point (Fig. I82, $a$ and $b, p$.$) , while the bulk of the spore-contents, still enclosed$ in the megaspore-wall, are occupied by large cells laden with food-material $(f)$. The few archegonia, which have very short, scarcely projecting necks (Fig. I82,e), are embedded in the small-celled apical region (Fig. I82, $a, b, a r$.$) .$

The fertilised egg divides transversely, the inner (lower) half giving rise to the embryo proper. The outer (upper) half, forming the so-called suspensor (Fig. I82, $b$ and $c, S$.), divides a few times and elongates considerably, so that the developing embryo is pushed fown into the large-celled nutritive tissue $(f$. $)$ below. The suspensor is a device characteristic of the higher seed-plants, but in other essential respects the embryology resembles that of Ferns. Absorption of the stored food takes place by means of a sucker or foot (Fig. I82, $c, f t$.), but the young plant acquires independence at an early stage (Fig. I82, g).

Regarded as a whole, the Pteridophyta exhibit a great variety of vegetative structure, and considerable specialisation in their reproductive processes. They offer a marked contrast to the Bryophyta in the relative importance of the sporeproducing phase, which is an independent plant highly adapted 
to a terrestrial existence. Associated with the last-named feature we see the differentiation of proper conducting tissue and of true roots. There is, moreover, a marked tendency towards

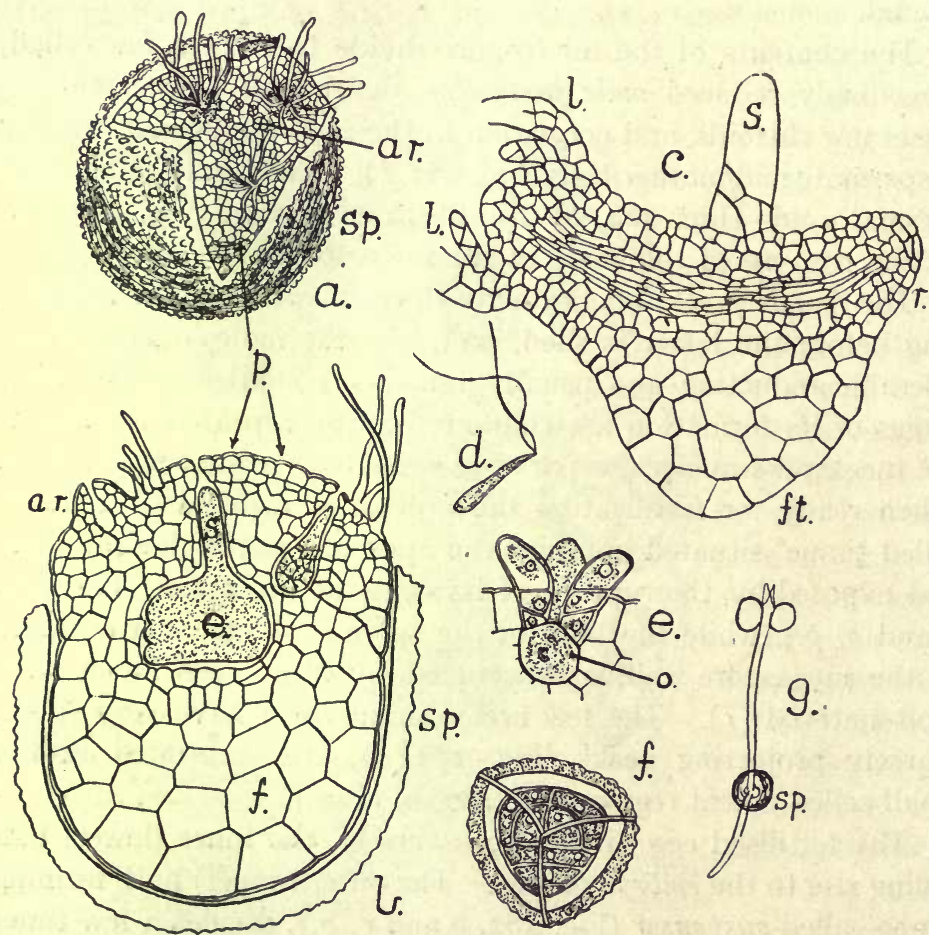

FIG. I82. - Selaginella, prothallia and embryology. $a$, front view, and $b$, vertical section of mature megaspore with female prothallus ; $c$, older embryo; $d$, spermatozoid ; $e$, archegonium in longitudinal section ; $f$, microspore with contained male prothallus; $g$, young plant still attached to the megaspore (sp.). ar., archegonia ; e., embryo (in $b)$; $f$., large-celled nutritive tissue (in $b$ ); $f$., foot (in $c$ ); $l$., leaves of embryo ; $o$., egg (in $e$ ); $p$, prothallus ; $r$., radicle ; $S$., suspensor ; $S p$., coat of megaspore. ( $a, e$, and $g$ after Bruchman; $d$ after Belajeff ; remainder after Pfeffer.)

division of labour, on the one hand between vegetative and reproductive leaves, on the other between the prothalli. In the Ferns all the latter are alike and bear both kinds of sexual organs, although there are occasional exceptions when the con- 
ditions of nutrition are abnormal (cf. p. 3r3). This tendency leads to the development of definite unisexual prothalli in the Horsetails, which, however, like the Ferns, have only one kind of spore, i.e. are homosporous. Lycopodium resembles the Ferns in these respects, but in Selaginella, not only are the prothalli unisexual, but they are produced from two kinds of spores, i.e. this genus is heterosporous.

Although heterospory involves the risk of the two sexes of prothalli not germinating in sufficiently close proximity to one another, certain advantages obviously accrue from it. The most conspicuous of these is similar to that which may have led to the evolution of oogamy (cf. p. 223), viz. the provision by the mother-plant of an abundant store of food for the development of the new sporophyte. These reserves are laid down in the megaspore before it is shed, and, as a consequence, the resulting prothallus can dispense with rhizoids and an assimilatory mechanism. Moreover, the embryo acquires additional protection during the early stages of its development from the coats of the megaspore, within which the greater part of the prothallus remains enclosed (cf. Fig. I82, b.). A further step would obviously be the retention of the megaspore within the sporangium until after fertilisation and during the development of the embryo, and this we shall find realised in the Phanerogams, the last great class of the Vegetable Kingdom. The microspores, requiring no appreciable amount of food-reserves, and being consequently of small dimensions, can be produced in large numbers. This affords an increased power of dispersal whereby the association of the two prothalli is rendered more probable. 


\section{CHAPTER XXII}

\section{The Cycads and Certain Extinct Seed-bearing Plants}

A GROUP may now be considered which, although portraying many of the characteristics of Flowering Plants, nevertheless shows several features reminiscent of Ferns. The plants in question are the Cycads, which have a wide distribution in the tropical regions of America, South Africa, Eastern Asia, and Australia, though most of the genera have a very restricted range. Fossil records show the group to be a very ancient one that played a particularly important part during the Mesozoic period, the present-day representatives appearing merely as relics. The Sago-palm (Cycas revoluta) is in most respects typical of the living forms.

Most Cycads (Fig. I83) have the appearance of Palms or Tree Ferns, the often huge pinnate leaves forming a crown at the top of the partly subterranean and tuberous, or overground and columnar, stems ; in the latter case the stems may attain a height of sixty feet. The trunk is rarely branched, and, in the older portion, its entire surface is covered with an armour formed by the persistent bases of the leaves of previous seasons. Fresh crops of leaves are produced at intervals, the outer ones being modified to form protective bud-scales. In some of the genera the unfolding leaves exhibit a spiral inrolling of the pinnæ (e.g. Cycas, Fig. 184), or of the midrib, similar to that characteristic of Ferns. Other resemblances to this group are to be found in the forked veining of the blades (Fig. I85, E) and in the structure of the petiole. The numerous vascular strands of the latter have the protoxylem embedded within the metaxylem, a feature especially characteristic of Ferns (cf. p. 293 and Fig. I6o, P.xy.), whereas in the vast majority of the Seed- 


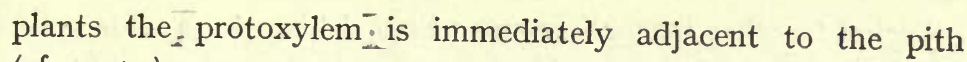
(cf. p. 79).

Cycads often attain a great age and their stems exhibit secondary growth, with the same general plan of construction as in a woody Dicotyledon, except for the absence of annual rings. The pith is very large, and, in the Sago-palm, contains

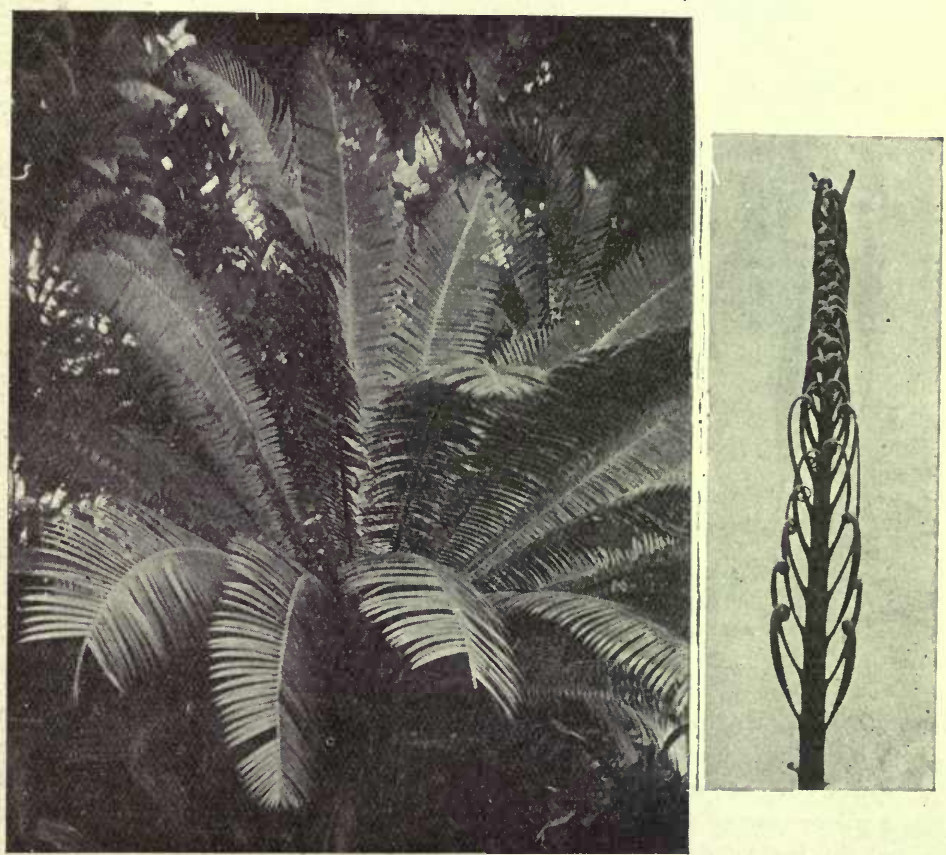

FIG. I83.-Young plant of Cycas circinalis. [Photo: E. J. S.]

FIG. 184.-Young leaf of Cycas, showing spiral inrolling of the pinnæ. [Photo: E. J. S.]

the stores of starch which are one of the sources of the sago of commerce.

The sporophylls are of two kinds, microsporophylls and megasporophylls, and are arranged in distinct male and female cones, borne on separate individuals. The microsporophylls are thick scales which are spirally arranged (Fig. I85, A), and bear, on their under-surface, numerous sporangia (Fig. I85, D), often 
collected together in small groups; they dehisce by a wide split. In most cases the megasporophylls are of a similar nature, although they produce only two megasporangia, usually placed one on either side of the stalk-like lower part (Fig. I86, B); the megasporangia are of a peculiar type and are known as ovules. In Cycas, however, the megasporophylls are very leaf-like, although relatively small, hairy, and brown in colour, and often bear
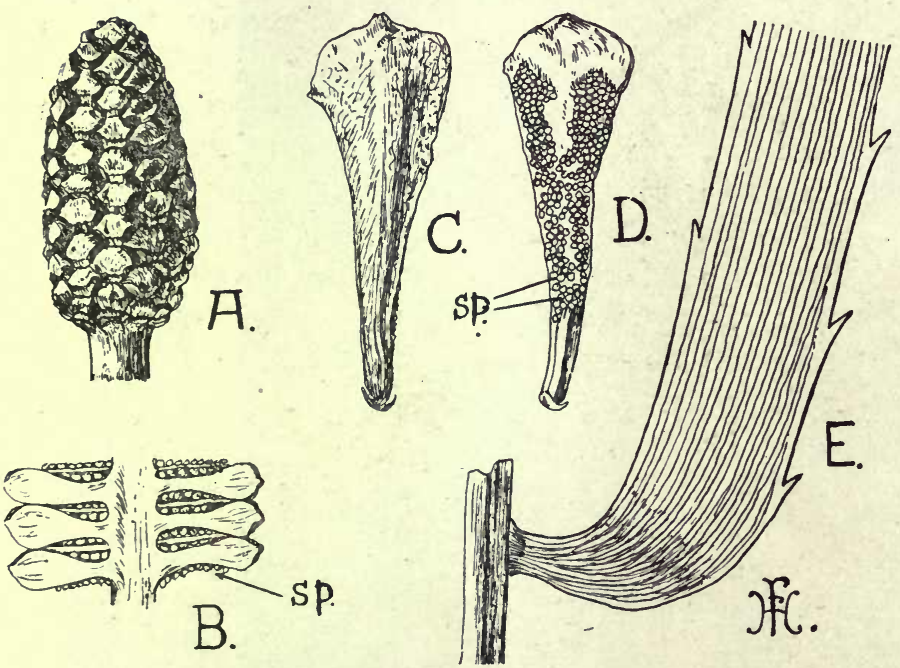

FIG. I 85.-A, Entire male cone of Bowenia spectabilis, and B, part of the same in longitudinal section, showing the microsporangia (sp.) on the lower surfaces of the sporophylls. C, Upper, and D, lower surface of a microsporophyll of Cycas. sp., microsporangia. E, Part of a pinna of the leaf of Encephalartos to show the veining.

more than two of these ovules (Fig. I86, A). The female plant of Cycas can, as a matter of fact, be profitably compared with such a Fern as Blechnum (cf. p. 308), since in both cases the sporangia occur on fronds which are but little modified. An examination of the megasporophylls of different Cycads shows all stages in the reduction of the lamina to a condition in which the leaf-like character is almost entirely obscured (e.g. Encephalartos, Fig. I86, B).

The ovules (megasporangia) (Fig. I86, C and D) are of con- 
siderable size, but contain only a single large megaspore $(p$. within the several-layered parenchymatous wall or mucellus (n.). The delicate texture of the latter can be related to the presence of a thick fleshy protective covering (o. and $i$.). This integument

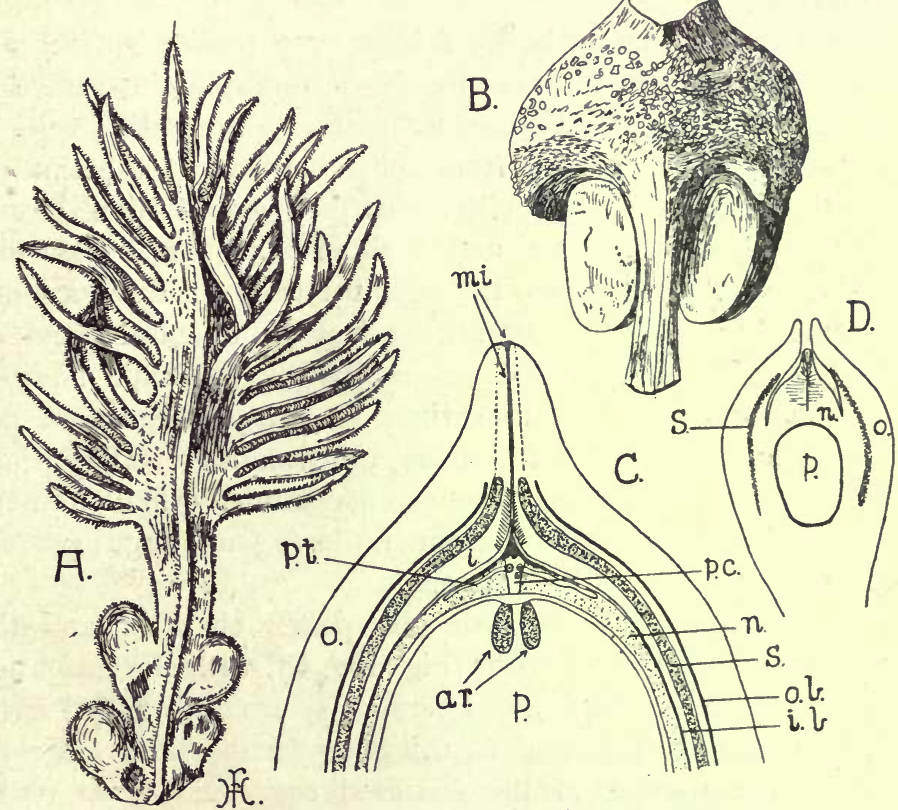

Fig. I 86.-A, Megasporophyll of Cycas revoluta with four ovules (about half natural size). B, Mature megasporophyll of Encephalartos hildenbrandtii, bearing two seeds (about half natural size). D, Diagram of longitudinal section of ovule of Bowenia spectabilis, and C, front end of same enlarged. ar., archegonia; $i$., inner fleshy layer of integument; i.b., vascular bundle of inner series; mi., micropyle; $n$., nucellus; $o$. , outer fleshy layer of integument; o.b., bundle of outer series; $p$. , female prothallus within megaspore; p.c., pollen chamber; p.t., pollen tube ; s., stony layer of integument. (A and B original ; $\mathrm{C}$ and $\mathrm{D}$ after Kershaw.)

completely envelops the nucellus, and is indeed fused with it except at its extreme apex. In this region the integument is pierced by a narrow canal, the micropyle (mi.), leading down to the tip of the nucellus, in which a conical cavity, the pollen chamber $(p . c$.$) , is formed by the breaking down of the tissue. The ovule is$ 
supplied by two vascular strands from the sporophyll. These fork at its base to form two systems of branches, extending almost to the extreme tip: those of the outer series (o.b.) traverse the peripheral layers of the integument, whilst those of the inner (i.b.) run close to the line of junction of the latter and the nucellus.

The wind- or insect-borne microspores (pollen grains) are drawn into the pollen chamber by the drying up and contraction of the mucilaginous fluid (formed by the disintegrating cells of the nucellus) which exudes from the micropyle at the time of pollination. Within the pollen chamber germination ensues (Fig. I86, C, and $187, \mathrm{C}$ ), and a short branching sucker-like pollen tube (p.t.) grows into the adjacent tissue of the nucellus. ${ }^{1}$ Subsequently two spermatozoids, each with a spiral band of cilia $^{2}$ (Fig. I87, B, s.), are developed within the main body of the microspore. In the meantime the large megaspore has become filled with a uniform tissue, the female prothallus (Figs. I86, $\mathrm{C}$, and $\mathrm{I} 87, \mathrm{C}, p$.) which produces a, commonly small, number of archegonia $(a r$.$) , with very minute necks (n$.) and large eggs (o.), at the end adjacent to the micropyle.

The nucellar tissue between the pollen chamber and the female prothallus breaks down (Fig. I87, C), with the formation of a slimy fluid in which the liberated spermatozoids (s.) swim to the archegonia (o.), and in this way fertilisation is accomplished. The nucleus of the fertilised egg divides repeatedly to form numerous nuclei which, at least in the lower part of the oospore, become separated by cell-walls. It is this region alone that gives rise to the embryo (Fig. I87, D, e.), whilst the remainder serves for nutrition.

In each ovule only one embryo ultimately develops, its growth taking place at the expense of the surrounding prothallus, into the centre of which it is carried by the marked elongation of a suspensor (Fig. I87, D, sp.). Upon reaching a certain stage, however, in which two cotyledons, plumule, and radicle can be distinguished, the embryo becomes dormant, the residue of the

1 The sequence of events in the germination of the microspores of Cycads is very similar to that in Conifers (cf. p. 348).

2 Apart from the Cycads, the Maiden-hair Tree (Ginkgo biloba) is the only seed-plant which has ciliated, free-swimming, sperms. 
female prothallus around forming a nutritive tissue, the endosperm. The whole is enveloped by the thick integument, now differentiated into three layers, which can even be recognised in an immature form in the young ovule. These layers comprise an inner (Fig. I86, C, i.), and a much thicker outer (o.), flesh, with an intervening very hard stony layer (s.). This product of megasporan gium, female prothallus, and e $\mathrm{mbryo}$ is known as a seed, and is a $\mathrm{p} 1 \mathrm{u} \mathrm{m}-1 \mathrm{ike}$ structure many times the size of the original ovule.

The Cycads obviously show many superficial resemblances to Ferns, but these are even more pronounced in another group of plants

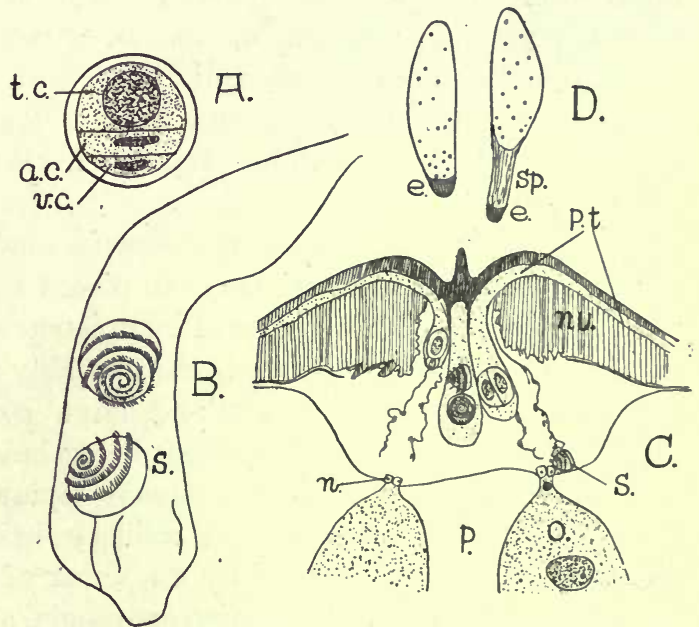

FIG. 187.-A, Germinating microspore of Cycas, showing vegetative cell (v.c.), antheridial cell (a.c.), and tube cell (t.c.). B, Pollen tube of same with the two spermatozoids (s.). C, Diagram of longitudinal section through apex of nucellus $(n u$.) and female prothallus $(p$.$) of Dioon edule, showing$ pollen tubes (p.t.) and pollen grains in various stages of development, spermatozoids (s.), and archegonia with eggs $(o$.$) and necks (n$.$) . D, Two$ proembryos of Dioon edule, the left-hand one younger than the right-hand one. e., embryo; sp., suspensor. (A and B after Ikeno; C and D from Chamberlain.)

(Pteridosperms) which, like the Calamites and Lepidodendrons of the Coal Measures, are known only as fossils. The members of this group, though closely resembling the Ferns in habit, show analogies with Cycads in their anatomy, their mode of reproduction, and especially in the possession of seeds.

A complete knowledge of such fossil plants is only acquired gradually and as a result of prolonged research. At first the 
separate fragments of stem, root, leaves, etc., are studied as unrelated structures, but subsequently patient toil pieces them together till a more or less complete picture of the whole is obtained. The external appearance of a fossil plant is best appreciated from casts or impressions preserved in consolidated mud, sand, etc. Not infrequently, however, the tissues have been impregnated with silica, calcium carbonate, etc., so that the internal structure is recognisable. Such petrified portions are cut up into thin slices, and these are ground down until sections, comparable to those prepared from a living plant, are obtained.

Lyginopteris (Lyginodendron) oldhamia, one of the commonest of the Coal Measure fossils, has been pieced together in this way until it is known with a degree of completeness only shared by a few living plants. Lyginopteris was probably a woody scrambler, with relatively slender stems and large compound Fern-like leaves (Fig. I88), the whole surface being beset with spines and large glandular hairs The leaves were separated by long internodes and produced occasional axillary branches, whilst the stem was attached to the soil by a number of adventitious roots arising near its base. The microsporangia and megasporangia (ovules) were borne on the ultimate ramifications of the ordinary foliage-leaves (cf. Fig. I90).

The stem underwent considerable secondary thickening. In transverse sections (Fig. I89) the parenchymatous pith, which included patches of sclerotic tissue, is seen to have been surrounded by five or more groups of primary wood $(X$.), some of which occurred in pairs; in each such strand of primary xylem the protoxylem occupied a more or less central position. Beyond was a prominent zone of secondary wood (Sec.), with wide parenchymatous rays, through which the leaf-trace bundles emerged (Bs.). The delicate cells of the cambium and phloem, external to the wood, are rarely well preserved. A layer of cork-like tissue (periderm) was usually developed in the pericyclic region, whilst the thin-walled inner cortex contained numerous secretory cells. A sharp contrast is afforded by the outer cortex, with its system of radial sclerenchymatous plates (S.), anastomosing at frequent intervals and giving mechanical support to the stem. 
Each leaf was supplied by one of the primary xylem strands which, as it passed outwards, became associated with phloem

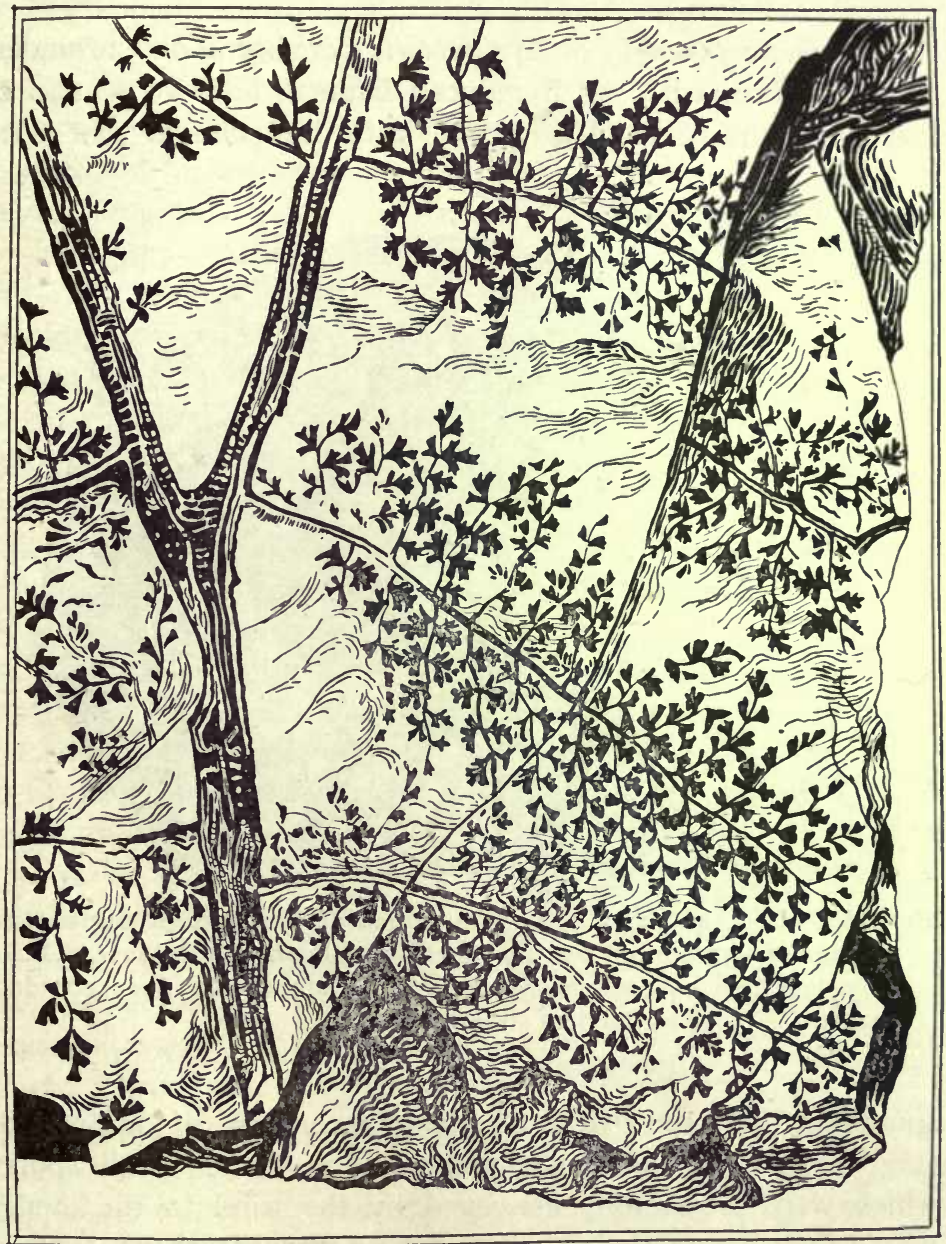

Frg. 188.- The foliage (formerly known as Sphenopteris hoeninghausii) of Lyginopteris oldhamia. (From a drawing, after Potonié, lent by Prof. F. W. Oliver, F.R.S.)

and, on entering the pericycle, divided into two. The double bundle (Fig. I89, Bs.) traversed several internodes before bending 
out into the leaf, whose detailed structure was much like that of many of the simpler Ferns. The root was similar to that of recent plants, possessing from two to eight xylemgroups.

The microsporangia of Lyginopteris were borne on the underside of pinnæ, with much reduced laminæ, and were more or less fused in pairs. The pinna, with its pendant elongated

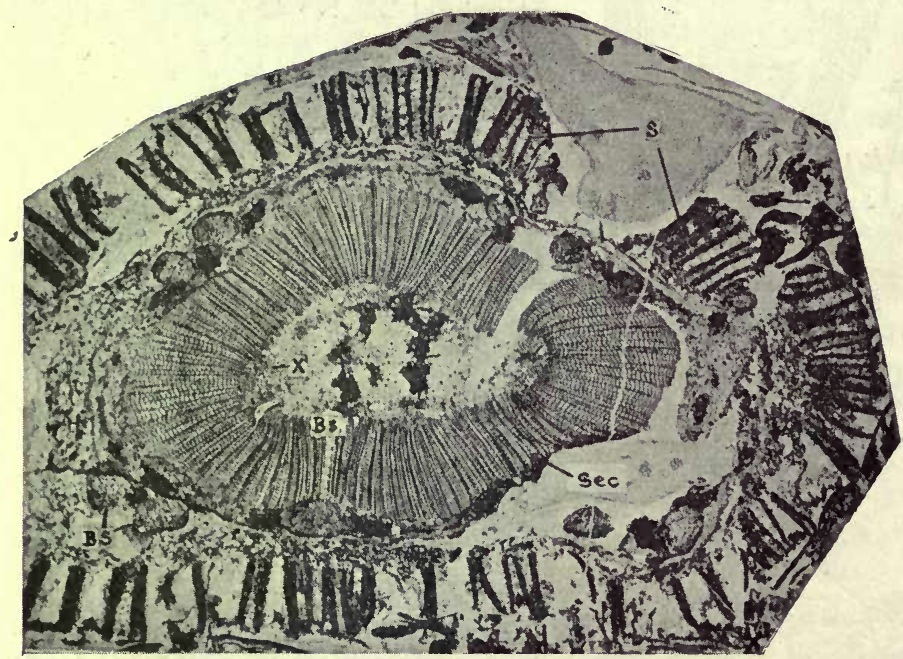

FIG. I89.-Photograph of transverse section of the stem of Lyginopteris oldhamia (reproduced by the courtesy of Prof. F. W. Oliver, F.R.S.). $B s$., leaf traces; $S$., sclerenchyma plates in outer cortex; Sec., secondary wood ; $X$., primary xylem strand.

sporangia, had somewhat of the appearance of an epaulet (Fig. I90). The sporangia produced numerous small spores, which were presumably conveyed by the wind to the ovules, where they became lodged within the pollen chamber. Of their further fate nothing is known, but it is probable that they developed a small male prothallus, giving rise to spermatozoids, somewhat like those of Cycads.

The ovules arose singly from the ends of truncated branches of the fronds, and showed the same plan of construction as in 
Cycads (Fig. I9IB), except that each possessed, in addition to the integument, a second protective covering. This took the form of a lobed cup-like structure $(C u$.), comparable with the cupule of a Hazel-nut, and beset with the same glands as occur on the vegetative organs of this plant (cf. Fig. IgIA). The robust integument (Fig. IgIB, I.) was fused with the nucellus (sporangium-wall, n.), except for the apical portion, which was pierced by the narrow canallike micropyle.

The tip of the nucellus was produced into a flaskshaped pollen chamber (Pc.), whose neck projected very slightly beyond the micropyle (cf. Fig. I9IA), so that the microspores reached the nucellus direct, a point of contrast to Cycads and other recent plants. The central portion of the pollen chamber was occupied by a dome of parenchymatous tissue, but between it and the surrounding wall was a narrow chinklike space (Fig. IgIB, Pc.) in which the pollen presumably germinated and liberation of the sperms ensued. The single huge megaspore $(m$. has been found filled with a uniform thin-walled prothallus, which

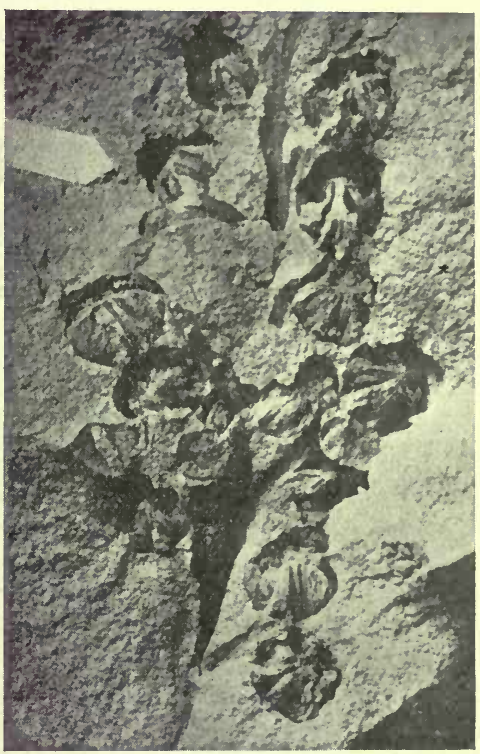

FIG. I90.-Ultimate pinnules of the foliage of Lyginopteris oldhamia, with microsporangia. (From a photograph by Mr. R. Kidston, F.R.S.)

doubtless bore the archegonia near its apex. The seed was supplied by a single vascular strand, which gave off branches to both envelopes. Those traversing the cupule (Fig. I9IB, Vb.) extended into its lobes, whilst those running in the integument (I.b.) penetrated to the neighbourhood of the micropyle, where the integument became free from the nucellus.

The plants described in this chapter are especially characterised by the method of fertilisation and the possession of seeds. 
As regards the former feature, the only essential difference from Selaginella lies in the germination of the microspores in close proximity to the megaspore, for which special devices such as the mucilaginous pollination drop and the pollen chamber are created. Moreover, with the help of the pollen tube some nourishment is obtained from the adjacent nucellus.

The seed may be looked upon as an extreme development of heterospory. Even in Selaginella it was noted that in some species there may be reduction to a single megaspore, which is retained within the sporangium till after fertilisation (cf. p. 3I9).

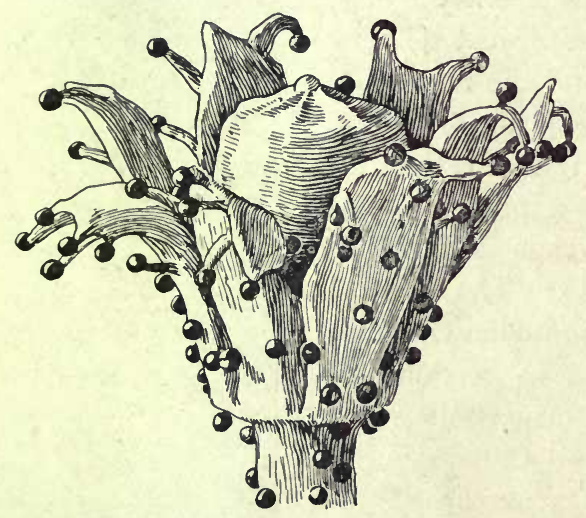

FIG. I9IA.-Restoration of the seed (Lagenostoma lomaxi) of Lyginopteris oldhamia, in its glandular cupule. (After Oliver.)

Such reduction and retention are eminently characteristic of the seed-habit, where dehiscence of the megasporangium is dispensed with. The added protection, furnished by the integument, admits of a change in the character of the sporangium-wall, which becomes a thin-walled tissue serving as intermediary between the vascular system of the ovule and the developing megaspore and female prothallus.

The possession of a vascular system, another characteristic of the ovule, facilitates nutrition and the storing up of foodreserves for the young embryo. It is probably in consequence of the increased food-supply that ovules and seeds in general attain so large a size as compared with the sporangia of lower 
plants. As a general rule (e.g. most Conifers and Flowering Plants), however, the vascular system of the ovule stops short at its base. The elaborate vascular supply of the early seedtypes, as contrasted with the more modern ones, may perhaps be related to the possession of motile spermatozoids, and to the relative degree of exposure and size of the ovules.

One important aspect of the permanent retention of the

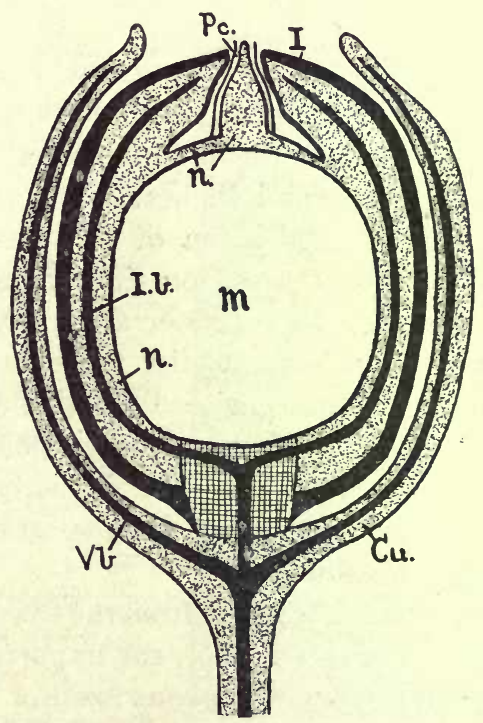

FIG. I9IB.-Reconstruction of a longitudinal section through the seed of Lyginopteris oldhamia (after Oliver). Cu., cupule; I., integument ; I.b., bundle traversing integument; $m$. , megaspore; $n$. , nucellus ; $P c$., pollen chamber; $V b$., bundle traversing cupule.

megaspore, involving considerable economy, is the possibility of postponing the accumulation of food-reserves to a relatively late stage, when fertilisation has taken place and the embryo has begun its development. The embryo is, moreover, more adequately protected, during its early phases, than is possible in Pteridophyta and Bryophyta, and, after attaining a certain stage, remains dormant and securely shielded until conditions arise suitable for its further growth. 


\section{CHAPTER XXIII}

\section{The Conifers}

All seed-plants, with the exception of certain fossil forms (e.g. Pteridosperms and some fossil Clubmosses), are grouped as Phanerogamia, the fourth and highest class of the Vegetable Kingdom. The further subdivision of this class into Gymnosperms and Angiosperms is based on the manner in which the ovules are borne; those of the former are freely exposed, as in the Cycads already considered, whilst those of the latter, the Flowering Plants proper, are enclosed in a structure called the ovary. There are, however, several other points of contrast, which will become apparent later. The Phanerogams as a whole are further distinguished by a higher differentiation of the strobili than is found among Pteridophyta.

The Gymnosperms include, apart from the Cycads, the Maidenhair Tree (Ginkgo biloba, cf. p. 326), the important group of the Conifers, and other forms known only as fossils. The Conifers, to which belong such familiar plants as the Scotch Fir (Pinus sylvestris, Fig. 200), Larch (Larix europaea, Fig. I93), Yew (Taxus baccata, Fig. 20r), 'etc., are essentially characteristic of temperate zones, some being the chief forest trees of colder regions (cf. p. 39I). They furnish some of the most important sources of timber (p. I29), turpentine, resin, etc. (p. 63). There are some 350 known species, of which more than a fifth belong to the genus Pinus.

All the Conifers are woody, and the majority are trees. The regular habit, so marked in the Spruce Fir (Picea excelsa) and Cypress (Cupressus), characterises the group and is an outcome of the monopodial branching. ${ }^{1}$ The feature which gives them their most distinctive stamp, however, is the foliage, which in general consists of small needle-like leaves (Figs. I92, I93, and 20I). 
These usually persist for several years, although the Larch, for instance, is deciduous.

The needles are either borne on the ordinary long shoots (e.g. Yew, Fig. 20I, $a$, and Silver Fir, Fig. I93, A), or, as in several common genera (Pinus, Larix, Cedrus, all members of the Abietineæ), are restricted to special dwarf-shoots. These arise in the axils of scale-leaves on the long shoots, and bear a few dark-coloured scales below and a variable number of foliage leaves above. In the Pines the number of the latter is limited (five in Pinus strobus, two in $P$. sylvestris, Fig. 200, and only one in $P$. monophylla), and no further leaves are produced after the first season, the whole dwarf-shoot being shed at the end of two or three years. On the other hand, in the Larch (Fig. I93, B) and Cedar (Cedrus), where the dwarfshoots are larger and bear a tuft of needles, quite a considerable number of new leaves is formed annually, although after some years this production

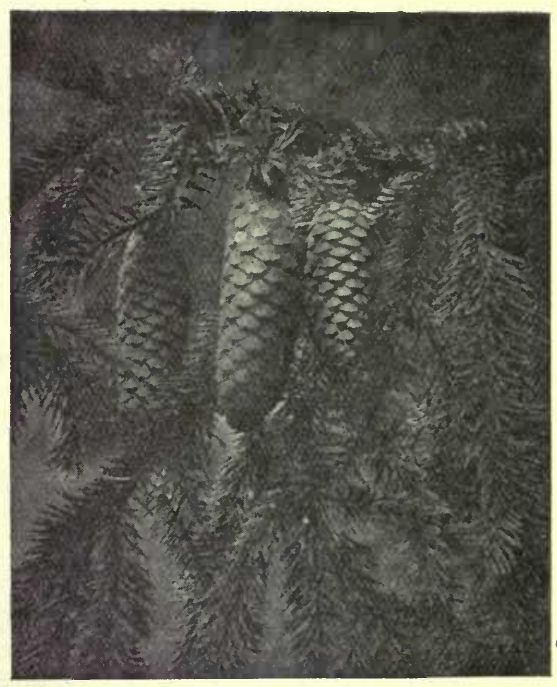

FIG. 192.-Branches of the Spruce Fir (Picea excelsa) with three ripe cones. [Photo: E. J. S.] ceases and the dwarf-shoots die away. These two Conifers also differ from the Pines in the fact that the needles are not entirely confined to the dwarf-shoots, since, during the first season's growth, they occur on the long shoots also. It will be realised that only the normal shoots contribute to the permanent branchsystem of the trees.

In most cases the leaves are sessile, although those of the Yew have a short stalk (Fig. 20I, a). Their bases are often fused with the stem for a short distance, and persist after the leaves have fallen, leaving characteristic scars (e.g. Spruce Fir, 
Picea); in the Scotch Fir similar scars are left by the decurrent bases of the dwarf-shoots.

The peculiar appearance of the Arbor Vitæ (Thuja, Fig. I94) and the Cypress (Cupressus), both belonging to the Cupressineæ, is due to the presence of minute leaves arranged in decussate pairs, and almost fused with the stem upon which they are
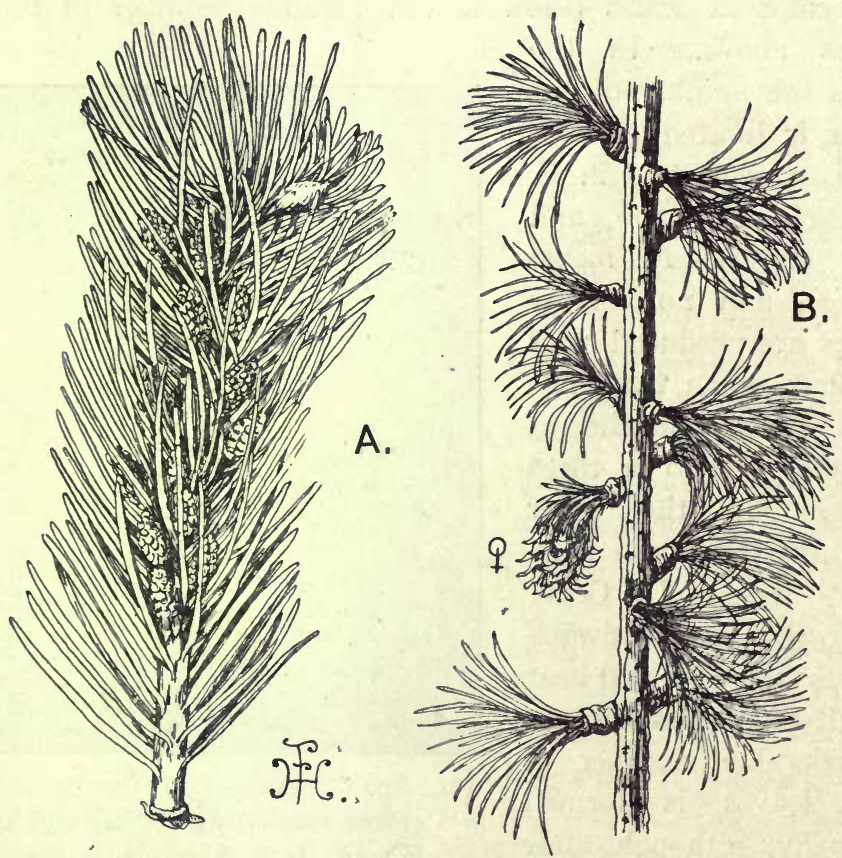

FIG. 193.-A, Branch of Silver Fir (Abies) with male cones. B, Branch of the Larch (Larix), showing several dwarf-shoots, one of them bearing a young female cone. (Both about natural size.)

borne. Moreover, owing to the larger size of the lateral leaves, and the restriction of most of the branches to their axils, the shoot as a whole acquires a flattened appearance. In the common Juniper (Juniperus), which is likewise a member of the Cupressinex, three leaves of the usual needle type arise at each node, and such a whorled arrangement is characteristic of this whole family. Large flattened leaves are seen in Araucaria, 
and a similar type of foliage is found in Podocarpus, which is allied to the Yew. Podocarpus is the most important genus of Conifers in the Southern Hemisphere, comprising some sixty species, of which several furnish valuable timber.

In spite of the considerable diversity in the mature structure, the seedlings of most Conifers exhibit great uniformity, the young stem bearing ordinary needle-leaves for some little distance above the cotyledonary node (Fig. I95). The dwarf-shoots of
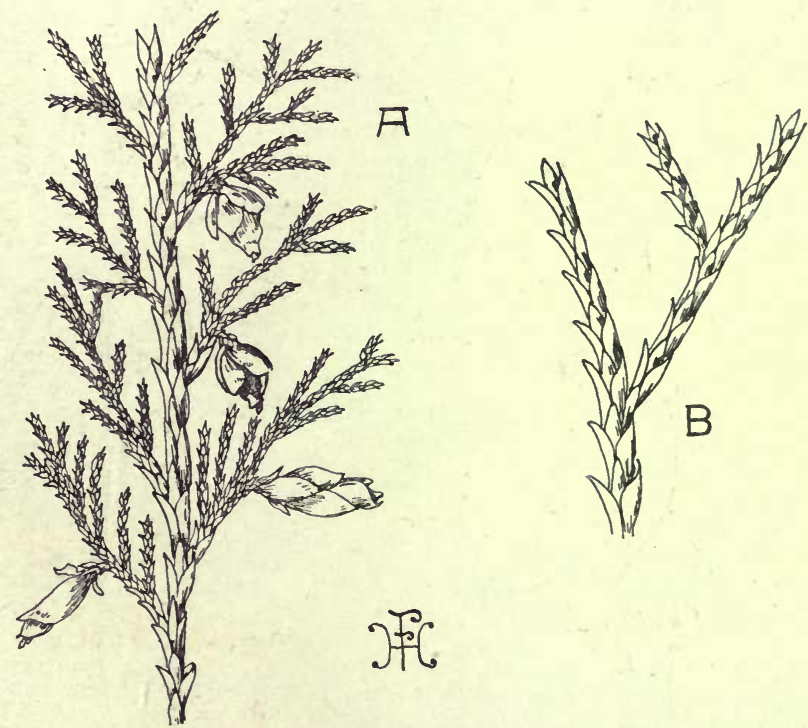

FIG. I94.-Arbor Vitæ (Thuja). A, Branch with ripe female cones (about natural size). B, Small part of a branch enlarged to show the leafarrangement.

Pinus and other Abietineæ, as well as the scale-like leaves found in the Cypress, etc., only appear at a later stage and as a secondary development. By appropriate means the juvenile foliage can be made to persist, even in the adult condition, as in some cultivated varieties of Thuja and Cupressus (the Retinospora of nurserymen). Even the deciduous habit of the Larch appears as a secondary acquisition, since in the seedling the leaves persist for some time. It is not always that the varied specialisation which a group has undergone, in evolving from a 
common ancestral type, is as plainly decipherable as in the Conifers.

The appearance of the stem in transverse section is very similar to that of a Dicotyledon having secondary growth. The Scotch Fir affords a typical example (Fig. I96, A). Here the
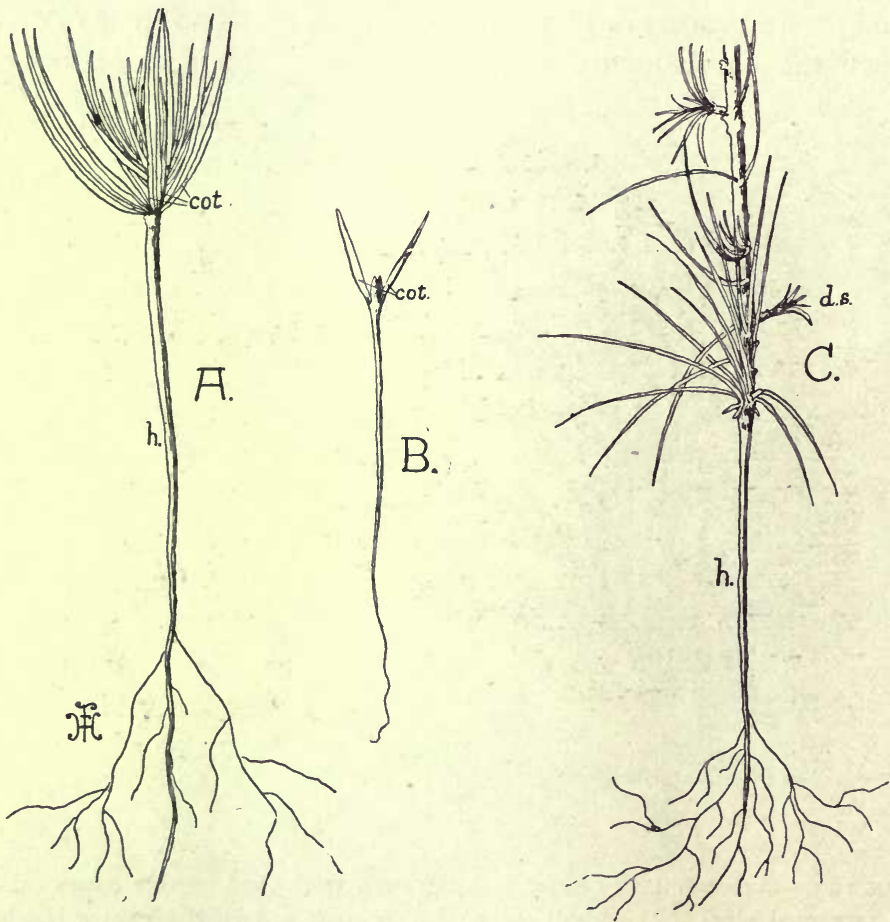

FIG. 195.- Seedlings of various Conifers. A, Scotch Fir (Pinus sylvestris). B, Cypress (Cupressus), showing the two cotyledons and plumule. C, Larch (Larix), older seedling which has already produced several dwarf-shoots. Cot., cotyledons; d.s., dwarf-shoot; $h$., hypocotyl.

irregular outline is due to the adherent bases of the scale-leaves which are occupied by a large-celled tissue (Fig. I96, B), on whose inner side the cork-cambium $(\mathrm{Ca}$.) arises. With the production of cork, therefore, the epidermis (e.) and the dwarf-shoots become exfoliated, so that the older branches have a relatively smooth surface. The narrow cortex is rather lacunar, and con- 
tains schizogenous resin-canals $(r$. ), whose structure has already been described (cf. p. I52). These resin-canals are particularly characteristic of Conifers, and are found in all parts of the plant; they are entirely absent only from the Yew.

The primary and secondary vascular tissues are not easily distinguished from one another, particularly in the case of the phloem, the broad zone of which is chiefly secondary in origin.

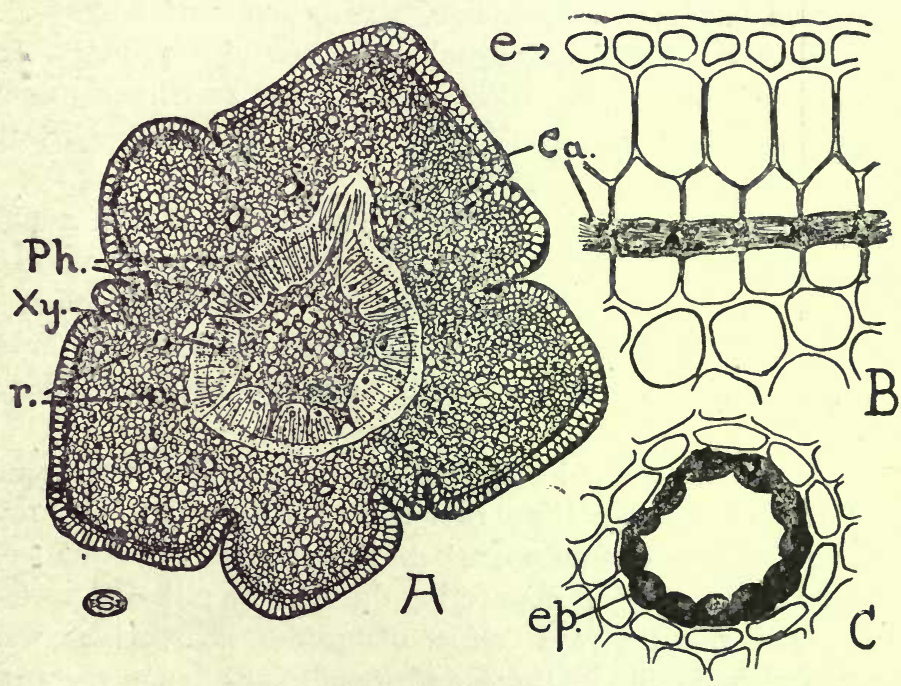

FIG. 196.- Structure of the young stem of the Scotch Fir (Pinus sylvestris) in transverse section. A, Entire section (diagrammatic). B, Small part of the peripheral tissues enlarged. C, Single resin-canal enlarged, showing the epithelium (ep.). Ca., cork cambium; e., epidermis ; $P h .$, phloem ; $r_{\text {., }}$ resin-canals ; $X y$., xylem.

This tissue $(P h$.$) is strikingly uniform, its elements, which have$ thick white shining walls, exhibiting a radial arrangement. The rows of more or less empty-looking sieve-tubes, which have no companion cells, are interspersed with a smaller number of irregular files of phloem-parenchyma, whose cells contain dense contents. In longitudinal sections the sieve-tubes appear as long tapering elements bearing sieve-areas (Fig. I97, E, si.) upon their sloping radial walls.

The secondary wood (Fig. I96, Xy.), internal to the narrow 
cambial zone, also exhibits a very uniform, radially seriated structure. Except for the narrow medullary rays and occasional resin-canals, it consists entirely of fibre-like tracheids, which are differentiated among themselves only in respect of the distinctions between spring- (Fig. I97, A, Sp.) and autumn-wood $(A u \text {. })^{1}$ (see p. I25). The tracheids bear a single row of large circular bordered pits on their radial walls, as can be recognised in transverse, but more readily in radial longitudinal (Fig. I97, C, b.), sections, when the pits themselves are seen in surface view; in the autumn-wood the tangential walls are also pitted. The groups of primary xylem, composed of spiral tracheids, project into the small pith and are separated from one another by the primary rays.

The structure just noted for the Scotch Fir is that typical of most Conifers, but resin-canals are absent from the wood in certain genera (being often replaced by resin-cells), whilst in the Araucarias, and occasionally in other members of the group (e.g. Pinus palustris), the tracheids bear two or more rows of bordered pits.

Radial and tangential longitudinal sections exhibit the same arrangement of the medullary rays as in Dicotyledons (Fig. I97). In some Conifers certain rays, which are relatively wide, are traversed by resin-canals connecting those of the pith and cortex. As a general rule the rays consist of uniform cells, whose walls often bear simple pits in Pinus and its allies, although elsewhere usually smooth. Several Abietineæ, including the Scotch Fir, show a complex differentiation of the rays, best seen in radial longitudinal sections. In the region of the wood the cells of the middle rows, which bear simple pits of exceptionally large size, are more particularly concerned with storage, and contain copious starch (Fig. I97, C, s.) ; on the other hand, the dead and empty. cells of the marginal rows $(t)$, which bear small bordered pits and often exhibit peg-like ingrowths of the walls, have a conducting function. Where the rays traverse the phloem, all the cells have thin walls and dense cytoplasm, but those at the margin (Fig. I97, E, a.) are often drawn out into finger-like processes which are insinuated between the sieve-tubes.

1 Annual rings are, however, absent from some Araucarias, and from most of the fossil representatives of this group. 
The root, which is generally diarch, shows quite the usual structure, although characterised by the presence of a large resin-canal within each protoxylem-group.

The leaves of most Conifers are traversed by a single vein

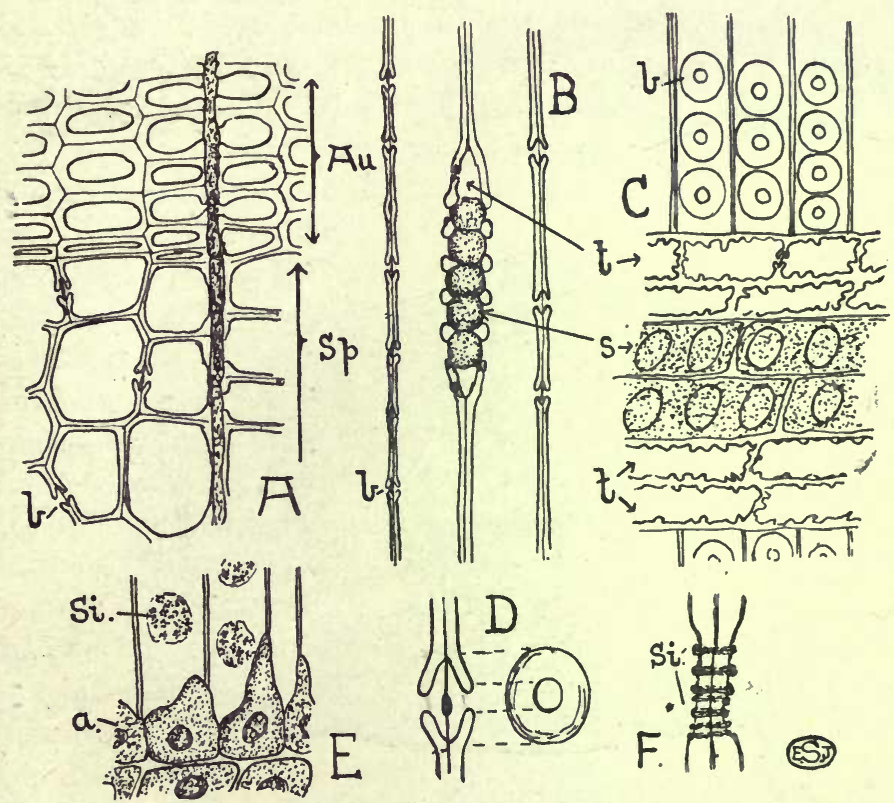

Fig. 197.- Structure of the stem of the Scotch Fir (Pinus sylvestris.) A, small part of the secondary wood in transverse section, showing spring- $(S p$.$) and autumn- (A u)$ wood; $\mathrm{B}$, the same in tangential longitudinal section showing a medullary ray; $\mathrm{C}$, the same in radial longitudinal section, with part of a medullary ray; $\mathrm{D}$, single bordered pit in section (on the left) and from the surface (on the right); $\mathrm{E}$, small part of the secondary phloem in radial longitudinal section; $\mathrm{F}$, single sieve-tube in tangential longitudinal section. $a$, albuminoid cells of medullary ray of phloem ; $b$, bordered pits; $s$, storage cells of medullary rays of wood; $S i$., sieve-plates; $t$, tracheidal cells of medullary rays of wood.

only, but, apart from that, their structure is somewhat varied. Its range can, however, be gauged from a consideration of the relatively simple dorsiventral leaf of the Yew (Taxus, Fig. I98) and the more complex centric one of the Scotch Fir (Pinus sylvestris, Fig. I99). In the former case the epidermis (Fig. 
I98, ep.) shows the thick cuticle and sunken stomata (Fig. I98, B) associated with transpiration-reduction ; the pronounced papillæ
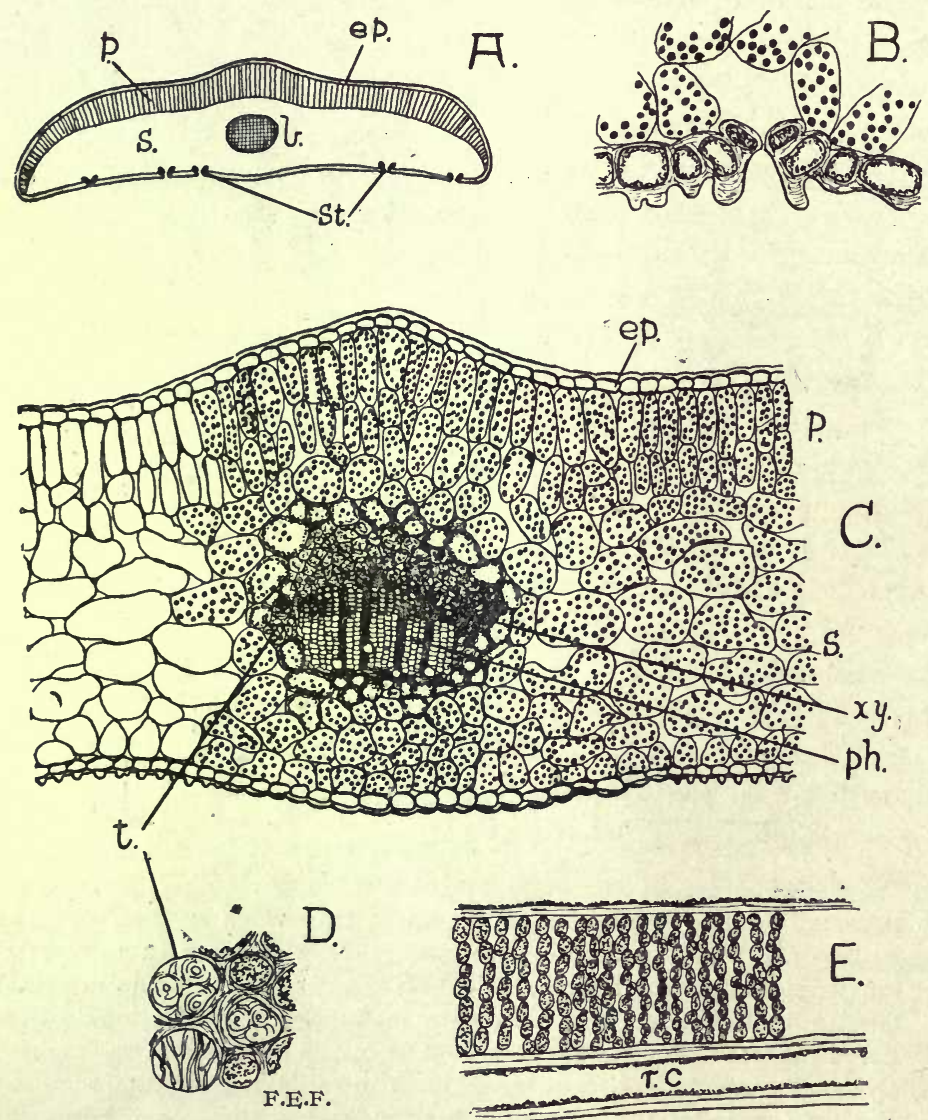

FIG. I98.-A-D, Structure of the leaf of theYew (Taxus baccata) in transverse section. A, Diagram of whole section; B, single stoma enlarged; $C$, central part of transverse section; $D$, a few cells of the transfusion tissue. b., bundle ; ep., epidermis ; p., palisade tissue ; ph., phloem ; $s .$, spongy tissue ; $S t$., stomata ; $t$., transfusion tissue ; $x y$. , xylem. E, Tangential longitudinal section of leaf of Pinus, showing a resincanal ( $\boldsymbol{r} . c$.$) and the transverse lamellæ of assimilating cells.$

on the lower surface (Fig. I98, B, C) are a special peculiarity. The mesophyll, comprising two layers of palisade cells $(p$.$) and$ 
spongy tissue (s.), is traversed by the single bundle (b.), which exhibits the usual dorsiventral structure. On either side of the xylem, however, are occasional spirally or reticulately thickened elements $(t$.), which sometimes bear bordered pits. These dead cells constitute what is called transfusion tissue, and may serve both for the lateral conduction of water, thus compensating for the absence of side-veins, and as water-reservoirs.

The epidermal cells of the Pinus-leaf (Fig. I99, A-D) also have a very pronounced cuticle $(C u$.), and, in this case, are so strongly thickened that their cavity is often reduced to a mere dot (ep.); beneath the epidermis is a strongly thickened hypoderm (h.). The deeply sunken stomata (St.) occur at intervals all round the leaf, as seen in transverse section, but, if the leaf be examined whole with a lens, they are seen to be arranged in longitudinal rows. The mesophyll contains several prominent resin-canals $(r$. $)$ and consists of more or less isodiametric cells, characterised by the presence of inwardly projecting folds of the wall $(i$.$) , which increase the surface for absorption of carbon$ dioxide (cf. p. IIO). It will be noticed that, apart from the respiratory cavities of the stomata, there are few intercellular spaces between the cells of the mesophyll. This tissue, however, consists of successive transverse lamellæ, and these are separated from one another by narrow air-spaces (Fig. I98, E). The twin-bundles (in some species of Pinus, as well as in Picea and Larix, there is only a single one) are connected by a group of thick-walled mechanical cells $(S c l$.) and are embedded in an extensive mass of tissue bounded by a wellmarked bundle-sheath (S.). This tissue consists, in large part, of ordinary living parenchymatous cells containing starchgrains. Scattered among these are the dead and empty cells of the transfusion tissue (cf. especially Fig. I99, C, tr.), in this case bearing small bordered pits (b.), which are seen both in section and from the surface. At the outer edge of the phloem of each bundle is a group of cells, whose dark contents are rich in proteins $(a$.$) , and which probably assist in the con-$ duction and storage of elaborated food-material.

IIt may be pointed out that, whilst many of the features of Coniferous leaves are those usually found in plants with reduced transpiration, others (such as the limited vascular supply and 
the transfusion tissue) are special peculiarities of the group. The necessity for the reduction of transpiration may well be connected with the relative inefficiency of the tracheids as waterconducting elements. ${ }^{1}$ The relation between the vascular system of stem and leaf in Conifers is similar to that which obtains in Flowering Plants (cf. p. II4).

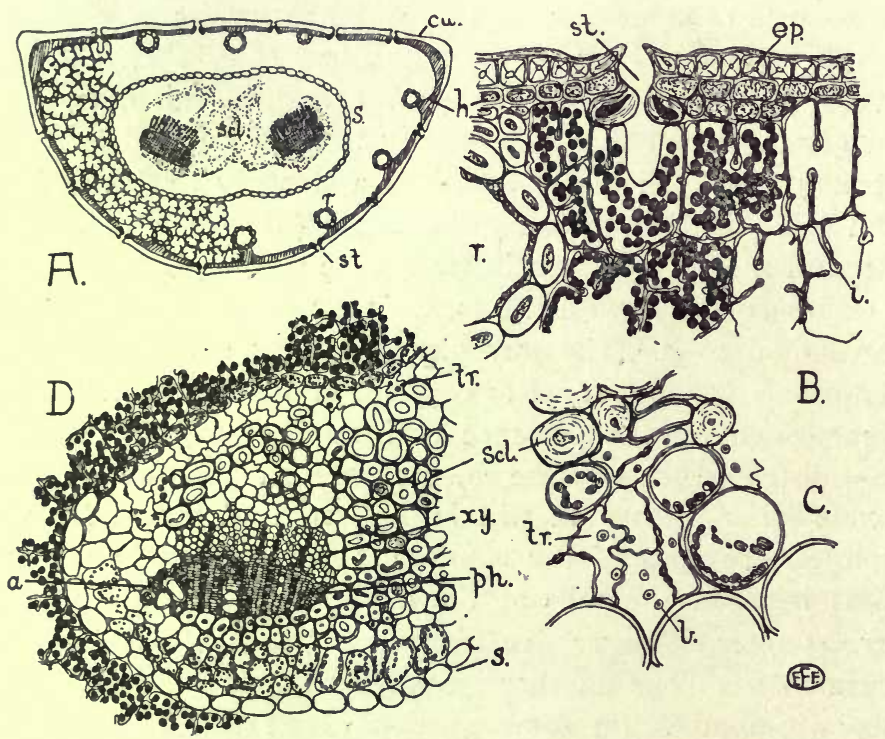

FIG. 199.- Structure of the leaf of Pinus in transverse section. A, Diagram of whole section. B, Small part of the superficial tissues, enlarged. C, A few cells of the transfusion tissue, etc., from the central vascular cylinder, greatly enlarged. D, About half the central vascular cylinder. $a$., albuminous cells ; $b$., bordered pit on transfusion cell ; $C u$., cuticle ; $e p .$, epidermis ; $h$., hypoderm; $i$., folds on walls of mesophyll-cells ; $p h .$, phloem ; $r_{\text {., }}$ resin-canal ; $S$., bundle-sheath ; Scl., sclerenchyma; st., stomata; tr., elements of transfusion tissue; $x y$., xylem.

The sporophylls of Conifers, like those of Cycads, are grouped in cones (Fig. 200), the two kinds being most commonly found on the same plant, though Taxus (Fig. 20I), for instance, furnishes an exception. The male cones, which often occur in clusters (Fig. 200, on the right), are yellow oval structures of diverse size, and frequently arise in the axils of the foliage(e.g. Taxus, Fig. 20I; e; Abies, Fig. I93, A) or scale-leaves 
(e.g. Scotch Fir and other species of Pinus). In Pinus the male cones replace the dwarf-shoots (Fig. 200), but in other cases (e.g. Larch) the latter bear leaves below the male cones.

The axis of the male cone (Fig. 202, A) supports large numbers of densely crowded microsporophylls (stamens), which mostly have the form of flattened scales, whose upturned overlapping tips are alone visible at the surface. They usually bear two large microsporangia (pollen sacs) on their under-surface (Fig. 202, B, ps.) although there are sometimes several (e.g. Cupressus). In Taxus the stamens are peltate in form, with several (5 to 9) pollen sacs pendant from the lower side of the lamina (Fig. 20I, d). In Araucaria also the

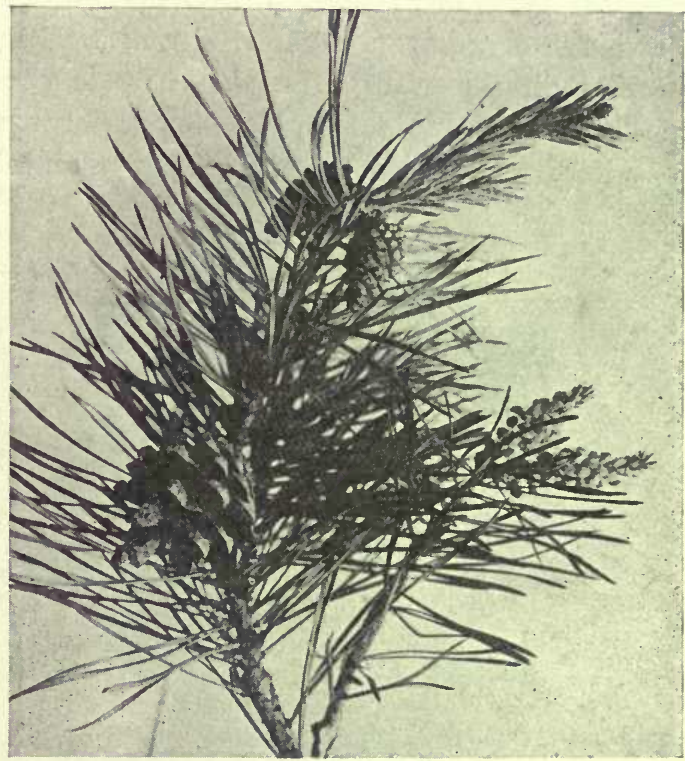

FIG. 200.-The Scotch Fir (Pinus sylvestris). On the right a branch bearing two clusters of male cones; on the left a branch with two fertilised female cones above, a mature cone from which the seeds have been shed below, and a young, just pollinated, cone at the extreme apex. On both branches the dwarf-shoots of the previous season areseen below, and young dwarf-shoots of the current year's growth above. (From a photo by W. B. Johnson.) microsporophylls bear numerous (Io to 20) pollen sacs.

The young microsporangia are provided with a wall of several layers which encloses large numbers of spore mother-cells, each dividing in the customary manner to form four microspores (pollen grains). The pollen sacs usually dehisce by means of a broad split, and the pollen is distributed by the wind. In Pinus and its allies the microspores develop a pair of blister-like en- 
largements, between the cuticle and the inner layer of the membrane ; these contain water, which subsequently evaporates, thus leaving two bladders which act as wings (Fig. 204, A, w.).

The ovules (megasporangia) of the Yew (Taxus) usually occur singly within small buds, which arise in the axils of the leaves and are generally spoken of as female cones; they closely resemble the ordinary vegetative buds. Each such cone bears several minute scales, the single ovule occupying a terminal position with the micropyle facing outwards. The ovule is peculiar in possessing, apart from the usual integument, a second
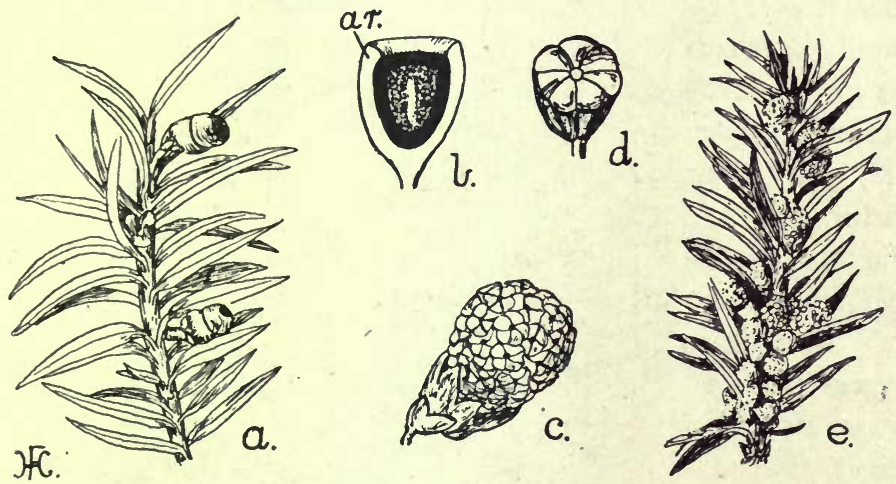

FIG. 201. -The Yew (Taxus baccata). $\quad a$, branch bearing two ripe seeds ; $b$, a ripe seed in longitudinal section, showing the aril (ar.); $c$, single male cone, enlarged ; $d$, single microsporophyll; $e$, branch with male cones. ( $d$ after Eichler; the rest original.)

protective'covering termed the aril, which remains inconspicuous till after fertilisation. It then develops into a bright-coloured fleshy cup investing the seed (Fig. 2or, $a, b$ ).

In most Conifers, however, more or less numerous ovules are found within each of the female cones, and these in the Monkey Puzzles (Araucaria), for instance, may attain considerable dimensions. The cones themselves occupy diverse positions. Thus, in the Scotch Fir (Fig. 200, on the left) they first appear as small reddish structures, situated at the ends of short stalks, just beneath the apical bud of the current year's growth. In the Larch, where they are larger and of a bright crimson colour, they terminate some of the dwarf-shoots, with a rosette of green 
needles at their base (Fig. I93, B). The small cones of the Cypress and Arbor Vitæ (Fig. I94) occupy a similar position.

The scale-like sporophylls generally show a spiral arrangement, although in Cupressus and Thuja they are decussate, like the foliage-leaves. In Pinus and other Abietineæ (Larix, Abies, etc.) the axis of the cone bears two different types of scales which are quite distinct from one another, and occur in superposed pairs (Fig. 202, D). The lower or bract scale (bs.) of each pair is smaller than the upper or ovuliferous scale $(O s$.$) . The latter$ appears to arise in the axil of the former, and bears, on its upper surface, two ovules $(O v$.) whose micropyles face towards the axis
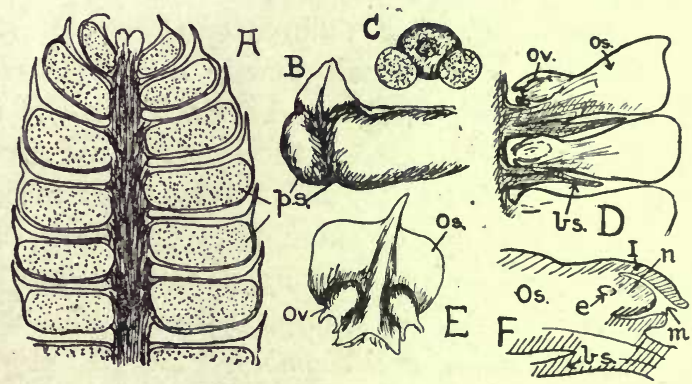

FIG. 202.-A-B, Structure of male cone of Pinus sylvestris. A, Upper part of cone, in longitudinal section; $\mathrm{B}$, Single sporophyll. ps., pollen sacs. C, Pollen grain. D, Longitudinal section through female cone of Pinus. E, Single megasporophyll of Pinus, from below. F, Part of $\mathrm{D}$, enlarged. $b s$. , bract scale; $e$. , megaspore; $I$, integument; $m$., micropyle ; $n$., nucellus ; Os., ovuliferous scale ; Ov., ovule.

of the cone (Fig. 202, E). The difference in size becomes more pronounced as the cone grows older, the ovuliferous scale enlarging to a much greater extent than the bract scale. In the Wellingtonia (Sequoia, Fig. 205, D) and Cupressineæ bract and ovuliferous scales are almost completely joined, whilst in some Conifers (e.g. Cryptomeria) the line of fusion is plainly recognisable. The scales in the Cupressineæ usually bear more than two ovules, situated with the micropyles directed outwards; not infrequently the uppermost scales are sterile (e.g. Thuja). The Monkey Puzzles are peculiar in having but a single ovule on each megasporophyll.

The ovules in all Conifers, apart from those of the Yew-group, possess a single thick integument (Fig. 202, F, I; Fig. 203, i.). This 
is partially fused on one side with the ovuliferous scale (Fig. 202, F), and, except in a few genera, the central nucellus is only free from the integument in the region of the micropyle (Fig. 203, $\mathrm{B}, m i$.). A single megaspore $(p$.$) is usually differentiated within$ the nucellus of the young ovule by the division of a mother-cell which arises hypodermally. This forms a linear tetrad of potential spores of which only the innermost survives. The functioning spore rapidly enlarges and eventually displaces the greater part of the nucellus, though a pronounced cap of tissue still remains in the free portion beneath the micropyle (Fig. 203, n.). The thin-walled parenchymatous female prothallus $(p$.$) , which is richly supplied with food-material,$ as a rule arises only after pollination has occurred. The archegonia (ar.), which are usually few in number, are generally differentiated at the micropylar end. Each consists of a relatively huge ovum $(o$.$) embedded in the tissue of the prothallus, a$ minute ventral canal cell (v.c.), and a very short inconspicuous neck (a.) composed of one or more tiers of cells. When the archegonia are numerous (as in most Cupressineæ) they are usually collected together in groups, opening into a common shallow depression (archegonial chamber), at the front end of the prothallus.

When the mature pollen is being scattered broadcast by the wind, the axis of the female cone elongates slightly, so that the cone-scales spread apart, thus giving access to the ovules. At this time each of the latter secretes a mucilaginous drop which oozes out from the micropyle, and serves to catch up the microspores as they are blown between the scales. By the gradual shrinking of the mucilage the pollen grains are sucked through the micropyle on to the surface of the nucellar cap, and, soon after this, the scales of the female cone enlarge and once again fit tightly together. This process of pollination must be sharply distinguished from that of fertilisation which occurs at a later stage - often only after a prolonged interval (see below).

When shed, the contents of the microspores have already undergone division, the cell, or cells, thus cut off from the general body lying against one side of the grain (Fig. 204, B) ; in most cases this comprises the single antheridial cell (a.c.) only, but in Pinus and related forms its formation is preceded by the cutting off 
of two very flat cells, which soon break down (Fig. 204, B, v.c.). The remaining and larger portion of the pollen grain forms the so-called tube cell $(t . n$.). On reaching the nucellus the outer membrane of the microspore is ruptured, and the tube cell grows out to form the pollen tube (Fig. 203, A, p.t.), into whose
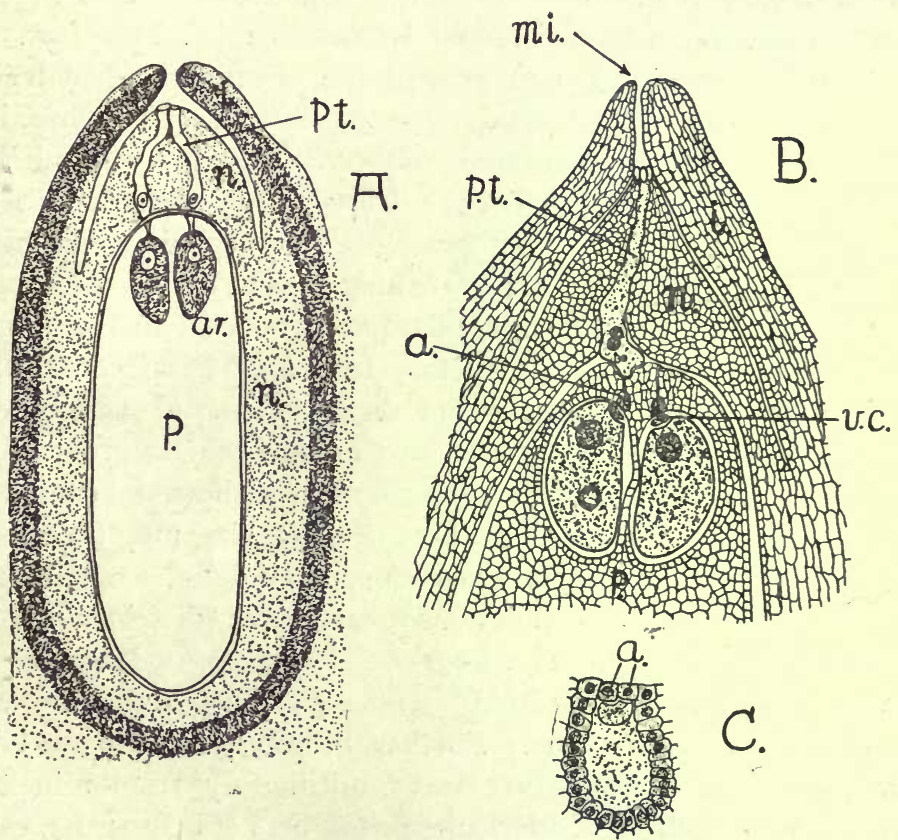

FIG. 203.-Structure of the Ovule of Pinus. A, Diagrammatic longitudinal section of mature ovule (after Coulter and Chamberlain). B, Front portion of same greatly enlarged, showing a pollen tube penetrating the nucellus (after Strasburger). C, Young archegonium showing the layer of nutritive cells investing the egg (after Ferguson). a., neck of archegonium; $a r_{\text {., }}$ archegonium; $i$. , integument ; mi., micropyle; n., nucellus ; 0. , egg ; p., female prothallus ; p.t., pollen tube ; s., stony layer of integument; v.c., ventral canal cell.

tip its nucleus wanders, whilst simultaneously the antheridial cell divides into two, the inner half (body cell) subsequently forming the two male cells.

The growth of the pollen tube into the nucellus soon ceases, and is not resumed till a short time before fertilisation. This interval between pollination and fertilisation is comparatively 
short (four to six weeks) where the seeds mature in the same year as that in which pollination occurs (e.g. Thuja, Picea), but is very prolonged (twelve to thirteen months) in the Pines, where pollination ensues in May or early June, whilst the seeds are not shed till the autumn of the following year. In the latter case there is very pronounced growth of the female cones in the interval between pollination and fertilisation (cf. Fig. 200).

With the resumption of growth the pollen tube penetrates deeper into the nucellus (Fig. 203, p.t.), frequently exhibiting slight lobing, and the two naked male cells (often merely nuclei), resulting from the division of the body cell, pass down into its apex (Fig. 204, C, ô). On reaching an archegonium the neck is crushed, and the pollen tube, breaking open at its tip, discharges its contents into the ovum. Subsequently one of the two male nuclei fuses with the egg-nucleus. In Pinus the second male nucleus is smaller (Fig. 204, C) and aborts, though in some genera (e.g. of Cupressineæ), where the archegonia occur in groups and the pollen tube discharges into the common archegonial chamber, both the male nuclei, which are here of equal size, may function.

The fertilised egg, becoming enveloped in a delicate membrane, almost immediately exhibits two successive divisions of its nucleus (Fig. 204, D). The four nuclei thus formed wander to the end of the egg remote from the micropyle, where they become grouped in a single plane. Further division, accompanied by the formation of separating walls, ultimately results in the development of three or four superposed tiers (Fig. 204, E), each usually consisting of four cells and occupying only a small part of the oospore. In this proembryo the tier furthest from the micropyle mostly gives rise to the new plant, ${ }^{1}$ whilst the cells of the adjacent tier elongate very considerably (Fig. 204, F, S.), and form a suspensor (cf. Selaginella, p. 3I9), which carries the developing embryo (em.) down into the middle of the prothallus.

The cells of the embryonic tier divide repeatedly to form an extensive mass of tissue. At the end away from the micropyle a number of lobes soon grow out and form the cotyledons (Fig. 204, G, cot.). These surround the developing plumule $(p$.$) ,$

$i$ In Pinus and some other genera the cells of the proembryo commonly separate, and as many as eight embryos may be formed, but, as in other conifers, one only reaches maturity. 
whilst the radicle $(r$.$) arises as a pointed structure at the opposite$ end, adjacent to the suspensor $(S$.$) . The number of cotyledons$ is very variable; there are only two in Taxus and Cupressinece (Fig. I95, B), whilst in Pinus (Fig. I95, A) there are from three to seventeen, according to the species.

As the embryo enlarges it absorbs the food-reserves stored
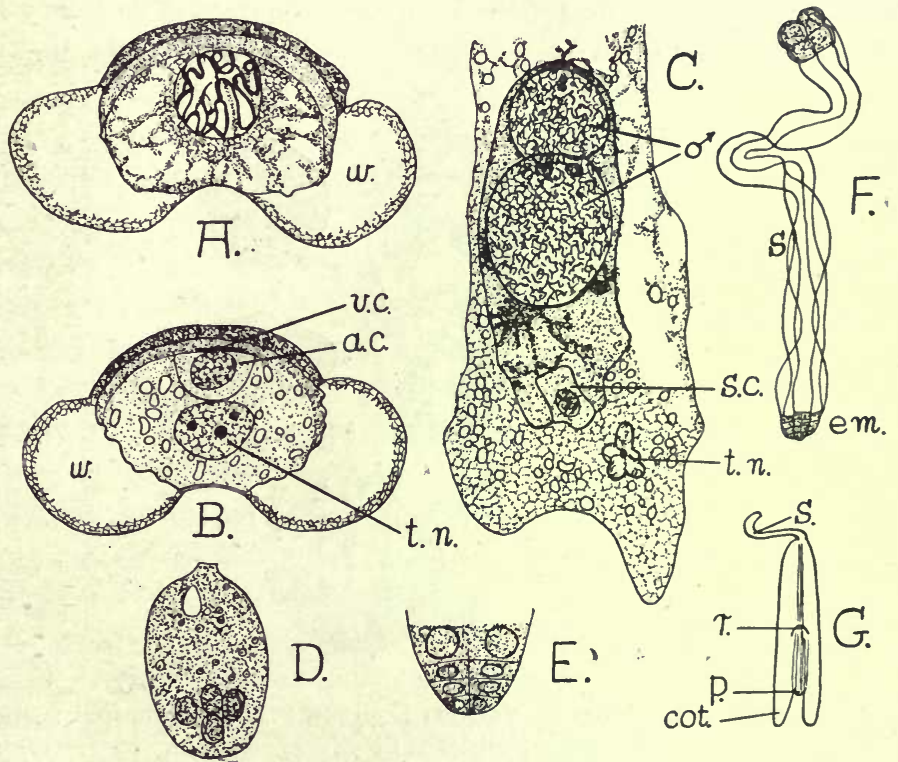

t. $n$.

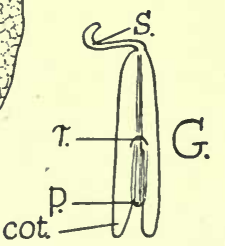

FIG. 204.-Pollen and embryology of Pinus. A, Mature pollen grain in which the nucleus has not yet divided; $B$, pollen grain at the time of pollination ; C, lower end of pollen tube a little before fertilisation. a.c., antheridial cell ; s.c., stalk cell; t.n., tube nucleus; v.c., vegetative cell; $w$., wing of pollen grain; $\hat{o}$ (in $\mathrm{C}$ ), the two male nuclei. D-G, Embryology. D, Fertilised ovum, with the nucleus divided into four: E, lower end of oospore, showing the young proembryo; F, much older proembryo; G, almost mature embryo.

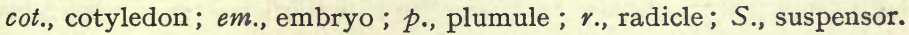
(A-D after Ferguson; the remainder after Strasburger.)

up in the prothallus and gradually displaces it. At the time when the seed is fully mature, however, a considerable part of this tissue still persists unaltered around the embryo and constitutes the endosperm (Fig. 205, A, e); the seed of Conifers is thus albuminous. The food-reserve is largely of the nature of oil, and 
is present in sufficient quantity to render the kernels of some species of Pines of nutritive value. The method of germination of the seeds is practically the same as that of a Castor Oil, the seedlings (Fig. I95) possessing long hypocotyls $(h$.) with epigeal cotyledons (cot.).

During the development of the embryo the entire ovule enlarges considerably, and at the same time the integument hardens to form the seed-coat, or testa (s.). The greater part of the

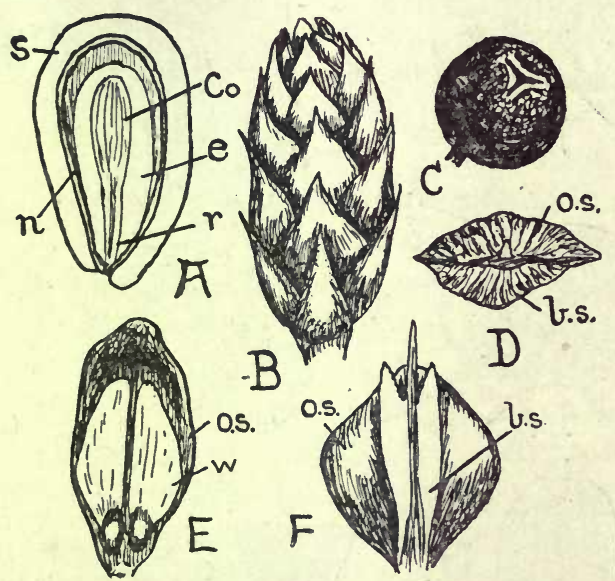

FIG. 205.- Seeds and cones of various Conifers. A, longitudinal section of ripe seed of the Stone Pine (Pinus pinea), showing the testa (s), the perisperm ( $n, \mathrm{cf} . \mathrm{p} .37 \mathrm{I})$, endosperm $(e)$, and the embryo with radicle $(r)$ and numerous cotyledons $(\mathrm{Co}) ; \mathrm{B}$, young female cone of Juniper (Juniperus) and C, ripe cone of same; D, outer edge of ripe conescale of Wellingtonia (Sequoia); E, single cone-scale of Pinus bearing the two seeds with wings $(w) ; F$, ripe cone-scales of Douglas Fir (Pseudotsuga). b.s., bract scale; o.s., ovuliferous scale. (B, after Antoine.)

latter comes to consist of very thick-walled tissue, foreshadowed as a dark zone in the integument of a young Pinus-ovule (Fig. 203, A, s.), and constituting the hard layer which is so conspicuous a feature in the seed of the Stone Pine (Pinus pinea). The micropyle appears on the testa as a minute opening, and it will be realised, from the foregoing description, that the tip of the radicle lies just beneath this. In Pinus and some of its allies, a thin membranous flake becomes detached from the 
ovuliferous scale and adheres to the ripe seed, forming a wing which aids in dispersal by the wind (Fig. 205, E, w). In Cephalotaxus and a few other genera the ripe seeds are plum-like, resembling those of Cycads.

The cones also undergo considerable enlargement in ripening, often becoming many times larger than they were prior to fertilisation (Fig. 200). The ripe cone-scales are usually woody, and gape apart to set free the seeds, although in the Cedar, for instance, they are shed with the latter. At this stage the bractscales in Pinus are no longer recognisable, though in some of the related forms (e.g. the Douglas Fir, Psendotsuga douglasii, Fig. $205, \mathrm{~F}$ ), they are conspicuous even in the mature cone. The Juniper is peculiar in that the cone-scales become fleshy, forming a berry-like structure (Fig. 205, C) in which the ripe seeds are embedded; the seeds are in this case distributed by birds.

In the life-history of the Conifers the method of fertilisation shows a considerable advance on that of Cycads, since the pollen tube, which is there but an organ for the absorption of nourishment from the nucellus (a function which it may also fulfil to a slight extent in the Conifers), is here utilised to convey the motionless male cells to the egg. Thereby the necessity for the presence of liquid water at the time of fertilisation is dispensed with, and we may see herein the loss of the last traces of the probable aquatic ancestry of the Vegetable Kingdom. 


\section{CHAPTER XXIV}

\section{The Angiosperms}

THE majority of the Angiosperms, the second subdivision of the Phanerogamia, are easily distinguished from the Gymnosperms by the possession of flowers, which are really highly specialised fertile shoots. In some cases, however, the flowers are relatively inconspicuous (e.g. Grasses and other windpollinated plants), and scarcely conform to the popular notion of these structures. As a matter of fact, the Angiosperms are much more markedly characterised by the enclosure of their ovules in a protective ovary, as well as by the possession of true vessels and of sieve-tubes of a special type (cf. p. 28).

The ordinary flower ${ }^{1}$ comprises stamens (microsporophylls) and carpels (megasporophylls) surrounded by one or two series of modified leaves, serving either for protection (calyx) or for the attraction of pollinating insects (corolla). These " nonessential" organs are not uncommonly reduced (e.g. apetalous flowers) or absent (naked flowers, e.g. Hazel). Though the majority of Angiosperms have hermaphrodite flowers, unisexuality is not uncommon, the two sexes occurring either on the same (monœcious, e.g. Hazel) or on different plants (diøcious, e.g. Willow).

In those flowers, which for many reasons are regarded as relatively unspecialised (e.g. Ranunculaceæ and its allies), the floral axis is elongated, bearing sepals, petals, stamens, and carpels at successively higher levels (hypogynous flowers), and these parts are wholly or in part spirally arranged; the stamens and carpels, moreover, are usually numerous, and not joined in

1 The reader is advised to familiarise himself fully with the broad features of floral structure (cf. F. and S., chapters xviii, xix) before passing on to the subsequent matter. 
any way. The further evolution of the flower has brought about increased efficiency and precision in pollination, and appears to have involved a gradual shortening of the floral axis, with the result that the different sets of organs became whorled and reduced in number. At the same time fusion of parts took place to a more or less marked extent. Thus, the commonest type of Dicotyledonous or Monocotyledonous flower possesses two whorls of stamens ( 3 to 5 in each whorl), and a syncarpous ovary formed by the fusion of a small number of carpels. Another development appears to have been concerned with the better protection of the ovary, the receptacle gradually becoming more and more concave (progressive stages can be found in the perigynous Rosaceæ), until the ovary is completely enveloped (epigynous flowers). Certain it is that the most highly specialised families (Umbelliferæ, Compositæ, Orchidaceæ) all possess such epigynous flowers, with the different members in whorls. The irregular (zygomorphic) flowers found in many families are no doubt a special development connected with the perfection of mechanisms for insect pollination (cf. p. 373).

The classification of Angiosperms into Monocotyledons and Dicotyledons is based on many morphological and anatomical features (leaf-form, number of cotyledons in the embryo, stemand root-structure, and number of floral parts), the Monocotyledons being an essentially herbaceous group exhibiting a high perfection of means for perennation and hibernation (e.g. bulbs, corms, etc.). The evidence that the Dicotyledons and Monocotyledons were derived from a common stock is almost overwhelming. This fact is plainly indicated, for example, by the widespread traces of the presence of a cambium in Monocotyledons, and the extremely few characters, either of development or structure, that are entirely restricted to the one or the other group (cf. also p. 367 ).

The Angiosperms are the predominant vascular plants on the earth's surface at the present day, and comprise some 125000 distinct species. In Britain this preponderance is especially marked. The native Angiosperms here number about 2,000 species and the Gymnosperms four species; amongst the Cryptogams, there are about 60 Pteridophyta, 600 Mosses, 250 Liverworts, several thousand species of Algæ, and some 5,000 
species of Fungi. The Angiosperms first appear in the geological succession in the Cretaceous period, but the study of these and of the abundant Tertiary fossils, though affording much that is of interest, has so far shed no light whatsoever on the origin of the group, which is merged in obscurity. The details of the life-history betray a very high degree of specialisation, but afford practically no evidence as to how this has been attained.

The ovary of the Angiosperm consists of one or more carpels or megasporophylls, whose nature is most obvious where they are distinct from one another, as in the Marsh Marigold and Pea. Here dissection shows the carpel to have a leaf-like structure, the midrib being marked by a well-defined vascular bundle, whilst the somewhat enlarged edges are joined and form two placentce, each bearing a row of ovules. Except, therefore, for the inrolling, such a carpel might be compared to the megasporophyll of Cycas (cf. p. 324).

Since the ovules are enclosed, the pollen cannot reach the micropyle direct as in Gymnosperms, but is received by the tip of the carpel, which is modified to form a special receptive surface, the stigma. Upon this germination of the pollen takes place. The stigma bears numerous papilla-like hairs, whose secretion may nourish the developing pollen. Very commonly the upper part of the carpel is produced into a stalk-like prolongation, termed the style, which serves to raise the stigma to a position more suitable for the reception of the pollen.

When, as is usually the case, several carpels are fused together to form a syncarpous ovary, the number of component carpels may be indicated by the number of loculi or by the number of lobes exhibited by the stigma. In most syncarpous ovaries the ovules retain their position on the margins of the carpels (axile or parietal placentation), though other arrangements obtain in some cases. Thus, in the Primulaceæ and Dock-family (Polygonacex) the ovules are borne on a prolongation of the floral axis (free central placenta of the former), whilst in the Flowering Rush (Butomus) and the White Water Lily (Nymphaa) they occur on the entire inner surface of the carpels. In many Angiosperms the ovary contains numerous ovules, but the number is not uncommonly reduced to one in each loculus (e.g. Umbelliferæ), or the whole ovary produces but a single ovule (e.g. Gramineæ, 
Compositæ). This last condition is often characteristic of some of the most advanced types of Flowering Plants.

The ovules of Angiosperms (Fig. 206), which in their general construction are like those of Gymnosperms, usually possess two integuments (inner, I.i., and outer, O.i.). A single one is the rule amongst the gamopetalous Dicotyledons (Sympetalæ) and a few polypetalous families (e.g. Umbelliferæ, Salicaceæ, etc.). In these latter, however, the single integument is generally relatively robust, a fact which suggests its possible origin from the fusion of two; a partial union of this character is, indeed, seen in some members of the Ranunculacex. ${ }^{1}$ The nucellus (n.), with very few exceptions (e.g. Bog Myrtle, Myrica), is only clearly separated from the integument at the apex, where it forms a cap of tissue extending between the large megaspore (embryo sac, e.s., see p. 365) and the micropyle $(m$.$) . The part of$ the nucellus in contact

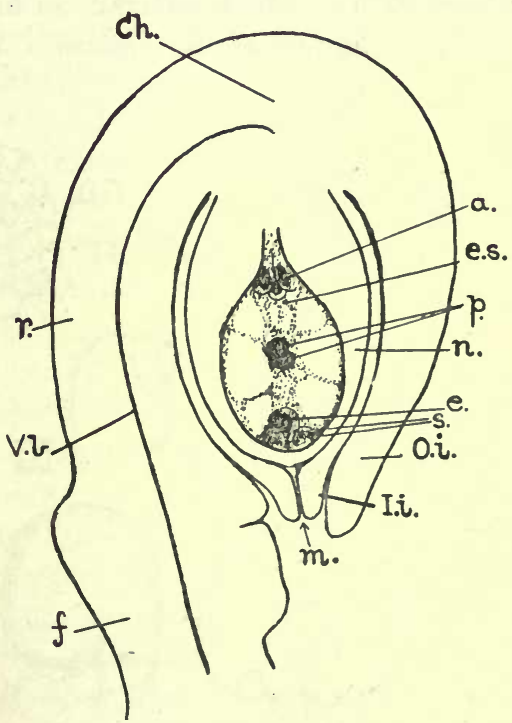

FIG. 206.-Diagrammatic longitudinal section through an anatropous ovule, greatly magnified. a., antipodal cells ; Ch., chalaza ; e., egg; e.s., embryo sac; $f$., funicle; $I . i$., inner integument; $m$., micropyle; $n$., nucellus; O.i., outer integument; p., polar nuclei ; $\boldsymbol{r}_{\text {, }}$ raphe ; $s$., synergidæ; $V . b .$, vascular bundle of raphe. with the integument can often be distinguished from the latter, in sections of the ovule, by the dense contents of its cells, which form a nutritive layer around the developing embryo sac.

By far the commonest type of ovule is the anatropous one (Figs. 206 and $207, \mathrm{C}$ ), in which the main body of the ovule is

1 In a few Rosaceæ (e.g. Geum) there is only a single integument due to failure of the inner one to develop. 
bent down against its stalk (funicle, $f$.), so that the micropyle $(m$.) is directed towards the placenta. The greater part of the funicle is in this case fused with the adjacent portion of the outer integument, which is in consequence not easily. distinguished on this side. The fused region, known as the raphe $(r$.$) , is traversed$ by the vascular bundle $(V . b$.) which enters the funicle from the placenta, and which extends as far as the base of the nucellus (a region known as the chalaza, $C h$.).

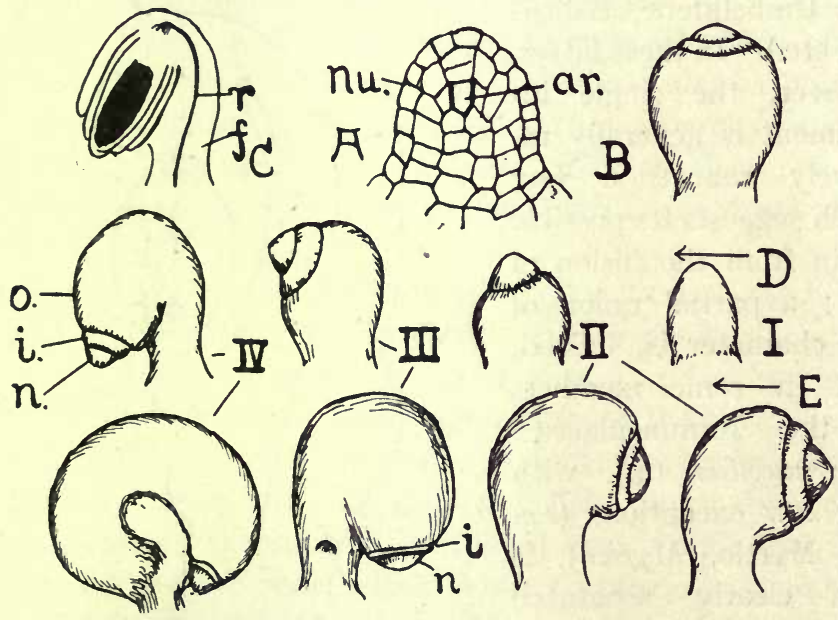

FIG. 207.-Ovules and stages in their development (after Le Maout and Decaisne). A, Young ovule, before the integuments appear, showing nucellus $(N u$.) and dividing archesporium $(a r$.$) . B, Orthotropous ovule$ of Polygonum. C, Anatropous ovule in longitudinal section, showing funicle $(f$.$) and raphe (r$.$) . D, I-IV (in the direction of the arrow),$ successive stages in the development of the anatropous ovule of Chelidonium. E, Successive stages (in the direction of the arrow) in the development of the campylotropous ovule of the Mallow (Malva). $i$., inner integument ; $n$., nucellus ; 0. , outer integument.

Erect or orthotropous ovules, in which the micropyle faces away from the placenta, and lies at the opposite end to the usually short funicle (Fig. .207, B), are much less frequent, but are found, for example, in the Knotweeds (Polygonum). Another rare type, the campylotropous ovule (Fig. 207, E), seen in many Caryophyllaceæ, is bent in such a way that the stalk appears to be attached midway between the chalaza and micropyle. 
All Angiospermous ovules are supplied by a vascular bundle which runs through the funicle, but terminates at the chalaza, except in those rare instances (e.g. Myrica) where the nucellus is not in intimate contact with the integument and hence provided with a special vascular supply.

The ovule invariably arises on the placenta as a small mound of thin-walled cells which represents the future nucellus (Fig. 207, A and D, I). Around the base of this the integuments subsequently develop as ring-like upgrowths, the inner being formed before the outer (II-IV). If a third integument, or aril, be present (as in the Spindle-tree, Euonymus), this is the last to arise, and only becomes conspicuous after fertilisation.

All ovules are at first erect. The anatropous form, characteristic of the majority, is due to excessive growth on one side of the chalazal region of the rudiment, so that the latter gradually becomes curved till the micropyle points towards the placenta (cf. Fig. 207, D). In the case of campylotropous ovules the mature form is due to even more extreme asymmetrical growth of the chalazal region (Fig. 207, E). Stages in the development of anatropous ovules can be readily studied in transverse sections through young ovaries of the Poppy.

In the case of the stamens or microsporophylls the leaf-like character is not so obvious as that of the carpels. The lamina is represented by the connective which bears the microsporangia (pollen sacs) on its margin, i.e. in the same position as the ovules. Clearer evidence of the foliar nature of the stamen is afforded by cases in which the connective is developed as a flat petal-like structure (as in the Pondweeds, Potamogeton, Fig. $208, \mathrm{~B}, \mathrm{Co}$.), or in which stipule-like outgrowths arise from the bases of the filaments (as in the Onions, Allium, Fig. 208, C). 
A single vascular bundle traverses filament and connective. In the vast majority of cases the anther bears four pollen sacs, two on either side of the connective (Fig. 209, A), although the stamens of the Mallow-family (Malvaceæ), for instance, have but two pollen sacs. In transverse sections through very young immature flower-buds, the anthers are seen to consist of uniform

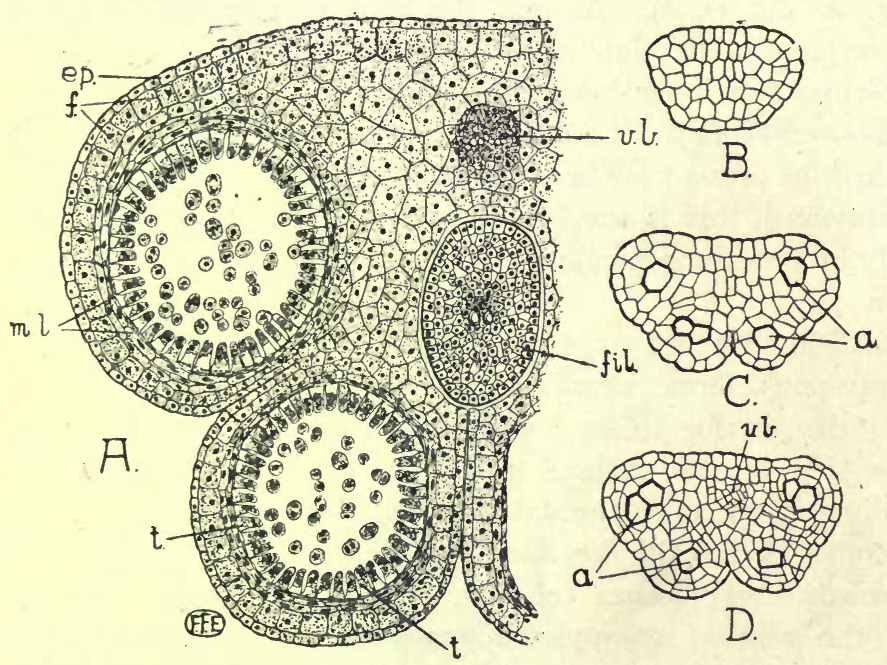

FIG. 209.-Development of the anther. A, Section of rather more than half the anther of a young stamen, showing the pollen-sacs at the mother-cell stage (the mother-cells are seen in various phases of division)., B, Section of young anther before the archesporia are differentiated. C and D, Later stages. a., dividing archesporial cells ; $e p .$, epidermis of anther ; $f$., fibrous layer ; fil., filament ; m.l., middle layer ; $t$., tapetum; $v . b$. , vascular bundle of connective. (A original, the remainder after Warming.)

thin-walled tissue bounded by a distinct epidermis, whilst a slight lobing foreshadows the future pollen sacs (Fig. 209, B). At a slightly later stage four subepidermal strips of varying width (often of only a single cell) become distinguishable, one in each lobe; these strips consist of cells with prominent nuclei and dense contents and constitute the archesporia (Fig. 209, C, a.), each of which extends as a narrow band of tissue for almost the whole 
length of the anther. Each archesporial cell divides tangentially (Fig. 209, C), and, from the inner halves (a.) thus formed, the actual pollen-producing tissue arises, so that the pollen mothercells are really subepidermal in origin. The outer half of each archesporial cell divides to form the wall of the pollen sac (Fig. 209, D), which comes to consist of several layers.

In transverse sections through older anthers (Fig. 209, A; Frontispiece, Fig. 2Io), the mass of spore mother-cells in the centre of each pollen sac is seen to be surrounded by a nutritive layer (tapetum, t.). This is composed of large, often palisadelike, cells with prominent nuclei and dense contents. Between the tapetum $(t$.$) and the epidermis (e p$.$) of the anther are two$ or more layers, the outermost of which generally consists of rather large, subsequently thickened cells (fibrous layer, $f$.), whilst the others (m.l.) are usually very much flattened.

Each pollen mother-cell, as in the groups previously studied, undergoes two divisions (cf. Fig. 209, A), the first of which is the reduction division. The four pollen grains, thus formed within each mother-cell, generally fall apart as the membrane of the latter becomes dissolved. But in a few cases the members of each tetrad remain in connection, when the pollen is shed (e.g. Typha, Neottia, Fig. 2II, D, a-c; Ericaceæ, Fig. 2II, D, d.), and a more extreme condition is seen in Orchids, where all the pollen grains formed by the single stamen cohere as two masses or pollinia (Fig. 2II, F). Such cohesion is, however, only met with in insect-pollinated flowers. During the development of the pollen grains the tapetum undergoes gradual disorganisation (Fig. $212, t$.$) , prior to which the cells often become bi- or$ multi-nucleate.

The mature pollen grain possesses a wall of two layers, the outer of which is thick and cuticularised, whilst the inner is delicate. Where the pollen is wind-borne, its surface is commonly smooth, 1 but when conveyed by insects it is usually sculptured in various ways (Fig. 2II, B, G). The outer layer

1 Similar smooth pollen is characteristic of plants in which pollination is effected under water (e.g. Hornwort). Such water-borne pollen is sometimes highly specialised, that of the Sea-Grass (Zostera), for example, being tubular, of the same specific gravity as sea-water, and without any cuticularised layer. 
is perforated, or rendered thin, by one or more pores or pits (Fig. 2II, C, o.), through one of which the pollen tube is subsequently protruded. In this respect the pollen of Angiosperms is more specialised than that of Gymnosperms, where the outer layer of the membrane is merely burst open in germination.

As the pollen matures the walls of the cells composing the
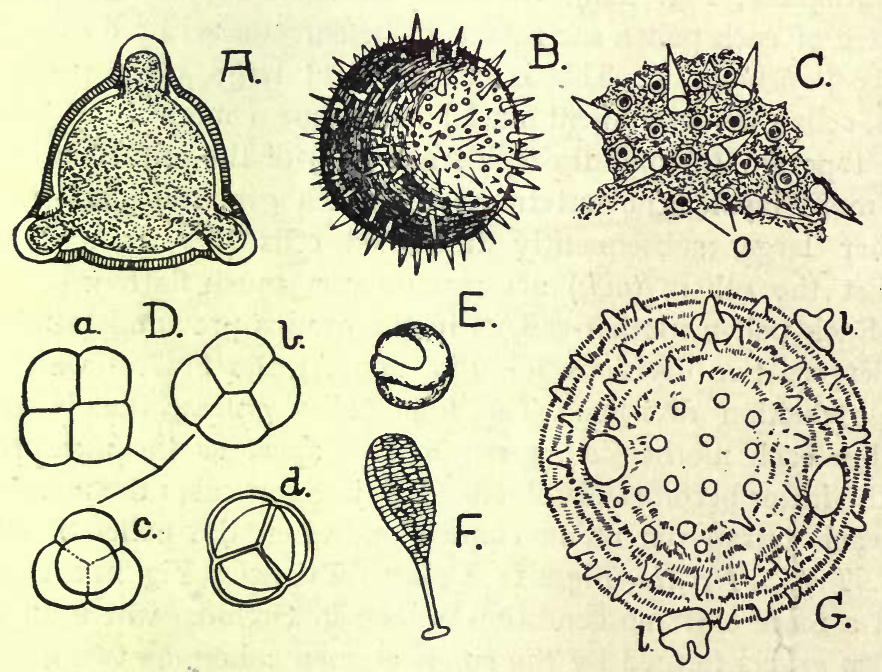

FIG. 2II.-Diverse types of pollen. A, Willow-herb (Epilobium), with three thin spots in the outer coat. B, Hollyhock (Althaa), with numerous pores. C, Small part of same enlarged, showing the pores $(o$.$) .$ D, Pollen-tetrads, $a-c$, of Bird's Nest Orchid (Neottia nidus avis), $d$, of Bilberry (Vaccinium myrtillus). E, Musk (Mimulus moschatus). F, Pollinium of Orchis morio. G, Pollen grain of Gourd (Cucurbita pepo), showing the embossed lids $(l$.$) of the outer coat, which become$ pushed off by the growing pollen tubes. (A-D after Sachs ; $d$ and $F$ after Wettstein; E after Mohl; G. after Schacht.)

fibrous layer become strengthened by a network of thickening bars, and simultaneously the cells loose their living contents (Fig. 212, en.) ; many of the cells in the region of the connective, moreover, develop similar thickenings. At this stage the two pollen sacs, in each half of the anther, become confluent through the drying up of the intervening thin-walled septa, and it is this same process of desiccation that ultimately leads to the setting 
free of the pollen. Drying is usually due to direct loss of moisture to the air, but may result from the withdrawal of water by neighbouring cells of high osmotic content, bclonging either to nectaries (e.g. Stellaria) or to sugar-containing tissue (e.g. Foxglove, Digitalis); in the latter case dehiscence of the stamens ensues, even in damp air.

Actual splitting usually takes place longitudinally, along the middle line of each antherlobe; the line of weakness is sometimes bounded on either side by a spindle-shaped group of enlarged epidermal cells (e.g. Lily). The radial thickenings of the cells of the fibrous layer (Fig. 2I2, en.) prevent contraction in this direction, so that the shrinkage during drying is mainly tangential, and it is probably the tension thus set up that finally results in rupture. In a few flowers dehiscence takes place in other ways, as, for instance, by apical pores (Ericaceæ), or by the formation of subapical valves which hinge backwards to allow the shedding of the pollen (e.g. Barberry).

Before the pollen grain is shed its nucleus divides into two (Fig. 215, B). One of these becomes surrounded by a small

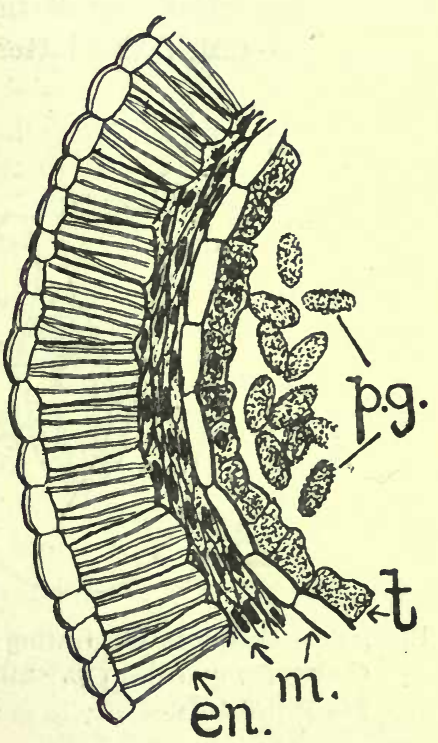

FIG. 212.-Transverse section through part of a pollen sac of a ripe anther. en., fibrous layer; $m$., middle layers ; $p . g$., poilen grains; $t_{\text {., }}$ disorganised tapetum. envelope of denser cytoplasm (generative cell, g.c.), whilst the other (tube nucleus, t.n.) lies freely in the general body of the grain. In this condition the latter is transferred, in one way or another, to the stigma of the same or of a different flower, and here germination takes place (Fig. 215, F). The tube nucleus passes into the tip of the pollen tube, and the naked generative 1 Cf. F. and S., chapters $\mathrm{xx}$, xxi. 
cell sooner or later follows suit (Fig. 215, C). The former usually remains undivided, whilst the latter ultimately produces two male nuclei (cf. below).

In its downward growth the pollen tube is sometimes nourished by the secretion of papillæ forming a continuous lining to the canal or canals traversing the style and extending uninterruptedly into the loculi of the ovary (e.g. Tulip and Violet). In many cases, however, the style is solid, the pollen tube penetrating through the axial core of thin-walled tissue, nourished by the sugary sap which the latter contains. In some cleistogamic

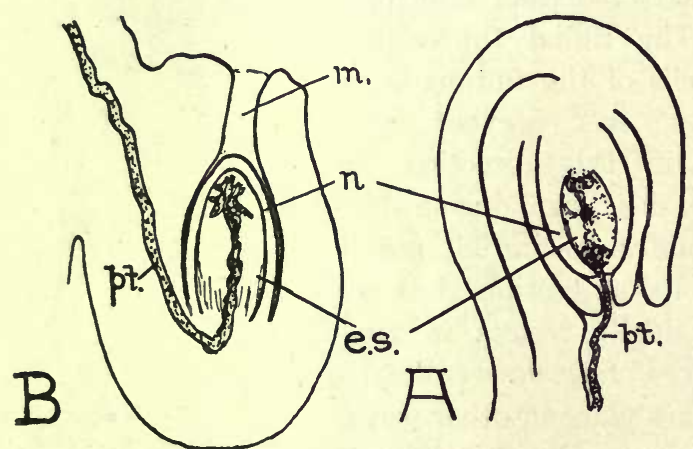

FIG. 213.-Diagrams illustrating normal fertilisation (porogamy, A) and chalazogamy (B). e.s., embryo sac ; m., micropyle ; n., nucellus ; $p t$., pollen tube.

flowers (e.g.' Purple Deadnettle) the pollen grains germinate in the anthers, and the pollen tubes grow from there to the stigma.

The period of time occupied by the pollen tube in growing down to the ovules varies greatly in different species, and bears no relation to the distance to be traversed. In the Crocus, where the style is 6-Io $\mathrm{cm}$. long, the interval is only from one to three days, whilst in the Meadow Saffron (Colchicum), with a style of about the same length, it is six months (from autumn, when pollination occurs, to the following spring). A similar prolonged interval not uncommonly obtains in woody plants (e.g. Hazel), though the distance traversed is short, and in some Oaks the ovules do not even commence development until after 
pollination. In most spring-flowering plants the anthers develop as far as the mother-cell stage in the previous autumn, although in the Hazel ripe pollen can be found in the male catkins during midwinter.

As a general rule the pollen tube traverses the cavity of the ovary, and thus reaches the micropyle of one of the ovules (porogamy, Fig. 2I3, A). But, in certain trees and shrubs (e.g. Elm, Beech, Hazel), it grows through the placental tissue and enters the ovule near the chalazal end (chalazogamy, Fig. 2I3, B). The growth of the pollen tube may therefore be compared to that of a fungal hypha, and its power to penetrate tissues, and eventually the megaspore membrane, is doubtless due to an analogous secretion of digestive enzymes.

The downward growth of the pollen tube, from the stigma into the ovary, is mainly determined by a chemotropic stimulus due to substances contained in the ovules or in the ovarywall. This fact can be experimentally demonstrated by sowing pollen grains of the Wild Hyacinth (Scilla) in a 5 per cent., or of Echeveria retusa in a I5 per cent., solution of cane-sugar around a fragment of the ovary, taking care to avoid the inclusion of air-bubbles. Observed under the microscope, the pollen tubes tend to grow in the direction of the piece of ovary.

The first steps towards the development of the megaspore are to be found in very young ovules, usually before the integuments have become plainly differentiated (Fig. 207, A). As in the case of the stamens, the archesporium (ar.) arisessubepidermally, but here it usually comprises but a single cell situated near the apex of the nucellus. This divides tangentially (Fig. 207, A, ar.), the inner half generally constituting the mother-cell, which as a rule, by two successive divisions, of which the first is the reduction division, gives rise to a row of four spores, forming a tetrad within the nucellus. It is usually the innermost member of the tetrad that becomes the functioning megaspore (generally known as the embryo sac), this cell subsequently increasing greatly in size so as to occupy the greater part of the nucellus (Fig. 206, e.s.). In the Mistletoe-family (Loranthaceæ) no proper ovules are differentiated; a parenchymatous outgrowth, which arises from the base of the ovary and almost fills the latter, develops a number of archesporia, from each of which an embryo 
sac results. This affords another instance of the reduction characteristic of parasites (cf. pp. I79, 23I).

Until it has reached a considerable size the embryo sac contains but a single nucleus, which usually lies in the middle of a strand of cytoplasm running from end to end of the sac and bridging the large central vacuole. Sooner or later, however, a nuclear division occurs, and the two resulting nuclei wander towards opposite ends of the embryo sac, where each again divides twice. Three of the four

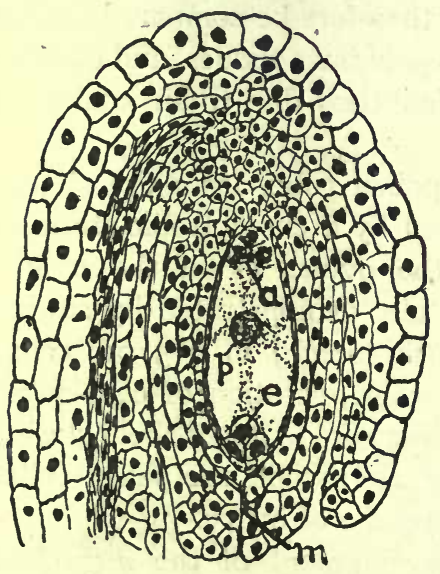

FIG. 214.-Longitudinal section of ovule of Marsh Marigold (Caltha), showing the structure of the mature embryo sac. $a$., antipodal cells; $e$., egg; $m$., micropyle ; $p$. , primary endosperm nucleus. nuclei thus formed, at the end remote from the micropyle, usually become separated by cellwalls and constitute the antipodal cells (Figs. 206, 2I4, 2I5, D, a.), which probably assist in the nourishment of the young embryo. They frequently enlarge after fertilisation, and, in some few cases (e.g. Burr-reed Sparganium), they may even divide to form an antipodal tissue.

Of the four nuclei at the micropylar end of the embryo. sac, three become surrounded by an envelope of specialised cytoplasm, and constitute a group of naked cells known as the eggapparatus (Fig. 206). This consists of the egg (e.) and two synergida (s.), the latter, which usually lie in front of the former, being supposed to aid in the passage of the male nucleus to the female cell. The remaining nuclei (one at each end, one of them the sisternucleus of the egg) pass back to the middle of the embryo sac, where these two polar nuclei (Figs. 206, p. ; 2I5, D, p.n.) meet and ultimately fuse to form the primary endosperm nucleus (Fig. 2I4, p.). This is the stage reached by the embryo sac at the time of fertilisation.

The sequence of events during this development and the 
resulting structure of the embryo sac are practically identical in the vast majority of the Angiosperms (both Monocotyledons and Dicotyledons) which have been investigated, and this uniformity constitutes one of the strongest arguments for the origin of the group from a common ancestry. Tubular sucker-like outgrowths
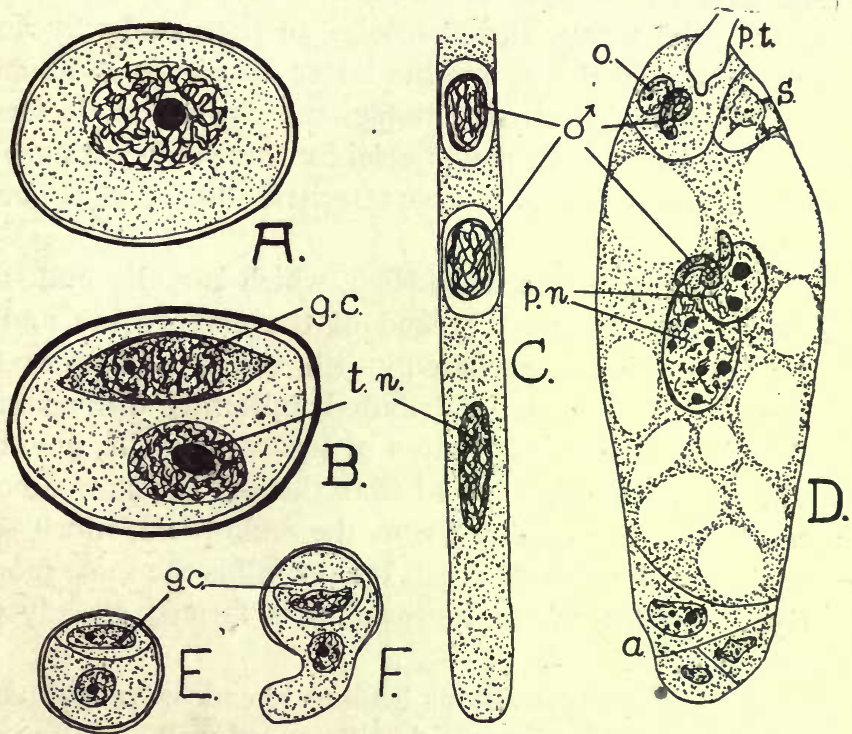

FIG. 21 5.-Germination of pollen and fertilisation. A-D, Lily (Lilium) (after Guignard). E-F, Tulip (Tulipa) (after Ernst). A, Mature pollen grain, showing the single nucleus. B and E, Later stage, showing generative cell (g.c.) and tube nucleus $(t . n$.). C, Tip of pollen tube with tube nucleus $(t . n$.$) and two male cells (\hat{o})$. D, Embryo sac at the moment of fertilisation, showing antipodals (a.), polar nuclei (p.n.), ovum (o.), one synergid (s.), and the two vermiform male cells ( $\hat{\sigma})$ discharged from the tip of the pollen tube (p.t.). F, Early stage in formation of pollen tube.

from the chalazal or both ends of the embryo sac are met with in the Beech, Hornbeam, and some Sympetalæ (Plantago lanceolata, Scrophulariaceæ), and probably aid in the transference of foodmaterial to the developing embryo. The same end is served by the haustorial outgrowths from the later-arising endosperm (see below) which occur in some genera.

Having passed through the micropyle, the pollen tube pene- 
trates the overlying nucellar cap, and thus reaches the embrya sac. Meanwhile the generative cell has divided to form two male nuclei (Fig. 2I5, C, ङ), which are extruded from the tip of the pollen tube, and, entering the embryo sac, fuse respectively $^{1}(a)$ with the egg, and (b) with the two polar nuclei (cf. Fig. 2I5, D), or with the nucleus formed by the fusion of the latter. The former fusion results, in the usual way, in the production of an embryo, but the latter also leads to abundant division, whereby a nutritive tissue, the endosperm, is formed. In the utilisation of both male nuclei for these different purposes, the Angiosperm exhibits a characteristic difference from the Gymnosperm.

The product of the second fusion, which is really built up of three nuclei (one from either end of the embryo sac and one male nucleus), divides repeatedly, till the sac becomes filled with numerous free nuclei distributed uniformly throughout its protoplasmic content. Formation of separating walls now takes place almost simultaneously, so that the sac becomes occupied by a continuous thin-walled tissue, the endosperm, which stores food for the developing embyro. It thus fulfils the same function as in Gymnosperms, where, however, it is formed already prior to fertilisation.

During the nuclear divisions leading to endosperm-formation, the fertilised egg, now provided with a cell-wall, enlarges considerably and commences to segment. The first division is unequal, a smaller cell situated at the end away from the micropyle being cut off from the large remaining portion. The latter forms the sac-like basal cell (Fig. 2I6, I-VI, b.) and plays no further part in the development of the embryo. The smaller segment undergoes successive transverse divisions, so as to give rise to a short elongating suspensor (Fig. 2I6, S.). Hereby the slightly larger hemispherical terminal cell (e.), which subsequently produces the embryo proper, is carried down into the heart of the developing endosperm. Young proembryos at this stage of development, with a suspensor composed of a number of flat cells, are readily squeezed out of very young seeds of the Shepherd's Purse, such as can be removed from the ovaries of flowers which have not yet faded; the seeds are best mounted

1 This process is often spoken of as " double fertilisation." 
in water, and gentle pressure applied by tapping the coverglass.

More advanced stages can be obtained in the same way from progressively older seeds. These show rather longer suspensors

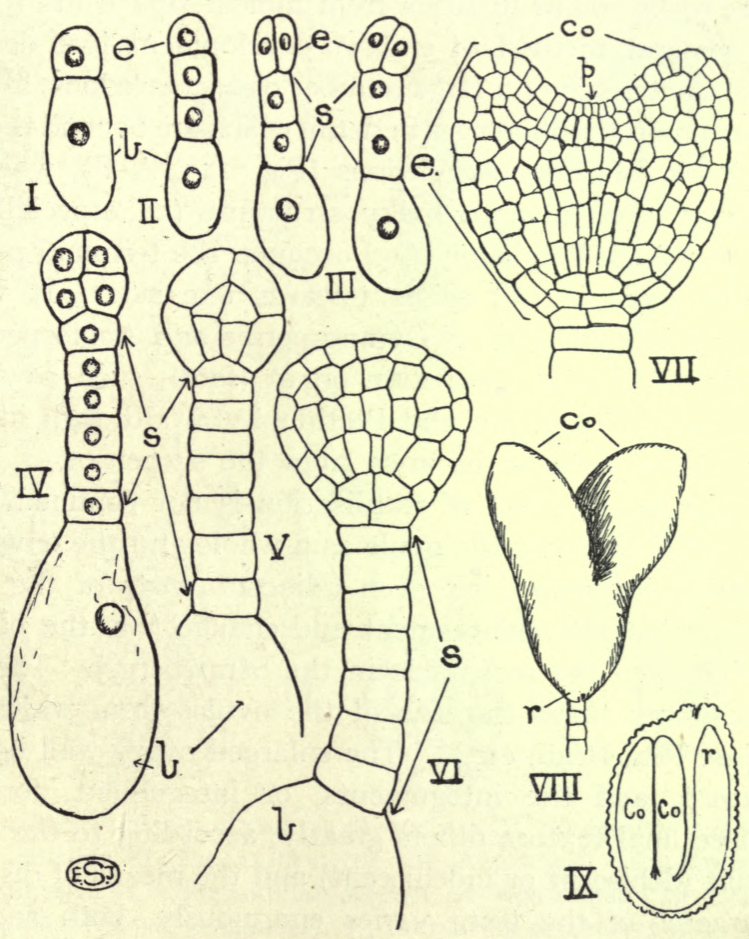

FIG. 2I6.-Embryology of the Shepherd's Purse (Capsella bursa-pastoris). The successive stages are numbered I-IX. In VII and VIII only the embryo and a small part of the adjacent suspensor are shown. IX is a longitudinal section of a mature seed, showing the coiled embryo. b., basal cell; Co., cotyledons ; e., embryo ; $p$. , plumule ; $r$, radicle ; S., suspensor.

and an increasing number of divisions in the enlarging embryonal cell (Fig. 2I6, IV-VI). The latter soon segments into octants, by three walls at right-angles to one another (III, IV), and subsequently dermatogen, periblem, and plerome are successively delimited by walls parallel to the surface (V, VI). Mean- 
while the embryo assumes a more or less flattened form, and, at its wider free end, the future cotyledons appear as two lobes (VII, Co.) between which the plumule $(p$.) develops. The attached end of the embryo invariably becomes the radicle (VIII, r.), which thus faces towards the micropyle. Such embryos are to be found in seeds from almost ripe fruits (cf. IX).

The general method of embryo-development just described is that characteristic of a large number of Dicotyledons, although there are numerous differences in detail which are beyond the scope of this book. In Monocotyledons (Fig. 2I7, A-E), where the suspensor is often a more bulky structure (cf. especially Fig. $2 \mathrm{I} 7, \mathrm{G})$, the single cotyledon $(\mathrm{Co}$.) occupies the terminal position, whilst the plumule $(p$.$) arises towards one side. It will be$ noticed that the embryos of Gymnosperms and Angiosperms do not develop a special absorptive organ (foot), such as characterises those of Bryophyta and Pteridophyta, although in a few cases haustorial outgrowths arise from the suspensor.

The stimulating effect of fertilisation is not confined to the embryo, but also affects the ovule as a whole and the enveloping ovary, whilst in some cases even adjacent parts of the flower undergo considerable enlargement and change (e.g. the perianth in the Mulberry, the receptacle in the Strawberry). The seeds are often many times the size of the ovules from which they sprang (e.g. Pea, Bean, etc.). The enlarged ovary-wall becomes the pericarp, and the integuments, or integument, form the testa, whose final texture differs greatly, according to the nature of the fruit (dehiscent or indehiscent) and the means of dispersal. The character of the testa varies enormously, both as to its surface, which is often sufficiently distinctive in its sculpturing (e.g. Corn Cockle, Agrostemma) to serve for purposes of identification, and as to its durability and thickness. The latter feature is probably responsible for the slow germination of some seeds. It may be noted in this connection that the seeds of the Hawthorn and others, which are normally swallowed and pass through the alimentary tract of animals, germinate much more readily after having been acted upon by the gastric juices.

The embryo may absorb all the food-materials in the endosperm before entering upon its resting-stage (exalbuminous seeds, as in the Shepherd's Purse, Pea, etc.), or part of the endosperm 
may persist up to germination (albuminous seeds, e.g. Buckwheat, Castor Oil, most Monocotyledons). In a few plants (e.g. many Caryophyllaceæ) the nucellus is not entirely displaced by the endosperm, a thin layer (perisperm) remaining, even in the ripe

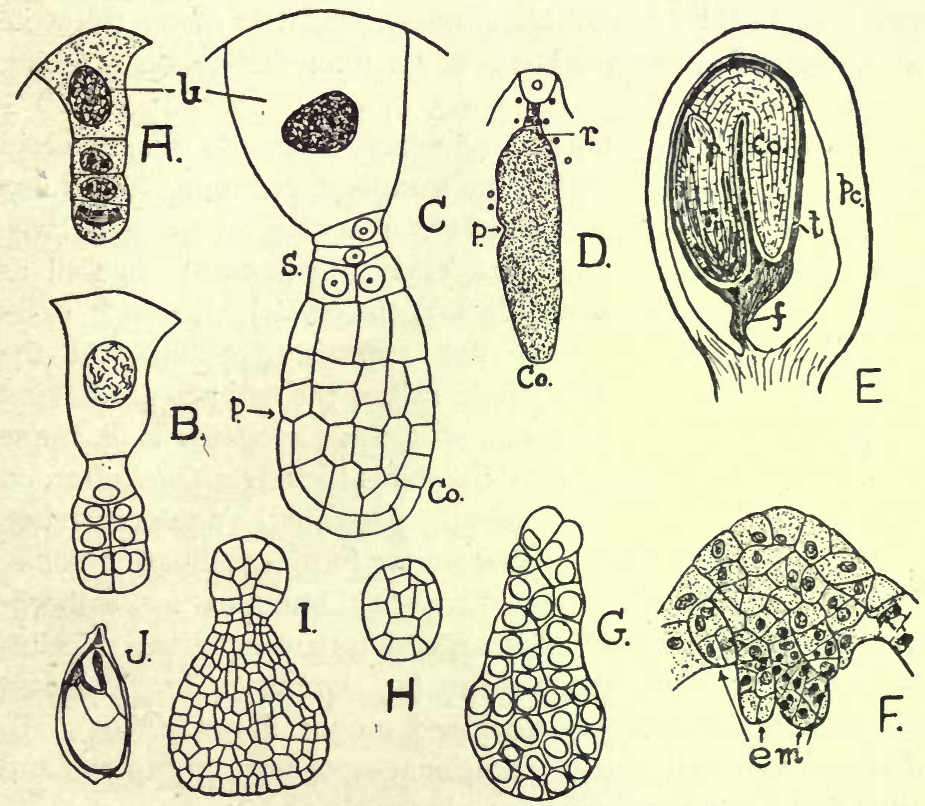

FIG. 217.-Embryology of various plants. A-D, Successive stages in the development of the embryo of the Arrowhead (Sagittaria) (after Schaffner). E, Longitudinal section of ripe achene of the Water Plantain (Alisma) (original). F, Formation of several embryos (em.) from a massive suspensor, by budding, in Erythronium americanum (Liliaceæ) (after Jeffrey). G, Proembryo, with massive suspensor, of Lilium (after Coulter). H, Embryo of Twayblade (Listera ovata) at time when seed is shed (after Pfitzer). I, Proembryo of Spartium junceum (Leguminosæ) (after Guignard). J, Longitudinal section of Orange pip (Citrus) (with two embryos) (after Wettstein). b., basal cell ; Co., cotyledon ; $f$., funicle ; $p$., plumule ; $p c$., pericarp ; $\boldsymbol{r}_{\text {., }}$ radicle ; $S$., suspensor ; $t$., testa.

seed, and functioning for the storage of food. The degree of differentiation attained by the embryo, at the time when it enters upon its resting-stage, varies considerably. Thus, in the Runner Bean (Phaseolus), even the venation of the first pair of plumular 
leaves is distinguishable, in the Castor Oil the plumule is merely a peg-like structure, whilst in the Lesser Celandine (Ficaria) the entire embryo is an undifferentiated mass of cells (see also Fig. 2I7, H), a condition likewise encountered in the embryos of most parasites. In some plants, moreover, there is no resting period (e.g. in the tropical Mangroves), whilst in many trees the seeds germinate most readily if sown immediately on reaching maturity.

Exceptions to the normal sequence of events described in the foregoing pages are by no means uncommon. Apogamy (cf. p. 305), for example, has been recorded in quite a large number of Compositæ (e.g. Dandelion, Hawkweed), as well as in some species of Lady's Mantle (Alchemilla). In such cases the reduction division does not appear to occur, and the embryo arises from an unfertilised cell of the embryo sac having the normal number of chromosomes. Such apogamy is of course akin to vegetative propagation, but gains the advantages afforded by the mechanism for seed-dispersal. Despite the non-occurrence of a sexual process in such apogamous forms, pollination sometimes appears to furnish a necessary stimulus for embryoformation. More rarely it is an ordinary cell of the nucellus that divides to form the embryo (e.g. Orange, Citrus), a case analogous to the apospory described among Ferns (p. 305). In the Hawkweeds all three conditions-apogamy, apospory, and normal fertilisation-have been observed.

The occasional presence of more than one embryo within a seed may be due to several causes. Sometimes more than one member of the tetrad, formed by the megaspore mother-cell, develops into an embryo sac, so that several embryos are present from the first. But more frequently accessory embryos arise by vegetative budding from the proembryo (Fig. 2I7, F). Orange pips frequently contain several embryos, of which one is the outcome of a sexual fusion, whilst the others are derived from nucellar cells which are presumably stimulated to growth as a result of fertilisation (Fig. 2I7, J).

The general course of the life-history in Angiosperms is obviously very similar to that of Gymnosperms. In both cases the young embryo lives, as a parasite, within the ovule, which forms a protective envelope around it until the time of germina- 
tion. As with the young Fern, however, its independence is soon established. In contrast to Gymnosperms, the most striking features are connected with the very efficient arrangements for the protection and nourishment of the developing embryos and seeds, and the highly perfected mechanism for pollination by virtue of which the most intimate relation often obtains between the flower and the pollinating agent. Not all flowers, however, are equally highly adapted, and almost all grades of specialisation can be found in relation to pollination by the agency of insects, wind, or water. The more advanced of the insect-pollinated flowers usually exhibit a fusion of both sepals and petals, a zygomorphic corolla, and a reduced output of pollen. In the most extreme cases only one type of insect is effective in pollination, or is most efficient in the pollination of that particular species. Wind-pollinated flowers are usually inconspicuous, produce an abundance of pollen, and often possess richly branched stigmas. Water-pollinated plants, which like wind-pollinated ones are commonly unisexual, exhibit floating devices either in the female flowers or pollen.

The pollen grains and embryo sacs of Angiosperms are obviously comparable to the microspores and megaspores of Gymnosperms and Selaginella, and within them divisions take place which lead to the formation of the male and female gametes respectively. The contents of the embryo sac may therefore be regarded as a female prothallus, and the contents of the microspore as a still more reduced male prothallus. By those who find such comparisons profitable, an alternation of generations, analogous to that of Pteridophyta and Bryophyta, is thus recognised. 


\section{CHAPTER XXV}

\section{HEREDITy AND EVOLUTION}

IT will be a matter of common knowledge that the offspring of either plants or animals resemble their parents very closely. This fact, though so obvious, really involves the fundamental principle of inheritance, i.e. the progeny inherit the characteristics of their parents. Thus, if we sow the seeds obtained from a self-pollinated flower of the Foxglove, the numerous resulting seedlings obviously inherit the same general characters. Closer observation, however, shows that there are many minute points of difference which may in the main be related to the fact that the conditions in the seed-bed are not uniform (cf. also Fig. 2I8). The features presented by any particular individual may, as a matter of fact, be regarded as the outcome or resultant of two sets of factors, being either inherited or due to the effect of the environment. Under environment we understand all the external influences-physical, chemical, and biological-to which the organism is subjected. In the case of plants, and still more in that of animals, the conditions of the environment are not constant throughout the life of the individual, but in general it is those experienced in the early stages of development which are most potent in moulding the organism.

Individual variations, though probably in the main correlated with differences in the environment, may well also result from changes in the internal conditions which are more difficult to

analyse. Such variations may be of two kinds. They are qualitative or substantive, when, for instance, they concern the shape or size of the entire plant (Fig. 2I8) or of any of its parts, the degree of hairiness, the type of colouration (Fig. 2I9), etc. On the other hand, they are quantitative or meristic, when they 
involve differences in the number of constituent parts, such as perianth-segments, leaflets in a compound leaf, etc.

It is easiest to study variation if some character is selected which is capable of exact measurement, as, for instance, the length of the leaf in the Privet, the length of the Runner Bean seed, or the number of ray-florets in the Daisy. Taking the first of these cases as an example, it will be found that if, say, a thousand leaves are measured, the difference between the length of the shortest and the longest is quite considerable, and that the majority of the leaves are of an average size. If all the thousand leaves be classified according to their lengths into separate groups, differing by increments of one millimetre, and the number of leaves in each group counted, those containing the smallest number will be found to be situated at the two extremes. Between these points the number of leaves of each particular length will be found to

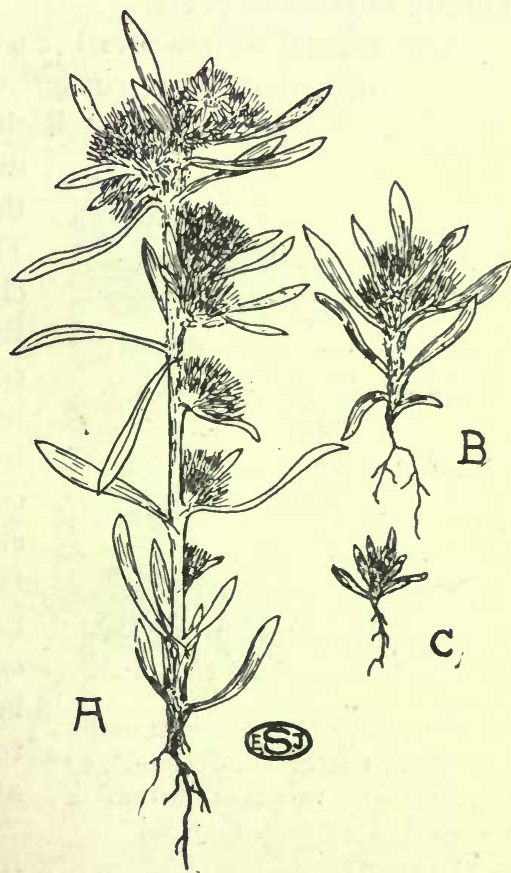

Fig. 218.-Three equal-aged individuals of the Marsh Cudweed (Gnaphalium uliginosum) from the same locality, showing individual variation. All three are in fruit. (Natural size.) increase with considerable regularity, as the size of its individuals approaches that exhibited by the majority. This most frequent size is termed the mode, and often corresponds very closely to the arithmetical mean of all the measurements.

By plotting a curve, in which the ordinates represent the number of individuals in each group, and the abscissæ the respective lengths, the variation can be graphically represented 
(Fig. 220). The larger the number of individuals taken into account, the smoother the outline of the curve. Such variation curves are most commonly symmetrical (Fig. 220, left), but they may be one-sided or asymmetrical (Fig. 220, right), as in the case of the meristic variation of the corolla-segments of many flowers, where there are often relatively few examples with less than the normal number of parts.

The normal symmetrical curve of variation agrees very closely with that representing variation depending on pure chance, a feature which in itself suggests that the manifold

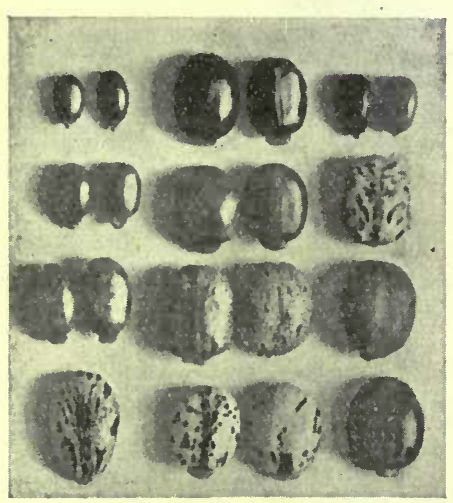

FIG. 219.-Seeds of different varieties of Castor Oil (Ricinus), to illustrate variation within a species. [Photo. E. J. S.] differences in the conditions of the environment are involved. The classical example of such chance variation is afforded by the repeated tossing up of two coins, the combination of one head and one tail being most frequent (forming about 50 per cent. of the cases), whilst the combinations two heads or two tails occur with about equal infrequency. A more illustrative curve of chance variation would be obtained if ten coins were tossed simultaneously for a large number of times in succession.

In cases of meristic variation it will be noticed that the difference between the extremes is much greater when the mode corresponds to a large number than when it coincides with a small one. Thus, if variation due to fission of parts (say of the corolla of a flower) be equal in two species, the one having a mode of five (i.e. usually five petals) and the other having a mode of ten (i.e. usually ten petals), there would be just double the chances of fission occurring in the latter as in the former. The extreme condition (i.e. ro and 20 petals) would be attained where all the petals underwent fission. That is, the range would be greater in the one case than in the other, although the actual frequency of fission of the individual segments was the same 
in the two species. Hence a comparison of variation in two cases, where the modes are dissimilar, can only be made by taking such dissimilarity into consideration, a comparison of the curves alone being misleading. The position of the mode can often be to some extent changed by modifying the environmental conditions, although the range of variation remains practically unaltered.

It appears that individual variations are not inherited, and that in a pure line of descent the average of the race is maintained. ${ }^{1}$ This has been established by sowing seeds from a single individual of pure descent which has been self-fertilised, the seed produced by the resulting plants (likewise self-fertilised) being sown in separate groups, and the same procedure followed for several successive generations. It was thus found that the

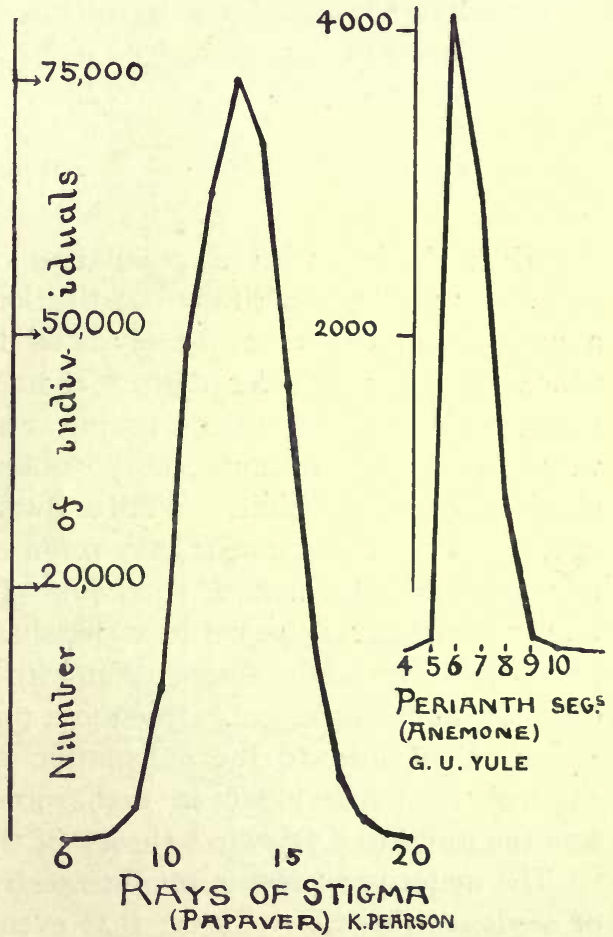

FIG. 220.-Variation curves, symmetrical on the left and asymmetrical on the right. average size of in-

dividuals derived from small parents is practically identical with that of individuals grown from tall parents; also that heavy seeds do not beget heavier-seeded offspring than those derived from light seeds. The following data, which serve

1 By a pure line is understood a pure-bred strain produced by selffertilisation from a single individual, 
to illustrate this point, are taken from Johansen's experiments with Beans which were self-pollinated:

Weight of seeds of parents.

$350-400 \mathrm{mg}$.
$450-500 \quad$,
$500-550 \quad$,
$550-600 \quad$,
$600-650 \quad$,
$650-700$,

Average weight of seeds of offspring.

$572 \mathrm{mg}$.
.535 ,,
. 570 ,,
. 565 ,.
566 ,,
. 555 ,

When dealing with a population consisting of members possessing varied hereditary constitution (i.e. not a pure line), a pure strain can often be obtained by selecting individuals which show a particular desired character and breeding from these, with due precautions against cross-pollination. In this way it might, for example, be possible by artificial selection to obtain pure lines, exhibiting a tall or dwarf habit. Under natural conditions the environment may often exercise such a selective influence (so-called natural selection). The possession of a particular character is sometimes decisive in determining which plant shall survive in competition with others, against adverse conditions of climate, etc. Even in a pure strain, some selective action might lead to the elimiration of all but the heaviest, shortest, etc., individuals in each successive generation. This was the essence of Darwin's theory of the origin of new species.

The majority of plants produce such an abundance of spores or seeds, as the case may be, that even with the most efficient dispersal it would usually be impossible for all the offspring to become established. Many a common plant would, indeed, if the means of dispersal were adequate, and all the progeny of successive generations survived, rapidly cover a large surface of the globe. That this does not happen is due to that ceaseless competition of living organisms with one another which is known as the struggle for existence. A Mullein plant, for example, may produce as many as 700,000 seeds. The resulting seedlings are not of equal vigour, and those first to succumb in the struggle for existence are obviously the weakest. The latter, applying 
the term in its widest sense, owe their lack of vigour partly to adverse environmental conditions and partly to inherited characteristics. Just as a human being with a "weak constitution" may have inherited his defect or owe it to the surroundings in which he grows up.

Unlike most animals, plants cannot choose their place of habitation. The seeds or spores are carried passively to a variety of situations, and their chances of developing into mature

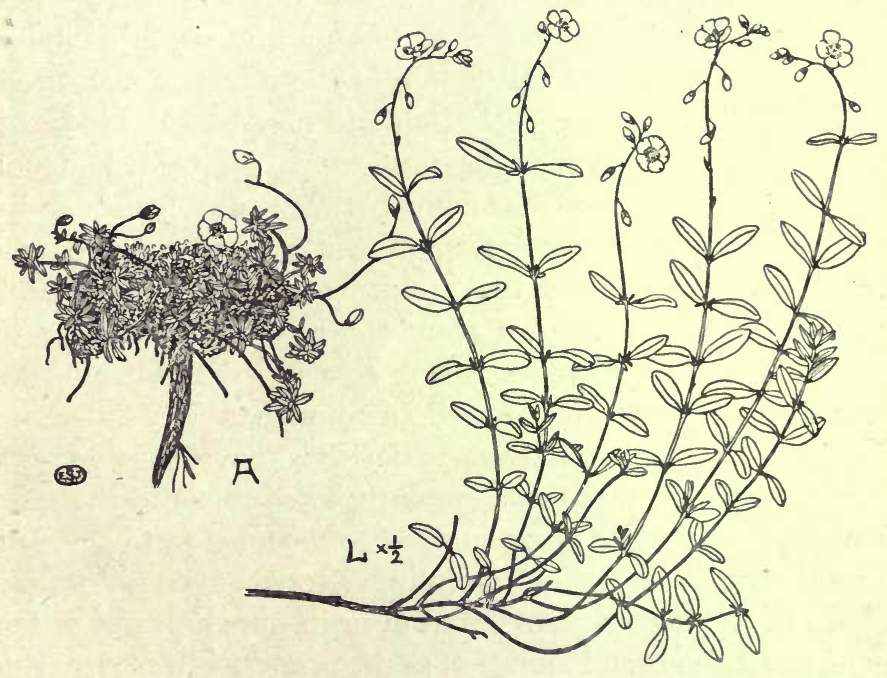

FIG. 221.-Alpine (A.) and lowland $(L$.) forms of the Rock Rose (Helianthemum vulgare). (After Bonnier.)

plants depend upon their power of accommodating themselves to the environment in which they may be placed. But many plants exhibit this power of adaptation to a very marked degree, as is well illustrated by those aquatics which can grow either totally submerged or on exposed mud (cf. p. I75), by the sunand-shade-forms of woodland plants (p. I68), and by the Alpine forms of lowland plants (p. I70, Fig. 22I).

It has long been a matter of dispute as to whether or no the changes, impressed upon an organism by the environment, bring about any corresponding internal modification by means 
of which the new characters can be transmitted to the offspring. So far all attempts to demonstrate satisfactorily the inheritance of such acquired characters have proved a failure. It is indeed difficult to conceive of any plausible means by which characters, acquired by the plant in the course of its lifetime, could affect and modify the hereditary mechanism which must obviously be contained within the fusing gametes. On the other hand, the marked adaptations of plants in the wild state to their normal environment, features often retained when the organism is transferred to another habitat, naturally suggest the possibility of the unstable acquired characters becoming ultimately stabilised. This view has led to the conception of the origin of new species, etc., through cumulative selection and hereditary transmission of favourable variations (or mutations, cf. below), tending towards more complete harmony between the plant and its environment. Another point, upon which there is much difference of opinion, is the actual influence of the environment in moulding the structure of a plant. Whilst some consider that adaptation to the environment is due to selection, others believe in a direct response to changed surroundings.

Seeing that the individual variations above considered are all encompassed within the apparently fixed range of the species (cf. p. 377), they could scarcely seem to have led to the evolution of new forms. But in carefully selected and self-fertilised cultures of the higher plants, and even in pure cultures of lower organisms, it has been found that slight or pronounced departures from the mode occasionally arise which breed true to their new characters from the very first, i.e. these are hereditarily transmitted. Such mutations, which are sometimes far more pronounced than the individual variations, and consequently obvious even to superficial observation (Fig. 222), may well be responsible for the origin of new species. As to the causes of mutation we are, however, in complete ignorance, though it is tempting to assume that the external environment is the stimulus that brings about the internal change. If this could be experimentally proved, many of the divergent views at present held could be harmonised.

A familiar instance of a mutation is afforded by the Irish Yew (Taxus baccata, var. fastigiata), which differs from the 
Common Yew, from whence it arose, in its darker foliage and cypress-like habit. Detailed studies of mutations were first made by De Vries on a species of Evening Primrose (Enothera lamarckiana), which occurred as an escape in a field near Amsterdam, where it was found producing a considerable number of

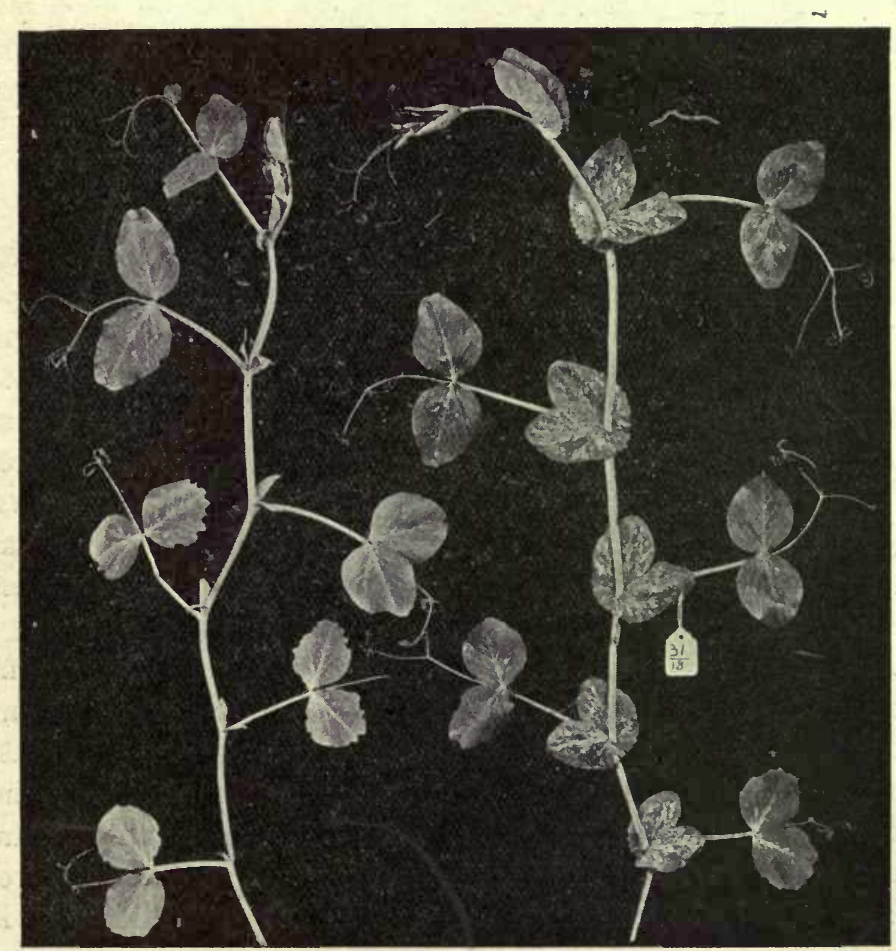

Fig. 222.-A new form or mutant which arose from a pure strain of Duke of Albany Pea and which differs in the narrow lanceolate stipules. The type on the right, the mutant on the left. [By permission of Prof. Bateson, F.R.S., and Miss Pellehew.]

new forms, which subsequently bred true. For instance, one form was distinguished by the possession of broad leaves, another by red-veined leaves, others by dwarf- or giant-habit, and so on, as the following epitome of some of De Vries' cultures shows. 


\section{Mutation in ENOTHERA LAMARCKIANA.}

(The horizontal lines show successive generations, invariably obtained only by sowing seeds of the ordinary form.)

\begin{tabular}{|c|c|c|c|c|c|c|c|}
\hline & $\begin{array}{l}\text { Giant } \\
\text { Form. }\end{array}$ & $\begin{array}{l}\text { White- } \\
\text { leaved } \\
\text { Form. }\end{array}$ & $\begin{array}{l}\text { Oblong- } \\
\text { leaved } \\
\text { Form. }\end{array}$ & $\begin{array}{l}\text { Red- } \\
\text { veined } \\
\text { Form. }\end{array}$ & $\begin{array}{l}\text { Normal } \\
\text { Form. }\end{array}$ & $\begin{array}{l}\text { Dwarf } \\
\text { Form. }\end{array}$ & $\begin{array}{c}\text { Broad } \\
\text { leaved } \\
\text { Form }\end{array}$ \\
\hline I & . - - & - & - & - & 9 & 一 & - \\
\hline II & . - & - & - & - & 15,000 & 5 & 5 \\
\hline III & . - - & - & - & I & 10,000 & 3 & 3 \\
\hline IV & I & I 5 & r 76 & 8 & $x_{4}, 000$ & 60 & 73 \\
\hline $\mathrm{V}$ & . - - & 25 & r 35 & 20 & 8,000 & 49 & 142 \\
\hline VI & . - - & I I & 29 & 3 & $\mathrm{I}, 800$ & 9 & 5 \\
\hline VII & . - - & - & 9 & - & 3,000 & I I & - \\
\hline
\end{tabular}

Since it has been suggested that De Vries' mutants sprang from an originally hybrid stock (cf. below), it may be mentioned that other instances of mutation have since been described in which the pure-bred character of the original strain appears to be beyond question. Moreover, mutations do not occur only in plants raised from seed, where as a consequence two gametes are involved. The propagation of vegetative mutations, or sports, which first appear only on a few branches of the plant, has given rise to the Copper Beech, and to the cut-leaved varieties of Alder, Beech (Fig. 223), etc.

Another mode of origin of new forms is due to the recombination of characters which takes place when two different races, varieties, or species are employed as the respective parents. In such cases the fertilised eggs are, of course, produced by the participation of both parents, one furnishing the female and the other the male sexual cell. Such " crossing " is often a failure, no seed being set, but when successful the next generation is found to show a mixture of the characters of the two parents, some belonging to the one and some to the other.

In respect to any single contrasting feature (e.g. height, hairiness, etc.), however, the character of one or other parent frequently altogether predominates (i.e. the offspring all show the one feature), although sometimes the hybrid is intermediate between the two (cf. p. 387). Since the characteristics of both parents must have been inherited, those of the one must, in the former case, be supposed to remain dormant, or, as is usually said, latent. As a matter of fact, if such hybrid plants 
are self-fertilised, the latent character reappears in a certain number of the individuals arising from the resulting seeds, a fact which proves that it must have been present, although not outwardly manifest.

An exact study of the phenomena of hybridisation was first undertaken by Mendel, an Austrian monk, by considering the behaviour of single pairs of characters only. In one of his earliest experiments he crossed tall and dwarf Peas, the whole of the resulting offspring (first generation) being tall. The seeds produced from these, by self-fertilisation, afforded two classes of individuals, threequarters being tall and onequarter dwarf. But of these tall Peas of the second generation (if again self-fertilised) only one-third bred true, the remainder behaving just like
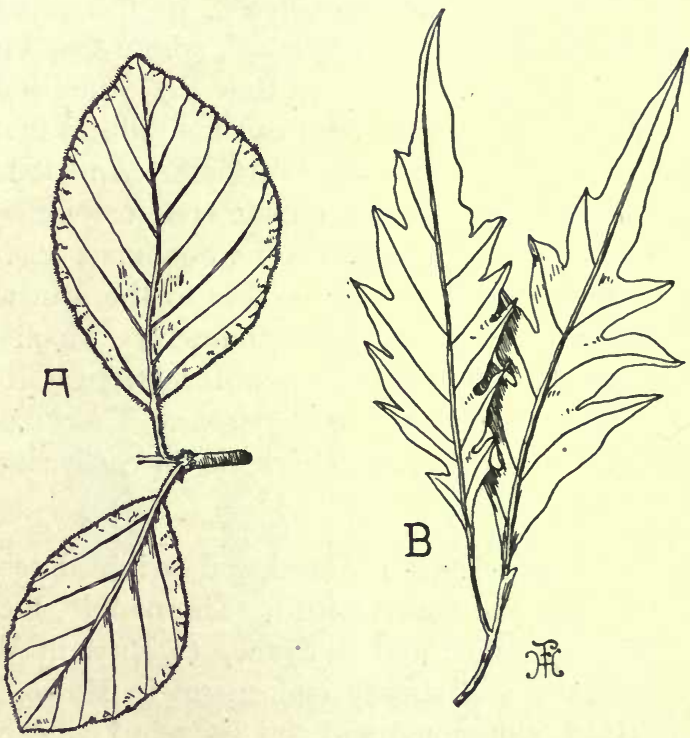

the original hybrids of the first generation, and giving rise to talls and dwarfs in the proportion of three to one. The dwarfs in every case bred true. In the second generation of such a cross, then, as regards any one particular pair of characters, half the offspring are pure (one quarter resembling one parent, another quarter the other parent), whilst the other half are impure and bear the characters of both. Representing the tall Pea by $T$ and the dwarf Pea by $d$, these results can be graphically expressed as follows: 
First generation: $T d$ (all tall).

Second generation: $\mathrm{I} T: \mathrm{I} d: 2 T d$ (one tall, one dwarf, two hybrids).

A considerable number of other pairs of characters have been studied in the same way, with identical results, though there are a number of exceptions, some of which are at present inexplicable.

The latent character is usually spoken of as the recessive and the other as the dominant. In the experiment with tall and dwarf Peas above described, where the difference is one of size, it is believed by many that the dwarf habit is due rather to the absence of a character causing tallness than to the presence of a special character for dwarfness. And it may well be that in all cases the recessive character is caused by the absence of something which determines the dominant (e.g. a glabrous form of a particular plant may be due to the absence of a character for hairiness, etc.). Pairs of characters, which thus combine in the first hybrid generation and segregate in the subsequent generations, are termed allelomorphs. The following are further examples, the dominant character in each case being that first named.

Yellow and green, round and wrinkled seeds in Peas.

Prickly and smooth fruits (Thornapple, Field Buttercup).

Susceptibility and resistance to Rust in Wheat.

Starchy and sugary endosperm in Maize.

Hard (glutenous) and soft (starchy) endosperm in Wheat.

A feature which may be of great significance is that almost all the mutants so far studied behave as recessives to the parent stocks from which they sprang. It may be added that recent research has shown that many apparently simple characters can be analysed into a number of subordinate ones, which, however, are very commonly inherited together.

The most important principle, demonstrated by the experiments of Mendel and subsequent workers in this field, is that the characters introduced by either parent do not become inextricably intermingled in the hybrid offspring, but become separated out again in the gametes; that is to say, each of 
the latter bears only one allelomorph. It is only on this basis that the reappearance of a latent character in the second generation can be explained. If the dominant and recessive characters of an allelomorphic pair are represented by $A$ and $a$ respectively, then the fertilised eggs produced by crossing will all have the constitution $A a$, and the resulting plants will all show the dominant character only; such individuals are spoken of as heterozygotes. It is believed that in these hybrids, during the formation of each tetrad of spores, and in conjunction with the rediction division (see p. 306), the characters $A, a$ become separated, so that each spore, and consequently each of the
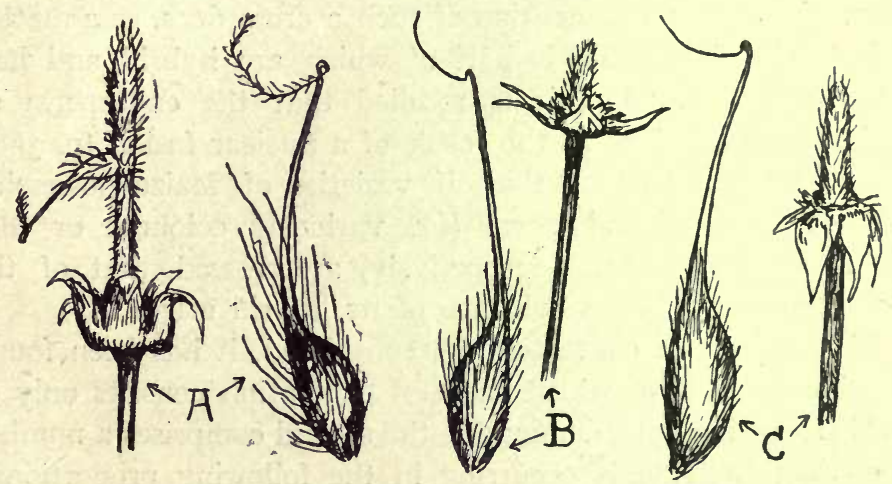

FIG. 224.-Geum rivale (A), Geum urbanum (C), and the hybrid between them, Geum intermedium (B). In each case the receptacle and calyx are shown, together with an enlargement of a single carpel.

gametes, to which it ultimately gives rise, bear either $A$ or $a$. It is probable, moreover, that the determinants for these characters are contained within the nuclei of the sexual cells (p. 390).

During self-fertilisation, and assuming the gametes to meet according to the laws of chance, there are four possible combinations: viz. (I) a male gamete bearing $A$ and a female bearing $A$; (2) a male bearing $A$ and a female bearing $a$; (3) a male bearing $a$ and a female bearing $A$; and (4) a male bearing $a$ and a female bearing $a$. That is to say, among each four fertilised eggs that result, there will probably be I $A A, 2 A a$, and I $a a$. Seeing that $A$ is invariably dominant, there will in the second generation be three individuals with the dominant to one indi- 
vidual showing the recessive character; of the former, however, two will be heterozygotes, which would exhibit segregation according to the same principle in the ensuing generation, whilst the other, as well as the individuals possessing the recessive character, are homozygotes.

Further evidence, in support of this hypothesis, is furnished by the result of crossing the hybrid of the first generation with one or other parent (i.e. $A a \times A A$ or $A a \times a a$ ). In this case only two kinds of combinations will be obtained, viz., taking the case in which the hybrid is crossed with the parent-form having the dominant character, $A A$ and $A a$, and there are obviously equal chances for either combination to occur. The second generation of such a cross does, as a matter of fact, afford individuals half of which are hybrid and half pure. Moreover, when it is recalled that the endosperm of Angiosperms develops as the result of a nuclear fusion (p. 368), it is of interest to note that, if varieties of Maize possessing different types of endosperm (i.e. variously coloured or containing sugar and starch respectively) are crossed, that of the resulting seeds exhibits evidence of its hybrid origin.

If two pairs of characters are considered, it has been found experimentally that, whilst the first generation consists only of hybrids showing both dominants, the second comprises a number of distinct individuals occurring in the following proportions: 9 with both dominant characters, 3 with one dominant and one recessive, 3 with the other dominant and the other recessive, and I showing both recessives. The relations will be plain if the diagram in Fig. 225 is studied. Thus, if yellow round Peas are crossed with green angular ones, the first generation all bear yellow round Peas; in the second generation there will be 9 yellow round, 3 yellow angular, 3 green round, I green angular. Of the nine individuals showing both dominant characters one only breeds true, as also does the one bearing both recessive characters. The remainder, on being self-fertilised, exhibit segregation according to the particular characters which they contain.

The dominance of a character is only important for the elucidation of the observed facts in the many cases where dominance occurs. But the principle of segregation is equally 
applicable where the heterozygote is intermediate between the two parents, or, as in some cases, even differs from both. A hybrid intermediate between the two parents is obtained, for instance, when the Star and Chinese Primroses are

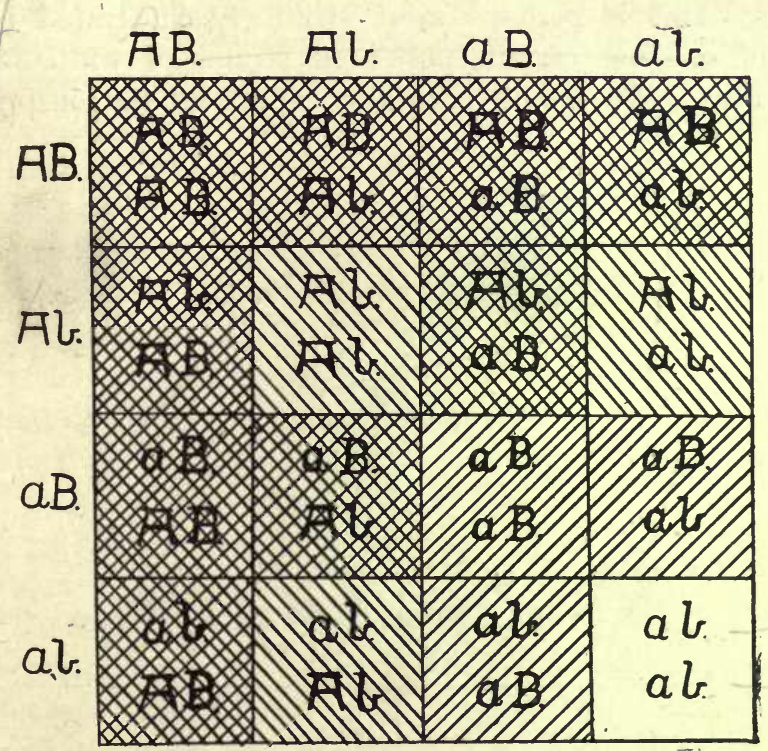

FIG. 225.-Scheme to illustrate the progeny of the second generation when two pairs of characters $(A a, B b)$ are taken into consideration. The individuals of the first generation will all have the constitution $A a B b$. When segregation occurs, the resulting gametes will have the four possible constitutions $A B, A b, a B, a b,{ }^{1}$ and the scheme shows the possible methods of combination of such gametes. When both dominants are present (in nine out of the sixteen cases) the squares are cross-hatched, when the dominant $A$ is present the shading slopes from left to right, when $B$ is present from right to left; when neither dominant occurs the square is left unshaded.

crossed. The petals in the first generation are intermediate in character between those of the two parents (Fig. 226); in the second generation segregation takes place in the usual way, the hybrid-individuals (one-half) still exhibiting flowers of the

1 The combinations $A a$ and $B b$ do not arise, since dominant and recessive characters are segregated. 
intermediate type, whilst the remainder consist of Star and Chinese Primroses in equal numbers.

Hybridisation experiments not only teach us the principle of segregation of characters, but also emphasise the fact that external appearance is no certain guide to the internal characteristics. This is perhaps most strikingly illustrated by the effect of crossing certain pure-bred strains of white-flowered Sweet Peas. We should naturally expect all the offspring to
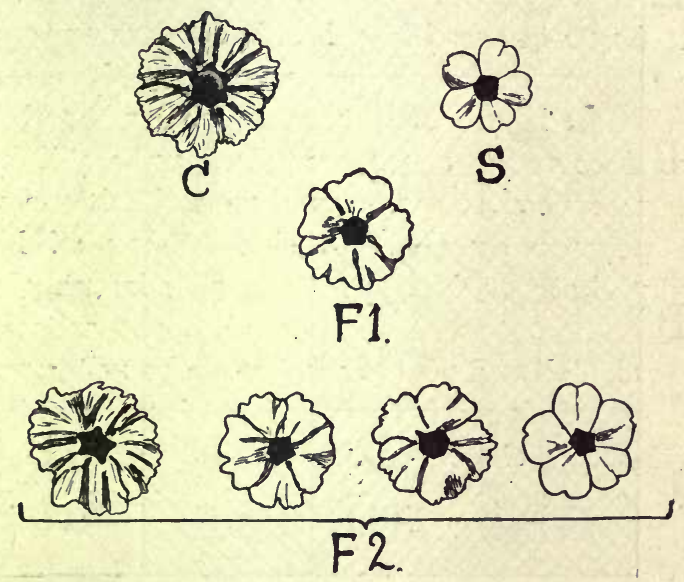

FIG. 226.-Hybrids between Chinese (Primula sinensis) and Star Primroses $(P$. stellata). At the top the two parents, the Chinese $(C$.) with large, rather wavy, much crenated petals, and the Star $(S$.$) with smaller flat$ petals exhibiting only a notch. The FI generation is intermediate between the two in these respects. In the F2 segregation takes place in the usual way. (After Punnett.)

be white-flowered too, but in reality all have coloured flowers, with a purple standard and blue wings. Here, clearly, the parents possess characters which in combination lead to the production of a coloured flower, though when separated they produce no visible effect. By breeding plants the limited knowledge of the constitution of any organism, which is obtained by mere examination of external or internal structure, can thus be to some éxtent supplemented.

When two varieties cross in nature, the appearance of the hybrid is of course influenced by all the characters present in 
the parents. Whether it resembles one or other parent, or is roughly intermediate between them, will depend on whether one parent contributes a greater proportion of dominant, or more conspicuous, characters than the other. Common examples of such hybrids in the wild state are afforded by various Willows, Geum intermedium (Fig. 224, B), Quercus intermedia, etc.

It has long been familiar to gardeners that shoots, which show a mingling of the characters of scion and stock, are sometimes produced as a result of grafting. The intermediate nature of these so-called graft-hybrids appears to be due to the fact that both scion and stock contribute to their development, the tissues of the one forming a skin over those of the other. The character of the seeds, and of the resulting offspring, is determined by the plant responsible for the formation of the subepidermal layer from which the archesporial tissue arises. This explains the fact that the seeds of graft-hybrids always breed true to the characters of either scion or stock. The commonest example of such a graft-hybrid is that known as Cytisus adami, which is due to the grafting of the Purple Broom (Cytisus purpureus) on the Laburnum (C. laburnum).

It has been repeatedly noted that the fusion of the nuclei of the gametes appears to be the most important step in sexual reproduction. This is supported by the fact that, in all the higher plants, the male cell consists of little else than the nucleus (cf. pp. 350, 368), and that, in hybrid-experiments, it is immaterial whether the one or the other parent is employed as the male. ${ }^{1}$ The nucleus has also been seen to play a very important part in the activity of the cell, and, when dividing, to pass through a very complex series of changes. These have as their outcome an equal distribution of the chromatic material, which indeed is the only part of the nucleus that remains recognisable throughout all the phases of division. It may therefore be reasonably supposed that, in some way or other, the chromatin is the carrier of the hereditary qualities of the organism.

Sexual fusion may then be regarded as operating in two ways, firstly as a stimulus leading to further development,

1 Some forms of Enothera and Epilobium appear to constitute an exception to this generalisation. 
secondly as a possible means of introducing new characters or of rearranging those already present in the two parents. The former result may be attained by other stimuli. Amongst animals, for instance, the eggs of the Frog have been caused to develop by mere pricking with a glass needle covered with blood-serum, and those of Sea-urchins by treatment with solutions having a higher osmotic pressure than sea-water. Amongst plants the polyembryonic seeds of the Orange (cf. p. 372) furnish an analogous example; here certain cells, apart from the fertilised egg, have developed into embryos, but there is no evidence that the apogamously produced seedlings are any less vigorous than those resulting from sexual fusion. It seems probable, therefore, that the chief advantage of sexual reproduction lies in the possibility of producing organisms, with a slightly different hereditary constitution, such as may survive under conditions that would be unfavourable to the pure parent strain. In other words, sexual reproduction provides material upon which natural selection can operate.

The segregation of characters above referred to is most probably effected during the reduction division in the spore mothercells. In this process the chromosomes, instead of splitting longitudinally, as in the vegetative divisions, separate in their entirety into two sets. These pass to the respective daughternuclei, so that, of the four resulting spores, two possess the characters borne by one set, and two those borne by the other set. If this hypothesis be true, the two separate sets of chromosomes probably represent the paternal and maternal chromatin respectively. In the case of an allelomorphic pair, it is assumed that the dominant character is present in one set and the recessive in the other. In the reduction division, therefore, the allelomorphs will become separated, so that pure recessives and pure dominants can be bred. Such a theoretical conception is incapable of proof, and it is only warranted because it tallies with the observed facts.

In vegetative propagation the offspring normally exhibit no change of character, as compared with the parent, and new forms can only arise by mutation. Mutations in vegetatively produced offspring, and even in certain branches of an individual, have indeed been occasionally observed (cf. p. 382), and may 
be the means of maintaining the race in harmony with its environment. Although vegetative mutation appears to be comparatively infrequent, it should be borne in mind that groups like Bacteria and Cyanophyceæ, which multiply exclusively by vegetative means, often do so with great rapidity. An exceptionally large number of generations is therefore formed in a short time, and so presumably the opportunities for mutation are proportionately great.

Intimately bound up with the subject of the origin of new forms, considered in the preceding pages, is that of the geographical distribution of plants. This is mainly related to differences of climate, the distribution of a species often depending on its tolerance of cold or heat, dryness or moisture, etc. Under more or less uniform climatic conditions, such as obtain in our own country, soil differences play a large part in determining the regional distribution of plants. Most frequently it is the physical properties of the soil that are important in thus modifying the effects of climate. Of two areas receiving the same annual rainfall, for instance, that with sandy soil may be a desert, whilst that with peaty soil forms a bog.

The effect of climate is well illustrated by the distribution of forest, grassland, and desert over the earth's surface. In general, forest occupies the regions of highest, and desert those of lowest, rainfall, whilst grassland occurs in areas of medium, rather uniformly distributed, rainfall; the influence of man or of winds may, however, profoundly modify the applicability of this generalisation. In northern latitudes forests are composed of such trees as Birch, Pine, and Spruce, whilst in temperate zones deciduous trees, such as Beech, Oak, and Ash, predominate. On the slopes of high mountains, like those of Switzerland, the same relative distribution occurs, with deciduous forests below and Coniferous ones above.

But, apart from such cases in which the range of species is limited by their inability to withstand competition under adverse climatic conditions, certain species, genera, or even families are found to be restricted to particular areas of the earth's surface, though others are equally well suited to their existence. This 
fact is constantly being illustrated by the successful spread of species after their first introduction into a country. For example, the Canadian Pondweed (Elodea canadensis), which was brought

Ireland 1836
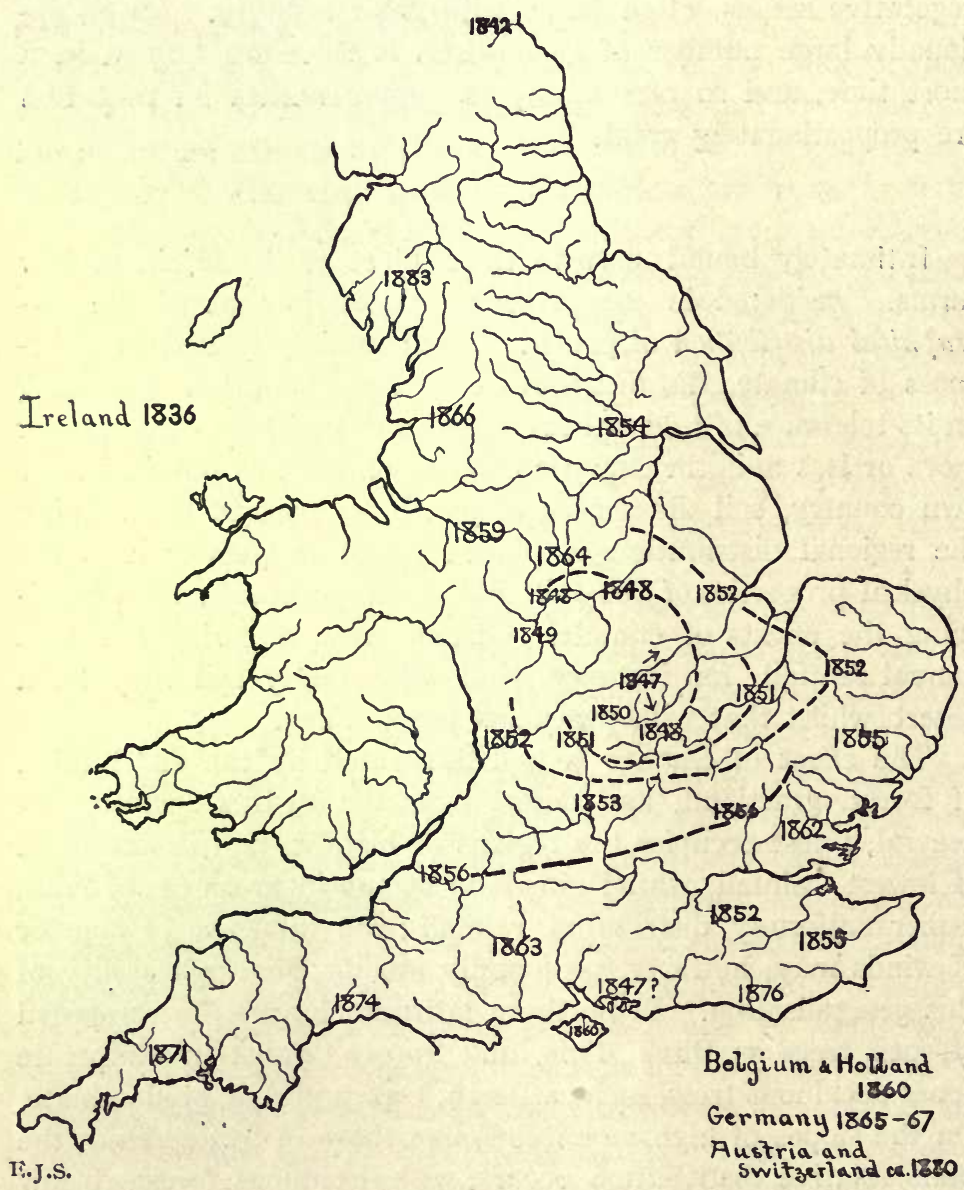

Fig. 227.-Map showing the spread of the Canadian Pondweed (Elodea canadensis) after its appearance in England in 1847. The broken lines connect identical years.

to England from America about I847, now occupies almost every waterway in this country (cf. Fig. 227). A similar phenomenon, on land, is presented by the Prickly Pear in Australia. In 
such cases it must be supposed that the ordinary agencies of dispersal have not sufficed to bring the species to all regions in which it can thrive successfully.

The natural barriers to the spread of plants are oceans, mountain ranges, deserts, etc., and it is significant that isolated islands (e.g. New Zealand) are peculiarly the home of species, so-called endemics, which are found there and there alone. There is, moreover, good reason for believing that, in a region devoid of natural barriers, the area over which a species is distributed is proportional to the age of the species or the time that has elapsed since its introduction. The capacity of a species for extending its geographical range, when the new territory is attained, depends on the efficiency of its seed-dispersal mechanism and the rapidity of its spread by this or by vegetative means.

The theory of evolution explains the resemblances between the members of a genus or family as the necessary consequence of their origin from a common ancestor or from closely related forms. It has already been seen how new types can arise as a result of mutation or hybridisation. Since these show a great resemblance to their known parents, it is reasonable to regard the many features in common, between species of a genus or between the genera of a family, as indications of a natural affinity between them. What has already been said, then, with regard to the distribution of species should also apply in a general way to genera and families, if these really comprise groups of forms with natural affinities. In many cases, indeed, the same principles are applicable ; for example, the genus Commidendron (a member of the Compositæ), with three species, is restricted to St. Helena, and whole families are sometimes largely confined to definite areas, as the Epacridaceæ (which are closely allied to the Heather-family) to Australia and Tasmania (cf. also Fig. 228).

Geological research has shown that oceans and continents have undergone manifold changes, even during the period of existence of many living species, and these secular changes probably afford the clue to the discontinuous distribution of many species and families. For certain groups, now represented only in widely separated areas over the earth's surface (e.g. the Cycads), are known to be ancient, and may well have attained their wide distribution before the present barriers were as pronounced or 
had even become established. A similar instance is furnished by the occurrence of the same Arctic species on the tops of many high mountains, although the present-day climate of the plains offers an insurmountable barrier to their dispersal from one chain to the other. These plants may, however, well represent the last remnants of a widespread flora of glacial times which, when the ice-sheet melted, found suitable conditions only on the mountain summits.

The conception of evolution regards the organic world, as we find it to-day, as consisting not of a number of immutable forms, but as presenting one phase in an everchanging series. The

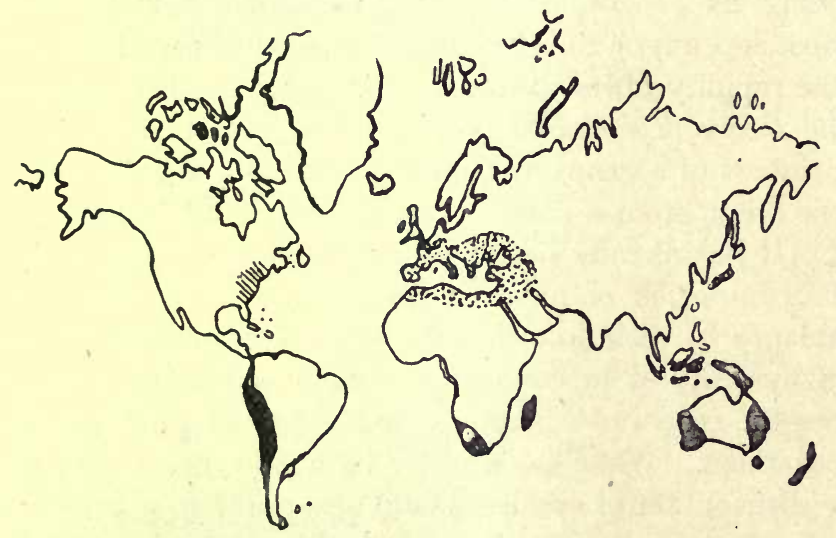

FIG. 228.-Outline map of the world, showing the distribution of Calycanthaceæ (indicated by shading), Proteaceæ (black), and Resedaceæ (dotted).

organisms of the present are the offspring of those of the past, and will themselves, in turn, give rise to the organisms of the future. Those animals or plants which have become extinct must be supposed to have failed to " make good" in the competitive struggle. From the fossil records it is known that whole floras and faunas have thus perished, leaving no living representatives or only much modified descendants (cf. Chapters XXI, XXII). Such disappearance may well be an outcome of the secular, but none the less profound, changes that have marked the history of the earth's surface since life first appeared. Organisms, unsuițed to the new conditions, would inevitably 
perish as a result of being handicapped in competition against, either their more adaptable contemporaries, or new forms which were better equipped to withstand the changed environment.

The evolutionist conceives of all life as having developed

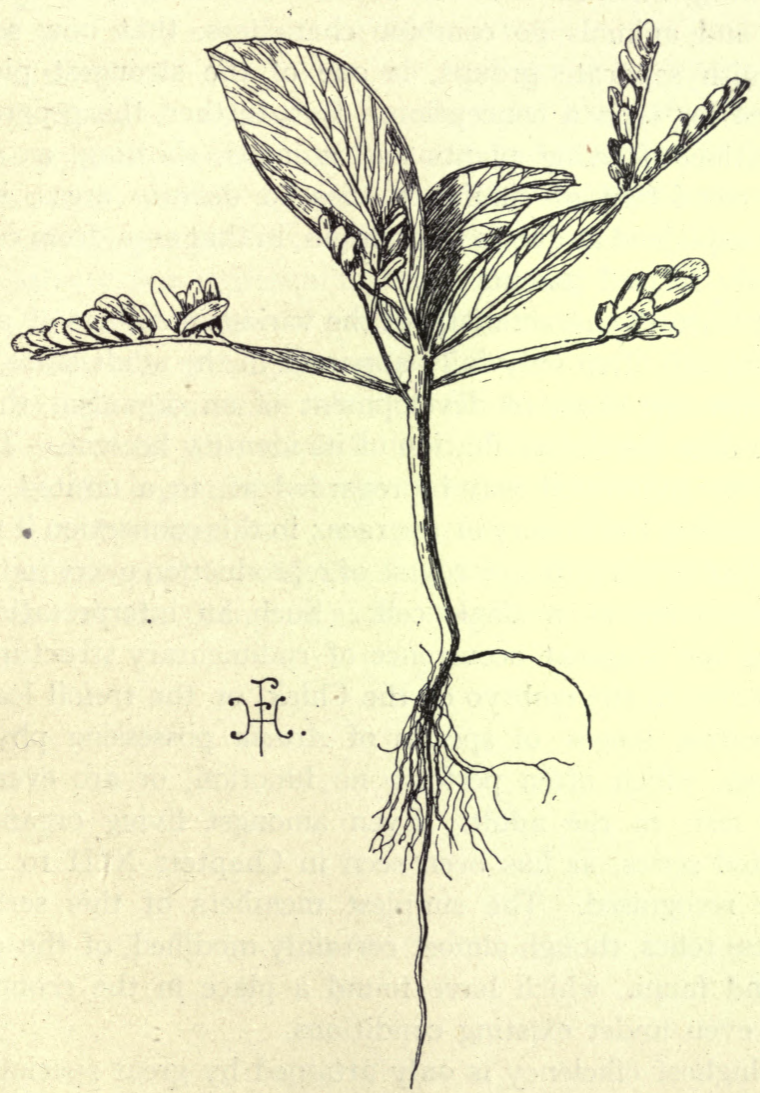

FIG. 229.- Seedling of an Acacia, showing the gradual differentiation of phyllodes, and suppression of the laminæ, in leaves of successive ages.

from relatively simple undifferentiated unicellular organisms, many of whose descendants, through the ages, have gradually acquired an increasing complexity of structure in relation to progressive division of labour. On this hypothesis, if our knowledge of all the extinct plants of former eras were complete, we 
could reconstruct the genealogical history (phylogeny) of the present-day forms. Those of the past would furnish the links connecting genus with genus, and family with family, till all were connected up to the primitive simple organisms from which they sprang when life first developed. The fact that many fossil plants and animals do combine characters, that now serve to distinguish separate groups, is one of the strongest pieces of evidence for such a conception. But, further, the geographical distribution of living plants and animals, showing, as already noted, restriction of similar species to definite areas, is most readily explained as due to their origin, in that area, from common or closely related parents.

The features characteristic of the various members of a genus or family are often only fully apparent in the adult state, whilst the earlier the stage of development of an organism, the more difficult does the determination of its identity become. The history of the individual may be regarded as, to a limited extent, recapitulating the history of the race; in this connection it may be noted that in the ordinary course of reproduction every individual commences life as a single cell. Such an interpretation also cxplains the frequent occurrence of rudimentary structures (e.g. the gill-slits in the embryo of the Chick, or the trefoil leaves in the seedling stages of species of Acacia possessing phyllodes, Fig. 229), which often perform no function, or are even completely lost, in the adult. Even amongst living organisms a graduated series, as has been seen in Chapters XIII to XXIV, can be recognised. The simplest members of this series are doubtless relics, though almost certainly modified, of the earliest flora and fauna, which have found a place in the economy of nature even under existing conditions.

The highest efficiency is only attained by great specialisation which proportionately diminishes the capacity for adaptation to a new environment. But the world of living things is a world of never-ceasing change, and hence the past history of the organic universe is the history of extinction of specialised races and individuals. The future of a group is thus seen to be dependent upon its less specialised, and thus at the moment less successful, members. But as in time, so too in space, the spread of a species may be handicapped by its lack of plasticity. 
The brief review of the groups here given is sufficient to indicate that the extinct Clubmosses and Horsetails, in the era of their success, were more specialised and more complex than their present descendants, which play so subordinate a rôle, and probably owe their survival to features which characterised the less successful members of these groups in the past. Such considerations lead one to suspect that the subordinate groups and individuals of any one age are the most likely starting-points for the dominant vegetation of the next, and so we can understand why the fossil record presents us with abundant examples of clearly defined groups (i.e. of the prevalent successful forms) and comparatively few representatives of groups "in the making." Indeed, the fossil plants of past ages and the living organisms of to-day combine to emphasise the rarity of the " missing link" which, like the thinker in advance of his age, is not sufficiently in harmony with the environment to command success, but yet marks the beginnings of the facies of the future. It is not, therefore, surprising that our progress in the reconstruction of the genealogical tree of the Vegetable Kingdom is slow, and that many of the groups remain in striking isolation from one another. 


\section{A P P E N D I X}

I. The Compound Microscope (Fig. 230).-This consists essentially of a stand which has for its purpose the appropriate support and adjustment of the optical parts. The latter comprise two systems of lenses, known respectively as ocular $(O c$.) and objective $(O b$.$) , whilst accessory structures are constituted by a mirror (M.)$ for reflecting light, and, in better instruments, a condenser $(C o$. for concentrating light upon the object.

The stand consists of a heavy foot or base $\left(F_{0}.\right)$, bearing a rigid upright pillar $(L$.$) . To the latter the remaining por-$ tions of the microscope are hinged in such a way that the whole can be employed either in a vertical or in an inclined position. The part actually hinged to the pillar is known as the limb, and to this the flat stage $(S$.) is attached, at right-angles, at the lower end, and the body tube ( $T$.) towards the upper end. The stage, which is usually square, is perforated by a central aperture through which the light from the mirror reaches the object. In transferring the microscope from place to place, it should always be carried by the non-movable parts.

The body tube is a hollow cylinder, usually of brass, and is adapted to take the ocular or eyepiece $(O c$.) at its upper, and the objectives $(\mathrm{Ob}$.$) at its lower, end. In order to secure rapid$ change of magnification; a nosepiece $(N$.$) , bearing two or three$ objectives, is screwed into the lower end of the body tube; by simply turning this a different objective can be brought to bear on the object. The distance between eyepiece and objective can be increased or decreased by pulling out or pushing in the draw-tube (D.t.), which is fitted into the upper end of the body tube. In order to focus the object clearly, the entire system of lenses can be moved nearer to, or farther from, the stage by means either of the coarse or fine adjustments. The coarse adjustment 
usually consists of two large milled heads $(C$.) on either side of the limb, and by turning either of these, which actuate a rack and pinion mechanism, a relatively large movement is brought about. The fine adjustment is generally operated by a single milled head $(F$.$) ,$ situated at the top of the limb, and by this means a very slight movement is effected, enabling great accuracy of focussing to be attained.

Below the stage is fixed an adjustable aperture, the diaphragm (D.), by means of which the amount of light reaching the object on the stage can be regulated. If a condenser is present, it is placed between the diaphragm and the stage, and, in the best instruments, its distance below the stage is adjustable by means of another milled head $(H$.$) .$ The mirror (M.), which is concave on

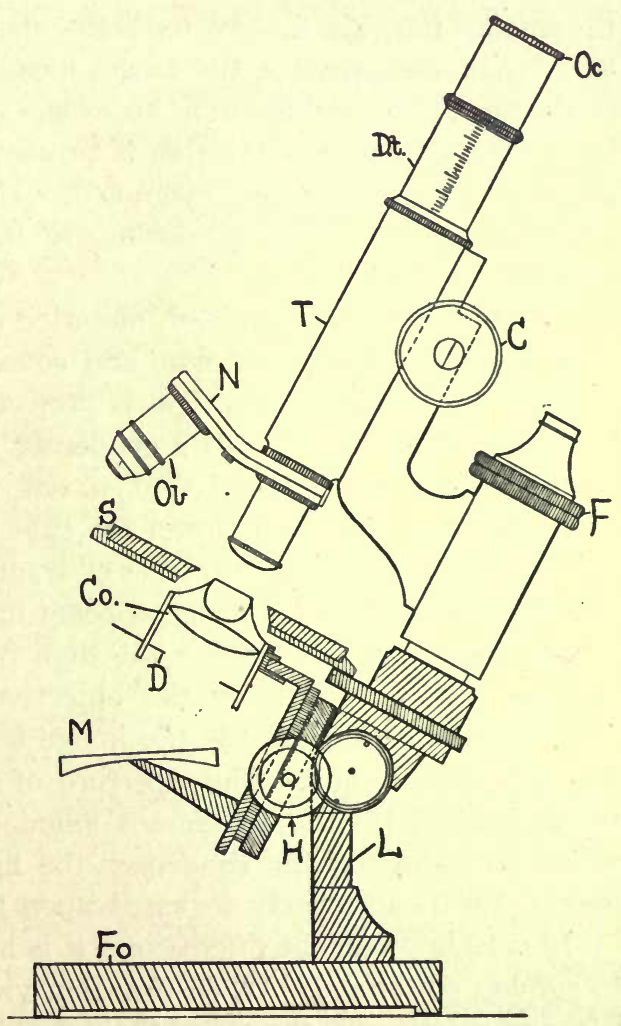

FIG. 230.-Diagrammatic representation of a compound microscope. C., Coarse adjustment ; Co., condenser ; D., diaphragm ; D.t., draw-tube; $F$., fine adjustment ; $F o$., foot ; $H$., milled head controlling substage adjustment for condenser; $L$., pillar : $M$., mirror ; $N$., nosepiece ; Ob., objective ; Oc., ocular or

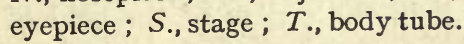
one surface and flat on the other, is either attached to the underside of the stage or (as in the type illustrated) forms part of the adjustable system bearing the condenser. 
The objectives most commonly in use are $\frac{2}{3}$ in. and $\frac{1}{6}$ in., ${ }^{1}$ which designations imply that, when focussed upon the object, they are approximately two-thirds and one-sixth inch respectively from the latter. These distances are the focal lengths, and the smaller they are the greater is the magnifying capacity of the lens. As a consequence, the longer focus lens is often spoken of as the low power, and the short focus lens as the high power. The image formed by the objective is projected on to the eyepiece, where it becomes further magnified. The amount of magnification of the eyepiece is commonly indicated by a number engraved upon it.

The object to be examined, mounted on a glass-slip in water or some other appropriate fluid and covered with a cover-glass, is placed on the stage, and light is projected on to it from below by means of the mirror. If a condenser is present, the flat side of the mirror is employed, but if not the concave side. To focus the object, gradually lower the tube by means of the coarse adjustment till the image becomes clear, and then turn the milled head to and fro until the image appears most distinct. Proceed in the same way when using the high power, but exercise the greatest care not to bring the objective in contact with the cover-glass, and immediately the image begins to appear use the fine adjustment only. The aperture of the diaphragm should be diminished till the maximum amount of detail is visible, whilst by adjusting the condenser the light reflected from the mirror can be accurately focussed upon the object.

In working with the microscope, it is best to accustom oneself to employ either eye. When drawing, view the object with the left, and sketch with the aid of the right, eye. As a first exercise in microscopic observation, it is well to examine the small air-bubbles almost invariably present in large numbers in a drop of water. Under the low power these appear as black dots or bright patches with broad dark margins ; this dark border is due to refraction. Adjust the slide so that one of the smaller bubbles is in the centre of the field of view, and turn the nosepiece so as to view the bubble with the high-power lens. Using the fine adjustment, it will be noted that at a high focus the

1 For the study of Bacteria and other minute organisms, higher powers are required, such as $\frac{1}{12}$ in. objectives. 
curved surface is seen and the outline appears shadowy, whilst at a lower focus only the circular equatorial portion (the optical section) is visible, and the outline becomes well defined.

II. Reagents.-Details as to the mode of preparation of the principal reagents mentioned in this book are given in the following :

Ammoniated Copper Oxide (Cuprammonia).-This reagent must be freshly prepared. Add ammonium chloride, and subsequently excess of sodium hydrate, to a solution of copper sulphate. The blue precipitate produced is filtered and washed thoroughly, and then dissolved in a small quantity of strong ammonia.

Eau de Javelle (mainly potassium hypochlorite).-Mix 20 parts of chloride of lime with Ioo parts of water. Allow to stand, and then add a solution of 15 parts of caustic potash in Ioo parts of water. Filter after some hours and use the filtrate.

Fehling's Solution (an alkaline solution of cupric oxide).According to Haas, this is best obtained by mixing equal quantities of a solution containing $69^{\circ} 28$ grams of pure crystallised copper sulphate in I litre, and of a solution containing 350 grams of Rochelle salt (potassic sodic tartrate) and Ioo grams of sodium hydrate in I litre. The resulting solution is of a clear dark blue colour. Ten cubic centimetres of this solution are reduced by $0^{\circ} 05$ gram of glucose.

Iodine Solution. - This is made by dissolving crystals of iodine in a strong solution of potassium iodide. For use this is diluted to a light brown colour.

Millon's Reagent.-This is a mixture of mercuric nitrate and nitrite. It can be prepared by dissolving I5 grams of mercury in 30 grams of cold nitric acid (sp. gr. I42), which operation should be performed in a fume cupboard. Dilute with twice the volume of distilled water, and filter after two hours. This reagent can also be bought ready made from the usual dealers in chemicals.

Phenylhydrazine Hydrochloride (after Mangham). - Prepare separate solutions, in ten times their weight of glycerine, of phenylhydrazine hydrochloride and sodium acetate respectively. Place the material to be investigated in equal drops of these two solutions, thoroughly mixed, and, after covering with a cover-glass, heat for one to several hours in an oven. 
Phloroglucin.-Prepare a saturated solution in alcohol. Treat material with this for a short time, and then mount in strong hydrochloric acid.

Scharlack Red.-Prepare a saturated solution in a mixture of 70 parts absolute alcohol and 30 parts water by volume. Filter and keep well stoppered.

Sulphuric Acid.-For cellulose tests it is usual to employ the concentrated acid. Great care must be exercised in its use, and strong ammonia should be at hand to neutralise any drops that may be spilled.

III. Artificial Sea-water.-For this purpose Tidman's sea-salt, dissolved in distilled water in appropriate concentrations, can be employed; or a solution can be made according to the following formula given by Osterhout:

I,000 parts sodium chloride (gram-molecular solution).

78 ", magnesium chloride (gram-molecular solution).

38 " magnesium sulphate (gram-molecular solution).

22 " potassium chloride (gram-molecular solution).

Io ", calcium chloride (gram-molecular solution).

This solution has an osmotic pressure of about 22.4 atmospheres; when diluted with an equal volume of distilled water, the osmotic pressure is halved, when diluted with twice its bulk of distilled water the osmotic pressure is one-third of that of the undiluted solution, and so on.

IV. Artificial Semipermeable Membranes.-For this purpose the membranous precipitate of copper ferrocyanide, formed when solutions of copper sulphate and potassium ferrocyanide meet, is often employed. The precipitate is customarily deposited in the wall of a small pot of unglazed porous porcelain, which is thoroughly washed so that it is impregnated with water. It is then nearly filled with a dilute solution of copper sulphate ( 2.5 grams per litre) and stood in a solution of potassium ferrocyanide (2.I grams per litre), where it is left for some time. Ultimately it is thoroughly washed and soaked in water. It is best to prepare several pots in this way, as some are sure to be faulty. To overcome this difficulty, Philip recommends depositing the copper ferrocyanide precipitate in a film of gelatine, formed 
over one end of a glass tube by dipping it in 20 per cent. gelatine to which a little potassium bichromate has been added; the latter has the effect of rendering the gelatine insoluble, if it is allowed to set in the light. After this the tube is again filled with the copper sulphate and the closed end allowed to dip into the potassium ferrocyanide solution until the gelatine has acquired the brown colour of the precipitate.

Still another method consists in allowing the copper ferrocyanide precipitate to form in a membrane of celloidin. This can be obtained by pouring a celloidin solution on a clean mercury surface contained in a Petri dish and allowing the solvent to evaporate away. The membrane which remains is then fitted over the open end of a thistle funnel, the overlapping portion being tied securely round the flange of the bulb. In drying, the membrane contracts slightly and becomes stretched taut. A very strong combination is obtained if two membranes of this kind are fitted over one another.

A piece of pig's bladder, which should be thoroughly dried before use, or a piece of parchment, stretched over the end of a thistle funnel, is often quite effective as a semipermeable membrane. In all cases the solution whose osmotic pressure is to be determined is placed in one of these osmometers, and the whole is immersed in water in such a way that diffusion between the two solutions can only take place by way of the semipermeable membrane. The pressure can then be calculated from the height to which the solution rises within a glass tube fitted on to the osmometer, or by means of a suitable manometer.

V. Equivalent Osmotic Pressures of Sea-water.-For the following data, which refer to strengths of Tidman's sea-salt, we are indebted to Mr. F. M. Haines, B.Sc. :

Concentration of solution Osmotic pressure in grams per roo c.c.

$\begin{array}{llll}0.01 & . & . & 0.08 \\ 0.02 & . & . & 0.16 \\ 0.03 & . & . & 0.24 \\ 0.04 & . & . & 0.32 \\ 0.05 & . & . & 0.40 \\ 0.06 & . & . & 0.48 \\ 0.07 & . & . & 0.56 \\ 0.08 & . & . & 0.64\end{array}$

Concentration of solution Osmotic pressure in grams per 100 c.c. in atmospheres.

$\begin{array}{llll}0.09 & . & . & 0.72 \\ 0.1 & . & . & 0.8 \\ 0.2 & . & . & 1.6 \\ 0.3 & . & . & 2.4 \\ 0.4 & . & . & 3.2 \\ 0.5 & . & . & 4.0 \\ 0.6 & . & . & 4.8 \\ 0.7 & . & . & 5.6\end{array}$


Concentration of solution
in grams per roo c.c.

$\begin{array}{cccc}0.8 & . & . & 6.4 \\ 0.9 & . & . & 7.15 \\ \mathrm{I} \cdot 0 & . & . & 7.95 \\ \mathrm{I} \cdot \mathrm{I} & . & . & 8.7 \\ \mathrm{I} \cdot 2 & . & . & 9.6 \\ \mathrm{I} \cdot 3 & . & . & \mathrm{I} 0.3 \\ \mathrm{I} \cdot 4 & . & . & \mathrm{II} \cdot \mathrm{I}\end{array}$

Concentration of solution Osmotic pressure in grams per roo c.c. in atmospheres.

$\begin{array}{llll}I \cdot 5 & . & . & \text { II.9 } \\ I .6 & . & . & I 2.7 \\ I \cdot 7 & . & . & I 3.5 \\ I .8 & . & . & I 4.3 \\ I \cdot 9 & . & . & I 5 . I \\ 2.0 & . & . & I 5.9\end{array}$

The osmotic pressure for concentrations between those given in this table are proportional to the values between which the particular concentration lies.

VI. Preserving and Staining.-To preserve material for anatomical investigation, ordinary methylated spirit will usually serve, provided there is at least four times the volume of liquid as of material. For showing nuclear structure, however, other fixatives are employed (cf. Chamberlain, Methods in Plant Histology), e.g. acetic alcohol, made by adding one part of glacial acetic acid to four parts of alcohol. After remaining in this for a few minutes up to several hours, according to the texture of the material, the latter is transferred to ordinary spirit.

For staining, the thinnest sections (cf. VIII below) should be placed in a few drops of safranin, ${ }^{1}$ on a slide, for from five to fifteen minutes, more safranin being added at intervals to replace that lost by evaporation. The excess of the stain is now removed by washing the sections with spirit, and then a few drops of Kleinenberg's hæmatoxylin are allowed to act for half a minute. After this the sections are washed with spirit, and permanent preparations are made in the following way:

The spirit is changed several times, and finally replaced by a few drops of absolute alcohol. In this way dehydration (i.e. removal of water) is effected. To the alcohol a drop or two of clove oil is then added, and this mixture is in turn replaced by pure clove oil. The sections should now become transparent, and, if this fails to occur, they have not been sufficiently dehydrated. After two to three minutes the oil is poured off, and Canada balsam, dissolved in xylol, added. A cover-glass is then carefully let down on to the sections, and the slide placed

1 Or methyl blue can be used, the sections being left in this for about half a minute. 
on one side till the balsam sets. Throughout all these processes the greatest care should be taken that the sections are never without a covering of liquid.

If permanent preparations are not required, such stains as phloroglucin, aniline chloride, etc., can be employed to differentiate the tissues, and the sections are usually mounted in glycerine diluted with an equal volume of water. Preparations can also be mounted in glycerine jelly, such mounts being much more rapidly and easily prepared than those with Canada balsam, but they often perish after a few years.

The liquid stains most commonly employed are prepared as follows :

Aniline Blue.-Saturated solution in alcohol or water, with a trace of acetic acid.

Bismarck Brown.-Dissolve 2 grams in Ioo c.c. of $70 \%$ alcohol. Stain for about $\mathrm{I} \frac{1}{2}$ minutes.

Eosin.- $\mathrm{r} \%$ solution in either water or alcohol. Stain for 3 to 5 minutes.

Gentian Violet.-I \% solution in water. Stain for from Io to 5 minutes, transfer to alcohol, and quickly counterstain with Bismarck brown.

Hamatoxylin.-Best bought prepared ready for use.

Methyl Blue.-Saturated aqueous solution. For live staining this is greatly diluted.

Safranin.- r $\%$ solution in $50 \%$ alcohol.

VII. Measurement under the Microscope.-This is accomplished by means of an eyepiece micrometer and a stage micrometer (both obtainable from the usual dealers in microscopic requisites). The stage micrometer is an ordinary slide on which is mounted a millimetre scale, divided into tenths and hundredths, and obtained by photographic reduction. The eyepiece micrometer fits into the ocular, and consists of a scale that is usually divided into a hundred equal parts. For each objective the value of a division of the eyepiece micrometer is determined in terms of the divisions of the stage micrometer (i.e. in hundredths of a millimetre). If subsequently any object is measured with the eyepiece scale, its actual size can be calculated.

VIII. Section-cutting.-During this operation both razor and material should be kept moist with either water (for fresh 
material) or spirit (for preserved material). Hold the object between the thumb and first finger of the left hand, and arrange the tips of the remaining fingers so as to form a rest on which the razor blade can be glided backwards and forwards through the material. Note that the razor must be gently drawn through the object, and not pressed, as in ordinary cutting. The greatest care should be taken that the axis of the object is either at rightangles (for transverse sections) or parallel to the razor blade (for longitudinal sections). For the latter only a very short length of the stem, etc., should be used. When very thin objects, such as leaves, are to be cut transversely, small rectangular pieces, including a vein, are embedded in a vertical incision made in a short length of Elder-pith. Sections are then cut of the pith, as well as of the embedded object.

After cutting, transfer the sections to a slide, on which a drop of water or dilute glycerine has previously been placed, by means of a well-moistened brush, and reject all but the two thinnest. Complete sections are in most cases quite unessential, whilst the small fragments will usually be the thinnest. Oblique sections, even if thin, are quite valueless. The razor should be carefully cleaned by wiping it from the back towards the edge.

IX. The following is an epitome of the main divisions of the Vegetable Kingdom :

I. Cryptogamia.

I. Thallophyta.

Flagellata (organisms related to Algæ, Fungi, and Protozoa).

Bácteria.

Peridineæ (motile organisms of fresh and salt water, possibly related to Diatomaceæ). Algæ.

(a) Cyanophyceæ or Myxophyceæ (Blue-green Algæ).

(b) Chlorophyceæ (Green Algæ).

(c) Phæophyceæ (Brown Algæ).

(d) Rhodophyceæ (Red Algæ).

(e) Diatomaceæ or Bacillarieæ (Diatoms).

Charales (Stoneworts).

Myxomycetes (Slime Fungi).

Fungi: 


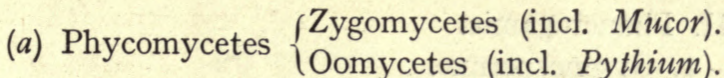

(b) Basidiomycetes.

(I) Uredineæ (Rust Fungi).

(2) Hemibasidii (Smuts and Bunt).

(3) Eubasidii $\left\{\begin{array}{c}\text { Hymenomycetes } \\ \text { and Mushrooms). } \\ \text { Gasteromycetes } \\ \text { Stinkhorns, etc.). }\end{array}\right.$ (Puffballs,

Ascomycetes (incl. Peziza, etc.).

(c) Ascomycetes Pyrenomycetes (incl. Claviceps, Erysiphaceæ, etc.).

(d) Lichenes.

2. Archegoniatæ.

A. Bryophyta.

(a) Hepaticæ (Liverworts).

(I) Marchantiales (incl. Riccia, Marchantia, etc.).

(2) Jungermanniales (incl. Cephalozia, Pellia, etc.).

(3) Anthocerotales.

(b) Musci (Mosses).

(I) Sphagnales (Bog-mosses).

(2) Andræales.

(3) Bryales (incl. most of the Mosses).

B. Pteridophyta.

Filicales (Ferns).

Psilophytales (only living genera Psilotum and Tmesipteris).

Sphenophyllales (Fossil).

Equisetales (Horsetail "Ferns" and Fossil

Calamites).

Lycopodiales [incl. Lycopodium, Selaginella, Lepido-

dendron (Fossil), and Isoetes].

Cycadofilices and Pteridospermæ. 
II. Phanerogamia.

I. Gymnospermæ.

Cycadales.

Bennettitales (Fossil only).

Cordaitales (Fossil only).

Ginkgoales (Maidenhair Tree).

Coniferales (Fir Trees, Yew, etc.).

2. Angiospermæ.

Monocotyledones (incl. Liliaceæ, Juncaceae, Gramineæ, Araceæ, etc.).

Dicatyledones.

(a) Archichlamydeæ.

(b) Sympetalæ (an artificial assemblage of the more specialised families).

The following list of works, dealing with some of the more advanced aspects of botanical science, may prove useful to those who desire to extend their reading or for purposes of reference :

\section{(a) Anatomy}

Haberlandt, "Physiological Plant Anatomy." (English translation by $M$. Drummond.) Macmillan \& Co.

\section{(b) Biochemistry}

Haas and Hill, "An Introduction to the Chemistry of Plant Products." Longmans, Green \& Co.

Monographs, on Plant Chemistry, Longmans, Green \& Co. :

Bayliss, "The Nature of Enzyme Action."

Osborne, "The Vegetable Proteins."

Armstrong, "The Simple Carbohydrates and the Glucosides." Russell, "Soil Conditions and Plant-growth."

\section{(c) Economic Botany}

Freeman and Chandler, "The World's Commercial Products." Pitman \& Sons.

Freeman, "Current Investigations in Economic Botany." New Phytologist, vols. iv and v, I905-6.

Common Commodities of Commerce Series. Pitman \& Sons. 
(d) Floras

West, "The British Freshwater Algæ." Cambridge University Press.

Macvicar and Jameson, "The Student's Handbook of British Hepatics." John Wheldon \& Co.

Dixon and Jameson, "The Student's Handbook of British Mosses." John Wheldon \& Co.

Hooker, "The Student's Flora of the British Isles." Macmillan $\&$ Co.

\section{(e) Fossil Botany}

Seward, "Fossil Plants." 4 vols. Cambridge University Press. Scott, "Studies in Fossil Botany." A. \& C. Black.

Scott, "The Present Position of Palæozoic Botany." Progressus Rei Botanicæ, i, I907, pp. I39 et seq.

Laurent, "Les Progrès de la paléobotanique angiospermique dans la dernière décade." Ibid., i, I907, pp. 3I9 et seq.

\section{(f) Heredity, etc.}

Darwin, "Origin of Species."

Punnett, "Mendelism." Macmillan \& Co.

Bateson, "Mendel's Principles of Heredity." Cambridge University Press.

Bateson, "The Progress of Genetics since the Rediscovery of Mendel's Papers." Progressus Rei Botanicæ, i, I907, pp. 368 et seq.

Lock, " Variation, Heredity, and Evolution." John Murray.

\section{(g) Morphology}

West, " Algæ." Cambridge Botanical Handbooks. Cambridge University Press.

Butler, "Fungi and Disease in Plants." Thacker, Spink \& Co. Harshburger, "A Textbook of Mycology and Plant Pathology." Churchill.

Ellis, " Outlines of Bacteriology." Longmans, Green \& Co. 
Cavers, "The Interrelationships of the Bryophyta." New Phytologist, vol. ix, IgIo.

Bower, "The Origin of a Land-flora.". Macmillan \& Co.

Coulter and Chamberlain, "Morphology of Gymnosperms." D. Appleton \& Co., New York.

Coulter and Chamberlain, "Morphology of Angiosperms."

D. Appleton \& Co., New York.

Willis, "Flowering Plants and Ferns." Cambridge University Press.
(h) CEcology of Plants

Tansley, "Types of British Vegetation." Cambridge University Press.

Warming, " Ecology of Plants." (English translation by P. Groom.) Clarendon Press.

Schimper, "Plant Geography." Clarendon Press.

(i) Physiology

Pfeffer, "Physiology of Plants." 3 vols. (English translation by $A$. J. Ewart.) Clarendon Press.

Darwin, "Lectures on the Physiology of Movement in Plants." New Phytologist, vol. vi, I907.

\section{(j) Technique}

Chamberlain, "Methods in Plant Histology." D. Appleton \& Co., New York.

(k) Microscopy

Spitta, "Microscopy." John Murray. 


\section{N D E X}

The principal references are printed in heavier type. 'Illustrations are indicated by ( $f$ ig.) after the paginal reference.

Abies, 335, 336 (fig.), 344, 347 ; A. pectinata, 129.

Abietineæ, 335, 337, 340, 347.

Abnormal growth, I42, I43 (fig.), 252,254 (fig.), 267.

Abscission-layer, I4I, I42 (fig.).

Absence-hypothesis, in hybridisation, 384 .

Absorption of carbon dioxide, 88 , I09, 259, 343.

of food by, cotyledons, I50 ; embryos, 282, 284, 302, 320, 35I, 370; Fungi, 230, 232, 236, 252 ; insectivorous plants, 150 ; parasitic Angiosperms, I79; plant-cells, I3, I5.

of mineral salts, 10, 13, I5, 72, 270.

of organic material, 72, 252.

of water, 147,158 ; by aerial roots, 73,74 ; by aquatics, 93 , I7I ; by Fern-prothalli, 301 ; by Lichens, 259 ; by Liverworts, 270; by Mosses, 274, 276 ; by mucilage, 38 ; by plant-cells, 10, 26 ; by roothairs, 67,72 .

Absorptive hairs, 150.

Acacia catechu, 39; A. senegal, 39. Acacia, False (Robinia pseudacacia), I I 5, I 26.

Acacia, seedlings of, 395 (fig.), 396. Accumulation of food-substances, 13, 40 .

Acer, I30; A. pseudoplatanus, Iо, 95, I I I 128 (fig.), I 42 (fig.); $A$. saccharinum, 45, I24; $A$. striatum, 135 .

Acetic acid, 265; - alcohol, 404. Achene, 37 I (fig.).

Achlya, 231.

Acids, organic, 49, 53, 54, 60, 265 , 302.

Aconitine, 6 I.
Aconitum napellus, $6 \mathrm{I}$.

Acorn (Quercus), 60.

Acorus calamus, stem-structure, 85 (fig.), 86.

Acquired characters, inheritance of, 380.

Activators, 57.

Active hydathodes, I 47.

Active principle of, Aniseed (Pimpinella anisum), 62; Bitter Almond (Prunus amygdalus), 48; Cinchona, 6r ; Cinnamon (Cinnamomum zeylanicum), 62 ; Cloves (Eugenia caryophyllata), 62 ; Cruciferæ, 48 ; Deadly Nightshade (Atropabelladonna), 6I ; Ergot (Claviceps purpurea), 255 ; Erythroxylon coca, 6I ; Eucalyptus, 62; Foxglove (Digitalis), 48; Ginger (Zingiber officinale), 63; Hemlock (Conium maculatum), 61 ; Henbane (Hyoscyamus niger), 61 ; Hop (Humulus lupulus), 63; Indigo-plant (Indigofera), 48 ; Ipecacuanha (Psychotria ipecacuanha), 6I ; Lavender (Lavendula), 62; Monkshood (Aconitum napellus), 61; Opium (Papaver somniferum), 6r, I55 ; Pepper (Piper), 63; Peppermint (Mentha piperita), 62.; Quillaia-bark, 48; Soapwort (Saponaria), 48; Solanaceæ, 61; Strychnos nux vomica, 61 ; Thornapple (Datura stramanium), 6I; Tobacco (Nicotiana tabacum), 61; Vanilla, 48; Woad (Isatis tinctoria), 48.

Adaptation, 379 (fig.), 380 ; to habitat, I 58-79 (figs.), 2 Io, $236,238,321$; to insectpollination, 373 .

Adiantum, 29I (fig.), 296. 
Adoxa moschatellina, epidermis, 95, 96 (fig.).

Adsorption, 56.

Advantage of, heterospory, 32I ; oogamy, 223; seed-habit, 332, 333.

Adventitious roots of, Ferns, 290, 303; Horsetails (Equisetum), 3II; Lycopodium, 3I5; Lyginopteris, 328; Selaginella, 317.

Ecidiospores, 247.

Æcidium, 246 (fig.), 247.

Egopodium podagraria, petiole, I 3 (fig.).

Aerating system, 26, 88, 96, 107, I08 (fig.), I09 (fig.), I ro, I39, r70 ; of aquatics, r75, I76, I77; of Lichens, 259; of marsh-plants, I74, I 75 (fig.), I78 ; of Moss-sporogonium, 284 ; of secondary wood, I24.

Aerenchyma, I 74, I75 (fig.).

Aerial roots, structure of, 73,74 , 75 (fig.).

Aerobic Bacteria, 263, 266.

Esculus, 52, 59 (fig.); hairs of, I02; stem-structure of, I20, I2I (fig.), I 23 (fig.), I 27.

Affinity between species, genera, etc., 393.

African rubber (Landolphia), 157.

Agar-agar, source of, 204; use of, 264.

Agaricus, 245; A. campestris, 248, 249 (fig.).

Age and geographical distribution, 393.

Agrostemma, I Iо, 370.

Air-bladders (of Algæ), 20 I (fig.), 204 (fig.).

Air-bubbles, appearance of, under microscope, 400 .

Air-canals of, aquatics, I74 (fig.), 175, I76; bog-plants, I78; Horsetails (Equisetum), 3II.

Air-spaces. See Intercellular spaces. Albumen, II, 23 I.

Albuminoid cells (of Conifers), 34I (fig.), 344 (fig.).

Albuminous seeds, $150,35 \mathrm{I}, 37 \mathrm{I}$.

Alchemilla, 372.

Alcohol, and protoplasmic movement, 4 ; use of, in determining width of stomatal aperture, 99, 100.

Alcoholic fermentation, 53, 255, 256, 257.
Alder (Alnus), 6o; cut-leaved, 382 ; root-nodules of, 268 ; vessels of, 28,35 (fig.).

Aleurone grains, 5I (fig.).

Aleurone layer (of Grasses), 52 (fig.). Algæ, 7, 1 3, 39, 181-229 (figs.), 231, 236,355 ; of Lichens, 257 . 259, 260 (fig.).

Algæ, chloroplasts of, I81, 207-ro (figs.) : classification of, 198, 199, 406; economic importance of, , 203, 204; sexual reproduction of, r84-6 (fig.), 213 , $2{ }_{4}$ (fig.), 220-28 (figs.); structure of, $180-207$ (figs).; vegetative reproduction of, $2 \mathrm{I} 2$, 216; zoospores of, 212-14 (figs.), 216-19 (figs.).

Alisma plantago, 100, 146 ; fruit of, 37 I (fig.).

Alkaloids, 60, 61, 62, 64, 155, 255 ; reactions of, $6 \mathbf{I}$.

Allelomorphs, 384, 385, 390.

Allium cepa, 97 (fig.), 359 (fig.).

Almond, Bitter (Prunus amygdalus), 48.

Alnus, 28, 35 (fig.), 60, 268.

Alpine plants, 63, 170, 379 (fig.), 394.

Alternation of generations, 288, $304,305,373$.

Althea, epidermis of, 95 ; pollen of, 362 (fig.).

Amanita muscaria, 255; A. phalloides, 255.

Amanitopsis vaginata, Plate II.

Amides, 50.

Amino-acids, 52, 53

Amitosis, 2r.

Ammonia, conversion to nitrates, 266.

Ammoniated copper oxide, 401 ; and callose, 82 ; and cellulose, 32 ; and cork, 137; and cuticle, 92 ; and lignified walls, 32.

Amygdalin, 48.

Anabana, 206 (fig.).

Anaerobic Bacteria, 263, 267 ; respiration, 257 .

Anæsthetics, 61 ; and motile lower organisms, 183; and protoplasmic movement, 4 .

Anatomy, and habitat, I58-79; of Alpine plants, I70; of Angiosperms, 65-I43 (figs.); of Avaucaria, 340; of bogplants, 178; of Coniferales, 
338-44 (figs.); of Cycadales, 322,323 ; of Equisetum, 31 I (fig.) ; of Filicales, 292-5 (figs.); of Fucus, 202, 225 (fig.); of Gleichenia, 294, 295; of Hepaticæ, 272, 273 (fig.); of Laminaria, 202; of Larix, 343; of Leucobryum, 277; of Lichens, 259, 260 (fig.); of Lycopodium, 315; of Lyginopteris, 328-30 (fig.) ; of Marchantia, 273 (fig.); of Mnium, 275 (fig.); of Musci, 275 (fig.), 276; of Nephrodium, 2.94 (fig.) ; of parasitic Angiosperms, I78 (fig.), I79; of Pellia, 272, 278 (fig.); of Picea, 343; of Pinus, 338-4I (fig.), 342 (fig.), 343, 344 (fig.); of Polytrichum, 276; of Pteris, 290 (fig.), 292-4 (fig.); of Selaginella, 317, 318; of Sphagnum, 275 (fig.), 276 ; of Taxus, 339, 34I, 342 (fig.) ; of water-plants, I 70-77 (figs.) ; of xerophytes, I 58-68 (figs.). See also Leaf-, Root-, and Stemstructure.

Anatropous ovules, 357 (fig.), $35^{8}$ (fig.), 359.

Andræales, 407.

Anemone nemorosa, 148, 248.

Angicsperms, I80, 334, 354-73 (figs.) ; classification of, 355 , 408 ; embryo sac of, 366 (fig.), 367 (fig.) ; embryology of, 368-72 (figs.) ; fertilisation of, 368 ; flowers of, 354, 355, 373 ; fossil, 356 ; leaf of, 9II 6 (figs.) ; ovary of, 356 ; ovules of, 357-9 (fig.) ; pollen sacs of, 360-63 (figs.); root of, $65-75$ (figs.), I3I (fig.), I32; seeds of, $370-72$; stamens of, 359 (fig.) ; stem of, 76-90 (figs.), I I 7-30 (figs.), I35-4I (figs.).

Animal galls, I43 (fig.).

Animals, compared with plants, I 4, 25, I8I, I88, 193, I94, 247, 307; differences between plants and, I88, I93, I94; diseases of, due to plants, 230, 23I, 236, 239, 252, 255; protection against, $39,48,58,64$, I04, I06, I26, I 57 .

Aniline blue, 405 ; and callose, 82 ; and mucilaginous walls, 38 .
Aniline salts, and lignified walls, 32.

Aniseed (Pimpinella anisum), 62.

Aniseed oil, 62.

Anisogamy, I85 (fig.), 186, 220.

Annual rings of Conifers, 340 ; of Dicotyledons, II9 (fig.), I23 (fig.), 125, I32.

Annular vessels, 35 (fig.), 36 .

Annularia sphenophylloides, 312 (fig.).

Annulus of Fern-sporangium, 297, 298 (fig.), 299 (fig.) ; of Mosssporogonium, 285 (fig.), 286 ; of Mushrooms, 248, 249 (fig.).

Anomalous secondary thickening, I 32-4 (fig.).

Antagonism, I4, I5.

Antheridial cell of, Conifers, 348, 35 I (fig.) ; Cycads, 327 (fig.).

Antheridial groups of, Liverworts, 270 (fig.), 279; Mosses, 274 (fig.), 279 (fig.).

Antheridium, 222; of Algæ, 22 I et seq. (fig.) ; of Ferns, 301 (fig.), 302, 303. (fig.) ; of Fucus, 221 (fig.), 225 ; of Fungi, 233 (fig.), 234,236 ; of Liverworts, 277 , 278 (fig.) ; of Mosses, 279 (fig.); of $E$ dogonium, 22 I (fig.), 222 ; of Vaucheria, 22 I (fig.), 224.

Anthers, 359 (fig.) ; dehiscence of, $363 ;$ structure of, $360-63$ (figs.).

Anthoceros, 260.

Anthocerotales, 407.

Anthocyanins, 63 .

Antipodal cells, 357 (fig.), 366 (fig.), 367 (fig.).

Antipyretics, 6r.

Antiseptics, 62, I55 ; in cork, 138 ; in heart-wood, 126.

Apetalous flowers, 354 .

Apical cells, I6-I8 (figs.) ; of Algæ, I6 (fig.), I7 (fig.), I95, 20I; of Dictyota, I7 (fig.) ; of Equisetum, I8 (fig.); of Filicales, I8, 29I, 302; of Hepaticæ, 270, 272 ; of Musci, I 8, 276, 288; of Sphacelaria, I6 (fig.), I7; tetrahedral, I8 (fig.).

Apocarpous ovary, 354.

Apocynaceæ, 157.

Apogamy in, Algæ, 2 I4 ; Angiosperms, 372, 390; Ferns, 305 ; Fungi, 236.

Apophysis, 284, 285 (fig.). 
Apospory in, Angiosperms, 273; Ferns, 305.

Apothecium, 239 (fig.), 240 (fig.), 24I, 258 (fig.), 259.

Apple (Pyrus malus), 3I, 55, 60, 137. Aquatic Fungi, 23I, 236.

Aquatics (excl. Algæ), 69, 72, 146, 170-77 (figs.), 379; aircanals of, I 74 (fig.), I 75, I 76 ; epidermis of, $91,92,93$, I 72 ; land- and water-forms of, $175-7$ (figs.); leaf of, I72, I77 (fig.) ; mechanics of, 88, I 70 , I 7 I ; pollen of, 361 ; stem of, I 70-74 (figs.); stomata of, IOI.

Aqueous tissue, 94 (fig.), I 59 (fig.), I 60,166, I 67, I 68 (fig.).

Araceæ, 408.

Arachis hypogea, 49.

Araucaria, 336, 340, 345, 346, 347.

Arbor-Vitæ (Thuja), 336, 337 (fig.), 347.

Archegonial chamber (of Cupressineæ), 348,350 ; - discs (of Liverworts), 270 (fig.), 280.

Archegoniatæ, 407.

Archegonium, 280 ; of Coniferales, 348,349 (fig.); of Cycadales, 325 (fig.), 326, 327 (fig.) ; of Filicales, 301 (fig.), 302, 303 (fig.); of Hepaticæ, 280, 28I (fig.); of Musci, 28o; of Selaginella, 319, 320 (fig.).

Archesporium, 282, 389 ; of Coniferales, $34^{8}$; of Filicales, 297. 299 (fig.); of Hepaticæ, 282 ; of Musci, 284, 285 (fig.) ; of ovules of Angiosperms, 358 (fig.), 365 ; of stamens, 360 (fig.), 36r.

Archichlamýdeæ, 408.

Arctic plants, 63, 394 .

Arctium, collenchyma, 3 I (fig.).

Arenaria peploides, 94.

Aril of Angiosperms, 359; of Taxus, 346 (fig.).

Aristolochia, I20.

Arm-palisade, I Io, I I I (fig.), 343 , 344 (fig.).

Armillaria mellea, Plate II.

Arrowhead (Sagittaria), embryology of, 37 I (fig.).

Artichoke, Jerusalem (Helianthus tuberosus), 44.

Artificial nectary, 149; sea-water, 402 ; semi-permeable membranes, 402, 403; stoma, 99.
Artificial selection, 378 .

Arum maculatum, II2.

Ascomycetes, 23I, 238-45 (figs.), $259,408$.

Ascospores, 240 ; development of, 245 ; of Ergot (Claviceps), 24I (fig.), 242; of Erysiphaceæ, 243, 244 (fig.) ; of Eurotium, 243 (fig.); of Lichens, 260 ; of Peziza, 240 (fig.).

Ascus, 240 (fig.), 24I (fig.), 242, 243 (fig.), 244 (fig.), 245.

Asexual reproduction, 212, 219 , 229, 288; of Algæ, 2I2-I4 (fig.), 2I6-20 (figs.); of Ascomycetes, 238 et seq. ; of Basidiomycetes, 245 et seq.; of Chlamydomonas, $\quad \mathrm{8} 82-4$ (fig.), I 88 ; of Coniferales, 344-8 (figs.) ; of Conjugatæ, 216 ; of Cyanophyceæ, 216; of Cycadales, 323-5 (figs.); of Ectocarpus, 218, 219 (fig.) ; of Equisetum, 3I2, 3 I 3 (fig.); of Filicales, 295-300 (figs.); of Fungi, 23I et seq. ; of Hepaticæ, 277, 282-4 (fig.) ; of Lichens, 260 (fig.); of Lycopodium, 3I5, 316 (fig.) ; of Musci, 277, 284-7 (figs.); of $C E d o-$ gonium, 216, 217 (fig.); of Phæophyceæ, 218 ; of Phycomycetes, 232 et seq.; of Rhodophyceæ, 228; of Selaginella, 318 (fig.) 319; of Ulothrix, 212-I4 (fig.); of Vaucheria, 2 I 7, 2 I 8 (fig.).

Ash (Fraxinus excelsior), 120, I22, I 28, I 29, 39I.

Asparagin, 50.

Aspergillus, 242, 243 (fig.).

Aspidium. See Nephrodium.

Asplenium, 296 (fig.) ; $A$. bulbiferum, 305 (fig.); $A$. ruta muraria, 296.

Assimilation. See Carbon dioxide assimilation.

Assimilatory stems, 88, I62 (fig.), I67, I 78,3 Iо.

Assimilatory tissues of, aquatics, 202 (fig.) ; centric leaves, I 65 (fig.), I66 ; cladodes, I59 (fig.) ; Conifers, 342 (fig.), 343, 344 (fig.) ; dorsiventral leaves, I07-I I (figs.) ; Ferns, 295, 298 (fig.) ; Fucus, 202, 225 (fig.) ; Liverworts, 273 (fig.), 278 (fig.) ; Mosses, 276, 284, 
285 (fig.) ; stems, 88, 162 (fig.), I67; sun- and shade-leaves, I68 (fig.), I69 (fig.).

Asterionella, I93 (fig.).

Astragalus, 39.

Athyrium filix-famina, 289, 29I, 305.

Atropa belladonna, 6r.

Atropine, 6r, 62.

Attaching organs of, Chloro. phycex, 197, 228; Cladophora, I96 (fig.) ; Fucus, 200 ; Laminaria, I99 (fig.) ; Edogonium, 217 (fig.); Pelvetia, 203 (fig.); Ulothrix, 213 (fig.) ; Vaucheria, 218 (fig.).

Attractive mechanisms, 58, I 49, 354.

Aulacomnion androgynum, 277.

Autumn-wood of Conifers, 340, 34 I (fig.) ; of Dicotyledons, I I 9 (fig.), I22, I 23 (fig.), 125.

Axile placentation, 356 .

Axillary buds, vascular supply of, II 4 .

Azotobacter, 266, 267 (fig.).

Bacillarieæ, 206, 207 (fig.), 406.

Bacillus, 262, 267 (fig.) ; $B$. amylobacter, $263 ; B$. anthracis, 263 (fig.) ; $B$. carotovorus, 265 ; $B$. cedamitis, 267 (fig.); $B$. radicicola, 267 (fig.) ; $B$. subtilis, 263; B. tetani, 263 (fig.), 264, 267 (fig.) ; B. typhosus, 263 (fig.).

Bacteria, 6I, 252, 261-8 (figs.), 406; cultures of, 264 ; physiology of, 26I, 263, 264-7 ; reproduction of, $262,263,267$ (fig.), 39I ; structure of, 262, 263 (fig.).

Bacterium, 262 ; B. aceticum, 265 ; $B$. denitrificans, 266.

Balanced solutions, I5.

Bamboo (Bambusa), 88.

Barberry (Berberis vulgaris), cork and lenticels of, I37, I39 (fig.) ; rust of (Puccinia graminis), 246 (fig.), 247 ; stamens of, 363.

Bark, I 9 (fig.), I20, 140, 141; chemical substances in, 60 , 61,62 .

Barley (Hordeum vulgare), 46, 257.

Barriers to plant-dispersal, 393 .

Bartsia, I 44.

Basal cell (of Angiosperm proembryo), 368,369 (fig.), 37 I (fig.).
Basidiomycetes, 23I, 240, 245-51 (figs.), 252, 255, 407 .

Basidiospores, 246 (fig.), 247, 249 (fig.).

Basidium, 249 (fig.).

Bast, 28, 34, 65. See Phloem

Bay Laurel (Laurus nobilis), I 5 I.

Bean, Broad (Vicia faba), 53, 148 ;

Runner -. (Phaseolus multi-

florus) , 93, 94 (fig.) , I I 5, I 44, I 45

(fig.), 147, 371.

Bean, protein of, 52 ; root of, $7 \mathrm{I}$ (fig.), 72 ; starch of, 42 .

Bear's Foot (Lycopodium clavatum), 314.

Bedstraw (Galium), 98.

Beech (Fagus sylvatica), 72, I29, I 40, I 70, 365, 367, 39I ; cutleaved -, 382, 383 (fig.) ; wood of, I2I, I22, 126.

Beer, 256.

Beetroot (Beta), chemical substances in, $31,45,50,63$; secondary thickening of, 133 .

Beet-sugar, 45.

Beggiatoa, 268.

Belladonna (Atropa belladonna), 6ז. Bending strains, resistance to, 33 , 87 , II 5, I 72 .

Bennettitales, 407 .

Berberis, I37, I39 (fig.), 247, 363.

Bergamot oil, 62 ; - orange (Citrus aurantium, var. bergamia), 62 .

Beta, I33.

Betula, 72, I22, I29, I37, I39, I4I. Betulin, 137.

Bicollateral bundles, 8x (fig.), 82 . Bilberry (Vaccinium myrtillus), pollen of, 362 (fig.) ; stem of, I67, I68 (fig.).

Biologic strains of, Bacteria, 268; Erysiphaceæ, 253; Uredineæ, $248,253$.

Birch (Betula), 72, 129, 39I ; bark of, I37, I39, I4I ; wood of, I22.

Bird's-Nest Orchid. (Neottia nidusavis), I79; mycorrhiza of, 72, 73 (fig.); pollen of, 361,362 (fig.).

Bishop's-weed (Egopodium podagraria), petiole of, II 3 (fig.).

Bismarck brown, 405; and cellwalls, 32 .

Bitter Almond (Prunus amygdalus), 48.

Black Bindweed (Polygonum convolvulus), II 3 (fig.), I 48 ; - Bryony (Tamus communis), 
86,87 (fig.), II2 ; - Currant (Ribes, nigrum), I05; - Mould (Mucor), 23I ; - Walnut (Juglans nigra), I 29.

Blackberry-rust (Phragmidium bulbosum), 248.

Bladder-hairs, 94 (fig.).

Bladderwort (Utricularia), I50.

Bladderwrack (Fucus vesiculosus), 38, 200-202 (figś.), 225 (fig.).

Blechnum spicant, 29I, 295, 308 (fig.), 324 .

Bleeding of plants, 124.

Blue-green Algæ (Cyanophyceæ), I92, 2I0, 257, 268, 406; reproduction of, 206 (fig.), 216 , 220 ; structure of, 204-6 (fig.).

Blue Gum (Eucalyptus globulus), I29; - Mould (Penicillium), 239.

Body cell (of Conifers), 349, 350.

Bog-moss (Sphagnum), 274, 275 (fig.), 276, 407; - Myrtle (Myrica gale), 268, 357, 359.

Bog-plants, structure of, I 77, I 78 .

Boletus, 245, 250, 25I (fig.).

Borage (Bovago), hairs of, I04.

Boraginaceæ, I02, 104.

Bordeaux mixture, 254.

Bordered pits, 35 (fig.), 36, 37 (fig.), 68, I $20,340,34 \mathrm{I}$ (fig.), 343 ; of Ferns, 293; of wood-fibres, 3.3, I2I ; one-sided, I22 ; rôle of, 37.

Bowenia spectabilis, 324 (fig.), 325 (fig.).

Box (Buxus), 86, 127.

Bracken (Pteris aquilina), extrafloral nectaries of, I48, I49 (fig.) ; habit of, 289, 290 (fig.) ; leaf of, '60, II2, I69, 291, 295 ; rhizome of, 290 (fig.), 292-4 (figs.) ; sori of, 296 (fig.).

Bracket-Fungus (Polyporus squamosus), 250 (fig.).

Bract-scale (of Conifers), 347 (fig.), 352 (fig.), 353.

Bramble (Rubus spp.), prickles of, I04.

Branched hairs, IOI, IO2 (fig.), IO3; in Algæ, 22 I (fig.), 225.

Branching, forked, 200, 201 (fig.), $269,275,290,315,317$; monopodial, 334 ; of Algæ, 195-8 (figs.) ; of Conifers, 334,335 ; of Cycads, 322 ; of Equisetum, 3IO, 3II (fig.); of
Ferns, 289, 290; of Liverworts, $269,-270$ (fig.) ; of Lycopodium, 3I5; of Lyginopteris, 328 ; of Mosses, 275 ; of rhizoids, 274 ; of roots, $65,7 \mathrm{I}$ (fig.); of Selaginella, 3I7 (fig.) ; of stems, 72,90 .

Brandy, 257.

Brazil Nut (Bertholletia excelsa), food-substances of, 49, 5 I.

Bread, 52.

Breathing, I78, 20I, 257; and protoplasmic movement, 4 ; of fat-containing seeds, 50 .

Breeding, 378, 382, 388 .

Bristle hairs, Io4.

Broad Bean (Vicia faba), 53; extrafloral nectaries of, 148 .

Brooklime (Veronica beccabunga), stoma of, I60 (fig.).

Broom (Cytisus scoparius), stem of, 88, I67.

Brown Algæ (Phæophyceæ), 406 ; reproduction of, $216,218,219$ (fig.), 22 I (fig.), 224-6 (fig.) ; structure of, $\mathrm{I}_{98-204}$ (figs.), 208, 2 I0.

Brownian movement, 2 Io, 262.

Brussels Sprouts, "Finger and Toe" disease of, 254 (fig.).

Bryales, 407.

Bryony, Black (Tamus communis), II 2 ; stem-structure of, 86,87 (fig.).

Bryophyta, 269-88 (figs.), 289, $305,307,321,333,370,373$, 407; antheridia of, $277-80$ (figs.) ; archegonia of, 280 , $28 \mathrm{r}$ (fig.) ; habit of, 269-72 (figs.), 274 (fig.) ; habitat of, 269; sporogonia of, $28 \mathrm{I}-7$ (figs.) ; structure of, 272,273 (fig.), 275 (fig.), 276, 278 (fig.) ; vegetative reproduction of, 277,287 (fig.), 288.

Bryum argenteum, 285 (fig.).

Buckthorn, Sea (Hippophe rhamnoides), hairs of, Io3 (fig.).

Buckwheat (Fagopyrum), 37I.

Bud-scales, I I I, 322.

Budding of suspensor, 37I (fig.), 372 ; of Yeast (Saccharomyces), 256 (fig.), 257.

Bulbils (of Lycopodium selago), 3 I 5 .

Bulbs, 40, 44, 45, 355.

Bulrush (Scirpus), I 78 .

Bundles, 76 ; bicollateral, 8 I (fig.), 82 ; collateral, 76,78 (fig.), 
79, 85 (fig.), II 2 ; concentric, 85 (fig.), 86, I33 (fig.), I34, 292 (fig.) ; cortical, 86 ; leaf-trace, II4, I73, 294 (fig.), 295 ; medullary, 86 ; secondary, I33 (fig.), I 34 .

Bundle-ends, 113, I45, I46, I47 (fig.).

Bundle-sheath, of Conifers, 343, 344 (fig.); of leaves, 107, 108 (fig.), II2, II3; of stems, 85 (fig.), 86.

Bunt, 407.

Burdock (Arctium), collenchyma, 3 I (fig.).

Burr-reed (Sparganium), 366.

Butcher's Broom (Ruscus aculeatus), cladode-structure, I59 (fig.), I66.

Butomus, 356.

Butter, cause of rancidity of, 265.

Buttercup (Ranunculus spp.), 384 ; leaf of, I 77 (fig.) ; nectaries of, I 48 ; root-structure of, 66-9 (figs.) ; stem-structure of, 86, 87.

Butterwort (Pinguicula), glands of, I 49, I 50 (fig.).

Buttress-roots, 125.

Butyric acid, 265, 267.

Buxus sempervirens, 86, 127.

By-products of plants, $3,40,58$ 64, I 38, I $44, I_{56}$

Cabbage, "Finger and Toe" disease of, 25I, 254; - Mildew (Peronospora parasitica), 243.

Cabinet-work, timber used in, 126, I 30.

Cactaceæ, 178.

Cactus, 167.

Caffein, 61; and tannins, 13, 60; permeability of protoplasm to, I3.

Calamites, 3I0, 3II, 3I 2 (fig.), 407.

Calcareous Algæ, 204.

Calcified hairs, I04; - walls, 39, 204.

Calcium oxalate, 58, 59 (fig.), I30, 237 ; reactions of, 59.

Callithamnion, 204.

Callitriche stagnalis, anatomy of, I 75-7 (figs.).

Callose, 82,231 ; reactions of, 82 .

Calluna, 72 ; leaf of, 162, I63 (fig.).

Callus, I 42 .

Caltha, 72, 356; hydathodes of, I 46 ; ovule of, 366 (fig.).
Calycanthaceæ, geographical distribution of, 394 (fig.).

Calyptra, 282 ; of Liverworts, 282, 283 (fig.) ; of Mosses, 274 (fig.), 284,285 (fig.).

Calyptrogen, 20.

Calyx, 354.

Cambium, 29 (fig.), 90, 117, 118 (fig.); of aquatics, I73; of cork, 135, I 36 (fig.), I 37, I 38 (fig.), I39 (fig.), I4I ; of leaves, I 2 ; of lenticels, I39, I40 (fig.) ; of Monocotyledons, II 7 , I33 (fig.), 355; of roots, 66 (fig.), 131 (fig.); of Coniferous stems, 328 ; of Dicotyledonous stems, 79, 8o (fig.), 8I (fig.), 83 (fig.), 117, 118 (fig.), I I9 (fig.), I 2 I (fig.).

Campanulaceæ, 44, I 53, I 54.

Camphor, 63.

Campion (Lychnis), 88.

Campylotropous ovules, $35^{8}$ (fig.), 359.

Canada Balsam, use of, 404, 405.

Canadian Pondweed (Elodea canadensis), cell-structure, 5, 6 (fig.); spread of, in British Isles, 392 (fig.).

Cane-sugar, 45, 46, 53, 54, 28I ; hydrolysis of, 46, 53, 55; osmotic pressure of, II ; reactions of, 47 .

Cannabis sativa, 34, 265.

Cantharellus, 25I (fig.).

Caoutchouc, I 55, I 57.

Caps (of $C$ dogonium), I95, 2 I 7 (fig.). Capsella bursa-pastoris, embryology of, 368-7o (fig.) ; hairs of, I02 (fig.); White Rust of, 23I, 233 (fig.).

Capsule of, Fern-sporangium, 297300 (figs.) ; Liverworts, 27 I (fig.), 282, 283 (fig.) ; Mosses, Ioo, 274 (fig.), 284-7 (fig.).

Carbohydrates, 3I, 40-48, 50, 82, 266,267 ; reactions of, $4 \mathrm{I}, 44$, 47 ; translocation of, 88 , I I0. Carbon dioxide, absorption of, 88 , I09, 259, 343; circulation of, 265,266 ; formation of, in alcoholic fermentation, 53, 256 .

Carbon dioxide assimilation, 40, 4I, I07; and light, 96 ; by Algæ, I8I, I83, 202, 207; by roots, 73,74 ; by stems, $88,162,167$; removal of products of, $4 \mathrm{I}$, IIO; sugars formed in, 46 . 
Carbonate of lime, deposition of, on cell-walls, 39,204 ; in vessels, I26.

Carotin, 64, 109.

Carpels, 354, 355, 356.

Carpinus, I 28, 367.

Carpospores (of Red Algæ), 205 (fig.), 228.

Carrageen (Chondrus crispus), 204, 205 (fig.).

Carrot (Daucus carota), 64; soft rot of (Bacillus carotovorus), 265.

Carteria, I 86, I 87 (fig.), I 88.

Carya, 130.

Caryophyllaceæ, 3.58, 37I.

Cassava (Manihot utilissima), 43.

Castanea sativa, I30.

Castilloa elastica, I 57.

Castor oil, 49.

Castor oil plant (Ricinus communis), aleurone grains of, 50, $5 \mathrm{I}$ (fig.); fat of, 49; hypocotyl of, I 8 (fig.) ; seeds of, $371,372,376$ (fig.).

Casuarina, stem-structure of, I62 (fig.).

Catalytic action of enzymes, 55, 56.

Catechu, 39.

Caterpillars, fungal disease of, 239 , 255.

Catharinea undulata, leaves of, 276.

Caustic potash and, callose, 82; cork, 136; fats, 49.

Ceara-rubber (Manihot glaziovii), I 57.

Cedar (Cedrus), 335, 353.

Cedar wood (Cedrela spp.), I29.

Cedrus, 335, 353.

Celandine, Greater (Chelidonium majus), laticiferous vessels of, I53, I54; ovule of, 358 (fig.).

Celandine, Lesser (Ficaria verna), embryo of, 372; hydathodes of, I 45 , I 46 (fig.), I 47 (fig.).

Celery (Apium), 27.

Cell-contents, living, $x-8$ (figs.) ; non-living, 40-64 (figs.).

Cell-division, $2 \mathrm{I}-5$ (figs.); effect of wounding on, 26, 142; in reproductive cells, I83, 213 (fig.), 2I 4, 305-8 (fig.).

Cell-plate, 24, 25 (fig.).

Cell-sap, 3, 6, Io, 26, 60, 63 ; osmotic pressure of, 8, IO, II, I2, 26.

Cell-wall, 1, 4, 9, 24, 29-39 (figs.) ; cuticularisation of, $9 \mathrm{I}$; formation of, 24, r96 (fig.); growth of, 29; incrustation of, 39 ; lignification of, 32 ; mucilaginous, $38,95,184,189$ (fig.), 202, 214, 262; of Bacteria, 262 ; of Diatoms, 206, 207 (fig.) ; of Fungi, 23I ; of hairs, IOI ; stratification of, 24,29 , 33 ; suberisation of, 136 ; thickening of, 29-37 (figs.), 47. Cells, apical, I6-I 8 (figs.) ; aqueous, I66 ; assimilatory, 107-I I (figs.) ; division of, 2I-5 (figs.), 305-8 (fig.) ; epidermal, 9I-6 (figs.); glandular, I48, I 49 (fig.) ; growth of, 28, 29; mechanical, 3I-4 (figs.); meristematic, I8 ; parenchymatous, 27; physiology of, 8-I5; plasmolysis of, 5 (fig.), 8 ; prosenchymatous, 27 ; sexual, I84, 214 ; structure of, I-8 (figs.); turgescence of, 9 .

Cellulose, 4, 24, 31, 9I, IOI, IO3; decomposition of, 265 ; reaction of, 4, 3I, 32 ; reserve $48,53$.

Central cylinder of, aquatics, I7074 (fig.) ; root, 66 (fig.), 68; stem, 78 .

Centric leaves, I I6, 165 (fig.), 166, 172; of Pinus, 343,344 (fig.).

Centric starch-grains, 42 (fig.).

Cephaeline, 6r.

Cephalotaxus, 353.

Cephalozia, 272, 407; C. bicuspidata, 27 I (fig.).

Ceramium, 204, 205 (fig.).

Cerastium, vascular system of, II 3 , I 4 (fig.).

Ceratophyllum demersum, anatomy of, I 70, I 7 I (fig.).

Cereals, 248 ; starch of, 43.

Cetraria islandica, 258, $26 \mathrm{I}$ (fig.).

Chalaza, 357 (fig.), 358, 359, 365, 367 .

Chalazogamy, 364 (fig.), 365 .

Chance variation, 376 .

Chara, 39, I98.

Charales, 406.

Cheese, production of, 265 .

Chelidonium majus, laticiferous vessels of, I 53, I54; ovule of, 358 (fig.).

Chemical changes in cell-wall, 29, $32,38,91,136$.

Chemistry of plants, $43-64$.

Chemotaxis, 222, 224, 232, 28I, 302. 
Chemotropism, 232, 365 .

Chenopodiaceæ, secondary thickening in, 133.

Cherry (Prunus cerasus), I4I.

Chestnut (Castanea sativa), I 30.

Chickweed, Mouse-ear (Cerastium), vascular system of, II3, II 4 (fig.).

Chile saltpetre, origin of, 266.

Chinese Primrose (Primula sinensis) glandular hairs of, 105 (fig.).

Chitin, 231.

Chlamydomonas, asexual reproduction of, I 82-4 (figs.), I 88 ; movements of, 182,183 ; Palmella-stage of, I 82 (fig.), I 84 ; sexual reproduction of, I84-6 (fig.), 220 ; structure of, I8I, I 82 (fig.), 207.

Chlamydomonas angulosa, I 82 (fig.) ; C. media, 182 (fig.) ; C. monadina, 182 (fig.), I 85 (fig.), I 86 , 220 ; $C$. pertyi, 185 (fig.) ; $C$. reinhardii, $\mathbf{1} 82$ (fig.), I 85 (fig.).

Chlamydospores, 238,248 .

Chlor-zinc-iodide, and callose, 82 ; and cellulose, 32 ; and cork, I36 ; and lignified walls, 32 .

Chlorella, 260.

Chloroform, and enzyme-action, 56 ; and protoplasmic movement, 4 .

Chlorophyceæ, I98, 406 ; chloroplasts of, 207-ro (fig.); colonial, I 88-92 (fig.); filamentous, 195-8 (figs.); sexual reproduction of, I84, I 85 (fig.), 213 (fig.), $214,220-24$ (fig.), 226-8 (fig.); unicellular, I81-8 (figs.), I92 (fig.), 208, 209 (fig.); zoospores of, 2 I2-I 4 (fig.), 2 I 6-I 8 (figs.); vegetative reproduction of, $212,216,217$ (fig.).

Chlorophyll, 4, 6, 109, 194, I98 ; and cork, I36; and light, I69; and starch-formation, 40, 4I ; formation of, $4 \mathrm{I}$; in roots, 73 ; in stems, 88, 162, 167, 178; in variegated leaves, I I I ; screening of, 102, I I0, I99.

Chloroplasts, 5; division of, 25 ; movement of, II2; of Algæ, 7 (fig.), 8, 198, 207-10 (fig.); of Bryophyta, 272, 278 (fig.); of Chlamydomonas, $181, \quad 182$ (fig.) ; of Cladophora, 196 (fig.), 208; of Desmids, 209 (fig.); of Diatoms, 208 ; of Ectocarpus,
219 (fig.) ; of epidermis, 9I, 169; of Hamatococcus, 187 (fig.) ; of higher plants, 5, 6 (fig.), 8, 88, 92 (fig.), 96, 107, I08 (fig.), 109 (fig.); of Hormidium, 2 I 5 (fig.); of $Q \mathrm{E} d o$ gonium, 208 (fig.), 217; of Phæophyceæ, 208; of Pleurococcus, 190, I92 (fig.); of Rhodophyceæ, 208 ; of Spirogyra, 7 (fig.); of Ulva, 2 I 5 (fig.) ; of Zygnema, 208, 227 (fig.) ; starch-formation in, 6 (fig.), 40, I8I, 207.

Chloroxylon swietenia, I 30 .

Chondrus crispus, 204, 205 (fig.).

Chromatin network, 22, 24, 306, 389 .

Chromoplasts, 64 .

Chromosomes, 2I-5 (figs.), 306, 307 (fig.), 372 ; segregation of, $306,390$.

Chroococcus, 205, 206 (fig.).

Cilia, I 82 (fig.); of motile Algæ, I 82 (fig.), I83, I 87 (fig.), I 89 (fig.) ; of spermatozoids, $22 \mathrm{I}$ (fig.), 224, 277, 278 (fig.), 302 , 303 (fig.), 320 (fig.), 326, 327 (fig.); of zoospores, 2 I3 (fig.), 2 I6, 2 I 7 (fig.), 2 I 8 (fig.), 2 I9 (fig.), 232, 233 (fig.).

Cinchona, $6 \mathrm{I}$.

Cinnamomum camphora, 63; C. zeylanicum, 62.

Cinnamon (Cinnamomum zeylanicum), 62.

Circaa lutetiana, 58, 59 (fig.), 146, I68.

Circulation of carbon dioxide, 265 , 266 ; of nitrogen, 263,266 , 267.

Citric acid, 60 .

Citrus, 37 I (fig.), 372 ; C. aurantium, var. bergamia, 62 .

Cladode, structure of, I59 (fig.).

Cladonia, 258 (fig.), 259, 260 (fig.).

Cladophora, chloroplast of, 196 (fig.), 208; habitat of, I95, 197; sexual reproduction of, 2I 5, 220; structure of, 195-7 (fig.) ; vegetative reproduction of, 2 I6; zoospores of, 196 (fig.), 215 .

Cladophora glomerata, 196 (fig.).

Classification of Vegetable Kingdom, 180, 18I, 230, 231, 238, $269,289,334,355,408-8$.

Clavaria, 250 ; C. cinerea, 25 I (fig.). 
Claviceps purpurea, 49, 239, 255 , 407 ; life-history of, 24 I (fig.), 242 .

Cleistogamic flowers, 364 .

Climate and plant-distribution, 39I, 394 .

Climbing hairs, 104.

Close-grained wood, 127.

Closing of stomata, 98-Ioo (fig.).

Closterium, 209 (fig.).

Clostridium pasteurianum, 267 (fig.).

Clove (Eugenia caryophyllata), 62 ; secretory cavities of, I 5 I, I52 (fig.).

Clubmoss (Lycopodium), I8o, 289, 310, 314-16 (fig.), 318, 32 .

Cluster crystals, 58, 59 (fig.).

Cluster-cups (æcidia), 246 (fig.), 247.

Coal Measures, plants of, 310, 3 I 2 (fig.), 327-33 (figs.).

Coarse adjustment (of microscope), $398,399$.

Coarse-grained wood, 127.

Cocaine, 6r.

Coccus-forms (of Bacteria), 262, 263 (fig.).

Cocoa (Theobroma cacao), 49, 53, 61 ; - butter, 49.

Coconut oil, 49; - Palm (Cocos nucifera), 49.

Coffee (Coffea spp.), 47, 61 .

Cola acuminata, 61.

Colchicum, 364.

Collateral bundles, 76,78 (fig.), 79, 85 (fig.), I I 2.

Collecting cells, I Io, I I I (fig.), I6I (fig.).

Collema, 258, 260.

Collenchyma, 3 I (fig.) ; of leaf, 108 (fig.), I12; of stem, 3I (fig.), 34 (fig.), 77 (fig), 79, 8I (fig.), 83 (fig.), 87, 88; mechanical value of, 33 .

Colloids, 43, 55, 56 .

Colonial Algæ, I 88 (fig.), I 89 (fig.), I92, 205, 206 (fig.).

Colonisation, 206, 257, 259.

Colouration of water by Algæ, I8I, I 88, I 92 .

Colours of flowers, 63 .

Coltsfoot (Tussilago farfara), 102.

Columella (of Moss-capsule), 284, 285 (fig.), 286.

Comfrey (Symphytum), I04.

Commidendron, 393.

Common bundles, i 4 (fig.), I 5 .

Companion cells, 28, 29 (fig.), 66 (fig.), 68, 78 (fig.), 79, 80 (fig.), $82,8_{3}, 8_{4}, 85$ (fig.), I 2 I (fig.), I 30 .

Competition among plants, 378 , 394.

Complementary tissue (of lenticels), I39.

Compositæ, 44, I 53, I 54, 355, 356, $372,393$.

Compound microscope, 398-400 (fig.) ; sieve-plates, I 30 ; starchgrains, 42 (fig.), 43.

Concentric bundles, of Angiosperms, 85 (fig.), 86, I 33 (fig.), I34 ; of Ferns, 292 (fig.).

Conceptacles (of Fucus), 201 (fig.), $202,224,225$ (fig.).

Condenser (of microscope), 398, $399,400$.

Conducting elements and tissues, $27,28,29$ (fig.), 35 (fig.); of Conifers, 339-4I (fig.); of Ferns, $292-5$ (figs.); of leaves, I08 (fig.), II 2-I 4 (fig.); of Mosses, 275 (fig.), 276; of roots, 66 (fig.), 68, 70 (fig.), I32; of stems, 78-86 (figs.), I $19-26$ (figs.).

Conduction of elaborated foodmaterial in, Angiosperms, 28, 65, 88, I IO, I I I, 112 ; aquatics, I 71 ; Conifers, 343; Mosses, 276; by laticiferous elements, I 56 ; by the xylem, I24.

Conduction of water and mineral salts in, Angiosperms, 27, 35, 37, $65,88,112,121,125$; Conifers, 343,344 ; Mosses, 276.

Cones of, Abies, 336 (fig:), 344, 347; Araucaria, 345, 346, 347 ; Cedrus, 353; Conifers, 344-7 (figs.), 352 (fig.) ; Cupressineæ, 337 (fig.), 345, 347 ; Cycads, $323, \quad 324$ (fig.) ; Equisetum, 3II (fig.), 3I2, 3I3 (fig.) ; Juniperus, $35^{2}$ (fig.), 353; Lavix, 336 (fig.), 345, 346, 347; Lycopodium, 3I4, 3I5, 316 (fig.); Pinus, 345 (fig.), 346, 347 (fig.), 352 ; Selaginella, $3 \mathrm{I} 7$ (fig.), 318 (fig.); Sequoia, 347, 352 (fig.); Taxus, $344,345,346$ (fig.) ; Thuja, 337 (fig.), 347.

Coniferous timber, 127, 129.

Conifers (Coniferales), 180, 333, 334-53 (figs.), 408; archegonia of, 348,349 (fig.) ; 
embryology of, $350-52$ (fig.) ; female cones of, 336 (fig.), 337 (fig.), 345-7 (figs.), 352 (fig.) ; fertilisation of, $349,350,353$; habit of, $334-6$ (figs.); leaf of, I6I, 335-7, 34I-4 (figs.) ; male cones of, 336 (fig.), 344-6 (figs.), 347 (fig.); ovules of, $333,348,349$ (fig.) ; pollen sacs (microsporangia) of, 345,347 (fig.) ; pollination of, 348 ; root of, $70,34 \mathrm{I}$; seedlings $\cdot$ of, 337 , 338 (fig.); seeds of, $35 \mathrm{I}, 3.52$ (fig.) ; stem of, II $7,33.5,338$ $4 \mathrm{I}$ (figs.); wood of, 37 (fig.), I27, 340, 34 I (fig.).

Coniine, 6r.

Conium maculatum, 6r.

Conjugatæ, 208 ; reproduction of, 216, 226-8 (fig.) ; structure of, 208-ro (fig.).

Conjugation, 226, 227 (fig.), 237 (fig.).

Connective, 359 (fig.), 360 (fig.), 362 .

Contractile vacuoles, 181 , I 82 (fig.), I 87 (fig.), I93, 2 I3.

Conversion of sewage, 265.

Convolvulus sepium, I53.

Copper Beech, 382 .

Copra, 49.

Coprinus, 245.

Coral-Fungus (Clavaria), 250, $25 \mathrm{I}$ (fig.).

Coral reefs and Algæ, 204.

Coral-spot disease (Nectria), 253.

Corallina, 204, 205 (fig.).

Corchorus, 34.

Cordaitales, 407.

Cordyceps, 239, 255.

Cork, I35-42 (figs.) ; and leaf-fall, I4I, I 42 (fig.); and wounds, I42; commercial sources of, I 40 ; formation of, I3I (fig.), 135-8 (figs.) ; functions of, I37, I 38 ; properties of, I37, 138.

Cork-cambium (phellogen), I 35-42 (figs.) ; and aerenchyma, I75 ; of Conifers, 338, 339 (fig.).

Cork Elm, I4I ; - Maple, I4I ; Oak (Quercus suber), I40.

Cork-wings, I 4 I.

Corms, 355 .

Corn Cockle (Agrostemma githago), I I0, 370.

Cornus sanguinea, galls of, 143 (fig.).
Corolla, 354, 373 .

Cortex, 65, 76; of aquatics, I 71 (fig.), I73 (fig.), I 75; of petiole, I I 4; of pulvinus, I I5: of root, 65 et seq., 295; of stem, 19, 30, 43, 76 et seq., I34, 338 .

Cortical bundles, 86 .

Cosmarium, 209 (fig.), 2 10; $C$. meneghini, 209 (fig.).

Cotton, IO3 ; - plant (Gossypium spp.), 102, 103.

Cotton-grass (Eriophorum), I 78 .

Cotton-seed oil, 49.

Cotyledons, digestive action of, I5O ; food-reserves of, 4O, 4I, 44, 50 ; of Angiosperms, 355 . 369 (fig.), 370, 37 I (fig.); of Conifers, 338 (fig.), 350, 35I (fig.), 352 (fig.) ; of Cycads, 326 ; structural features of, 30 , I 53 .

Covering hairs, IO2 (fig.).

Cow-wheat (Melampyrum), I79.

Crassulaceæ, I 78 .

Creeping Buttercup (Ranunculus repens), root-structure of, 66-9 (figs.).

Crenothrix, 268.

Cress (Lepidium), 48 ; “ damping off " disease of, 235 (fig.).

Crocus, 364 ; C. sativus, 64 .

Crops, fungal diseases of, 230, 231 , 234, 235 (fig.), 24 I (fig.), 243 (fig.), 245, 246 (fig.), 247, 248, 252-4.

Crossing (hybridisation), 382-9.

Crowberry (Empetrum nigrum), leaf of, I63 (fig.).

Cruciferæ, 48, 63, 69, I03, I04, 23 I.

Cryptogamia, 355, 406.

Cryptomeria, 347.

Crystal-sand, 58.

Crystalloid, 50, 5I (fig.).

Crystals of, inulin, 44,45 (fig.) ; osazones, 47 (fig.); oxalate of lime, 58, 59 (fig.), I 30,237 ; pigments, 64 .

Cuckoo-pint (Arum maculatum), I I 2.

Cucumber-family (Cucurbitaceæ), 8I ; - mildew (Erysiphe polygoni), 243.

Cucurbita, hairs of, 102 (fig.); phloem of, 29 (fig.), 82, I30; stem-structure of, $80-83$ (figs.), 87 ; tyloses of, 127 (fig.); vessels of, 35 (fig.), 83 .

Cucurbita pepo, pollen of, 362 (fig.). 
Cultures (of Bacteria), 264.

Cup-Fungus (Peziza), 239 (fig.), 240 (fig.).

Cuprammonia. See Ammoniated copper oxide.

Cupressineæ, 336, 347, 348, 350, 351.

Cupressus, cones of, 345, 347; habit of, 334, 336, 337; seedling of, 337,338 (fig.).

Cupule (of Lyginopteris), 331, 332 (fig.), 333 (fig.).

Curcuma longa, 64 .

Curing of rubber, 157 ; of tobacco, 265.

Currant (Ribes), I05, I I5 ; cork of, 137, 138 (fig.).

Curves of variation, 375-7 (fig.).

Cuscuta, I 78 (fig.), I 79 .

Cushion (of Fern-prothallus), 30I, 302.

Cut-leaved Beech, 382, 383 (fig.).

Cuticle, 9I-3 (fig.), 95; function of, 92,93 ; of aquatics, I72 ; of Coniferous leaves, 342, 343, 344 (fig.) ; of plants of dry habitats, I58, I 59, I60 ; of shade-leaves, I69.

Cuticular transpiration, 93, I6I.

Cuticularisation, 91, 92, 300, 36r.

Cuticularised layers (of epidermis), 9 I.

Cuttings, callus-formation in, 142 .

Cyanophyceæ, 192, $210,257,268$, 406; reproduction of, 206 (fig.), $216,220,391$; structure of, 204-6 (fig.).

Cycadofilices, 407.

Cycads (Cycadales), 322-7 (figs.), 393, 407; anatomy of, 323; cones of, 323,324 (fig.) ; embryology of, 326,327 (fig.) ; fertilisation of, 326,327 (fig.), 353; habit of, 322, 323 (fig.); ovules of, 324, 325 (fig.) ; pollination of, 326 ; seeds of, 327 ; spermatozoids of, 326 , 327 (fig.).

Cycas, 324 (fig.), 327 (fig.), 356 ; C. circinalis, 323 (fig.); $C$. revoluta, 322,325 (fig.).

Cylindrocystis, 209 (fig.).

Cymbella, 207 (fig.).

Cynoglossum, hairs of, 102 (fig.).

Cyperaceæ, stoma of, roo.

Cypress (Cupressus), cones of, 345 . 347 ; habit of, $334,336,337$; seedling of, 337,338 (fig.).
Cystococcus, 259.

Cystoliths, 38 (fig.), 39.

Cystopus, 23I-4 (fig.); C. candidus, 233 (fig.).

Cytase, 53.

Cytisus adami, 389; C. scoparius, I67.

Cytoplasm, I-7; composition of, 2 , 50; growth of, 25 ; movement of, 4, 6, IO4; permeability of, $9,12,13,15$; properties of, 3 ; structure of, 3,4 . Cytoplasmic connections, 24, 30, r9o.

Dedalea quercina, 250.

Dahlia, inulin of, 44,45 (fig.).

Damp habitats, structural features of plants of, 96, 103, 160, 166, I77, 276, 296.

"Damping off" Fungus (Pythium), 23I, 252, 253; structure and life-history of, 234, 235 (fig.), 236.

Dandelion (Taraxacum), I 54, I70, 372; flowers of, Plate I (Frontispiece).

Darwin's "Origin of Species," 378.

Date (Phonix dactylifera), endosperm of, 30 (fig.), 47.

Datura stramonium, 61, 62 (fig.), 384 .

Daughter-cells, I7, 24, I83; colonies, I 88 (fig.), I 89 (fig.), r9o ; - nuclei, 2I, 23, 24, 25 (fig.), 390.

Deal, Red (Pinus sylvestris), I29; White (Picea excelsa), I 29.

Dead-nettle (Lamium), 4, I05, 364; collenchyma of, 31 (fig.), 34 (fig.) ; leaf of, 168, I69 (fig.).

Deadly Nightshade (Atropa belladonna), 6r.

Death Cap (Amanita phalloides), 255.

Decay due to Bacteria, 252, 263, 265, 266; due to Fungi, 230, 252 ; prevention of, 126.

Dehiscence of, antheridia, 277, 280, 302, 303 (fig.) ; Fern-sporangia, 298 (fig.), 299, 300 ; Liverwortsporogonium, 27I (fig.), 282 ; microsporangia of Conifers and Cycads, 324, 345 ; Moss-sporogonium, 285 (fig.), 286, 287 ; stamens, 363 .

Dendrobium, aerial roots of, 75 (fig.). Denitrification, 266. 
Dentifrices, 207.

Depression of stomata, 96, 158, I6o (fig.), I65, I70.

Dermatogen, I9, 20, 74, 95, 369.

Desert, distribution of, 39I.

Desert-plants, 178 .

Desmidium, 209 (fig.).

Desmids (Desmidiaceæ), 192, 2 II ; reproduction of, 209 (fig.), $216,220,228$; structure of, 208-10 (fig.).

Deutzia, hairs of, I02 (fig.), I03.

Development of, anther, 360 (fig.), 36I ; ascospores, 240 (fig.), 245 ; cambium, I I 7, I I 8 (fig.) ; cork-cambium, I35, I36 (fig.), 137 ; Fern-leaf, 290 (fig.), 29I ; Fern-prothallus, 299 (fig.), 300; Fern-sporangium, 29I-9 (fig.) ; gametes, I 84 (fig.), 2 I 3 (fig.), 2 I 4 ; gonidia, 232, 243 (fig.) ; laticiferous cells, I53 ; laticiferous vessels, I54; lateral branches of root, $65,7 \mathrm{I}$ (fig.) ; lateral branches of stem, 90 ; leaves, I9 (fig.), 90 ; lenticels, I39; Moss-plant, 287 (fig.), 288; Mushroom (Agaricus), 248, 249 (fig.) ; ovules of Angiosperms, $35^{8}$ (fig.), 359 ; pollen grains, 360 (fig.), 36I ; procambial strands, 89 ; rootcap, I8, 20 (fig.), 65; secretory cavities, $15 \mathrm{I}$; sieve-tubes, 28 ; spermatozoids, 277 ; spores, 305-8 (fig.) ; sporogonium of Liverworts, 282 ; sporogonium of Mosses, 284, 285 (fig.); stamens, 360 (fig.), 36I ; starch - grains, 4I ; stomata, 98 ; tyloses, I 26; vascular tissues, 89,90 ; vessels, 28 ; zoospores, 2 I2, 2 I3 (fig.), 2I6, 2 I 7 (fig.), 218 (fig.), 2 I9 (fig.). See also under Embryology.

De Vries, 38r.

Dextrin, II, 46, 47, 53 .

Dextrose, 45 . See also Glucose.

Dialysis, 57.

Diaphragm, of aquatics, 175 ; of microscope, 399,400 ; of Mosscapsule, 285 (fig.), 286.

Diarch roots, 69, 70, I3I (fig.), 295 , $34 \mathrm{r}$.

Diastase, 46, 53, 56; extraction of, 53,54 .

Diatomaceous earth, 207.
Diatoms (Diato maceæ), 39, 192 I93 (fig.), 206, 207 (fig.), 208 2 I I, 406 ; reproduction of, 2 I 6 $219,228$.

Dicotyledonous timbers, I29, I30.

Dicotyledons, $355,367,408$; embryology of, 368-7I (fig.) ; epidermis of, 93 ; leaf of, 93 , 98, 107-I4 (figs.) ; root of, 65-7I (figs.), I 31 (fig.), I32; stem of, 76-84 (figs.), 86, I I 7-30 (figs.), 135-4I (figs.).

Dicranella heteromella, protonema of, 287 (fig.).

Dictyota, I 7 (fig.).

Differentiation of $\operatorname{sex}, 186,2 \mathrm{I}$, $220,227,228$.

Diffusion in plant-cells, 6, 12, 13, 52.

Digestive glands, I44, I49, I50 (fig.).

Digitalin, 48 .

Digitalis, 48,363 .

Diœcious, 223, 225, 354 .

Dioon edule, 327 (fig.).

Diospyros, I 26.

Direct nuclear division, 21.

Disaccharides, $44,45$.

Disc of Umbelliferæ, $\mathbf{I}_{4} 8$.

Discomycetes, 407.

Diseases of plants, and immunity, 253; control of, 247, 253, 254; due to animals, r43; due to Bacteria, 265; due to Fungi, 230, 23I, 235, 236, 242, 243, $245,248,25$ I, 252, 253.

Dispersal, 219,392 (fig.), 393; barriers to, 393 ; of gonidia, 232, 242 ; of microspores, $326,330,345$; of oospores, 223; of seeds, 46 , $50,103,353,372$; of soredia of Lichens, 26I ; of spores, $238,242,245,249,253,282$, $287,300,313,321$; of zygospores, 186.

Dissipation of energy, 265.

Distribution of plants, 39I-3.

Division of, cells, I6, $2 \mathrm{I}-5$ (figs.) ; chloroplasts, 25 ; nuclei, 20-2 5 (figs.) ; spore mother-cells, 306-8 (fig.).

Division of labour, 395; in $\mathrm{A}^{\prime} \mathrm{g} æ$, 190, 197, 198, 199, 210, 223 ; in Ferns, 308 ; in he:erosporous plants, $32 \mathrm{I}$.

Dock (Rumex), I I 3 .

Dock-family (Polygonaceæ), 356.

Dodder (Cuscuta), I 78 (fig.), I79. 
Dog's Mercury (Mercurialis perennis), leaf of, 37 (fig.), 59 (fig.), I68, I69; petiole of, I I3 (fig.).

Dogwood (Cornus sanguinea), galls of, I 43 (fig.).

Dominant characters, 384 et seq.

Dormant characters (of hybrids), 382.

Dorsiventral leaves, structure of, 98, I07-I I (figs.), I I6, 295, 34 I, 342 (fig.).

Double chromosomes, 306.

Double fertilisation, 368 .

Douglas Fir (Pseudotsuga douglasii), I28, I29; cone of, 352 (fig.), 353.

Dragon Tree (Dracana), secondary thickening of, I33 (fig.), I34.

Draparnaldia, I97 (fig.).

Drosera, tentacles of, I 50.

Drought, resistance to, I86, I9I, 205, 215, 269, 271 .

Dry habitats, structural features of plants of, $38,88,92,94,96$, I I I, I 58-66.

"Dry rot" of timber (Merulius lacrymans), 252.

Duckweed (Lemna), II2, 260.

Dunes, I63.

Dwarf-males (of $E$ dogonium), 22 I (fig.), 223.

Dwarf-shoots (of Conifers), 335, 336 (fig.), 337, 338.

Dyes, vegetable, 64, I 26, 26I.

Dynamite and Diatoms, 207.

Eau de Cologne, 62.

Eau de Javelle, 40I ; and lignified walls, 32 .

Ebony (Diospyros), I26.

Echeveria retusa, 365 .

Economic plants, 34, 35, 39, 43, 45, $48,49,53,62$, I03, I 24, 1 26, I 40,155, I $57,203,204,207$, $255,256,257,26 \mathrm{I}, 276,323$, $334,337,352$.

Economic utilisation of, Algæ, 203, 204; alkaloids, 6I ; Bacteria, 265 ; Bog-moss (Sphagnum), 276 ; Conifers, 127, I29, 334, 337,352 ; cork, I36, I 40 ; Diatoms, 207; ethereal oils, 62; fats, 49; fibres, 34 , 35; Fungi, 255, 256, 257; glucosides, 48; gums, 39 ; hairs, I03; latex, I 55, I57 ; Lichens, 26I ; starches, 43; sugars, 45, I24; timber, 126,
127-30; vegetable pigments, $64,126$.

Economy of water, I 58-66.

Ectocarpus, I99; habitat of, I95 ; sexual reproduction of, 2I 2, 2 I 9 (fig.), 220; structure of, 198, 219 (fig.) ; zoospores of, 2 I8, 219 (fig.).

Ectocarpus littoralis, 2 I9 (fig.).

Ectotrophic mycorrhiza, 72.

Edible plants, 43, 44, 45, 48, 49, $52,53,60,203,204,255,26 \mathrm{I}$, 352 .

Egg (ovum), 220; of Algæ, 220, 221 (fig.), 222, 224, 226; of Angiosperms, 357 (fig.), 366 (fig.), 367 (fig.), 368 ; of Conifers, 348,349 (fig.) ; of Cycads, 326, 327 (fig.) ; of Ferns, 302, 303 (fig.) ; of Fungi, 233 (fig.), 234,236 ; of Liverworts, 280 , 28I (fig.); of Mosses, 280; of Selaginella, 3I9, 320 (fig.).

Egg-apparatus (of Angiosperms), 366 (fig.).

Elaborated food-material, conduction of, in Angiosperms, 28, 65, 88, IIO, III, 112; aquatics, I7I ; Conifers, 343 ; Mosses, 276 ; by laticiferous elements, I 56 ; by the xylem, I24.

Elaagnus, I03.

Elasticity, of collenchyma, 3I, 33 ; of cork, I37; of fibres, 33 ; of wood, 127, 128.

Elaters (of Liverworts), 27 I (fig.), 282,283 (fig.).

Elder (Sambucus), cork of, 136 (fig.) ; leaf of, IIO, III (fig.) ; lenticels of, I40 (fig.) ; pith of, 30 (fig.), I $5 \mathrm{I}$.

Elm (Ulmus), I30, I 39, I4I, 365 ; Cork -, I4I ; secondary wood of, $120,126$.

Elodea canadensis, cell-structure, 5, 6 (fig.) ; spread of, in British Isles, 392 (fig.).

Elongation, zone of, in root, 65 .

Embryo of, Angiosperms, 367, 36872 (figs.) ; Conifers, 350-52 (fig.), 370 ; Cycads, 326, 327 (fig.), 332, 333 ; Dicotyledons, 368-7I (figs.); Ferns, 294, 302-4 (fig.); Fucus, $22 \mathrm{I}$ (fig.), 226 ; Liverworts, 282 ; Monocotyledons, 370, 37 I (fig.); Mosses, 284, 285 (fig.) ; parasitic Angiosperms, I79, 372 ; 
Selaginella, 319, 320 (fig.), 321.

Embryo sac, 357 (fig.), 364 (fig.), 367 (fig.), 372, 373 ; development of, 365,366 (fig.).

Emetics, 6I.

Empetrum nigrum, leaf of, I63 (fig.).

Empusa musca, 231, 238.

Emulsin, 48, 53.

Encephalartos, 324 (fig.) ; E. hildenbrandtii, 325 (fig.).

Enchanter's Nightshade (Circaa lutetiana), 58, 59 (fig.), I46, I 68 .

Endemics, 393.

Endodermis, 68, 69, I50 ; function of, 69; of aquatics, 69, I 7I (fig.), I73 (fig.), I74 (fig.) ; of Ferns, 292 (fig.) ; of roots, 66 (fig.), 68, 69, 70 (fig.), 7I, 75 (fig.); of Selaginella, 318.

Endogenous, 71, 72.

Endosperm, 327, 351, 384 ; foodreserves of, 30 (fig.), 40, 44,50 , 5 I (fig.), 52, I50 ; hybrid, 386 ; of Angiosperms, $367,368,370$, 371, 386 ; of Conifers, 351, 352 (fig.) ; of Cycads, 327.

Endotrophic mycorrhiza, 72, 73 (fig.).

Energy, liberation of, in fermentation, $257,265,266,268$.

Environment, 374, 395 ; adaptation to, 158-79, 203, 210,236 , 238,321 ; influence of, 168 , I69, I $75-7,374,376,377$, $378,379,380$.

Enzymes, 53-7, 63; digestive, 52, I 49, I50, 365; extraction of, 53, 54; fermenting, 55, 256, 257 ; mode of action of, 55, 56,256 ; of Fungi, 55, 230; oxidising, 55, I55 ; proteolytic, $52,53,54,149$.

Eosin, 43, 405; and sieve-plates, 82.

Epacridaceæ, geographical distribution of, 393 .

Epidermis, 65, 9I-IO6 (figs.), I 58 63 (figs.); functions of, 65 , 77,95 ; glandular, I48, I 50 ; modification of, 94,95 ; of anthers, 360 (fig.), 363; of aquatics, 172, I $_{7} 6$ (fig.), 177 ; of leaf, I, 2 (fig.), 4, 90, 91-101 (figs.), I0 7, I08 (fig.), I I I (fig.), I I , I 58-63 (figs.), I69, 295 , 298 (fig.), 34I, 342 (fig.), 343,
344 (fig.) ; of Moss-capsule, 285 (fig.), 286 ; of root, 66,74 ; of stem, I9 (fig.), 76, 77, 79, 8I (fig.), 83 (fig.), 93, 94 (fig.), 98, I 36 (fig.), I37, I 59 (fig.).

Epigynous flowers, 355.

Epilobium, 389; pollen of, 362 (fig.) ; $E$. hirsutum, 174 .

Epiphytes, 73, 272 (fig.), 309 ; among Algæ, 206.

Epithelium, I5I, I52 (fig.), 339 (fig.).

Epithem, I46, I47 (fig.), I48.

Equimolecular solutions, I I.

Equisetales, 289, 3 IO-I3 (figs.), 407.

Equisetum, 39, 180, 289, 310-13 (figs.), 32I ; growing point of, I 8 (fig.).

Equisetum arvense, 3II, 312; E. limosum, 3I I (fig.); E. maximum, I8 (fig.), 313 (fig.); $E$. palustre, 3 II (fig.).

Equivalent osmotic pressures of sea-water, 403.

Erect ovules, $35^{8}$ (fig.).

Erepsin, 52.

Ergot (Claviceps purpurea), 49, 239, 255 ; life-history of, $24 \mathrm{I}$ (fig.), 242.

Ericaceæ, 178, 361, 363.

Eriodendron anfractuosum, 103.

Eriophorum, I78.

Eryngium maritimum, 93; petiole of, II3 (fig.).

Erysiphaceæ, 230, 239, 252, 253 , 407 ; structure and life-history of, 242-4 (fig.).

Erysiphe graminis, 243; E. polygoni, 243.

Erythronium americanum, 37 I (fig.). Erythroxylon coca, 6I.

Esters, 49.

Ethereal oils, 62, 63, 64, I05, I06, I 26, I $5 \mathrm{I}$.

Ethyl alcohol, 256.

Euastrum, 209 (fig.).

Eubasidii, 407.

Eucalyptus, 62 ; E. globulus, I29; E. marginata, I 30 .

Eudorina elegans, I88 (fig.), 192.

Eugenia caryophyllata, 62; secretory cavities of, I.5I, I 52 (fig.)

Euonymus, I39, 359.

Euphorbia, laticiferous cells of, I 53 . I 54 (fig.), r 55 ; leaf of, I Io.

Euphorbia amygdaloides, I IO, I53.

Euphorbiaceæ, I53, I57.

Eurotium, 242, 243 (fig.). 
Evening Primrose (Enothera), mutation in, 381,382 .

Evergreen leaves, II2, 335 .

Evolution, $210,337,338,355,367$, 393-6 ; of flowers, 35.5 ; of new species, etc., 378,380 , 38 I (fig.).

Exalbuminous seeds, 370.

Excentric starch-grains, 4I, 42 (fig.).

Exodermis, 67 (fig.), 69, 72, 74, 75 (fig.).

Exogenous, 90.

Extinct plants, 310, 3 I 2 (fig.), 313, 314 (fig.), 319, 322, 327-33 (figs.), 334, 340, 356, 394, 407.

Extraction of, enzymes, 53, 54; fibres, 34; oils, 49; rubber, I 57 ; sugars, 45,46 .

Extrafloral nectaries, I44, 148, I 49 (fig.).

Exudation of water, I44-8, I49.

Eyepiece (of microscope), 398.

Eye-spot, 181, I93 ; of Chlamydomonas, $18 \mathrm{I}$, I 82 (fig.) ; of Hamatococcus (Sphaerella), 187 (fig.) ; of zoospores, $2 \mathrm{r}_{3}$ (fig.), 2 I9 (fig.).

Fagus sylvatica, 72, 129, I40, 170, $365,367,382,383$ (fig.) ; wood of, I $21,122,126$.

Fall of leaves, etc., I4I, I42 (fig.).

False Acacia (Robinia pseudacacia), I I 5, I 26.

False plasmolysis, $\mathrm{r}_{3}-\mathrm{r} 5$; - tissues, 239, 24I, 248, 249 (fig.).

Fats, 49, 50, 53, 263 ; formation of, 50 ; reactions of, 49.

Fatty acids, 49, 53, 54; - bodies, 9I, 92,$136 ; 223$.

Favus, cause of, 253.

Febrifuges, 6I.

Fegatella, 270 (fig.), 279.

Fehling's solution, 40I ; and sugars, 47,401 ; and tannins, 60.

Female sexual cells, I $86,220,22$ I (fig.), 233 (fig.), 234, 244 (fig.). See also Egg.

Fennel (Føniculum), 6I.

Fennel-leaved Pondweed (Potamogeton pectinatus), stem of, 172 .

Fermentation, alcoholic, 255, 256 ; due to Bacteria, 265.

Ferments. See Enzymes.

Ferns (Filicales), I80, I93, 289-309 (figs.), 310, 321, 322, 407 ; antheridia of, 301 (fig.), 302, 303 (fig.) ; archegonia of, 30I (fig.), 302, 303 (fig.) ; embryo of, 294, 302-4 (fig.), 373 ; fertilisation of, 302 ; growing point of, 18, 295; habit of, 289-9I; leaf of, 290-92 (figs.), 295, 303; petiole of, 295 ; prothallus of, $300-302$ (fig.), 304, 305, 313; root of, 290, 295 ; sporangia of, 295300 (figs.), 303, 308, 309 (fig.) ; stem of, 289,290 (fig.), 292-5 (figs.) ; vegetative reproduction of, 305 (fig.).

Ferrugineous waters, 268.

Fertilisation, 222, 223, 289, 306, 332,382 ; in Algæ, 221 (fig.), 222, 223, 226 ; in Angiosperms, 364 (fig.), 366, 367 (fig.), 368, 372 ; in Conifers, 349,350 , 353 ; in Cycads, 326, 327 (fig.) ; in Ferns, 302 ; in Fungi, 233 (fig.), 234; in Liverworts, 280, 28I; in Mosses, 28I; in Selaginella, 319.

Fibre-yielding plants, 34, 35 .

Fibres, 32-4 (fig.), 73, 78 (fig.), 86, I I 167, I 68 (fig.) ; borderedpitted, 33, 37, I2 I ; mechanical value of, 33; of Ferns, 292, 293 (fig.); of secondary phloem, I3o; of secondary wood, 33, I2I, 123 (fig.).

Fibres of nuclear spindle, 23.

Fibrous layer (of anthers), 360 (fig.), $361,362,363$ (fig.).

Fibrous tracheids, 37 (fig.), I34, 340, 34 I (fig.).

Ficaria verna, embryo of, 372 ; hydathodes of, I45, I 46 (fig.), I 47 (fig.).

Ficus, 125; F. elastica, 39, 95, I I0, I 57.

Field Horsetail (Equisetum arvense), 3II, 3 I2.

Fig (Ficus), 39.

" Figure" (of wood), I27, I 28.

Filaments of, Algæ, 7, I95-8 (fig.), 205, 206 (fig.); Bacteria, 262, 263 (fig.), 268; Fungi, 230, 231 ; stamens, 359 (fig.), 360 (fig.).

Filicales, 310, 407. See Ferns.

Filmy Ferns (Hymenophyllaceæ), 296, 297 (fig.), 301.

Fine adjustment (of microscope), 399. 
"Finger and Toe" disease (Plasmodiophora brassica), I43, 251, 254 (fig.).

Fir Clubmoss (Lycopodium selago), 314.

Fir, Douglas (Pseudotsuga douglasii), I28, I 29, 352 (fig.), 353; Scotch - (Pinus sylvestris), 37, I 4I, I 52, I66, 334 et seq. (figs.); Silver - (Abies), 335, 336 (fig.), 344, 347; Spruce - (Picea excelsa), 63, 334, 335 (fig.).

Fixation of nitrogen, 266, 267.

Flagellata, 406.

Flax (Linum usitatissimum), 34, 265; New Zealand - (Phormium tenax), 34 .

Fleshy roots, structure of, 132, 133, I 54, I55 (fig.).

Flexibility (of petiole), I I5.

Floating Algæ (Plankton), I92, 193 (fig.), 207, 210.

Floating leaves, structure of, IOI, I74, I 77 .

Floating Pondweed (Potamogeton natans), stem-structure of, I 72 , I74 (fig.).

Floral axis, 354, 355, 370 ; - nectaries, I44, 148, 149, 363.

Flowering Plants. See Angiosperms.

Flowering Rush (Butomus), 356.

Flowers, 354-6, 373; cleistogamic, 364 ; colour of, 63 ; detachment of, I42 ; nectaries of, I $44,148,149,363$; odour of, $62 ;$ zygomorphic, 355 , 373.

Flowers of $\tan$ (Fuligo), $25 \mathrm{I}$.

Flowing water, plants of, I95, 197.

Fly-disease (Empusa musca), 23I, 238.

Fly Toadstool (Amanita muscaria), 255.

Focusing under the microscope, 400.

Foliose Liverworts (Jungermanniales), $27 \mathrm{I}$; antheridia of, 279 ; archegonia of, 280 ; protonema of, 288 ; sporogonia of, 27 I (fig.), 282 ; structure of, 27 I (fig.), 272; vegetative reproduction of, 277.

Food-content of plant-products, 53 .

Food-plants, 43, 44, 45, 48, 49, $52,53,60,203,204,255,26 \mathrm{I}$, 323,352 .

Food-reserves, 40-52, 242, 319; accumulation of, 13 ; in seeds,
$40,44,45,47,49,50,52,333$, $35 \mathrm{I}$; in storage-organs other than seeds, $44,45,50$; nitrogenous, 50 ; storage of, 40, 64 , 88 , I32; translocation of, 6 , $4 \mathrm{I}, 46,52$, I 24, I32, 282.

Food-storage, $\mathrm{r}_{3}$; in Fungi, 23I : in Liverworts, 273; in oospores, 223 ; in roots, 132 ; in seeds, $40,44,45,47,49,50,52$, $333,35 \mathrm{I}$; in spores, $319,32 \mathrm{I}$; in zygospores, 185 .

Foot, 282, 370 ; of Ferns, 302 ; of Liverworts, 282, 283 (fig.); of Mosses, 284, 285 (fig.); of Selaginella, 320 (fig.).

Forests, distribution of, 334, 39I.

Forget-me-not (Myosotis), 102 ; - mildew (Oidium), 243.

Forked branching of, Algæ, 200, $20 \mathrm{I}$ (fig.) ; Ferns, 290; Lycopodium, 3I5; Mosses, 269, 275 ; Selaginella, 3I5.

Forked venation, 292, 322.

Fossil plants, 310, 3 I 2 (fig.), 313, 3I 4 (fig.), 319, 322, 327-33 (figs.), 334, 340, 356, 394, 407. Foxglove (Digitalis), 48, 363 .

Fragmentation, in Algæ, 212, 2 I 5 (fig.), 216; in Bryophyta, 277.

Fraxinus excelsior, I20, I22, I28, I 29.

Free central placenta, 356 ; - nuclear division, 245, 319, 368.

Fronds of, Brown Algæ, I99-204 (figs.) ; Ferns, 289-9I (fig.), 297 (fig.), 304 (fig.), 305 (fig.), 308 (fig.), 309 (fig.).

Fructose (fruit-sugar), 45, 46, 53 , 55 ; reactions of, 47.

Fruit-bodies of, Ascomycetes, 239 (fig.), 240 (fig.), 242, 243 (fig.), 244 (fig.) ; Basidiomycetes, 248, 249 (fig.), 250 (fig.), 25 I (fig.) ; Lichens, 258 (fig.), 259 , 260.

Fruits, 31, 33, 46, 61, 63, 93, 103, 370 ; unripe, 60.

Frullania tamariscini, 272 (fig.).

Fuchsia, hydathodes of, 146 ; leafstructure of, IO7-ro (figs.).

Fucus, conceptacles of, 201 (fig.), 202, 224, 225 (fig.); habitat of, 200 (fig.), 203; sexual reproduction of, 220, $22 \mathrm{I}$ (fig.), 224-6 (fig.), 307; thallus of, 200202 (figs.), 225 (fig.); vegetative reproduction of, 216 . 
Fucus platycarpus, 224; $F$. serratus, 201 (fig.), 202, 224; $F$. vesiculosus, 38, 200-202 (figs.), 225 (fig.).

Funaria, antheridia of, 279 (fig.); development of, 287 (fig.); habit of, 274; leaf of, 276 ; sporogonium of, 285 (fig.).

Function of, aerenchyma, I 75 ; alkaloids, 64 ; antipodal cells, 366 ; bordered pits, 37 ; cambium, II7 ; chromoplasts, 64 ; chromosomes, 307, 390; cilia, I82 ; collenchyma, $3 \mathrm{I}$; contractile vacuoles, I8I; cork, I35, I37, I38; cuticle, 92 ; elaters of Liverworts, 282 ; endodermis, 69; epidermis, $65,77,95$; ethereal oils, 64 ; exodermis, 67 ; extrafloral nectaries, I49; eye-spot, I8I ; glucosides, 48 ; hairs, IOI, IO2, 104, I06, I65; hydathodes, I47; latex, I55, I56; lenticels, I39; leucoplasts, 4I ; medullary rays, I24; mucilage, 38 ; nectaries, I49, 363 ; nuclei, 3. 389; palisade tissue, 107; phloem, 88 ; resins, 64 ; root-hairs, 72 ; sclerenchyma, 33; spongy tissue, I07, II0; stomata, I00 ; synergidæ, 366; tannins, 64 ; transfusion tissue of Conifers, 343 ; veins of leaf, I 2 ; velamen, 74 ; vestibule of stomata, 96, 160; xylem, 88 ; relation of structure and, $72-4,87,88$, I09, III, II 2 , I I 5, I 44 et seq.

Fungi, 1 80, 230-57 (figs.) ; classification of, 23I, 406, 407; economic' importance of, 255 , 256, 257; physiology of, 55, 72 , I00, 252-7; protection against attacks of, I42, 254 ; reproduction of, 232-5I (figs.); structure of, 230, 23I; zOOspores of, 232, 233 (fig.), 235 (fig.), 236.

Fungicides, 254.

Funicle, 357 (fig.), $35^{8}$ (fig.), 359.

Furniture, timber used for, I $29,130$. Fusion of, cells, 28, I 42, I 54 ; floral members, 355,373 ; gametes, I 84,185 (fig.), 2 I 3 (fig.), 2I4, 219 (fig.), 237 (fig.), 389 (see also Fertilisation); integuments, 357.
Galactose, 53.

Galium aparine, hairs of, 104.

Galls, due to Fungi, 254 (fig.); due to insects, 60, I42, I 43 (fig.). Gametangium, 219 (fig.), 220.

Gametes of, Chlamydomonas, I84, I85 (fig.), I 86, 220 ; Cladophora, 215, 220; Ectocarpus, 219 (fig.), 220; Mucor, 237 (fig.), 238; Pleurococcus, I9I ; Ulothrix, 213 (fig.), 2I4. See also Egg and Spermatozoids. Gametophyte, .288, 289, $301,306$. Gamopetalous Dicotyledons, 357. Gaseous exchange, mechanism of, in Angiosperms, 67, 88, 96-I0I (figs.), 107, 108, 139, I40 (fig.), I 75,178 ; in Conifers, 343 ; in Liverworts, 273.

Gasteromycetes, 407 .

Gelatinous Lichens, 258.

Gemmæ of, Liverworts, 270 (fig.), 273 (fig.), 277; Mosses, 277, 287 (fig.).

Generative cell (of Angiosperms), 363,367 (fig.), 368 .

Gentian violet, 405 ; and lignified walls, 32; and starch-grains, 43.

Geoglossum, 239 (fig.), 240.

Geographical distribution of plants, $310,322,334,337,391-3$.

Geological changes and plant-distribution, 393.

Geotropic stimuli, perception of, 75,78 .

Geranium, Garden (Pelargonium), hairs of, 105.

Germination of, Fucus, 221 (fig.), 226; oospores, 223, 224, 233 (fig.); Pelvetia, 226; pollen, $356,362,363,367$ (fig.) ; seeds, $352,370,372$; spores, 238,246 (fig.), 247, 287 (fig.), 299 (fig.), 300, 326,348 ; zoospores, 213 (fig.), 2 I 4,217 (fig.), 2 I 8 (fig.), 232; zygospores, I86, 213 (fig.), $214,228$.

Geum, ovules of, 357 ; hybridisation in, 385 (fig.).

Geum intermedium, 385 (fig.), 389. Gills (of Mushrooms), 248, 249 (fig.). Ginger (Zingiber officinale), 63.

Gingerbeer, 265.

Ginkgo biloba, 326, 334 .

Ginkgoales, 408.

Gipsywort (Lycopus europaus), I74.

Glacial period, flora of, 394.

Glands, digestive, I49, I50 (fig.) ; 
oil-secreting, 105 (fig.), I 5I-3 (fig.) ; sugar-secreting, I48, I 49 (fig.) ; water-secreting (hydathodes), I44-8 (figs.).

Glandular cells, I48, I 49 (fig.).

Glandular hairs, I05 (fig.), I06, 298 (fig.), 299 (fig.), 328.

Gleichenia, 294, 295, 3 I0.

Globe animalcule (Volvox), I 89 (fig.), I90.

Globoid (of aleurone grains), 5I (fig.).

Gloeocapsa, 205, 206 (fig.).

Glucose, 45, 46, 48, 53, 55, 63 ; osmotic pressure of, II; reactions of, 47 .

Glucosides, 48, 63, 64; hydrolysis of, 48,53 .

Glutamin, 50 .

Glycerine, 49, 53.

Glycerine jelly, use of, 405 .

Glycine, 49.

Glycogen, 23I, 255, 262 ; reactions of, $23 I$.

Gnaphalium uliginosum, 375 (fig.).

Gonidia of, Claviceps purpurea, 24 I (fig.), 242 ; Cystopus, 232, 233 (fig.) ; Erysiphaceæ, 243, 244 (fig.); Eurotium (Aspergillus), 243 (fig.) ; Penicillium, 243 (fig.); Phytophthora, 234, 235 (fig.); Pythium, 234, 235 (fig.), 236 ; Rust-Fungi, 247. Gonidiophore, 243 (fig.).

Gonium, I89, I9I (fig.).

Gooseberry (Ribes grossularia), 3I ; - Mildew (Sphaerotheca morsuva), $230,242$.

Goosegrass (Galium aparine), hairs of, 104.

Goosefoot, Silver (Obione portulacoides), hairs of, 94 (fig.) ; secondary thickening of, I 33 .

Gorse (Ulex), I67 ; epidermis of, 92 , I60 (fig.).

Gossypium, 49, I02.

Gourd (Cucurbita pepo), pollen of, 362 (fig.).

Graft-hybrids, 389 .

Grafting, I 42, 389.

Grain (of wood), 127.

Gramineæ, 39, 44, 80, 88, I I 7, I63, $354,356,408$; diseases of, $24 \mathrm{I}, 243,245$; grains of, 52 (fig.), I 50 ; hydathodes of, I 45 ; intercalary meristems of, 21 ; stomata of, 97 (fig.), Ioo, IOI.
Graminin, 44.

Grape (Vitis vinifera), 60, 257; - mildew (Plasmopora viticola), 243.

Grape sugar. See Glucose.

Grasses. See Gramineæ.

Grassland, distribution of, 39r.

Grasswrack (Zostera), pollen of, 361 ; stem-structure of, I72.

Gravity, perception of, 75, 78 .

Greater Celandine (Chelidonium majus), laticiferous vessels of, I 53, I 54 ; ovule of, 358 (fig.). Green Algæ, 406. See Chlorophyceæ.

"Green cells" of animals, 260.

Green manuring, 267.

Green stems, structure of, 88, I62 (fig.), I67, I 78, 3 Io.

Ground Ivy (Nepeta glechoma), I68; epidermis of, 94 (fig.).

Ground tissue, 65, 88; of aquatics, I 7 I (fig.), I 73 (fig.), I 75 ; of leaf, ro7-II (figs.) ; of petiole, II4; of root, 66,67 ; of stem, I9-30, 33, 76, 78, 338; secondary, I33, I 34, I37.

Growing point, 16-20 (figs.), 26, 89,90 ; intercalary, 21 ; of Algæ, I6 (fig.), I7 (fig.), I95, 201 ; of Dictyota, I7 (fig.); of Equisetum, I 8 (fig.); of Fernprothallus, 300, 30I (fig.) ; of Ferns, 18, 291, 302; of leaf, I 8 (fig.), 29I ; of Liverworts, 270,272 ; of Mosses, 18,276 288 ; of root, 18, 20 (fig.), 65 ; of Sphacelaria, I6 (fig.), I7; of stem, I8 (fig.), I9 (fig.); unicellular, I6-I8 (figs.).

Growth, 16-20, 26, 31, 36, 40, 86 ; of Algæ, 195, 198, I99, 201 ; of cells, 25, 26, 28, 29, 80, 93, II7, I 53, I83, I9I ; of pollen tubes, $350,364,365$; secondary, II 7 et seq.

Guaiacum officinale, I30.

Guard cells, 96-IOI (figs.) ; change of shape of, 98-roo (fig.); development of, 98; raised, 96 ; sunken, 96 , г6o.

Guelder Rose (Viburnum opulus), extrafloral nectaries of, 148 .

Gum arabic, 39; - tragacanth, 39.

Gums, 38, 39, I 55.

Gutta percha, I 57.

Gymnosperms, I 80, 322-7 (figs.), 334-53 (figs.), 354, 355, 356, $362,368,370,372,373,407$. 
Habit of, Abies, 335, 336 (fig.) ; Athyrium filix-fomina, 289 , 29I ; Blechnum spicant, 29I, 308 (fig.) ; Cedrus, 335 ; Cephalozia, 27I (fig.), 272; Cetraria islandica, 26I (fig.) ; Chondrus crispus, 205 (fig.); Cladonia, 258 (fig.) ; Cladophora, I96 (fig.) ; Conifers, 334-6 (figs.) ; Cupressus, 336; Cycads, 322, 323 (fig.) ; Draparnaldia, I97 (fig.) ; Ectocarpus, 2 I9 (fig.); Equisetum, 3I0, 3 I I (fig.) Fegatella, 270 (fig.); Frullania, 272 (fig.) ; Fucus, 200-202 (figs.) ; Funaria, 274; Hepaticæ, 268-72 (figs.) ; Hymenophyllaceæ, 296, 297 (fig.); Hурпит, 274 (fig.), 275; Isoetes, 3I5 (fig.); Juniperus, 336; Laminaria, I99 (fig.) ; Larix, 335, 336 (fig.) ; Lepidodendron, 3 I 4 (fig.); Lichens, 258 (fig.); Lycopodium, 3I4, 3 I6 (fig.); Lvginopteris, 328, 329 (fig.); Marchantia, 270 (fig.) ; Mnium, 274 (fig.); Mosses, 274 (fig.) ; Nephrodium filix-mas, 290 ; Osmunda regalis, 289, 309 (fig.) ; Parmelia physodes, 258 (fig.); Pellia, 270 (fig.), 283 (fig.) ; Pelvetia, 203 (fig.) ; Phæophyceæ, 199-204 (figs.); Picea, 334, 3.35 (fig.) ; Pinus, 335, 345 (fig.) ; Polypodium, 29I (fig.); Polytrichum, 274 (fig.); Pteris aquilina, 290 (fig.), 29I ; Rhizocarpon confervoides, 258 (fig.) ; Rhodophyceæ, 205 (fig.) ; Sargassum, 204 (fig.); Scolopendrium, 291 (fig.) ; Selaginella, 316, 317 (fig.); Sphagnum, 275 (fig.); Taxus, 335, 346 (fig.); Thuja, 336, 337 (fig.); Tortula muralis, 274 (fig.); Tree Ferns, 28o ; Ulva, I98; Usnea barbata, 258 (fig.); Xanthoria parietina, 258 (fig.).

Habitat, adaptation to, I68, I69 (fig.), I 70, I 75-7 (figs.), 379 (fig.), 380.

Hæmatochrome, 188.

Hamatococcus, I86, I87 (fig.) ; $H$. droebakensis, 187 (fig.); $H$. pluvialis, 187 (fig.).

Hæmatoxylin, 404, 405; and cellwalls, 32 ; source of, 126 .
Hamatoxylon campechianum, 126.

Hair-moss (Polytrichum), 269, 274 (fig.), 276, 286 .

Hairs (trichomes), 4, 5 (fig.), 93 , 101-6 (figs.), I 58, I62, I63, I69, I78; bristle -, I04; climbing, I04; covering, I02 (fig.); function of, I01, I02, I04, I06, I65 ; glandular, 63, I05 (fig.), I06; peltate, IO3 (fig.), I05; root -, 65, 66, 67 (fig.) ; stellate, I02 (fig.), IO3; stinging, I04, I05 (fig.); water-storing, 94 (fig.) ; water-secreting, I44-6 (fig.)

Hairs of, Algæ, 202, 2 I9 (fig.), 22 I (fig.), 224, 225 (fig.) ; Bartsia, I 44 ; Black Currant, I05 ; Bladderwort, I 50 ; Boraginaceæ, IO2, I04 ; Brambles, IO4 ; Broad Bean, 148 ; Butterwort, I 49, I 50 (fig.) ; Chinese Primrose, I05 (fig.) ; Coltsfoot, IO2; Cotton-plant, IO2, IO3; Cruciferæ, IO3, I04; Cynoglossum, 102 (fig.) ; Deutzia, 102 (fig.), I03; Elaagnus, I03 ; Ferns, 29I (fig.) ; Fungi, 240 (fig.), 244 ; Garden Geranium, I05; Goosegrass, I04; Hop, IO2 (fig.), IO4, I05; Horse Chestnut, I02; Ice-plant, 94; Labiatæ, I05 ; Malvaceæ, Iо3; Mosses, 279 (fig.), 280 ; Mullein, I03; Oleander, I6I (fig.), I62; Pitcher-plant, I49; Plane, I03; Rose, I04; Runner Bean, I 44, I 45 (fig.), I 47 ; Sea Buckthorn, IO3 (fig.) ; semiparasites, I 44, I45 (fig.) ; Shepherd's Purse, I02 (fig.); Silk-cotton tree, I03; Silver Goosefoot, 94 (fig.) ; Silverweed, I02; Stinging Nettle, I04, I05 (fig.); Sundew, I50 ; Thistle, I02; Tiliaceæ, I03; Toothwort, I45 (fig.) ; Vegetable Marrow, IO2 (fig.); Yellow Rattle, I44, I45 (fig.). Hakea, leaf-structure of, I66.

Hard Fern (Blechnum spicant), 29I, 295, 308 (fig.), 324 .

Hard wood, I 27.

Harebell family (Campanulaceæ), 44, I 53, I 54 .

Hart's-tongue Fern (Scolopendrium vulgare), 29 I (fig.), 296 (fig.).

Haustoria of, embryo sac, 367 ; 
Fungi, 232, 233 (fig.), 243 ; parasitic Angiosperms, 178 (fig.), I79.

Hawkweed (Hieracium), 372.

Hawthorn (Cratagus), I37, 370.

Hay, fermentation of, 261.

Hazel (Corylus), 354; pollen tube of, 364,365 .

Heart-wood, 125, 126.

Heat, and enzyme-action, 56 ; and protoplasmic movement, 4; evolution of, in fermentation, 26x, 265.

Heat-rays, protection against, 64 , $95,102,138$.

Heath-plants, 178, 257, 269, 277.

Heather (Calluna), 72 ; leaf of, I62, I63 (fig.).

Heather-family (Ericaceæ), I78, $361,363$.

Hedera helix, Io; secretory canals of, I52 (fig.).

Helianthemum vulgare, 379 (fig.).

Helianthus, 49, II 3 ; stem-structure of, 76-80 (figs.), 87.

Helleborus foetidus, cuticle of, 91 .

Hemibasidii, 407.

Hemlock (Conium maculatum), 6I.

Hemp (Cannabis sativa), 34, 265.

Henbane (Hyoscyamus niger), 6I.

Hepaticæ, I80, I93, 269-73 (figs.), $277,278,355,407$; antheridia of, 277,278 (fig.), 279 ; archegonia of, 280, 28I (fig.) ; embryo of, 282 ; fertilisation in, 280,281 ; gemmæ of, 270 (fig.), 273 (fig.), 277; growing point of, 270 , 272 ; habitat of, 269, 272; sporogonia of, 271 (fig.), 282-4 (fig.) ; thallus of, 269-73 (figs.), 278 (fig.).

Herbaceous plants, stability of, 9, 87.

Heredity, 374-91 ; nucleus and, 389.

Hermaphrodite flowers, 354.

Heterocysts (of Blue-green Algæ), 206 (fig.).

Heterospory, 321, 332.

Heterozygote, 385 .

Hevea brasiliensis, I 56 (fig.), 157.

Hickory (Carya), I30.

High power (of microscope), 400.

Hilum (of starch-grains), 4I, 42 (fig.), 43.

Hinge-cells (of Grasses), I63, I64 (fig.).

Hippopha rhamnoides, hairs of, 103 (fig.).
Hippuris, growing point of, I9 (fig.); leaf of, I77; stemstructure of, I72, I73 (fig.).

Hogweed (Heracleum), $3 \mathrm{I}$; nectary of, 148 .

Holcus mollis, II2.

Holdfasts. See Attaching organs.

Hollow stems, 80, 8I (fig.), I 26.

Holly (Ilex aquifolium), I27; leafstructure of, 92 (fig.), 95, I I I, I 3 , I60 (fig.).

Hollyhock (Althaa), epidermis of, 95 ; pollen of, 362 (fig.) ; rust of (Puccinia malvacearum), $248,253$.

Homospory, $32 \mathrm{I}$.

Homozygote, 386 .

Honey, 46, I 48.

Hop (Humulus lupulus), 63, 257 ; fibres of, 34 (fig.); hairs of, IO2 (fig.), I04, I05; Mildew of (Sphaerotheca castagnei), 244 (fig.).

Hormidium, 208, 2 I 5 (fig.), 2 I6.

Hornbeam (Carpinus betulus,) 128, 367.

Hornwort (Cevatophyllum), anatomy of, I 70, I II (fig.); pollen of, $36 r$.

Horse Chestnut (Esculus hippocastanum), 52, 59 (fig.) ; hairs of, 102 ; stem-structure of, I20, I2I (fig.), I23 (fig.), I 27.

Horse Radish (Cochlearia armoracia), 48.

Horsetail (Equisetum), 39, I80, 289, 310-13 (figs.), 321, 407; growing point of, 18 (fig.).

Host, I 79, 230, 232, 245, 247, 248, $252,253,264$.

Hot-beds, 261.

Hot-springs, organisms of, 204, 26 I.

House-leek (Sempervivum tectorum), 98 , III.

Humidity, influence of, on plantstructure, I68, I 70.

Humulus lupulus, 63, 257; fibres of, 34 (fig.) ; hairs of, 102 (fig.), I04, 105; Mildew of (Sphaerotheca castagnei), 244 (fig.).

Humus, 239, 248, 252, 266; and mycorrhiza, 72, 252, 316.

Hyacinth (Scilla), 44, 365; growing point of root of, 20 (fig.).

Hybrid endosperm, 386.

Hybrids, 253, 382-9; graft 389 ; intermediate between 
parents, $382,387,388$ (fig.) ; natural, 385 (fig.), 388, 389; result of crossing with parent, 386 ; segregation of characters in, $384-8,390$.

Hydathodes, I 44-8 (figs.), I 49, I79 : function of, I47; mechanism of, 147.

Hydnum, 250; H. repandum, 251 (fig.).

Hydra viridis, "green cells" of, 260.

Hydrocarbons, 62.

Hydrocotyle, I 5 .

Hydrolysis, 46, 54, 55

Hylocomium, leaf of, 27.5 (fig.).

Hymenium of, Ascomycetes, 240 (fig.), 242; Basidiomycetes, 248, 249 (fig.), 250; Cystopus, 232 ; Rust-Fungi, 247.

Hymenomycetes, 407 .

Hymenophyllaceæ, 296, 301.

Hymenophyllum tunbridgense, 296, 297 (fig.).

Hyoscyamine, 61, 62.

Hyoscyamus niger, 61.

Hypericum perforatum, secretory cavities of, ${ }_{5}$ I.

Hypertrophy, 232.

Hyphæ (of Fungi), 230, 231, 232, 233 (fig.), 24I, 243 (fig.), 248. Hypnum, 274 (fig.), 275 (fig.), 276. Hypocotyl, structure of, 89 (fig.), I 8 (fig.).

Hypoderm, 92 (fig.), 95, I I I, I62, 343,344 (fig.).

Hypogynous flowers, 354.

Iceland Moss (Cetraria islandica), $25^{8}, 26$ L (fig.).

Ice-plant (Mesembryanthemum crystallinum), 94, 167.

Ilex aquifolium, 127 ; leaf-structure of, 92 (fig.), 95, I I I, II 3, I60 (fig.).

Illumination. See Light.

Imbibition water, 10, 26, 42 .

Immunity, 253, 384.

Incrustation of cell-walls, 39, 204 206.

Indiarubber, I 57.

Indiarubber-plant (Ficus elastica), I 57 ; leaf of, 39,95 , I IO.

Indican, 48 .

Indigo-plant (Indigofera), 48.

Indirect nuclear division (mitosis), 22-5 (figs.).
Individual variations, $374^{-8}$ (figs.), 380.

Indusium (of Ferns), 296 (fig.), 297 (fig.), 298 (fig.), 299.

Infection, 232, 234, 235, 242, 246, $247,253,267$.

Inheritance, 374, 379; in pure lines, 377,378 ; of acquired characters, 380 .

Injury, effects of, 26,48 , I I6, I 42, $216,252$.

Ink, 60 .

Insect-galls, 6o, I42, I43 (fig.).

Insect-pollination, $148,326,354$, $355,361,373$.

Insectivorous plants, 144 ; digestive glands of, I 49, I 50 (fig.).

Integument of, Angiosperms, 357 (fig.), $35^{8}$ (fig.), 359, 370 ; Conifers, 346, 347, 349 (fig.) ; Cycads, 325 (fig.), 326, 327 ; Lyginopteris, 331, 332, 333 (fig.).

Intercalary meristems, 21 .

Intercellular spaces, development of, 26 ; of aerenchyma, I74, I 75 (fig.) ; of aquatics, I7I (fig.), I75, I76 (fig.), I77 ; of bog-plants, 178 ; of leaves, 96, I07 et seq. (figs.), I70, 343; of Lichens, 259; of marsh-plants, I74, I75 (fig.) ; of Moss-sporogonium, 284; of root, 66 (fig.), 67; of secondary wood, 124; of stems, 88; secretory - , I5I, I52 (fig.).

Interfascicular cambium, I 8 (fig.), I 20.

Interval between pollination and fertilisation, in Angiosperms, 364 ; in Conifers, 350.

Intrafascicular cambium, I 8 (fig.). Introduction of new forms, 392, 393.

Inulase, 46, 53 .

Inulin, I I, 44, 45, 53; hydrolysis of, $46,53,55$; reactions of, 44, 47; sphere-crystals of, 44,45 (fig.).

Invert sugar, 46 .

Invertase, $46,53,55,256$; extraction of, 54 .

Involucre, of Liverworts, $280,28 \mathrm{I}$ (fig.), 283 (fig.); of Mosses, 279,280 .

Iodine, in Seaweeds, I 5 .

Iodine-solution, 40I; and alkaloids, 6I ; and cellulose, 31 ; 
and cuticle, 92 ; and dextrin, 46 ; and glycogen, 231 ; and inulin, 44; and lignified walls, 32 ; and proteins, 3,50 ; and starch, $4 \mathrm{I}$; and tannins, 60.

Ions, I 4, I 5.

Ipecacuanha (Psychotria ipecacuanha), 6I.

Iris, 44; leaf-structure of, 96, 97 (fig.), 99, II5, II6, I6o ; rootstructure of, 70 (fig.).

Iris germanica, 10, 97 (fig.).

Irish Yew (Taxus baccata, var. fastigiata), 380 .

Irisin, 44.

Iron-Bacteria, 268.

Irregular flowers, 355, 373.

Irritability, of guard-cells, Ioo ; of sexual cells, 222, 224, 28I, 302 ; of zoospores, 21 $4,232$.

Isatis tinctoria, 48.

Island floras, 393.

Isobilateral leaves, structure of, 97, I 6 .

Isoetes lacustris, 314, 315 (fig.), 407.

Isogamy, in Algæ, 185 (fig.), 186, 213 (fig.), 2I 4, 220, 227; in Fungi, 237 (fig.), 238.

Ivy (Hedera helix), Io; secretory canals of, I 52 (fig.).

Jarrah (Eucalyptus marginata), I30. Jerusalem Artichoke (Helianthus tuberosus), 44.

Johansen, 378 .

Joinery, timber used in, 129.

Jointed Rush (Juncus articulatus), leaf-structure of, I65 (fig.), I 66.

Juglans nigra, 129.

Juncaceæ, 408.

Juncus articulatus, leaf-structure of, I 65 (fig.), I 66.

Jungermanniales, 407 .

Juniper (Juniperus), 336, 352 (fig.), 353.

Jute (Corchorus), 34, 265.

Juvenile forms, of Angiosperms, 395 (fig.), 396 ; of Conifers, 337,338 (fig.).

Kapok (Eriodendron anfractuosum), I03.

Karyokinesis, 22.

Knots (in timber), I29.

Knotweed (Polygonum), ovules of, 358 (fig.).

Kola (Cola acuminata), 6r.
Labiatæ, 2I, 6I, 80, I05.

Laburnum (Cytisus laburnum), I37, 389.

Lacquer-tree (Rhus vermicifera), 155.

Lactic acid Bacteria, 265.

Lactose, 265.

Ladder-like perforations (of vessels), 35 (fig.).

Lady Fern (Athyrium filix-famina), 289, 29I, 305.

Lady's Mantle (Alchemilla), 372.

Lagenostoma lomaxi, 332 (fig.).

Laminaria, habitat of, 199, 203 ; reproduction of, $2 \mathrm{I} 8$; thallus of, I99 (fig.), 200, 202.

Laminaria digitata, I99 (fig.) ; $L$. saccharina, 199.

Lamium, 4, 105, 364 ; collenchyma of, 3 I (fig.), 34 (fig.).

Lamium galeobdolon, sun- and shadeleaves of, I68, r69 (fig.).

Land-forms of aquatics, 93, I 75-7 (figs.).

Landolphia, I 57.

Larch (Larix europaa), I29, 334 ; cones of, 336 (fig.), 345, 346, 347 ; habit of, 335,336 (fig.) ; leaf of, 343 ; seedlings of, 337 , 338 (fig.).

Lastrcea filix-mas. See Nephrodium filix-mas.

Latent characters (of hybrids), $382,383,384,385$.

Lateral branches, of roots, 65, 7 I (fig.) ; of stems, 72, I 29.

Lateral conjugation, 227 (fig.).

Latex, I 53-7.

Lathrca, hydathodes of, I45 (fig.).

Laticiferous cells, I 53, I 54 (fig.) ; - vessels, I 54, I 55 (fig.), I 57.

Laurel (Laurus nobilis), I 5I.

Lavender (Lavendula), 61, 62, 106; oil of, 62 .

Leaf, anatomy of, 9I-II6 (figs.) ; connection with stem, I I4 (fig.), 294 (fig.), 295, 344 ; development of, 90 ; effect of environment on, I68-70 (fig.), I77 (fig.) ; evergreen, I I 2, 335 ; growing point of, I 8 (fig.); movements of, II5, II6 ; position in relation to light of, II2 ; reduced, III ; reduction of, $158,165,178$; rolled, I63-4 (figs.) ; shedding of, I4I, I42 (fig.); succulent, I66; support of, II5; variegated, I I I. 
Leaf of, Conifers, 334-7 (figs.) ; Cycads, 322, 323 (fig.), 324 (fig.) ; Equisetum, 3I0, 3I I (fig.) ; Ferns, 290-92 (figs.), 303; foliose Liverworts, $27 \mathrm{I}$ (fig.), 272 (fig.) ; Lycopodium, 3I4, 3I5, 3I6 (fig.); Lyginopteris, 328, 329 (fig.) ; Mosses, 274, 275 (fig.) ; Selaginella, 3I6, 317 (fig.), 318.

Leaf-fall, 59, I 4 I, I 42 (fig.).

Leaf-gaps (of Ferns), 294 (fig.), 295.

Leaf-scars, I 42.

Leaf-stalk. See Petiole.

Leaf-structure of, Alpines, I70 ; aquatics, 91, 92, 93, IOI, 172, I77 (fig.) ; Bracken (Pteris), I 48, I49 (fig.), I69; Brooklime (Veronica beccabunga), I6o (fig.); Catharinea undulata, 276 ; Clubmoss (I-ycopodium), 3I5 ; Conifers, 34I-4 (figs.); Corn Cockle (Agrostemma githago), IIo ; Crowberry (Empetrum nigrum), I63 (fig.); Cycads, 322 ; Dicotyledons, 93, 98, 107 et seq. (figs.); Elder (Sambucus), I Io, II I (fig.) ; Enchanter's Nightshade (Circaa), I46 ; Euphorbia amygdaloides, IIo; evergreens, I I2, 335 ; Ferns, 29.5, 298 (fig.) ; Ficus elastica, 95, I I0 ; Fuchsia, I07-Io (figs.), I46 ; Ground Ivy (Nepeta glechoma), 94 (fig.) ; Hakea, I66; Heather (Calluna), I62, I63 (fig.) ; Holly (Ilex aquifolium), 92 (fig.), 95. I I I, I I3, I60 (fig.) ; Hornwort (Ceratophyllum), I72; House-leek (Sempervivum), III ; Ice-plant (Mesembryanthemum), 167; Iris, 96, 97 (fig.), I I 5, I I6, I60; Jointed Rush (Juncus articulatus), I65 (fig.), I66; leafy Liverworts, 273 ; Lesser Celandine (Ficaria verna), I 45 , I 46 (fig.), I 47 (fig.); Lyginopteris, 329, 330; Male Fern (Nephrodium), 298 (fig.) ; Marsh Marigold (Caltha), I 46 ; Monocotyledons, 93, 98, II 2 ; Mosses, II2, 275 (fig.), 276 ; Myrtle (Myrtus), I 5I; Nettle (Urtica), 38 (fig.) ; Oleander (Nerium oleander), IIo, I6I (fig.) ; Onion (Allium), 94 (fig.), 97 (fig.) ; Pinus, 152,
I66, 343, 344 (fig.) ; Prickly Saltwort-(Salsola), I 66 ; Psamma arenaria, 92, I63, I64 (fig.) ; Ranunculus auricomus, 92 (fig.) ; Rue (Ruta), I5I ; St. John's Wort (Hypericum), I5I ; Sca-blite (Suceda), I66; Selaginella, 318 ; shadeplants, 9I, 95, I68, I69 (fig.) ; Silver Goosefoot (Obione portulacoides), 94 (fig.); Sphagnum, 275 (fig.), 276; Stonecrop (Sedum), I66 ; succulents, I16, I66; Wallflower (Cheiranthus cheiri), III; Water Buttercup (Ranunculus aquatilis), I77 (fig.) ; Water Starwort (Callitriche), I77; Wild Strawberry (Fragaria), I46; Yellow Dead-nettle (Lamium galeobdolon), I68, I69 (fig.) ; Yellow Pimpernel (Lysimachia nemorum), I60 (fig.); Yew (Taxus), 34I, 342 (fig.).

Leaf-traces of, Angiosperms, 86, II4 ; aquatics, I73 ; Ferns, 294 (fig.), 295; Lyginopteris, 329,330 (fig.).

Leafy Liverworts(Jungermanniales). See Foliose I iverworts.

Leathery leaves, structure of, 160.

Leguminosæ, 30, 72, 371 ; rootnodules of, $267,268$.

Lemna, II2.

Lemon (Citrus limonum), 60.

Lenticels, 77, 139, I 40 (fig.), I4I.

Lepidodendron, $3 \mathrm{I} 2$ (fig.), 3 I 4 (fig.), 3 I 5,407 .

Leptothrix, 268.

L.esser Celandine (Ficaria verna), embryo of, 372 ; hydathodes of, I 45, I 46 (fig.), I 47 (fig.).

Lettuce (Latuca), 53.

Leucin, 52.

Leucobryum, 277.

Leucocytes and Bacteria, 265.

Leucoplasts, 4I, 43.

Levulose, 45.

Lianes, structure of, I34.

Lichens, 407; habitat of, 257. 258 ; reproduction of, $25^{8}$ (fig.), 260 (fig.), 26I ; structure of, 259,260 (fig.) ; thallus of, 258 (fig.).

Lid (of Moss-capsule), 284, 285 (fig.), 286.

Iife-histories. See Reproduction. Light and, anthocyanin, 63 ; chloro- 
phyll-formation, 4I, 74; chloroplasts, I IO, I II, I I 2 ; enzyme-action, 56 ; leaf-structure, III, I68-70, I 72; position of leaves, II2; sleepmovements, II5; stomatal mechanism, I0o; zoospores, etc., I83, 214.

Light, concentration of, 96 (fig.) ; perception of, 95, I8I ; protection against, 95, I02, I I0, . I I I, I I 2, 199.

Jignification, 32 .

Lignified walls, $32,33,68$; reactions of, 32 .

Lignin, 32.

Lignum vitæ (Guaiacum officinale), I30.

Iigule (of Selaginella), 318 (fig.).

Ligulifloræ, I 53.

Liliaceæ, 37I, 408.

Lily (Lilium), I Io; embryo of, 37 I (fig.) ; pollen and embryo sac of, 367 (fig.) ; stamen of, 363 .

Lime (Tilia), secondary phloem of, I30; secondary wood of, 28 , I20, 127.

Lime-family (Tiliaceæ), Io3.

Limestone rocks, rôle of Algæ in formation of, 204.

Linaceæ, 6r.

Linseed oil, 49.

Linum usitatissimum, 34, 49.

Lipase, 49, 53; extraction of, 54.

Listeva ovata, embryo of, 37 I (fig.).

Litmus, 261.

Liverworts, I80, I93, 269, 355, 407. See Hepaticæ.

Lloyd's alcohol method (for determining stomatal aperture), 99, 100.

Lockjaw Bacillus (Bacillus tetani), 263 (fig.).

Loculi (of ovary), 356, 364 .

Loganiaceæ, 6r.

Logwood (Hamatoxylon campechianum), I 26.

Lophocolea, 272, 280.

Loranthaceæ, ovary of, 365 .

Loss of sexuality, $214,236,245$, $305,372$.

Low power (of microscope), 400.

Lowland forms of Alpine plants, 379. Lupine (Lupinus), 30, 47, 52.

Lycoperdon, 245, 250, 25 I (fig.).

Lycopodiales, 289, 310, 313-20 (figs.), 407.

Lycopodium, 180, 289, 3I0, 314,
I6 (fig.), 318, 32I, 407; $L$. clavatum. 314, 315, 316 (fig.) ; L. inundatum, 315 ; L. selago, 3I 4 , 3I 5.

Lycopus europaus, 174.

Lyginopteris (Lyginodendron) oldhamia, 328-33 (figs.) ; anatomy of, 328-30 (fig.) ; microsporophylls of, 330, 331 (fig.) ; ovules and seeds of, 33I-3 (figs.) ; vegetative organs of, 328,329 (fig.).

Lyngbya, 205, 206 (fig.).

Lysigenous secretory cavities, I5I, I 52 (fig.).

Lysimachia nemorum, stoma of, 160 (fig.).

Lythrum salicaria, I74.

Macrocystis, 200.

Madder (Rubia peregrina), stoma of, 97 (fig.), 98.

Mahogany (Swietenia mahagoni), I26, 130.

Maiden Hair Fern (Adiantum), sori of, $29 \mathrm{I}$ (fig.), 296.

Maiden Hair Tree (Ginkgo biloba), $326,334,408$.

Maize (Zea mais), disease of, 248 ; grains of, $45,52,53,384$; hybrid-endosperm of, 386 ; leaf of, II 7 ; root-structure of, 73 , 74 (fig.) ; stem-structure of, 84-6 (figs.).

Male Fern (Nephrodium filix-mas), sorus of, 296 (fig.), 298 (fig.); sporangium of, 299 (fig.) ; sporeformation in, 307 (fig.) ; stem of, 290, 294 (fig.).

Male sexual cells, $186,220,389$; of Angiosperms, 364, 367 (fig.), 368 ; of Conifers, $349,350,35^{\text {I }}$ (fig.), 353 ; of Fungi, 234, 236 , 244 (fig.). See also Spermatozoids.

Malic acid, 60, 302.

Mallow (Malva), I30, I52; ovule of, 358 (fig.).

Mallow-family (Malvaceæ), hairs of, 102, 103 ; stamens of, 360 .

Malt, 46, 256, 257; - sugar, 45 .

Maltase, 47, 53 .

Maltose, $45,46,53,56$; hydrolysis of, 47,53 ; reactions of, 47 .

Malva, 130, 152; ovule of, $35^{8}$ (fig.).

Malvaceæ, hairs of, I02, 103 ; stamens of, 360 . 
Mandioc (Manihot utilissima), 43.

Mangrove (Rhizophora), 372.

Manihot glaziovii, I57; M. utilissima, 43.

Mannose, 53.

Manure, fermentation of, 26I ; use of Algæ for, 203.

Maple (Acer campestre), 28, I30, I3.5 ; Cork-, I4I.

Maple sugar, 124.

Marchantia, 407; antheridia of, 279; archegonia of, 280, 28I (fig.) ; gemmæ of, 270 (fig.), 273 (fig.), 277 ; fertilisation of, 281 ; habit of, 270 (fig.) ; sporogonia of, 281, 282 ; structure of, 27I, 273 (fig.).

Marchantiales, 407.

Mare's Tail (Hippuris), growing point of, I9 (fig.); leaf of, I77; stem-structure of 172 , 173 (fig.).

Margarine, 49.

Marine Algæ. See Seaweeds.

Marram-grass (Psamma arenaria), leaf-structure of, 92, I63, I64 (fig.).

Marrow (Cucurbita), hairs of, 102 (fig.) ; phloem of, 29 (fig.), 82, I 30 ; stem-structure of, $80-83$ (figs.) ; tyloses of, 127 (fig.) ; vessels of, 35 (fig.), 83.

Marsh Cudweed (Gnaphalium uliginosum), 375 (fig.); - Marigold (Caltha palustris), 72, I46, 356, 366 (fig.) ; - Pennywort ( $H y$ drocotyle), I 5 ; - Samphire (Salicornia), I 75 (fig.).

Marsh-plants, structure of, 72, I00, I 74, I75 (fig.).

Meadow Rue (Thalictrum), stemstructure of, 86.

Meadow Saffron (Colchicum), 364.

Meadowsweet (Spirca ulmaria), I69.

Measurement under the microscope, 405.

Mechanical elements and tissues, 27, 31-6 (figs.); of Ferns, 292, 293 (fig.); of Fucus, 202 ; of leaves, I I2, I I4, I I5, I66: of Lyginopteris, 329, 330 (fig.) ; of Mosses, 276; of roots, 69, 72,73 ; of secondarily thickened stems, I 21, I 23 (fig.), I 25, I37 ; of young stems, 87,88 .

Mechanics of, aquatics, I70, I 7 I, I 72 ; leaves, 92, 95, I I 2 , I I 5 ,
II6 ; petioles, II5 ; roots, $69,72,73$; secondarily thickened branches, I25, I 37 ; young stems, 87,88 .

Medicinal plants, $48,49,60,6$ I, I 55 . $255,276$.

Medulla (of Algæ), 202, 225 (fig.).

Medullary bundles, 86 .

Medullary rays, of Conifers, 340, 34 I

(fig.); of Lyginopteris, 328, 330 (fig.) ; of root, I32; of secondary phloem, I30; of secondary wood , I I9 (fig.), I 20, 122-4 (fig.), 128 (fig.) ; primary -, 76, 77 (fig.), 78 (fig.), 1 I 8.

Medullary sheath, 79, 124.

Megasporangium, 318 (fig.), 319, 324,325 (fig.), 328, 346.

Megaspore, 32I, 332; of Angiosperms, $357,365,373$; of Conifers, 348, 349 (fig.); of Cycads, 325 (fig.), 326, 327 (fig.) ; of Lyginopteris, 33I, 333 (fig.); of Selaginella, 318 (fig.), 3 I9.

Megasporophylls, of Angiosperms, 3.54, 356; of Conifers, ' 347 (fig.); of Cycads, 324, 325 (fig.).

Meiosis, 305-7 (fig.).

Melampyrum, I 79 .

Melosira, I93 (fig.), 207 (fig.).

Membrane, cell-, see Cell-wall ; semi-permeable - 8,9 .

Mendel's law, 383-7.

Mentha piperita, 62 .

Menthol, 62.

Mercurialis perennis, leaf of, 37 (fig.), 59 (fig.), I68, I69; petiole of, II 3 (fig.).

Meristems, intercalary, 21, 199 ; primary, 18-21 (figs.), 65, 79, 89 ; secondary, I I 7, I I 8 , I 35 , 137.

Meristic variations, 374,376 .

Merulius lacrymans, 252.

Mesembryanthemum crystallinum, $94,167$.

Mesophyll of, Angiosperms, 107I 2 (figs.); aquatics, I72, I 77 (fig.) ; Conifers, 342 (fig.), 343. 344 (fig.) ; Ferns, 295, 298 (fig.) ; shade-leaves, I69 (fig.), I 70 .

Mesozoic period, plants of, 322 .

Metabolism, 58 .

Metaxylem, 68, 69; of Ferns, 292 (fig.), 293 (fig.); of roots, 68, 70 (fig.); of stems, 78 (fig.), 79 , 
80 (fig.), 8 I (fig.), 83 (fig.), 85 (fig.), 86, 87 (fig.).

Methyl blue, 404, 405; and cellulose, 32 ; penetration of, into living plant-cells, $\mathbf{I} 2$.

Metroxylon, 44.

Micrasterias, 209 (fig.), 2 ro.

Micrococcus, 262, 264.

Micrometers, 405 .

Micropyle of Angiosperms, 357 (fig.), 364 (fig.); of Conifers, 347 (fig.), 348, 349 (fig.); of Cycads, 325 (fig.), 326 ; of Lyginopteris, $33 \mathrm{I}$.

Microscope, construction of, 398400 (fig.).

Microspora comma, 263 (fig.).

Microsporangium, 318 (fig.), 319 , 324 (fig.), 328, 330, 331 (fig.), 359.

Microspores, 321, 332 ; of Angiosperms, 373 ; of Conifers, 345 , 347 (fig.), 348, 349; of Cycads, 326, 327 (fig.) ; of Lyginopteris, 330; of Selaginella, 318 (fig.), 319.

Microsporophylls of, Angiosperms, 354,359 ; Conifers, 345,346 (fig.), 347 (fig.); Cycads, 323 , 324 (fig.) ; Lyginopteris, 33I (fig.).

Middle lamella, 24, 27, 30 (fig.), 3 I (fig.), 36, 37 (fig.), 68, 83, I4I.

Middle layer (of anther-wall), 360 (fig.), 363 (fig.).

Midrib of, Algæ, 200, 201 (fig.) ; carpels, 356 ; leaves, 31 , 108 (fig.), 113, I I5; Liverworts, 270 (fig.), 272; Mosses, 274, 275 (fig.), 276.

Mildews (Erysiphaceæ), 230, 239, 252,253 ; structure and lifehistory of, 242-4 (fig.).

Milk, souring of, 265 .

Millet-grass (Millium effusum), stoma of, 97 (fig.).

Millon's reagent, 40I; and proteins, 50 .

Mimosa pudica, movements of, II5. II 6.

Mimulus moschatus, pollen of, 362 (fig.).

Mineral deposits in cell-walls, 39.

Mineral salts, absorption of, Io, I3, I5, 72, 270; conduction of, $65,88,112$; toxic effect of, I4, 15 .

Mint (Mentha), 6r.
Mistletoe (Viscum album), 30.

Mistletoe-family (Loranthacea), ovary of, 365 .

Mitosis, in spore-mother cells, 305-7 (fig.); in vegetative cells, 20-25 (figs.).

Mnium, 274 (fig.), 275 (fig.), 276.

Mnium hornum, stem-structure of, 275 (fig.).

Mode (in variation), $375,376,377$, 380.

Molassine meal, 276.

Molecular solutions, I I.

Monarch roots, 318.

Monkey Puzzle (Araucaria), 346, 347.

Monkshood (Aconitum), 6I.

Monocotyledons, 44, 58, 355, 367, 408; embryology of, 370,37 I (fig.) ; epidermis of, 93 ; flowers of, 355 ; leaf of, 93, 98, I I2, II4, II6, I46; root of, 67,70 (fig.), $7 \mathrm{I}$; secondary thickening of, I 33 (fig.), I 34; stem of, $36,82,84-6$ (figs.), 87 , I I 7 ; seeds of, 371.

Monœcious, 223, 354.

Monopodial branching, 334 .

Monosaccharides, $44,45,46,53$.

Morel (Morchella), 239 (fig.), 240, 255.

Morphine, 6I, I 55.

Moschatel (Adoxa moschatellina), epidermis of, 95, 96 (fig.).

Mosses (see Musci), 180, 193, 257. $269,355,407$.

Mother-cells of, megaspores, 319 , $348,365,372$; pollen grains, 345,360 (fig.) ; spermatozoids, $277,302,303$ (fig.) ; spores, $282,284,285$ (fig.), 297, 305-7 (fig.).

Mother - of - Thousands (Saxifraga sarmentosa), 8, 63 .

Moulds, 6I, 230, 23I, 237 (fig.), 239, 242,243 (fig.), 252.

Mountains, distribution of forest on, 391 .

Mounting of sections, etc., 400, 404.

Mouse-ear Chickweed (Cerastium), vascular system of, II3, I I4 (fig.).

Movements of, chloroplasts, I I2 ; cytoplasm, 4, 6, 104; guardcells, 98, 99 (fig.) ; motile Algæ, I81, I82, 183, 189, I90, I93 ; spermatozoids, 222, 226, 280 , 302, 326 ; zoospores, 214,217 , 2I 8,232 ; sleep 一, II 5 . 
Mucilage, 38, 58, I 49, I 50, I67, 28I, 302 ; of Algæ, I88, 205, 213. $217,222,225$.

Mucilage-cavities, I 52 ; - cells, 273 (fig.) ; - glands, I50 (fig.).

Mucilaginous walls, 38, 95, I 84, I 89 (fig.), 202, 2I 4, 262.

Mucor, 231, 252, 257, 406 ; structure and life-history of, 236-8 (fig.).

Mucor-yeast, 257.

Mulberry (Morus), 370.

Mullein (Verbascum), 103, 378.

Multicellular, I.

Multiciliate zoospores, 2 I 8 (fig.).

Multinucleate structures, I 53, I98, 208, 218 (fig.), 224, 234, 326, 361,368 .

Musci, 269, 274-7 (figs.), 304, 407.; antheridia of, 279 (fig.); archegonia of, 28o ; branching of, 274,275 ; embryo of, 284 , 285 (fig.); fertilisation in, 28I ; growing point of, I8, 276 ; habitat of, 269,277 ; leaves of, II2, 274, 275 (fig.) ; protonema of, 287 (fig.), 288 ; rhizoids of, 274; sporogonia of, $284-7$ (fig.); stem of, 275 (fig.), 276; stomata of, IOO, 284,285 (fig.) ; vegetative reproduction of, 277,287 (fig.), 288.

Mushroom (Agaricus), 245, 255, 407; structure and reproduction of, 248, 249 (fig.).

Musk (Mimulus moschatus), pollen of, 362 (fig.).

Mustard (Sinapis), 69.

Mustard oil, 48, 63.

Mutants, 381 (fig.), 382, 384.

Mutation, $330-82$ (fig.); vegetative, 382,383 (fig.), 390.

Mycelium, 230, 236, 237 (fig.), 239, $243,248$.

Mycorrhiza, 72, 252, 316 ; of Bird'sNest Orchid (Neottia), 72, 73 (fig.).

Myrica gale, 268; ovule of, 357 , 359.

Myriophyllum, I 7 I.

Myronate of potash, 48 .

Myrosin, 48, 53 .

Myrtaceæ, 62.

Myrtle (Myrtus communis), secretory cavities of, I $5 \mathrm{I}$.

Myxomycetes, 250, 25I, 406.

Myxophyceæ, 406. See Cyanophyceæ
Naked cells, I84, I86, 213, 250, 366 ; - flowers, 354 .

Narcotics, 6I.

Nasturtium, Garden (Tropaolum), 63. II5.

Natural hybrids, 385 (fig.), 388 , 389 .

Natural selection, 378,390 .

Navicula, 207 (fig.).

Neck of, archegonium of, Conifers, 348,349 (fig.) ; Cycads, 326, 327 (fig.) ; Ferns, 302, 303 (fig.) ; Liverworts, 280, 28I (fig.); Mosses, 280; Selaginella, 319,320 (fig.).

Neck-canal cells, 280, 302 .

Nectaries, I 44, 363; mechanism of, I 49; structure of, I48, I 49 (fig.).

Nectria, 239, 242, 253.

Needle-shaped crystals, 58, 59 (fig.), 237.

Negative pressure, 35, 69 .

Neottia nidus-avis, I79; mycorrhiza of, 72,73 (fig.) ; pollen of, 361 , 362 (fig.).

Nepeta glechoma, I68; epidermis of, 94 (fig.).

Nepenthes, glands of, I49.

Nephrodium filix-mas, sorus of, 296 (fig.), 298 (fig.); sporangium of, 299 (fig.) ; spore-formation in, 307 (fig.); stem of, 290, 294 (fig.).

Nerium oleander, leaf-structure of, I IO, I6I (fig.), I62.

Nettle (Urtica), hairs of, I04, 105 (fig.) ; leaf-structure of, 38 (fig.), 39; root of, 132 .

New species, origin of, 378,380 , 381, 382, 393.

New Zealand Flax (Phormium tenax), 34:

Nicotiana tabacum, $6 \mathbf{I}$.

Nicotine, 6I.

Nightshade, Deadly (Atropa belladonna), 6I ; Enchanter's (Circaa lutetiana), 58, 59 (fig.), I $46,168$.

Nitrate-Bacteria (Nitrobacter), 266.

Nitric acid, and proteins, 50.

Nitrite-Bacteria (Nitrosomonas), 266. Nitrobacter, 266.

Nitrogen-fixation, 266, 267.

Nitrogen-fixing Bacteria, 266, 267 (fig.).

Nitrogenous food-reserves, 50-52.

Nitrosomonas, 266. 
Nitzschia, 207 (fig.).

Nodules of Leguminosæ, etc., 267 , 268.

Non-essential organs of flowers, 354.

Non-reducing sugars, 47.

Non-vascular plants, I80 et seq.

Nosepiece (of microscope), 398.

Nostoc, 205; 206 (fig.), 259.

Nucellus of, Angiosperms, 357 (fig.), 358 (fig.), 3.59, 365, 37I, 372 ; Conifers, 348, 349 (fig.), 350, 353 ; Cycads, 325 (fig.), 326, 327 (fig.) ; Lyginopteris, 33I, 333 (fig.).

Nuclear division, direct, 21 ; indirect, 20-25 (figs.) ; in sporemother cells, 305-7 (fig.), 385; in vegetative cells, 24,25 .

Nuclear membrane, 3, 22, 24.

Nuclear sap, 22.

Nuclear spindle, 2 I (fig.), 23 (fig.), 306, 307 (fig.).

Nuclei, 2 (fig.), 3, 4, 5 (fig.), 7 (fig.), 50 ; function of, $3,385,389$; fusion of, $184,222,234,238$, $245,247,281,306,350,366$, 368,389 ; and heredity, 389 , 390 .

Nuclei of, ascus, 245; Bacteria, 262 ; Cladophora, I96 (fig.), 208; Cyanophyceæ, 205, 210 ; Fungi, 23I; laticiferous elements, I53; Edogonium, 208, 217 (fig.); Rust Fungi, 247; spermatozoids, 277,278 (fig.) ; Vaucheria, I98, 218 (fig.); Yeast (Saccharomyces), 255 , 256 (fig.).

Nucleolus, 2 (fig.), 3, 2 I (fig.), 22, $24,306$.

Nutrition, 40, I83, I88, 220 ; and sex of Equisetum - prothalli, 3I3; in plants and animals compared, I94; of embryos, $302,320,32 \mathrm{I}, 326,332,35 \mathrm{I}$, $367,370,373$; of Moss-sporogonium, 284; of secondary wood, 124.

Nymphaa, 101, 356.

Oak (Quercus), 130, 364, 391 ; bark of, 60, I4I ; cork of, I40 ; wood of, 28, 35 (fig.), I22, I28 (fig.).

Oat (Avena), 53; fungal diseases of, 241,248 ; starch of, 43 .

Obelia, I93.
Obione portulacoides, hairs of, 94. (fig.) ; secondary thickening of, 133 .

Objective, 398, 400

Ocular, 398.

Edogonium, habitat of, I95 ; sexual reproduction of, 217 (fig.), 220-23 (fig.), 226 ; structure of, 195, 197, 208, 217 (fig.) ; zoospores of, 216,217 (fig.).

Edogonium concatenatum, 2 I 7 (fig.), 22 I (fig.); $O$. diplandrum, 22 I (fig.) ; $O$. landsboroughi, $22 \mathrm{I}$ (fig.) ; O. pachyandrium, 2 I 7 (fig.), 22 I (fig.).

Enanthe crocata, stem-structure of, 86.

Enothera, 389; O. lamarckiana, mutation in, $38 \mathrm{I}, 382$.

Oidium, 243.

Oil-cake, 50.

Oils, $40,49,50,53,155,35 \mathrm{I}$; in Fungi, 23I ; in oospores, 223 ; in Vaucheria, 208; in zygopores, I85; reactions of, 49.

Oils, volatile or ethereal, $62,63,64$, 105, 106, 126, I 5 I.

Old Man's Beard (Usnea), 258 (fig.).

Olea europaa, 49.

Oleander (Nerium oleander), leafstructure of, I IO, I6I (fig.), I62.

Oleic acid, 49.

Olive (Olea europaea), 49.

Olive oil, 49.

One-sided bordered pits, I 22.

Onion (Allium cepa), 45; cellstructure of, $\mathrm{I}, 2$ (fig.), 3 , 26 ; epidermis of, 94 (fig.), 97 (fig.), 99; stamens of, 359 (fig.).

Oogamy, 220, 22 I (fig.), 223, 226, $234,238$.

Oogonium of, Cystopus, 233 (fig.), 234; Fucus, 22I (fig.), 224, 225 (fig.) ; Edogonium, 2 I 7 (fig.), 221 (fig.), 222 ; Pelvetia, 226; Vaucheria, 22 I (fig.), 224.

Oomycetes, 23I-6 (figs.), 238, 406.

Oospore of, Algæ, 221 (fig.), 223 , 224, 226; Angiosperms, 368; Conifers, 350 ; Cycads, 326; Ferns, 302; Fungi, 233 (fig.), 234, 236; Liverworts and Mosses, 28I; Selaginella, 3 I9. Open grain of wood, 127.

Opening of stomata, 98-Ioo (fig.).

Opium, 6r. 
Opium Poppy (Papaver somniferum) 6I, I 55.

Optical section, 6 (fig.), 401.

Orange (Citrus aurantium), 62, 151 ; polyembryony of, $37 \mathrm{I}$ (fig.), $372,390$.

Orchids (Orchidacea), 355; aerial roots of, 73-5 (fig.); pollen of, 361 .

Orchis morio, pollen of, 362 (fig.).

Oregon Pine (Pseudotsuga douglasii), I 29.

Organic acids, 60.

Organic material, absorption of, by Fungi, 230, 232, 236, 237, 259; by insectivorous plants, I 49 , I5O; by mycorrhiza, 72, 252; by parasitic Angiosperms, I79. Origin of new species, $378,380,381$, $382,393$.

Orthotropous ovules, 358 (fig.).

Oryza sativa,43, 53, 257; starch of, 42 (fig.), 43.

Osazones, 47 (fig.).

Oscillatoria, 205.

Osmic acid, and fats, 49.

Osmometers, 402, 403 .

Osmosis, 8.

Osmotic pressure, 8,10 ; determination of, IO, II, I 2, I5 ; of different strengths of sea-water, 402, 403; of guard-cells, IOo; of food-reserves, II, 44, 45; rôle of, in growth of plant-cells, 26.

Osmunda regalis, habit of, 289, 309 (fig.); prothallus of, 304 ; sporangia of, 300, 309 (fig.).

Osterhout, I3, 402 .

Ovary (of Angiosperms), 334, 354-6, 364,365 .

Ovules of, Angiosperms, 354, 356, 357-9 (figs.), 365,366 (fig.); Conifers, 334, 336, 347 (fig.), 348,349 (fig.) ; Cycads, 324, 325 (fig.) ; Lyginopteris, 328, 330, 33I; parasites, I79, 365.

Ovuliferous scale (of Conifers), 347 (fig.), 348, 352 (fig.), 353.

Ovum. See Egg.

Oxalate of lime, 58,59, 1 30,237 ; reactions of, 59 .

Oxalic acid, 58.

Oxidases (oxidising enzymes), 55, I 55 .

Oxygen and Bacteria, 263; and protoplasmic movement, 4 .
Pæony (Paonia), 8.

Palæozoic period, plants of, 3II.

Palisade tissue, 38 (fig.), 107, 108 (fig.), 109, 110, I6I (fig.); arm -, IIO, III; function of, 107, I09; of Alpines, I70; of centric leaves, II6, I66; of stems, I67 ; of sun- and shadeleaves, I69 (fig.) ; of Taxusleaf, 342 (fig.).

Palmella-stage of, Chlamydomonas, I82 (fig.), I84; Ulothrix, 2 I4, 2 I 5 (fig.).

Palmitic acid, 49.

Palms, 44, II 7.

Papaver somniferum, 6I, I55.

Papaveraceæ, I 53, I 54 .

Paper, 35, I 29.

Papillæ, I03, I48, 342 (fig.) ; stigmatic, 356,364 .

Para-rubber (Hevea brasiliensis), I 56, I 57 .

Parallel venation, II 2.

Parasites, protection against, 138 , 142.

Parasitic Angiosperms, I 78 (fig.), I79, 231, 365, 372 ; Bacteria, 263; Fungi, 230 et seq., 252-5.

Parasitism, 23I, 247, 248, 252, 259, $264,281,288,372$; and immunity, 253.

Parenchyma, 27, 33, 69.

Parenchyma-sheath, I I2, II 3.

Parenchymatous cells, 27, 32, 65 , 76.

Parietal placentation, 356 .

Parmelia physodes, $25^{8}$ (fig.).

Passage-cells of, endodermis, 70 (fig.), 7 I, 75 (fig.) ; exodermis, $67,74,75$ (fig.).

Passive hydathodes, I 48.

Pathogenic Bacteria, 263, 264.

Pea (Pisum), 356, 370 ; hybrids of, $383,384,386$; mutation in, 381 (fig.) ; protein of, 50, 52 ; root of, 72,74 ; starch of, 42 (fig.).

Pea-mildew (Erysiphe polygoni), 243.

Pea-nut (Arachis hypogáa), 49, 53.

Pear (Pyrus), 6o; stone-cells of, 30 (figs.), 32, 33 .

Pectic substances, 31 .

Pectose, 231.

Pediastrum, I9I (fig.), I92.

Pelargonium, hairs of, I05.

Pellia, 407; anatomy of, 272,278 (fig.) ; antheridia of, 277,278

(fig.) ; archegonia of, 280, 28I 
(fig.) ; habit of, 270 (fig.) ; sporogonium of, 282,283 (fig.).

Pellionia, starch-formation in, 4I, 42 (fig.).

Peltate hairs, ro3 (fig.), ro5; leaves, XI 5 .

Peltigera, 259.

Pelvetia canaliculata, conceptacles of, 226, 269 ; habitat of, 202, 203 ; thallus of, 203 (fig.).

Penicillium, 239; gonidia of, 242, 243 (fig.).

Pentarch roots, 69, 7 I (fig.).

Pepper (Piper nigrum), 63.

Peppermint (Mentha piperita), 62 ; - oil, 62.

Pepsin, 52, 54, 57.

Peptones, 52, 53.

Perception of, gravity, 75, 78; light, 95, I8I.

Perforations of vessels, 28, 35 (fig.), 83,121 .

Perfumes, 62, 106.

Perianth, 370.

Periblem, I9, 20, 90, 95, 369.

Pericarp, 370, 371 (fig.).

Pericycle, 69, 78, 137; of aquatics, I7I ; of Ferns, 292 (fig.) ; of Lyginopteris, 328, 330 ; of roots, 66 (fig.), 68, 69, 70 (fig.), 7 I (fig.), I3I ; of stems, 78, I 18.

Pericyclic fibres, 78 (fig.), 79, 80 (fig.), 87, I I 8 (fig.), I 2 I (fig.).

Periderm (of Lyginopteris), 328.

Peridineæ, 406.

Perigynous flowers, 355 .

Periodicity of Algæ, 192, 2 I I.

Perisperm of Angiosperms, 371 ; of Conifers, 352 (fig.).

Peristome of Mosses, 285 (fig.), 286; mechanism of, 287 .

Perithecium, 24I (fig.), 242.

Permanent mounting, 404, 405.

Permeability of cell-walls, 9, 29, $31,32,36,67,71,91,93$, I36.

Permeability of cytoplasm, 9, I2, I3, I5; and guard-cells, Ioo; and leaf-movements, II 5 ; demonstration of, 12, I3.

Peronospora parasitica, 243.

Petals, 63, 103, 354, 373.

Petiole, and leaf-fall, I4I ; movement of, II5, II6; nectaries on, I48; of Angiosperms, II 3 (fig.), II 5 ; of Cycads, 322 ; of Ferns, 295.

Petrifactions, 328.
Peziza, 231, 239 (fig.), 240 (fig.), 407.

Phæophyceæ, 406; reproduction of, 216, 218, 219 (fig.), 22 I (fig.), 224-6 (fig.) ; structure of, 198-204 (figs.), 208, 210.

Phanerogamia, 307, 32I, 334-73 (figs.), 407.

Phaseolus multiflorus, II5, 37I ; epidermis of stem of, 93, 94 (fig.) ; hydathodes of, I44, I 45 (fig.), I 47.

Phelloderm, 135, I36 (fig.), I37, I38 (fig.), I39.

Phellogen, I35-42 (figs.) ; and aerenchyma, I75; of Conifers, 338, 339 (fig.).

Phenylhydrazine hydrochloride, 47, 401 .

Phloem, 28, 29 (fig.), 82 ; function of, $28,65,88$; secondary, I 8 , I I9 (fig.), I 20, I 2 I (fig.), 130,134 .

Phloem of, aquatics, I7I (fig.), I72, I 73 (fig.) ; Conifers, 339 (fig.), 340, 34I, (fig.) ; Dicotyledons, 28, 29 (fig.), 65, 82; Ferns, 292 (fig.), 293 (fig.) ; leaves, I07, II2, II3; Monocotyledons, 84,85 (fig.), 86; parasitic Angiosperms, I79; petioles, II3 (fig.) ; roots, 66 (fig.), 68, 69,70 (fig.) ; stems, 76, 77 (fig.), 79, 8I (fig.), 82, 84 (fig.), 85 (fig.), 86, I 8 (fig.).

Phloem-bundles, 86.

Phloem-parenchyma, 29 (fig.), 78 (fig.), $79,82,83,84,86,88$, I30, I32; of Conifers, 339 ; of Ferns, 292 (fig.), 293 (fig.).

Phloroglucin, 402; and lignified walls, 32 .

Phormium tenax, 34.

Phosphorescence of meat, 265.

Photosyn thesis. See Carbon dioxide assimilation.

Phototaxis, 183, 214.

Phragmidium bulbosum, 248.

Phycomycetes, 231-8 (figs.), 240, $243,406$.

Phylogeny, 210, 396.

Physiology of, cells, 8-I5; Bacteria, 26I, 263, 264-7; Fungi, $55,72,100,252-7$.

Phytophthora infestans, 230, 231 , 253,254 ; structure and reproduction of, 234, 235 (fig.), 236. 
Picea excelsa, 63, 129, 350 ; habit of, 334, 335 (fig.); leaf of, 343.

Pigments, 6o, 64, 150 ; of Bacteria, 264; of chloroplasts, 109; of cork, 137 ; of flowers, 63 ; of Fungi, 23I ; of Hamatococcus, I 88; of heart-wood, I 25, I26; of Lichens, 26I ; of Seaweeds, I98, 199.

Piliferous layer, 66, 67 (fig.), 69, $72,77$.

Pimpernel, Yellow (Lysimachia nemorum), stoma of, 160 (fig.).

Pimpinella anisum, 62.

Pine, Oregon (Pseudotsuga douglasii), 129; Pitch - (Pinus palustris), I 29; Stone - (Pinus Pinea), 352 (fig.).

Pinguicula, glands of, I49, 150 (fig.).

Pinus, archegonia of, 348,349 (fig.) ; economic importance of, 63, I 29 ; embryology of, 350, 35 I (fig.); female cones of, 345 (fig.), 346,347 (fig.) ; fertilisation of, 350 ; habit of, 334 , 335; leaf-structure of, I 52, I 66, 342-4 (figs.); male cones of, 344, 345 (fig.), 347 (fig.) ; ovules of, 348,349 (fig.) ; pollen of, 345 , 347 (fig.), 35I (fig.) ; pollination of, 348,350 ; seeds of, $35 \mathrm{I}, 352$ (fig.) ; seedlings of, 337,338 (fig.); stem-structure of, 338-4I (figs.) ; wood of, 37 (fig.), 340, 34I (fig.).

Pinus monophylla, 335; $P$. palustris, I 29, 340; $P$. pinaster, 63; P. pinea, 352 (fig.) ; $P$. strobus, 129,$335 ; P$. sylvestris, 37 (fig.) , 129, 152, 166, 334 et seq. (figs.), 39r.

Piper nigrum, 63.

Pit, simple, 30 (fig.), 33, I2I, I22, 276; bordered, 33,35 (fig.), $36,37,68,120,293,340,34 \mathrm{I}$ (fig.).

Pit-canals, 33.

Pit-membrane, 30 (fig.), 36, 26.

Pitch Pine (Pinus palustris), I 29.

Pitcher-plant (Nepenthes), glands of, 149.

Pith of, petiole, II 4 ; root, 69,70 (fig.), 72, 90; stem, 19, 30, 43, 45, 76, 77 (fig.), 80 (fig.), I 72,173 (fig.), 323, 340 .

Pith-cavity, 80, 8I (fig.).

Pitted vessels, 35 (fig.), 36 ,
Placenta of, Angiosperms, 356, 359, 365 ; Ferns, 295, 296, 298 (fig.). Plane (Platanus), bark of, I4I ; hairs of, 102, 103.

Plankton, 192, I93 (fig.), 207, 2 I0.

Plantago lanceolata, 367.

Plants, compared with animals, I4, 25, I8I, I88, I93, I94, 247, 307; differences between animals and, 188, 193, 194.

Plasmatic membrane, 3, 4, 8, 9, I I, I 2, I 4 .

Plasmodiophora brassica, 25I, 254 (fig.).

Plasmodium (of Slime-Fungi), 250.

Plasmolysis, false, I3, I4, I 5 ; nature of, 9 ; true, 5 (fig.), 8, I I, I 5 .

Plasmopora viticola, 243.

Plastids, 4, 25, 41, 50, 64 .

Platycerium, 309.

Plerome, 19, 20, 89, 369.

Pleurococcus, I, 190-92 (fig.).

Pleurotanium, 209 (fig.).

Pleurotus ostreatus, Plate II.

Plimmer, 54 .

Plocamium, 204.

Plum (Prunus), 93; Silver-leaf disease of (Stereum purpureum), 250, 252.

Plumule of, Angiosperms, 369 (fig.), 370,37 I (fig.), 372 ; Conifers, 338 (fig.), 350, 35I (fig.) ; Cycads, 326.

Pneumococcus, 263 (fig.).

Podocarpus, 337.

Poisonous plants, 48, 60, 6I, 255 .

Poisons, and protoplasmic movement, 4 .

Polar nuclei (of embryo sac), 357 (fig.), 366, 367 (fig.), 368 .

Polarised light and starch-grains, 43.

Poles (of nuclear spindle), 23.

Pollarded trees, 142.

Pollen mother-cells, 360 (fig.), 36r.

Pollen of, Angiosperms, 356, 361, 362 (fig.), 367 (fig.), 373 ; Conifers, 345, 348, 349, 35I (fig.) ; Cycads, 326 ; waterplants, 361 .

Pollen-chamber, of Cycads, 325 (fig.), 326; of Lyginopleris, 330, $331,332,333$ (fig.).

Pollen-sacs of, Angiosperms, 359 , 360-63 (figs.) ; Conifers, 345, 347 (fig.) ; Cycads, 323, 324 (fig.). 
Pollen tube of, Angiosperms, 363, 364 (fig.), 365, 367 (fig.); Conifers, 349 (fig.), 350, 35I (fig.), 353 ; Cycads, 325 (fig.), 326, 327 (fig.), 332.

Pollination, by insects, 46, 36I, 373 ; by water, 361 ; by wind, 361, 373; of Angiosperms, $355,356,363,372$; of Conifers, $345,348,349,350$; of Cycads, 326 ; of Lyginopteris, 330, 33I.

Pollination-drop, 332 ; of Conifers, 348 ; of Cycads, 326.

Pollinia (of Orchids), 361, 362 (fig.).

Polyarch roots, 69, 70 (fig.).

Polyembryony, 37I (fig.), 372, 390.

Polygonaceæ, 356.

Polygonum, ovule of, 358 (fig.).

Polygonum convolvulus, extrafloral nectaries of, 148 ; petiole of, I 3 (fig.).

Polypetalous Dicotyledons, 357.

Polypody (Polypodium), 309; sori of, 29 I (fig.), 295.

Polyporus, 255; P. squamosus, 250 (fig.).

Polysaccharides, 3I, 43, 44, 48, 231; hydrolysis of, 46,48 .

Polystictis versicolor, Plate II.

Polytrichum, 274 (fig.), 276, 286 ; P. juniperinum, 269.

Pond-scums (Algæ), I95 et seq.

Pond weed(Potamogeton), stamen of, 359 (fig.) ; stem-structure of, I 72 , 174 (fig.).

Pondweed - family (Potamogetonaceæ), I 78 .

Poplar (Populus), I30, I4I, I42 ; vessels of, 28,35 (fig.).

Poppy (Papaver), 6I, I 55, 359.

Poppy-family (Papaveraceæ̇), I53, I 54.

Populations, inheritance among, 378.

Populus, I30, I4I, 142 ; vessels of, 28,35 (fig.).

Pores of, Liverworts, 273 (fig.); pollen-grains of Angiosperms, 362 (fig.); sieve-plates, 28 ; stomata, 96, 98, 99 (fig.), Ioo.

Porogamy, 364 (fig.), 365.

Porometer, 99, Ioo.

Potamogeton, 359 (fig.) ; P. lucens, I72 ; $P$. natans, I72, I 74 (fig.) ; $P$. pectinatus, 172 .

Potamogetonaceæ, 178 .

Potash, and callose, 82 ; and cork, I 36 ; and fats, 49 .
Potato (Solanum tuberosum), foodcontent of, 4I, 42 (fig.), 43, 50, 52,53 ; starch of, 41,42 (fig.).

Potato-blight (Phytophthora infestans), 230, 231, 253, 254; structure and reproduction of, 234, 235 (fig.), 236.

Potato-family (Solanacex), 58, 6o, $6 \mathrm{I}, 8 \mathrm{I}$.

Preservation of fossil-plants, 312, 328 ; of plant-material, 404 .

Prickles, I04.

Prickly Pear, 392.

Prickly Saltwort (Salsola kali), leaf of, 94, I66.

Primary endosperm nucleus (of Angiosperms), 366. (fig.).

Primary medullary rays, 76,78 (fig.), I I 8 ; of Conifers, 340 ; of secondarily thickened stems, I 19 (fig.), I 20, I 22, I 24; of secondarily thickened roots, 132.

Primrose (Primula), hairs of, 105 (fig.) ; hybrids of, 387,388 (fig.).

Primulaceæ, 356.

Procambial strands, 89, 90, II 7, I $7 \mathbf{I}$.

Proembryo of, Angiosperms, 368, 369 (fig.), 371 (fig.), 372 ; Conifers, 350, 35I (fig.) ; Cycads, 327 (fig.).

Prop-roots, structure of, 73, 74 (fig.). Prosenchymatous cells, 27, 32, 37 . Proteaceæ, distribution of, 394 (fig.). Proteases, 53.

Protection against, animals, 39.48 , 58,64, I04, I06, I 26, I 57 ; excessive transpiration, $64,9 \mathrm{I}$, 92, I06, I37, 158-65, 203; heat-rays, $64,95,102, \mathrm{I}_{3} 8$; injury, 92, II $5, I_{42}, I_{55}$; intense light, 95, I02, I IO, I II, II 2, I99; parasites, I38, I42.

Protection of embryos, 321, 332, 333,373 ; of lateral roots, 72 .

Protective tissues, 9I et seq., I35 et seq.

Proteins, 2, 7, I 4, 40, 50-52, 53. I 43, 262, 343 ; decomposition of, by Bacteria, 265 ; hydrolysis of, 52 ; in latex, 155 ; reactions of, 3,49 ; soluble, 50 ; translocation of, 52,88 .

Proteolytic enzymes, 52, 53, 54 . I 49.

Prothallus of, Angiosperms, 373 ; 
Conifers, $\quad 348,349$ (fig.) ; Cycads, 325 (fig.), 326, 327 (fig.) ; Equisetum, 313; Ferns, 299 (fig.), 300-302 (fig.), 304, 305, 313; Lycopodium, 3I6 (fig.) ; Lyginopteris, 330, 33I ; Selaginella, 3I9, 320 (fig.), 32 I. Protococcus (Hamatococcus), 186.

Protonema, 287 (fig.), 288.

Protophloem, 90.

Protoplasm, 2, 3 ; composition of, 2 , 50 ; growth of, 25 ; movement of, 4,6 ; permeability of, 9, I2, I3, I5; properties and structure of, 3,4 .

Protoplasmic connections, 24, 30, I90.

Protoplast, 2, 3 .

Protoxylem, $36,68,89$; of Cycads, 322 ; of Ferns, 292 (fig.), 293 ; of leaf, II2, II4; of Lyginopteris, 328 ; of root, 66 (fig.), 68, 69, 70 (fig.), 3I 8, 34I ; of stem, 78 (fig.), 79, 80 (fig.), 8r (fig.), 83 (fig.), 84 (fig.), 85 (fig.), 86, I 8 (fig.).

Protoxylem canal, 85 (fig.), 86.

Prussic acid, 48; and enzymeaction, 56.

Psamma arenaria, leaf-structure of, 92, I63, I64 (fig.).

Pseudomonas, 262, 263 (fig.).

Pseudotsuga douglasii, I28, 129; cone of, 352 (fig.), 353.

Psilophytales, 407.

Psilotum, 407.

Psychotria ipecacuanha, 6r.

Pteridophyta, 289-321 (figs.), 333, $334,355,370,373,407$.

Pteridosperms, 327-33 (figs.), 334, 407.

Pteris aquilina, extrafloral nectaries of, I48, I 49 (fig.) ; habit of, 289,290 (fig.); leaf of, 60 , I I , I69, 291, 295 ; rhizome of 290 (fig.), 292-4 (figs.) ; sori of, 296 (fig.)

Ptomaines, 6r.

Puccinia anemones, 247, 248; $P$. graminis, 245-7 (fig.) ; P. malvacearum, 248, 253

Puff-ball (Lycoperdon), 245, 250, 25 I (fig.), 407.

Pulling-strains, resistance to, in aquatics, 88 , I 70 ; in roots, 72 .

Pulses, 43.

Pulvinus, structure of, II5, II6 (fig.).
Pure cultures (of Bacteria), 264; - lines, 377,378 .

Purine-derivatives, $6 \mathrm{r}$.

Purple Dead-nettle (Lamium purpureum), 364; - Loosestrife (Lythrum salicaria), I74.

Putrefaction, 265.

Pyrenoids, 7 (fig.), 8, I81, I82 (fig.) I 87 (fig.), I96 (fig.), 207, 208, 209 (fig.), 217 (fig.), 227 (fig.).

Pyrenomycetes, 407.

Pythium debaryanum, 23I, 252, 253, 406; structure and lifehistory of, 234, 235 (fig.), 236.

Qualitative variations, 374, 375 (fig.), 376 (fig.).

Quantitative variations, 374 .

Quercus, 130, 364; bark of, 60, I 4I ; cork of, I4O ; wood of, 28, 35 (fig.), 122, 128 (fig.).

Quercus intermedia, $389 ; Q$. suber, I 40.

Quillaia-bark, 48.

Quillwort (Isoetes lacustris), 3 I 4 , 3 I 5 (fig.).

Quinine, 6r.

Radiation, prevention of, 138 .

Radicle of, Angiosperms, 369 (fig.), 370,37 I (fig.); Conifers, 350 , 35I (fig.), 352 (fig.) ; Cycads, 326 ; Selaginella, 320 (fig.).

Raffia-tape, 34 .

Rainfall and plant-distribution, 39r. Raising of stomata, 96, I60 (fig.), I6I, I62, I70.

Ramentum (of Ferns), 29I (fig.).

Rancidity of butter, cause of, 265 .

Ranunculaceæ, 60, 354, 357.

Ranunculus aquatilis, I77 (fig.) ; $R$. auricomus, 92 (fig.); $R$. repens, 66.

Raphe (of anatropous ovules), 357 (fig.), $35^{8}$ (fig.).

Raphia, 35.

Raphides, 58, 59 (fig.).

Rattle, Yellow (Rhinanthus), hydathodes of, I44, I45 (fig.).

Rays, of Conifers, 340,34 I (fig.) ; of Lyginopteris, 328, 330 (fig.) ; of root, $\mathrm{r} 32$; of secondary phloem, I30; of secondary wood, II9 (fig.), I20, 122-4 (fig.), I 28 (fig.); primary -, 76, 77 (fig.), 78 (fig.), II 8.

Rcactions of, alkaloids, $6 \mathrm{I}$; callose, 82 ; cellulose, $4,31,32$; 
cuticle, 92 ; dextrin, 46, 47; ethereal oils, 63 ; fats, 49 ; glycogen, 231; inulin, 44,47 ; lignified walls, 32 ; mucilage, 38,95 ; oils, 49 ; oxalate of lime, 59; proteins, $3,49,50$; starch, 8, 4I, 43; suberised walls, I36, I37; sugars, 47 ; tannins, 49, 60; terpenes, 63 . Reagents, microscopic, $4 \mathrm{OI}, 402$.

Receptacle of flower, 354, 355, 370 . Reception of pollen, in Angiosperms, 356 ; in Gymnosperms, 326, 348 ; in Lyginopteris, $33 \mathrm{I}$.

Receptive spot, 221 (fig.), 222, 224. Recessive characters, 384 et seq.

Red Algæ (Rhodophyceæ), 203, 406; reproduction of, 205 (fig.), 228; structure of, 203, 204, 205 (fig.), 208.

Red Deal (Pinus sylvestris), I29; - Currant (Ribes), II5.

Red Snow, I88.

Reducing sugars, 47.

Reduction of, flowers, 354, 355 ; leaf-surface, I 58 , I 78 ; number of ovules, 356 ; number of spores, 319, 332 ; pollen-output, 373; vascular system, I $7 \mathrm{I}-3,3 \mathrm{I}$.

Reduction division, 305-7 (fig.), $361,365,372$; and segregation in hybrids, 385,390 .

Remedies against parasitic Fungi, 254.

Reproduction, I90, I93, I95, I97, $207,2 I I, 212,2 I 5,2 I 6,228$, $229,320,32 \mathrm{I}$.

Reproduction of, Abies, 336 (fig.), 344, 347; Adiantum, 296; Agaricus, 248, 249 (fig.); Algæ, 212-29 (figs.); Angiosperms, 357-73 (figs.); Araucaria, 345, 346, 347; Ascomycetes, 238-45 (figs.); Asplenium, 296 (fig.), 305 (fig.); Athyrium, 305; A ulacomnion, 277; Bacteria, 262, 267 (fig.) ; Basidiomycetes, 245-5I (figs.) ; Blechnum, 295; Boletus, 250, 25I (fig.); Bryophyta, 27788 (figs.); Cedrus, 353; Cephalozia, 27 I (fig.) ; Chlamydomonas, I82-6 (figs.), I 88, 220; Cladophora, I96 (fig.), 197, 2I2, 2I5, 2I6, 220 ; Clavaria, 250, 25I (fig.); Claviceps purpurea, 24I, 242 (fig.) ; Conifers, 344-53 (fig.) ; Conjugatæ, 216, 226-8 (fig.) ; Cryptomeria, 347 ; Cupressineæ, $347,348,350$; Cupressus, 345 . 347 (fig.) ; Cyanophyceæ, 206 (fig.), 2I6, 220; Cycads, 323-7 (figs.) ; Cystopus, 23I-4 (fig.) ; Desmids, 209 (fig.) 2I6, 220, 228; Diatoms, 216 219, 228; Ectocarpus, 212 , 218, 219 (fig.), 220; Empusa, 238; Equisetum, 312, 313 (fig.) ; Erysiphaceæ, 242-4 (fig.); Eudorina, I88 (fig.), I89; Eurotium (Aspergillus), 242, 243 (fig.) ; Fegatella, 270, (fig.), 279; Ferns, 29I (fig.), 295-309 (figs.) ; Fucus, 201 (fig.), 216, 220, 221 (fig.), 224-6 (fig.) ; Funaria, 279, 285 (fig.), 287 (fig.) ; Geoglossum, 239 (fig.), 240; Hamatococcus (Sphaerella), I88; Hepaticæ, 277-9 (fig.), 280, 28I (fig.), 282-4 (fig.), 288; Hormidium, 2 I 5 (fig.), 216 ; Hydnum, 250, 25 I (fig.); Hymenophyllaceæ, 296, 297 (fig.), 301 ; Juniperus, 352 (fig.), 353 ; Laminaria, 218 ; Lavix, 336 (fig.), 345, 346, 347; Leafy Liverworts, 277, 279, 280, 282, 288 ; Lichens, 259, 260 (fig.), 26I ; Lycoperdon, 250, $25 \mathrm{I}$ (fig.) ; Lycopodium, 3I5, 3 I6 (fig.); Lyginopteris, 330-33 (figs.); Marchantia, 270 (fig.), 273 (fig.), 277, 279, 28o, 28I (fig.), 282; Morchella, 239 (fig.), 240; Mucor, 236-8 (fig.); Musci, 277, 279 (fig.), 280, 284-8 (figs.) ; Myxomycetes, 25I ; Nectria, 242 ; Nephrodium filix-mas, 296 (fig.), 297, 298 (fig.), 299 (fig.), 300; Edogonium, 216, 217 (fig.), 220-23 (fig.) ; Oomycetes, $23 \mathrm{I}-6$ (figs.), 238 ; Osmunda, 300, 304, 308, 309 (fig.); parasitic Angiosperms, I79, 365; Pediastrum, 192; Pellia, 270 (fig.), 277, 278 (fig.), 280, 281 (fig.), 282, 283 (fig.); Pelvetia, 226; Penicillium, 242, 243 (fig.); Peziza, 239 (fig.), 240 (fig.); Phycomycetes, 23I-8 (figs.); Phytophthora, 234, 235 (fig.), 236 ; 
Picea, 350; Pinus, 344, 345(fig.), 346,347 (fig.), 348, 349 (fig.), 350, 35 I (fig.), 352 (fig.), 353 ; Pleurococcus, I9I, 192 (fig.) ; Polypodium, 296; Polyporus, 250 (fig.) ; Polytrichum, 274, (fig.), 286; Pteris, 296 (fig.), 300 ; Puccinia graminis, 245-7 (fig.) ; Pythium, 234, 235 (fig.), 236; Rhodophyceæ, 205 (fig.), 228 ; Riccia, 284; Saccharomyces, 256 (fig.); Saprolegnia, 235 (fig.), 236; Scenedesmus, I92 ; Sclerotinia, 239 (fig.) 24I ; Scolopendrium, 296 (fig.) ; Selaginella, 318-20 (fig.); Sequoia, 347, 352 (fig.) ; Sphagnum, 275 (fig.); Spirogyra, $2 \mathrm{x} 6,226-8$ (fig.) ; Taxus, $344,345,346$ (fig.), 347 ; Thuja, 337 (fig.), 347, 350 ; Tuber, 240, 24I ; Ulothrix, 2 I2I 5 (figs.), 220; Uredineæ, 245-8 (fig.) ; Ustilagineæ, 248; Vaucheria, 2I7, 2 I 8 (fig.), 220, $22 \mathrm{I}$ (fig.), 223, 224; Volvox, 190; Xylaria, 239 (fig.), 242 ; Zygnema, 226-8 (fig.) ; Zygomycetes, 236-8 (fig.). Reproductive cells, asexual, 2 I2, 219. See Gonidia, Spores, and Zoospores.

Reproductive cells, sexual, I84, 214, 220. See Egg, Gametes, and Spermatozoids.

Resedaceæ, distribution of, 394 (fig.). Reserve-cellulose, 48, 53 ; hydrolysis of, 53.

Reserve-substances, 40-52, 242, 319; accumulation of, 13 ; in seeds, $40,44,45,47,49,50$, $52,333,351$; in storage-organs other than seeds, $44,45,50$; nitrogenous, 50 ; storage of, $40,88,132$; translocation of, $6,41,46,52,124,132,282$.

Resin-cells, 340.

Resin-passages, 63, 339 (fig.), 340, 341,342 (fig.), 343, 344 (fig.).

Resins, 63, 64, I25, I 5 I, I 55, 334 .

Resistance to, bending, I I 5, I 72 ; disease, 265, 384; drought, I86, I9I, 205, 2I5; pulling strains, 72,88, I 70 ; tearing, 93 , I I 5 .

Respiration, 178, 20I, 257; and protoplasmic movement, 4 ; of fat-containing seeds, 50 .
Respiratory cavity, 97, I08 (thg.), I Io, I64 (fig.), I66, 343.

Resting nucleus, 2 I (fig.), 22.

Resting stages of, Chlamydomonas, I88; Cladophora, 216; Fungi, $24 \mathrm{I}$ (fig.); Hamatococcus (Sphaevella), I87 (fig.), I88 ; Mucor, 238; Ulothrix, 2 I 5 (fig.) ; Yeast, 256 (fig.). See also Oospores and Zygospores. Retention of megaspores, 32I, 332, 333.

Reticulate tracheids, II 3 ; - veins, I 12 ; - vessels, 35 (fig.), 36,83 (fig.).

Retinospora, 337.

Retting of fibres, 265.

Rheum rhaponticum, Ir3.

Rhinanthus, hydathodes of, I44, I45 (fig.).

Rhizocarpon confervoides, 258 (fig.).

Rhizoids of, Fern-prothallus, 299 (fig.), 300, 301 (fig.) ; Liverworts, 270 (fig.), 27I, 272, 273 (fig.), 278 (fig.); Mosses, 274, 287 (fig.), 288.

Rhizomes, 82, 88, I I I ; of Ferns, 289, 290 (fig.); of Horsetails (Equisetum), 3II (fig.); of Mosses, 274.

Rhizophores (of Selaginella), 317 (fig.), 318.

Rhizopus stolonifer, 237.

Rhodophyceæ, 203, 406 ; reproduction of, 205 (fig.), 228 ; structure of, 203, 204, 205 (fig.), 208. Rhubarb (Rheum rhaponticum), I I3. Rhus vermicifera, 155.

Ribes, 105, II 5 ; cork of, 137, 138 (fig.).

Riccia, 284, 407.

Rice (Oryza sativa), 43, 53, 257 ; starch of, 42 (fig.), 43.

Ricinus communis, aleurone grains of, 50, 5I (fig.); fat of, 49 ; hypocotyl of, I 8 (fig.) ; seeds of, $371,372,376$ (fig.).

Ring-bark, I $4 \mathrm{I}$.

Ringworm, cause of, 253.

Rivularia, 206 (fig.).

Robinia pseudacacia, II5, I26.

Roccella, 26 I.

Rock-pools, plants of, 195, 203, 204.

Rock Rose (Helianthemum vulgave), 379 (fig.).

Rolled leaves, I 78 ; structure of, I63 (fig.), I64 (fig.). 
Root-cap, I8, 20 (fig.), 65, 7 I (fig.), 74.

Root-hairs, 3, 10, 13, 65, 66, 67 (fig.) ; function of, 72 .

Root-nodules of Leguminosæ, 267 , 268.

Roots, aerial, 73, 74, 75 (fig.) ; anomalous thickening of, 133 ; cork-formation in, I3I (fig.), I 37 ; fleshy -, I 32, I33, I 54, I55 (fig.) ; growing point of, I6, I8, 20 (fig.) ; lateral - 65 , $7 \mathrm{I}$ (fig.) ; of Ferns, 290, 303 ; of Horsetails (Equisetum), 3 II ; of Lycopodium, 3I5; of Lyginopteris, 328; of Selaginella, 317 (fig.) ; prop-, 73, 74 (fig.); relation of structure and function in, 72 ; secondary thickening of, I3I (fig.), I32 ; transition from stem to, 89 (fig.).

Root-structure of, Bean (Phaseolus), 7 I (fig.); Beet (Beta), I33; Conifers, 34I ; Creeping Buttercup (Ranunculus repens), 66-9 (figs.); Dandelion (Taraxacum), I54; Dendrobium, 75 (fig.); Dicotyledons, 65-70 (figs.) ; Ferns, 295; Iris, 70 (figs.) ; Leguminosæ, 72 ; $L y$ ginopteris, 330; Maize (Zea mais), 73, 74 (fig.); Monocotyledons, 70 (fig.), 7 I ; Nettle (Urtica), I32; Salsify (Tragopogon), 44, I 32, I 54, I 55 (fig.) ; Selaginella, 318; Wallflower (Cheiranthus cheiri), 70; White Mustard (Sinapis alba), 69.

Rope, 34 .

Rosaceæ, I37, 355, 357.

Rose (Rosa), 104, I37, 389; - Mildew (Sphaerotheca pannosa), 243.

Rosette-habit of Alpines, I70.

Rosin, 63.

Rotation of crops, 248, 267.

Royal Fern (Osmunda regalis), habit of, 289, 309 (fig.) ; prothallus of, 304; sporangia of, 300, 309 (fig.).

Rubia peregrina, stoma of, 97 (fig.), 98.

Rubiaceæ, 6r.

Rubber, I 57.

Rue (Ruta graveolens), secretory cavities of, I 5 I.

Rumex, II 3 .

Runner Bean (Phaseolus), I15,
371; epidermis of stem of, 93, 94 (fig.) ; hydathodes of, I 44, I 45 (fig.), I 47.

Ruscus aculeatus, cladode-structure of, 159 (fig.), I 66.

Rush (Juncus), I 78 ; leaf-structure of, I65 (fig.), I 66.

Rust-Fungi (Uredineæ), I 43, 245-8 (fig.), 252, 253, 407.

Rust of Wheat (Puccinia graminis), 384 ; structure and life-history of, 245-7 (fig.).

Ruta graveolens, secretory cavities of, 151 .

Rye (Secale), fungal diseases of, 239, 241.

Saccharomyces, physiology of, 53, $54,56,57,256,257,265$; structure and reproduction of, 255,256 (fig.).

Saccharose, 45.

Saccharum officinarum, 45, 46 .

Saffron, 64.

Safranin, 404, 405.

Sagittaria, embryology of, 37 I (fig.).

Sago, 43, 323.

Sago-Palm (Cycas revoluta), 322, 323.

St. John's Wort (Hypericum), secretory organs of, I5I, I 53 .

Saké, 257.

Salicaceæ, 357.

Salicase, 48 .

Salicin, 48 .

Salicornia, aerenchyma of, $\mathbf{1} 75$ (fig.).

Saligenin, 48 .

Salmon-disease (Saprolegnia ferox), $230,236$.

Salsify (Tragopogon), root-structure of, $44,132,154,155$ (fig.).

Salsola kali, leaf of, 94, 166.

Saltmarsh plants, 178,216 ; roothairs of, Io.

Sambucus, cork of, I 36 (fig.) ; leaf of, I Io, I I I (fig.); lenticels of, I 40 (fig.) ; pith of, 30 (fig.), I 5 I.

Samphire, Marsh (Salicornia), aerenchyma of, I 75 (fig.).

Sand-dune plants, 163 .

Sandalwood (Santalum album), I30.

Sap, cell, 3, 6, Iо, 26, 60, 63 ; nuclear - 22.

Sap-wood, 125, 126.

Saponaria, 48 .

Saponification of fats, 49 . 
Saponins, 48 .

Sapotaceæ, laticiferous elements of, I 57.

Saprolegnia, 23I, 235 (fig.); S. ferox, 236.

Saprophytic Algæ, 206 ; - Angiosperms, I78, I79; - Fungi, 230 et seq., 252, 253.

Sarcina, 262.

Sargasso Sea, 203.

Sargassum bacciferum, 203, 204 (fig.).

Satinwood (Chloroxylon swietenia), I30.

Saxifraga sarmentosa, 8, 63.

Saxifrages (Saxifraga), chalk-glands of, $\mathrm{I} 47$.

Scalariform tracheids, 293 (fig.).

Scale-bark, I4I.

Scale-leaves, structure of, III.

Scales of Liverworts, 271, 272, 273 (fig.) ; of Mosses, 274.

Scars, I 4 I, I 42.

Scenedesmus, I9I (fig.),. I92, 207 ; $S$. obliquus, I9I (fig.); $S$. quadricauda, I9I (fig.).

Scharlack Red, 402; and cork, I36; and cuticle, 92 ; and fats, 49.

Schizogenous secretory cavities, I5I, I52 (fig.).

Schultze's solution. See Chlor-zinciodide.

Scilla, 44, 365; growing point of root of, 20 (fig.) ; S. nutans, 44.

Scion, I42, 389 .

Scirpus, 178 .

Sclerenchyma, 32-4 (fig.) ; of assimilatory stems, I62 (fig.); of Ferns, 290 (fig.), 292, 293 (fig.), 294 (fig.), 295; of Hakealeaf, I66; of leaf, II2; of Lyginopteris, 328, 330 (fig.); of secretory canals, I 52 (fig.); of stem, 8o, 8I (fig.), 83 (fig.), 87 (fig.), I 39 (fig.).

Scleroderma vulgare, 25 I (fig.).

Sclerotinia, 239 (fig.), 24 I.

Sclerotium, 239 (fig.), 241 (fig.), $242,255$.

Scolopendrium vulgare, leaf and sorus of, 29I (fig.), 296 (fig.).

Scotch Fir (Pinus sylvestris), archegonia of, 348, 349 (fig.) ; embryology of, 350, 35I (fig.) ; female cones of, 345 (fig.), 346, 347 (fig.) ; fertilisation of, 350 ; habit of, 334,335 ; leaf- structure of, I52, I66, 342-4 (figs.) ; male cones of, 344, 345 (fig.), 347 (fig.) ; ovules of, 348,349 (fig.) ; pollen of, 345,347 (fig.), 35 I (fig.); pollination of, 348,350 ; seeds of, $35 \mathrm{x}, 352$; seedlings of, 337,338 (fig.) ; stem-structure of, 338-4I (figs.) ; wood of, 37 (fig.), 340, 34I (fig.).

Scrophulariaceæ, 60, 367 .

Scutellum (of Grasses), epidermis of, I 50 .

Sea Blite (Sucda maritima), I66; - Buckthorn (Hippopha rhamnoides), 103 (fig.); - Grass (Zostera), I72, 36I ; - Holly (Eryngium maritimum), 93, I I 3 (fig.) ; - Lettuce (Ulva), r98, 2 I 5 (fig.); - Purslane (Arenaria peploides), 94.

Seasoning of timber, I28, 129.

Seawater, artificial, 402 ; equivalent osmotic pressures of, 403; use of, in determining osmotic pressure, II, I5; in plasmolysis, 8,15 .

Seaweeds, 38, I80, I95, 198-205 (figs.), 2 I I ; ash of, I5.

Secondary bundles, I33 (fig.), 134 . Secondary medullary rays of, Conifers, 340, 34I (fig.) ; Dicotyledons, I I9 (fig.), I20, 122-4 (fig.), I 28 (fig.), I 30 ; roots, I 32 . Secondary phloem of, Conifers, 339, $34 \mathrm{I}$ (fig.) ; roots, I3I, I32; stems, I I 8 , I I9 (fig.), I 20 , I 2 I (fig.), 130.

Secondary thickening, II 7-26 (figs.), I30-43 (figs.) ; anomalous -, I32-4 (fig.) ; effects of, II9, I20, I35, I 37 ; of Chenopodiaceæ, 133; of Conifers, 338, 339 (fig.); of Cycads, 323 ; of Dicotyledonous stems, I I 726 (figs.) ; of Dracana, I33 (fig.), I34; of fossil Pteridophyta, 312, 3I5; of leaf, II2; of Lyginopteris, 328, 330 (fig.); of Monocotyledons, I33 (fig.), I34; of roots, I3I (fig.), I32. Secondary wood of, Conifers, 127 , 339-4I (figs.); Cycads, 323; Lyginopteris, 328, 330 (fig.); root, I3I, I32; stem, II 8 , I $19-26$ (figs.).

Secretion, 58, I44-57; in glandular hairs, I04-6; of 
digestive juices, I 49, I50 ; of sugar, 148, 149: of water, $144-8$.

Secretory canals, 152, 339 (fig.), 340, 34I, 342 (fig.), 344 (fig.); - cavities, 63, 151, I52 (fig.); - cells, 63, I30, 151, 328, 340 ; - hairs, ro5 (fig.), ro6, I44, I 45 (fig.), I49, I50 (fig.).

Section-cutting, 405, 406.

Sedatives, 6r.

Sedge (Carex), 88.

Sedge-family (Cyperaceæ), stoma of, 100.

Sedum, leaf of, II6, I66; stoma of, 97 (fig.), 98.

Sedum album, II6; S. spectabile, 97 (fig.).

Seed-coat, 352 (fig.), 370, 37 I (fig.).

Seed-plants, 322-73 (figs.).

Seedlings, diseases of, $23 \mathrm{r}, 235$ (fig.); of Conifers, 337, 338 (fig.), 352 ; of Ferns, 294, 304 (fig.); of Selaginella, 320 (fig.).

Seeds, 332,333 ; dispersal of, 46, $50,203,353,372$; food-

(969 Teserves of, 40, 44, 45, 47, 49, 50, 150; of Angiosperms, 37 I-3; of Conifers, 35r, 352 (fig.); of Cycads, 325 (fig.), 327; of graft-hybrids, 389 ; of Lyginopteris, 33I-3 (figs.) ; of parasites, I 79, 372 ; of Taxus, 346 (fig.).

Segments (of growing point), I6 (fig.), I 7 (fig.), I8, 195 .

Segregation of characters in hybrids $384-8,390$.

Selaginella, 314, 32 r, 332, 373, 407; anatomy of, 317,318 ; archegonia of, 319, 320 (fig.) ; cones of, 318 (fig.); embryology of, 319,320 (fig.) ; habit of, 316, 317 (fig.); prothalli of, 3I9, 320 (fig.) ; spermatozoids of, 319,320 (fig.); sporangia of, 318. (fig.), 319.

Selaginella kraussiana, 317; S. martensii, 317 (fig.) ; S. rupestris, 319; $S$. spinosa, 316, 317 (fig.); S. umbrosa, 318 (fig.).

Selection, $378,380,390$.

Self-fertilisation, 224, 385 ; - pol$\therefore$ lination, $374 ;-$ - pruning, I 42 . Semiparasites, I 44,147 .

Semipermeable membranes; 8,9 , artificial - 402, 403.
Sensitive Plant (Mimosa pudica), pulvini of, I I 5, I I6 (fig.).

Sepals, 354, 373.

Septa (of vessels), 28, 83, I20, I2I. Septate wood-fibres, I 2 r.

Sequoia, cone of, 347,352 (fig.).

Serrated Wrack (Fucus serratus), 201 (fig.), 202.

Seta (of sporogonium) of Liverworts, 282, 283 (fig.) ; of Mosses, 284, 285 (fig.).

Sewage, conversion of, 265 .

Sexual cells. See Egg, Gametes, Spermatozoids.

Sexual differentiation, 186, 212 , $220,227,228$.

Sexual fusion, 184, I 85 (fig.), 2 I 3 (fig.), 214,219 (fig.), 237 (fig.). $\mathrm{See}$ also Fertilisation.

Sexual organs and reproduction, of Algæ, I84-6. (fig.), I88, 2 r3, 214 (fig.), 220-28 (figs.), 269; Angiosperms, $365,366,368$; Chlamydomonas, I 84-6 (fig.); Conifers, $34^{8}-5 \mathrm{I}$ (figs.) ; Cycads, 326, 327 (fig.) ; Cystopus, 233 (fig.), 234; Equisetum, 313; Ferns, 30I-3 (figs.) ; Fucus, 22 r (fig.), 224-6 (fig.) ; Fungi, 233 (fig.), 234, 236, 237 (fig.), 238, 242, 244 (fig.), 245; Liverworts, 277-9 (fig.); Mucor, 237 (fig.), 238; Mosses, 279 (fig.): Edogonium, 22I - 3 (fig.); Selaginella, 319, 320 (fig.); Ulothrix, $2 \mathrm{I} 3$ (fig.), $2 \mathrm{I}_{4}$; Vaucheria, 221 (fig.), 223, 224. Sexual reproduction, $288,289,306$, $307,389,390$.

Shade-leaves, I I2 ; structure of, 9I, 95, 96, 100, 168-70 (fig.), 295 , 379.

She-Oak (Casuarina), stem-structure of; $x 62$ (fig.).

Sheath, bundle-, of Conifers, 343 , 344 (fig.) ; of leaves, 107, 108 (fig.), II2, II3; of stems, 85 (fig.), 86.

Sheath of Blue-green Algæ, 206 (fig.).

Shepherd's. Purse (Capsella bursapastoris), embryology of, 36870 (fig.); hairs of, 102 (fig.); White Rust of, 23I, 233 (fig.). Shingle beach, Lichens of, 258 (fig.). Sieve plates, compound, I30; of Angiosperms, 28, 29 (fig.), 78 
(fig.), 79, 82; of Brown Algæ, 202; of Conifers, 34I (fig.).

Sieve tubes, function of, 28,88 ; of Angiosperms, 28, 29 (fig.), 80 (fig.), 82,85 (fig.); of aquatics, I7I, I74 (fig.); of Conifers, 339, 340, 34I (fig.) ; of Ferns, 292 (fig.), 293 (fig.) ; of secondary phloem of Dicotyledons, I 2 I (fig.), I30.

Sigillaria, $3^{\text {I }} 4$ (fig.).

Siliceous deposits due to Diatoms, 207.

Silicification of cell-wall, 39, 206.

Silicified hairs, I04.

Silk-Cotton Tree (Eriodendron anfractuosum), 103.

Silkworm disease, 230.

Silver Goosefoot (Obione portulacoides), 94 (fig.), I33; - Fir (Abies), 335, 336 (fig.), 344, 347.

Silver Leaf Disease of Plum (Stereum purpureum), 250, 252.

Silverweed (Potentilla anserina), I02. Simple pits, 30 (fig.), 33, I2I, I 22, 276.

Sinigrin, 48 .

Skeletal tissues, 3I-6 (figs.), 72, 87 , II 5 .

Skin-diseases due to Fungi, 253.

Sleep-movements, II 5 .

Slime-Fungi (Myxomycetes), 250, 25I, 406.

Smuts (Ustilagineæ), 230, 245, 248, 252, 407.

Soap, 50.

Soapwort (Saponaria), 48.

Soft rot of Carrots (Bacillus carotovorus), 265.

Soft rubber, I57; - wood, 127.

Soil Algæ, I98, 204, 208, 216 ; - Bacteria, 266, 267 (fig.).

Soil-differences and plant-distribution, 39I.

Soja-beans (Glycine), 49.

Solanaceæ, 58, 60, 6I, 8I.

Solitary crystals, 58,59 (fig.).

Soredia (of Lichens), 260 (fig.), $26 \mathrm{I}$.

Sorrel, Wood- (Oxalis acetosella), 95.

Sori (of Ferns), 29 I (fig.), 295, 296 (fig.), 297 (fig.).

" Souring" of milk, 265.

Space-parasites, 260.

Spartium junceum, proembryo of, 37 I (fig.).

Spawn (of Mushrooms), 248.

Spermatozoid mother-cells, 277, 278 (fig.), 302, 303 (fig.) 2319
Spermatozoids, 220, 236, 330, 333 ; of Algæ, 220; 221 (fig.), 222, 224, 225; of Cycads, 326,327 (fig.) ; of Ferns, 302, 303 (fig.); of Liverworts, 277, 278 (fig.) ; of Mosses, 280 ; of Selaginella, 319, 320 (fig.).

Spermogonia (of Rusts), 246 (fig.), 247.

Sphacelaria, growing point of, I6 (fig.), I 7.

Sphaerella (Hamatococcus), I86, I 87 (fig.).

Sphaerotheca castagnei, 244 (fig.) ; S. mors-uva, 243; S. pannosa, 243.

Sphagnales, 407.

Sphagnum, 274, 275 (fig.), 276.

Sphenophyllales, 407.

Sphenopteris honinghausii, 329.(fig.).

Sphere-crystals (of inulin), 44, 45 (fig.).

Spices, 62.

Spiderwort (Tradescantia), cellstructure of, 4, 5 (fig.).

Spinach (Spinacia oleracea), stem of, 86.

Spinach-family (Chenopodiaceæ), secondary thickening in, 133 .

Spindle-fibres, 23 (fig.), 24, 25 (fig.)

Spindle-tree (Euonymus europaus), I39, 359.

Spirca ulmaria, r69.

Spiral flowers, $354 ;$ - tracheids, 37 (fig.), II3, 293, 340; - vessels, 35 (fig.), 36, 68, 80.(fig.), 83 (fig.), 89, I72.

Spireme, 2 I (fig.), 22.

Spirillum, 262, 263 (fig.) ; $S$. rubrum, 263 (fig.).

Spirogyra, I6, I95, 307; cellstructure of, 7 (fig.), 208; filaments of, 195, 197, 210; fragmentation of, $2 \times 6$; sexual reproduction (conjugation) of, 226-8 (fig.).

Spirogyra bellis, 227 (fig.) ; S. weberi 227 (fig.).

Sponges, "green cells" of, 260.

Spongy parenchyma, 38 (fig.), 92 (fig.), 107, 108 (fig.), 109 (fig.), 110; function of, IO7, IIO ; of shade-leaves, I 70 :

Sporangia of, Algæ, 218, 228 ; Conifers, $345-8$ (figs.); Cycads, 323-6 (figs.), Equisetum, 3I2, 313 (fig.); Ferns, 295-300 (figs.), 303, 308, 309 
(fig.) ; Fungi, 235 (fig.), 236, $237^{\circ}$ (fig.), 238, 240 (fig.); Lycopodium, 315, 316 (fig.); Lyginopteris, $328, \quad 330-33$ (figs.) ; Selaginella, 317 (fig.), 318 (fig.), 319; Slime-Fungi, $25 \mathrm{I}$.

Spore-mother cells, 282, 390.

Spore-mother cells of, Angiosperms, 361,365 ; Conifers, 345,348 ; Ferns, 297, 305, 307, 308; Liverworts, 282 ; Mosses, 284 , 285 (fig.) ; Selaginella, 319.

Spores of; Algæ, 206 (fig.), 213 , 215-20 (figs.), 228; Bacteria, 262, 263, 267 (fig.) ; Conifers, 345,348 ; Cycads, 326, 327 ; Equisetum, 313 (fig.); Ferns, 299 (fig.), 300, 305, 306, 307 (fig.), 308; Fungi, 237 (fig.), 238, 240 (fig.), 245, 246 (fig.), 248, 249 (fig.), 253; Lycopodium, 3 I 6 (fig.) ; Mosses, 286, 287 (fig.); Selaginella, 318 (fig.), 3 I9.

Sporogonium, 28I ; of Liverworts, 270 (fig.), 27 I (fig.), 282-4 (fig.); of Mosses, 274 (fig.), 275 (fig.), 284-7 (fig.).

Sporophylls of, Angiosperms, 354, 356,359 ; Conifers, 344-7 (figs.); Cycads, 323-5 (figs.); Equisetum, 312, 313 (fig.); Ferns, 308 (fig.); Lycopodium, 315, 316 (fig.); Selaginella, 317 (fig.), 318 (fig.).

Sporophyte, 288, 289, 303, 321 .

Sports, 382.

Spraying, 254.

Spread of species, 392 (fig.).

Spring-wood, of Conifers, 340, $34 \mathrm{I}$ (fig.) ; of Dicotyledons, I I9 (fig.), I 23 (fig.), 125.

Spruce Fir (Picea excelsa), 63, 129, 350,391 ; habit of, 334,335 (fig.) ; leaf of, 343 .

Spurge (Euphorbia), laticiferous cells of, I53, I54 (fig.), I 55 ; leaf of, rio.

Spurge-family (Euphorbiaceæ), r 53 , I57.

Stability of herbaceous plants, 9, 87 .

'Stag's Horn Fungus (Xylaria), 239 (fig.), 242.

Staining of sections, 404 .

Stains, 405; for cellulose, 32 ; for lignified walls, 32 ; for sieve plates, 82 ; for starch 43 .
Stalk-cell (of Conifers), 35 I (fig.).

Stamen, of Angiosperms, 354, 355. 359-63 (figs.); of Conifers, 345, 347 (fig.).

Staphylococcus, $262 ;$ S. aureus, 263 (fig.).

Starch, I3, 40-44; economic importance of, 43, 53; hydrolysis of, 46,53 ; reactions of, 8 , 41 .

Starch-grains, 40-43 (figs.), 66 (fig.), 67 (fig.), 96 ; and pyrenoids, 7 (fig.), 8, 181, 207; centric, 42 (fig.); compound, 42 (fig.), 43; development of, 4I, 42 (fig.), 43; excentric, 4I, 42 (fig.); in secondary wood, r24; stains for, 43; transitory, 6 (fig.), 40, 4I, 42 (fig.); under polarised light, 43 .

Starch-grains of, Cereals, 43; Leguminosæ, 42 (fig.) ; Oat (Avena), 43; Pellionia, 4I, 42 (fig.); Potato (Solanum), 4I, 42 (fig.), 43; Rice (Oryza), 42 (fig.), 43 ; root-cap, 74 ; Spurge (Euphorbia), I 54, I55; starch-sheath 77,80 (fig.).

Starch-sheath, 78, 79, 80 (fig.), I I 8 (fig.).

Starch-solution, 43, 46.

Starwort, Water (Callitriche), anatomy of, 175-7 (figs.).

Staurastrum, 209 (fig.).

Stele of, Angiosperms, 78, 84; Ferns, 290 (fig.), 292-5 (figs.) ; Lycopodiales, 3I5, 317.

Stellaria, 363.

Stellate hairs, I02 (fig.), I03.

Stem, anatomy of unthickened, $76-88$ (figs.) ; anomalous secondary thickening of, 132, I 33 (fig.) ; assimilatory -, I 62 (fig.), I67, 178, 310; connection with leaf, II 4 (fig.), 294 (fig.), 295 ; cork-formation in, I3542 (figs.) ; epidermis of, 76 , 93 ; growing point of, 16, I8 (fig.), I9 (fig.); lateral branching of, 72 ; relation of structure and function, 87,88 ; secondary thickening of, 1 I 7-27 (figs.) ; succulent, 167 ; transition from root to, 89 (fig.).

Stem of, Calamites, 310, 3II ; Conifers, 334, 335; Cycads, 322, 323; Equisetum, 310, 3 II (fig.); Ferns, 289, 290 (fig.) : 
Lycopodium, 3I4, 3 I6 (fig.) ; Lyginopteris, 328 ; Mosses, 274 (fig.), 275; Selaginella, 3 I 7 (fig.).

Stem-structure of, aquatics, 88 , I70-74 (figs.); Aristolochia, I20; Ash (Fraxinus), I22; Bamboo (Bambusa), 88; Barberry (Berberis), I37, I39 (fig.) ; Beech (Fagus), I2I, I22, I40; Birch (Betula), I 22, I37, I39, I4I; Black Bryony (Tamus), 86, 87 (fig.) ; Box (Buxus), 86, I27; Bracken (Pteris), 292-5(figs.) ; Broom (Cytisus), 88, I67; Butcher's Broom (Ruscus), I59 (fig.) ; Buttercup (Ranunculus), 86, 87; Cactus, I67; Campion (Lychnis), 88; Castor Oil Plant (Ricinus), I I 8 (fig.) ; Chenopodiaceæ, I33 ; Cherry (Prunus), I4I ; Conifers, 338-4I (figs.) ; Cucurbitaceæ, 8I ; Currant (Ribes), 137, 138 (fig.) ; Cycads, 323; Dicotyledons, 76-84 (figs.), 86, II7 et seq. (figs.); Dodder (Cuscuta), I78 (fig.), I79 ; Dracana, I33 (fig.), I34 ; Elder (Sambucus), I36 (fig.), r40 (fig.), I5I; Elm (Ulmus), I20, I26, I39, I4I; Equisetum, 3II (fig.) ; Ferns, 292-5 (figs.) ; Gleichenia, 294 ; Gorse (Ulex), I67 ; Grasses (Gramineæ), 80, 88; Grasswrack (Zostera), I72; Holly (Ilex), r27 ; Hornwort (Ceratophyllum), I70, I7I (fig.); Horse Chestnut (Esculus), I20, I2I (fig.), I23 (fig.), I27; Labiatæ, 80; Laburnum (Cytisus), I 37 ; lianes, I34; Lime (Tilia), I20, I27, I30; Lycopodium, 3I5; Lyginopteris, 328-30 (fig.); Maize $(Z e a), \quad 84-6$ (figs.); Male Fern (Nephrodium), 294 (fig.), 295; Mallow (Malva), I30; Maple (Acer), I35, I4I ; Mare's Tail (Hippuris), I72, I73 (fig.) ; Marrow (Cucurbita), 80-83 (figs.), 87, I26, I27 (fig.), I 30 ; Meadow Rue (Thalictrum), 86; Monocotyledons, 84-6 (figs.), 87; Oak (Quercus), I22, I28 (fig.), I40, I4I ; Plane (Platanus), I4I ; Pondweed (Potamogeton), I72, I74 (fig.) ; Pop- lar (Populus), I4I ; Rosaceæ, I37; Scotch Fir (Pinus), I4I, 338-4I (figs.) : Sedge (Carex), 88; Selaginella, 3I7, 318; She-Oak (Casuarina) I62 (fig.) ; Silver Goosefoot (Obione), I33; Spinach (Spinacia), 86 ; Spindle Tree (Euonymus), I39; Spurge (Euphorbia), I53, I54 (fig.) ; Solanaceæ, 8I ; succulents, I67; Sunflower (Helianthus), 76-8o (figs.), 87; Sweet Flag (Acorus), 85 (fig.) ; Sycamore (Acer), , I2I, I 28 (fig.) ; Tree Ferns, 294; Umbelliferæ, 80,88 ; Water Dropwort (Oenanthe), 86; Water Milfoil (Myriophyllum), I7I ; Water Starwort (Callitriche), I75, I76 (fig.) ; Whortleberry (Vaccinium), I67, I68 (fig.) ; Willow (Salix), I21, I24, I26, I37, I39.

Stentor, 3.

Stereum purpureum, 250, 252.

Sterilisation, 264

Stigma (eye-spot), I8I, r93. See also Eye-spot.

Stigma (of Angiosperms), 64, 356, $364,365,373$.

Stimulants, $48,6 \mathrm{I}$.

Stimulus of environment, 380 ; of sexual fusion, 222, 223, 370, $389,390$.

Stinging Nettle (Urtica), hairs of, I04, I05 (fig.) ; leaf-structure of, 38 (fig.), 39 ; root of, 132.

Stinkhorn (Phallus), 407.

Stipules, nectaries on, 148 ; on stamens, 359 (fig.); vascular supply of, 90 , I I 4 :

Stock (Matthiola), 104

Stomata, 94 (fig.), 96-101 (figs.), I07, I ro, 298 (fig.) ; artificial, 99; development of, 98 ; influence of external conditions on, 99, Ioo; mechanism of, 98-roo (fig.); plugging of, I6I ; raised, 96, I60 (fig.), I6I, I62, I70 ; sunken, 96, I $_{5} 8$, I6o (fig.), I65, I70.

Stomata of, Brooklime (Veronica), I6o (fig.) ; Butcher's Broom (Ruscus), I59 (fig.) ; Conifers, 342 (fig.), 343, 344 (fig.) ; floating leaves, Ior, I74 ; Gorse (Ulex), I60 (fig.); Graminea and Cyperaceæ, 97 (fig.), Ioo, 
Ior ; Holly (Ilex), I6o (fig.) : Iris, 96, 97 (fig.) ; land-forms of aquatics, 177 ; Madder (Rubia), 97 (fig.), 98 ; Mosses, Ioo, 284, 285 (fig.); Stonecrop (Sedum), 97 (fig.), 98; Yellow Pimpernel (Lysimachia), I60 (fig.).

Stomatal chambers, $\mathrm{I} 6 \mathrm{I}-3$ (figs.) ; - grooves, I62 (fig.).

Stomium (of Fern-sporangium), 297, 299 (fig.), 300.

Stone-cells, 30 (fig.), 32, 33.

Stone-crop (Sedum), leaf of, II6, I66 ; stoma of, 97 (fig.), 98.

Stonecrop - family (Crassulaceæ), 178.

Stone Pine (Pinus pinea), seed of, 352 (fig.).

Stonewort (Chara), 39, I98, 406.

Storage of food-reserves, 13, 40, $44,48,50,64,88,132,155,231$, $273,276,343$; of water, 38,94 (fig.), 95, I 58, 166, 167, $272,343$.

Storage-organs and tissues, 88, 132, I33 ; in Algæ, 202, 225 (fig.) : in Fungi, 242 ; in Mosses; 276.

Stratification, of cell-wall, 29, 33, 208 ; of starch-grains, 4I, 42 (fig.).

Strawberry (Fragaria), 370; hydathodes of, 146 .

Streaming of cytoplasm, 4, 6, ro4.

Streptococcus, $262 ; S$. pyogenes, 263 (fig.).

Striation of cuticle, 92.

Strobilus, 3I2, 334. See Cones.

Structure. See Anatomy, Leaf, Root, Stem.

Struggle for existence, 378, 394.

Strychnine, 6r.

Strychnos nux-vomica, 6r.

Style, 356,364 .

Suceda, 166.

Suberisation, I36, 137 .

Suberised walls of, cork, 136,137 , I38, I42 ; endodermis, 68, 69; I 7 I ; exodermis, 67 .

Sub-hymenium, 240 (fig.), 249 (fig.). Submerged aquatics, structure of, 91, 92, IOI, 170-77 (figs.).

Subsidiary cells, 97 (fig.), 98, rог.

Substantive variations, 374,375 (fig.), 376 (fig.).

Subterranean parasites, 254.

Succulent fruits, 3 I, 46 ; - plants, I $6,166,167$, I 78 ; - storageorgans, 44,50 .
Sucrose, 45, 46; hydrolysis of, 46 ; reactions of, 47 .

Sugar Beet (Beta), 45.

Sugar Cane (Saccharum officinarum), 45, 46; - Maple (Acer saccharinum), 45.

Sugars, I3, 40, 4I, 44-7, I 55, 364 ; fermentation of, 256, 257; hydrolysis of, 46 ; in foliageleaves, 46; in wood, I 24 ; microchemical tests for, 47 ; non-reducing, 47 ; of nectaries, 46, I 49; reactions of, 47; reducing, 47.

Sulphur-Bacteria, 268.

Sulphuretted hydrogen, 268 ; influence on enzyme-action, 56 .

Sulphuric acid and cell-walls, 3, 4, $32,68,92,137,402$.

Sundew (Drosera), tentacles of, I.50.

Sunflower (Helianthus), 49, II3 ; stem-structure of, 76-80 (figs.), 87.

Sun-leaves, II I, 169 (fig.), 170.

Support of plant, 73, I 70.

Susceptibility to disease, 253, 384 . Suspensor of, Angiosperms, 368, 369 (fig.), 370, 37 I (fig.) ; Conifers, 350, 35I (fig.) ; Cycads, 326, 327. (fig.) ; Selaginella, 319, 320 (fig.).

Sweet Corn (Zea), 45 .

Sweet Flag (Acorus calamus), stemstructure of, 85 (fig.), 86.

Sweet Pea (Lathyrus), hybrids of, 388 .

Swietenia mahogoni, 130.

Sycamore (Acer pseudoplatanus), epidermis of, 95 ; leaf-fall in, I 42 (fig.) ; secondary wood of, I 2 I, 128 (fig.).

Symbiosis, 257-6o.

Sympetalæ, 357, 367, 408.

Synapsis, 306, 307 (fig.).

Syncarpous ovary, $355,356$.

Synergidæe, 357 (fig.), 366, 367 (fig.).

Taniophyllum, 74.

Tamus communis, II2; stemstructure of, 86,87 (fig.).

Tannic acid and alkaloids, 61 .

Tannin, 60, 64, I 25, I 37, I 55 ; reactions of, $12,13,49,60$.

Tannin-sacs, 130, r51, 166.

Tanning, 39, 60 .

Tap-root, $7 \mathbf{I}$. 
Tapetum, of Angiosperms, 360 (fig.), 36I; 363 (fig.) ; of Ferns, 297, 299 (fig.).

Tapioca, 43.

Taraxacum, 154, I70, 372; flowers of, Plate I (Frontispiece).

Tartaric acid, 60.

Taxus baccata, 128, 334; female cones of, 346. (fig.); habit of, 335, 346 (fig.) ; leaf-structure of, $34 \mathrm{I}, 342$ (fig.) ; male cones of, 344, 345, 346 (fig.) ; ovules of, 346 ; seeds of, 346 (fig.), $35 \mathrm{I}$; stem of, 339.

Taxus baccata, var. fastigiata, 380 . Tea (Thea sinensis), 6I, 63.

Teak (Tectona grandis), I 26, I30.

Tearing, protection against, 93, II 5 .

Tectona grandis, I 26, I 30.

Teleutospores, 246 (fig.), 248.

Tension, resistance to, $72,88,170$.

Terpenes, 62, 63.

Terrestrial Algæ, I90, I98, 204, 205, 208.

Tertiary fossil-plants, 356 .

Testa, 352 (fig.), 370, 371 (fig.).

Tetrads, of pollen grains, $36 \mathrm{I}, 362$ (fig.) ; of spores, 307 (fig.), 318 (fig.), 319, 348, 365, 372 .

Tetrahedral apical cells, 18 (fig.), 288, 297.

Tetrarch roots, 66 (fig.), 69, I3I (fig.).

Tetraspora, I90, I9I (fig.).

Tetraspores of Red Algæ, 205 (fig.), 228.

Textiles, 34, ro3.

Thalictrum, stem-structure of, 86 . Thallophyta, 180-268 (figs.), 307, 406. See Algæ and Fungi.

Thallus of, Algæ, I80, I95-207 (figs.), 225 (fig.) ; Fungi, 230, 23I ; Lichens, 258-6I (figs.); Liverworts, 269-73 (figs.).

Theobroma cacao, 49.

Theobromine, 6I.

Thermophilic Bacteria, 26r.

Thickening of cell-walls, 29-37 (figs.); annular, 35 (fig.), 36 ; reticulate, 35 (fig.), $36,74,83$ (fig.), I20, 273; spiral, 35 (fig.), 36, 37 (fig.), 74, 80 (fig.), 83 (fig.), 120.

Thistle, I02.

Thornapple (Datura stramonium), $6 \mathrm{r}, 62$ (fig.), 384.

Thread-Bacteria, 268.

Thuidium, 276.
Thuja, 347, 350 ; habit of, 336,337 (fig.).

Tilia, secondary phloem of, 130 ; secondary wood of, 28, I20, I 27.

Tiliaceæ, 103.

Timber, I27-30; commercial sources of, I29, I $30,162,334$, 337 ; " Dry Rot " of (Merulius lacrymans), 252.

Tissues, I, 27, 65 ; epidermal, 91Io6 (figs.) ; false, 239 ; glandular, .148, I49; ground, 65 . 88, I07-I I ; mechanical, 3I-6 (figs.) ; meristematic, I8, II 7 , I 35 ; secondary, 120-42 (figs.) ; secretory, I 5 I-5 (figs.); storage, 88 , 132; vascular, $67-70$ (figs.), 78-86 (figs.), I โ2, I 13.

Tissue-tensions, I 2, 87 .

Tmesipteris, 407.

Toadstools, 230, 245, 255, 407 .

Tobacco (Nicotiana tabacum), 6I, 265.

Tolypothrix, 205, 206 (fig.).

Tomato (Solanum lycopersicum), 63.

Toothwort (Lathraa); hydathodes of, 145 (fig.).

Tortula, 274; T. muralis, 269,274 (fig.).

Torus (of bordered pits), 36, 37, 34I (fig.).

Toxic effect, of Bacteria, 265; of mineral salts, I4, I5.

Tracheidal cells (of Conifers), 34I (fig.).

Tracheids, 37 (fig.), I 79 ; of Conifers, 37 (fig.), I27, 340, 34I (fig.), 344 ; of Ferns, 293 (fig.), 296, 298 (fig.) ; of foliage-leaves, 37 (fig.), I I 3, I46 ; of secondary wood of Dicotyledons, I2O, I2I, I34.

Tradescantia, cell-structure of, 4, 5 (fig.).

Tragopogon, root-structure of, 44, I 32, I 54, I 55 (fig.).

Transfusion tissue, 342 (fig.), 343 , 344 (fig.).

Transition from stem to root, 89 (fig.).

Transitory starch, 6 (fig.), 40, 4I, 42 (fig.), 46.

Transmission of acquired characters, 380 .

Transpiration, $35,158,203$; and anthocyanins, 63; and hyda- 
thodes, I47; control of, 92, 95, I00, I37; cuticular - 93, I6r; of Alpines, I70 ; of bogplants, 177 ; of rolled leaves, I63; of shade-leaves, I7o; of succulents, I67; of woodland plants, I78; reduction of, $58,64,93,95$, ror, 106, 15865 (figs.), 342, 343, 344 .

Tree Ferns, 289 , 294.

Triarch roots, 69.

Tropaolum, 63, I I 5 .

Tropical plants, $73,75,103,125$, I 53, I 57, 204, 289, 294, 296, $309,372$.

Truffle (Tuber), 240, 241, 255.

Tube-cell of, Angiosperms, 363, 367 (fig.) ; Conifers, 349, 35 I (fig.) ; Cycads, 327 (fig.).

Tubers, $40,43,44,50,88$; of Equisetum, 3 II.

Tulip (Tulipa), pollen and stamen of, 359 (fig.), 364, 367 (fig.).

Turgescence, $\mathbf{9}, \mathbf{1 0}, 87,98$, 1 00, 149, I52, I98; and sleep - movements, I I 5 , II 6 .

Turmeric, 64 .

Turnip, "Finger and Toe" disease of (Plasmodiophora), 254.

Turpentine, 63, 334 .

Twayblade (Listera ovata), embryo of, 37 I (fig.).

Tyloses, 126, I27 (fig.), I42.

Tyndall, 64 .

Typha, 36r.

Tyrosin, 52 .

Ulex europaus, I67; epidermis of, 92, 160 (fig.).

Ulmus, I30, I39, I4I, 365; secondary wood of, 120, 126.

Ulothrix, 212-15 (figs.) ; filaments of, 197, 207, 208, 210, 213 (fig.); habitat of, I95; $\mathrm{Pal}$ mella-stages of, 214, 215 (fig.) ; sexual reproduction of, $2 \mathrm{I} 3$ (fig.), 214, 220; vegetative reproduction of, 212, 215 (fig.) ; zoospores of, 2I2-I4 (fig.).

Ulothrix zonata, 213 (fig.).

Ulva, 198, 215 (fig.).

Umbelliferæ, 60, 61, 80, 88, I48, I 53, 355, 356, 357 .

Underground parasites, 254.

Undulation of epidermal walls, 93.

Unicellular Algæ, I, I81-92 (figs.), I 95, 205, 206 (fig.), 208-10 (figs.) ; - Fungi, 255, 256

(fig.) ; - growing points, 16-18

(figs.) ; - hairs, IOI, IO3, 104.

Unisexual flowers, 354, 373; prothalli, 313, 319, 321 .

Uredineæ, I 43, 245-8 (fig.), 252, 253 , 407.

Uredospores, 245, 246 (fig.), 247.

Urtica, hairs of, I04, IO5 (fig.) : leaf-structure of, 38 (fig.), 39 ; root of, 132 .

Usnea barbata, $25^{8}$ (fig.).

Ustilagineæ, 230, 245, 248, 252.

Ustilago longissima, 25 I (fig.).

Utricularia, I 50 .

Vaccinium myrtillus, pollen of, 362 (fig.); stem of, I67, I68 (fig.).

Vacuoles, 2 (fig.), 3, 5 (fig.), 6 (fig.), $26,5 \mathrm{I}$; contractile - , 181, I82 (fig.), 187 (fig.), 193, 213; of Desmids, 209; of Fungi, 231 ; of Yeast, 255.

Vanilla planifolia, 48 .

Vanillin, 48.

Variation, 374-80 (figs.) ; measurement of, 375-7 (fig.).

Variation-curves, 376,377 (fig.).

Variegated leaves, I I I.

Varnish, I 55 .

Vascular bundles, 76 ; bicollateral, 8 I (fig.), 82 ; collateral, 76,78 (fig.), $79,84,85$ (fig.), II 2 ; concentric, 85 (fig.), 86, I33 (fig.), I 34, 292 (fig.); cortical, 86 ; medullary, 86 ; secondary, I 33 (fig.), I 34 .

Vascular cylinder, of aquatics, I 70-74(figs.) ; of root, 66 (fig.), 68 ; of stem, 78 .

Vascular system, 65; differentia. tion of, 89 .

Vascular system of, aquatics, I7074 (figs.), 176 (fig.) ; cladodes, I60 ; Conifers, 339-4I (figs.), 343 ; Cycads, 322 ; Equisetum, 3 II (fig.) ; Ferns, 292-5 (figs.) ; leaves, 90, 107, II $2-$ I4 (fig.) ; Lycopodium, 3I5 ; Lyginopteris, 328-30 (fig.); Mosses, 276 ; ovules, 325 (fig.), $326,331,332,333$ (fig.), 357, $358, \quad 359$; parasites, I79; petioles, I13 (fig.) ; pulvini, I 1 5, I I6 (fig.) ; roots, 66 (fig.), 68, 70 (fig.); Selaginella, 3 I 7 ; 
stamens, $360 \cdot($ fig.) ; stems, $77-86$ (figs.), II 7 et seq. stipules, 90, II 4 .

Vaucheria, 49; habitat of, I98; sexual : reproduction of, 220 , 221 (fig.) $223,224,226$; thallus of, I98, 208, 2 I 8 (fig.) ; zoospores of, 217,218 (fig.).

Vaucheria sessilis, 22 I (fig.).

Vegetable Caterpillar (Cordyceps), $239,255$.

Vegetable Kingdom, classification of, I8o, I8I, 230, 23I, 238, 269, $289,334,355,406-8$; compared with Animal Kingdom, I 4, 25, I8I, I 88,193, I94, 247, 307.

Vegetable Marrow (Cucurbita); hairs of, IO2 (fig.) ; phloem of, 29 (fig.), 82, I30 ; stem-structure of, $80-83$ (figs.), 87 ; tyloses of; 127 (fig.) ; vessels of, 35 (fig.), 83 .

Vegetative cell of pollen of Conifers, 349, 35 I (fig.); of Cycads, 327 (fig.).

Vegetative mutations, 382,383 (fig.), 390.

Vegetative reproduction, I83, 390.

Vegetative reproduction of, Algæ, I 82 (fig.), I83, I 84, I 88 (fig.), I9I, I92 (fig.), 212, 216 ; Ferns, 305 (fig) $;:$ Hormidium, 215 (fig.), 216; Liverworts, 277; Lycopodium selago, 3I5; Marchantia, 270 (fig.), 277 ; Mosses, 277, 287 (fig.), 288; Yeast, 256 (fig.).

Veins, I07, IIO, III, 112, II5.

Velamen, 74, 75 (fig.).

Venation of, Angiosperms, I I2; Conifers, 34I ; Cycads, 322, 324 (fig.) ; Ferns, 29I, 292.

Venter (of archegonium), 280, 282, 302, 319.

Ventral canal cell of Bryophyta, 280, 28I (fig.) ; of Conifers, 348 , 349 (fig.); of Ferns, 302, 303 (fig.).

Veronica beccabunga, stoma of, 160 (fig.).

Vessels, 27, 28, 35-7 (fig.), 354; of metaxylem, 36,66 (fig.), 68, 78 (fig.), 79, 80 (fig.); of protoxylem, 36, 66 (fig.), 68, 79,80 (fig.); of secondary wood, 120, 121, I23 (fig.), 125, I26, I27, I28 (fig.), I32.
Vesséls, laticiferous, I 54, I55 (fig.), I 57.

Vestibule, 96, 159, I60 (fig.).

Viburnum opulus, extrafloral nectaries of, 148 .

Vicia faba, 53 ; extrafloral nectaries of, 148.

Vine (Vitis vinifera), I2I.

Vinegar, 265.

Violet (Viola), style of, 364 .

Volatile oils, 62, 63, 64, I05, I06; I26, 15 I.

Volutin, 255, 256 (fig.), 262.

Volvox, I89 (fig.), I90; V. aureus, I 89 (fig.) ; V.globator, I 89 (fig.).

Vulcanisation of rubber, I 57.

Vulcanite, 157.

Wall Moss (Tortula muralis), 269, 274 (fig.) ; - Rue (Asplenium ruta-muraria), 296.

Wall of cell, see Cell-wall ; of hairs; I03, 104.

Wallflower (Cheiranthus cheiri), teaf - of, III ; root of; 70 .

Walnut (Juglans), I26, 129.

Warmth, and enzyme-action. 56 ; and protoplasmic movement 4 :

Wart-disease of Potato, 254.

Waste-products of plants, 3,40 , 58-64, I 38, I 44, I 56 .

Water, absorption of, ro, 26, 38, 67, 72, 73, 74, 93, I 47, I 58, I 7 I; 259, 270, 276, 301 ; conduction of, $27,35,37,65,88,112,121$, I25, 276, 343, 344; exudation of, I $44-8$, I 49 ; storage of, 38,94 (fig.), 95, I 58, 166, 167, $272,343$.

Water-bloom, cause of, 192 .

Water Buttercup (Ranunculus aquatilis), I 77 (fig.) ; - Dropwort (Oenanthe crocata), 86; - (Lily (Nymphaa), IoI, 356; - Milfoil (Myriophyllum), I 7I ;

- Plantain (Alisma plantago), Ioo, I 46, 37 I (fig.) ; - Starwort (Callitriche), I75-7 (fig.).

Water-containing hairs, 94 (fig.).

Water-cultures, absorption from, Io.

Water-forms (of aquatics), 93, I 75-7 (figs.).

Water-plants. See Aquatics.

Water-pollinated plants, 36I, 373.

Water-pores, I45-8 (figs.).

Water-secreting hairs (hydathodes), I44, I 45 (fig.). 
Wax, 93, IOI, I6I, I74.

Wellingtonia (Sequoia), cone of, 347,352 (fig.).

Wetting of leaf-surface, prevention of, 93 , IOI, IO3.

Wheat (Triticum), grains of, 52 (fig.), 53, 384; - mildew (Ery siphe graminis), 243; -- rust (Puccinia graminis), 245-7 (fig.), 253, 384; - smut (Ustilago), 230. 248 .

Whisky, 257.

White Convolvulus (C. sepium), I53; - Dead Nettle (Lamiam album), 4, 105: - Deal (Picea excelsa), $129 ;-$ Lily (Lilium), I 10; - Mustard (Sinapis alba), $69 ;$ - Pine (Abies pectinatá), I29; - Rust of Cruciferæ (Cystopus candidus), $23 \mathrm{I}-4$ (fig.); - Stonecrop (Sedum album), II6.

Wholemeal bread, 52.

Whorled branching, 3 Io, 3 I I (fig.) ; - flowers, 355.

Whortleberry (Vaccinium myrtillus), pollen of, 362 (fig.) ; stem of, I67, I 68 (fig.).

Wild Hyacinth (Scilla nutans), 44, 365 ; - Strawberry (Fragaria vesca), 146 .

Willow (Salix), 48, I03, I37, I39, 354,389 ; wood of, I 21 , I24, I26, 128.

Willow-herb (Epilobium), I03, I74 ; pollen of, 362 (fig.).

Wilting and stomata, Ioo.

Wind-pollinated plants, 326, 330, $345,348,354,36 \mathrm{I}, 373$.

Wine, 256.

Winged stems, 167.

Woad (Isatis tinctoria), 48.

Wood. See Xylem.

Wood Anemone ( $A$. nemorosa), I48, 248; - Sorrel (Oxalis acetosella), 95; - Spurge (Euphorbia amygdaloides), I 53.

Wood-fibres, 33, 121, I23 (fig.), I25, 127, 128 (fig.).

Wood-parenchyma, $79,83,86$; of Ferns, 292 (fig.), 293 (fig.); of leaf, II2, II 3 ; of secondary wood, I2I, 122, I 23 (fig.), I 24 , I26, 132.

Wood-pulp, 35.

Wood-vessels. See Vessels.

Woodland plants, structure of, I68, I69, I78, 379 .
Woody perennials, $40,-364$; phloem of, 82 ; root of, 70 ; secondary growth of, I 7 et seq.

Wound parasites, $252 ; 253$

Wounds, effect of 26,252 ; healing of, $64,141,142,155$.

Xanthophyll, ro9.

Xanthoprotein reaction, 50 .

Xanithoria parietina, 258 (fig.), 260 (fig.).

Xerophytes, I58. See also Dry habitats, plants of.

Xylaria hypoxylon, 239 (fig.).

Xylem, 27, 32, 33, 36, 37, 65; function of, 65,88 , II 2 ; primary, 66 et seq., 78 et seq., II2 et seq.; secondary, 33 , I $8,119-26$ (figs.).

Xylem of, aquatics, 171 (fig.), 172, I 73 (fig.), I 74 (fig.), I 76 (fig.); Conifers, 37 (fig.), 127, 339 (fig.) ; Ferns, 292-4 (figs.); leaf of Angiosperms, 107, I I2, I 3 ; parasites and saprophytes, I78, I79; petiole, II3 (fig.); root of Angiosperms, 66 et seq. (figs.) ; stem of Angiosperms, 78 et seq. (figs.).

Xylem-canal of aquatics, 171 (fig.), 172, 174 (fig.); Monocotyledons, 36,85 (fig.), 86.

Yeast (Saccharomyces), physiology of, $53,54,56,57,256,257$, 265 ; structure and reproduction of, 255, 256 (fig.).

Yellow Dead-nettle (Lamium gale. obdolon), I68, 169 (fig.) ; - Pimpernel (Lysimachia nemorum), I6o (fig.) :- Pine (Pinus strobus), I29; - Rattle (Rhinanthus), I 44, I 45 (fig.).

Yew (Taxus baccata), I28, 334, 408 ; female cone of, 346 (fig.): habit of, 335,346 (fig.) ; leafstructure of, 34I, 342 (fig.) ; male cones of, $344,345,346$ (fig.) ; ovules of, 346 ; seeds of, 346 (fig.), 351; stem of, 339.

Yorkshire Fog (Holcus mollis), II 2.

Zea mais, disease of, 248; grains of, $45,52,53,384$; hybridendosperm of 386 ; leaf of, 
II 7; root-structure of, 73,74 (fig.) ; stem-structure of, $84^{-6}$ (figs.).

Zingiber officinale, 63 .

Zonation of Seaweeds, 199, 200, 202, 203.

Zone of elongation of root, 65 .

Zooglœa-stages (of Bacteria), 262.

Zoosporangia of Algæ; I96 (fig.) ; Fungi, 218 (fig.), 2 I9 (fig.).

Zoospores, 214, $219,238$.

Zoospores of, Cladophora, 215; Cystopus, 232, 233 (fig.); Ectocarpus, 218, 219 (fig.); $C$ dogonium, 216, 217 (fig.) ; Pleurocaccus, I9I; Saprolegnia, 235 (fig.), 236; Ulothrix, 213 (fig.), 214; Vaucheria, 2I7, 218 (fig.).
Zostera, pollen of, $36 \mathrm{I}$; stemstructure of, $\mathbf{r} 72$.

Zygnema, cell-structure of, 208 . sexual reproduction of, $226-8$ (fig.).

Zygnema pectinatum, 227 (fig.).

Zygomorphic flowers, $355,373$.

Zygomycetes, 236-8 (fig.), 406 .

Zygospores, I86, 307 ; dispersal of, I 86,2 I $4,228$.

Zygospores of, Chlamydomonas, I85 (fig.), I86; Conjugatæ, 227 (fig.), 228 ; Desmids, 209 (fig.), 228; Ectocarpus, 2 i9 (fig.), 220; Mucor, 237 (fig.), 238; Ulothrix, 213 (fig.), 214.

Zygote, 184, I85 (fig.), I86, 2 I3 (fig.), 214.

Zymase, 53, 57, 256. 
Demy 8vo. Fourth Edition. With over 250 Illustrations. 7 s. 6 . net.

\section{An Introduction to the Study of Plants}

This Volume completely covers the London Matriculation Syllabus.

Educational Times: "This book is one of a very small class, the kind that makes teachers wonder whatever their pupils did in the past without them."

Journal of Education: "Certainly one of the very best of the elementary textbooks of botany we have seen."

Crown 8vo. Fourth Edition. IVith over 150 Illustrations. 3s. 6d. net.

\section{Elementary Studies in Plant Life}

This short course in Plant Physiology has been prepared to meet the need, expressed by many teachers, for a more elementary textbook than the same authors' "Introduction to the Study of Plants." The present book meets the requirements of the Oxford and Cambridge Locals, the Northern Universities' Matriculation, and similar examinations. It is fully illustrated from drawings by the authors and specially prepared photographs.

Nature: "It would not be easy to find a book better suited to the needs of junior students."

School World: "There is plenty of practical work, the style is easy and natural, the illustrations are good and numerous. Altogether this is one of the soundest and most attractive books for beginners we have seen."

\section{Elementary Botany}

By Percy Groom, M.A. (Cantab. et Oxon), D.Sc. (Oxon), F.L.S., F.R.H.S., Professor of the Technology of Woods and Fibres at the Imperial College of Science and Technology. With 275 Illustrations. Sixteenth Edition, Revised and Enlarged. Crown 8vo. 5 s.

"To attempt the study of Botany without the practical examination of plants is futile. Students of plant-life must look at plants, and this book is specially designed for use during the process. ... The section on Physiology has been so written that no knowledge of the histology of plants is assumed - a feature which is, I believe, here introduced for the first time. Though by no means a ' crambook' for elementary examinations, a thorough knowledge of the contents of this book will enable a candidate to pass with distinction." - From the Preface.

\section{Botany for Schools and Colleges}

By George Francis Atrinson, Ph.B., Professor of Botany in Cornell University. With 4I 3 Illustrations. Crown 8vo. 5s. net.

This book is intended for the first or second year in the high school.

\section{The Botanist's Pocket-book}

By W. R. Hayward. Fourteenth Edition, Revised and Enlarged by G. Claridge DRuce, Fielding Curator in the University of Oxford, Hon. Sec. Bot. Exch. Club of British Isles. F'cap 8vo, cloth limp. 5s. net.

Containing, in a tabulated form, the Chief Characteristics of British Plants, with the botanical names, soil or situation, colour, growth, and time of flowering of every plant, arranged under its own order; with a copious index. 


\section{Practical Plant Physiology}

By Frederick Keeble, Sc.D., Sherardian Professor of Botany, Oxford University, assișted by. M. C. RAYNER, B.Sc., formerly Lecturer in Botany at University College, Reading. With numerous Illustrations. SECOND Edition. Crown 8vo." 4s. $6 d$.

The book covers the necessary ground for a biological course in nature study in the higher classes in schools, and for the first-year class in the University: The numerou's illustrations have in almost every case been made specially for this work.

\section{London Catalogue of British Plants}

Part I, containing the British Phænogamia, Filices, Equisetaceæ, Lyco: pođiaceæ, Selaginellaceæ, Marsileaceæ, and Characeæ.

Tenth Edition, revised. Demy 8vo, Is, net.

\section{An Introduction to the Study of the Com= parative Anatomy of Animals}

By Gilbert C. Bourne, M.A., D.Sc., Linacre Professor of Comparative Anatomy; Oxford. 2 vols., Crown 8 vo. Illustrated, 7s. 6d. each.

Vol. I. Animal Organisation. The Protozoa and Celenterata. SixTh EDition.

Vol. II. The Colomate Metazoa. Ninth Edition.

Nature: "This admirable little book is designed to meet the requirements of the elementary examinations of the leading Universities of Great Britain."

\section{Biology}

By G. N. Calkins, Ph. D., Professor of Protozoology in Columbia University.

Demy 8 vo. ros. $6 d$. net.

Nature: "This introduction to general biology has a pleasing freshness, a quality difficult to attain in these days of many books. It considers general biology as having to do with fundamental facts and principles. Prof. Calkins' treatment is beautifully clear throughout, and he drives his nails home. .... . A work of "great excellence, which is sure to be found very useful."

\section{A Manual of Zoology}

By Richard Hertwig, Professor of Zoology in the University at Munich. From the Fifth German Edition. Translated and Edited by J.S. KingsLey, Professor of Zoology in Tuft's College. 8vo. Profusely Illustrated. I 2s. $6 \mathrm{~d}$. net.

\section{A Textbook of Vertebrate Zoology}

By J. S. Kingsley, Professor of Zoology in Tuft's College. With 378 Diagrams. 8vo. 12s. net.

R. L. (in "Nature"): "The work is thoroughly comprehensive in its scope; and is, in fact, for the most part exactly what such a treatise should be. We have no hesitation in saying that Prof. Kingsley's little volume is worthy of a hearty welcome on this side of the Atlantic on the part of both teachers and pupils."

LONDON: G. BELL AND SONS LTD.

YORK HOUSE, PORTUGAL, STREET, W.C.2 







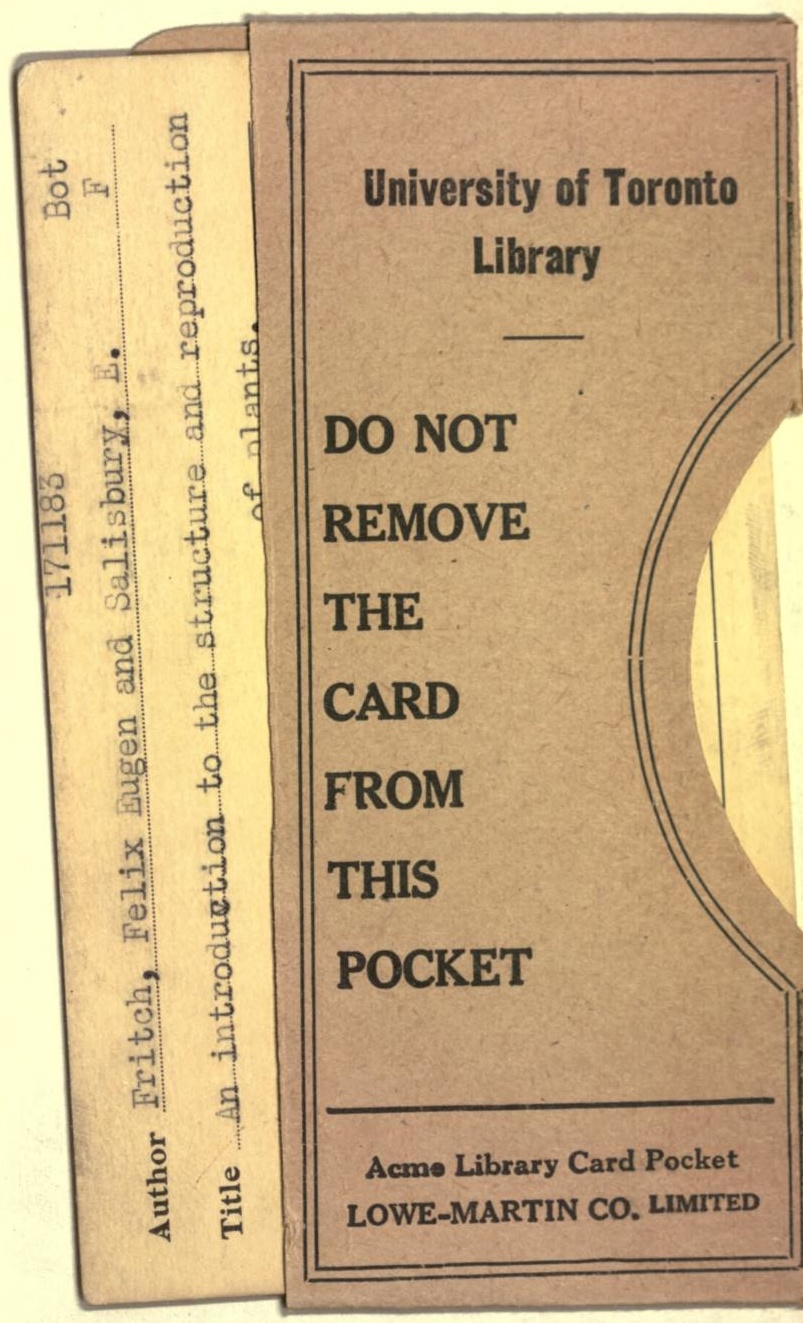


